\title{
GROUND-WATER DATA FOR GEORGIA, \\ 1987
}
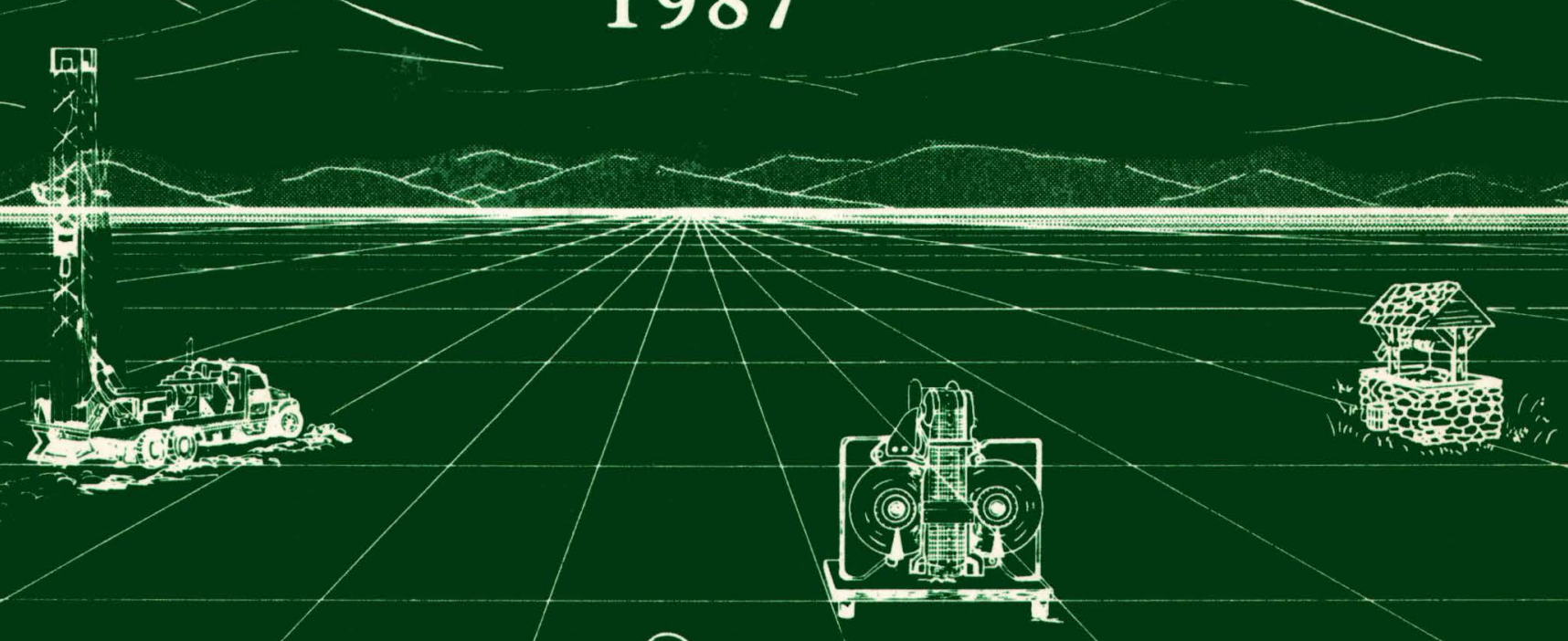
GROUND-WATER DATA FOR GEORGIA, 1987

By C.N. Joiner, M.S. Reynolds, W.L. Stayton, and F.G. Boucher

U.S. GEOLOGICAL SURVEY

Open-File Report 88-323

Prepared in cooperation with the

GEORGIA DEPARTMENT OF NATURAL RESOURCES

ENV IRONMENTAL PROTECTION DIVISION

GEORGIA GEOLOGIC SURVEY

Doraville, Georgia

1988 


\section{DEPARTMENT OF THE INTERIOR \\ DONALD PAUL HODEL, Secretary}

U.S. GEOLOGICAL SURVEY

Dallas L. Peck, Director

For additional information write to:

District Chief

U.S. Geological Survey

Suite B

6481 Peachtree Industrial Boulevard

Doraville, Georgia 30360
Copies of this report can be purchased from:

U.S. Geological Survey Books and Open-File Reports Federal Center, Building 41 Box 25425

Denver, Colorado 80225 


\section{PREFACE}

This report was prepared in cooperation with the State of Georgia; Chatham County; Glynn County; the cities of Brunswick and Valdosta; and the Albany Water, Gas, and Light Commission.

This report is the culmination of a concerted effort by dedicated personnel of the U.S. Geological Survey who collected, compiled, analyzed, verified, and organized the data, and who edited and assembled the report. In addition to the authors, who had primary responsibility for assuring that the information contained herein is accurate, complete, and adheres to Geological Survey policy and established guidelines, the following individuals contributed significantly to the collection, processing, and tabulation of the data:

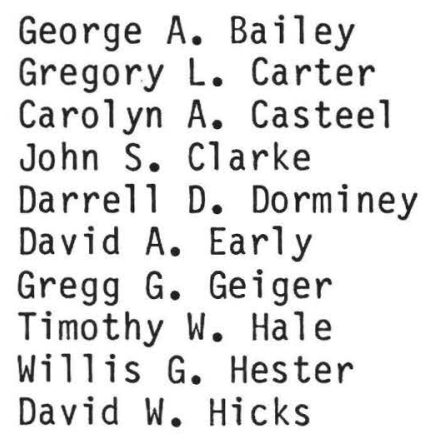

\author{
Stephen $H_{\text {. Jones }}$ \\ Richard E. Krause \\ Coleman D. Leach \\ Keith W. McFadden \\ Barbara J. Milby \\ R. Terry Nichols \\ Michael F. Peck \\ John W. Tyler \\ Blaine T. White
}

Records of all water-level measurements and water-quality data used in this report may be obtained upon request from the U.S. Geological Survey, Water Resources Division, 6481 Peachtree Industrial Boulevard, Suite B, Doraville, GA 30360 . 
ABSTRACT ........................................ 1

1.0 INTRODUCTION........................................ 2

1.1 Major aquifers................................... 4

2.0 GROUND-WATER LEVELS................................. 6

2.1 Paleozoic rock aquifers............................ 8

2.2 Crystalline rock aquifers.......................... 12

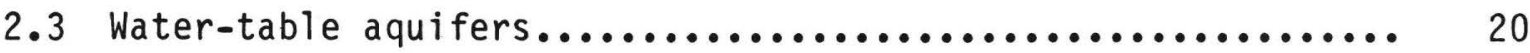

2.4 Cretaceous aquifer system......................... 30

2.4.1 Providence aquifer........................... 34

2.4.2 Dublin, Midville, and Dublin-Midville aquifer

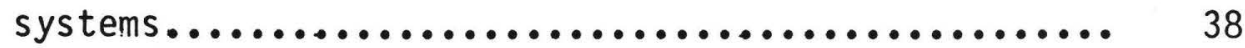

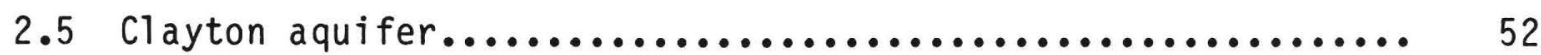

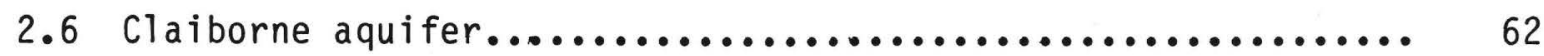

2.7 Upper Floridan aquifer.......................... 70

2.7 .1 Southwest area.............................. 72

2.7 .2 South-central area........................... 88

2.7.3 East-central area........................... 100

2.7 .4 Coastal area............................... 108

2.7.4.1 Savannah area.......................... 110

2.7.4.2 Jesup-Riceboro area..................... 122

2.7.4.3 Brunswick area.......................... 136

2.7.4.4 Kings Bay-Okefenokee Swamp area............. 146

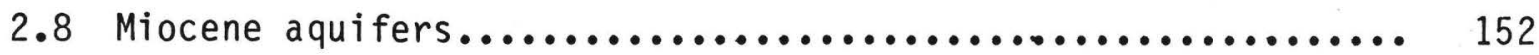


3.0 GROUND-WATER QUALITY................................ 158

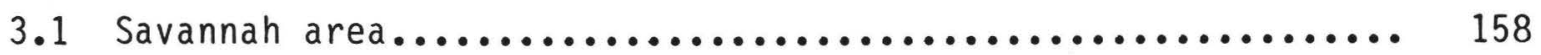

3.2 Brunswick area................................. 162

4.0 SELECTED REFERENCES................................. 168

\section{LIST OF ILLUSTRATIONS}

Figure 1.1-1. Map showing areas of utilization of major aquifers and block diagram showing major aquifers and physiographic provinces of Georgia..............

2.0-1. Map showing locations of observation wells for which hydrographs are included in this report............

2.1-1. Map showing location of observation well in the

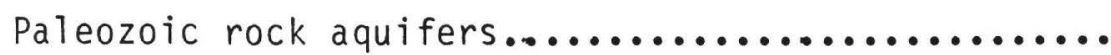

2.1-2. Hydrographs showing the water level in observation

wel1 03Pp01, walker County.................... 11

2.2-1. Map showing location of observation wells in the

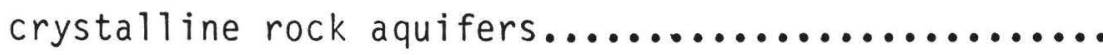

2.2-2. Hydrographs showing the water level in observation

well 100002, Fulton County..................... 15

2.2-3. Hydrographs showing the water level in observation

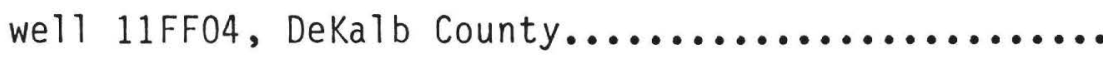

2.2-4. Hydrographs showing the water-level in observation

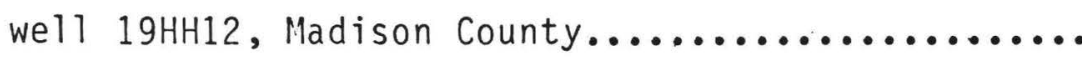




\section{LIST OF ILLUSTRATIONS}

Page

Figure 2.3-1. Map showing locations of observation wells in

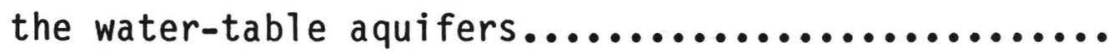

2.3-2. Hydrographs showing water levels in observation

well 11AA01, Spalding County.

2.3-3. Hydrographs showing water levels in observation

well 13M007, Worth County

2.3-4. Hydrographs showing water levels in observation

well 07H003, Miller County

2.3-5. Hydrographs showing water levels in observation

well 35P094, Chatham County.

2.4-1. Map showing locations of observation wells in the

Cretaceous aquifer system.

2.4-2. Hydrographs showing the water level in observation

well 06S001, Chattahoochee County................

2.4.1-1. Map showing the location of observation well 12L021

and the water level in the Providence aquifer,

October 1987

2.4.1-2. Hydrographs showing the water level in observation

well 12L021, Dougherty County..................

2.4.2-1. Map showing locations of observation wells and the water level in the Dublin and Dublin-Midville

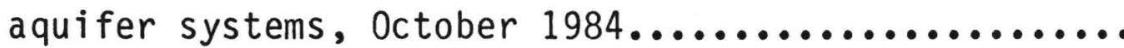

2.4.2-2. Hydrographs showing water levels in observation wel1 18u001, Twiggs County................... 


\section{LIST OF ILLUSTRATIONS--Continued}

Page

Figure 2.4.2-3. Hydrographs showing water levels in observation

well 18T001, Pulaski County...................

2.4.2-4. Hydrographs showing water levels in observation

we11 21U004, Laurens County....................

2.4.2-5. Hydrographs showing water levels in observation

wel1 24v001, Johnson County...................

2.4.2-6. Hydrographs showing water levels in observation

well 28x001, Burke County.....................

2.4.2-7. Hydrographs showing water levels in observation

well 30AA04, Richmond County..................

2.5-1. Map showing observation well locations and the

water level in the Clayton aquifer, October 1987...

2.5-2. Hydrographs showing water levels in observation

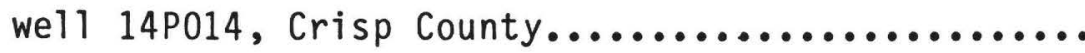

2.5-3. Hydrographs showing water levels in observation

well 07N001, Randolph County....................

2.5-4. Hydrographs showing water levels in observation

wel1 11L002, Dougherty County...................

2.5-5. Hydrographs showing water levels in observation

well 13L002, Dougherty County...................

2.6-1. Map showing observation well locations and the water

level in the Claiborne aquifer, October 1987.......

2.6-2. Hydrographs showing water levels in observation

well 11L001, Dougherty County................... 65 


\section{LIST OF ILLUSTRATIONS--Continued}

Page

Figure 2.6-3. Hydrographs showing water levels in observation

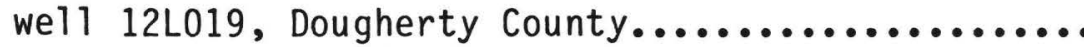

2.6-4. Hydrographs showing water levels in observation

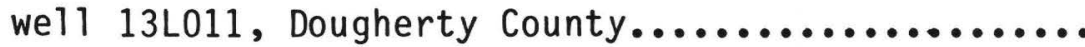

2.7-1. Map showing water level in the Upper Floridan aquifer, May 1985

2.7.1-1. Map showing observation well locations and the water level in the Upper Floridan aquifer in the southwest area, October $1987 \ldots \ldots \ldots \ldots \ldots \ldots \ldots \ldots$

2.7.1-2. Hydrographs showing water levels in observation

wel1 13L003, Dougherty County

2.7.1-3. Hydrographs showing water levels in observation wel1 13L012, Dougherty County

2.7.1-4. Hydrographs showing water levels in observation well 13J004, Mitchell County.

2.7.1-5. Hydrographs showing water levels in observation well 10G313, Mitchell County.

2.7.1-6. Hydrographs showing water levels in observation well 09F520, Decatur County

2.7.1-7. Hydrographs showing water levels in observation well 08G001, Miller County.

2.7.1-8. Hydrographs showing water levels in observation well 06F001, Seminole County 
Figure 2.7.2-1. Map showing observation well locations and the water level in the Upper Floridan aquifer in the south-central area, May 1985....................

2.7.2-2. Hydrographs showing water levels in observation well 15L020, Worth County.................... 91

2.7.2-3. Hydrographs showing water levels in observation wel1 18K049, Tift County.......................

2.7.2-4. Hydrographs showing water levels in observation well 18H016, Cook County..................... 95

2.7.2-5. Hydrographs showing water levels in observation well 19F039, Lowndes County.................. 97

2.7.2-6. Hydrographs showing water levels in observation wel1 19E009, Lowndes County.................... 99

2.7.3-1. Map showing observation well locations and the water level in the Upper Floridan aquifer in the east-central area, May 1985.

2.7.3-2. Hydrographs showing water levels in observation we11 21T001, Laurens County...

2.7.3-3. Hydrographs showing water levels in observation wel1 250001, Montgomery County.

2.7.3-4. Hydrographs showing water levels in observation we11 26R001, Toombs County..................... 107

2.7.4-1. Map showing water level in the Upper Floridan aquifer in the coastal area, May 1985. 


\section{LIST OF ILLUSTRATIONS--Continued}

Figure 2.7.4.1-1. Map showing observation well locations and the

water level in the Upper Floridan aquifer in

the Savannah area, May 1985.

2.7.4.1-2. Hydrographs showing water levels in observation

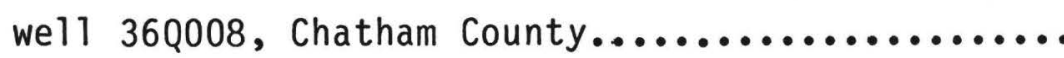

2.7.4.1-3. Hydrographs showing water levels in observation

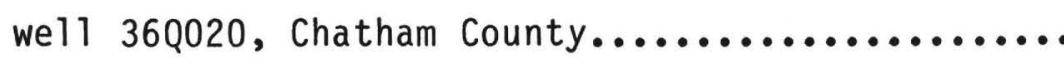

2.7.4.1-4. Hydrographs showing water levels in observation

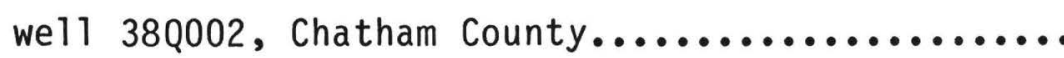

2.7.4.1-5. Hydrographs showing water levels in observation

we11 39Q003, Chatham County....................

2.7.4.1-6. Hydrographs showing water levels in observation

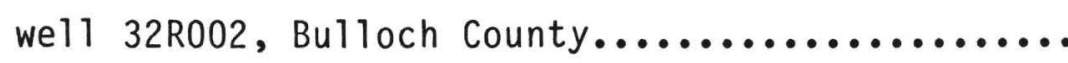

2.7.4.2-1. Map showing observation well locations and the water level in the Upper Floridan aquifer in

the Jesup-Riceboro area, May $1985 . . . . . . . . . . . . .$.

2.7.4.2-2. Hydrographs showing water levels in observation

wel1 30L003, Wayne County....................

2.7.4.2-3. Hydrographs showing water levels in observation

we11 32L015, Wayne County.....................

2.7.4.2-4. Hydrographs showing water levels in observation

we11 33M004, Long County.....................

2.7.4.2-5. Hydrographs showing water levels in observation well 34M054, Liberty County...................

2.7.4.2-6. Hydrographs showing water levels in observation

well 34N089, Liberty County.................... 
Figure 2-7.4.2-7. Hydrographs showing water levels in observation well 35M013, McIntosh County................. 135

2.7.4.3-1. Map showing observation well locations and the water level in the Upper Floridan aquifer in the Brunswick area, October 1987.

2.7.4.3-2. Hydrographs showing water levels in observation wel1 33H127, lower water-bearing zone, Glynn County.

2.7.4.3-3. Hydrographs showing water levels in observation wel1 33H133, upper water-bearing zone, Glynn County.

2.7.4.3-4. Hydrographs showing water levels in observation well 34H391, brackish-water zone, Glynn County.

2.7.4.3-5. Hydrographs showing water levels in observation well 33J044, brackish-water zone, Glynn County 145

2.7.4.4-1. Map showing observation well locations and the water level in the Upper Floridan aquifer in the Kings Bay-Okefenokee Swamp area, May 1985.....

2.7.4.4-2. Hydrographs showing water levels in observation we11 33E027, Camden County.....................

2.7.4.4-3. Hydrographs showing water levels in observation well 27E004, Charlton County. 
Figure 2.8-1. Map showing observation well locations in the Miocene aquifers

2.8-2. Hydrographs showing water levels in observation well 310009, Bulloch County................... 155

2.8-3. Hydrographs showing water levels in observation wel1 32L016, Wayne County.

3.1-1. Map showing locations of chloride-monitoring wells in the Savannah area. 159

3.1-2. Graphs showing chloride concentrations in the

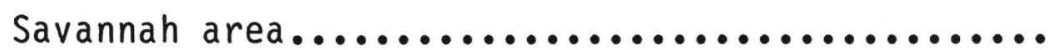

3.2-1. Map showing locations of the chloride-monitoring wells and chloride concentrations in the upper water-bearing zone in the Brunswick area, October 1987

3.2-2. Graphs showing chloride concentrations in the Bay Street area of Brunswick.

3.2-3. Graphs showing chloride concentrations in the north Brunswick area 
List of observation wells for which water-level

hydrographs are included in this report

\begin{tabular}{|c|c|c|c|c|}
\hline County & Aquifer & $\begin{array}{c}\text { Wel1 } \\
\text { number }\end{array}$ & $\begin{array}{l}\text { Wel1 } \\
\text { name }\end{array}$ & $\underline{\text { Page }}$ \\
\hline Burke & $\begin{array}{l}\text { Midville aquifer } \\
\text { system }\end{array}$ & $28 \times 001$ & Midville Exp. Station & 49 \\
\hline Bulloch & Upper Floridan & 32R002 & Bulloch South, TW 1 & 121 \\
\hline But1och & Miocene & 310009 & Hopeulikit, TW 2 & 155 \\
\hline Camden & Upper Floridan & 33Е027 & Kings Bay & 149 \\
\hline Charlton & Upper Floridan & 27E004 & Test we11 0K9 & 151 \\
\hline Chatham & Water table & 35 P094 & UGA & 29 \\
\hline Chatham & Upper Floridan & 360008 & Layne-Atlantic & 113 \\
\hline Chatham & Upper Floridan & $36 Q 020$ & Morrison & 115 \\
\hline Chatham & Upper Floridan & 380002 & Pilot House & 117 \\
\hline Chatham & Upper Floridan & $39 Q 003$ & Test well 7 , point 3 & 119 \\
\hline Chattahoochee & $\begin{array}{l}\text { Cretaceous aquifer } \\
\text { system }\end{array}$ & 065001 & Fort Benning & 3 \\
\hline Cook & Upper Floridan & $18 \mathrm{H} 016$ & Ade 1 & 95 \\
\hline Crisp & Clayton & $14 P 014$ & $\begin{array}{l}\text { Veteran's Memorial Park } \\
\text { TW1 }\end{array}$ & 55 \\
\hline Decatur & Upper Floridan & $09 F 520$ & Bolton & 83 \\
\hline DeKalb & Crystalline rock & $11 \mathrm{FF} 04$ & GAR, TW 5 & 17 \\
\hline Dougherty & Providence & 12L021 & Test well 10 & 37 \\
\hline Dougherty & Clayton & 11L002 & Al bany Nursery & 59 \\
\hline Dougherty & Clayton & 13L002 & Turner City & 61 \\
\hline Dougherty & Claiborne & 11L001 & Test well 4 & 65 \\
\hline Dougherty & Claiborne & 12L019 & Test well 5 & 67 \\
\hline Dougherty & Claiborne & 13L011 & Test wel1 2 & 69 \\
\hline
\end{tabular}


List of observation wells for which water-level hydrographs are included in this report--Continued

County

Dougherty

Dougherty

Fulton

Glynn

Glynn

Glynn

Glynn

Johnson

Laurens

Laurens

Liberty

Liberty

Long

Lowndes

Lowndes

Madison

McIntosh

Miller

Miller

Mitchel1

Mitchell

Montgomery
Upper Floridan

Upper Floridan

Crystalline rock

Upper Floridan

Upper Floridan

Upper Floridan

Upper Floridan

Midville aquifer system

Upper Floridan

Midville aquifer system

Upper Floridan

Upper Floridan

Upper Floridan

Upper Floridan

Upper Floridan

Crystalline rock

Upper Floridan

Water table

Upper Floridan

Upper Floridan

Upper Floridan

Upper Floridan

$\begin{array}{cc}\begin{array}{c}\text { We11 } \\ \text { number }\end{array} & \text { We11 } \\ \text { name }\end{array}$

Page

13L003 A1bany-Dougherty Co.

75

13 L012 Test well 3

77

10DD02 Fort McPherson

15

33 H127 Test well 3

139

33 H133 Test well 6

141

33J044 Test well 27

145

34H391 Test well 16

143

24V001 Test well 1

$21 T 001$ Hogan

103

21 U004 Test well 3

$34 M 054$ Test well 2

131

34N089 Test well 1

133

33M004 Test wel1 3

129

19E009 Valdosta 99

19F039 Valdosta 8

19HH12 Meadowlake Estates 19

$35 M 013$ Harris Neck

135

$07 \mathrm{H} 003 \quad \mathrm{DP}-3$

08G001 Fleet

85

10G313 Meinders

13 J004 Wright

79

250001 Uvalda School 
List of observation wells for which water-level hydrographs are included in this report--Continued

County

Pulaski

Randolph

Richmond

Seminole

Spalding

Tift

Toombs

Twiggs

Walker

Wayne

Wayne

Wayne

Worth

Worth
Aquifer

Midville aquifer system

Clayton

Dubl in-Midville aquifer system

Upper Floridan

Water table

Upper Floridan

Upper Floridan

Dubl in aquifer system

Paleozoic rock

Upper Floridan

Upper Floridan

Miocene

Water table

Upper Floridan $\begin{array}{cc}\begin{array}{c}\text { Well } \\ \text { number }\end{array} & \text { We11 } \\ \text { name }\end{array}$

Page

18 T001 Arrowhead test

well 1

07N001 Cuthbert

30AA04 McBean 2

06F001 Roddenberry

87

11AA01 Experiment Station

18K049 Test well 1

26R001 Vidalia 2

107

18 U001 Test well 3

03PP01 Fort 0glethorpe

30L003 Johnson

32L015 Gardi TW 1

32L016 Gardi TW 2

$13 M 007$ DP-9

25

15L020 Sylvester 
Multiply inch-pound units

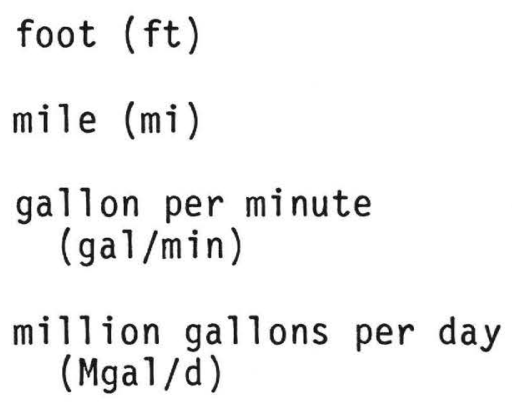

mile (mi)

gallon per minute (gal/min)

million gallons per day $(\mathrm{Mgal} / \mathrm{d})$

million gallons per day (Mgal/d)

43.81

\section{Concentration}

parts per million

(ppm)
By

0.3048

1.609

0.06308

0.04381

1
To obtain SI units

meter (m)

kilometer $(\mathrm{km})$

liter per second

( L/s)

cubic meter per second $\left(\mathrm{m}^{3} / \mathrm{s}\right)$

liter per second

$(\mathrm{L} / \mathrm{s})$

milligram per liter $(\mathrm{mg} / \mathrm{L})$

Sea level

In this report "sea level" refers to the National Geodetic Vertical Datum of 1929 (NGVD of 1929)--a geodetic datum derived from a general adjustment of the first-order level nets of both the United States and Canada, formerly called "Mean Sea Level of 1929." 
GROUND-WATER DATA FOR GEORGIA, 1987

By

Charles N. Joiner, Mark S. Reynolds,

Welby L. Stayton, and Frank G. Boucher

ABSTRACT

Continuous water-level records from 142 wells and water-level measurements from an additional 481 wells in Georgia during 1987 provide the basic data for this report. Hydrographs for selected wells illustrate the effects that changes in recharge and discharge have had on the ground-water reservoirs in the State. Daily mean water levels are shown in hydrographs for 1987 . Monthly mean water levels are shown for the 10-year period 1978-87. Annual mean water levels were from 3.9 feet lower to 5.1 feet higher than in 1986. Throughout most of the State, water levels showed a significant recovery from the lows measured during the 1986 drought. By early spring, water levels had recovered 1 foot to 50 feet from the lows and record lows measured during the 1986 drought. Although water levels generally recovered, record lows were measured in seven wells tapping the crystalline rock aquifers, the Midville aquifer system, and the Upper Floridan and Miocene aquifers in late 1987. The 1987 lows were from 0.1 to 0.8 foot lower than the previous record lows. At the end of 1987, water levels were from 5.7 feet higher to 14.2 feet lower than at the end of 1986 .

Water-quality samples are collected periodically throughout Georgia and are analyzed as part of areal and regional ground-water studies. Periodic monitoring of water quality in the Savannah and Brunswick areas indicates that the chloride concentration in the Upper Floridan aquifer generally has remained stable. 


\subsection{INTRODUCTION}

Monitoring water levels and water quality is essential to the management of a ground-water reservoir or aquifer. Fluctuations and long-term trends in water levels occur as a result of recharge to and discharge from the aquifer. Recharge varies in response to precipitation, evapotranspiration, and surfacewater infiltration into the aquifer. Discharge occurs as natural flow from the aquifer to streams and springs, evapotranspiration, and withdrawal from wells.

Ground-water levels have been monitored in Georgia for about a hundred years. In the early years, the water-level data were used in areal reconnaissance studies, and published, usually as tables and a few graphs that showed water-level trends. These data had limited value for resource management purposes, especially considering the timelag between data collection and publication.

As part of the cooperative ground-water investigations undertaken by the U.S. Geological Survey and the State of Georgia, a statewide water-level measurement program to monitor long-term trends was begun in 1938. This program initially consisted of an observation-well network to provide long-term data on changes in ground-water storage and quality in the coastal area. Other wells were added in areas where changes in water levels might forewarn of potential water-resources problems. More than 950 water-level measurements were made in Georgia during 1987, and an additional 142 network and project wells were monitored continuously. 
This report continues a series of publications that annually presents both ground-water level and ground-water quality data for Georgia. Hydrographs from 57 wells have been selected to illustrate the effects that changes in recharge and discharge have had on the various aquifers in the State. Daily mean water levels are shown in hydrographs for 1987. Monthly mean water levels, as well as chloride concentrations for selected areas along the coast, are shown for the 10-year period 1978-87. Because the 1987 hydrographs are plotted from daily mean values, a record low or record high water level that occurred on a given day would have been lower or higher than that shown on the hydrograph.

The report also includes maps that show the potentiometric surfaces of the Upper Floridan, Claiborne, Clayton, Providence, and Dublin-Midville aquifers. The potentiometric surface of an aquifer is an imaginary surface that represents the altitude to which water would rise in tightly cased wells that penetrate the aquifer. The potentiometric surface is highest in areas of recharge and lowest in areas of discharge, indicating that ground water flows from recharge areas to discharge areas. Where discharge is concentrated and exceeds recharge, the potentiometric surface is lowered, forming a cone of depression.

The cooperation and assistance of the following agencies in collecting water-level and water-quality data during 1987 are gratefully acknowledged:

Georgia Department of Natural Resources, Georgia Geologic Survey

Glynn County

City of Brunswick

City of Valdosta, and

Albany Water, Gas, and Light Commission. 


\subsection{Major Aquifers}

Differing geologic features and landforms of the several physiographic provinces of Georgia cause significant differences in ground-water conditions from one part of the State to another. The most productive aquifers in Georgia are in the Coastal Plain province, which includes the southern half of the State. The Coastal Plain is underlain by alternating layers of sand, clay, and limestone that dip and thicken to the southeast. In the Coastal Plain, aquifers generally are confined, except near their northern limit where they are exposed or are near land surface. Major aquifers of the Coastal Plain include the predominantly limestone Upper Floridan aquifer, the sandy Claiborne aquifer, the limestone Clayton aquifer, and the sandy Cretaceous aquifer system. The predominantly clastic Miocene aquifers overlie the Upper Floridan aquifer in most of the Floridan's area of occurrence, but herein are not considered major aquifers. The Piedmont and the Blue Ridge provinces in the northern half of Georgia are underlain by massive igneous and metamorphic rocks that form aquifers of low permeability. The Valley and Ridge and the Appalachian Plateaus provinces in the northwestern corner of Georgia, are underlain by sandstone, limestone, dolostone, and shale of Paleozoic age. Water-table conditions occur where the aquifers are unconfined and near land surface. For a more complete discussion of aquifers, see the reports listed in "Selected References." 

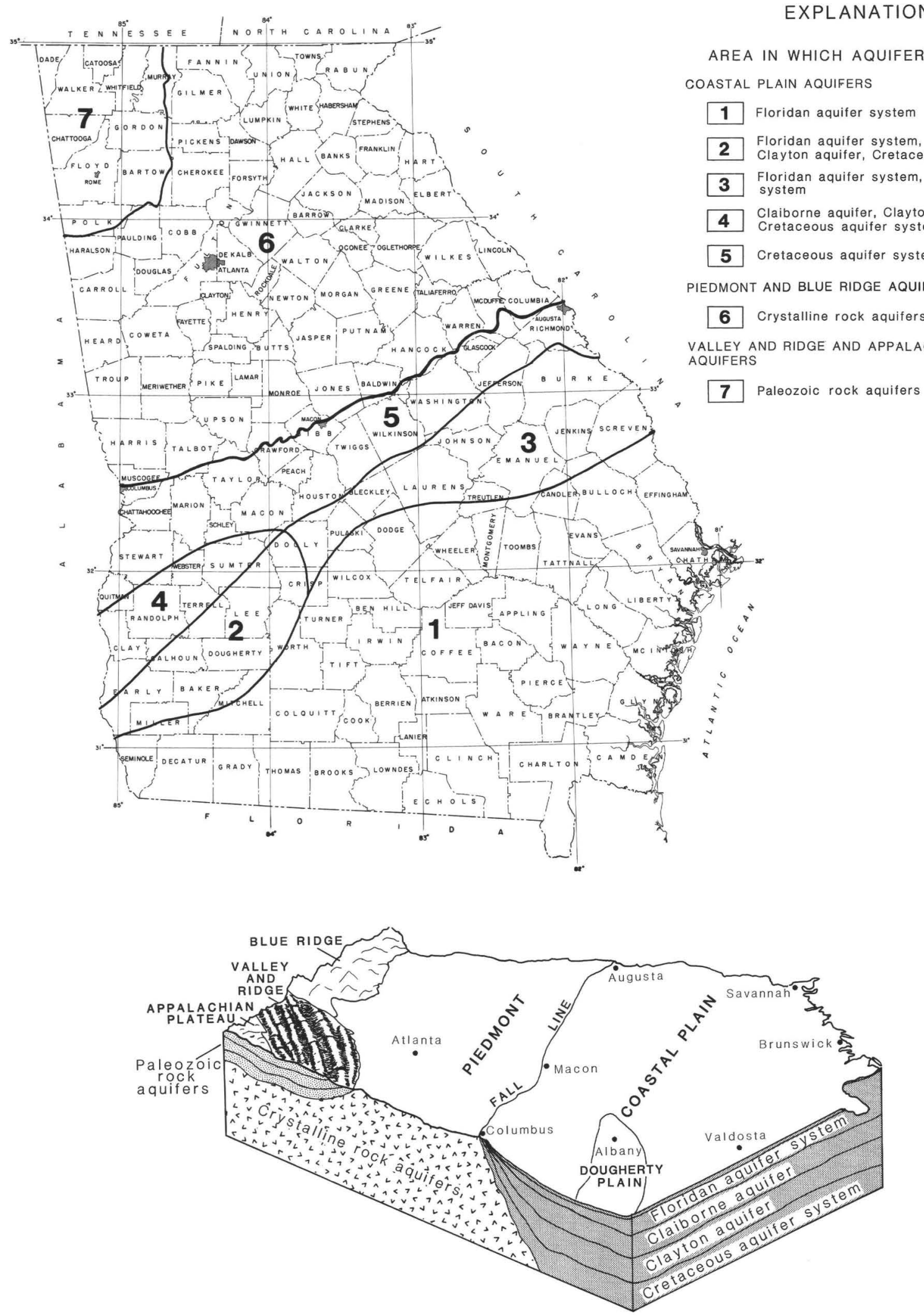

Figure 1.1-1.- Areas of utilization of major aquifers and block diagram showing major aquifers and physiographic provinces of Georgia. 


\subsection{GROUND-WATER LEVELS}

Annual mean ground-water levels were from $3.9 \mathrm{ft}$ lower to $5.1 \mathrm{ft}$ higher than in 1986. Of the 57 wells having continuous water-level records selected for this report, 23 had annual mean water levels that were lower in 1987 than in 1986, 32 had water levels that were higher, and two remained about the same. Record low water levels were measured in seven wells during late 1987 that were from 0.1 to $0.8 \mathrm{ft}$ lower than the previous record lows. The new record lows were measured during December in the crystalline rock aquifers; during November and December in the Midville aquifer system; and during December in the Upper Floridan and Miocene aquifers of the Coastal Plain province. 


\section{EXPLANATION}

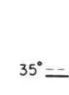

$35^{\circ}=$

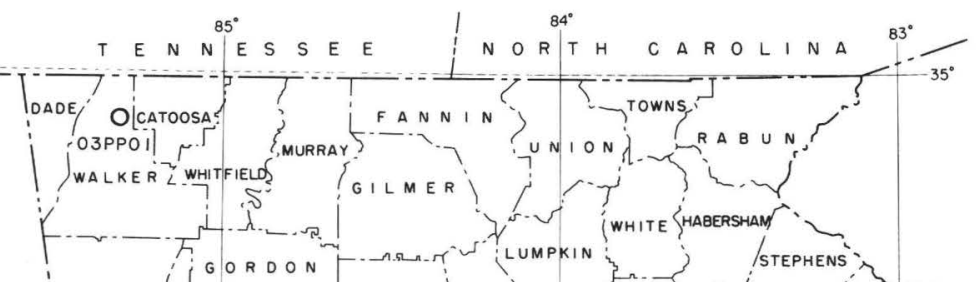

AQUIFER DESIGNATION AND WELL IDENTIFICATION NUMBER

9HHI

$03 \mathrm{PPOI}$

Water table

Crystalline rock

Paleozoic rock

o Miocene aquifer

26ROOI Upper Floridan aquifer

Cretaceous aquifer system

Clayton

Claiborne

$34^{\circ}$

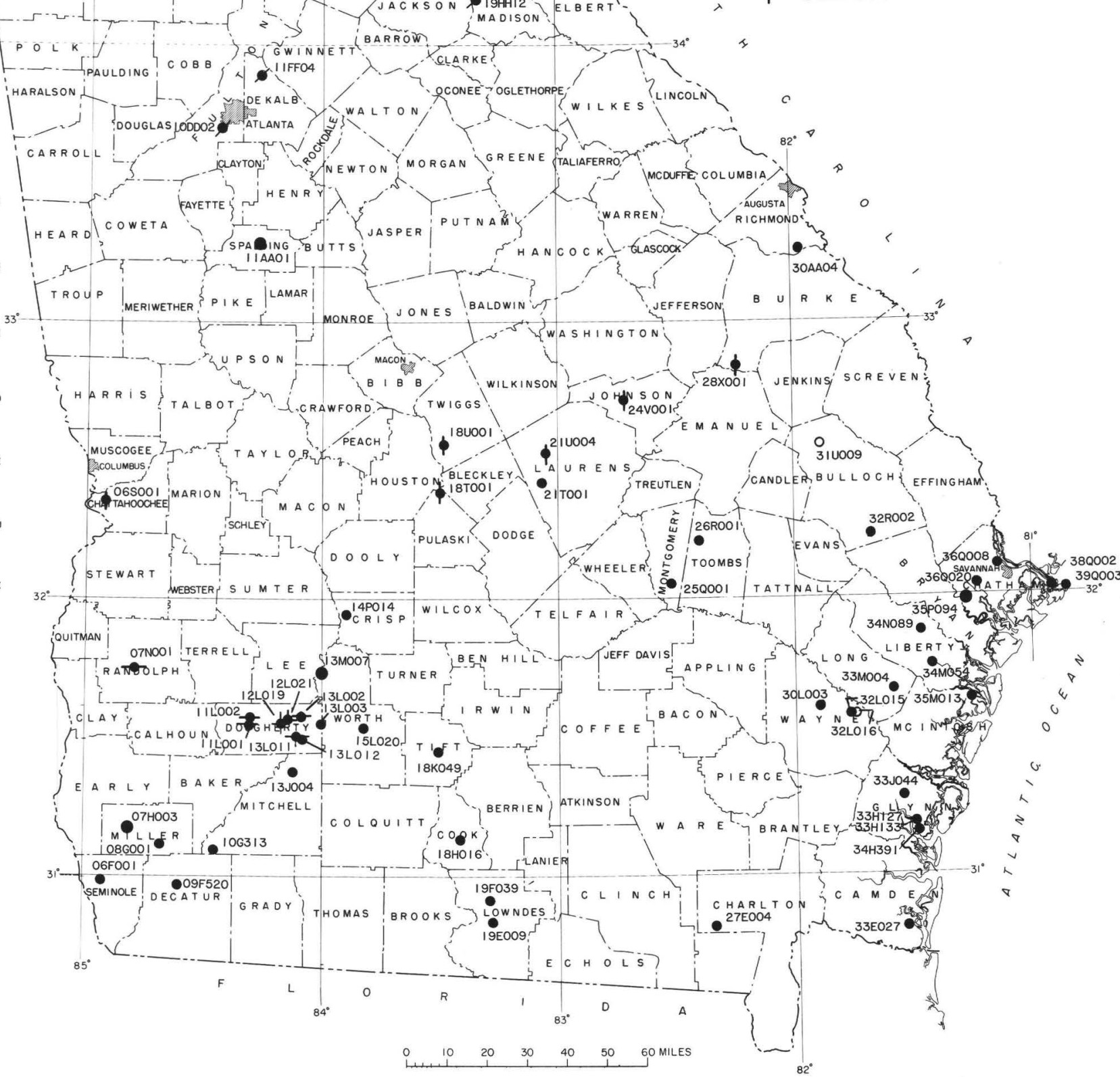

Figure 2.0-1.-Locations of observation wells for which hydrographs are included in this report. 


\subsection{Paleozoic Rock Aquifers}

During 1985, about $29 \mathrm{Mgal} / \mathrm{d}$ (Turlington and others, 1987) was withdrawn from the Paleozoic rock aquifers, primarily for industrial supply. Water in the Paleozoic rock aquifers generally occurs under water-table conditions, and storage is limited mainly to the residuum and to joints, fractures, and solution openings in the bedrock.

Ground-water levels in the Paleozoic rock aquifers are affected mainly by precipitation. Rainfall in the area generally is heavy in winter and midsummer and relatively light in spring and fall. Water levels generally are at their highest for the year in March or April and at their lowest for the year in October or November.

Wells in areas having a thin soil cover commonly show a rapid response to rainfall, and water levels may rise several feet within a few minutes or hours. In areas having a thick soil cover, wells may show little response to individual rains, but undergo a gradual rise in water level during wet periods. The water level in most wells declines slowly between rains.

The hydrographs for observation well 03 PP01 in Walker County illustrate the effect that precipitation has on water levels in areas of thin soil cover. The mean water level in well 03PP01 during 1987 was $1.2 \mathrm{ft}$ lower than in 1986. However, by the end of February, the water level had recovered about $17.3 \mathrm{ft}$ from the low measured during the 1986 drought. Although there was some recovery during the year, the water level at the end of 1987 was $3.2 \mathrm{ft}$ lower than at the end of 1986. 


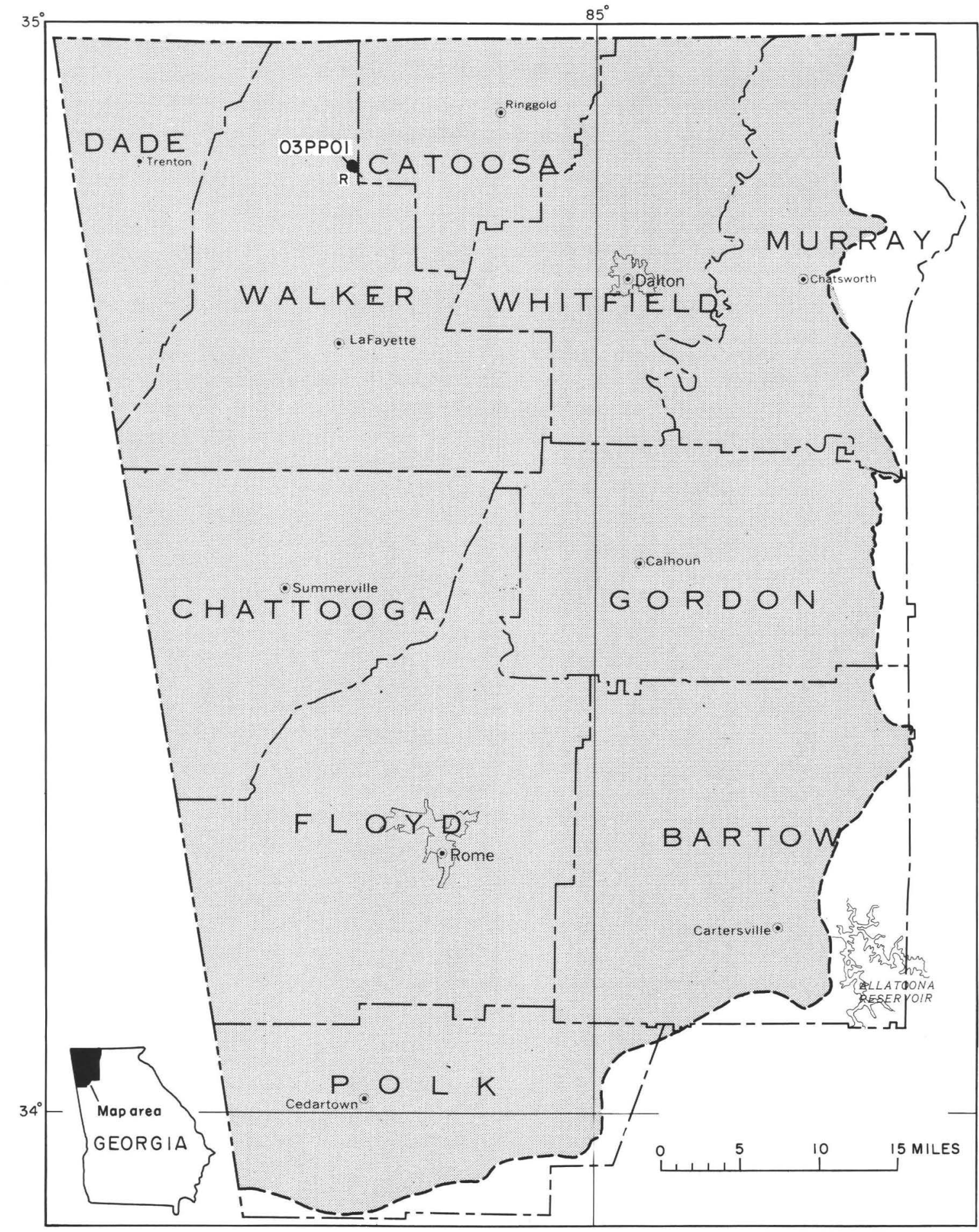

EXPLAN A T IO N AREA OF PALEOZOIC ROCK
AQUIFERS O3PPOI

PPOI OBSERVATION WELL AND IDENTIFICATION NUMBER-Equipped with recorder; hydrograph included in this report

Figure 2.1-1.-Location of observation well in the Paleozoic rock aquifers. 
345403085160001 Local number, 03PP01.

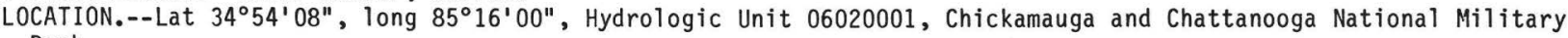
Park.

Owner: National Park Service, Fort Oglethorpe.

AQUIFER.--Paleozoic Rock (Chickamauga Limestone).

WELL CHARACTERISTICS.--Cable-tooled, observation well, diameter $8 \mathrm{in}$., depth $72 \mathrm{ft}$.

DATUM.--Elevation of land-surface datum is $730 \mathrm{ft}$

Measuring point: Pointer on recorder shelter, $2.09 \mathrm{ft}$ above land surface.

REMARKS.--Well sounded 0ctober 18, 1977.

PERIOD OF RECORD.--1977 to current year.

EXTREMES FOR PERIOD OF RECORD.--Highest water level, $1.97 \mathrm{ft}$ below land-surface datum, March 9, 1978; 1owest, $21.70 \mathrm{ft}$ below land-surface datum, August $5,1978$.

DEPTH BELOW LAND SURFACE (WATER LEVEL) (FEET) CALENDAR YEAR JANUARY 1987 TO DECEMBER 1987 MEAN VALUES

\begin{tabular}{|c|c|c|c|c|c|c|c|c|c|c|c|c|}
\hline DAY & JAN & FEB & MAR & APR & MAY & JUN & JUL & AUG & SEP & OCT & NOV & DEC \\
\hline $\begin{array}{l}1 \\
2 \\
3 \\
4 \\
5\end{array}$ & $\begin{array}{l}10.05 \\
10.18 \\
10.35 \\
10.53 \\
10.73\end{array}$ & $\begin{array}{l}9.82 \\
8.66 \\
9.57 \\
9.75 \\
9.79\end{array}$ & $\begin{array}{l}6.85 \\
9.45 \\
9.70 \\
9.76 \\
9.79\end{array}$ & $\begin{array}{l}9.67 \\
9.75 \\
9.27 \\
9.54 \\
9.72\end{array}$ & $\begin{array}{l}10.50 \\
10.66 \\
10.83 \\
10.42 \\
10.11\end{array}$ & $\begin{array}{l}14.01 \\
14.16 \\
14.27 \\
14.36 \\
14.51\end{array}$ & $\begin{array}{l}15.36 \\
15.50 \\
15.63 \\
15.73 \\
15.83\end{array}$ & $\begin{array}{l}18.60 \\
18.65 \\
18.72 \\
18.77 \\
18.25\end{array}$ & $\begin{array}{l}19.38 \\
19.41 \\
19.45 \\
19.49 \\
19.52\end{array}$ & $\begin{array}{l}19.51 \\
19.53 \\
19.58 \\
19.60 \\
19.60\end{array}$ & $\begin{array}{l}20.24 \\
20.25 \\
20.25 \\
20.25 \\
20.27\end{array}$ & $\begin{array}{l}19.63 \\
19.77 \\
19.83 \\
19.90 \\
19.95\end{array}$ \\
\hline $\begin{array}{r}6 \\
7 \\
8 \\
9 \\
10\end{array}$ & $\begin{array}{l}10.89 \\
11.07 \\
11.28 \\
11.44 \\
11.54\end{array}$ & $\begin{array}{l}9.81 \\
9.82 \\
9.85 \\
9.90 \\
9.91\end{array}$ & $\begin{array}{l}9.82 \\
9.82 \\
9.77 \\
7.40 \\
9.51\end{array}$ & $\begin{array}{l}9.77 \\
9.79 \\
9.81 \\
9.83 \\
9.84\end{array}$ & $\begin{array}{l}10.22 \\
10.33 \\
10.51 \\
10.71 \\
10.89\end{array}$ & $\begin{array}{l}14.64 \\
14.74 \\
14.82 \\
14.90 \\
15.04\end{array}$ & $\begin{array}{l}15.86 \\
15.87 \\
16.13 \\
16.87 \\
16.94\end{array}$ & $\begin{array}{l}18.11 \\
18.48 \\
18.56 \\
18.55 \\
18.62\end{array}$ & $\begin{array}{l}19.55 \\
19.56 \\
19.58 \\
19.61 \\
19.62\end{array}$ & $\begin{array}{l}19.64 \\
19.77 \\
19.83 \\
19.86 \\
19.88\end{array}$ & $\begin{array}{l}20.30 \\
20.30 \\
20.31 \\
20.32 \\
18.22\end{array}$ & $\begin{array}{l}19.99 \\
20.04 \\
20.07 \\
20.08 \\
20.10\end{array}$ \\
\hline $\begin{array}{l}11 \\
12 \\
13 \\
14 \\
15\end{array}$ & $\begin{array}{l}11.77 \\
11.94 \\
12.07 \\
12.17 \\
12.26\end{array}$ & $\begin{array}{l}9.90 \\
9.90 \\
9.95 \\
9.95 \\
9.69\end{array}$ & $\begin{array}{l}9.75 \\
9.77 \\
9.79 \\
9.80 \\
9.82\end{array}$ & $\begin{array}{l}9.85 \\
9.86 \\
9.88 \\
9.36 \\
8.01\end{array}$ & $\begin{array}{l}11.11 \\
11.30 \\
11.40 \\
11.54 \\
11.69\end{array}$ & $\begin{array}{l}15.13 \\
15.23 \\
15.32 \\
15.40 \\
15.49\end{array}$ & $\begin{array}{l}17.00 \\
17.05 \\
17.10 \\
17.18 \\
17.25\end{array}$ & $\begin{array}{l}18.68 \\
18.76 \\
18.81 \\
18.86 \\
18.89\end{array}$ & $\begin{array}{l}19.54 \\
17.69 \\
17.52 \\
17.77 \\
18.34\end{array}$ & $\begin{array}{l}19.89 \\
19.91 \\
19.95 \\
19.99 \\
20.01\end{array}$ & $\begin{array}{l}19.35 \\
19.74 \\
19.91 \\
20.01 \\
20.09\end{array}$ & $\begin{array}{l}20.12 \\
20.15 \\
20.21 \\
19.50 \\
15.11\end{array}$ \\
\hline $\begin{array}{l}16 \\
17 \\
18 \\
19 \\
20\end{array}$ & $\begin{array}{r}12.35 \\
12.40 \\
4.37 \\
3.81 \\
8.98\end{array}$ & $\begin{array}{l}5.35 \\
9.06 \\
9.71 \\
9.78 \\
9.82\end{array}$ & $\begin{array}{l}9.84 \\
9.85 \\
7.79 \\
6.78 \\
9.45\end{array}$ & $\begin{array}{l}9.58 \\
9.73 \\
9.79 \\
9.82 \\
9.84\end{array}$ & $\begin{array}{l}11.83 \\
11.97 \\
12.12 \\
12.26 \\
12.81\end{array}$ & $\begin{array}{l}15.33 \\
15.41 \\
15.55 \\
15.65 \\
15.71\end{array}$ & $\begin{array}{l}17.33 \\
17.41 \\
17.49 \\
17.63 \\
17.71\end{array}$ & $\begin{array}{l}18.94 \\
18.92 \\
18.65 \\
18.75 \\
18.82\end{array}$ & $\begin{array}{l}18.64 \\
18.75 \\
18.86 \\
18.93 \\
18.99\end{array}$ & $\begin{array}{l}20.03 \\
20.06 \\
20.08 \\
20.10 \\
20.11\end{array}$ & $\begin{array}{l}20.12 \\
16.13 \\
17.81 \\
18.80 \\
19.16\end{array}$ & $\begin{array}{l}15.89 \\
16.57 \\
17.27 \\
17.90 \\
18.08\end{array}$ \\
\hline $\begin{array}{l}21 \\
22 \\
23 \\
24 \\
25\end{array}$ & $\begin{array}{l}9.70 \\
9.61 \\
9.53 \\
9.58 \\
9.19\end{array}$ & $\begin{array}{l}9.82 \\
7.92 \\
8.40 \\
9.67 \\
9.75\end{array}$ & $\begin{array}{l}9.71 \\
9.77 \\
9.80 \\
9.82 \\
9.81\end{array}$ & $\begin{array}{l}9.85 \\
9.86 \\
9.87 \\
9.89 \\
9.93\end{array}$ & $\begin{array}{l}12.97 \\
13.12 \\
13.26 \\
13.40 \\
13.52\end{array}$ & $\begin{array}{l}15.05 \\
14.24 \\
14.55 \\
14.64 \\
14.59\end{array}$ & $\begin{array}{l}17.77 \\
17.83 \\
17.91 \\
18.09 \\
18.16\end{array}$ & $\begin{array}{l}18.89 \\
18.94 \\
18.99 \\
19.04 \\
19.09\end{array}$ & $\begin{array}{l}19.05 \\
19.11 \\
19.15 \\
19.19 \\
19.26\end{array}$ & $\begin{array}{l}20.03 \\
20.10 \\
20.13 \\
20.14 \\
20.15\end{array}$ & $\begin{array}{l}19.34 \\
19.45 \\
19.54 \\
19.58 \\
19.61\end{array}$ & $\begin{array}{l}17.78 \\
17.82 \\
18.28 \\
15.91 \\
14.69\end{array}$ \\
\hline $\begin{array}{l}26 \\
27 \\
28 \\
29 \\
30 \\
31\end{array}$ & $\begin{array}{l}9.14 \\
9.57 \\
9.73 \\
9.76 \\
9.76 \\
9.81\end{array}$ & $\begin{array}{r}8.26 \\
2.64 \\
3.37 \\
--- \\
--- \\
---\end{array}$ & $\begin{array}{l}9.82 \\
9.84 \\
9.86 \\
9.86 \\
6.03 \\
8.71\end{array}$ & $\begin{array}{c}10.01 \\
10.07 \\
10.16 \\
10.26 \\
10.35 \\
\ldots . .\end{array}$ & $\begin{array}{l}13.66 \\
13.80 \\
13.94 \\
14.08 \\
13.97 \\
13.87\end{array}$ & $\begin{array}{r}14.74 \\
14.86 \\
15.04 \\
15.12 \\
15.24 \\
-.-\end{array}$ & $\begin{array}{l}18.21 \\
18.28 \\
18.33 \\
18.41 \\
18.48 \\
18.55\end{array}$ & $\begin{array}{l}19.13 \\
19.17 \\
19.21 \\
19.35 \\
19.36 \\
19.35\end{array}$ & $\begin{array}{r}19.31 \\
19.35 \\
19.39 \\
19.41 \\
19.47 \\
-\ldots\end{array}$ & $\begin{array}{l}20.15 \\
20.16 \\
20.19 \\
20.20 \\
20.22 \\
20.24\end{array}$ & $\begin{array}{c}19.64 \\
19.66 \\
19.64 \\
19.18 \\
19.42 \\
\ldots .-\end{array}$ & $\begin{array}{l}14.32 \\
12.49 \\
11.59 \\
12.22 \\
12.84 \\
13.20\end{array}$ \\
\hline MEAN & 10.18 & 8.92 & 9.28 & 9.77 & 12.03 & 14.92 & 17.19 & 18.84 & 19.10 & 19.96 & 19.56 & 17.46 \\
\hline CAL & & & & $\mathrm{HIGH}$ & 2.64 & & & & & & & \\
\hline
\end{tabular}




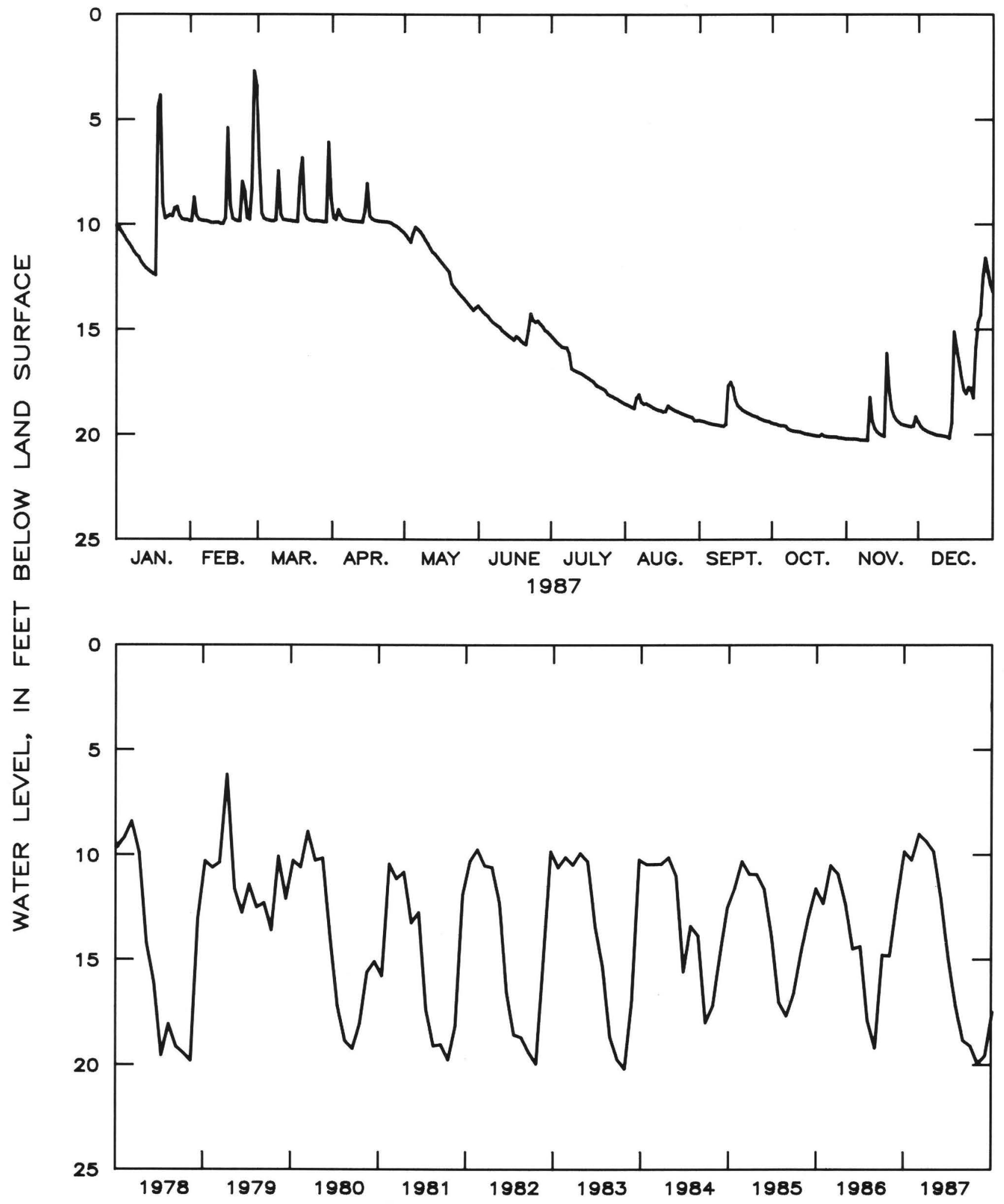

Figure 2.1-2.--Water level in observation well 03PP01, Walker County.

11 


\subsection{Crystalline Rock Aquifers}

Although individual crystalline rock aquifers are not laterally extensive; collectively they yielded an estimated $91 \mathrm{Mgal} / \mathrm{d}$ in 1985 (Turlington and others, 1987), primarily for rural supply. Ground-water storage occurs in unconsolidated material overlying the crystalline rock and in joints, fractures, and other types of secondary openings within the rock (Cressler and others, 1983).

Ground-water levels in the crystalline rock aquifers are affected mainly by precipitation and evapotranspiration. Rainfall in the area is heavy in winter and midsummer and relatively light in spring and fall. The driest season of the year is fall. Ground-water levels rise rapidly with the onset of late winter rains and reduced evapotranspiration, and generally reach their highest levels for the year in March or April. Increases in evapotranspiration and decreases in rainfall during the spring and early summer cause groundwater levels to decline. Heavy rainfall in midsummer results in small rises in ground-water levels, but a lack of recharge in the fall causes water levels to decline to the annual lows, which generally occur in October or November.

During 1987, the mean water levels at wells 100002 in Fulton County, 11FF04 in DeKalb County, and $19 \mathrm{HH} 12$ in Madison County were from 0.2 to $1.3 \mathrm{ft}$ higher in 1987 than in 1986. By the end of March, water levels in the wells had recovered 1.5 to $4.8 \mathrm{ft}$ from the record lows measured during the 1986 drought. However, a new record low was measured at well 10DD02 in early December. The decline was in response to local pumping at the end of 1987 , and water levels were from about the same to $1.6 \mathrm{ft} l o w e r$ than at the end of 1986. 


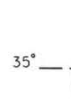

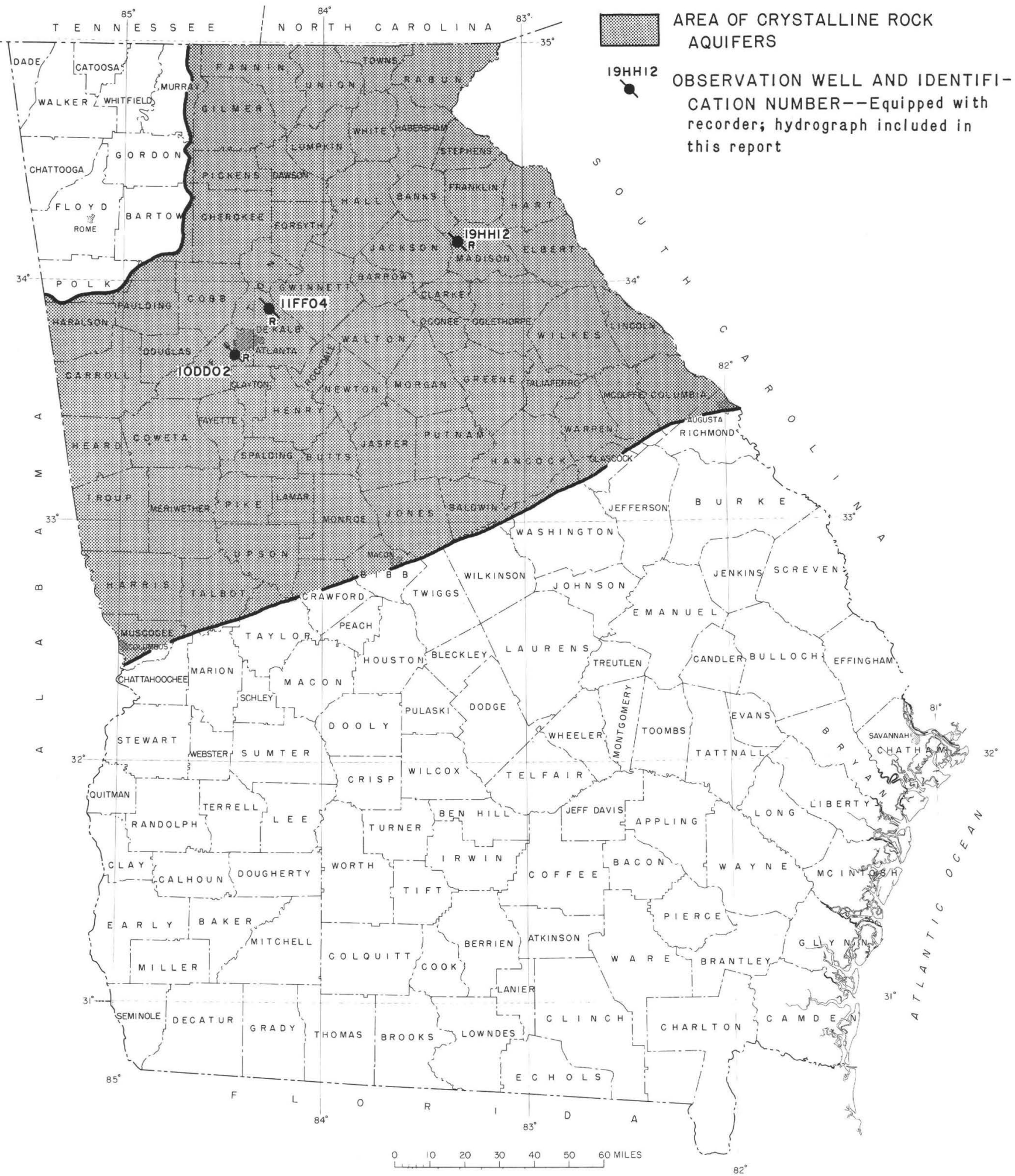

Figure 2.2-1.-Location of observation wells in the crystalline rock aquifers. 
334207084254801 Local number, 10DD02.

LOCATION.--Lat $33^{\circ} 42^{\prime} 07^{\prime \prime}$, long $84^{\circ} 25^{\prime} 48^{\prime \prime}$, Hydrologic Unit $03130002,100 \mathrm{ft}$ east of parking $10 \mathrm{t}$ at main entrance. Owner: U.S. Army, Fort McPherson.

AQUIFER.--Biotite gneiss.

WELL CHARACTERISTICS.--Drilled unused supply well, diameter $12 \mathrm{in.}$, depth $338 \mathrm{ft}$, cased to $41 \mathrm{ft}$, open hole. DATUM.--Elevation of land-surface datum is $1,013 \mathrm{ft}$.

Measuring point: At land-surface datum.

REMARKS.--We11 pumped and sounded February 14, 1976, to a depth of $338 \mathrm{ft}$, well pumped and water sample collected November 26, 1985. Borehole geophysical survey conducted November 19, 1974. Water levels for period of missing record, January 19-27, were estimated.

PERIOD OF RECORD.--November 1973 to current year.

EXTREMES FOR PERIOD OF RECORD.--Highest water level, $0.10 \mathrm{ft}$ below land-surface datum, March 30, 1980; lawest, $10.09 \mathrm{ft}$ below land-surface datum, December $6-7,1987$.

DEPTH BELOW LAND SURFACE (WATER LEVEL) (FEET) CALENDAR YEAR JANUARY 1987 TO DECEMBER 1987 MEAN VALUES

\begin{tabular}{|c|c|c|c|c|c|c|c|c|c|c|c|}
\hline DAY & JAN & FEB & MAR & APR & MAY & JUN & JUL & AUG & SEP & OCT & NOV \\
\hline $\begin{array}{l}1 \\
2 \\
3 \\
4 \\
5\end{array}$ & $\begin{array}{l}8.07 \\
8.13 \\
8.20 \\
8.16 \\
8.17\end{array}$ & $\begin{array}{l}7.32 \\
7.20 \\
7.29 \\
7.40 \\
7.48\end{array}$ & $\begin{array}{l}6.19 \\
6.41 \\
6.60 \\
6.69 \\
6.77\end{array}$ & $\begin{array}{l}6.44 \\
6.36 \\
6.29 \\
6.28 \\
6.23\end{array}$ & $\begin{array}{l}6.29 \\
6.31 \\
6.39 \\
6.44 \\
6.50\end{array}$ & $\begin{array}{l}7.48 \\
7.58 \\
7.63 \\
7.66 \\
7.71\end{array}$ & $\begin{array}{l}7.96 \\
7.92 \\
7.92 \\
7.91 \\
7.91\end{array}$ & $\begin{array}{l}8.56 \\
8.54 \\
8.57 \\
8.59 \\
8.58\end{array}$ & $\begin{array}{l}9.40 \\
9.47 \\
9.52 \\
9.55 \\
9.58\end{array}$ & $\begin{array}{r}9.85 \\
9.87 \\
9.96 \\
10.01 \\
9.90\end{array}$ & $\begin{array}{l}9.88 \\
9.86 \\
9.85 \\
9.82 \\
9.89\end{array}$ \\
\hline $\begin{array}{r}6 \\
7 \\
8 \\
9 \\
10\end{array}$ & $\begin{array}{l}8.22 \\
8.17 \\
8.23 \\
8.22 \\
8.14\end{array}$ & $\begin{array}{l}7.42 \\
7.21 \\
7.04 \\
7.22 \\
7.27\end{array}$ & $\begin{array}{l}6.76 \\
6.67 \\
6.54 \\
6.46 \\
6.61\end{array}$ & $\begin{array}{l}6.26 \\
6.24 \\
6.28 \\
6.27 \\
6.28\end{array}$ & $\begin{array}{l}6.47 \\
6.39 \\
6.39 \\
6.48 \\
6.51\end{array}$ & $\begin{array}{l}7.74 \\
7.82 \\
7.85 \\
7.88 \\
7.99\end{array}$ & $\begin{array}{l}7.90 \\
7.85 \\
7.82 \\
7.84 \\
7.84\end{array}$ & $\begin{array}{l}8.63 \\
8.61 \\
8.60 \\
8.58 \\
8.57\end{array}$ & $\begin{array}{l}9.57 \\
9.54 \\
9.57 \\
9.65 \\
9.70\end{array}$ & $\begin{array}{l}8.99 \\
8.94 \\
9.03 \\
9.07 \\
9.07\end{array}$ & $\begin{array}{r}10.05 \\
10.07 \\
10.05 \\
10.00 \\
9.89\end{array}$ \\
\hline $\begin{array}{l}11 \\
12 \\
13 \\
14 \\
15\end{array}$ & $\begin{array}{l}8.27 \\
8.30 \\
8.32 \\
8.31 \\
8.28\end{array}$ & $\begin{array}{l}7.25 \\
7.13 \\
7.15 \\
7.12 \\
7.12\end{array}$ & $\begin{array}{l}6.72 \\
6.71 \\
6.65 \\
6.61 \\
6.59\end{array}$ & $\begin{array}{l}6.30 \\
6.35 \\
6.40 \\
6.38 \\
6.31\end{array}$ & $\begin{array}{l}6.51 \\
6.51 \\
6.60 \\
6.64 \\
6.63\end{array}$ & $\begin{array}{l}8.08 \\
8.17 \\
8.16 \\
8.11 \\
8.04\end{array}$ & $\begin{array}{l}7.83 \\
7.84 \\
7.84 \\
7.84 \\
7.89\end{array}$ & $\begin{array}{l}8.56 \\
8.59 \\
8.63 \\
8.70 \\
8.75\end{array}$ & $\begin{array}{l}9.74 \\
9.74 \\
9.73 \\
9.73 \\
9.72\end{array}$ & $\begin{array}{l}9.03 \\
8.96 \\
9.11 \\
9.25 \\
9.33\end{array}$ & $\begin{array}{r}9.97 \\
10.06 \\
10.02 \\
10.03 \\
10.08\end{array}$ \\
\hline $\begin{array}{l}16 \\
17 \\
18 \\
19 \\
20\end{array}$ & $\begin{array}{l}8.30 \\
8.31 \\
8.00 \\
7.93 \\
7.86\end{array}$ & $\begin{array}{l}7.09 \\
7.16 \\
7.23 \\
7.33 \\
7.34\end{array}$ & $\begin{array}{l}6.61 \\
6.68 \\
6.60 \\
6.52 \\
6.54\end{array}$ & $\begin{array}{l}6.29 \\
6.32 \\
6.43 \\
6.52 \\
6.55\end{array}$ & $\begin{array}{l}6.64 \\
6.70 \\
6.74 \\
6.77 \\
6.82\end{array}$ & $\begin{array}{l}7.95 \\
7.91 \\
7.94 \\
7.94 \\
7.91\end{array}$ & $\begin{array}{l}7.96 \\
8.08 \\
8.21 \\
8.28 \\
8.32\end{array}$ & $\begin{array}{l}8.77 \\
8.82 \\
8.95 \\
9.01 \\
9.09\end{array}$ & $\begin{array}{l}9.65 \\
9.63 \\
9.60 \\
9.63 \\
9.63\end{array}$ & $\begin{array}{l}9.37 \\
9.42 \\
9.45 \\
9.48 \\
9.57\end{array}$ & $\begin{array}{r}10.07 \\
9.96 \\
9.98 \\
9.99 \\
9.94\end{array}$ \\
\hline $\begin{array}{l}21 \\
22 \\
23 \\
24 \\
25\end{array}$ & $\begin{array}{l}7.79 \\
7.71 \\
7.64 \\
7.57 \\
7.50\end{array}$ & $\begin{array}{l}7.28 \\
7.19 \\
7.23 \\
7.34 \\
7.39\end{array}$ & $\begin{array}{l}6.53 \\
6.55 \\
6.58 \\
6.59 \\
6.63\end{array}$ & $\begin{array}{l}6.53 \\
6.53 \\
6.52 \\
6.56 \\
6.64\end{array}$ & $\begin{array}{l}6.92 \\
7.00 \\
7.05 \\
7.07 \\
7.13\end{array}$ & $\begin{array}{l}7.91 \\
7.92 \\
7.88 \\
7.86 \\
7.76\end{array}$ & $\begin{array}{l}8.36 \\
8.33 \\
8.28 \\
8.34 \\
8.49\end{array}$ & $\begin{array}{l}9.20 \\
9.27 \\
9.23 \\
9.30 \\
9.33\end{array}$ & $\begin{array}{l}9.67 \\
9.73 \\
9.73 \\
9.73 \\
9.85\end{array}$ & $\begin{array}{l}9.63 \\
9.62 \\
9.61 \\
9.63 \\
9.61\end{array}$ & $\begin{array}{r}9.97 \\
10.06 \\
10.05 \\
10.08 \\
10.04\end{array}$ \\
\hline $\begin{array}{l}26 \\
27 \\
28 \\
29 \\
30 \\
31\end{array}$ & $\begin{array}{l}7.43 \\
7.36 \\
7.29 \\
7.28 \\
7.21 \\
7.30\end{array}$ & $\begin{array}{c}7.33 \\
7.03 \\
6.48 \\
--- \\
--- \\
---\end{array}$ & $\begin{array}{l}6.63 \\
6.56 \\
6.50 \\
6.50 \\
6.35 \\
6.38\end{array}$ & $\begin{array}{c}6.73 \\
6.73 \\
6.60 \\
6.43 \\
6.17 \\
-\ldots\end{array}$ & $\begin{array}{l}7.22 \\
7.27 \\
7.35 \\
7.35 \\
7.39 \\
7.41\end{array}$ & $\begin{array}{c}7.74 \\
7.86 \\
7.93 \\
8.02 \\
8.02 \\
---\end{array}$ & $\begin{array}{l}8.53 \\
8.54 \\
8.57 \\
8.69 \\
8.70 \\
8.67\end{array}$ & $\begin{array}{l}9.35 \\
9.38 \\
9.40 \\
9.38 \\
9.46 \\
9.45\end{array}$ & $\begin{array}{r}9.95 \\
10.05 \\
10.07 \\
9.95 \\
9.82 \\
-\cdots\end{array}$ & $\begin{array}{l}9.59 \\
9.62 \\
9.65 \\
9.74 \\
9.78 \\
9.86\end{array}$ & $\begin{array}{r}9.93 \\
9.90 \\
9.87 \\
9.84 \\
9.82 \\
---\end{array}$ \\
\hline MEAN & 7.92 & 7.22 & 6.57 & 6.41 & 6.77 & 7.88 & 8.14 & 8.92 & 9.70 & 9.48 & 9.97 \\
\hline
\end{tabular}

$\begin{array}{lllllll}\text { CAL YR } 1987 & \text { MEAN } & 8.25 & \text { HIGH } & 6.17 & \text { LOW } & 10.09\end{array}$ 

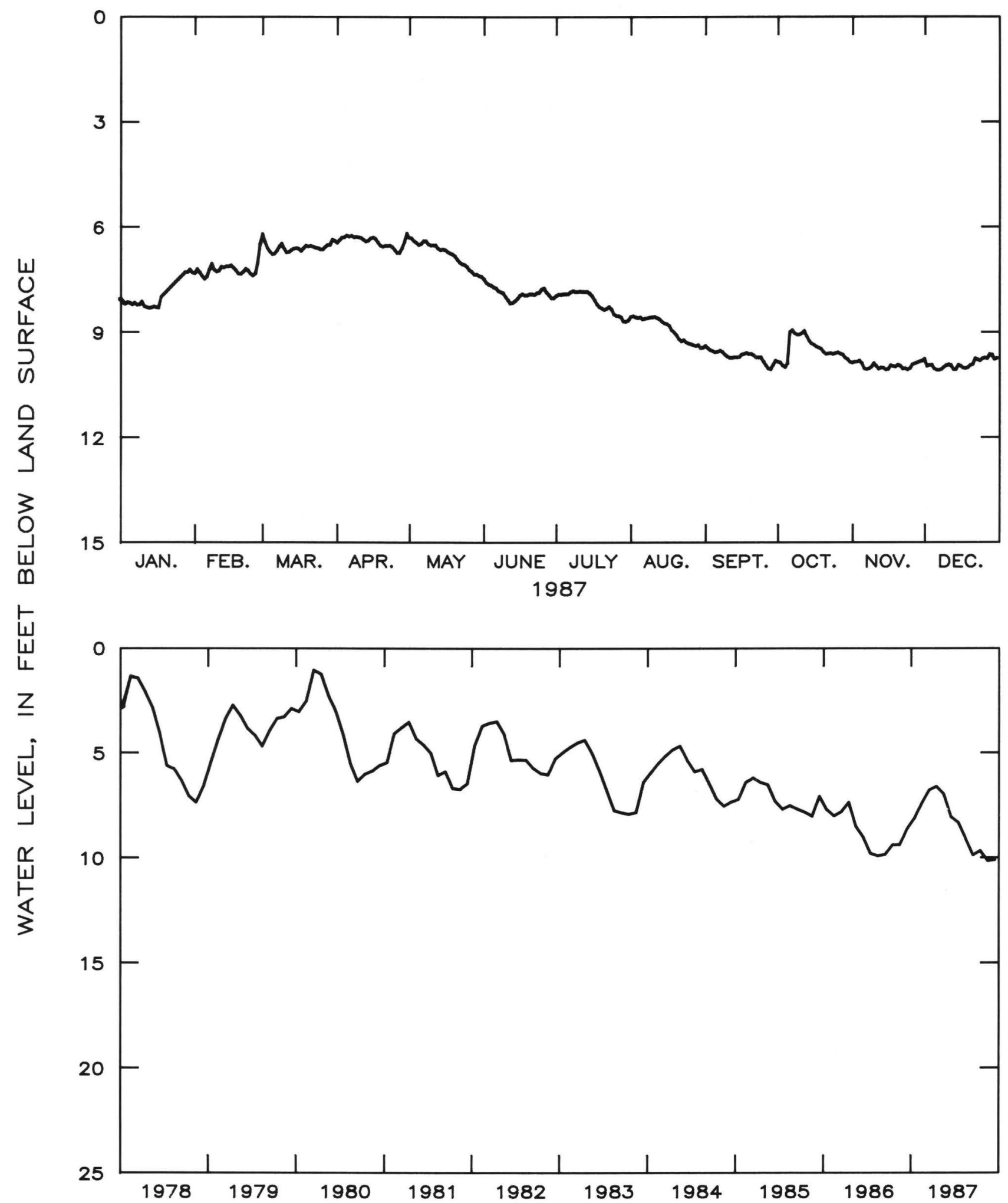

Figure 2.2-2.--Water level in observation well 10DDO2, Fulton County. 
335517084164001 Local number, 11 FF04.

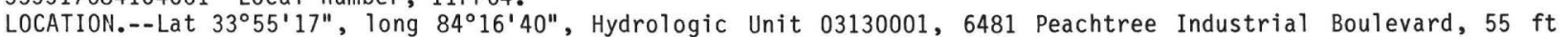
south of southeastern corner of building.

Owner: U.S. Geological Survey

AQUIFER.--Crystalline rock.

WELL CHARACTERISTICS.--Drilled observation well, diameter 6 in., depth $620 \mathrm{ft}$, cased to $36 \mathrm{ft}$, open hole. DATUM.--Elevation of land-surface datum is $950 \mathrm{ft}$.

Measuring point: Floor of recorder shelter, $3.0 \mathrm{ft}$ above land-surface datum.

REMARKS.--Well sounded to a depth of $620 \mathrm{ft}$. Borehole geophysical survey conducted April 18 , 1980.

PERIOD OF RECORD.--February 1980 to January 1984. October 1984 to current year. Water levels for period of missing record, February 28 to March 5, were estimated.

EXTREMES FOR PERIOD OF RECORD.--Highest water level, $5.46 \mathrm{ft}$ below land-surface datum, February 2 , 1983 ; 1 owest, $7.58 \mathrm{ft}$ below land-surface datum, August 25, 1986.

DEPTH BELOW LAND SURFACE (WATER LEVEL) (FEET) CALENDAR YEAR JANUARY 1987 TO DECEMBER 1987 MEAN VALUES

\begin{tabular}{|c|c|c|c|c|c|c|c|c|c|c|c|c|}
\hline DAY & JAN & FEB & MAR & APR & MAY & JUN & JUL & AUG & SEP & OCT & NOV & DEC \\
\hline $\begin{array}{l}1 \\
2 \\
3 \\
4 \\
5\end{array}$ & $\begin{array}{l}6.89 \\
6.98 \\
7.00 \\
6.99 \\
7.01\end{array}$ & $\begin{array}{l}6.69 \\
6.59 \\
6.64 \\
6.72 \\
6.76\end{array}$ & $\begin{array}{l}6.41 \\
6.45 \\
6.48 \\
6.51 \\
6.54\end{array}$ & $\begin{array}{l}6.53 \\
6.52 \\
6.28 \\
6.45 \\
6.52\end{array}$ & $\begin{array}{l}6.76 \\
6.71 \\
6.73 \\
6.82 \\
6.85\end{array}$ & $\begin{array}{l}7.00 \\
7.01 \\
7.03 \\
6.98 \\
7.03\end{array}$ & $\begin{array}{l}6.61 \\
6.68 \\
6.72 \\
6.72 \\
6.75\end{array}$ & $\begin{array}{l}7.09 \\
6.95 \\
6.97 \\
6.44 \\
6.65\end{array}$ & $\begin{array}{l}7.25 \\
7.26 \\
7.30 \\
7.33 \\
7.31\end{array}$ & $\begin{array}{l}7.26 \\
7.29 \\
7.35 \\
7.34 \\
7.31\end{array}$ & $\begin{array}{l}7.37 \\
7.35 \\
7.34 \\
7.31 \\
7.31\end{array}$ & $\begin{array}{l}7.15 \\
7.21 \\
7.17 \\
7.21 \\
7.23\end{array}$ \\
\hline $\begin{array}{r}6 \\
7 \\
8 \\
9 \\
10\end{array}$ & $\begin{array}{l}7.01 \\
7.01 \\
7.04 \\
6.94 \\
6.92\end{array}$ & $\begin{array}{l}6.67 \\
6.39 \\
6.60 \\
6.67 \\
6.70\end{array}$ & $\begin{array}{l}6.57 \\
6.45 \\
6.38 \\
6.34 \\
6.49\end{array}$ & $\begin{array}{l}6.53 \\
6.55 \\
6.60 \\
6.61 \\
6.63\end{array}$ & $\begin{array}{l}6.84 \\
6.84 \\
6.87 \\
6.90 \\
6.91\end{array}$ & $\begin{array}{l}7.05 \\
7.08 \\
7.09 \\
7.10 \\
7.12\end{array}$ & $\begin{array}{l}6.74 \\
6.77 \\
6.84 \\
6.89 \\
6.90\end{array}$ & $\begin{array}{l}6.86 \\
6.46 \\
6.67 \\
6.77 \\
6.83\end{array}$ & $\begin{array}{l}7.24 \\
7.23 \\
7.10 \\
7.15 \\
7.20\end{array}$ & $\begin{array}{l}7.30 \\
7.33 \\
7.39 \\
7.40 \\
7.40\end{array}$ & $\begin{array}{l}7.36 \\
7.38 \\
7.36 \\
7.33 \\
7.05\end{array}$ & $\begin{array}{l}7.24 \\
7.25 \\
7.24 \\
7.22 \\
7.14\end{array}$ \\
\hline $\begin{array}{l}11 \\
12 \\
13 \\
14 \\
15\end{array}$ & $\begin{array}{l}7.03 \\
7.04 \\
7.06 \\
7.05 \\
7.01\end{array}$ & $\begin{array}{l}6.70 \\
6.70 \\
6.72 \\
6.72 \\
6.73\end{array}$ & $\begin{array}{l}6.55 \\
6.52 \\
6.54 \\
6.54 \\
6.55\end{array}$ & $\begin{array}{l}6.61 \\
6.63 \\
6.65 \\
6.57 \\
6.55\end{array}$ & $\begin{array}{l}6.86 \\
6.88 \\
6.57 \\
6.40 \\
6.58\end{array}$ & $\begin{array}{l}7.08 \\
7.08 \\
6.84 \\
6.83 \\
6.78\end{array}$ & $\begin{array}{l}6.93 \\
6.95 \\
6.95 \\
6.97 \\
6.98\end{array}$ & $\begin{array}{l}6.86 \\
6.89 \\
6.92 \\
6.95 \\
6.97\end{array}$ & $\begin{array}{l}7.25 \\
7.23 \\
7.24 \\
7.26 \\
7.28\end{array}$ & $\begin{array}{l}7.37 \\
7.36 \\
7.37 \\
7.39 \\
7.37\end{array}$ & $\begin{array}{l}7.20 \\
7.26 \\
7.26 \\
7.27 \\
7.30\end{array}$ & $\begin{array}{l}7.16 \\
7.18 \\
7.23 \\
6.98 \\
6.80\end{array}$ \\
\hline $\begin{array}{l}16 \\
17 \\
18 \\
19 \\
20\end{array}$ & $\begin{array}{l}6.96 \\
6.92 \\
6.09 \\
6.12 \\
6.50\end{array}$ & $\begin{array}{l}6.61 \\
6.65 \\
6.71 \\
6.76 \\
6.78\end{array}$ & $\begin{array}{l}6.56 \\
6.59 \\
6.47 \\
6.44 \\
6.51\end{array}$ & $\begin{array}{l}6.59 \\
6.62 \\
6.67 \\
6.70 \\
6.71\end{array}$ & $\begin{array}{l}6.75 \\
6.79 \\
6.82 \\
6.83 \\
6.83\end{array}$ & $\begin{array}{l}6.51 \\
6.71 \\
6.80 \\
6.49 \\
6.34\end{array}$ & $\begin{array}{l}7.00 \\
7.04 \\
7.06 \\
7.07 \\
7.09\end{array}$ & $\begin{array}{l}6.98 \\
6.98 \\
7.00 \\
6.85 \\
6.80\end{array}$ & $\begin{array}{l}7.29 \\
7.29 \\
7.31 \\
7.26 \\
7.27\end{array}$ & $\begin{array}{l}7.37 \\
7.37 \\
7.37 \\
7.37 \\
7.38\end{array}$ & $\begin{array}{l}7.26 \\
6.98 \\
7.12 \\
7.13 \\
7.17\end{array}$ & $\begin{array}{l}7.04 \\
7.13 \\
7.16 \\
7.16 \\
7.10\end{array}$ \\
\hline $\begin{array}{l}21 \\
22 \\
23 \\
24 \\
25\end{array}$ & $\begin{array}{l}6.56 \\
6.41 \\
6.52 \\
6.53 \\
6.15\end{array}$ & $\begin{array}{l}6.77 \\
6.70 \\
6.70 \\
6.76 \\
6.77\end{array}$ & $\begin{array}{l}6.58 \\
6.56 \\
6.59 \\
6.55 \\
6.53\end{array}$ & $\begin{array}{l}6.70 \\
6.71 \\
6.72 \\
6.73 \\
6.76\end{array}$ & $\begin{array}{l}6.84 \\
6.88 \\
6.90 \\
6.92 \\
6.94\end{array}$ & $\begin{array}{l}6.49 \\
6.68 \\
6.76 \\
6.38 \\
6.50\end{array}$ & $\begin{array}{l}7.09 \\
7.10 \\
7.13 \\
7.12 \\
7.09\end{array}$ & $\begin{array}{l}6.86 \\
6.88 \\
6.89 \\
6.90 \\
6.90\end{array}$ & $\begin{array}{l}7.32 \\
7.35 \\
7.36 \\
7.37 \\
7.38\end{array}$ & $\begin{array}{l}7.40 \\
7.42 \\
7.41 \\
7.41 \\
7.39\end{array}$ & $\begin{array}{l}7.20 \\
7.24 \\
7.25 \\
7.26 \\
7.24\end{array}$ & $\begin{array}{l}7.04 \\
7.01 \\
7.08 \\
7.06 \\
7.02\end{array}$ \\
\hline $\begin{array}{l}26 \\
27 \\
28 \\
29 \\
30 \\
31\end{array}$ & $\begin{array}{l}6.26 \\
6.47 \\
6.54 \\
6.61 \\
6.61 \\
6.67\end{array}$ & $\begin{array}{r}6.64 \\
6.35 \\
6.38 \\
-.- \\
\ldots- \\
\ldots-\end{array}$ & $\begin{array}{l}6.55 \\
6.52 \\
6.58 \\
6.54 \\
6.32 \\
6.44\end{array}$ & $\begin{array}{r}6.79 \\
6.79 \\
6.76 \\
6.82 \\
6.81 \\
-.-\end{array}$ & $\begin{array}{l}6.97 \\
7.00 \\
6.98 \\
6.98 \\
6.98 \\
6.99\end{array}$ & $\begin{array}{r}6.57 \\
6.75 \\
6.70 \\
6.79 \\
6.81 \\
\ldots\end{array}$ & $\begin{array}{l}7.09 \\
7.10 \\
7.12 \\
7.18 \\
7.14 \\
7.11\end{array}$ & $\begin{array}{l}7.07 \\
7.24 \\
7.25 \\
7.27 \\
7.28 \\
7.27\end{array}$ & $\begin{array}{r}7.40 \\
7.43 \\
7.34 \\
7.24 \\
7.07 \\
. . .\end{array}$ & $\begin{array}{l}7.38 \\
7.26 \\
7.33 \\
7.36 \\
7.37 \\
7.38\end{array}$ & $\begin{array}{l}7.12 \\
7.15 \\
7.16 \\
7.13 \\
7.10 \\
. .-\end{array}$ & $\begin{array}{l}7.05 \\
6.91 \\
6.81 \\
7.00 \\
7.03 \\
7.01\end{array}$ \\
\hline MEAN & 6.74 & 6.66 & 6.50 & 6.64 & 6.84 & 6.81 & 6.97 & 6.93 & 7.28 & 7.36 & 7.23 & 7.10 \\
\hline
\end{tabular}



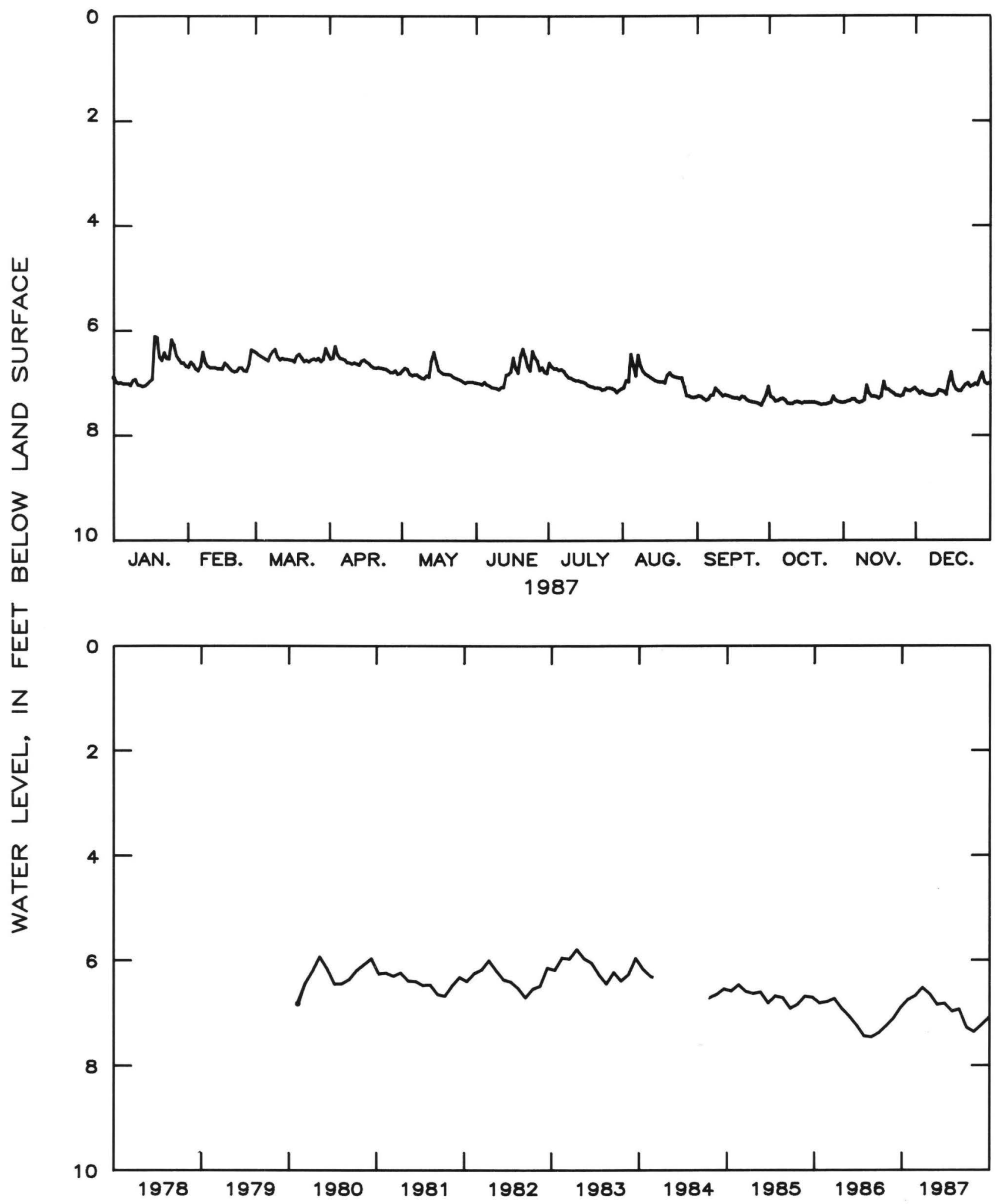

Figure 2.2-3.--Water level in observation well 11FFO4, Dekalb County. 


\section{HH12 MEADOWLAKE ESTATES MADISON COUNTY}

341020083201701 Local number, 19 HH12.

LOCATION.--Lat $34^{\circ} 10^{\prime} 20^{\prime \prime}$, long $83^{\circ} 20^{\prime} 17^{\prime \prime}$, Hydrologic Unit $03060104,2.5 \mathrm{mi}$ west of the intersection of Georgia Highways 98 and 106 in Ila, approximately $0.8 \mathrm{mi}$ south of Georgia Highway 98.

Owner: Meadowlake Estates.

AQUIFER.--Crystalline rock.

WELL CHARACTERISTICS.--Drilled unused supply well, diameter $6 \mathrm{in}$., depth $185 \mathrm{ft}$, cased to $50 \mathrm{ft}$, open hole. DATUM.--Elevation of land-surface datum is $800 \mathrm{ft}$

Measuring point: Floor of recorder shelter, $3.0 \mathrm{ft}$ above land-surface datum.

REMARKS.--None.

PERIOD OF RECORD.--October 1983 to current year.

EXTREMES FOR PERIOD OF RECORD.--Highest water level, $6.69 \mathrm{ft}$ below land-surface datum, April 14, 1984; 1owest, $15.23 \mathrm{ft}$ below land-surface datum, October 6,1986 .

DEPTH BELOW LAND SURFACE (WATER LEVEL) (FEET) CALENDAR YEAR JANUARY 1987 TO DECEMBER 1987 MEAN VALUES

\begin{tabular}{|c|c|c|c|c|c|c|c|c|c|c|c|c|}
\hline DAY & JAN & FEB & MAR & APR & MAY & JUN & JUL & AUG & SEP & ОСT & NOV & DEC \\
\hline $\begin{array}{l}1 \\
2 \\
3 \\
4 \\
5\end{array}$ & $\begin{array}{l}12.89 \\
12.94 \\
12.96 \\
12.96 \\
12.99\end{array}$ & $\begin{array}{l}11.75 \\
11.74 \\
11.81 \\
11.86 \\
11.88\end{array}$ & $\begin{array}{l}10.41 \\
10.41 \\
10.47 \\
10.56 \\
10.64\end{array}$ & $\begin{array}{l}10.74 \\
10.68 \\
10.64 \\
10.65 \\
10.63\end{array}$ & $\begin{array}{l}10.86 \\
10.88 \\
10.91 \\
10.94 \\
10.97\end{array}$ & $\begin{array}{l}11.49 \\
11.49 \\
11.59 \\
11.63 \\
11.67\end{array}$ & $\begin{array}{l}12.17 \\
12.20 \\
12.21 \\
12.23 \\
12.27\end{array}$ & $\begin{array}{l}13.04 \\
13.07 \\
13.06 \\
13.05 \\
13.05\end{array}$ & $\begin{array}{l}13.44 \\
13.47 \\
13.51 \\
13.54 \\
13.54\end{array}$ & $\begin{array}{l}13.91 \\
13.93 \\
13.97 \\
13.98 \\
13.98\end{array}$ & $\begin{array}{l}14.25 \\
14.25 \\
14.24 \\
14.23 \\
14.25\end{array}$ & $\begin{array}{l}14.17 \\
14.18 \\
14.16 \\
14.18 \\
14.20\end{array}$ \\
\hline $\begin{array}{r}6 \\
7 \\
8 \\
9 \\
10\end{array}$ & $\begin{array}{l}12.99 \\
13.00 \\
13.03 \\
13.02 \\
13.02\end{array}$ & $\begin{array}{l}11.85 \\
11.81 \\
11.80 \\
11.87 \\
11.85\end{array}$ & $\begin{array}{l}10.69 \\
10.69 \\
10.67 \\
10.67 \\
10.76\end{array}$ & $\begin{array}{l}10.64 \\
10.64 \\
10.65 \\
10.67 \\
10.69\end{array}$ & $\begin{array}{l}10.96 \\
10.97 \\
11.01 \\
11.06 \\
11.07\end{array}$ & $\begin{array}{l}11.70 \\
11.73 \\
11.76 \\
11.78 \\
11.81\end{array}$ & $\begin{array}{l}12.30 \\
12.31 \\
12.34 \\
12.37 \\
12.39\end{array}$ & $\begin{array}{l}13.04 \\
12.94 \\
12.93 \\
12.93 \\
12.94\end{array}$ & $\begin{array}{l}13.52 \\
13.50 \\
13.51 \\
13.53 \\
13.55\end{array}$ & $\begin{array}{l}14.00 \\
14.03 \\
14.07 \\
14.09 \\
14.09\end{array}$ & $\begin{array}{l}14.28 \\
14.28 \\
14.27 \\
14.26 \\
14.22\end{array}$ & $\begin{array}{l}14.21 \\
14.21 \\
14.20 \\
14.20 \\
14.19\end{array}$ \\
\hline $\begin{array}{l}11 \\
12 \\
13 \\
14 \\
15\end{array}$ & $\begin{array}{l}13.07 \\
13.06 \\
13.09 \\
13.09 \\
13.09\end{array}$ & $\begin{array}{l}11.83 \\
11.82 \\
11.85 \\
11.84 \\
11.87\end{array}$ & $\begin{array}{l}10.78 \\
10.77 \\
10.77 \\
10.79 \\
10.80\end{array}$ & $\begin{array}{l}10.71 \\
10.74 \\
10.77 \\
10.77 \\
10.65\end{array}$ & $\begin{array}{l}11.08 \\
11.08 \\
11.12 \\
11.13 \\
11.12\end{array}$ & $\begin{array}{l}11.82 \\
11.83 \\
11.84 \\
11.85 \\
11.89\end{array}$ & $\begin{array}{l}12.42 \\
12.46 \\
12.48 \\
12.50 \\
12.53\end{array}$ & $\begin{array}{l}12.99 \\
13.01 \\
13.03 \\
13.05 \\
13.06\end{array}$ & $\begin{array}{l}13.56 \\
13.56 \\
13.57 \\
13.59 \\
13.61\end{array}$ & $\begin{array}{l}14.10 \\
14.11 \\
14.13 \\
14.15 \\
14.15\end{array}$ & $\begin{array}{l}14.26 \\
14.25 \\
14.25 \\
14.26 \\
14.26\end{array}$ & $\begin{array}{l}14.20 \\
14.23 \\
14.25 \\
14.24 \\
14.21\end{array}$ \\
\hline $\begin{array}{l}16 \\
17 \\
18 \\
19 \\
20\end{array}$ & $\begin{array}{l}13.10 \\
13.11 \\
12.97 \\
12.30 \\
12.10\end{array}$ & $\begin{array}{l}11.86 \\
11.89 \\
11.91 \\
11.94 \\
11.93\end{array}$ & $\begin{array}{l}10.84 \\
10.88 \\
10.86 \\
10.87 \\
10.89\end{array}$ & $\begin{array}{l}10.57 \\
10.56 \\
10.60 \\
10.62 \\
10.63\end{array}$ & $\begin{array}{l}11.15 \\
11.17 \\
11.18 \\
11.20 \\
11.23\end{array}$ & $\begin{array}{l}11.89 \\
11.91 \\
11.93 \\
11.93 \\
11.93\end{array}$ & $\begin{array}{l}12.59 \\
12.64 \\
12.65 \\
12.68 \\
12.71\end{array}$ & $\begin{array}{l}13.07 \\
13.08 \\
13.09 \\
13.12 \\
13.15\end{array}$ & $\begin{array}{l}13.62 \\
13.63 \\
13.66 \\
13.68 \\
13.68\end{array}$ & $\begin{array}{l}14.16 \\
14.18 \\
14.20 \\
14.22 \\
14.23\end{array}$ & $\begin{array}{l}14.25 \\
14.22 \\
14.25 \\
14.23 \\
14.22\end{array}$ & $\begin{array}{l}14.24 \\
14.24 \\
14.25 \\
14.23 \\
14.24\end{array}$ \\
\hline $\begin{array}{l}21 \\
22 \\
23 \\
24 \\
25\end{array}$ & $\begin{array}{l}12.07 \\
12.01 \\
12.01 \\
11.98 \\
11.88\end{array}$ & $\begin{array}{l}11.91 \\
11.88 \\
11.95 \\
11.96 \\
11.95\end{array}$ & $\begin{array}{l}10.89 \\
10.92 \\
10.93 \\
10.93 \\
10.94\end{array}$ & $\begin{array}{l}10.63 \\
10.66 \\
10.67 \\
10.70 \\
10.75\end{array}$ & $\begin{array}{l}11.27 \\
11.29 \\
11.30 \\
11.32 \\
11.36\end{array}$ & $\begin{array}{l}11.94 \\
11.96 \\
11.98 \\
12.01 \\
12.02\end{array}$ & $\begin{array}{l}12.74 \\
12.77 \\
12.80 \\
12.84 \\
12.86\end{array}$ & $\begin{array}{l}13.18 \\
13.19 \\
13.22 \\
13.26 \\
13.27\end{array}$ & $\begin{array}{l}13.70 \\
13.73 \\
13.75 \\
13.77 \\
13.79\end{array}$ & $\begin{array}{l}14.26 \\
14.27 \\
14.26 \\
14.25 \\
14.24\end{array}$ & $\begin{array}{l}14.24 \\
14.25 \\
14.25 \\
14.25 \\
14.25\end{array}$ & $\begin{array}{l}14.24 \\
14.24 \\
14.26 \\
14.24 \\
14.22\end{array}$ \\
\hline $\begin{array}{l}26 \\
27 \\
28 \\
29 \\
30 \\
31\end{array}$ & $\begin{array}{l}11.66 \\
11.62 \\
11.64 \\
11.65 \\
11.66 \\
11.74\end{array}$ & $\begin{array}{r}11.95 \\
11.79 \\
11.12 \\
--- \\
--- \\
---\end{array}$ & $\begin{array}{l}10.94 \\
10.93 \\
10.95 \\
10.95 \\
10.87 \\
10.83\end{array}$ & $\begin{array}{c}10.78 \\
10.77 \\
10.78 \\
10.81 \\
10.82 \\
-\ldots\end{array}$ & $\begin{array}{l}11.40 \\
11.42 \\
11.44 \\
11.46 \\
11.48 \\
11.49\end{array}$ & $\begin{array}{c}12.02 \\
12.06 \\
12.11 \\
12.14 \\
12.16 \\
. .-\end{array}$ & $\begin{array}{l}12.89 \\
12.91 \\
12.95 \\
12.99 \\
13.01 \\
13.02\end{array}$ & $\begin{array}{l}13.29 \\
13.31 \\
13.34 \\
13.37 \\
13.40 \\
13.42\end{array}$ & $\begin{array}{r}13.81 \\
13.84 \\
13.86 \\
13.85 \\
13.86 \\
-.-\end{array}$ & $\begin{array}{l}14.24 \\
14.24 \\
14.25 \\
14.25 \\
14.25 \\
14.26\end{array}$ & $\begin{array}{r}14.24 \\
14.24 \\
14.23 \\
14.23 \\
14.21 \\
-.-\end{array}$ & $\begin{array}{l}14.22 \\
14.22 \\
14.16 \\
14.16 \\
14.16 \\
14.13\end{array}$ \\
\hline EN & 12.54 & 11.84 & 10.78 & 10.69 & 11.17 & 11.86 & 12.59 & 13.13 & 13.64 & 14.14 & 14.25 & 4. \\
\hline
\end{tabular}



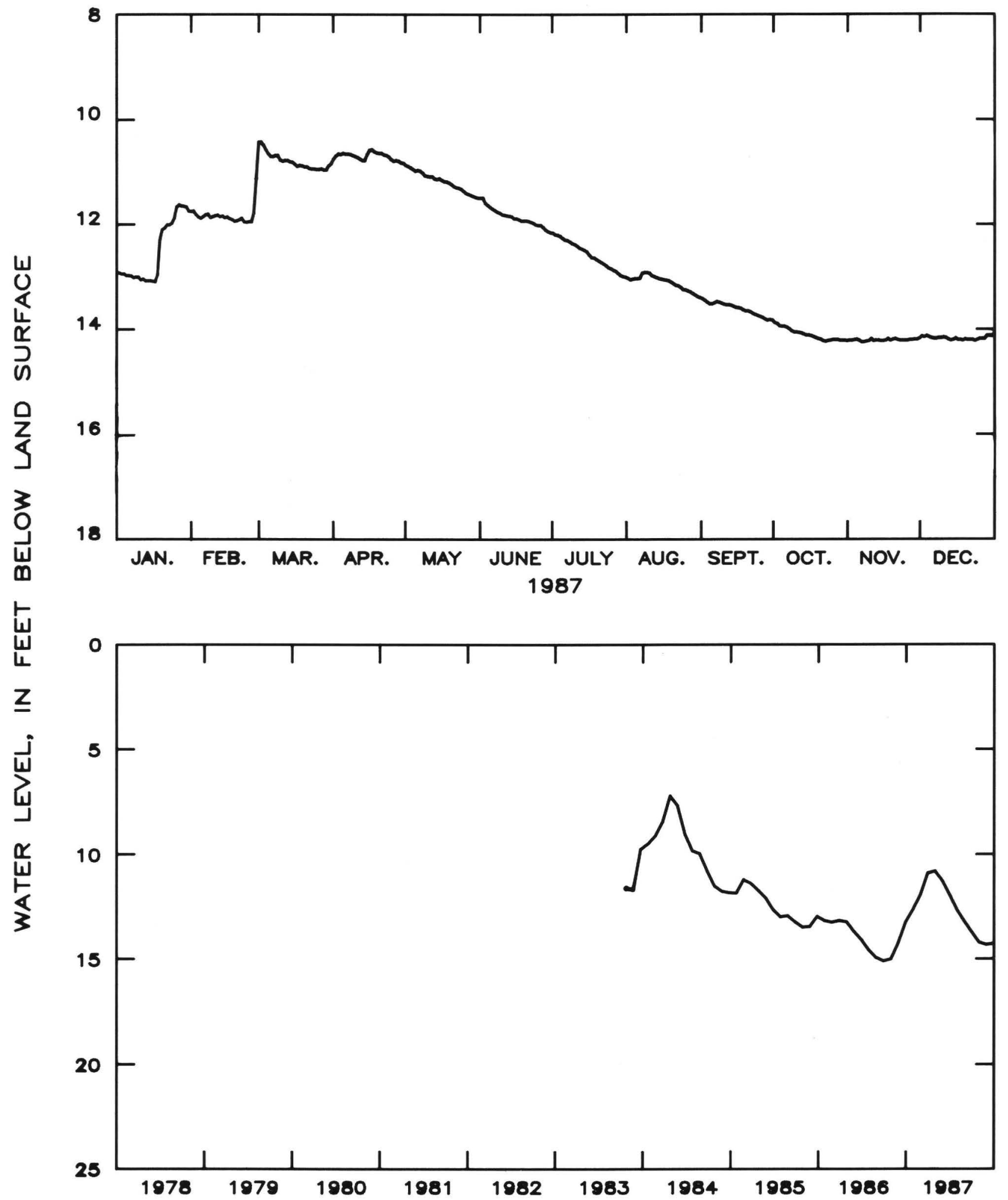

Figure 2.2-4.--Water level in observation well 19HH12, Madison County. 


\subsection{Water-Table Aquifers}

Shallow water-table aquifers are used for domestic and stock supplies in most areas of Georgia. In the Piedmont and the Blue Ridge provinces, the aquifers consist of residual soils derived from weathering of crystalline rocks. In the southwestern part of the Coastal Plain province, the aquifers consist of undifferentiated sand, clay, and limestone that range in thickness from less than $10 \mathrm{ft}$ to about $125 \mathrm{ft}$ (Hayes and others, 1983). Water-table aquifers in the Savannah area consist of sand, silt, and clay containing some shell and gravel beds.

Water-level fluctuations in these aquifers are caused mainly by changes in precipitation. Water levels generally rise rapidly during wet periods and decline slowly during dry periods. Prolonged droughts may cause water levels, particularly on hill tops and steep slopes, to decline below pump intakes in dug, bored, or shallow drilled wells, which result in temporary well failures. Generally, the well yields are restored with the return of precipitation.

The mean water levels in four wells tapping shallow water-table aquifers were from 0.8 to $2.8 \mathrm{ft}$ higher in 1987 than in 1986. During 1987, the mean water level in well 11AA01 in Spalding County in the Piedmont province was about $2.3 \mathrm{ft}$ higher than in 1986. By the end of March, the water level in well 11AA01 had recovered $9.2 \mathrm{ft}$ from the record low measured during the 1986 drought. Although there was some recovery, at the end of 1987 the water level was $3.0 \mathrm{ft}$ lower than at the end of 1986.

In the southwestern part of the Coastal Plain province (Dougherty Plain), the mean water levels in wells 13 M007 in Worth County and 07H003 in Miller County were from 0.8 to $2.8 \mathrm{ft}$ higher in 1987 than in 1986. In the Coastal Plain province near Savannah, the mean water level in well 35 P094 was about $2.4 \mathrm{ft}$ higher in 1987 than in 1986. In early 1987, water levels in the three wells had recovered 7.5 to $17.0 \mathrm{ft}$ from the lows measured during the 1986 drought. Although there was some recovery, water levels at the end of 1987 were from 2.2 to $4.8 \mathrm{ft}$ lower than at the end of 1986 . 


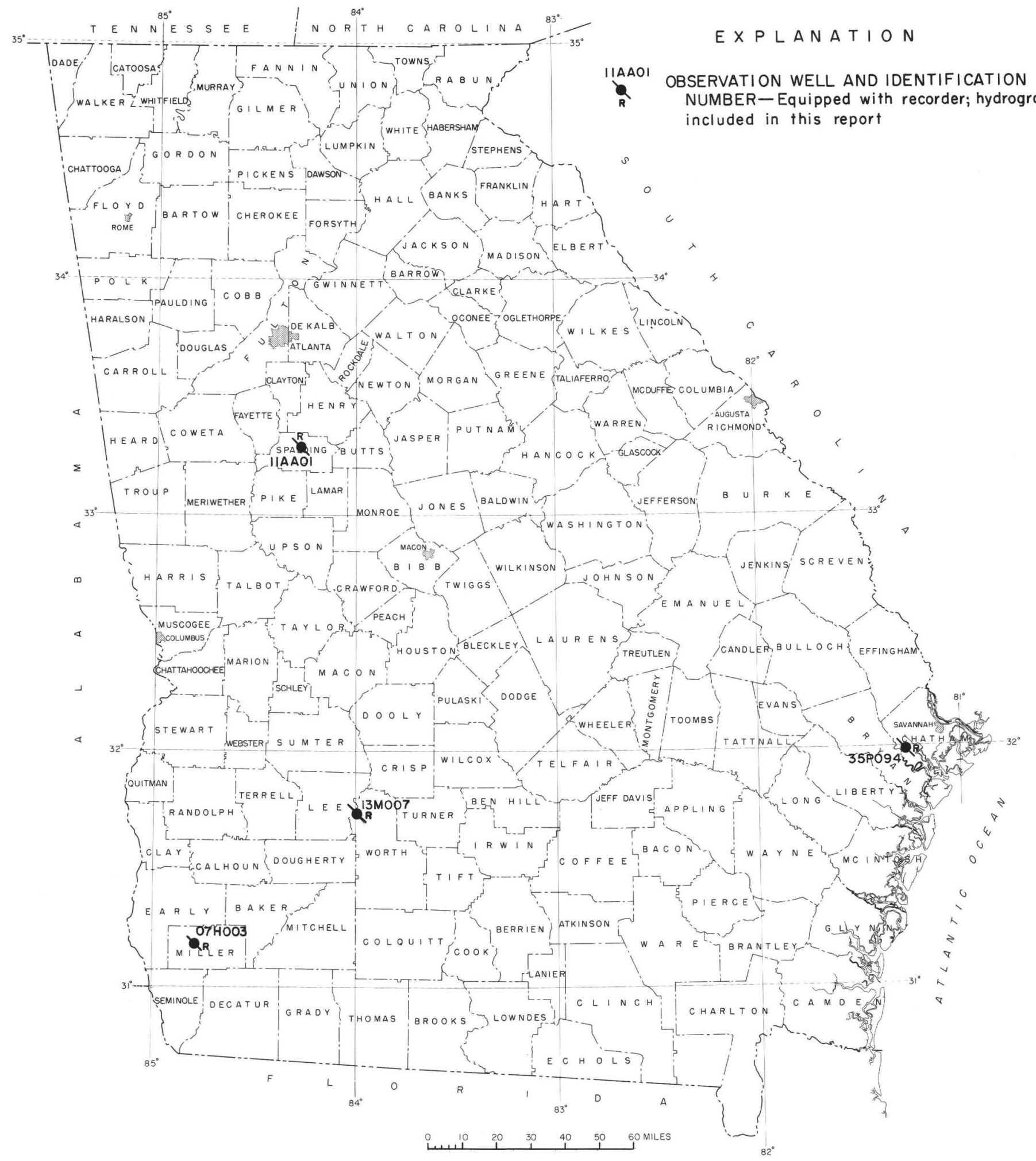

Figure 2.3-1.- Locations of observation wells in the water-table aquifers. 
331507084171801 Local number, 11AA01.

LOCATION.--Lat $33^{\circ} 15^{\prime} 54^{\prime \prime}$, long $84^{\circ} 16^{\prime} 56^{\prime \prime}$, Hydrologic Unit 03070103, University of Georgia Experiment Station, Experiment, Ga.

Owner: University of Georgia.

AQUIFER.--Residuum.

WELL CHARACTERISTICS.--Dug unused water-table well, size $4 \times 4 \mathrm{ft}$, depth $30 \mathrm{ft}$, open hole. DATUM.--Elevation of land-surface datum is $960 \mathrm{ft}$.

Measuring point: Hole in floor of recorder sheiter, $3.1 \mathrm{ft}$ above land-surface datum.

REMARKS.--Water level for period of missing record, September 28, was estimated.

PERIOD OF RECORD.--October 1943 to current year

EXTREMES FOR PERIOD OF RECORD.---Highest water leve1, $8.26 \mathrm{ft}$ below land-surface datum, March 19, 1948; 1owest, $21.82 \mathrm{ft}$ below land-surface datum, November $18-19,1986$.

DEPTH BELOW LAND SURFACE (WATER LEVEL) (FEET) CALENDAR YEAR JANUARY 1987 TO DECEMBER 1987
MEAN VALUES

\begin{tabular}{|c|c|c|c|c|c|c|c|c|c|c|c|c|}
\hline DAY & JAN & FEB & MAR & APR & MAY & JUN & JUL & AUG & SEP & OCT & NOV & $\mathrm{DE}$ \\
\hline $\begin{array}{l}1 \\
2 \\
3 \\
4 \\
5\end{array}$ & $\begin{array}{l}17.75 \\
17.77 \\
17.77 \\
17.72 \\
17.74\end{array}$ & $\begin{array}{l}15.95 \\
15.84 \\
15.90 \\
15.92 \\
15.91\end{array}$ & $\begin{array}{l}14.25 \\
14.07 \\
13.92 \\
13.83 \\
13.77\end{array}$ & $\begin{array}{l}12.93 \\
12.73 \\
12.64 \\
12.66 \\
12.61\end{array}$ & $\begin{array}{l}13.74 \\
13.80 \\
13.86 \\
13.91 \\
13.98\end{array}$ & $\begin{array}{l}15.14 \\
15.20 \\
15.27 \\
15.31 \\
15.38\end{array}$ & $\begin{array}{l}15.82 \\
15.82 \\
15.83 \\
15.84 \\
15.85\end{array}$ & $\begin{array}{l}16.90 \\
16.94 \\
17.00 \\
17.03 \\
17.06\end{array}$ & $\begin{array}{l}17.76 \\
17.80 \\
17.85 \\
17.90 \\
17.94\end{array}$ & $\begin{array}{l}19.31 \\
19.35 \\
19.40 \\
19.44 \\
19.45\end{array}$ & $\begin{array}{l}20.17 \\
20.19 \\
20.23 \\
20.25 \\
20.30\end{array}$ & $\begin{array}{l}20.7 \\
20.8 \\
20.8 \\
20.8 \\
20.8\end{array}$ \\
\hline $\begin{array}{r}6 \\
7 \\
8 \\
9 \\
10\end{array}$ & $\begin{array}{l}17.74 \\
17.71 \\
17.72 \\
17.71 \\
17.68\end{array}$ & $\begin{array}{l}15.82 \\
15.73 \\
15.61 \\
15.66 \\
15.57\end{array}$ & $\begin{array}{l}13.73 \\
13.61 \\
13.48 \\
13.46 \\
13.56\end{array}$ & $\begin{array}{l}12.62 \\
12.62 \\
12.64 \\
12.65 \\
12.68\end{array}$ & $\begin{array}{l}13.98 \\
13.99 \\
14.04 \\
14.15 \\
14.17\end{array}$ & $\begin{array}{l}15.44 \\
15.51 \\
15.55 \\
15.59 \\
15.64\end{array}$ & $\begin{array}{l}15.86 \\
15.85 \\
15.87 \\
15.89 \\
15.90\end{array}$ & $\begin{array}{l}17.11 \\
17.05 \\
16.96 \\
16.90 \\
16.87\end{array}$ & $\begin{array}{l}17.98 \\
18.01 \\
18.05 \\
18.10 \\
18.15\end{array}$ & $\begin{array}{l}19.47 \\
19.53 \\
19.61 \\
19.66 \\
19.68\end{array}$ & $\begin{array}{l}20.36 \\
20.39 \\
20.42 \\
20.45 \\
20.46\end{array}$ & $\begin{array}{l}20.9 \\
20.9 \\
20.9 \\
20.9 \\
20.9\end{array}$ \\
\hline $\begin{array}{l}11 \\
12 \\
13 \\
14 \\
15\end{array}$ & $\begin{array}{l}17.76 \\
17.75 \\
17.76 \\
17.77 \\
17.76\end{array}$ & $\begin{array}{l}15.46 \\
15.38 \\
15.37 \\
15.32 \\
15.33\end{array}$ & $\begin{array}{l}13.60 \\
13.56 \\
13.50 \\
13.49 \\
13.49\end{array}$ & $\begin{array}{l}12.72 \\
12.79 \\
12.85 \\
12.86 \\
12.82\end{array}$ & $\begin{array}{l}14.19 \\
14.19 \\
14.25 \\
14.31 \\
14.32\end{array}$ & $\begin{array}{l}15.72 \\
15.76 \\
15.80 \\
15.83 \\
15.87\end{array}$ & $\begin{array}{l}15.91 \\
15.94 \\
15.97 \\
16.01 \\
16.07\end{array}$ & $\begin{array}{l}16.85 \\
16.87 \\
16.91 \\
16.96 \\
16.99\end{array}$ & $\begin{array}{l}18.19 \\
18.21 \\
18.25 \\
18.29 \\
18.31\end{array}$ & $\begin{array}{l}19.70 \\
19.73 \\
19.80 \\
19.85 \\
19.89\end{array}$ & $\begin{array}{l}20.53 \\
20.56 \\
20.58 \\
20.62 \\
20.66\end{array}$ & $\begin{array}{l}20.9 \\
20.9 \\
21.0 \\
21.0 \\
21.0\end{array}$ \\
\hline $\begin{array}{l}16 \\
17 \\
18 \\
19 \\
20\end{array}$ & $\begin{array}{l}17.78 \\
17.81 \\
17.75 \\
17.67 \\
17.63\end{array}$ & $\begin{array}{l}15.30 \\
15.37 \\
15.40 \\
15.47 \\
15.45\end{array}$ & $\begin{array}{l}13.51 \\
13.56 \\
13.52 \\
13.50 \\
13.52\end{array}$ & $\begin{array}{l}12.84 \\
12.92 \\
13.05 \\
13.14 \\
13.18\end{array}$ & $\begin{array}{l}14.36 \\
14.41 \\
14.44 \\
14.47 \\
14.53\end{array}$ & $\begin{array}{l}15.90 \\
15.94 \\
15.97 \\
15.96 \\
15.97\end{array}$ & $\begin{array}{l}16.13 \\
16.18 \\
16.22 \\
16.25 \\
16.30\end{array}$ & $\begin{array}{l}17.03 \\
17.07 \\
17.11 \\
17.14 \\
17.19\end{array}$ & $\begin{array}{l}18.34 \\
18.37 \\
18.40 \\
18.43 \\
18.48\end{array}$ & $\begin{array}{l}19.93 \\
19.96 \\
20.01 \\
20.04 \\
20.09\end{array}$ & $\begin{array}{l}20.69 \\
20.70 \\
20.70 \\
20.69 \\
20.65\end{array}$ & $\begin{array}{l}21.0 \\
21.0 \\
21.0 \\
21.1 \\
21.1\end{array}$ \\
\hline $\begin{array}{l}21 \\
22 \\
23 \\
24 \\
25\end{array}$ & $\begin{array}{l}17.50 \\
17.30 \\
17.25 \\
17.10 \\
16.82\end{array}$ & $\begin{array}{l}15.38 \\
15.33 \\
15.42 \\
15.42 \\
15.40\end{array}$ & $\begin{array}{l}13.51 \\
13.55 \\
13.58 \\
13.58 \\
13.64\end{array}$ & $\begin{array}{l}13.18 \\
13.21 \\
13.24 \\
13.31 \\
13.40\end{array}$ & $\begin{array}{l}14.60 \\
14.66 \\
14.69 \\
14.72 \\
14.77\end{array}$ & $\begin{array}{l}15.92 \\
15.86 \\
15.83 \\
15.81 \\
15.80\end{array}$ & $\begin{array}{l}16.34 \\
16.37 \\
16.42 \\
16.48 \\
16.53\end{array}$ & $\begin{array}{l}17.25 \\
17.28 \\
17.30 \\
17.35 \\
17.41\end{array}$ & $\begin{array}{l}18.53 \\
18.58 \\
18.60 \\
18.62 \\
18.67\end{array}$ & $\begin{array}{l}20.15 \\
20.21 \\
20.24 \\
20.29 \\
20.31\end{array}$ & $\begin{array}{l}20.66 \\
20.67 \\
20.68 \\
20.71 \\
20.71\end{array}$ & $\begin{array}{l}21.1 \\
21.1 \\
21.0 \\
21.0 \\
21.0\end{array}$ \\
\hline $\begin{array}{l}26 \\
27 \\
28 \\
29 \\
30 \\
31\end{array}$ & $\begin{array}{l}16.67 \\
16.51 \\
16.34 \\
16.20 \\
16.04 \\
16.03\end{array}$ & $\begin{array}{r}15.38 \\
15.18 \\
14.74 \\
-.- \\
-.-\end{array}$ & $\begin{array}{l}13.66 \\
13.58 \\
13.53 \\
13.43 \\
13.22 \\
13.11\end{array}$ & $\begin{array}{r}13.50 \\
13.50 \\
13.56 \\
13.69 \\
13.68 \\
---\end{array}$ & $\begin{array}{l}14.83 \\
14.89 \\
14.94 \\
14.99 \\
15.03 \\
15.09\end{array}$ & $\begin{array}{r}15.79 \\
15.82 \\
15.88 \\
15.86 \\
15.82 \\
-\ldots\end{array}$ & $\begin{array}{l}16.57 \\
16.62 \\
16.64 \\
16.74 \\
16.84 \\
16.88\end{array}$ & $\begin{array}{l}17.45 \\
17.49 \\
17.56 \\
17.61 \\
17.66 \\
17.71\end{array}$ & $\begin{array}{r}18.73 \\
18.77 \\
18.99 \\
19.22 \\
19.24 \\
---\end{array}$ & $\begin{array}{l}20.19 \\
19.94 \\
20.00 \\
20.05 \\
20.09 \\
20.13\end{array}$ & $\begin{array}{r}20.72 \\
20.74 \\
20.76 \\
20.77 \\
20.77 \\
.\end{array}$ & $\begin{array}{l}20.98 \\
20.98 \\
20.95 \\
20.88 \\
20.85 \\
20.78\end{array}$ \\
\hline MEAN & 17.37 & 15.50 & 13.59 & 13.01 & 14.40 & 15.70 & 16.19 & 17.16 & 18.36 & 19.85 & 20.56 & 20.9 \\
\hline CAL $Y$ & & & & $\mathrm{HIGH}$ & 12.61 & & & & & & & \\
\hline
\end{tabular}



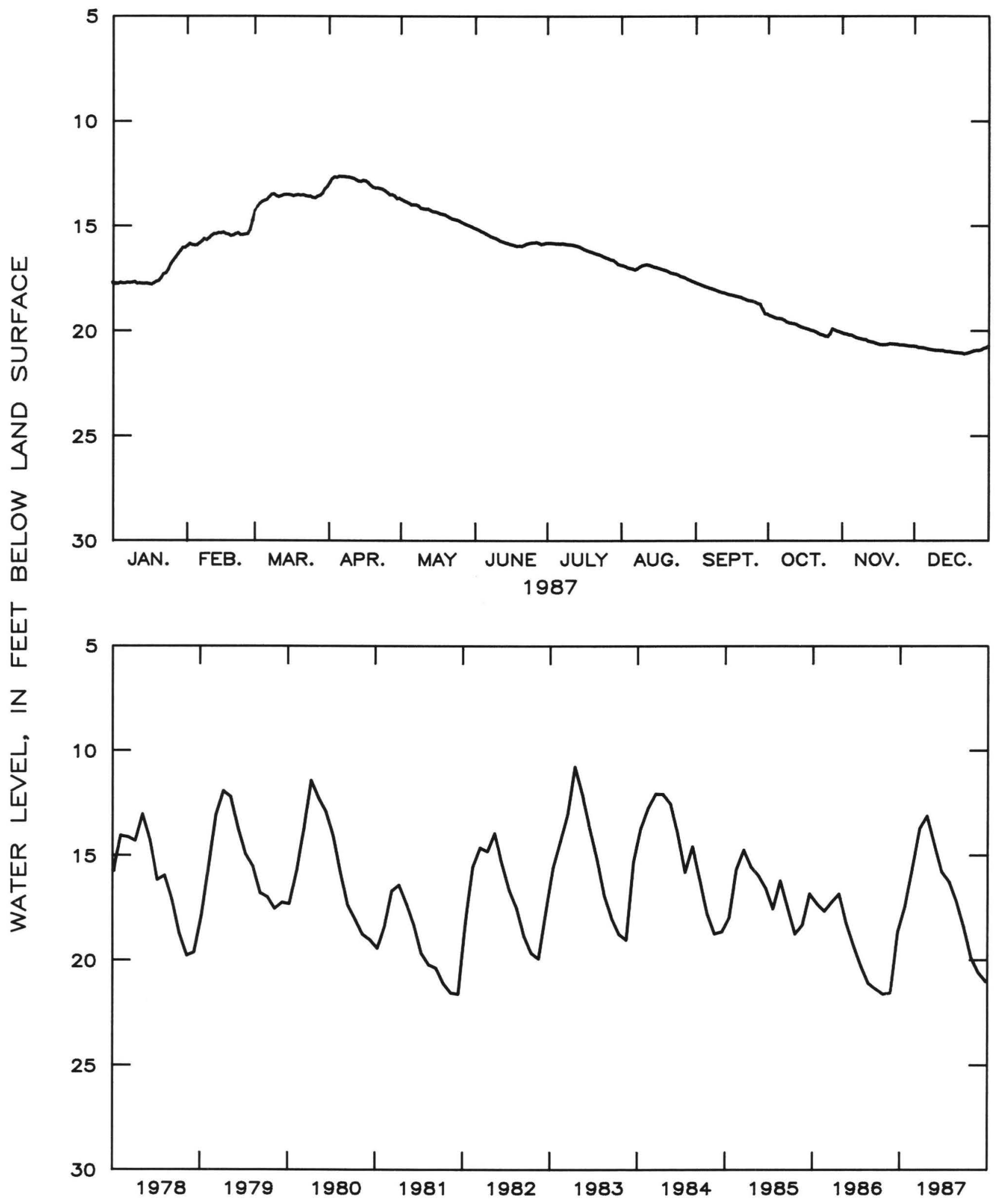

Figure 2.3-2.--Water level in observation well 11AA01, Spalding County. 
314330084005403 Local number, 13 M007.

LOCATION.--Lat $31^{\circ} 43^{\prime} 30^{\prime \prime}$, long $84^{\circ} 00^{\prime} 54^{\prime \prime}$, Hydrologic Unit $03130006,1,400 \mathrm{ft}$ east of the Flint River on the north side of State Highway 32.

Owner: U.S. Geological Survey, test well DP-9.

AQUIFER.---Eocene residuum.

WELL CHARACTERISTICS.--Drilled observation well, diameter $4 \mathrm{in}$., depth $25 \mathrm{ft}$, cased to $10 \mathrm{ft}$, open hole. DATUM.--Elevation of land-surface datum is $230 \mathrm{ft}$.

Measuring point: Top of 4-in. casing, $1 \mathrm{ft}$ above land-surface datum.

REMARKS.--Water level for period of missing record, November 24, was estimated.

PERIOD OF RECORD.--April 1980 to current year.

EXTREMES FOR PERIOD OF RECORD.--Highest water level, $3.48 \mathrm{ft}$ below land-surface datum, March 7, 1984; lowest, $13.03 \mathrm{ft}$ below land-surface datum, October 22, 1981 .

DEPTH BELOW LAND SURFACE (WATER LEVEL) (FEET) CALENDAR YEAR JANUARY 1987 TO DECEMBER 1987 MEAN VALUES

\begin{tabular}{|c|c|c|c|c|c|c|c|c|c|c|c|c|}
\hline DAY & JAN & FEB & MAR & APR & MAY & JUN & JUL & AUG & SEP & OCT & NOV & DEC \\
\hline $\begin{array}{l}1 \\
2 \\
3 \\
4 \\
5\end{array}$ & $\begin{array}{l}7.36 \\
7.39 \\
7.36 \\
7.28 \\
7.24\end{array}$ & $\begin{array}{l}5.25 \\
5.26 \\
5.32 \\
5.35 \\
5.37\end{array}$ & $\begin{array}{l}4.79 \\
4.68 \\
4.69 \\
4.74 \\
4.78\end{array}$ & $\begin{array}{l}4.82 \\
4.79 \\
4.79 \\
4.84 \\
4.86\end{array}$ & $\begin{array}{l}5.95 \\
6.00 \\
6.05 \\
6.11 \\
6.16\end{array}$ & $\begin{array}{l}7.34 \\
7.39 \\
7.44 \\
7.48 \\
7.53\end{array}$ & $\begin{array}{l}7.11 \\
7.15 \\
7.19 \\
7.21 \\
7.12\end{array}$ & $\begin{array}{l}8.18 \\
8.23 \\
8.29 \\
8.33 \\
8.37\end{array}$ & $\begin{array}{l}9.28 \\
9.27 \\
9.28 \\
9.30 \\
9.30\end{array}$ & $\begin{array}{l}10.10 \\
10.15 \\
10.19 \\
10.22 \\
10.23\end{array}$ & $\begin{array}{l}11.11 \\
11.14 \\
11.16 \\
11.18 \\
11.21\end{array}$ & $\begin{array}{l}11.57 \\
11.59 \\
11.60 \\
11.61 \\
11.62\end{array}$ \\
\hline $\begin{array}{r}6 \\
7 \\
8 \\
9 \\
10\end{array}$ & $\begin{array}{l}7.13 \\
7.01 \\
6.95 \\
6.90 \\
6.87\end{array}$ & $\begin{array}{l}5.35 \\
5.32 \\
5.16 \\
5.16 \\
5.16\end{array}$ & $\begin{array}{l}4.82 \\
4.80 \\
4.76 \\
4.69 \\
4.72\end{array}$ & $\begin{array}{l}4.90 \\
4.94 \\
4.99 \\
5.03 \\
5.07\end{array}$ & $\begin{array}{l}6.20 \\
6.24 \\
6.28 \\
6.35 \\
6.39\end{array}$ & $\begin{array}{l}7.57 \\
7.63 \\
7.67 \\
7.71 \\
7.77\end{array}$ & $\begin{array}{l}7.08 \\
7.05 \\
7.07 \\
7.11 \\
7.16\end{array}$ & $\begin{array}{l}8.42 \\
8.47 \\
8.52 \\
8.57 \\
8.61\end{array}$ & $\begin{array}{l}9.32 \\
9.34 \\
9.36 \\
9.39 \\
9.41\end{array}$ & $\begin{array}{l}10.26 \\
10.32 \\
10.38 \\
10.41 \\
10.43\end{array}$ & $\begin{array}{l}11.26 \\
11.27 \\
11.29 \\
11.30 \\
11.32\end{array}$ & $\begin{array}{l}11.63 \\
11.64 \\
11.65 \\
11.66 \\
11.66\end{array}$ \\
\hline $\begin{array}{l}11 \\
12 \\
13 \\
14 \\
15\end{array}$ & $\begin{array}{l}6.93 \\
6.92 \\
6.93 \\
6.96 \\
6.96\end{array}$ & $\begin{array}{l}5.15 \\
5.16 \\
5.21 \\
5.25 \\
5.29\end{array}$ & $\begin{array}{l}4.78 \\
4.81 \\
4.83 \\
4.86 \\
4.90\end{array}$ & $\begin{array}{l}5.12 \\
5.16 \\
5.20 \\
5.23 \\
5.24\end{array}$ & $\begin{array}{l}6.43 \\
6.46 \\
6.51 \\
6.57 \\
6.59\end{array}$ & $\begin{array}{l}7.82 \\
7.86 \\
7.89 \\
7.91 \\
7.93\end{array}$ & $\begin{array}{l}7.22 \\
7.26 \\
7.32 \\
7.38 \\
7.44\end{array}$ & $\begin{array}{l}8.65 \\
8.68 \\
8.72 \\
8.75 \\
8.77\end{array}$ & $\begin{array}{l}9.44 \\
9.47 \\
9.49 \\
9.52 \\
9.54\end{array}$ & $\begin{array}{l}10.43 \\
10.46 \\
10.51 \\
10.54 \\
10.55\end{array}$ & $\begin{array}{l}11.36 \\
11.38 \\
11.39 \\
11.41 \\
11.44\end{array}$ & $\begin{array}{l}11.67 \\
11.69 \\
11.73 \\
11.76 \\
11.78\end{array}$ \\
\hline $\begin{array}{l}16 \\
17 \\
18 \\
19 \\
20\end{array}$ & $\begin{array}{l}6.99 \\
6.94 \\
6.80 \\
6.43 \\
5.91\end{array}$ & $\begin{array}{l}5.30 \\
5.34 \\
5.36 \\
5.40 \\
5.41\end{array}$ & $\begin{array}{l}4.94 \\
4.98 \\
4.99 \\
4.96 \\
4.95\end{array}$ & $\begin{array}{l}5.27 \\
5.32 \\
5.37 \\
5.43 \\
5.46\end{array}$ & $\begin{array}{l}6.63 \\
6.66 \\
6.70 \\
6.75 \\
6.80\end{array}$ & $\begin{array}{l}7.87 \\
7.52 \\
7.32 \\
7.17 \\
7.10\end{array}$ & $\begin{array}{l}7.49 \\
7.54 \\
7.58 \\
7.63 \\
7.68\end{array}$ & $\begin{array}{l}8.79 \\
8.81 \\
8.83 \\
8.85 \\
8.90\end{array}$ & $\begin{array}{l}9.56 \\
9.59 \\
9.61 \\
9.65 \\
9.69\end{array}$ & $\begin{array}{l}10.57 \\
10.66 \\
10.69 \\
10.73 \\
10.75\end{array}$ & $\begin{array}{l}11.45 \\
11.46 \\
11.47 \\
11.44 \\
11.42\end{array}$ & $\begin{array}{l}11.84 \\
11.85 \\
11.87 \\
11.88 \\
11.89\end{array}$ \\
\hline $\begin{array}{l}21 \\
22 \\
23 \\
24 \\
25\end{array}$ & $\begin{array}{l}5.76 \\
5.45 \\
5.14 \\
5.08 \\
5.01\end{array}$ & $\begin{array}{l}5.39 \\
5.36 \\
5.32 \\
5.26 \\
5.23\end{array}$ & $\begin{array}{l}4.94 \\
4.97 \\
5.01 \\
5.03 \\
5.06\end{array}$ & $\begin{array}{l}5.49 \\
5.54 \\
5.59 \\
5.63 \\
5.67\end{array}$ & $\begin{array}{l}6.84 \\
6.88 \\
6.92 \\
6.96 \\
7.00\end{array}$ & $\begin{array}{l}7.05 \\
7.02 \\
6.99 \\
6.95 \\
6.92\end{array}$ & $\begin{array}{l}7.74 \\
7.78 \\
7.82 \\
7.86 \\
7.89\end{array}$ & $\begin{array}{l}8.93 \\
8.96 \\
8.98 \\
9.01 \\
9.06\end{array}$ & $\begin{array}{l}9.74 \\
9.77 \\
9.80 \\
9.83 \\
9.87\end{array}$ & $\begin{array}{l}10.79 \\
10.83 \\
10.86 \\
10.89 \\
10.92\end{array}$ & $\begin{array}{l}11.45 \\
11.46 \\
11.47 \\
11.49 \\
11.51\end{array}$ & $\begin{array}{l}11.90 \\
11.91 \\
11.92 \\
11.93 \\
11.94\end{array}$ \\
\hline $\begin{array}{l}26 \\
27 \\
28 \\
29 \\
30 \\
31\end{array}$ & $\begin{array}{l}5.05 \\
5.08 \\
5.09 \\
5.13 \\
5.14 \\
5.21\end{array}$ & $\begin{array}{c}5.23 \\
5.20 \\
5.12 \\
--- \\
--. \\
---\end{array}$ & $\begin{array}{l}5.07 \\
5.08 \\
5.12 \\
5.14 \\
4.99 \\
4.87\end{array}$ & $\begin{array}{r}5.74 \\
5.77 \\
5.81 \\
5.86 \\
5.90 \\
-.--\end{array}$ & $\begin{array}{l}7.05 \\
7.11 \\
7.16 \\
7.20 \\
7.24 \\
7.29\end{array}$ & $\begin{array}{l}6.91 \\
6.93 \\
6.97 \\
7.02 \\
7.06 \\
---\end{array}$ & $\begin{array}{l}7.93 \\
7.98 \\
8.01 \\
8.06 \\
8.12 \\
8.15\end{array}$ & $\begin{array}{l}9.11 \\
9.14 \\
9.18 \\
9.21 \\
9.25 \\
9.27\end{array}$ & $\begin{array}{r}9.93 \\
9.97 \\
10.00 \\
10.02 \\
10.04 \\
\ldots\end{array}$ & $\begin{array}{l}10.92 \\
10.94 \\
10.98 \\
11.01 \\
11.05 \\
11.08\end{array}$ & $\begin{array}{r}11.51 \\
11.53 \\
11.53 \\
11.54 \\
11.55 \\
-.-\end{array}$ & $\begin{array}{l}11.94 \\
11.95 \\
11.95 \\
11.96 \\
11.98 \\
11.98\end{array}$ \\
\hline MEAN & 6.34 & 5.27 & 4.90 & 5.29 & 6.63 & 7.39 & 7.52 & 8.77 & 9.59 & 10.61 & 11.38 & 11.7 \\
\hline
\end{tabular}

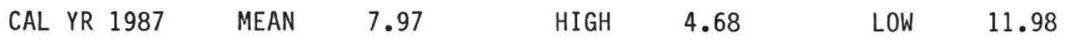



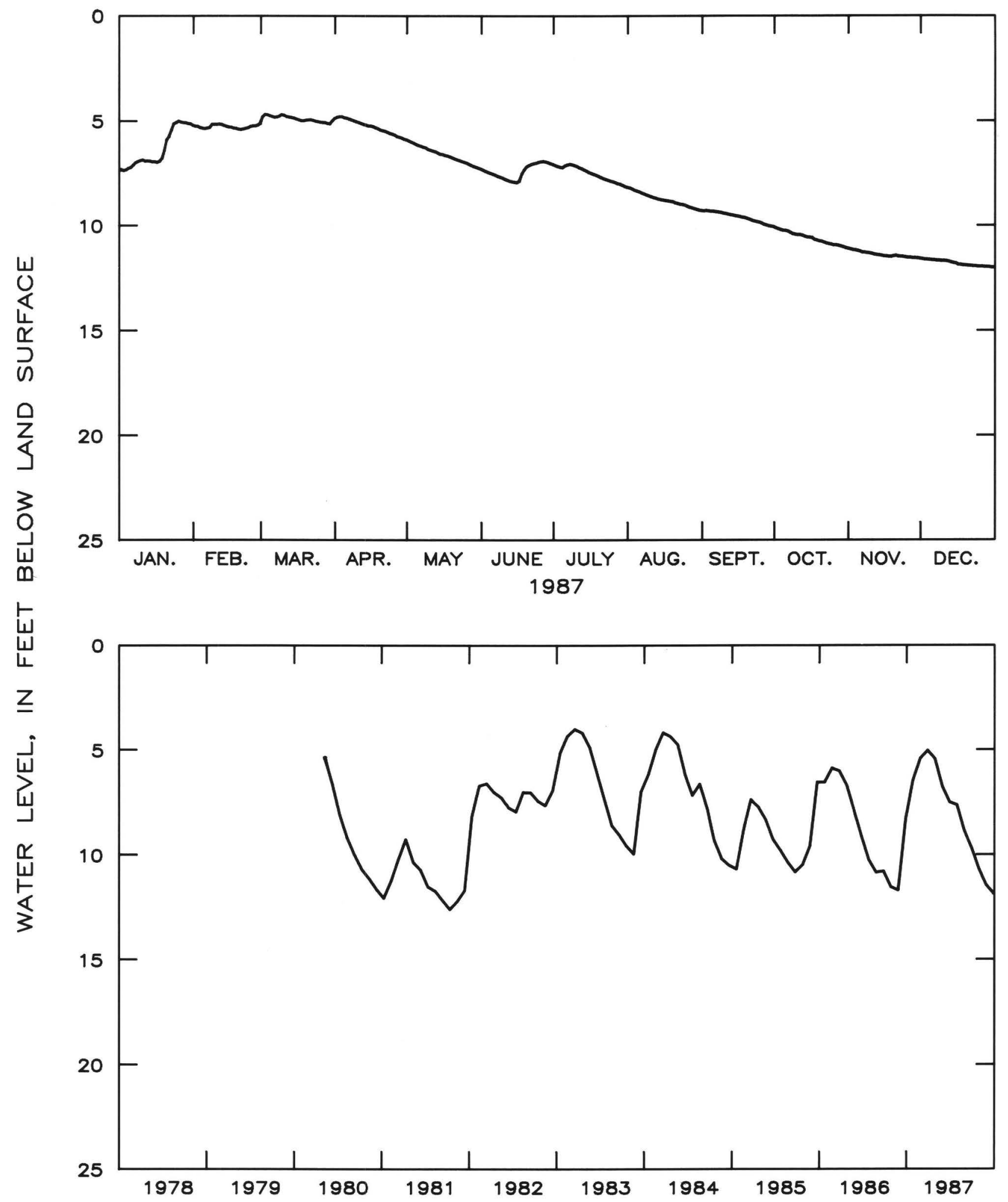

Figure 2.3-3.--Water level in observation well $13 \mathrm{M} 007$, Worth County. 
311009084495503 Local number, $07 \mathrm{H003}$

LOCATION.--Lat $31^{\circ} 10^{\prime} 08^{\prime \prime}$, long $84^{\circ} 49^{\prime} 54^{\prime \prime}$, Hydrologic Unit 03130010 , 0.2 mi north on dirt road off Georgia Highway $273,2.75 \mathrm{mi}$ west of intersection of Georgia Highways 273 and 91 .

Owner: U.S. Geological Survey

AQUIFER.--Residuum.

WELL CHARACTERISTICS.--Drilled observation well, diameter 4 in., depth $40 \mathrm{ft}$, perforated casing 30 to $40 \mathrm{ft}$. DATUM.--Elevation of land-surface datum is $180 \mathrm{ft}$.

Measuring point: Floor of recorder shelter, $3.0 \mathrm{ft}$ above land-surface datum.

REMARKS.--None.

PERIOD OF RECORD.--February 1980 to current year.

EXTREMES FOR PERIOD OF RECORD.--Highest water level, $0.52 \mathrm{ft}$ below land-surface datum, March 6 , 1984 ; lowest, $24.19 \mathrm{ft}$ below land-surface datum, November $10,1981$.

DEPTH BELOW LAND SURFACE (WATER LEVEL) (FEET) CALENDAR YEAR JANUARY 1987 TO DECEMBER 1987 MEAN VALUES

\begin{tabular}{|c|c|c|c|c|c|c|c|c|c|c|c|c|}
\hline DAY & JAN & FEB & MAR & APR & MAY & JUN & JUL & $A \cup G$ & SEP & OCT & NOV & $\mathrm{DEC}$ \\
\hline $\begin{array}{l}1 \\
2 \\
3 \\
4 \\
5\end{array}$ & $\begin{array}{l}9.71 \\
9.84 \\
9.56 \\
7.86 \\
4.48\end{array}$ & $\begin{array}{l}3.70 \\
3.74 \\
3.38 \\
3.55 \\
3.79\end{array}$ & $\begin{array}{l}2.19 \\
2.59 \\
2.92 \\
3.20 \\
3.43\end{array}$ & $\begin{array}{l}2.19 \\
2.55 \\
2.80 \\
2.97 \\
3.21\end{array}$ & $\begin{array}{l}8.15 \\
8.41 \\
8.69 \\
8.94 \\
9.23\end{array}$ & $\begin{array}{l}7.94 \\
8.23 \\
8.56 \\
8.85 \\
8.98\end{array}$ & $\begin{array}{l}5.50 \\
5.79 \\
5.81 \\
4.57 \\
2.53\end{array}$ & $\begin{array}{l}4.45 \\
4.49 \\
4.71 \\
5.00 \\
5.44\end{array}$ & $\begin{array}{l}11.25 \\
11.21 \\
11.19 \\
11.23 \\
11.24\end{array}$ & $\begin{array}{l}11.44 \\
11.56 \\
11.65 \\
11.76 \\
11.79\end{array}$ & $\begin{array}{l}13.86 \\
13.93 \\
13.98 \\
14.03 \\
14.08\end{array}$ & $\begin{array}{l}15.05 \\
14.96 \\
14.87 \\
14.76 \\
14.65\end{array}$ \\
\hline $\begin{array}{r}6 \\
7 \\
8 \\
9 \\
10\end{array}$ & $\begin{array}{l}4.90 \\
5.77 \\
6.82 \\
7.69 \\
8.29\end{array}$ & $\begin{array}{l}3.87 \\
3.55 \\
3.14 \\
3.39 \\
3.66\end{array}$ & $\begin{array}{l}3.64 \\
3.77 \\
3.32 \\
3.18 \\
3.44\end{array}$ & $\begin{array}{l}3.43 \\
3.63 \\
3.82 \\
3.99 \\
4.16\end{array}$ & $\begin{array}{r}9.60 \\
10.01 \\
9.11 \\
8.63 \\
8.80\end{array}$ & $\begin{array}{r}9.20 \\
9.49 \\
9.83 \\
10.21 \\
10.62\end{array}$ & $\begin{array}{l}2.90 \\
3.33 \\
3.70 \\
4.02 \\
4.30\end{array}$ & $\begin{array}{l}5.93 \\
6.39 \\
6.81 \\
7.19 \\
7.57\end{array}$ & $\begin{array}{l}11.29 \\
11.34 \\
11.35 \\
11.38 \\
11.40\end{array}$ & $\begin{array}{l}11.82 \\
11.90 \\
12.03 \\
12.15 \\
12.22\end{array}$ & $\begin{array}{l}14.17 \\
14.29 \\
14.38 \\
14.45 \\
14.50\end{array}$ & $\begin{array}{l}14.57 \\
14.50 \\
14.43 \\
14.38 \\
14.34\end{array}$ \\
\hline $\begin{array}{l}11 \\
12 \\
13 \\
14 \\
15\end{array}$ & $\begin{array}{l}8.92 \\
9.20 \\
9.34 \\
9.51 \\
9.70\end{array}$ & $\begin{array}{l}3.88 \\
4.06 \\
4.25 \\
4.41 \\
4.57\end{array}$ & $\begin{array}{l}3.70 \\
3.84 \\
3.89 \\
4.00 \\
4.15\end{array}$ & $\begin{array}{l}4.33 \\
4.51 \\
4.68 \\
4.83 \\
4.95\end{array}$ & $\begin{array}{l}9.00 \\
9.17 \\
9.36 \\
9.52 \\
8.25\end{array}$ & $\begin{array}{r}10.97 \\
11.24 \\
11.19 \\
9.39 \\
6.73\end{array}$ & $\begin{array}{l}4.58 \\
4.85 \\
5.14 \\
5.51 \\
5.70\end{array}$ & $\begin{array}{l}7.96 \\
8.38 \\
8.52 \\
8.31 \\
8.14\end{array}$ & $\begin{array}{r}11.41 \\
11.11 \\
8.66 \\
7.79 \\
8.13\end{array}$ & $\begin{array}{l}12.25 \\
12.27 \\
12.34 \\
12.47 \\
12.57\end{array}$ & $\begin{array}{l}14.57 \\
14.66 \\
14.73 \\
14.79 \\
14.85\end{array}$ & $\begin{array}{l}14.30 \\
14.26 \\
14.25 \\
14.26 \\
14.24\end{array}$ \\
\hline $\begin{array}{l}16 \\
17 \\
18 \\
19 \\
20\end{array}$ & $\begin{array}{l}8.83 \\
3.28 \\
3.31 \\
1.84 \\
2.22\end{array}$ & $\begin{array}{l}4.56 \\
4.48 \\
4.53 \\
4.67 \\
4.79\end{array}$ & $\begin{array}{l}4.29 \\
4.43 \\
3.91 \\
2.37 \\
2.73\end{array}$ & $\begin{array}{l}5.08 \\
5.25 \\
5.45 \\
5.64 \\
5.80\end{array}$ & $\begin{array}{l}6.74 \\
6.16 \\
5.75 \\
5.53 \\
5.52\end{array}$ & $\begin{array}{l}5.65 \\
4.71 \\
2.88 \\
2.36 \\
2.79\end{array}$ & $\begin{array}{l}5.96 \\
6.28 \\
6.59 \\
6.88 \\
7.15\end{array}$ & $\begin{array}{l}8.17 \\
8.21 \\
8.21 \\
8.46 \\
8.84\end{array}$ & $\begin{array}{r}8.75 \\
9.12 \\
9.44 \\
9.75 \\
10.02\end{array}$ & $\begin{array}{l}12.64 \\
12.70 \\
12.77 \\
12.83 \\
12.92\end{array}$ & $\begin{array}{l}14.91 \\
14.90 \\
14.78 \\
14.73 \\
14.74\end{array}$ & $\begin{array}{l}14.21 \\
14.22 \\
14.20 \\
14.14 \\
14.06\end{array}$ \\
\hline $\begin{array}{l}21 \\
22 \\
23 \\
24 \\
25\end{array}$ & $\begin{array}{l}2.46 \\
1.08 \\
1.71 \\
2.05 \\
2.12\end{array}$ & $\begin{array}{l}4.45 \\
2.69 \\
1.98 \\
2.37 \\
2.63\end{array}$ & $\begin{array}{l}3.08 \\
3.37 \\
3.61 \\
3.81 \\
3.81\end{array}$ & $\begin{array}{l}5.96 \\
6.15 \\
6.34 \\
6.53 \\
6.76\end{array}$ & $\begin{array}{l}5.63 \\
5.82 \\
5.83 \\
5.91 \\
6.12\end{array}$ & $\begin{array}{l}3.19 \\
3.43 \\
2.85 \\
3.16 \\
3.56\end{array}$ & $\begin{array}{l}7.41 \\
7.65 \\
7.90 \\
8.23 \\
8.57\end{array}$ & $\begin{array}{r}9.25 \\
9.71 \\
10.12 \\
10.43 \\
10.63\end{array}$ & $\begin{array}{l}10.27 \\
10.47 \\
10.63 \\
10.75 \\
10.87\end{array}$ & $\begin{array}{l}13.01 \\
13.12 \\
13.20 \\
13.28 \\
13.34\end{array}$ & $\begin{array}{l}14.78 \\
14.84 \\
14.91 \\
14.98 \\
15.04\end{array}$ & $\begin{array}{l}13.98 \\
13.87 \\
13.78 \\
13.70 \\
13.59\end{array}$ \\
\hline $\begin{array}{l}26 \\
27 \\
28 \\
29 \\
30 \\
31\end{array}$ & $\begin{array}{l}2.35 \\
2.63 \\
2.88 \\
3.15 \\
3.35 \\
3.51\end{array}$ & $\begin{array}{r}2.60 \\
2.52 \\
2.62 \\
-.- \\
-.- \\
-.-\end{array}$ & $\begin{array}{l}3.72 \\
3.85 \\
3.86 \\
3.57 \\
1.72 \\
1.70\end{array}$ & $\begin{array}{l}7.00 \\
7.18 \\
7.38 \\
7.66 \\
7.91 \\
-.--\end{array}$ & $\begin{array}{l}6.35 \\
6.61 \\
6.86 \\
7.11 \\
7.37 \\
7.67\end{array}$ & $\begin{array}{r}3.90 \\
4.23 \\
4.57 \\
4.88 \\
5.18 \\
\ldots .-\end{array}$ & $\begin{array}{r}8.88 \\
9.20 \\
9.59 \\
10.06 \\
9.84 \\
5.43\end{array}$ & $\begin{array}{l}10.78 \\
10.89 \\
11.00 \\
11.11 \\
11.22 \\
11.31\end{array}$ & $\begin{array}{r}10.98 \\
11.10 \\
11.24 \\
11.30 \\
11.34 \\
\ldots\end{array}$ & $\begin{array}{l}13.38 \\
13.42 \\
13.50 \\
13.61 \\
13.70 \\
13.79\end{array}$ & $\begin{array}{r}15.09 \\
15.12 \\
15.16 \\
15.13 \\
15.11 \\
\ldots\end{array}$ & $\begin{array}{l}13.48 \\
13.38 \\
13.25 \\
13.05 \\
12.63 \\
11.96\end{array}$ \\
\hline MEAN & 5.43 & 3.64 & 3.39 & 5.07 & 7.61 & 6.63 & 6.25 & 8.31 & 10.53 & 12.63 & 14.65 & 14.04 \\
\hline
\end{tabular}



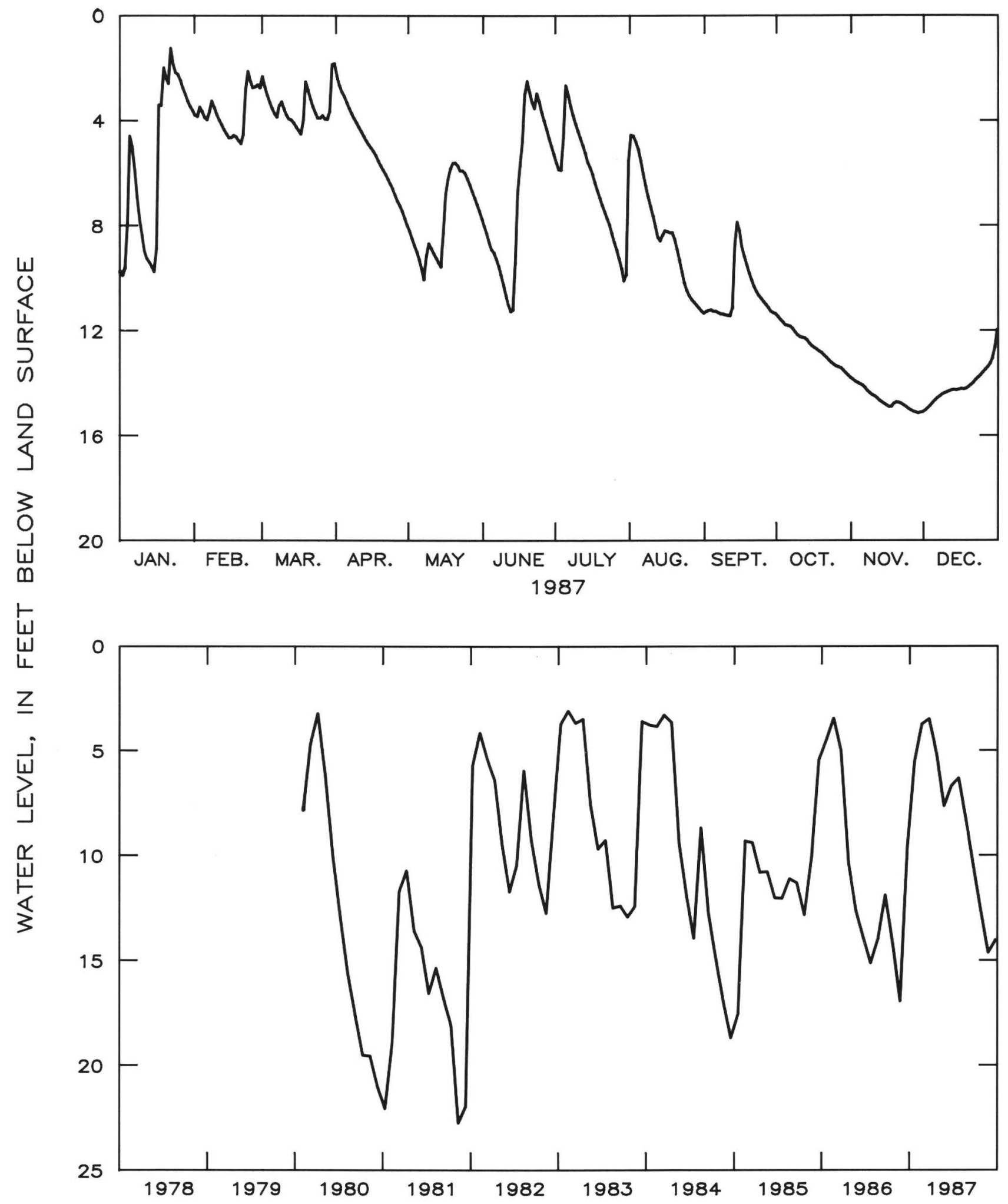

Figure 2.3-4.--Water level in observation well 07H0O3, Miller County. 
315950081161201 Local number, 35P094.

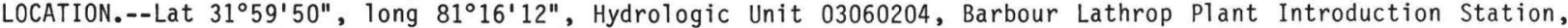
10 miles south of Savannah, north of the intersection of U.S. Highway 17 and Argyle Rd.

Owner: University of Georgia, formerly U.S. Department of Agriculture.

AQUIFER.--Sands of Holocene and Pleistocene age.

WELL CHARACTERISTICS.---Bored observation we11, diameter $30 \mathrm{in}$., depth $15 \mathrm{ft}$, cased to $15 \mathrm{ft}$, open end. DATUM.--Elevation of land-surface datum is $18.67 \mathrm{ft}$.

Measuring point: Iron bracket on recorder shelter, $3.3 \mathrm{ft}$ above land-surface datum.

REMARKS.--Responds quickly to precipitation. Water level for period of missing record, September 2, was estimated. PERIOD OF RECORD.--August 1942 to current year.

EXTREMES FOR PERIOD OF RECORD.--Highest water level, $0.05 \mathrm{ft}$ below land-surface datum, September 26, 1953; 10west, $12.28 \mathrm{ft}$ below land-surface datum, November $30,1972$.

DEPTH BELOW LAND SURFACE (WATER LEVEL)

(FEET) CALENDAR YEAR JANUARY 1987 TO DECEMBER MEAN VALUES

\begin{tabular}{|c|c|c|c|c|c|c|c|c|c|c|c|c|}
\hline DAY & JAN & FEB & MAR & APR & MAY & JUN & JUL & AUG & SEP & OCT & NOV & $\mathrm{DEC}$ \\
\hline $\begin{array}{l}1 \\
2 \\
3 \\
4 \\
5\end{array}$ & $\begin{array}{r}2.97 \\
2.50 \\
2.66 \\
2.51 \\
.89\end{array}$ & $\begin{array}{l}2.44 \\
2.14 \\
1.99 \\
2.32 \\
2.43\end{array}$ & $\begin{array}{l}1.59 \\
1.90 \\
2.24 \\
2.44 \\
2.59\end{array}$ & $\begin{array}{l}2.25 \\
2.46 \\
2.59 \\
2.69 \\
2.84\end{array}$ & $\begin{array}{l}4.54 \\
4.59 \\
4.63 \\
4.75 \\
4.76\end{array}$ & $\begin{array}{l}4.83 \\
4.92 \\
5.03 \\
4.96 \\
4.24\end{array}$ & $\begin{array}{l}5.47 \\
5.47 \\
5.45 \\
4.44 \\
4.14\end{array}$ & $\begin{array}{l}5.86 \\
6.12 \\
6.35 \\
6.51 \\
6.63\end{array}$ & $\begin{array}{l}7.39 \\
7.18 \\
6.98 \\
6.76 \\
4.57\end{array}$ & $\begin{array}{l}3.17 \\
3.34 \\
3.44 \\
3.54 \\
3.64\end{array}$ & $\begin{array}{l}5.86 \\
5.92 \\
5.99 \\
5.97 \\
5.69\end{array}$ & $\begin{array}{l}6.83 \\
6.93 \\
6.91 \\
6.92 \\
7.07\end{array}$ \\
\hline $\begin{array}{r}6 \\
7 \\
8 \\
9 \\
10\end{array}$ & $\begin{array}{l}1.28 \\
1.57 \\
1.85 \\
2.08 \\
1.70\end{array}$ & $\begin{array}{l}1.68 \\
1.05 \\
1.44 \\
1.99 \\
2.26\end{array}$ & $\begin{array}{r}2.69 \\
2.61 \\
.83 \\
1.14 \\
1.57\end{array}$ & $\begin{array}{l}2.95 \\
3.04 \\
3.07 \\
3.14 \\
3.20\end{array}$ & $\begin{array}{l}4.83 \\
4.80 \\
4.92 \\
5.06 \\
5.16\end{array}$ & $\begin{array}{l}4.22 \\
4.22 \\
4.30 \\
4.27 \\
4.28\end{array}$ & $\begin{array}{l}4.10 \\
3.76 \\
3.80 \\
3.74 \\
3.88\end{array}$ & $\begin{array}{l}6.72 \\
6.82 \\
6.88 \\
6.98 \\
7.13\end{array}$ & $\begin{array}{l}1.59 \\
1.05 \\
1.62 \\
1.92 \\
1.90\end{array}$ & $\begin{array}{l}3.72 \\
3.83 \\
3.92 \\
4.02 \\
4.02\end{array}$ & $\begin{array}{l}5.64 \\
5.61 \\
5.62 \\
5.65 \\
5.70\end{array}$ & $\begin{array}{l}7.13 \\
7.16 \\
7.18 \\
7.21 \\
7.22\end{array}$ \\
\hline $\begin{array}{l}11 \\
12 \\
13 \\
14 \\
15\end{array}$ & $\begin{array}{l}1.71 \\
2.08 \\
2.31 \\
2.43 \\
2.41\end{array}$ & $\begin{array}{l}2.39 \\
2.49 \\
2.60 \\
2.66 \\
2.73\end{array}$ & $\begin{array}{l}1.94 \\
2.05 \\
2.24 \\
2.41 \\
2.53\end{array}$ & $\begin{array}{l}3.26 \\
3.26 \\
3.32 \\
3.37 \\
3.40\end{array}$ & $\begin{array}{l}5.22 \\
5.35 \\
5.28 \\
4.72 \\
4.59\end{array}$ & $\begin{array}{l}4.33 \\
4.39 \\
4.47 \\
4.54 \\
4.59\end{array}$ & $\begin{array}{l}4.06 \\
4.14 \\
4.47 \\
4.98 \\
5.17\end{array}$ & $\begin{array}{l}6.15 \\
5.71 \\
5.76 \\
5.85 \\
5.95\end{array}$ & $\begin{array}{l}2.32 \\
2.06 \\
1.86 \\
2.32 \\
2.57\end{array}$ & $\begin{array}{l}4.11 \\
4.22 \\
4.31 \\
4.15 \\
4.37\end{array}$ & $\begin{array}{l}5.85 \\
5.91 \\
5.99 \\
6.08 \\
6.15\end{array}$ & $\begin{array}{l}7.18 \\
7.27 \\
7.40 \\
7.27 \\
7.35\end{array}$ \\
\hline $\begin{array}{l}16 \\
17 \\
18 \\
19 \\
20\end{array}$ & $\begin{array}{r}1.72 \\
1.11 \\
.93 \\
1.02 \\
1.41\end{array}$ & $\begin{array}{l}1.87 \\
1.90 \\
2.17 \\
2.38 \\
2.41\end{array}$ & $\begin{array}{l}2.45 \\
2.65 \\
2.78 \\
2.48 \\
2.66\end{array}$ & $\begin{array}{l}3.43 \\
3.54 \\
3.70 \\
3.79 \\
3.78\end{array}$ & $\begin{array}{l}4.58 \\
4.59 \\
4.67 \\
4.53 \\
3.74\end{array}$ & $\begin{array}{l}4.63 \\
4.75 \\
4.76 \\
4.83 \\
4.80\end{array}$ & $\begin{array}{l}5.31 \\
5.42 \\
5.54 \\
5.59 \\
5.65\end{array}$ & $\begin{array}{l}6.07 \\
6.20 \\
6.31 \\
6.43 \\
6.58\end{array}$ & $\begin{array}{l}2.56 \\
2.45 \\
2.70 \\
2.61 \\
2.25\end{array}$ & $\begin{array}{l}4.47 \\
4.50 \\
4.62 \\
4.73 \\
4.80\end{array}$ & $\begin{array}{l}6.17 \\
6.21 \\
6.23 \\
6.22 \\
6.27\end{array}$ & $\begin{array}{l}7.46 \\
7.54 \\
7.56 \\
7.46 \\
7.50\end{array}$ \\
\hline $\begin{array}{l}21 \\
22 \\
23 \\
24 \\
25\end{array}$ & $\begin{array}{r}1.53 \\
.33 \\
.74 \\
1.12 \\
.94\end{array}$ & $\begin{array}{l}1.83 \\
1.38 \\
1.27 \\
1.73 \\
2.01\end{array}$ & $\begin{array}{l}2.80 \\
2.91 \\
2.97 \\
2.99 \\
2.77\end{array}$ & $\begin{array}{l}3.75 \\
3.91 \\
3.89 \\
3.99 \\
4.09\end{array}$ & $\begin{array}{l}3.72 \\
3.81 \\
3.88 \\
3.87 \\
4.00\end{array}$ & $\begin{array}{l}4.92 \\
4.81 \\
4.75 \\
4.70 \\
4.78\end{array}$ & $\begin{array}{l}5.80 \\
5.94 \\
6.11 \\
6.24 \\
6.22\end{array}$ & $\begin{array}{l}6.70 \\
6.79 \\
6.89 \\
7.05 \\
7.19\end{array}$ & $\begin{array}{l}2.56 \\
2.75 \\
2.81 \\
2.91 \\
3.02\end{array}$ & $\begin{array}{l}4.89 \\
5.01 \\
5.11 \\
5.18 \\
5.25\end{array}$ & $\begin{array}{l}6.38 \\
6.43 \\
6.49 \\
6.54 \\
6.56\end{array}$ & $\begin{array}{l}7.59 \\
7.65 \\
7.73 \\
7.74 \\
7.74\end{array}$ \\
\hline $\begin{array}{l}26 \\
27 \\
28 \\
29 \\
30 \\
31\end{array}$ & $\begin{array}{l}1.02 \\
1.48 \\
1.73 \\
1.97 \\
2.09 \\
2.32\end{array}$ & $\begin{array}{c}2.09 \\
1.99 \\
2.19 \\
--- \\
--- \\
---\end{array}$ & $\begin{array}{l}2.36 \\
2.49 \\
2.61 \\
2.74 \\
2.43 \\
2.02\end{array}$ & $\begin{array}{r}4.15 \\
4.21 \\
4.33 \\
4.39 \\
4.47 \\
---\end{array}$ & $\begin{array}{l}4.12 \\
4.25 \\
4.37 \\
4.48 \\
4.54 \\
4.73\end{array}$ & $\begin{array}{l}5.22 \\
5.15 \\
5.18 \\
5.27 \\
5.35 \\
---\end{array}$ & $\begin{array}{l}6.30 \\
6.39 \\
6.51 \\
6.31 \\
5.90 \\
5.98\end{array}$ & $\begin{array}{l}7.27 \\
7.35 \\
7.47 \\
7.57 \\
7.69 \\
7.78\end{array}$ & $\begin{array}{r}3.14 \\
3.24 \\
3.24 \\
3.24 \\
3.22 \\
----\end{array}$ & $\begin{array}{l}5.34 \\
5.52 \\
5.60 \\
5.67 \\
5.74 \\
5.82\end{array}$ & $\begin{array}{l}6.61 \\
6.64 \\
6.68 \\
6.71 \\
6.75 \\
-.--\end{array}$ & $\begin{array}{l}7.76 \\
7.79 \\
7.78 \\
7.81 \\
7.82 \\
7.78\end{array}$ \\
\hline MEAN & 1.69 & 2.07 & 2.35 & 3.48 & 4.55 & 4.72 & 5.23 & 6.67 & 3.16 & 4.52 & 6.15 & 7.41 \\
\hline CAL $Y$ & & & & HIGH & .33 & & & & & & & \\
\hline
\end{tabular}



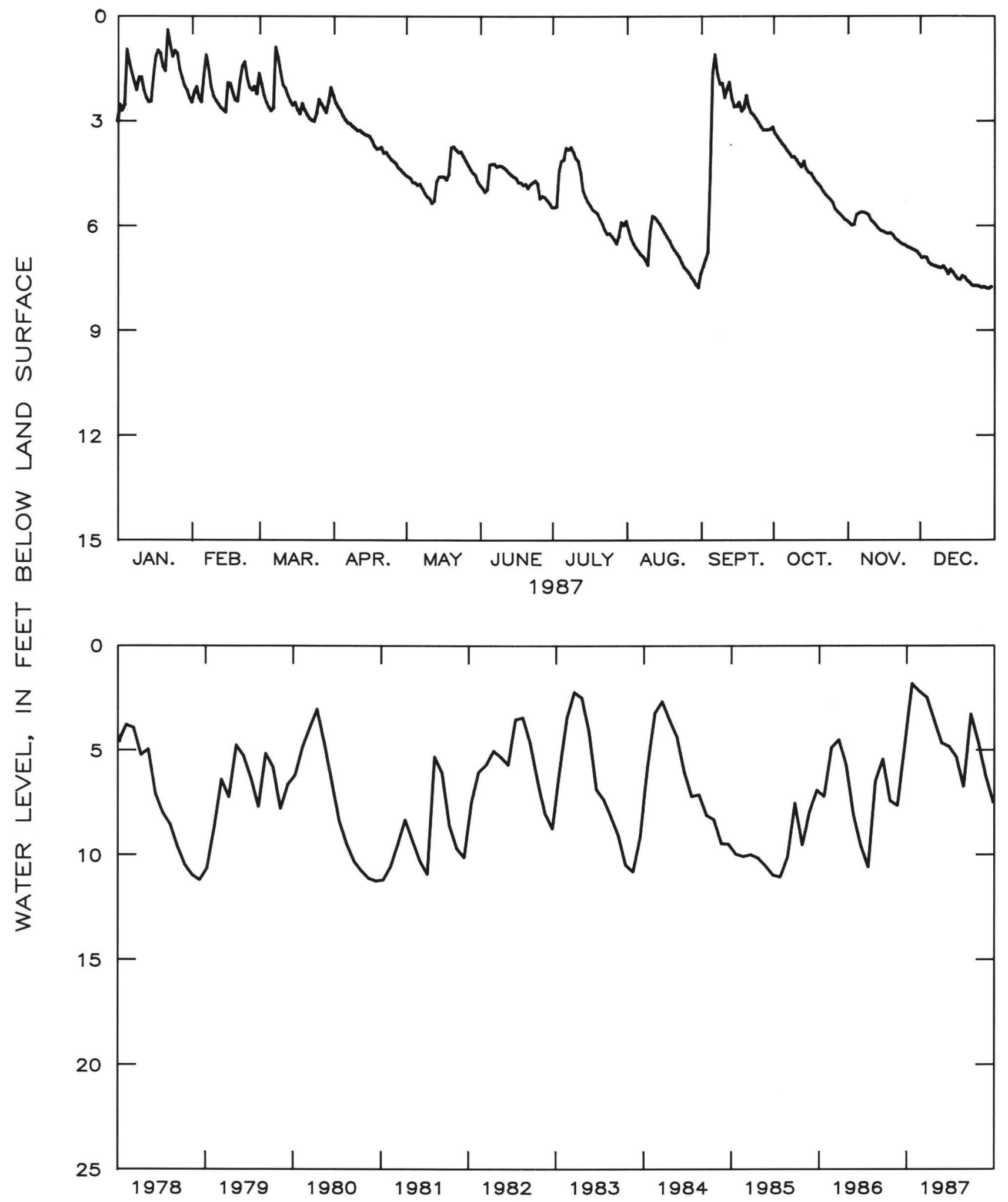

Figure 2.3-5.--Water level in observation well 35P094, Chatham County. 


\subsection{Cretaceous Aquifer System}

The Cretaceous aquifer system in the Coastal Plain of Georgia supplied more than $135 \mathrm{Mgal} / \mathrm{d}$ in 1985, primarily for municipal and industrial use (Turlington and others, 1987). The aquifer system consists of sand and gravel that locally contains layers of clay and silt that act as confining beds. In parts of the area these confining beds separate the aquifer system into two or more aquifers. In southwestern Georgia, the Cretaceous aquifer system includes the Providence aquifer and sandy units in the Cusseta, Blufftown, Eutaw, and Tuscaloosa Formations. In east-central Georgia, the Cretaceous aquifer system is divided into three subsystems: the Dublin aquifer system, the Midville aquifer system, and the Dublin-Midville aquifer system (Clarke and others, 1985a).

The major source of recharge to the Cretaceous aquifer system is rainfall in areas where the aquifers crop out at land surface or where they are overlain by permeable surface material. Rainfall that enters the aquifers directly or infiltrates through the surface material generally moves downgradient through the system toward the southeast. Most of the natural discharge from the aquifer system is to streams and rivers that cross the outcrop area of the aquifers.

Water-level fluctuations in the cretaceous aquifer system are related primarily to changes in precipitation and pumping. In western Georgia, the mean water level in well 065001 in Chattahoochee County was about $3.7 \mathrm{ft}$ lower in 1987 than in 1986. This decline continued a downward trend of the water level in that area. At the end of 1987 the water level in well 065001 was $4.9 \mathrm{ft}$ lower than at the end of 1986. 


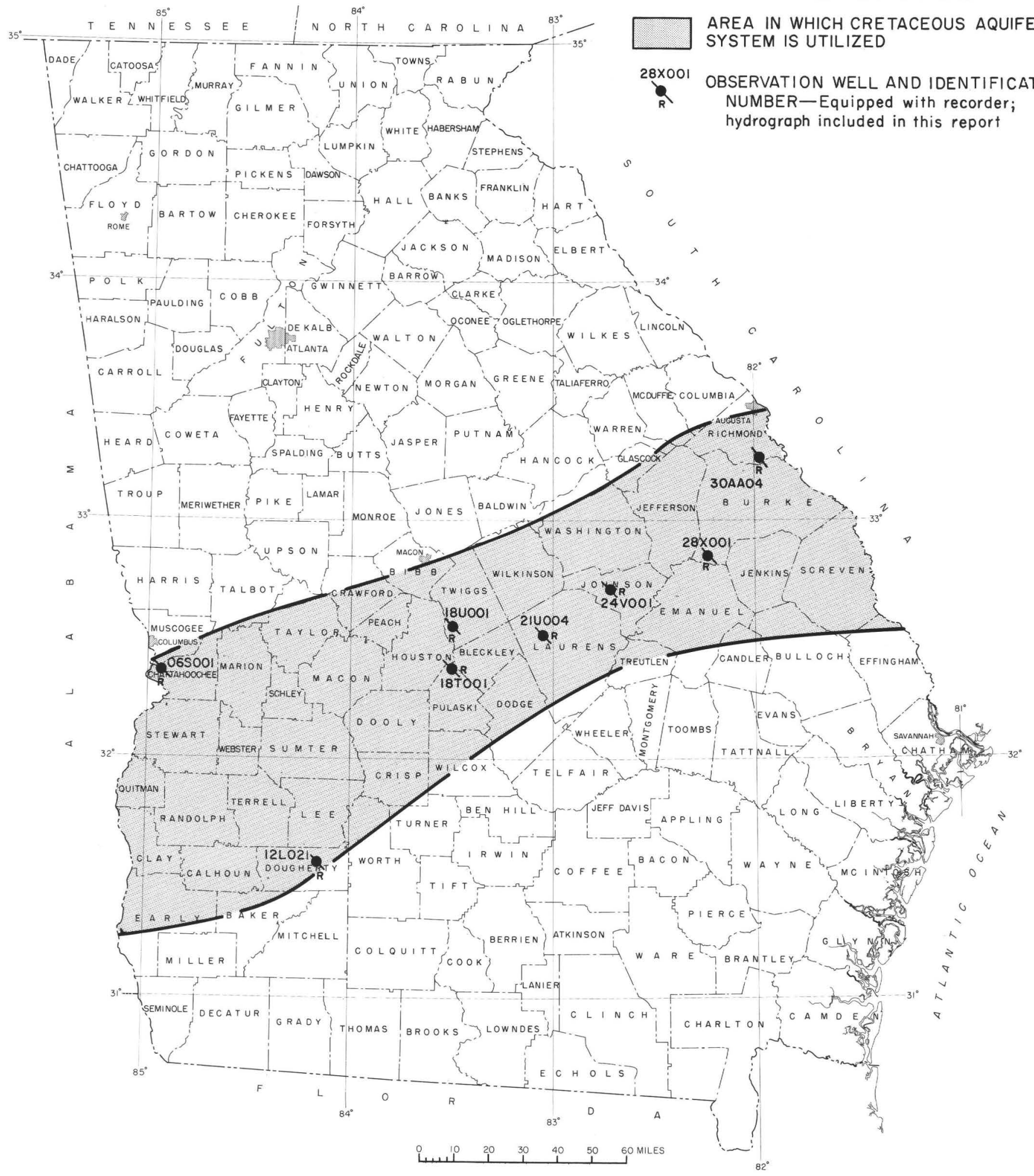

Figure 2.4-1.-Locations of observation wells in the Cretaceous aquifer system. 
322036084590301 Local number, 065001

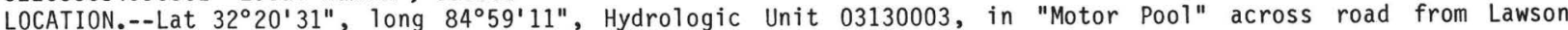
Airfield main building. Owner: U.S. Army.

AQUIFER.--Upper Cretaceous (Blufftown, Eutaw, and Tuscaloosa Formations).

WELL CHARACTERISTICS.--Drilled unused supply well, diameter $12 \mathrm{in.,}$ depth $568 \mathrm{ft}$, screened interval 215-220 ft, $230-235 \mathrm{ft}, 280-290 \mathrm{ft}, 540-550 \mathrm{ft}$

DATUM.--Elevation of land-surface datum is $255 \mathrm{ft}$.

Measuring point: Floor of recorder shelter, $2.80 \mathrm{ft}$ above land-surface datum.

REMARKS.--Well pumped June 1978; water-quality sample collected at conclusion of pumping.

PERIOD OF RECORD.--May 1950 to current year.

EXTREMES FOR PERIOD OF RECORD.---Highest water level, $0.37 \mathrm{ft}$ below land-surface datum, April 10, 1964; 10west, $29.73 \mathrm{ft}$ below land-surface datum, September 10, 1958.

DEPTH BELOW LAND SURFACE (WATER LEVEL) (FEET) CALENDAR YEAR JANUARY 1987 TO DECEMBER 1987 MEAN VALUES

\begin{tabular}{|c|c|c|c|c|c|c|c|c|c|c|c|c|}
\hline DAY & JAN & FEB & MAR & APR & MAY & JUN & JUL & AUG & SEP & OCT & NOV & $\mathrm{DEC}$ \\
\hline $\begin{array}{l}1 \\
2 \\
3 \\
4 \\
5\end{array}$ & $\begin{array}{l}23.80 \\
23.86 \\
23.89 \\
23.84 \\
23.87\end{array}$ & $\begin{array}{l}23.59 \\
23.53 \\
23.57 \\
23.62 \\
23.65\end{array}$ & $\begin{array}{l}23.57 \\
23.57 \\
23.60 \\
23.64 \\
23.69\end{array}$ & $\begin{array}{l}23.92 \\
23.90 \\
23.88 \\
23.93 \\
23.93\end{array}$ & $\begin{array}{l}24.36 \\
24.36 \\
24.39 \\
24.43 \\
24.47\end{array}$ & $\begin{array}{l}24.98 \\
25.00 \\
25.02 \\
25.02 \\
25.06\end{array}$ & $\begin{array}{l}25.54 \\
25.55 \\
25.58 \\
25.58 \\
25.58\end{array}$ & $\begin{array}{l}26.40 \\
26.43 \\
26.46 \\
26.45 \\
26.47\end{array}$ & $\begin{array}{l}27.37 \\
27.38 \\
27.41 \\
27.44 \\
27.46\end{array}$ & $\begin{array}{l}27.94 \\
27.98 \\
28.04 \\
28.07 \\
28.06\end{array}$ & $\begin{array}{l}28.65 \\
28.63 \\
28.60 \\
28.57 \\
28.59\end{array}$ & $\begin{array}{l}28.62 \\
28.68 \\
28.67 \\
28.67 \\
28.71\end{array}$ \\
\hline $\begin{array}{r}6 \\
7 \\
8 \\
9 \\
10\end{array}$ & $\begin{array}{l}23.66 \\
23.52 \\
23.54 \\
23.54 \\
23.51\end{array}$ & $\begin{array}{l}23.60 \\
23.56 \\
23.56 \\
23.65 \\
23.68\end{array}$ & $\begin{array}{l}23.72 \\
23.67 \\
23.60 \\
23.61 \\
23.68\end{array}$ & $\begin{array}{l}23.93 \\
23.94 \\
23.95 \\
23.96 \\
23.96\end{array}$ & $\begin{array}{l}24.49 \\
24.50 \\
24.52 \\
24.58 \\
24.61\end{array}$ & $\begin{array}{l}25.11 \\
25.16 \\
25.19 \\
25.21 \\
25.23\end{array}$ & $\begin{array}{l}25.61 \\
25.62 \\
25.65 \\
25.68 \\
25.71\end{array}$ & $\begin{array}{l}26.52 \\
26.56 \\
26.60 \\
26.62 \\
26.64\end{array}$ & $\begin{array}{l}27.48 \\
27.50 \\
27.50 \\
27.52 \\
27.55\end{array}$ & $\begin{array}{l}28.04 \\
28.09 \\
28.17 \\
28.22 \\
28.25\end{array}$ & $\begin{array}{l}28.67 \\
28.69 \\
28.69 \\
28.66 \\
28.62\end{array}$ & $\begin{array}{l}28.73 \\
28.73 \\
28.73 \\
28.71 \\
28.68\end{array}$ \\
\hline $\begin{array}{l}11 \\
12 \\
13 \\
14 \\
15\end{array}$ & $\begin{array}{l}23.60 \\
23.61 \\
23.61 \\
23.62 \\
23.59\end{array}$ & $\begin{array}{l}23.67 \\
23.65 \\
23.67 \\
23.65 \\
23.65\end{array}$ & $\begin{array}{l}23.76 \\
23.78 \\
23.78 \\
23.79 \\
23.81\end{array}$ & $\begin{array}{l}23.98 \\
24.02 \\
24.04 \\
24.03 \\
24.01\end{array}$ & $\begin{array}{l}24.62 \\
24.61 \\
24.62 \\
24.60 \\
24.58\end{array}$ & $\begin{array}{l}25.27 \\
25.30 \\
25.30 \\
25.24 \\
25.25\end{array}$ & $\begin{array}{l}25.73 \\
25.76 \\
25.78 \\
25.80 \\
25.85\end{array}$ & $\begin{array}{l}26.66 \\
26.69 \\
26.71 \\
26.75 \\
26.79\end{array}$ & $\begin{array}{l}27.58 \\
27.58 \\
27.59 \\
27.62 \\
27.64\end{array}$ & $\begin{array}{l}28.24 \\
28.22 \\
28.27 \\
28.33 \\
28.35\end{array}$ & $\begin{array}{l}28.68 \\
28.71 \\
28.70 \\
28.71 \\
28.74\end{array}$ & $\begin{array}{l}28.67 \\
28.68 \\
28.75 \\
28.74 \\
28.66\end{array}$ \\
\hline $\begin{array}{l}16 \\
17 \\
18 \\
19 \\
20\end{array}$ & $\begin{array}{l}23.59 \\
23.59 \\
23.54 \\
23.53 \\
23.58\end{array}$ & $\begin{array}{l}23.61 \\
23.66 \\
23.71 \\
23.77 \\
23.78\end{array}$ & $\begin{array}{l}23.83 \\
23.85 \\
23.81 \\
23.78 \\
23.80\end{array}$ & $\begin{array}{l}24.00 \\
24.02 \\
24.08 \\
24.14 \\
24.16\end{array}$ & $\begin{array}{l}24.59 \\
24.62 \\
24.63 \\
24.64 \\
24.67\end{array}$ & $\begin{array}{l}25.25 \\
25.27 \\
25.31 \\
25.32 \\
25.32\end{array}$ & $\begin{array}{l}25.90 \\
25.96 \\
26.00 \\
26.03 \\
26.07\end{array}$ & $\begin{array}{l}26.83 \\
26.87 \\
26.91 \\
26.94 \\
26.99\end{array}$ & $\begin{array}{l}27.65 \\
27.66 \\
27.67 \\
27.69 \\
27.73\end{array}$ & $\begin{array}{l}28.36 \\
28.37 \\
28.38 \\
28.39 \\
28.41\end{array}$ & $\begin{array}{l}28.73 \\
29.24 \\
29.13 \\
28.89 \\
28.83\end{array}$ & $\begin{array}{l}28.71 \\
28.74 \\
28.74 \\
28.73 \\
28.71\end{array}$ \\
\hline $\begin{array}{l}21 \\
22 \\
23 \\
24 \\
25\end{array}$ & $\begin{array}{l}23.54 \\
23.41 \\
23.46 \\
23.47 \\
23.39\end{array}$ & $\begin{array}{l}23.76 \\
23.72 \\
23.77 \\
23.80 \\
23.82\end{array}$ & $\begin{array}{l}23.80 \\
23.83 \\
23.84 \\
23.85 \\
23.88\end{array}$ & $\begin{array}{l}24.16 \\
24.16 \\
24.18 \\
24.20 \\
24.24\end{array}$ & $\begin{array}{l}24.71 \\
24.74 \\
24.76 \\
24.77 \\
24.80\end{array}$ & $\begin{array}{l}25.33 \\
25.32 \\
25.32 \\
25.34 \\
25.35\end{array}$ & $\begin{array}{l}26.10 \\
26.11 \\
26.12 \\
26.16 \\
26.18\end{array}$ & $\begin{array}{l}27.05 \\
27.09 \\
27.12 \\
27.15 \\
27.19\end{array}$ & $\begin{array}{l}27.77 \\
27.79 \\
27.81 \\
27.81 \\
27.83\end{array}$ & $\begin{array}{l}28.46 \\
28.51 \\
28.53 \\
28.54 \\
28.53\end{array}$ & $\begin{array}{l}28.84 \\
28.83 \\
28.83 \\
28.83 \\
28.72\end{array}$ & $\begin{array}{l}28.69 \\
28.65 \\
28.68 \\
28.68 \\
28.66\end{array}$ \\
\hline $\begin{array}{l}26 \\
27 \\
28 \\
29 \\
30 \\
31\end{array}$ & $\begin{array}{l}23.45 \\
23.50 \\
23.51 \\
23.52 \\
23.51 \\
23.57\end{array}$ & $\begin{array}{r}23.82 \\
23.77 \\
23.64 \\
--- \\
--- \\
---\end{array}$ & $\begin{array}{l}23.90 \\
23.88 \\
23.91 \\
23.92 \\
23.85 \\
23.89\end{array}$ & $\begin{array}{r}24.30 \\
24.31 \\
24.32 \\
24.35 \\
24.36 \\
---\end{array}$ & $\begin{array}{l}24.83 \\
24.86 \\
24.88 \\
24.91 \\
24.93 \\
24.95\end{array}$ & $\begin{array}{r}25.35 \\
25.39 \\
25.44 \\
25.49 \\
25.53 \\
. .--\end{array}$ & $\begin{array}{l}26.19 \\
26.21 \\
26.24 \\
26.27 \\
26.33 \\
26.38\end{array}$ & $\begin{array}{l}27.22 \\
27.24 \\
27.26 \\
27.29 \\
27.32 \\
27.35\end{array}$ & $\begin{array}{r}27.86 \\
27.90 \\
27.92 \\
27.90 \\
27.88 \\
-.-\end{array}$ & $\begin{array}{l}28.51 \\
28.50 \\
28.55 \\
28.59 \\
28.61 \\
28.64\end{array}$ & $\begin{array}{r}28.65 \\
28.64 \\
28.62 \\
28.61 \\
28.60 \\
-.--\end{array}$ & $\begin{array}{l}28.66 \\
28.66 \\
28.62 \\
28.68 \\
28.73 \\
28.72\end{array}$ \\
\hline MEAN & 23.59 & 23.68 & 23.77 & 24.08 & 24.65 & 25.26 & 25.91 & 26.86 & 27.65 & 28.33 & 28.73 & 28.69 \\
\hline CAL & & & & HIGH & 23.39 & & & & & & & \\
\hline
\end{tabular}



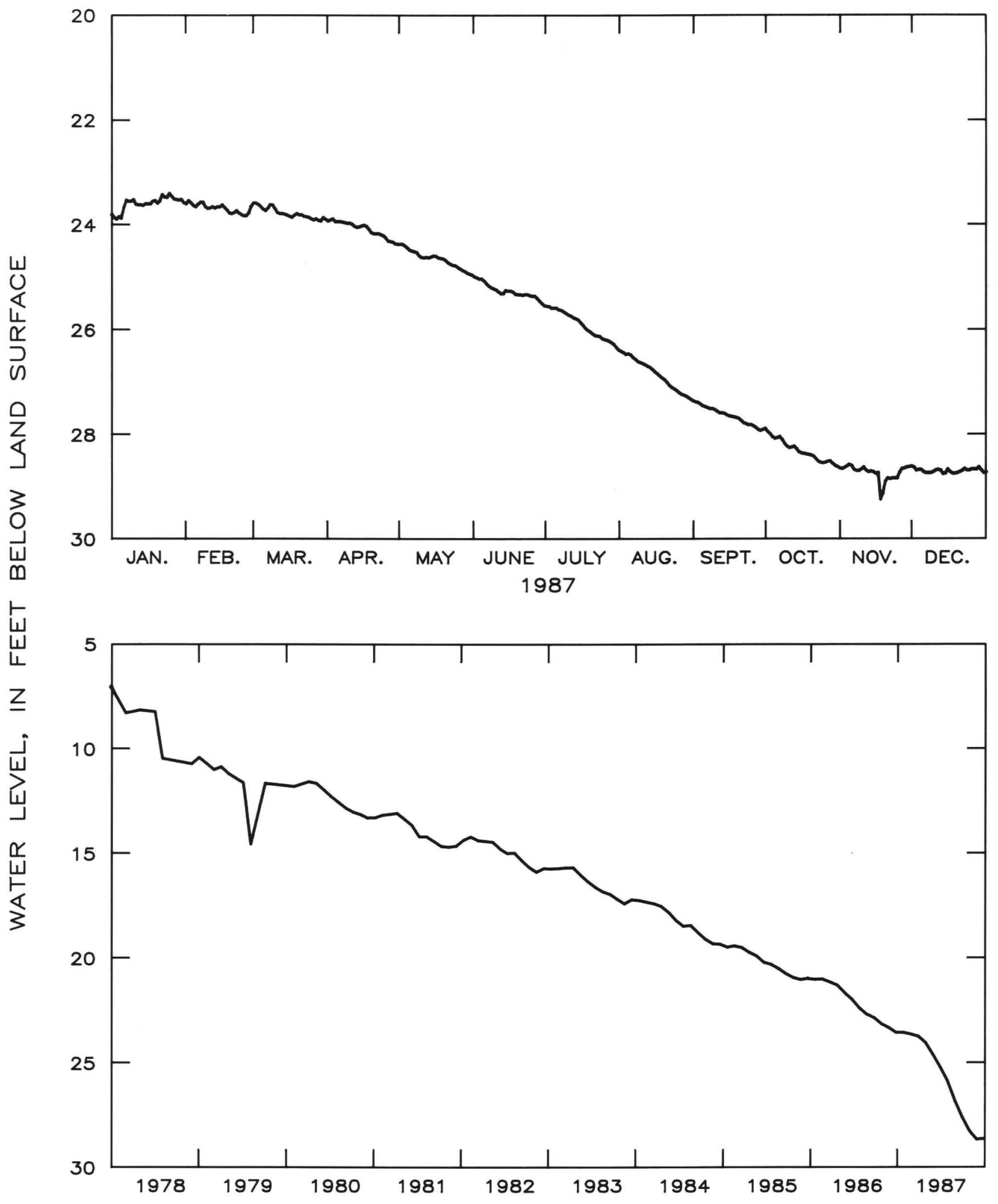

Figure 2.4-2.--Water level in observation well 065001, Chattahoochee County. 


\subsubsection{Providence Aquifer}

The Providence aquifer consists of sand of Late Cretaceous age and supplies about $9 \mathrm{Mgal} / \mathrm{d}$ for municipal, industrial, and agricultural use in southwestern Georgia (Clarke and others, 1983). The water level in the Providence aquifer is affected primarily by changes in local pumping.

During late October and early November, water levels were measured in 32 wells tapping the Providence aquifer. Data from these water-level measurements were used to update the potentiometric surface of Clarke and others (1983). Pumping from the aquifer has resulted in the development of cones of depression at Albany and Americus. According to Clarke and others (1983), the water level in the Providence aquifer near Albany declined more than 100 ft during the period 1950-80.

The mean water level in well 12 L021 at Albany was about $1.0 \mathrm{ft}$ higher in 1987 than in 1986. Increased rainfall during early 1987 and decreased pumping resulted in water-level rises in well 12L021. By the end of April, the water level in the well had recovered $28.3 \mathrm{ft}$ from the record low measured in July 1986. At the end of 1987, the water level in well 12 L021 was $5.7 \mathrm{ft}$ higher than at the end of 1986 . 


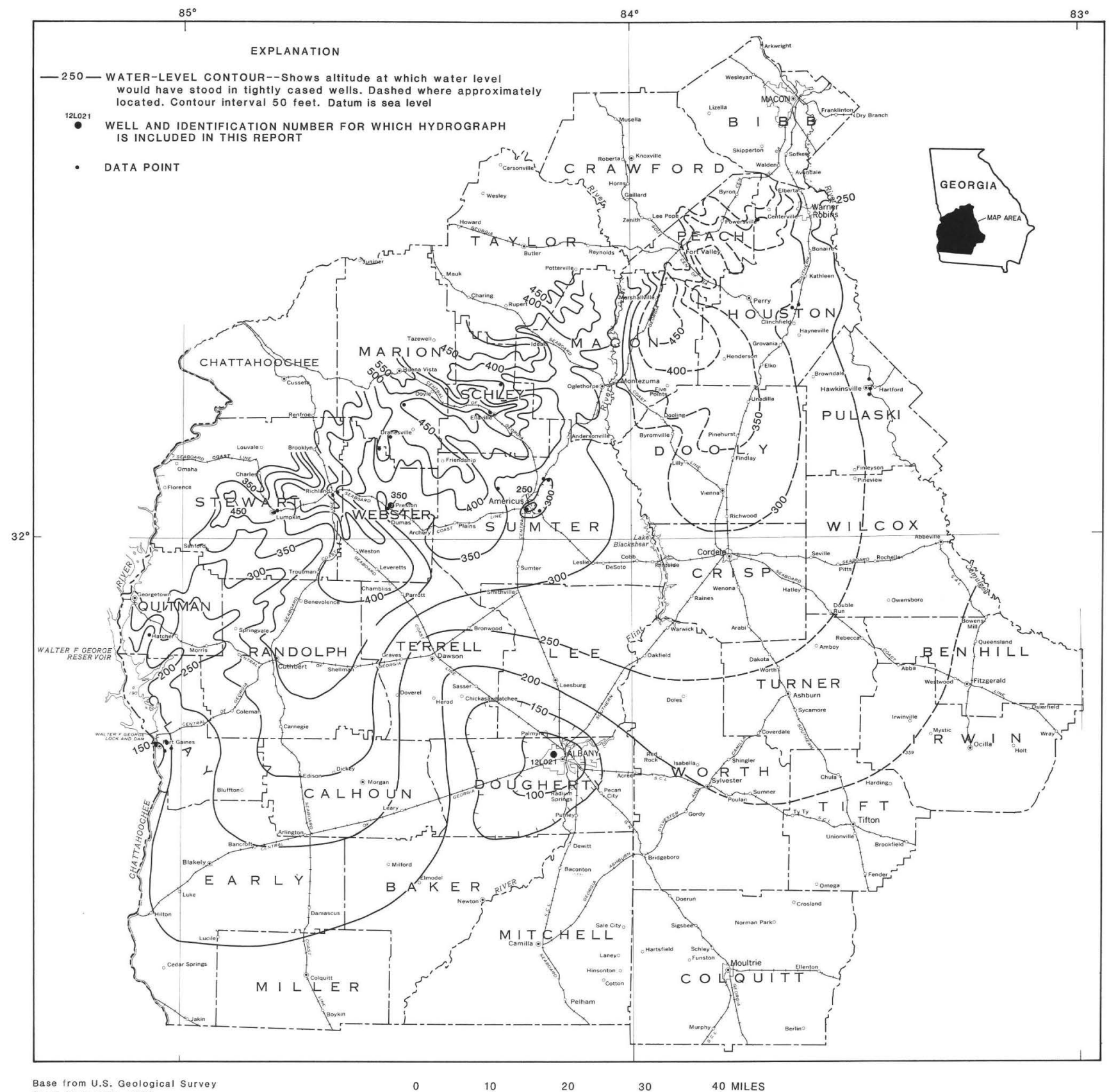

Base from U.S. Geological Survey

$0 \quad 10$

Figure 2.4.1-1.--Location of observation well $12 \mathrm{~L} 021$ and the water level in the Providence aquifer, October 1987. 
313534084103003 Local number, 12 L021.

LOCATION.--Lat $31^{\circ} 35^{\prime} 37^{\prime \prime}$, long $84^{\circ} 10^{\prime} 30^{\prime \prime}$, Hydrologic Unit 03130008 , located in park at intersection of Slappey Drive and Fifth Avenue.

Owner: U.S. Geological Survey, test well 10.

AQUIFER.--Providence (Upper Cretaceous).

WELL CHARACTERISTICS.---Drilled observation well, depth 1,346 ft, cased to $797 \mathrm{ft}$.

DATUM.--Elevation of land-surface datum is $198 \mathrm{ft}$.

Measuring point: Floor of recorder shelter, $3.0 \mathrm{ft}$ above land-surface datum.

REMARKS.--Water levels for period of missing record, January 27 to February 24 , were estimated.

PERIOD OF RECORD.--December 1978 to current year.

EXTREMES FOR PERIOD OF RECORD.--Highest water level, $101.59 \mathrm{ft}$ below land-surface datum, Apri1 26, 1984; lowest, $156.36 \mathrm{ft}$ below land-surface datum, July $26,1986$.

DEPTH BELOW LAND SURFACE (WATER LEVEL) (FEET) CALENDAR YEAR JANUARY 1987 TO DECEMBER 1987 MEAN VALUES

\begin{tabular}{|c|c|c|c|c|c|c|c|c|c|c|c|c|}
\hline DAY & JAN & FEB & MAR & APR & MAY & JUN & JUL & AUG & SEP & ОСТ & NOV & DEC \\
\hline $\begin{array}{l}1 \\
2 \\
3 \\
4 \\
5\end{array}$ & $\begin{array}{l}134.80 \\
134.75 \\
135.20 \\
135.68 \\
136.49\end{array}$ & $\begin{array}{l}135.98 \\
136.17 \\
135.79 \\
135.36 \\
135.17\end{array}$ & $\begin{array}{l}136.78 \\
136.37 \\
136.48 \\
136.89 \\
136.42\end{array}$ & $\begin{array}{l}130.65 \\
131.25 \\
131.52 \\
131.97 \\
132.45\end{array}$ & $\begin{array}{l}134.65 \\
134.70 \\
135.11 \\
135.71 \\
136.42\end{array}$ & $\begin{array}{l}135.33 \\
135.82 \\
136.30 \\
136.83 \\
137.54\end{array}$ & $\begin{array}{l}136.19 \\
135.64 \\
134.87 \\
134.29 \\
134.24\end{array}$ & $\begin{array}{l}141.43 \\
141.71 \\
142.06 \\
142.34 \\
142.60\end{array}$ & $\begin{array}{l}141.41 \\
140.93 \\
140.32 \\
140.05 \\
139.68\end{array}$ & $\begin{array}{l}141.02 \\
141.43 \\
141.91 \\
141.83 \\
141.34\end{array}$ & $\begin{array}{l}143.95 \\
144.71 \\
144.60 \\
144.00 \\
143.45\end{array}$ & $\begin{array}{l}135.61 \\
135.47 \\
135.15 \\
134.62 \\
134.12\end{array}$ \\
\hline $\begin{array}{r}6 \\
7 \\
8 \\
9 \\
10\end{array}$ & $\begin{array}{l}136.95 \\
136.37 \\
135.71 \\
135.28 \\
136.03\end{array}$ & $\begin{array}{l}134.98 \\
135.39 \\
135.73 \\
136.34 \\
136.68\end{array}$ & $\begin{array}{l}136.02 \\
136.04 \\
136.27 \\
136.30 \\
136.77\end{array}$ & $\begin{array}{l}132.43 \\
131.60 \\
130.66 \\
129.70 \\
129.12\end{array}$ & $\begin{array}{l}137.03 \\
137.17 \\
136.90 \\
136.82 \\
136.92\end{array}$ & $\begin{array}{l}138.10 \\
138.31 \\
138.37 \\
138.43 \\
138.48\end{array}$ & $\begin{array}{l}134.23 \\
134.57 \\
134.66 \\
135.02 \\
134.55\end{array}$ & $\begin{array}{l}142.30 \\
142.72 \\
142.85 \\
143.12 \\
143.38\end{array}$ & $\begin{array}{l}139.03 \\
138.79 \\
139.40 \\
139.39 \\
138.97\end{array}$ & $\begin{array}{l}141.66 \\
142.04 \\
142.32 \\
142.90 \\
143.13\end{array}$ & $\begin{array}{l}143.40 \\
143.20 \\
142.34 \\
141.47 \\
140.78\end{array}$ & $\begin{array}{l}133.62 \\
133.22 \\
132.85 \\
132.89 \\
133.26\end{array}$ \\
\hline $\begin{array}{l}11 \\
12 \\
13 \\
14 \\
15\end{array}$ & $\begin{array}{l}136.86 \\
137.07 \\
137.08 \\
136.90 \\
136.25\end{array}$ & $\begin{array}{l}136.40 \\
136.34 \\
136.43 \\
136.48 \\
136.29\end{array}$ & $\begin{array}{l}136.82 \\
136.20 \\
136.77 \\
137.47 \\
137.93\end{array}$ & $\begin{array}{l}129.29 \\
129.06 \\
128.45 \\
128.72 \\
128.77\end{array}$ & $\begin{array}{l}137.57 \\
138.15 \\
137.74 \\
136.92 \\
136.27\end{array}$ & $\begin{array}{l}138.78 \\
139.47 \\
139.62 \\
138.93 \\
138.25\end{array}$ & $\begin{array}{l}134.94 \\
135.55 \\
136.12 \\
136.67 \\
137.18\end{array}$ & $\begin{array}{l}143.67 \\
143.64 \\
143.10 \\
142.75 \\
143.15\end{array}$ & $\begin{array}{l}138.84 \\
138.72 \\
138.32 \\
138.57 \\
138.48\end{array}$ & $\begin{array}{l}143.40 \\
143.95 \\
144.55 \\
144.60 \\
144.03\end{array}$ & $\begin{array}{l}140.73 \\
140.73 \\
140.60 \\
141.55 \\
142.10\end{array}$ & $\begin{array}{l}133.51 \\
133.85 \\
133.67 \\
132.89 \\
132.43\end{array}$ \\
\hline $\begin{array}{l}16 \\
17 \\
18 \\
19 \\
20\end{array}$ & $\begin{array}{l}135.81 \\
135.45 \\
134.80 \\
134.50 \\
135.18\end{array}$ & $\begin{array}{l}136.03 \\
136.37 \\
136.54 \\
136.92 \\
137.17\end{array}$ & $\begin{array}{l}137.58 \\
136.32 \\
134.97 \\
134.08 \\
134.38\end{array}$ & $\begin{array}{l}128.06 \\
128.48 \\
129.18 \\
129.25 \\
128.77\end{array}$ & $\begin{array}{l}135.72 \\
135.63 \\
135.10 \\
134.36 \\
134.87\end{array}$ & $\begin{array}{l}137.68 \\
137.45 \\
137.67 \\
138.12 \\
138.77\end{array}$ & $\begin{array}{l}137.69 \\
138.15 \\
138.60 \\
139.09 \\
139.56\end{array}$ & $\begin{array}{l}143.58 \\
143.83 \\
143.50 \\
142.50 \\
141.63\end{array}$ & $\begin{array}{l}138.74 \\
138.93 \\
139.68 \\
140.55 \\
141.30\end{array}$ & $\begin{array}{l}143.70 \\
143.76 \\
143.81 \\
143.72 \\
143.77\end{array}$ & $\begin{array}{l}142.44 \\
141.62 \\
140.53 \\
139.68 \\
139.56\end{array}$ & $\begin{array}{l}132.21 \\
131.27 \\
131.58 \\
132.00 \\
132.48\end{array}$ \\
\hline $\begin{array}{l}21 \\
22 \\
23 \\
24 \\
25\end{array}$ & $\begin{array}{l}135.16 \\
134.74 \\
134.72 \\
134.65 \\
134.47\end{array}$ & $\begin{array}{l}137.00 \\
137.03 \\
137.39 \\
137.57 \\
137.82\end{array}$ & $\begin{array}{l}134.93 \\
134.67 \\
133.58 \\
132.73 \\
133.04\end{array}$ & $\begin{array}{l}128.25 \\
128.82 \\
129.78 \\
130.57 \\
131.35\end{array}$ & $\begin{array}{l}135.28 \\
135.55 \\
135.29 \\
135.07 \\
134.89\end{array}$ & $\begin{array}{l}138.80 \\
138.27 \\
137.44 \\
137.53 \\
137.60\end{array}$ & $\begin{array}{l}140.02 \\
140.15 \\
140.37 \\
140.87 \\
140.35\end{array}$ & $\begin{array}{l}141.78 \\
141.70 \\
141.76 \\
142.24 \\
142.68\end{array}$ & $\begin{array}{l}142.00 \\
142.37 \\
142.36 \\
142.08 \\
141.61\end{array}$ & $\begin{array}{l}144.20 \\
144.08 \\
143.78 \\
144.43 \\
144.77\end{array}$ & $\begin{array}{l}140.18 \\
139.88 \\
139.16 \\
139.40 \\
139.20\end{array}$ & $\begin{array}{l}132.99 \\
133.37 \\
133.25 \\
132.34 \\
131.65\end{array}$ \\
\hline $\begin{array}{l}26 \\
27 \\
28 \\
29 \\
30 \\
31\end{array}$ & $\begin{array}{l}134.45 \\
134.11 \\
134.16 \\
133.61 \\
134.08 \\
135.23\end{array}$ & $\begin{array}{r}137.57 \\
137.50 \\
137.27 \\
--- \\
--- \\
---\end{array}$ & $\begin{array}{l}133.58 \\
134.00 \\
133.75 \\
132.73 \\
131.68 \\
130.94\end{array}$ & $\begin{array}{r}132.10 \\
132.28 \\
133.25 \\
133.66 \\
134.30 \\
---\end{array}$ & $\begin{array}{l}134.77 \\
134.67 \\
134.82 \\
133.84 \\
134.27 \\
134.82\end{array}$ & $\begin{array}{r}137.20 \\
137.62 \\
137.57 \\
136.69 \\
136.11 \\
. .-\end{array}$ & $\begin{array}{l}141.70 \\
142.01 \\
142.30 \\
142.63 \\
142.18 \\
141.37\end{array}$ & $\begin{array}{l}142.56 \\
141.89 \\
141.44 \\
141.93 \\
141.92 \\
141.40\end{array}$ & $\begin{array}{r}141.83 \\
141.20 \\
140.78 \\
141.19 \\
141.15 \\
-.-\end{array}$ & $\begin{array}{l}144.55 \\
144.25 \\
143.67 \\
143.12 \\
143.00 \\
143.25\end{array}$ & $\begin{array}{r}138.31 \\
137.60 \\
136.95 \\
136.41 \\
135.88 \\
---\end{array}$ & $\begin{array}{l}131.59 \\
130.81 \\
130.00 \\
129.42 \\
129.06 \\
129.40\end{array}$ \\
\hline MEAN & 35.37 & 136.49 & 135.24 & 130.46 & 135.71 & 137.77 & 137.93 & 142.49 & 140.22 & 143.29 & 140.81 & 32.60 \\
\hline
\end{tabular}

$\begin{array}{lllllll}\text { CAL YR } 1987 & \text { MEAN } & 137.37 & \text { HIGH } & 128.06 & \text { LOW } & 144.77\end{array}$ 

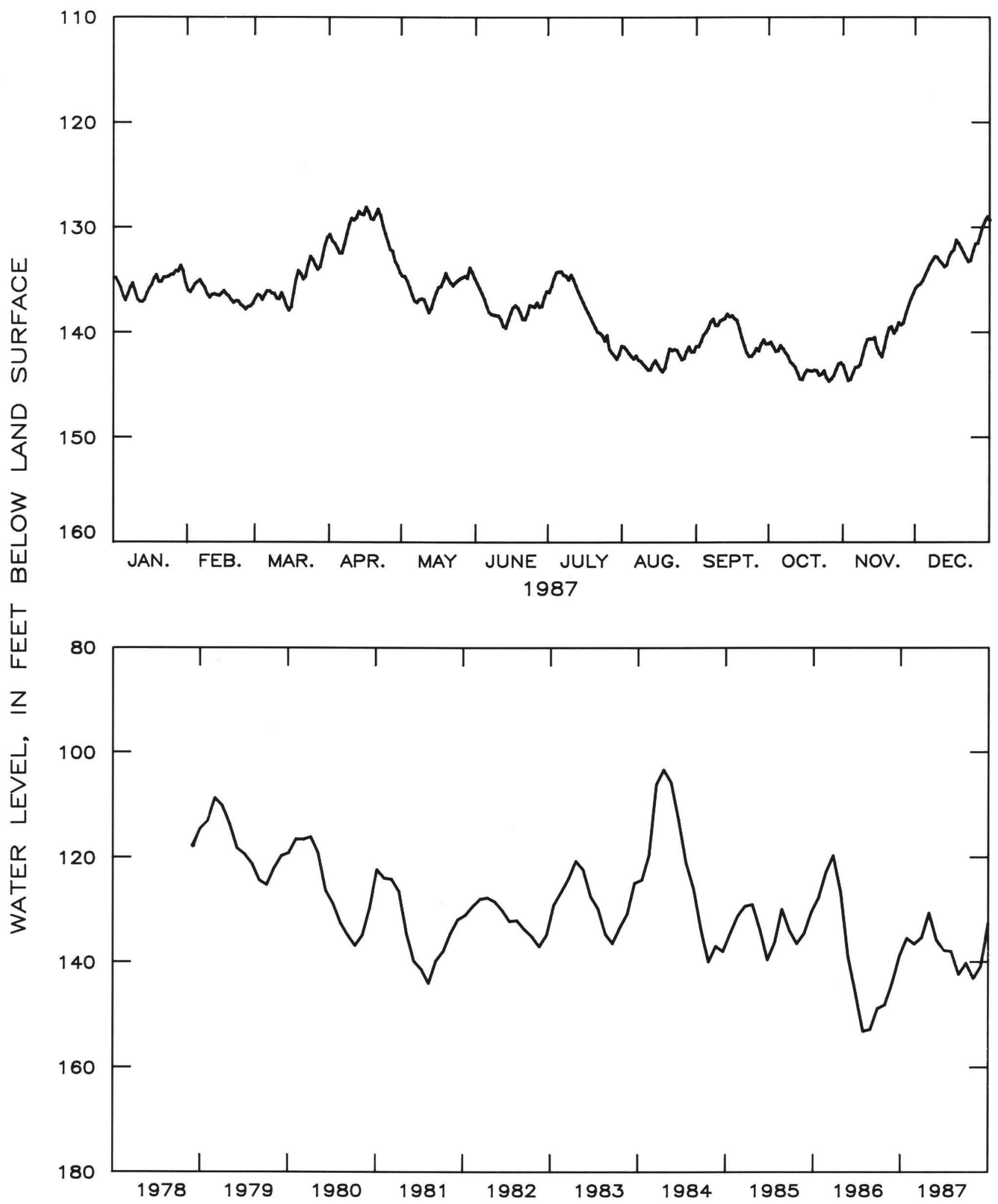

Figure 2.4.1-2--Water level in observation well 12LO21, Dougherty County. 
2.4.2 Dublin, Midville, and Dublin-Midville aquifer systems

In east-central Georgia, interlayered sand and clay of Paleocene and Late Cretaceous age form the Dublin and Midville aquifer systems. In the northern quarter of the Coastal Plain, the systems combine to form the DublinMidville aquifer system. During 1985, the aquifer systems supplied an estimated $126 \mathrm{Mgal} / \mathrm{d}$, about 60 percent of which was withdrawn for kaolin mining and processing, and other industries (Turlington and others, 1987).

The water level in the Dublin aquifer system in southern Twiggs County is affected by rainfall and by pumping in eastern Houston and western Twiggs Counties, where pumpage exceeded $37 \mathrm{Mgal} / \mathrm{d}$ in 1985 (Turlington and others, 1987). Although the water-level trend in the area has been generally downward since 1984, the 1987 mean water level in well 180001 was about the same as in 1986. By the end of April the water level had recovered about $2.6 \mathrm{ft}$. During the year, the water level in well 180001 showed a recovery from the record low recorded in August 1986.

The water level in the Midville aquifer system is affected primarily by local and regional pumping. During 1987, the mean water levels in four wells tapping the Midville aquifer system were from $0.1 \mathrm{ft}$ higher to $1.0 \mathrm{ft}$ lower than in 1986. By the end of April, water levels in four wells tapping the Midville aquifer system had recovered 1.1 to $2.4 \mathrm{ft}$ from the lows and record lows measured during the 1986 drought. Record low water levels were measured during November and December in wells 21U004, 24V001, and 28x001. By the end of 1987 water levels were 0.4 to $1.4 \mathrm{ft}$ lower than at the end of 1986 . These declines continue the downward water-level trend in the Midville aquifer system.

The water level in the Dublin-Midville aquifer system in Richmond County is influenced primarily by rainfall (Clarke and others, 1985a). Rainfall in this area was below normal from mid-1984 through the first half of 1986, and is reflected by a water-level decline in well 30AA04. Rainfall was above normal from mid-1986 to early 1987 and is reflected by a slight rise in water level. The mean water level in the well was about the same in 1987 as in 1986, and by mid-March, the water level had recovered about $3.1 \mathrm{ft}$ from the low measured during the 1986 drought. Although there was some recovery from the drought, the water level in well 30AA04 was $0.9 \mathrm{ft}$ lower at the end of 1987 than at the end of 1986. 
DUBLIN-MIDVILLE AOUIFEA SYY

SYREA

AREA IN WHICH DUBLIN AND MIDVILLE AQUIFER SYSTEM
Contours in this area are for the Dublin aquifer system

POTENTIOMETRIC CONTOUR-Shows altitude at which water level would have
stood in tightly cased wells. Dashed where approximately loato

180001 WELL AND IDENTIFICATION NUMBER FOR WHICH HYDROGRAPH IS
INCLUDED IN THIS REPORT

- data point

D-

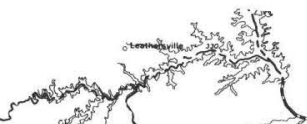

(1)

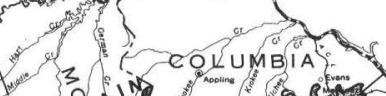

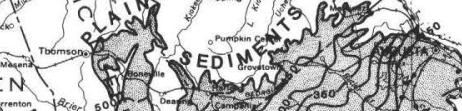

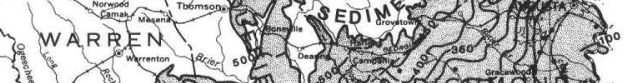

2or ह

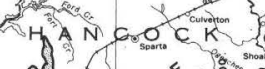

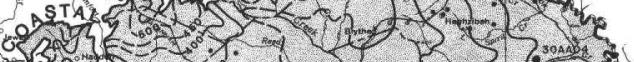

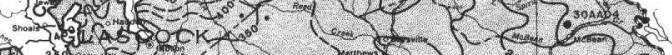

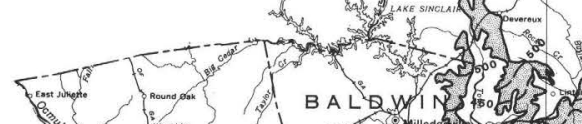

$3^{\circ}$
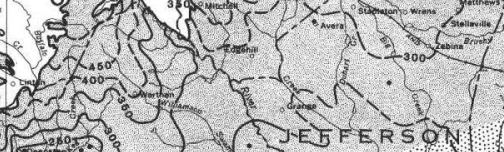

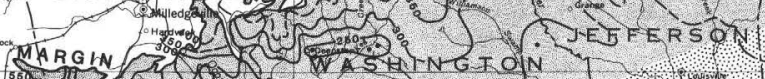

.

M

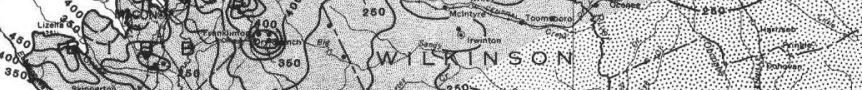

(1)

T.

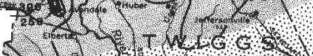

D.

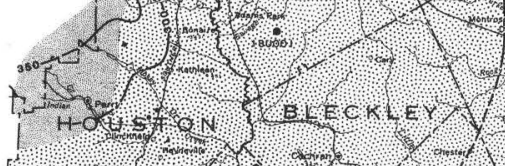

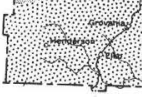

. $1.1 .1 .0 .0 \%$
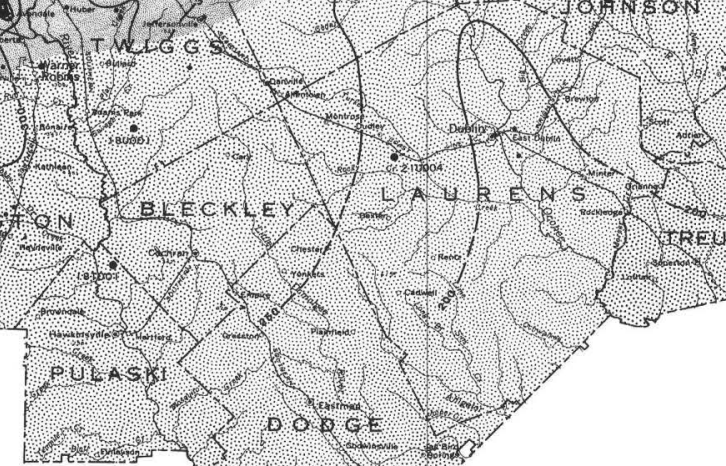

.

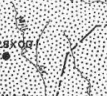

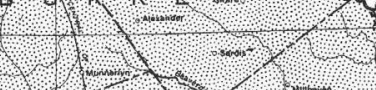

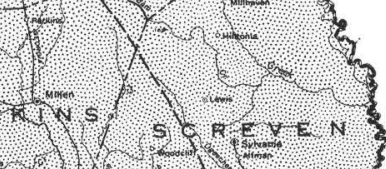

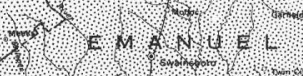

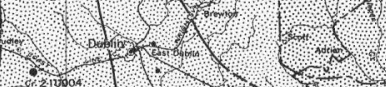
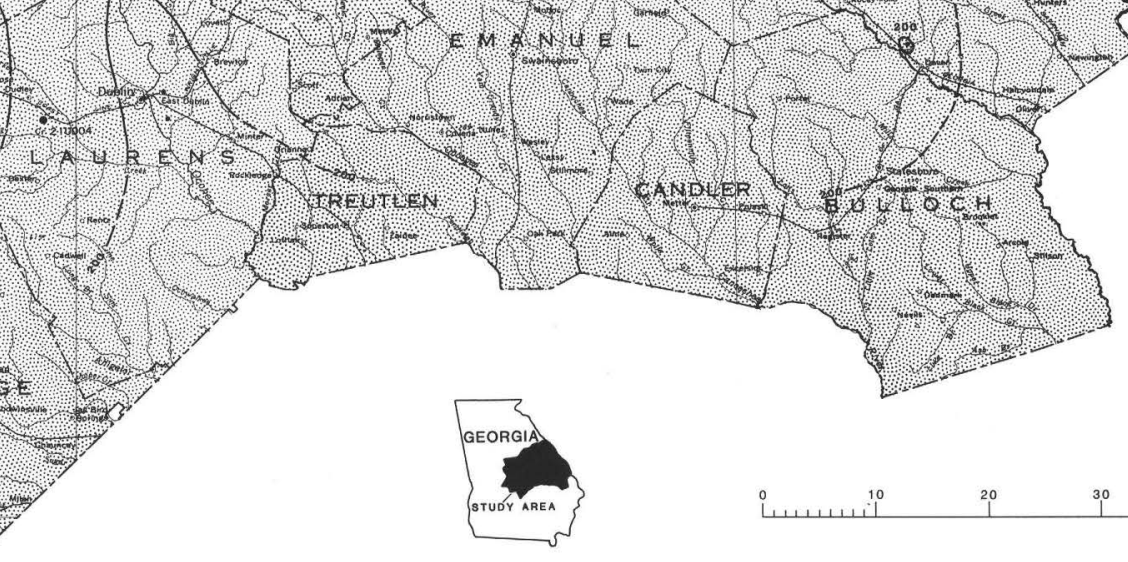

Base Irom U.S. Ge Geological Surve
State base maos, $1: 5000000$.

Outcrop area from Geologic Map of Georgia, 1976

Figure 2.4.2-1.-Observation well locations and the water level in the Dublin and Dublin-Midville aquifer systems, October 1984. 
323302083263401 Local number, 184001.

LOCATION.--Lat $32^{\circ} 33^{\prime} 02^{\prime \prime}$, long $83^{\circ} 26^{\prime} 34^{\prime \prime}$, Hydrologic Unit $03070104,0.6$ mi north of intersection of U.S. Highways 23 and 12 and Georgia Highway 96, 100 feet west of highway near Woods Road West.

Owner: Georgia Kraft.

AQUIFER.--Dublin aquifer system.

WELL CHARACTERISTICS.--Drilled observation well, diameter $6 \mathrm{in}$., depth $590 \mathrm{ft}$; 3 in., depth 586-616 ft, cased to $616 \mathrm{ft}$.

DATUM.--Elevation of land-surface datum is $442 \mathrm{ft}$.

Measuring point: Floor of recorder shelter, $2.6 \mathrm{ft}$ above land-surface datum.

REMARKS.--Borehole geophysical survey conducted. Water quality analysis June 10, 1975 . Water level for period of missing record, August 10 , were estimated.

PERIOD OF RECORD.--JUT y 1975 to current year.

EXTREMES FOR PERIOD OF RECORD.--Highest water level, $162.00 \mathrm{ft}$ below land-surface datum, April 4, 1977; 1owest, $166.39 \mathrm{ft}$ below land-surface datum, August 10-11, 1986.

DEPTH BELOW LAND SURFACE (WATER LEVEL) (FEET) CALENDAR YEAR JANUARY 1987 TO DECEMBER 1987 MEAN VALUES

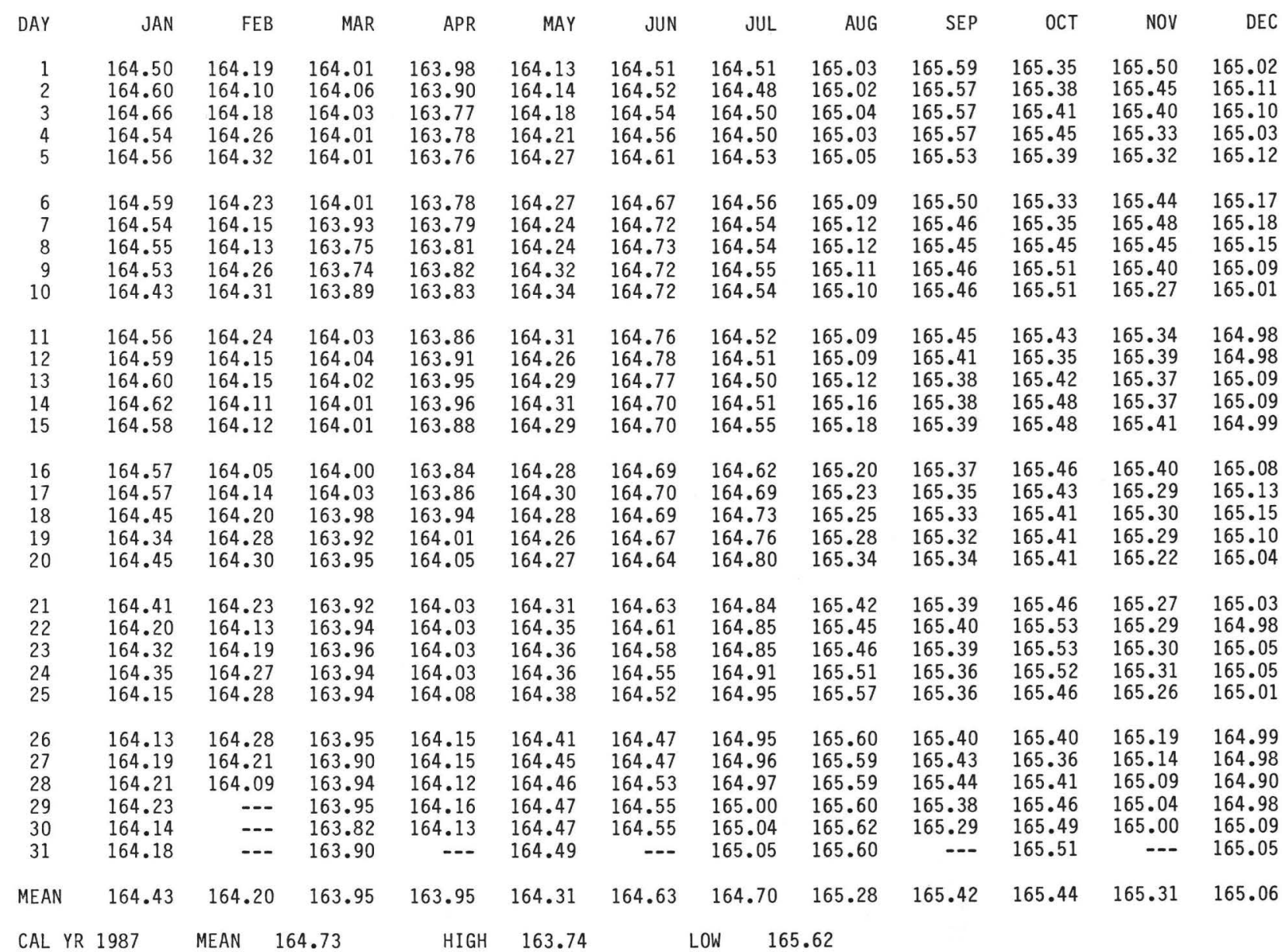




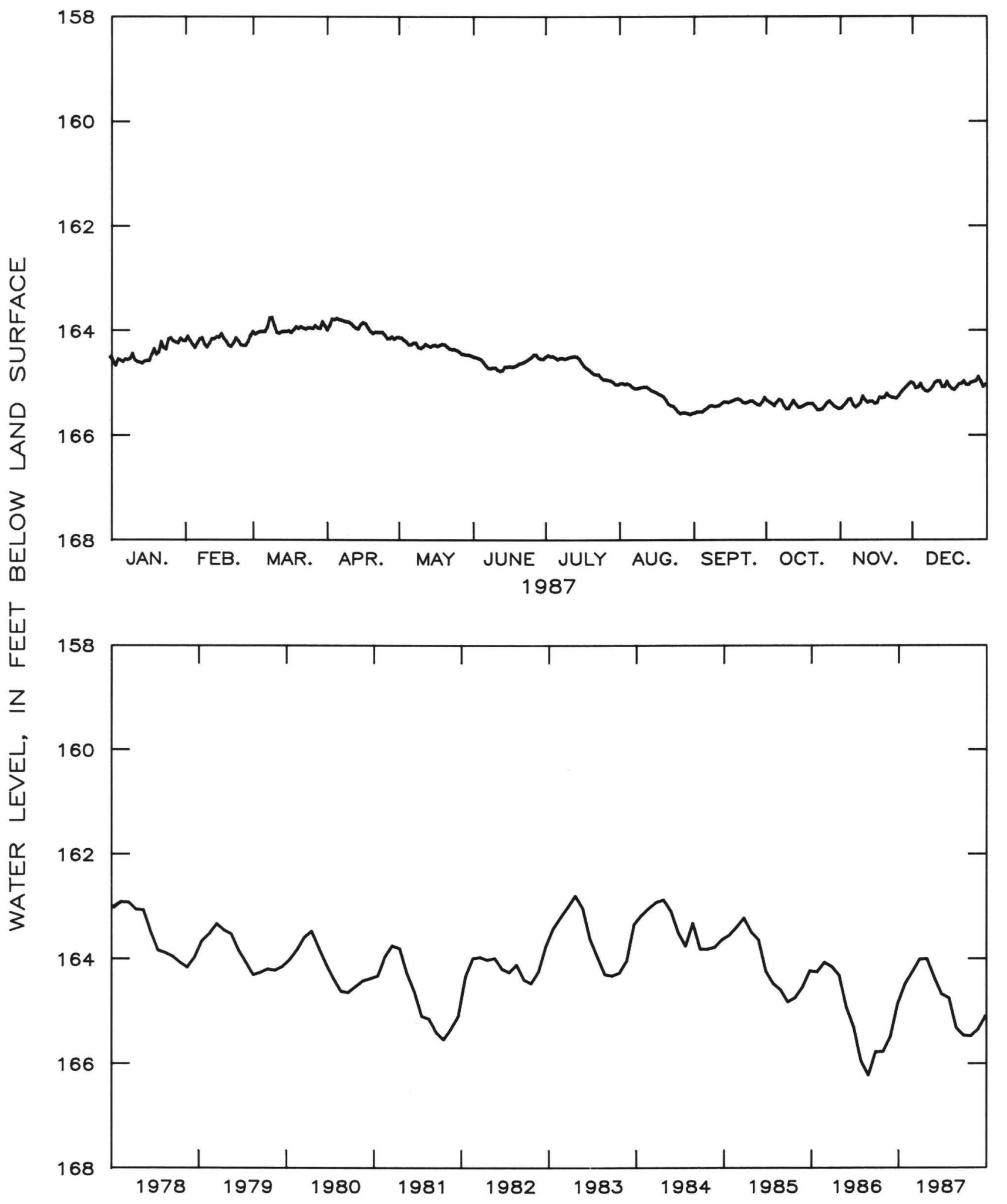

Figure 2.4.2-2--Water level in observation well 184001, Twiggs County. 


\section{$18 T 001$ ARROWHEAD TEST WELL 1 PULASKI COUNTY}

322245083290101 Local number, 18T001.

LOCATION.--Lat $32^{\circ} 22^{\prime} 45^{\prime \prime}$, long $83^{\circ} 29^{\prime} 01^{\prime \prime}$, Hydrologic Unit 03070104 , about 8.5 mi west of Cochran off State Highway 126, at Georgia Forestry Commission Tree Nursery. Owner: U.S. Geological Survey, test well 1

AQUIFER.--Midville aquifer system.

WELL CHARACTERISTICS.--Drilled observation we11, depth 1,555 ft cased to $1,555 \mathrm{ft}$, screened interval $970-980 \mathrm{ft}$, $1,110-1,130 \mathrm{ft}$, and $1,270-1,280 \mathrm{ft}$

DATUM.--Elevation of land-surface datum is $334 \mathrm{ft}$.

Measuring point: Floor of recorder shelter, $3.0 \mathrm{ft}$ above land-surface datum.

REMARKS.--Borehole geophysical survey conducted January 28 and April 15, 1981. Water quality analysis May 12, 1981.

PERIOD OF RECORD.--June 1981 to current year.

EXTREMES FOR PERIOD OF RECORD.--Highest water level, $55.48 \mathrm{ft}$ below land-surface datum, April 12, 1983; 10west, $59.41 \mathrm{ft}$ below land-surface datum, August 22, 1986 .

DEPTH BELOW LAND SURFACE (WATER LEVEL) (FEET) CALENDAR YEAR JANUARY 1987 TO DECEMBER 1987 MEAN VALUES

\begin{tabular}{|c|c|c|c|c|c|c|c|c|c|c|c|c|}
\hline DAY & JAN & FEB & MAR & APR & MAY & JUN & JUL & AUG & SEP & OCT & NOV & DEC \\
\hline $\begin{array}{l}1 \\
2 \\
3 \\
4 \\
5\end{array}$ & $\begin{array}{l}57.93 \\
58.00 \\
58.07 \\
57.96 \\
57.95\end{array}$ & $\begin{array}{l}57.77 \\
57.71 \\
57.80 \\
57.89 \\
57.94\end{array}$ & $\begin{array}{l}57.40 \\
57.41 \\
57.11 \\
57.03 \\
57.12\end{array}$ & $\begin{array}{l}57.52 \\
57.42 \\
57.17 \\
57.18 \\
57.29\end{array}$ & $\begin{array}{l}57.69 \\
57.70 \\
57.74 \\
57.77 \\
57.83\end{array}$ & $\begin{array}{l}58.10 \\
58.10 \\
58.11 \\
58.12 \\
58.15\end{array}$ & $\begin{array}{l}58.24 \\
58.23 \\
58.24 \\
58.24 \\
58.26\end{array}$ & $\begin{array}{l}58.40 \\
58.39 \\
58.42 \\
58.43 \\
58.43\end{array}$ & $\begin{array}{l}58.65 \\
58.66 \\
58.68 \\
58.70 \\
58.70\end{array}$ & $\begin{array}{l}58.77 \\
58.81 \\
58.85 \\
58.89 \\
58.83\end{array}$ & $\begin{array}{l}58.90 \\
58.85 \\
58.79 \\
58.70 \\
58.69\end{array}$ & $\begin{array}{l}58.51 \\
58.60 \\
58.56 \\
58.51 \\
58.60\end{array}$ \\
\hline $\begin{array}{r}6 \\
7 \\
8 \\
9 \\
10\end{array}$ & $\begin{array}{l}57.97 \\
57.94 \\
57.97 \\
57.96 \\
57.88\end{array}$ & $\begin{array}{l}57.81 \\
57.68 \\
57.63 \\
57.79 \\
57.83\end{array}$ & $\begin{array}{l}57.27 \\
57.39 \\
57.41 \\
57.48 \\
57.64\end{array}$ & $\begin{array}{l}57.42 \\
57.49 \\
57.53 \\
57.54 \\
57.55\end{array}$ & $\begin{array}{l}57.81 \\
57.78 \\
57.78 \\
57.87 \\
57.89\end{array}$ & $\begin{array}{l}58.20 \\
58.24 \\
58.25 \\
58.24 \\
58.22\end{array}$ & $\begin{array}{l}58.27 \\
58.22 \\
58.21 \\
58.22 \\
58.21\end{array}$ & $\begin{array}{l}58.46 \\
58.49 \\
58.51 \\
58.50 \\
58.49\end{array}$ & $\begin{array}{l}58.70 \\
58.66 \\
58.67 \\
58.70 \\
58.72\end{array}$ & $\begin{array}{l}58.77 \\
58.80 \\
58.90 \\
58.97 \\
58.96\end{array}$ & $\begin{array}{l}58.83 \\
58.86 \\
58.84 \\
58.79 \\
58.67\end{array}$ & $\begin{array}{l}58.64 \\
58.65 \\
58.62 \\
58.56 \\
58.48\end{array}$ \\
\hline $\begin{array}{l}11 \\
12 \\
13 \\
14 \\
15\end{array}$ & $\begin{array}{l}58.00 \\
58.00 \\
58.00 \\
58.00 \\
57.95\end{array}$ & $\begin{array}{l}57.71 \\
57.58 \\
57.60 \\
57.62 \\
57.65\end{array}$ & $\begin{array}{l}57.79 \\
57.80 \\
57.75 \\
57.70 \\
57.69\end{array}$ & $\begin{array}{l}57.58 \\
57.62 \\
57.64 \\
57.64 \\
57.53\end{array}$ & $\begin{array}{l}57.89 \\
57.86 \\
57.87 \\
57.89 \\
57.87\end{array}$ & $\begin{array}{l}58.25 \\
58.26 \\
58.24 \\
58.18 \\
58.17\end{array}$ & $\begin{array}{l}58.20 \\
58.20 \\
58.20 \\
58.20 \\
58.23\end{array}$ & $\begin{array}{l}58.48 \\
58.50 \\
58.52 \\
58.55 \\
58.57\end{array}$ & $\begin{array}{l}58.75 \\
58.73 \\
58.71 \\
58.73 \\
58.73\end{array}$ & $\begin{array}{l}58.88 \\
58.80 \\
58.88 \\
58.94 \\
58.93\end{array}$ & $\begin{array}{l}58.76 \\
58.80 \\
58.78 \\
58.79 \\
58.84\end{array}$ & $\begin{array}{l}58.44 \\
58.44 \\
58.54 \\
58.53 \\
58.44\end{array}$ \\
\hline $\begin{array}{l}16 \\
17 \\
18 \\
19 \\
20\end{array}$ & $\begin{array}{l}57.93 \\
57.92 \\
57.80 \\
57.61 \\
57.67\end{array}$ & $\begin{array}{l}57.59 \\
57.68 \\
57.74 \\
57.83 \\
57.83\end{array}$ & $\begin{array}{l}57.67 \\
57.69 \\
57.62 \\
57.54 \\
57.56\end{array}$ & $\begin{array}{l}57.48 \\
57.49 \\
57.56 \\
57.65 \\
57.68\end{array}$ & $\begin{array}{l}57.89 \\
57.92 \\
57.93 \\
57.93 \\
57.95\end{array}$ & $\begin{array}{l}58.13 \\
58.14 \\
58.14 \\
58.14 \\
58.14\end{array}$ & $\begin{array}{l}58.29 \\
58.34 \\
58.36 \\
58.36 \\
58.39\end{array}$ & $\begin{array}{l}58.58 \\
58.60 \\
58.61 \\
58.61 \\
58.64\end{array}$ & $\begin{array}{l}58.73 \\
58.72 \\
58.70 \\
58.69 \\
58.71\end{array}$ & $\begin{array}{l}58.91 \\
58.88 \\
58.86 \\
58.85 \\
58.85\end{array}$ & $\begin{array}{l}58.82 \\
58.71 \\
58.73 \\
58.70 \\
58.65\end{array}$ & $\begin{array}{l}58.53 \\
58.57 \\
58.58 \\
58.54 \\
58.50\end{array}$ \\
\hline $\begin{array}{l}21 \\
22 \\
23 \\
24 \\
25\end{array}$ & $\begin{array}{l}57.71 \\
57.51 \\
57.58 \\
57.62 \\
57.44\end{array}$ & $\begin{array}{l}57.74 \\
57.64 \\
57.70 \\
57.77 \\
57.77\end{array}$ & $\begin{array}{l}57.52 \\
57.53 \\
57.54 \\
57.51 \\
57.54\end{array}$ & $\begin{array}{l}57.66 \\
57.64 \\
57.62 \\
57.61 \\
57.66\end{array}$ & $\begin{array}{l}57.98 \\
58.03 \\
58.03 \\
58.03 \\
58.05\end{array}$ & $\begin{array}{l}58.16 \\
58.17 \\
58.13 \\
58.12 \\
58.11\end{array}$ & $\begin{array}{l}58.39 \\
58.37 \\
58.35 \\
58.38 \\
58.38\end{array}$ & $\begin{array}{l}58.70 \\
58.71 \\
58.68 \\
58.70 \\
58.74\end{array}$ & $\begin{array}{l}58.76 \\
58.78 \\
58.78 \\
58.76 \\
58.77\end{array}$ & $\begin{array}{l}58.90 \\
58.96 \\
58.96 \\
58.94 \\
58.88\end{array}$ & $\begin{array}{l}58.72 \\
58.75 \\
58.77 \\
58.78 \\
58.72\end{array}$ & $\begin{array}{l}58.51 \\
58.47 \\
58.53 \\
58.52 \\
58.48\end{array}$ \\
\hline $\begin{array}{l}26 \\
27 \\
28 \\
29 \\
30 \\
31\end{array}$ & $\begin{array}{l}57.31 \\
57.42 \\
57.60 \\
57.74 \\
57.68 \\
57.73\end{array}$ & $\begin{array}{r}57.76 \\
57.67 \\
57.49 \\
-.- \\
-- \\
---\end{array}$ & $\begin{array}{l}57.55 \\
57.50 \\
57.52 \\
57.53 \\
57.39 \\
57.47\end{array}$ & $\begin{array}{r}57.74 \\
57.73 \\
57.70 \\
57.73 \\
57.69 \\
-.-\end{array}$ & $\begin{array}{l}58.07 \\
58.09 \\
58.10 \\
58.09 \\
58.08 \\
58.09\end{array}$ & $\begin{array}{r}58.07 \\
58.06 \\
58.15 \\
58.21 \\
58.24 \\
. .-\end{array}$ & $\begin{array}{l}58.36 \\
58.35 \\
58.34 \\
58.35 \\
58.40 \\
58.42\end{array}$ & $\begin{array}{l}58.75 \\
58.74 \\
58.74 \\
58.74 \\
58.74 \\
58.68\end{array}$ & $\begin{array}{r}58.82 \\
58.86 \\
58.86 \\
58.80 \\
58.70 \\
. \ldots-\end{array}$ & $\begin{array}{l}58.81 \\
58.77 \\
58.83 \\
58.88 \\
58.90 \\
58.92\end{array}$ & $\begin{array}{r}58.65 \\
58.62 \\
58.58 \\
58.53 \\
58.48 \\
. \ldots\end{array}$ & $\begin{array}{l}58.46 \\
58.46 \\
58.39 \\
58.49 \\
58.57 \\
58.53\end{array}$ \\
\hline MEAN & 57.80 & 57.72 & 57.51 & 57.56 & 57.92 & 58.16 & 58.30 & 58.58 & 58.73 & 58.87 & 58.74 & 58.52 \\
\hline
\end{tabular}




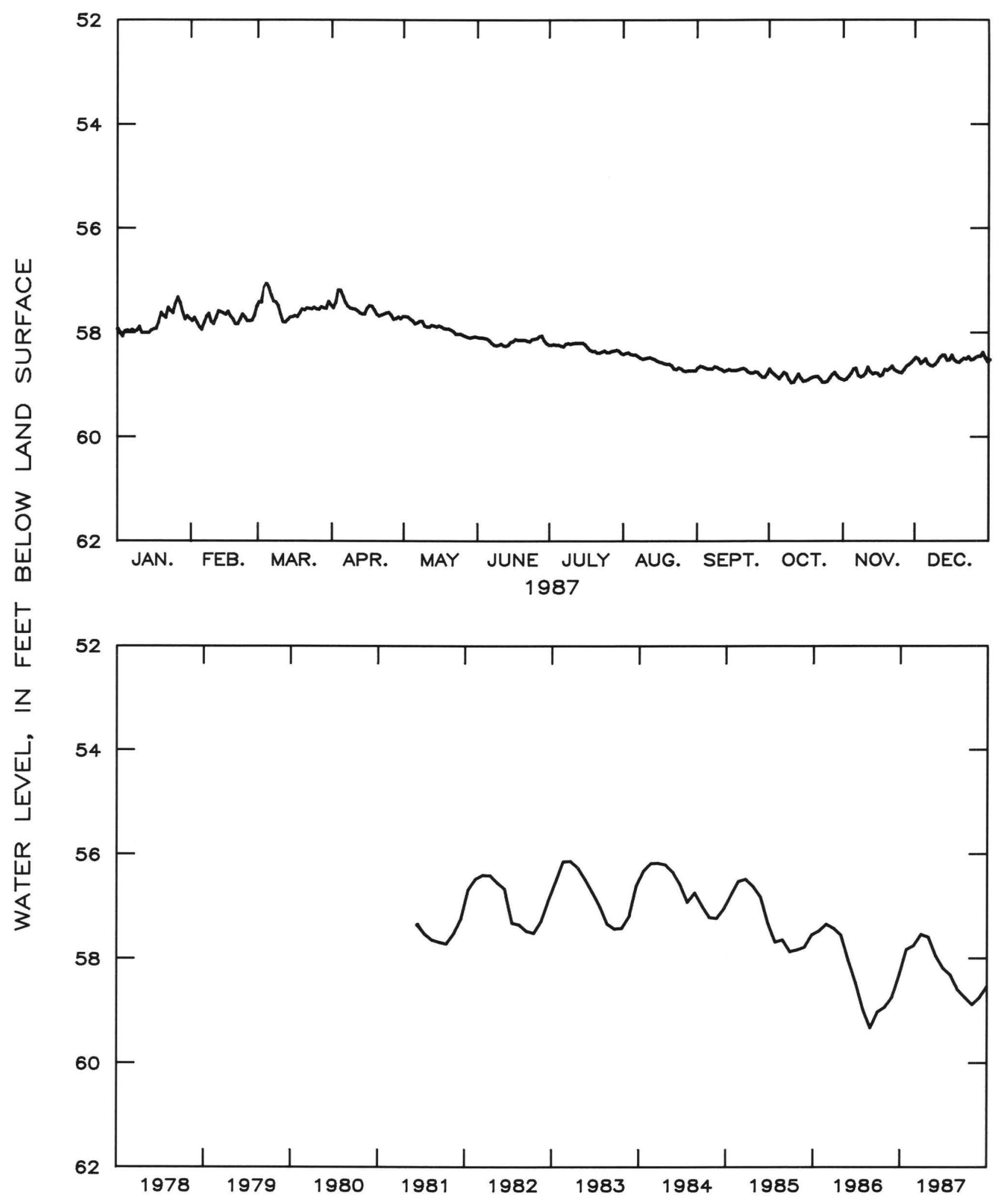

Figure 2.4.2-3.--Water level in observation well 18T001, Pulaski County.

43 
323030083030003 Local number, 210004.

LOCATION.--Lat $32^{\circ} 30^{\prime} 28^{\prime \prime}$, long $83^{\circ} 02^{\prime} 45^{\prime \prime}$, Hydrologic Unit 03070102 , at rest area No. 87 on U.S. Highway I-16 (east).

Owner: U.S. Geological Survey.

AQUIFER.--Midville aquifer system.

WELL CHARACTERISTICS.--Drilled observation we11, depth 1,685 ft, cased to 1,685 ft, screened interval 1,060-1,080 $\mathrm{ft}$, and $1,220-1,240 \mathrm{ft}$.

DATUM.--Elevation of land-surface datum is $282 \mathrm{ft}$.

Measuring point: Floor of recorder shelter, $3.0 \mathrm{ft}$ above land-surface datum.

REMARKS.--Borehole geophysical survey conducted. Water quality analysis January 28, 1982. Water levels for period of missing record, June 8 to August 11, were estimated.

PERIOD OF RECORD.--February 1982 to current year.

EXTREMES FOR PERIOD OF RECORD.--Highest water level, $35.17 \mathrm{ft}$ below land-surface datum, April 3, 1983; lowest, $38.35 \mathrm{ft}$ below land-surface datum, December 30, 1987.

DEPTH BELOW LAND SURFACE (WATER LEVEL) (FEET) CALENDAR YEAR JANUARY 1987 TO DECEMBER 1987 MEAN VALUES

\begin{tabular}{|c|c|c|c|c|c|c|c|c|c|c|c|c|}
\hline DAY & JAN & FEB & MAR & APR & MAY & JUN & JUL & AUG & SEP & OCT & NOV & DEC \\
\hline $\begin{array}{l}1 \\
2 \\
3 \\
4 \\
5\end{array}$ & $\begin{array}{l}37.44 \\
37.44 \\
37.53 \\
37.44 \\
37.42\end{array}$ & $\begin{array}{l}37.32 \\
37.23 \\
37.26 \\
37.34 \\
37.42\end{array}$ & $\begin{array}{l}37.00 \\
37.10 \\
37.19 \\
37.25 \\
37.30\end{array}$ & $\begin{array}{l}37.17 \\
37.12 \\
37.05 \\
37.06 \\
37.06\end{array}$ & $\begin{array}{l}37.16 \\
37.17 \\
37.18 \\
37.21 \\
37.26\end{array}$ & $\begin{array}{l}37.27 \\
37.27 \\
37.28 \\
37.27 \\
37.30\end{array}$ & $\begin{array}{l}37.34 \\
37.33 \\
37.30 \\
37.29 \\
37.29\end{array}$ & $\begin{array}{l}37.49 \\
37.47 \\
37.44 \\
37.41 \\
37.41\end{array}$ & $\begin{array}{l}37.72 \\
37.72 \\
37.73 \\
37.74 \\
37.73\end{array}$ & $\begin{array}{l}37.94 \\
37.99 \\
38.03 \\
38.09 \\
38.03\end{array}$ & $\begin{array}{l}38.32 \\
38.28 \\
38.23 \\
38.14 \\
38.14\end{array}$ & $\begin{array}{l}38.07 \\
38.18 \\
38.16 \\
38.11 \\
38.21\end{array}$ \\
\hline $\begin{array}{r}6 \\
7 \\
8 \\
9 \\
10\end{array}$ & $\begin{array}{l}37.50 \\
37.47 \\
37.48 \\
37.45 \\
37.37\end{array}$ & $\begin{array}{l}37.34 \\
37.23 \\
37.16 \\
37.30 \\
37.40\end{array}$ & $\begin{array}{l}37.32 \\
37.19 \\
37.03 \\
36.94 \\
37.06\end{array}$ & $\begin{array}{l}37.04 \\
37.02 \\
37.02 \\
37.02 \\
37.02\end{array}$ & $\begin{array}{l}37.26 \\
37.21 \\
37.18 \\
37.26 \\
37.27\end{array}$ & $\begin{array}{l}37.34 \\
37.39 \\
37.46 \\
37.42 \\
37.40\end{array}$ & $\begin{array}{l}37.32 \\
37 \cdot 34 \\
37.35 \\
37.37 \\
37.36\end{array}$ & $\begin{array}{l}37.46 \\
37.52 \\
37.56 \\
37.55 \\
37.54\end{array}$ & $\begin{array}{l}37.72 \\
37.70 \\
37.71 \\
37.75 \\
37.78\end{array}$ & $\begin{array}{l}37.97 \\
38.00 \\
38.12 \\
38.20 \\
38.19\end{array}$ & $\begin{array}{l}38.29 \\
38.34 \\
38.32 \\
38.26 \\
38.15\end{array}$ & $\begin{array}{l}38.27 \\
38.29 \\
38.25 \\
38.20 \\
38.11\end{array}$ \\
\hline $\begin{array}{l}11 \\
12 \\
13 \\
14 \\
15\end{array}$ & $\begin{array}{l}37.45 \\
37.48 \\
37.48 \\
37.48 \\
37.45\end{array}$ & $\begin{array}{l}37.36 \\
37.28 \\
37.26 \\
37.22 \\
37.20\end{array}$ & $\begin{array}{l}37.19 \\
37.21 \\
37.18 \\
37.18 \\
37.18\end{array}$ & $\begin{array}{l}37.03 \\
37.07 \\
37.10 \\
37.08 \\
36.98\end{array}$ & $\begin{array}{l}37.24 \\
37.22 \\
37.22 \\
37.25 \\
37.22\end{array}$ & $\begin{array}{l}37.39 \\
37.41 \\
37.40 \\
37.37 \\
37.36\end{array}$ & $\begin{array}{l}37.35 \\
37.34 \\
37.33 \\
37.32 \\
37.32\end{array}$ & $\begin{array}{l}37.53 \\
37.53 \\
37.57 \\
37.60 \\
37.61\end{array}$ & $\begin{array}{l}37.78 \\
37.77 \\
37.75 \\
37.78 \\
37.80\end{array}$ & $\begin{array}{l}38.11 \\
38.02 \\
38.11 \\
38.18 \\
38.18\end{array}$ & $\begin{array}{l}38.24 \\
38.29 \\
38.27 \\
38.28 \\
38.32\end{array}$ & $\begin{array}{l}38.08 \\
38.08 \\
38.20 \\
38.21 \\
38.10\end{array}$ \\
\hline $\begin{array}{l}16 \\
17 \\
18 \\
19 \\
20\end{array}$ & $\begin{array}{l}37.40 \\
37.40 \\
37.32 \\
37.17 \\
37.22\end{array}$ & $\begin{array}{l}37.14 \\
37.16 \\
37.24 \\
37.32 \\
37.37\end{array}$ & $\begin{array}{l}37.17 \\
37.21 \\
37.14 \\
37.09 \\
37.12\end{array}$ & $\begin{array}{l}36.93 \\
36.92 \\
36.98 \\
37.06 \\
37.12\end{array}$ & $\begin{array}{l}37.21 \\
37.22 \\
37.21 \\
37.17 \\
37.18\end{array}$ & $\begin{array}{l}37.35 \\
37 \cdot 34 \\
37.34 \\
37.34 \\
37.33\end{array}$ & $\begin{array}{l}37.35 \\
37.41 \\
37.43 \\
37.44 \\
37.45\end{array}$ & $\begin{array}{l}37.62 \\
37.63 \\
37.64 \\
37.64 \\
37.67\end{array}$ & $\begin{array}{l}37.80 \\
37.80 \\
37.78 \\
37.78 \\
37.81\end{array}$ & $\begin{array}{l}38.17 \\
38.14 \\
38.13 \\
38.13 \\
38.14\end{array}$ & $\begin{array}{l}38.31 \\
38.22 \\
38.25 \\
38.23 \\
38.19\end{array}$ & $\begin{array}{l}38.20 \\
38.27 \\
38.30 \\
38.27 \\
38.21\end{array}$ \\
\hline $\begin{array}{l}21 \\
22 \\
23 \\
24 \\
25\end{array}$ & $\begin{array}{l}37.25 \\
37.09 \\
37.18 \\
37.32 \\
37.22\end{array}$ & $\begin{array}{l}37.33 \\
37.20 \\
37.22 \\
37.32 \\
37.35\end{array}$ & $\begin{array}{l}37.11 \\
37.10 \\
37.14 \\
37.14 \\
37.13\end{array}$ & $\begin{array}{l}37.10 \\
37.08 \\
37.07 \\
37.05 \\
37.12\end{array}$ & $\begin{array}{l}37.22 \\
37.26 \\
37.26 \\
37.25 \\
37.25\end{array}$ & $\begin{array}{l}37.33 \\
37.31 \\
37.28 \\
37.26 \\
37.25\end{array}$ & $\begin{array}{l}37.46 \\
37.46 \\
37.45 \\
37.49 \\
37.50\end{array}$ & $\begin{array}{l}37.72 \\
37.73 \\
37.70 \\
37.73 \\
37.77\end{array}$ & $\begin{array}{l}37.88 \\
37.91 \\
37.91 \\
37.90 \\
37.92\end{array}$ & $\begin{array}{l}38.19 \\
38.28 \\
38.29 \\
38.28 \\
38.23\end{array}$ & $\begin{array}{l}38.25 \\
38.26 \\
38.26 \\
38.29 \\
38.27\end{array}$ & $\begin{array}{l}38.20 \\
38.16 \\
38.24 \\
38.25 \\
38.21\end{array}$ \\
\hline $\begin{array}{l}26 \\
27 \\
28 \\
29 \\
30 \\
31\end{array}$ & $\begin{array}{l}37.21 \\
37.32 \\
37.34 \\
37.36 \\
37.30 \\
37.32\end{array}$ & $\begin{array}{r}37.34 \\
37.28 \\
37.11 \\
\ldots- \\
. .- \\
. .-\end{array}$ & $\begin{array}{l}37.12 \\
37.08 \\
37.10 \\
37.12 \\
36.99 \\
37.04\end{array}$ & $\begin{array}{r}37.19 \\
37.22 \\
37.18 \\
37.23 \\
37.18 \\
\ldots .-\end{array}$ & $\begin{array}{l}37.28 \\
37.30 \\
37.30 \\
37.28 \\
37.28 \\
37.27\end{array}$ & $\begin{array}{r}37.23 \\
37.22 \\
37.24 \\
37.30 \\
37.34 \\
\ldots \ldots\end{array}$ & $\begin{array}{l}37.48 \\
37.47 \\
37.46 \\
37.46 \\
37.48 \\
37.50\end{array}$ & $\begin{array}{l}37.78 \\
37.76 \\
37.77 \\
37.76 \\
37.78 \\
37.77\end{array}$ & $\begin{array}{c}37.98 \\
38.02 \\
38.04 \\
37.97 \\
37.86 \\
-\ldots\end{array}$ & $\begin{array}{l}38.17 \\
38.12 \\
38.19 \\
38.25 \\
38.30 \\
38.33\end{array}$ & $\begin{array}{r}38.20 \\
38.15 \\
38.11 \\
38.06 \\
38.03 \\
. .-\end{array}$ & $\begin{array}{l}38.20 \\
38.19 \\
38.13 \\
38.22 \\
38.35 \\
38.33\end{array}$ \\
\hline MEAN & 37.36 & 37.28 & 37.13 & 37.08 & 37.23 & 37.33 & 37.39 & 37.62 & 37.82 & 38.15 & 38.23 & 38.20 \\
\hline
\end{tabular}

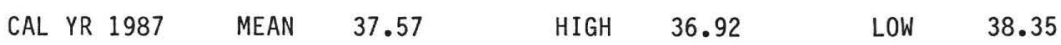



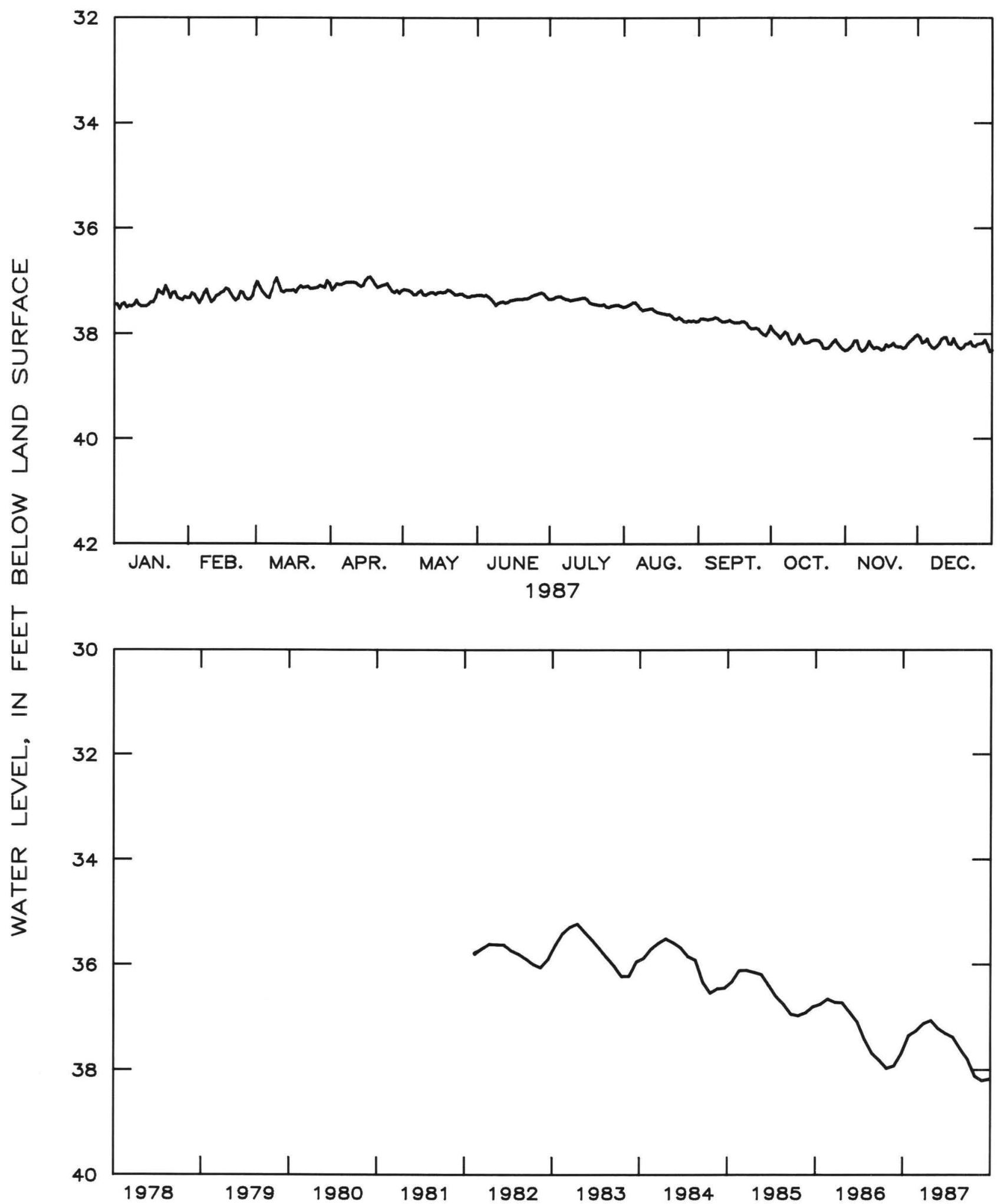

Figure 2.4.2-4.--Water level in observation well 210004 , Laurens County. 
324209082430201 Local number, 24V001.

LOCATION.--lat $32^{\circ} 42^{\prime} 09^{\prime \prime}$, long $82^{\circ} 43^{\prime} 02^{\prime \prime}$, Hydrologic Unit 03070107 , about $500 \mathrm{ft}$ west of State Highway $15,1.8 \mathrm{mi}$ south of intersection of State Highways 15 and 57, at Georgia Forestry Commission Firetower.

Owner: U.S. Geological Survey, test well 1

AQUIFER.--Midville aquifer system.

WELL CHARACTERISTICS.--Drilled observation we11, depth $1,780 \mathrm{ft}$, cased to $1,780 \mathrm{ft}$, screened interval 1,120-1,140 $\mathrm{ft}, 1,260-1,280 \mathrm{ft}$, and $1,320-1,340 \mathrm{ft}$.

DATUM.--Elevation of land-surface datum is $355 \mathrm{ft}$.

Measuring point: Floor of recorder shelter, $3.0 \mathrm{ft}$ above land-surface datum.

REMARKS.---Borehole geophysical survey conducted July 15 and August 18, 1980. Water-quality analysis August 29, 1980. Water levels for period of missing record, March 16 to 18, were estimated.

PERIOD OF RECORD.--September 1980 to current year.

EXTREMES FOR PERIOD OF RECORD.--Highest water level, $129.30 \mathrm{ft}$ below land-surface datum, March 5, 1981; 10west, $135.92 \mathrm{ft}$ below land-surface datum, November 7, 1987.

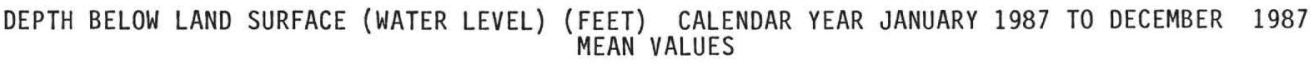

\begin{tabular}{|c|c|c|c|c|c|c|c|c|c|c|c|c|}
\hline DAY & JAN & FEB & MAR & APR & MAY & JUN & JUL & AUG & SEP & OCT & NOV & $\mathrm{DEC}$ \\
\hline $\begin{array}{l}1 \\
2 \\
3 \\
4 \\
5\end{array}$ & $\begin{array}{l}134.26 \\
134.32 \\
134.38 \\
134.34 \\
134.36\end{array}$ & $\begin{array}{l}134.11 \\
134.05 \\
134.02 \\
134.08 \\
134.14\end{array}$ & $\begin{array}{l}133.73 \\
133.78 \\
133.86 \\
133.93 \\
133.97\end{array}$ & $\begin{array}{l}133.70 \\
133.71 \\
133.66 \\
133.63 \\
133.63\end{array}$ & $\begin{array}{l}133.70 \\
133.70 \\
133.70 \\
133.71 \\
133.73\end{array}$ & $\begin{array}{l}133.92 \\
133.92 \\
133.92 \\
133.93 \\
133.93\end{array}$ & $\begin{array}{l}134.20 \\
134.20 \\
134.18 \\
134.18 \\
134.19\end{array}$ & $\begin{array}{l}134.62 \\
134.61 \\
134.59 \\
134.57 \\
134.58\end{array}$ & $\begin{array}{l}135.19 \\
135.18 \\
135.18 \\
135.18 \\
135.19\end{array}$ & $\begin{array}{l}135.51 \\
135.53 \\
135.59 \\
135.65 \\
135.66\end{array}$ & $\begin{array}{l}135.91 \\
135.90 \\
135.87 \\
135.82 \\
135.80\end{array}$ & $\begin{array}{l}135.63 \\
135.70 \\
135.72 \\
135.70 \\
135.74\end{array}$ \\
\hline $\begin{array}{r}6 \\
7 \\
8 \\
9 \\
10\end{array}$ & $\begin{array}{l}134.38 \\
134.37 \\
134.37 \\
134.34 \\
134.33\end{array}$ & $\begin{array}{l}134.10 \\
133.99 \\
133.92 \\
134.02 \\
134.10\end{array}$ & $\begin{array}{l}133.98 \\
133.88 \\
133.77 \\
133.73 \\
133.79\end{array}$ & $\begin{array}{l}133.63 \\
133.63 \\
133.62 \\
133.62 \\
133.62\end{array}$ & $\begin{array}{l}133.74 \\
133.72 \\
133.71 \\
133.73 \\
133.75\end{array}$ & $\begin{array}{l}133.95 \\
134.04 \\
134.12 \\
134.09 \\
134.08\end{array}$ & $\begin{array}{l}134.22 \\
134.25 \\
134.27 \\
134.30 \\
134.30\end{array}$ & $\begin{array}{l}134.64 \\
134.71 \\
134.75 \\
134.75 \\
134.75\end{array}$ & $\begin{array}{l}135.19 \\
135.19 \\
135.19 \\
135.20 \\
135.22\end{array}$ & $\begin{array}{l}135.63 \\
135.62 \\
135.68 \\
135.74 \\
135.76\end{array}$ & $\begin{array}{l}135.88 \\
135.92 \\
135.91 \\
135.89 \\
135.83\end{array}$ & $\begin{array}{l}135.77 \\
135.80 \\
135.80 \\
135.78 \\
135.73\end{array}$ \\
\hline $\begin{array}{l}11 \\
12 \\
13 \\
14 \\
15\end{array}$ & $\begin{array}{l}134.36 \\
134.36 \\
134.37 \\
134.37 \\
134.36\end{array}$ & $\begin{array}{l}134.10 \\
134.06 \\
134.01 \\
134.00 \\
133.96\end{array}$ & $\begin{array}{l}133.87 \\
133.89 \\
133.87 \\
133.86 \\
133.86\end{array}$ & $\begin{array}{l}133.62 \\
133.64 \\
133.66 \\
133.66 \\
133.62\end{array}$ & $\begin{array}{l}133.77 \\
133.77 \\
133.77 \\
133.76 \\
133.74\end{array}$ & $\begin{array}{l}134.08 \\
134.10 \\
134.10 \\
134.08 \\
134.08\end{array}$ & $\begin{array}{l}134.30 \\
134.30 \\
134.30 \\
134.30 \\
134.30\end{array}$ & $\begin{array}{l}134.75 \\
134.76 \\
134.80 \\
134.84 \\
134.86\end{array}$ & $\begin{array}{l}135.26 \\
135.28 \\
135.27 \\
135.29 \\
135.32\end{array}$ & $\begin{array}{l}135.75 \\
135.70 \\
135.71 \\
135.76 \\
135.78\end{array}$ & $\begin{array}{l}135.83 \\
135.87 \\
135.88 \\
135.88 \\
135.89\end{array}$ & $\begin{array}{l}135.67 \\
135.66 \\
135.70 \\
135.74 \\
135.70\end{array}$ \\
\hline $\begin{array}{l}16 \\
17 \\
18 \\
19 \\
20\end{array}$ & $\begin{array}{l}134.33 \\
134.30 \\
134.14 \\
134.03 \\
134.07\end{array}$ & $\begin{array}{l}133.90 \\
133.90 \\
133.95 \\
134.02 \\
134.05\end{array}$ & $\begin{array}{l}133.83 \\
133.79 \\
133.75 \\
133.72 \\
133.71\end{array}$ & $\begin{array}{l}133.57 \\
133.56 \\
133.56 \\
133.60 \\
133.64\end{array}$ & $\begin{array}{l}133.73 \\
133.74 \\
133.75 \\
133.75 \\
133.78\end{array}$ & $\begin{array}{l}134.08 \\
134.08 \\
134.09 \\
134.10 \\
134.09\end{array}$ & $\begin{array}{l}134.34 \\
134.41 \\
134.44 \\
134.46 \\
134.48\end{array}$ & $\begin{array}{l}134.89 \\
134.91 \\
134.93 \\
134.95 \\
134.98\end{array}$ & $\begin{array}{l}135.34 \\
135.35 \\
135.35 \\
135.35 \\
135.36\end{array}$ & $\begin{array}{l}135.78 \\
135.78 \\
135.77 \\
135.76 \\
135.76\end{array}$ & $\begin{array}{l}135.90 \\
135.84 \\
135.81 \\
135.84 \\
135.80\end{array}$ & $\begin{array}{l}135.70 \\
135.76 \\
135.78 \\
135.80 \\
135.78\end{array}$ \\
\hline $\begin{array}{l}21 \\
22 \\
23 \\
24 \\
25\end{array}$ & $\begin{array}{l}134.04 \\
133.95 \\
134.02 \\
134.14 \\
134.08\end{array}$ & $\begin{array}{l}134.04 \\
133.96 \\
133.92 \\
133.99 \\
134.02\end{array}$ & $\begin{array}{l}133.71 \\
133.70 \\
133.71 \\
133.72 \\
133.72\end{array}$ & $\begin{array}{l}133.65 \\
133.65 \\
133.65 \\
133.64 \\
133.66\end{array}$ & $\begin{array}{l}133.82 \\
133.86 \\
133.85 \\
133.86 \\
133.86\end{array}$ & $\begin{array}{l}134.10 \\
134.09 \\
134.07 \\
134.06 \\
134.06\end{array}$ & $\begin{array}{l}134.50 \\
134.51 \\
134.50 \\
134.55 \\
134.57\end{array}$ & $\begin{array}{l}135.07 \\
135.10 \\
135.09 \\
135.10 \\
135.14\end{array}$ & $\begin{array}{l}135.39 \\
135.43 \\
135.45 \\
135.45 \\
135.45\end{array}$ & $\begin{array}{l}135.80 \\
135.86 \\
135.88 \\
135.88 \\
135.87\end{array}$ & $\begin{array}{l}135.82 \\
135.86 \\
135.87 \\
135.88 \\
135.87\end{array}$ & $\begin{array}{l}135.76 \\
135.74 \\
135.73 \\
135.75 \\
135.75\end{array}$ \\
\hline $\begin{array}{l}26 \\
27 \\
28 \\
29 \\
30 \\
31\end{array}$ & $\begin{array}{l}134.08 \\
134.14 \\
134.16 \\
134.20 \\
134.14 \\
134.10\end{array}$ & $\begin{array}{r}134.03 \\
133.96 \\
133.82 \\
--- \\
--- \\
---\end{array}$ & $\begin{array}{l}133.73 \\
133.72 \\
133.70 \\
133.70 \\
133.62 \\
133.61\end{array}$ & $\begin{array}{r}133.71 \\
133.77 \\
133.72 \\
133.72 \\
133.70 \\
-.--\end{array}$ & $\begin{array}{l}133.87 \\
133.90 \\
133.91 \\
133.91 \\
133.91 \\
133.91\end{array}$ & $\begin{array}{r}134.05 \\
134.05 \\
134.07 \\
134.14 \\
134.19 \\
-.-\end{array}$ & $\begin{array}{l}134.56 \\
134.56 \\
134.56 \\
134.57 \\
134.60 \\
134.62\end{array}$ & $\begin{array}{l}135.17 \\
135.17 \\
135.18 \\
135.17 \\
135.19 \\
135.20\end{array}$ & $\begin{array}{r}135.48 \\
135.52 \\
135.56 \\
135.57 \\
135.54 \\
---\end{array}$ & $\begin{array}{l}135.84 \\
135.82 \\
135.80 \\
135.84 \\
135.87 \\
135.91\end{array}$ & $\begin{array}{c}135.84 \\
135.79 \\
135.76 \\
135.70 \\
135.63 \\
---\end{array}$ & $\begin{array}{l}135.74 \\
135.73 \\
135.68 \\
135.69 \\
135.78 \\
135.80\end{array}$ \\
\hline MEAN & 134.23 & 134.01 & 133.78 & 133.65 & 133.79 & 134.06 & 134.39 & 134.90 & 135.33 & 135.75 & 135.84 & 135.74 \\
\hline CAL & 87 & EAN & 63 & HIGH & 133.5 & & & & & & & \\
\hline
\end{tabular}




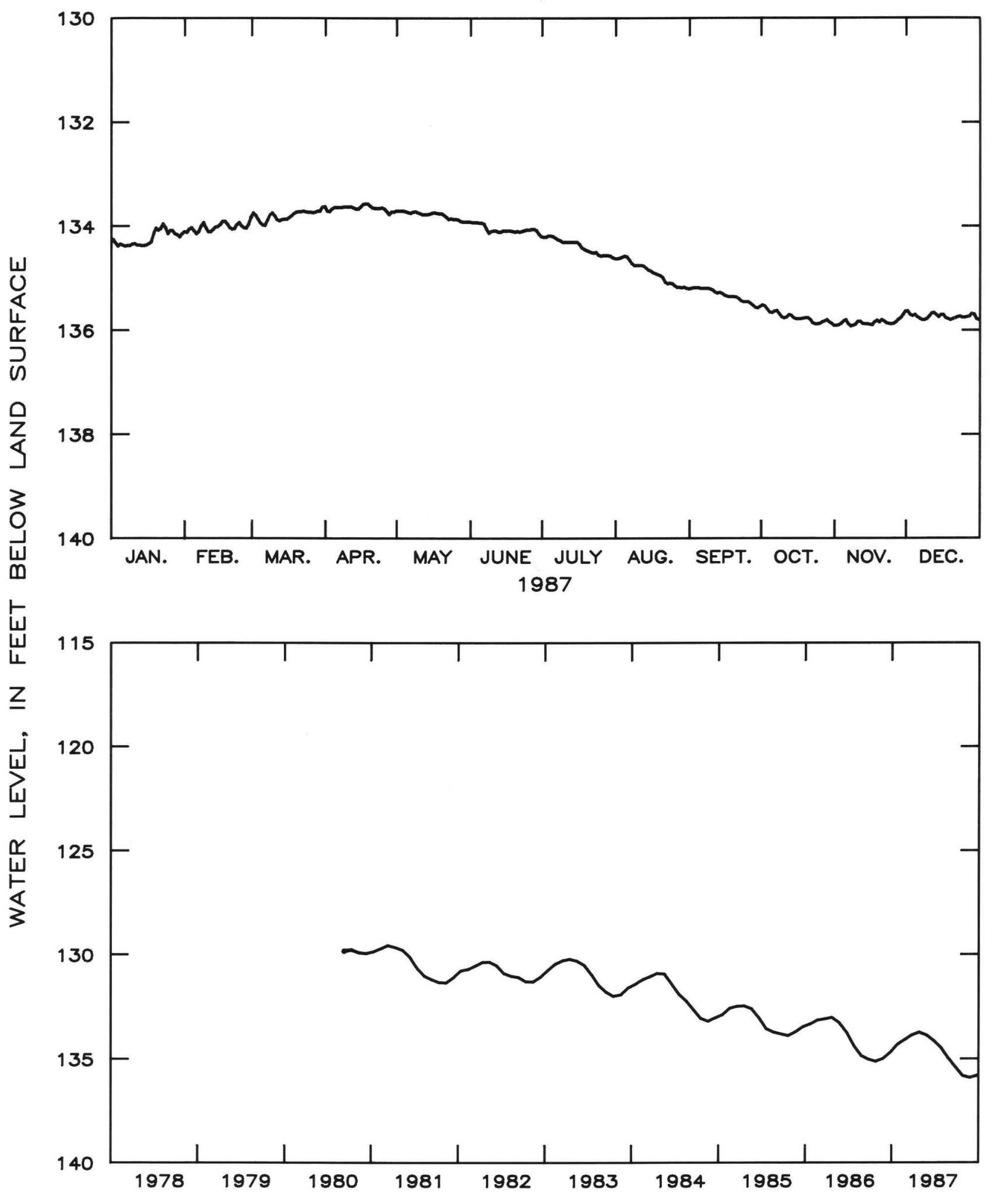

Figure 2.4.2-5.--Water level in observation well 24V001, Johnson County. 
325232082131501 Local number, $28 \times 001$.

LOCATION.--Lat $32^{\circ} 52^{\prime} 32^{\prime \prime}$, long $82^{\circ} 13^{\prime} 15^{\prime \prime}$, Hydrologic Unit $03060201,4.2$ mi north of Midville off State Highway 56 at Southeastern Experiment Station.

Owner: U.S. Geological Survey.

AQUIFER.--Midville aquifer system.

WELL CHARACTERISTICS.--Drilled observation well, diameter $4 \mathrm{in}$., depth $1,045 \mathrm{ft}$, cased to $1,025 \mathrm{ft}$, screened. DATUM.--Elevation of land-surface datum is $269 \mathrm{ft}$.

Measuring point: Floor of recorder platform, $3.04 \mathrm{ft}$ above land-surface datum.

REMARKS.--Borehole geophysical survey conducted March 8 and April 22, 1980. Water quality analyses May $23,1980$. PERIOD OF RECORD.---June 1980 to current year.

EXTREMES FOR PERIOD OF RECORD.---Highest water level, $49.07 \mathrm{ft}$ below land-surface datum, June 4, 1980; lowest, $58.54 \mathrm{ft}$ below land-surface datum, December $30,1987$.

DEPTH BELOW LAND SURFACE (WATER LEVEL) (FEET) CALENDAR YEAR JANUARY 1987 TO DECEMBER 1987 MEAN VALUES

\begin{tabular}{|c|c|c|c|c|c|c|c|c|c|c|c|c|}
\hline DAY & JAN & FEB & MAR & APR & MAY & JUN & JUL & AUG & SEP & ОСТ & NOV & DEC \\
\hline $\begin{array}{l}1 \\
2 \\
3 \\
4 \\
5\end{array}$ & $\begin{array}{l}57.50 \\
57.59 \\
57.64 \\
57.56 \\
57.52\end{array}$ & $\begin{array}{l}57.32 \\
57.23 \\
57.25 \\
57.33 \\
57.41\end{array}$ & $\begin{array}{l}56.99 \\
57.07 \\
57.12 \\
57.14 \\
57.18\end{array}$ & $\begin{array}{l}57.03 \\
57.00 \\
56.95 \\
56.92 \\
56.93\end{array}$ & $\begin{array}{l}56.95 \\
56.95 \\
56.96 \\
56.98 \\
57.02\end{array}$ & $\begin{array}{l}57.04 \\
57.03 \\
57.05 \\
57.05 \\
57.08\end{array}$ & $\begin{array}{l}56.98 \\
56.98 \\
56.97 \\
56.98 \\
57.00\end{array}$ & $\begin{array}{r}57.22 \\
57.23 \\
57.20 \\
57.19 \\
.--\end{array}$ & $\begin{array}{l}--- \\
--- \\
--- \\
---\end{array}$ & $\begin{array}{l}--- \\
--- \\
-\cdots \\
--- \\
---\end{array}$ & $\begin{array}{l}58.42 \\
58.38 \\
58.34 \\
58.26 \\
58.29\end{array}$ & $\begin{array}{l}58.32 \\
58.40 \\
58.38 \\
58.36 \\
58.43\end{array}$ \\
\hline $\begin{array}{r}6 \\
7 \\
8 \\
9 \\
10\end{array}$ & $\begin{array}{l}57.55 \\
57.51 \\
57.52 \\
57.51 \\
57.44\end{array}$ & $\begin{array}{l}57.34 \\
57.23 \\
57.17 \\
57.30 \\
57.36\end{array}$ & $\begin{array}{l}57.19 \\
57.09 \\
56.96 \\
56.89 \\
56.98\end{array}$ & $\begin{array}{l}56.93 \\
56.93 \\
56.92 \\
56.92 \\
56.92\end{array}$ & $\begin{array}{l}57.04 \\
57.00 \\
56.99 \\
57.04 \\
57.08\end{array}$ & $\begin{array}{l}57.11 \\
57.14 \\
57.14 \\
57.11 \\
57.10\end{array}$ & $\begin{array}{l}57.02 \\
57.04 \\
57.05 \\
57.05 \\
57.05\end{array}$ & $\begin{array}{l}--- \\
--- \\
--- \\
---\end{array}$ & $\begin{array}{l}--- \\
--- \\
--- \\
---\end{array}$ & $\begin{array}{l}--- \\
-\cdots \\
--- \\
--- \\
--\end{array}$ & $\begin{array}{l}58.40 \\
58.45 \\
58.44 \\
58.39 \\
58.30\end{array}$ & $\begin{array}{l}58.47 \\
58.50 \\
58.48 \\
58.43 \\
58.36\end{array}$ \\
\hline $\begin{array}{l}11 \\
12 \\
13 \\
14 \\
15\end{array}$ & $\begin{array}{l}57.48 \\
57.52 \\
57.54 \\
57.56 \\
57.54\end{array}$ & $\begin{array}{l}57.32 \\
57.22 \\
57.22 \\
57.18 \\
57.19\end{array}$ & $\begin{array}{l}57.11 \\
57.11 \\
57.08 \\
57.08 \\
57.07\end{array}$ & $\begin{array}{l}56.93 \\
56.97 \\
56.99 \\
57.02 \\
56.94\end{array}$ & $\begin{array}{l}57.04 \\
57.00 \\
57.02 \\
57.03 \\
57.00\end{array}$ & $\begin{array}{l}57.11 \\
57.10 \\
57.08 \\
57.02 \\
57.00\end{array}$ & $\begin{array}{l}57.04 \\
57.03 \\
57.03 \\
57.03 \\
57.05\end{array}$ & $\begin{array}{l}--- \\
--- \\
--- \\
---\end{array}$ & $\begin{array}{l}--- \\
--- \\
--- \\
---\end{array}$ & $\begin{array}{l}--- \\
--- \\
--- \\
--- \\
--\end{array}$ & $\begin{array}{l}58.36 \\
58.43 \\
58.41 \\
58.42 \\
58.46\end{array}$ & $\begin{array}{l}58.32 \\
58.32 \\
58.40 \\
58.44 \\
58.35\end{array}$ \\
\hline $\begin{array}{l}16 \\
17 \\
18 \\
19 \\
20\end{array}$ & $\begin{array}{l}57.52 \\
57.52 \\
57.46 \\
57.34 \\
57.42\end{array}$ & $\begin{array}{l}57.14 \\
57.14 \\
57.22 \\
57.28 \\
57.33\end{array}$ & $\begin{array}{l}57.06 \\
57.10 \\
57.03 \\
56.98 \\
56.98\end{array}$ & $\begin{array}{l}56.86 \\
56.84 \\
56.88 \\
56.94 \\
56.98\end{array}$ & $\begin{array}{l}56.99 \\
57.02 \\
57.00 \\
56.99 \\
56.98\end{array}$ & $\begin{array}{l}57.00 \\
57.01 \\
57.03 \\
56.98 \\
56.97\end{array}$ & $\begin{array}{l}57.09 \\
57.15 \\
57.20 \\
57.21 \\
57.23\end{array}$ & $\begin{array}{l}--- \\
--- \\
--- \\
---\end{array}$ & $\begin{array}{l}--- \\
--- \\
--- \\
---\end{array}$ & $\begin{array}{l}--- \\
--- \\
--- \\
---\end{array}$ & $\begin{array}{l}58.48 \\
58.39 \\
58.40 \\
58.41 \\
58.36\end{array}$ & $\begin{array}{l}58.42 \\
58.48 \\
58.52 \\
58.50 \\
58.44\end{array}$ \\
\hline $\begin{array}{l}21 \\
22 \\
23 \\
24 \\
25\end{array}$ & $\begin{array}{l}57.36 \\
57.21 \\
57.27 \\
57.35 \\
57.24\end{array}$ & $\begin{array}{l}57.29 \\
57.19 \\
57.19 \\
57.29 \\
57.32\end{array}$ & $\begin{array}{l}56.97 \\
56.97 \\
56.98 \\
56.99 \\
56.98\end{array}$ & $\begin{array}{l}56.98 \\
56.96 \\
56.94 \\
56.91 \\
56.94\end{array}$ & $\begin{array}{l}56.98 \\
57.02 \\
57.02 \\
57.01 \\
57.02\end{array}$ & $\begin{array}{l}56.96 \\
56.96 \\
56.94 \\
56.95 \\
56.92\end{array}$ & $\begin{array}{l}57.25 \\
57.22 \\
57.22 \\
57.23 \\
57.25\end{array}$ & $\begin{array}{l}--- \\
--- \\
--- \\
---\end{array}$ & $\begin{array}{l}--- \\
--- \\
--- \\
---\end{array}$ & $\begin{array}{l}--- \\
--- \\
--- \\
--- \\
--\end{array}$ & $\begin{array}{l}58.43 \\
58.47 \\
58.49 \\
58.50 \\
58.46\end{array}$ & $\begin{array}{l}58.43 \\
58.40 \\
58.44 \\
58.47 \\
58.44\end{array}$ \\
\hline $\begin{array}{l}26 \\
27 \\
28 \\
29 \\
30 \\
31\end{array}$ & $\begin{array}{l}57.20 \\
57.30 \\
57.21 \\
57.32 \\
57.25 \\
57.28\end{array}$ & $\begin{array}{r}57.32 \\
57.26 \\
57.10 \\
- \\
-- \\
-\end{array}$ & $\begin{array}{l}56.97 \\
56.94 \\
56.96 \\
56.99 \\
56.90 \\
56.92\end{array}$ & $\begin{array}{r}57.02 \\
57.02 \\
56.97 \\
56.99 \\
56.97 \\
---\end{array}$ & $\begin{array}{l}57.03 \\
57.06 \\
57.07 \\
57.06 \\
57.04 \\
57.04\end{array}$ & $\begin{array}{r}56.86 \\
56.84 \\
56.90 \\
56.97 \\
56.98 \\
.-\end{array}$ & $\begin{array}{l}57.23 \\
57.20 \\
57.19 \\
57.17 \\
57.22 \\
57.24\end{array}$ & $\begin{array}{l}--- \\
--- \\
--- \\
--- \\
---\end{array}$ & $\begin{array}{l}--- \\
--- \\
--- \\
--- \\
---\end{array}$ & $\begin{array}{l}58.23 \\
58.28 \\
58.34 \\
58.38 \\
58.42\end{array}$ & $\begin{array}{r}58.42 \\
58.38 \\
58.36 \\
58.31 \\
58.27 \\
.\end{array}$ & $\begin{array}{l}58.42 \\
58.42 \\
58.37 \\
58.42 \\
58.54 \\
58.53\end{array}$ \\
\hline MEAN & 57.43 & 57.26 & 57.03 & 56.95 & 57.01 & 57.01 & 57.12 & 57.21 & -.- & 58.33 & 58.40 & 58.4 \\
\hline
\end{tabular}




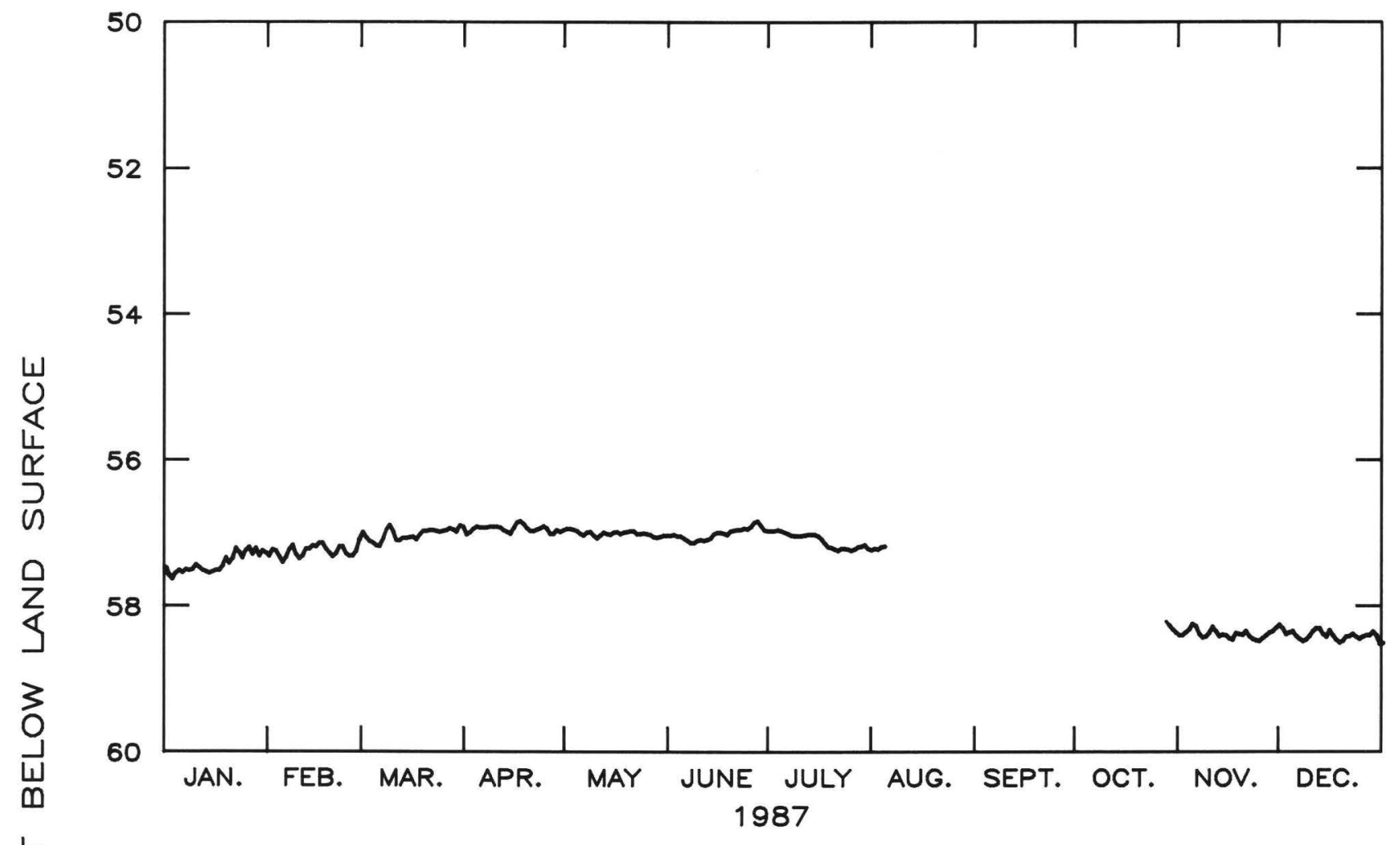

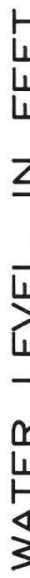

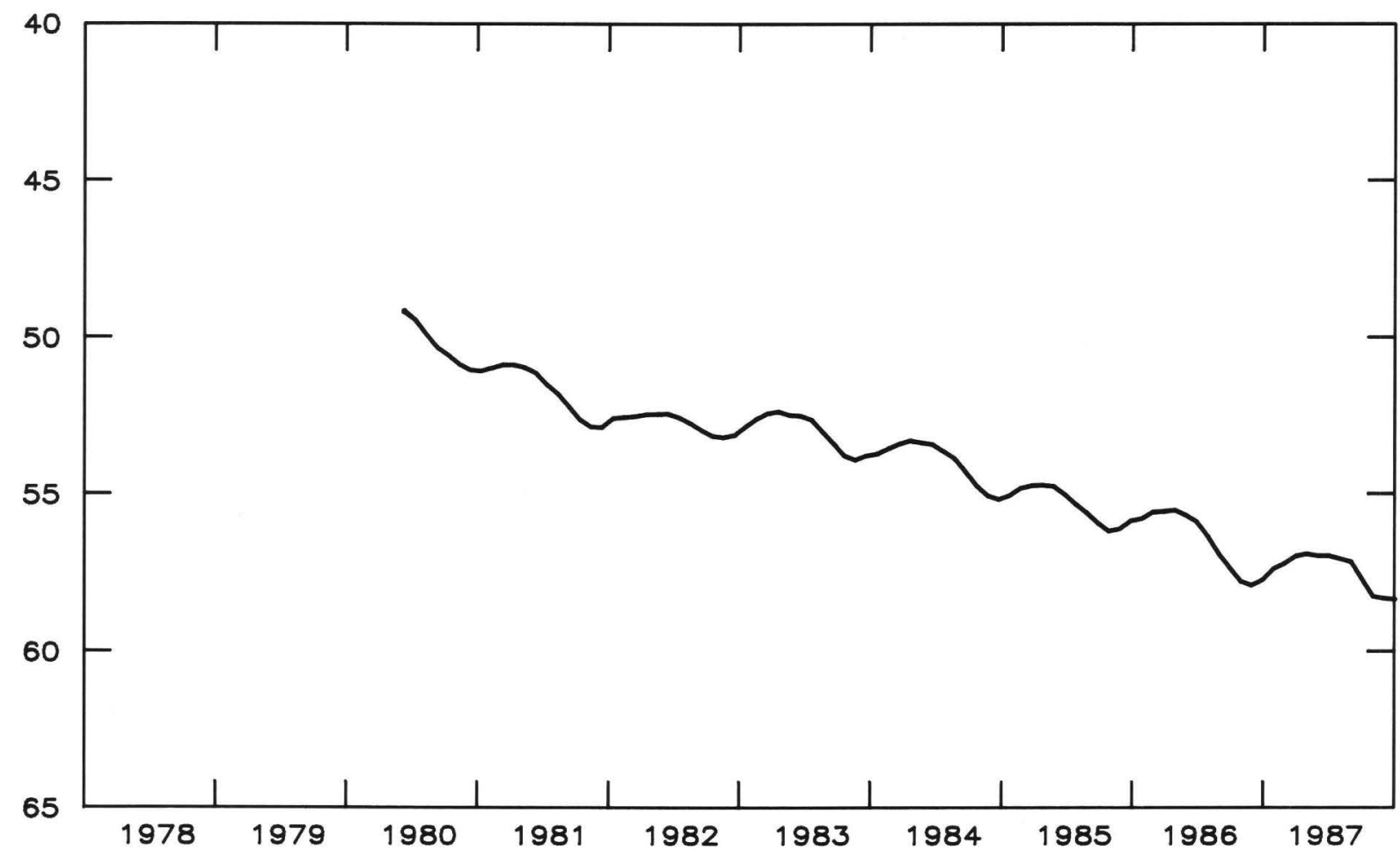

Figure 2.4.2-6.--Water level in observation well 28x001, Burke County. 
331711081573701 Local number, 30AA04.

LOCATION.--lat $33^{\circ} 15^{\prime} 25^{\prime \prime}$, long $81^{\circ} 57^{\prime} 47^{\prime \prime}$, Hydrologic Unit $03060106,1.5$ mi north of McBean, 0.65 mi south of Little McBean Creek, 0.5 mi west of Georgia Highway 56.

Owner: Richmond County water system.

AQUIFER.--Dublin-Midville aquifer system.

WELL CHARACTERISTICS.--Drilled unused municipal well, diameter $6 \mathrm{in}$., depth $496 \mathrm{ft}$, cased to $174 \mathrm{ft}$, screened. DATUM.--Elevation of land-surface datum is $293 \mathrm{ft}$.

Measuring point: Top of 6-in. casing, $1.5 \mathrm{ft}$ above land-surface datum.

REMARKS.--Borehole geophysical survey conducted October 23, 1967 . Water-quality sample collected November 26, 1967.

PERIOD OF RECORD.--June 1979 to current year.

EXTREMES FOR PERIOD OF RECORD.--Highest water level, $116.70 \mathrm{ft}$ below land-surface datum, May 30, 1984; lowest, $129.06 \mathrm{ft}$ below land-surface datum, August 3, 1986.

DEPTH BELOW LAND SURFACE (WATER LEVEL) (FEET) CALENDAR YEAR JANUARY 1987 TO DECEMBER 1987 MEAN VALUES

\begin{tabular}{|c|c|c|c|c|c|c|c|c|c|c|c|c|}
\hline DAY & JAN & FEB & MAR & APR & MAY & JUN & JUL & AUG & SEP & OCT & NOV & DEC \\
\hline $\begin{array}{l}1 \\
2 \\
3 \\
4 \\
5\end{array}$ & $\begin{array}{l}127.52 \\
127.40 \\
127.36 \\
127.36 \\
127.35\end{array}$ & $\begin{array}{l}126.82 \\
126.77 \\
126.72 \\
126.73 \\
126.74\end{array}$ & $\begin{array}{l}126.11 \\
126.09 \\
126.13 \\
126.16 \\
126.19\end{array}$ & $\begin{array}{l}126.37 \\
126.36 \\
126.37 \\
126.36 \\
126.35\end{array}$ & $\begin{array}{l}126.74 \\
126.74 \\
126.75 \\
126.92 \\
127.04\end{array}$ & $\begin{array}{l}127.73 \\
127.90 \\
128.04 \\
127.90 \\
127.60\end{array}$ & $\begin{array}{l}127.26 \\
127.48 \\
127.56 \\
127.45 \\
127.41\end{array}$ & $\begin{array}{l}128.24 \\
128.10 \\
128.03 \\
128.01 \\
128.04\end{array}$ & $\begin{array}{l}128.47 \\
128.40 \\
128.38 \\
128.37 \\
128.37\end{array}$ & $\begin{array}{l}128.40 \\
128.40 \\
128.41 \\
128.43 \\
128.43\end{array}$ & $\begin{array}{l}128.71 \\
128.68 \\
128.66 \\
128.66 \\
128.58\end{array}$ & $\begin{array}{l}128.34 \\
128.36 \\
128.37 \\
128.36 \\
128.38\end{array}$ \\
\hline $\begin{array}{r}6 \\
7 \\
8 \\
9 \\
10\end{array}$ & $\begin{array}{l}127.36 \\
127.36 \\
127.36 \\
127.36 \\
127.33\end{array}$ & $\begin{array}{l}126.70 \\
126.60 \\
126.53 \\
126.54 \\
126.57\end{array}$ & $\begin{array}{l}126.2 \\
126.1 \\
126.0 \\
125.9\end{array}$ & $\begin{array}{l}126.34 \\
126.34 \\
126.34 \\
126.34 \\
126.34\end{array}$ & 6.91 & $\begin{array}{l}127.56 \\
127.60 \\
127.46 \\
127.45 \\
127.64\end{array}$ & 7.35 & $\begin{array}{l}127.96 \\
127.96\end{array}$ & $\begin{array}{l}128.32 \\
128.31\end{array}$ & 128.42 & $\begin{array}{l}128.57 \\
128.58 \\
128.57 \\
128.55 \\
128.51\end{array}$ & $\begin{array}{l}128.43 \\
128.45 \\
128.45 \\
128.43 \\
128.41\end{array}$ \\
\hline $\begin{array}{l}11 \\
12 \\
13 \\
14 \\
15\end{array}$ & $\begin{array}{l}127.30 \\
127.30 \\
127.31 \\
127.32 \\
127.30\end{array}$ & $\begin{array}{l}126.58 \\
126.57 \\
126.54 \\
126.50 \\
126.48\end{array}$ & $\begin{array}{l}126.37 \\
126.71 \\
126.74 \\
126.73\end{array}$ & & & & & & & $\begin{array}{l}128.48 \\
128.52\end{array}$ & $\begin{array}{l}128.52 \\
128.56 \\
128.59 \\
128.62 \\
128.62\end{array}$ & $\begin{array}{l}128.38 \\
128.38 \\
128.42 \\
128.45 \\
128.42\end{array}$ \\
\hline $\begin{array}{l}16 \\
17 \\
18 \\
19 \\
20\end{array}$ & $\begin{array}{l}127.28 \\
127.26 \\
127.19 \\
127.03 \\
126.96\end{array}$ & $\begin{array}{l}126.44 \\
126.41 \\
126.44 \\
126.47 \\
126.49\end{array}$ & $\begin{array}{l}126.70 \\
126.64 \\
126.58 \\
126.52 \\
126.50\end{array}$ & $\begin{array}{l}126.52 \\
126.48 \\
126.46 \\
126.52 \\
126.57\end{array}$ & & & & & & & $\begin{array}{l}128.62 \\
128.52 \\
128.43 \\
128.44 \\
128.43\end{array}$ & $\begin{array}{l}128.42 \\
128.45 \\
128.48 \\
128.50 \\
128.48\end{array}$ \\
\hline $\begin{array}{l}21 \\
22 \\
23 \\
24 \\
25\end{array}$ & $\begin{array}{l}126.98 \\
126.89 \\
126.86 \\
126.89 \\
126.89\end{array}$ & $\begin{array}{l}126.50 \\
126.45 \\
126.42 \\
126.45 \\
126.47\end{array}$ & $\begin{array}{l}126.48 \\
126.48 \\
126.48 \\
126.48 \\
126.47\end{array}$ & $\begin{array}{l}126.58 \\
126.72 \\
126.92 \\
126.89 \\
126.80\end{array}$ & $\begin{array}{l}127.30 \\
127.23 \\
127.28 \\
127.48 \\
127.41\end{array}$ & $\begin{array}{l}127.27 \\
127.25 \\
127.24 \\
127.24 \\
127.22\end{array}$ & & & $\begin{array}{l}128.34 \\
128.40 \\
128.48 \\
128.50 \\
128.46\end{array}$ & $\begin{array}{l}128.62 \\
128.66 \\
128.79 \\
128.83 \\
128.78\end{array}$ & $\begin{array}{l}128.45 \\
128.48 \\
128.48 \\
128.48 \\
128.47\end{array}$ & $\begin{array}{l}120.45 \\
128.48 \\
128.48\end{array}$ \\
\hline $\begin{array}{l}26 \\
27 \\
28 \\
29 \\
30 \\
31\end{array}$ & $\begin{array}{l}126.84 \\
126.85 \\
126.85 \\
126.86 \\
126.84 \\
126.82\end{array}$ & $\begin{array}{r}126.49 \\
126.43 \\
126.26 \\
--- \\
--- \\
---\end{array}$ & $\begin{array}{l}126.47 \\
126.46 \\
126.44 \\
126.44 \\
126.40 \\
126.36\end{array}$ & $\begin{array}{r}126.86 \\
126.80 \\
126.72 \\
126.85 \\
126.82 \\
---\end{array}$ & $\begin{array}{l}127.41 \\
127.52 \\
127.51 \\
127.60 \\
127.89 \\
127.88\end{array}$ & $\begin{array}{r}127.09 \\
126.98 \\
127.03 \\
127.11 \\
127.17 \\
. .-\end{array}$ & $\begin{array}{l}128.02 \\
127.96 \\
128.06 \\
128.30 \\
128.34 \\
128.31\end{array}$ & $\begin{array}{l}128.68 \\
128.54 \\
128.45 \\
128.45 \\
128.61 \\
128.58\end{array}$ & $\begin{array}{r}128.43 \\
128.43 \\
128.43 \\
128.44 \\
128.43 \\
.--\end{array}$ & $\begin{array}{l}128.64 \\
128.55 \\
128.53 \\
128.64 \\
128.73 \\
128.73\end{array}$ & $\begin{array}{r}128.43 \\
128.38 \\
128.35 \\
128.32 \\
128.32 \\
---\end{array}$ & $\begin{array}{l}128.47 \\
128.47 \\
128.44 \\
128.43 \\
128.48 \\
128.52\end{array}$ \\
\hline EAN & 127.15 & 126.54 & 126.38 & 126.55 & 127.15 & 127.48 & 127.86 & 128.32 & 128.33 & 128.56 & 128.52 & 128.4 \\
\hline
\end{tabular}

$\begin{array}{lllllll}\text { CAL YR } 1987 & \text { MEAN } & 127.61 & \text { HIGH } & 125.99 & \text { LOW } & 128.83\end{array}$ 


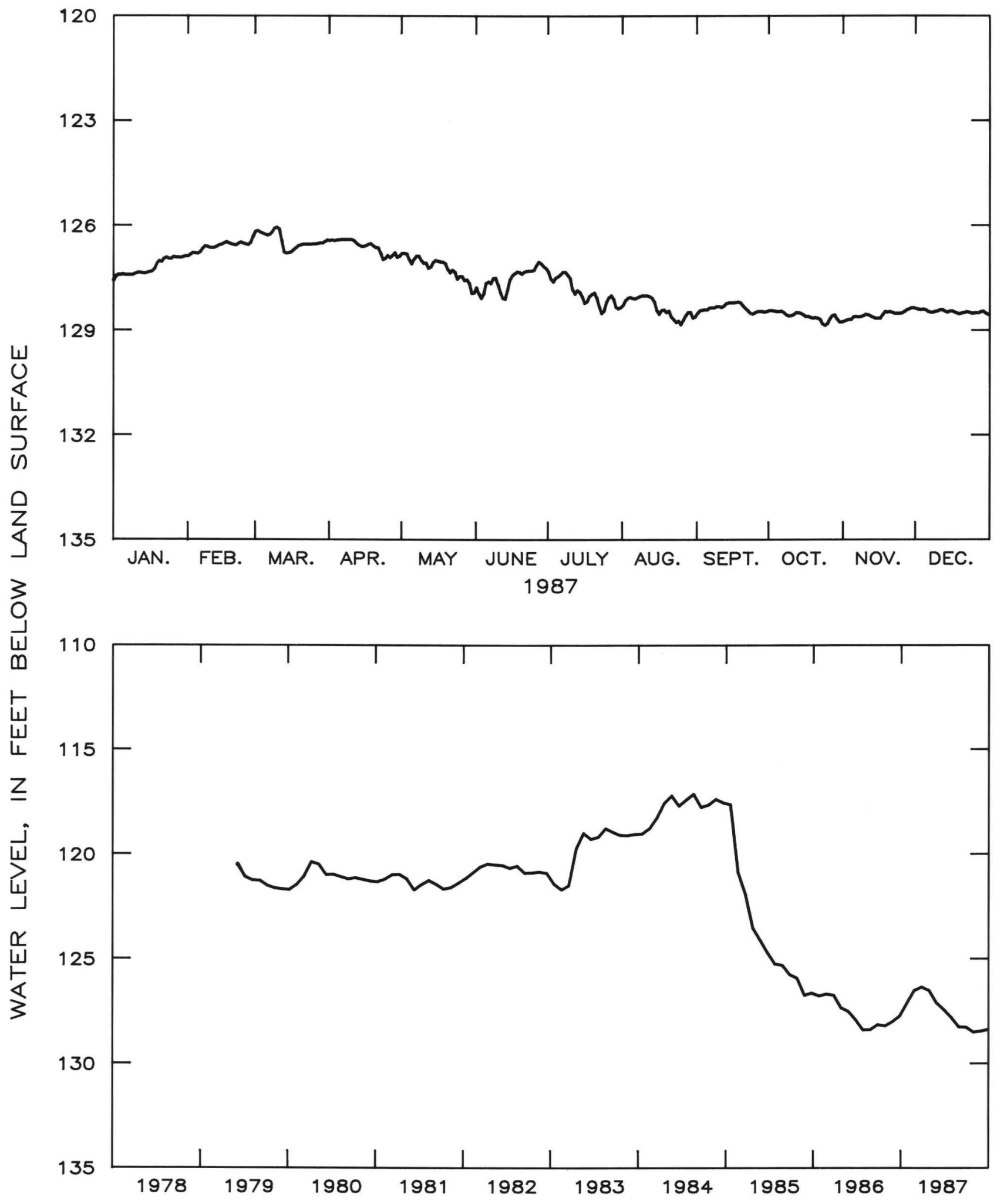

Figure 2.4.2-7 Water level in observation well 3OAAO4, Richmond County. 


\subsection{Clayton Aquifer}

The Clayton aquifer consists of limestone and sand, and supplies more than $20 \mathrm{Mgal} / \mathrm{d}$ for municipal and agricultural use in the area between the Chattahoochee and Flint Rivers in southwestern Georgia (Clarke and others, 1984a). Pumping from the Clayton has resulted in the development of a cone of depression centered at Albany.

During October and November, water levels were measured in 68 wells tapping the Clayton aquifer. From these measurements, a map showing the configuration of the potentiometric surface was prepared.

In the Cordele, Cuthbert and Albany areas, the water level in the Clayton aquifer is primarily affected by seasonal changes in local and regional pumping. In 1987, above normal precipitation during the first six months caused water levels to recover from the effects of the 1986 drought. By the end of April, water levels had recovered from the record lows set in late summer and early fall of 1986. Increases in water levels were from $4.2 \mathrm{ft}$ in well 14P014 to $49.6 \mathrm{ft}$ in well 11L002. By the end of 1987, water levels in the Albany and Cordele areas had recovered 0.1 to $2.8 \mathrm{ft}$ from the 1986 yearend water levels. At the end of 1987, well 07 N001 near Cuthbert was $0.2 \mathrm{ft}$ lower than the 1986 year-end water level.

The mean water level in well 07 N001 near Cuthbert was $0.4 \mathrm{ft}$ lower in 1987 than in 1986. These declines probably were a result of the decrease in rainfall in the latter six months of 1987, and to an increase in local pumping.

Although water levels showed some recovery, mean water levels in the Cordele and the Cuthbert areas were lower in 1987 than in 1986. In the Cordele area, the mean water level in well $14 \mathrm{P} 014$ was $3.9 \mathrm{ft}$ lower than in 1986. Further south in the Albany area, the mean water level in well 11 L002 in western Dougherty County was $1.7 \mathrm{ft}$ higher than in 1986. In wel1 13 L002 within the Albany city limits, the mean water level was $1.4 \mathrm{ft}$ higher than in 1986. These rises reflect reductions in local pumping. 


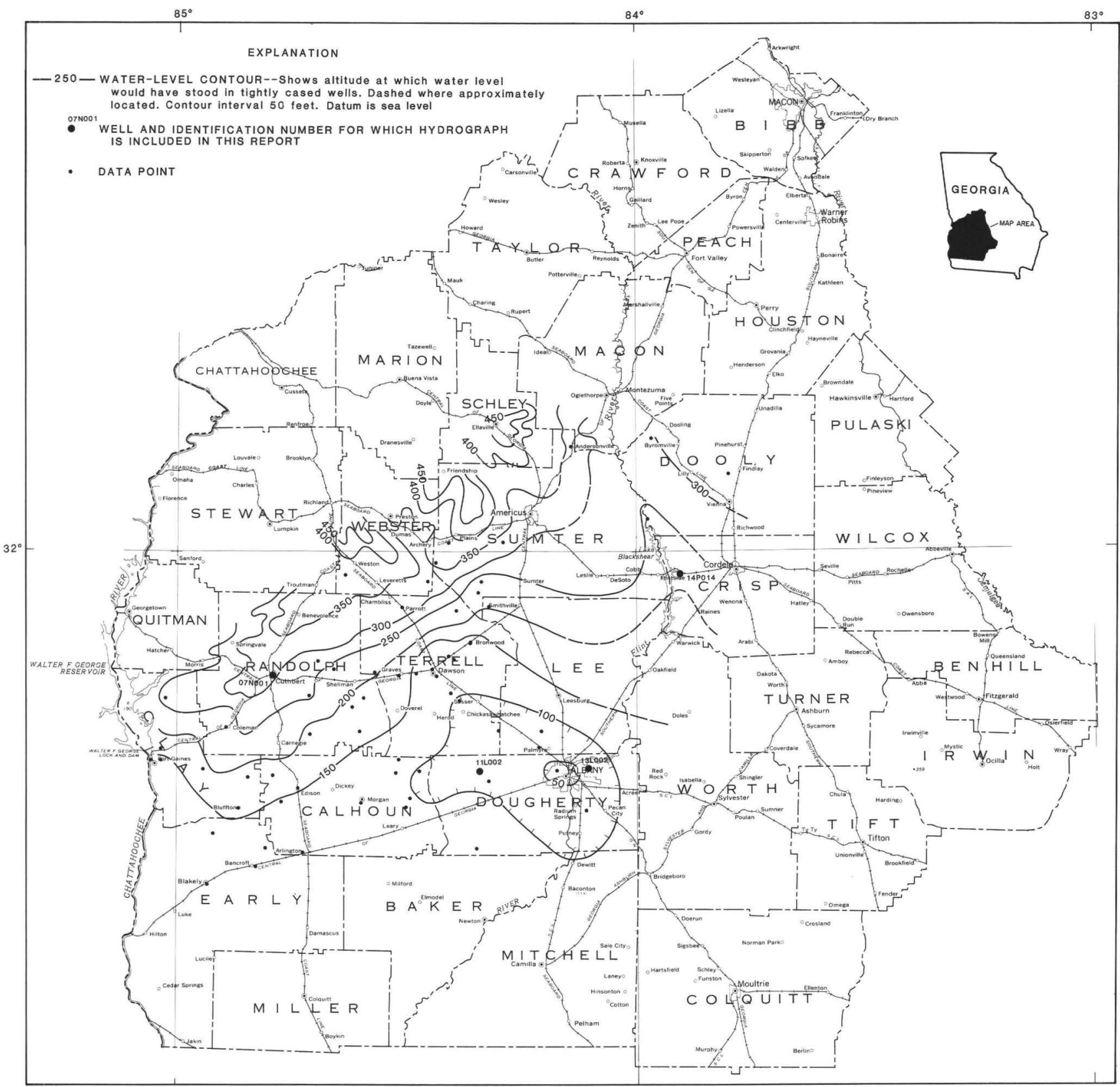




\section{P014 GEORGIA VETERANS TW1 CRISP COUNTY}

315731083542301 , Local number, 14P014.

LOCATION.--Lat 31 $57^{\prime} 31^{\prime \prime}$, long $83^{\circ} 54^{\prime} 23^{\prime \prime}$, Hydrologic Unit 03130006, in the Georgia Veterans Memorial State Park, about $7.5 \mathrm{mi}$ west of Cordele.

Owner: State of Georgia.

AQUIFER.--Clayton.

WELL CHARACTERISTICS.--Drilled observation well, diameter $6 \mathrm{in}$., depth $550 \mathrm{ft}$, cased to $500 \mathrm{ft}$, open hole. DATUM.--Elevation of land-surface datum is $252 \mathrm{ft}$.

Measuring point: Floor of recorder shelter, $3.1 \mathrm{ft}$ above land-surface datum.

REMARKS.--Well pumped May 20, 1982. Borehole geophysical survey conducted February 22, 1982.

PERIOD OF RECORD.--September 1984 to current year.

EXTREMES FOR PERIOD OF RECORD.--Highest water level, $37.16 \mathrm{ft}$ below land-surface datum, September 2, 1984; lowest, $48.91 \mathrm{ft}$ below land-surface datum, November $14,1986$.

DEPTH BELOW LAND SURFACE (WATER LEVEL) (FEET), CALENDAR YEAR JANUARY 1987 TO DECEMBER 1987 MEAN VALUES

\begin{tabular}{|c|c|c|c|c|c|c|c|c|c|c|c|c|}
\hline DAY & JAN & FEB & MAR & APR & MAY & JUN & JUL & AUG & SEP & OCT & NOV & DEC \\
\hline $\begin{array}{l}1 \\
2 \\
3 \\
4 \\
5\end{array}$ & $\begin{array}{l}48.28 \\
48.42 \\
48.44 \\
48.26 \\
48.32\end{array}$ & $\begin{array}{l}47.70 \\
47.57 \\
47.65 \\
47.74 \\
47.75\end{array}$ & $\begin{array}{l}46.89 \\
47.00 \\
47.07 \\
47.10 \\
47.07\end{array}$ & $\begin{array}{l}46.29 \\
46.19 \\
46.10 \\
46.15 \\
46.07\end{array}$ & $\begin{array}{l}45.49 \\
45.44 \\
45.42 \\
45.40 \\
45.42\end{array}$ & $\begin{array}{l}44.95 \\
44.98 \\
45.02 \\
45.00 \\
44.99\end{array}$ & $\begin{array}{l}44.83 \\
44.85 \\
44.88 \\
44.88 \\
44.88\end{array}$ & $\begin{array}{l}45.18 \\
45.19 \\
45.23 \\
45.23 \\
45.25\end{array}$ & $\begin{array}{l}45.97 \\
45.96 \\
45.98 \\
46.04 \\
46.07\end{array}$ & $\begin{array}{l}47.04 \\
47.10 \\
47.18 \\
47.24 \\
47.20\end{array}$ & $\begin{array}{l}48.08 \\
48.06 \\
48.02 \\
47.95 \\
47.99\end{array}$ & $\begin{array}{l}48.20 \\
48.33 \\
48.31 \\
48.28 \\
48.39\end{array}$ \\
\hline $\begin{array}{r}6 \\
7 \\
8 \\
9 \\
10\end{array}$ & $\begin{array}{l}48.35 \\
48.30 \\
48.30 \\
48.26 \\
48.16\end{array}$ & $\begin{array}{l}47.57 \\
47.46 \\
47.42 \\
47.60 \\
47.63\end{array}$ & $\begin{array}{l}47.04 \\
46.88 \\
46.67 \\
46.64 \\
46.74\end{array}$ & $\begin{array}{l}46.04 \\
46.02 \\
46.01 \\
46.00 \\
45.96\end{array}$ & $\begin{array}{l}45.38 \\
45.34 \\
45.30 \\
45.37 \\
45.33\end{array}$ & $\begin{array}{l}45.01 \\
45.02 \\
45.00 \\
44.96 \\
44.93\end{array}$ & $\begin{array}{l}44.91 \\
44.92 \\
44.97 \\
45.00 \\
45.00\end{array}$ & $\begin{array}{l}45.32 \\
45.35 \\
45.35 \\
45.35 \\
45.35\end{array}$ & $\begin{array}{l}46.08 \\
46.05 \\
46.10 \\
46.19 \\
46.26\end{array}$ & $\begin{array}{l}47.17 \\
47.26 \\
47.41 \\
47.50 \\
47.49\end{array}$ & $\begin{array}{l}48.18 \\
48.23 \\
48.21 \\
48.14 \\
48.03\end{array}$ & $\begin{array}{l}48.43 \\
48.43 \\
48.40 \\
48.35 \\
48.29\end{array}$ \\
\hline $\begin{array}{l}11 \\
12 \\
13 \\
14 \\
15\end{array}$ & $\begin{array}{l}48.30 \\
48.31 \\
48.32 \\
48.28 \\
48.19\end{array}$ & $\begin{array}{l}47.55 \\
47.45 \\
47.42 \\
47.36 \\
47.31\end{array}$ & $\begin{array}{l}46.85 \\
46.85 \\
46.79 \\
46.74 \\
46.73\end{array}$ & $\begin{array}{l}45.95 \\
45.97 \\
45.93 \\
45.91 \\
45.81\end{array}$ & $\begin{array}{l}45.29 \\
45.22 \\
45.19 \\
45.19 \\
45.13\end{array}$ & $\begin{array}{l}44.94 \\
44.93 \\
44.88 \\
44.82 \\
44.80\end{array}$ & $\begin{array}{l}44.98 \\
44.97 \\
44.97 \\
44.96 \\
44.99\end{array}$ & $\begin{array}{l}45.38 \\
45.42 \\
45.45 \\
45.53 \\
45.54\end{array}$ & $\begin{array}{l}46.32 \\
46.30 \\
46.30 \\
46.32 \\
46.38\end{array}$ & $\begin{array}{l}47.41 \\
47.36 \\
47.50 \\
47.61 \\
47.64\end{array}$ & $\begin{array}{l}48.15 \\
48.21 \\
48.20 \\
48.21 \\
48.26\end{array}$ & $\begin{array}{l}48.26 \\
48.27 \\
48.40 \\
48.38 \\
48.29\end{array}$ \\
\hline $\begin{array}{l}16 \\
17 \\
18 \\
19 \\
20\end{array}$ & $\begin{array}{l}48.13 \\
48.09 \\
48.02 \\
47.91 \\
47.98\end{array}$ & $\begin{array}{l}47.23 \\
47.34 \\
47.38 \\
47.45 \\
47.43\end{array}$ & $\begin{array}{l}46.67 \\
46.67 \\
46.59 \\
46.53 \\
46.53\end{array}$ & $\begin{array}{l}45.72 \\
45.69 \\
45.77 \\
45.81 \\
45.79\end{array}$ & $\begin{array}{l}45.11 \\
45.11 \\
45.09 \\
45.07 \\
45.08\end{array}$ & $\begin{array}{l}44.75 \\
44.76 \\
44.78 \\
44.78 \\
44.74\end{array}$ & $\begin{array}{l}45.04 \\
45.11 \\
45.11 \\
45.12 \\
45.17\end{array}$ & $\begin{array}{l}45.54 \\
45.60 \\
45.63 \\
45.65 \\
45.70\end{array}$ & $\begin{array}{l}46.42 \\
46.46 \\
46.46 \\
46.48 \\
46.56\end{array}$ & $\begin{array}{l}47.67 \\
47.67 \\
47.66 \\
47.69 \\
47.73\end{array}$ & $\begin{array}{l}48.23 \\
48.14 \\
48.23 \\
48.21 \\
48.20\end{array}$ & $\begin{array}{l}48.42 \\
48.45 \\
48.43 \\
48.36 \\
48.29\end{array}$ \\
\hline $\begin{array}{l}21 \\
22 \\
23 \\
24 \\
25\end{array}$ & $\begin{array}{l}47.92 \\
47.74 \\
47.87 \\
47.89 \\
47.71\end{array}$ & $\begin{array}{l}47.32 \\
47.18 \\
47.25 \\
47.28 \\
47.28\end{array}$ & $\begin{array}{l}46.44 \\
46.41 \\
46.41 \\
46.38 \\
46.41\end{array}$ & $\begin{array}{l}45.74 \\
45.72 \\
45.66 \\
45.62 \\
45.65\end{array}$ & $\begin{array}{l}45.07 \\
45.00 \\
44.95 \\
44.93 \\
44.93\end{array}$ & $\begin{array}{l}44.74 \\
44.73 \\
44.74 \\
44.76 \\
44.76\end{array}$ & $\begin{array}{l}45.19 \\
45.21 \\
45.23 \\
45.30 \\
45.25\end{array}$ & $\begin{array}{l}45.79 \\
45.82 \\
45.81 \\
45.84 \\
45.90\end{array}$ & $\begin{array}{l}46.64 \\
46.73 \\
46.77 \\
46.77 \\
46.80\end{array}$ & $\begin{array}{l}47.81 \\
47.89 \\
47.92 \\
47.92 \\
47.87\end{array}$ & $\begin{array}{l}48.28 \\
48.29 \\
48.31 \\
48.34 \\
48.29\end{array}$ & $\begin{array}{l}48.24 \\
48.21 \\
48.30 \\
48.30 \\
48.25\end{array}$ \\
\hline $\begin{array}{l}26 \\
27 \\
28 \\
29 \\
30 \\
31\end{array}$ & $\begin{array}{l}47.77 \\
47.87 \\
47.84 \\
47.81 \\
47.66 \\
47.74\end{array}$ & $\begin{array}{r}47.25 \\
47.15 \\
46.98 \\
--- \\
---\end{array}$ & $\begin{array}{l}46.39 \\
46.31 \\
46.31 \\
46.29 \\
46.15 \\
46.26\end{array}$ & $\begin{array}{r}45.70 \\
45.64 \\
45.56 \\
45.58 \\
45.51 \\
-.-\end{array}$ & $\begin{array}{l}44.96 \\
44.98 \\
44.99 \\
44.98 \\
44.95 \\
44.94\end{array}$ & $\begin{array}{r}44.70 \\
44.70 \\
44.79 \\
44.83 \\
44.86 \\
. \ldots-\end{array}$ & $\begin{array}{l}45.20 \\
45.17 \\
45.19 \\
45.23 \\
45.27 \\
45.22\end{array}$ & $\begin{array}{l}45.94 \\
45.97 \\
46.00 \\
46.03 \\
46.03 \\
46.02\end{array}$ & $\begin{array}{r}46.86 \\
46.90 \\
46.95 \\
46.94 \\
46.90 \\
-.--\end{array}$ & $\begin{array}{l}47.82 \\
47.81 \\
47.92 \\
48.02 \\
48.07 \\
48.11\end{array}$ & $\begin{array}{r}48.24 \\
48.21 \\
48.17 \\
48.15 \\
48.13 \\
.--\end{array}$ & $\begin{array}{l}48.23 \\
48.23 \\
48.15 \\
48.27 \\
48.39 \\
48.32\end{array}$ \\
\hline MEAN & 48.08 & 47.42 & 46.64 & 45.85 & 45.16 & 44.86 & 45.06 & 45.59 & 46.43 & 47.60 & 48.18 & 48.32 \\
\hline
\end{tabular}




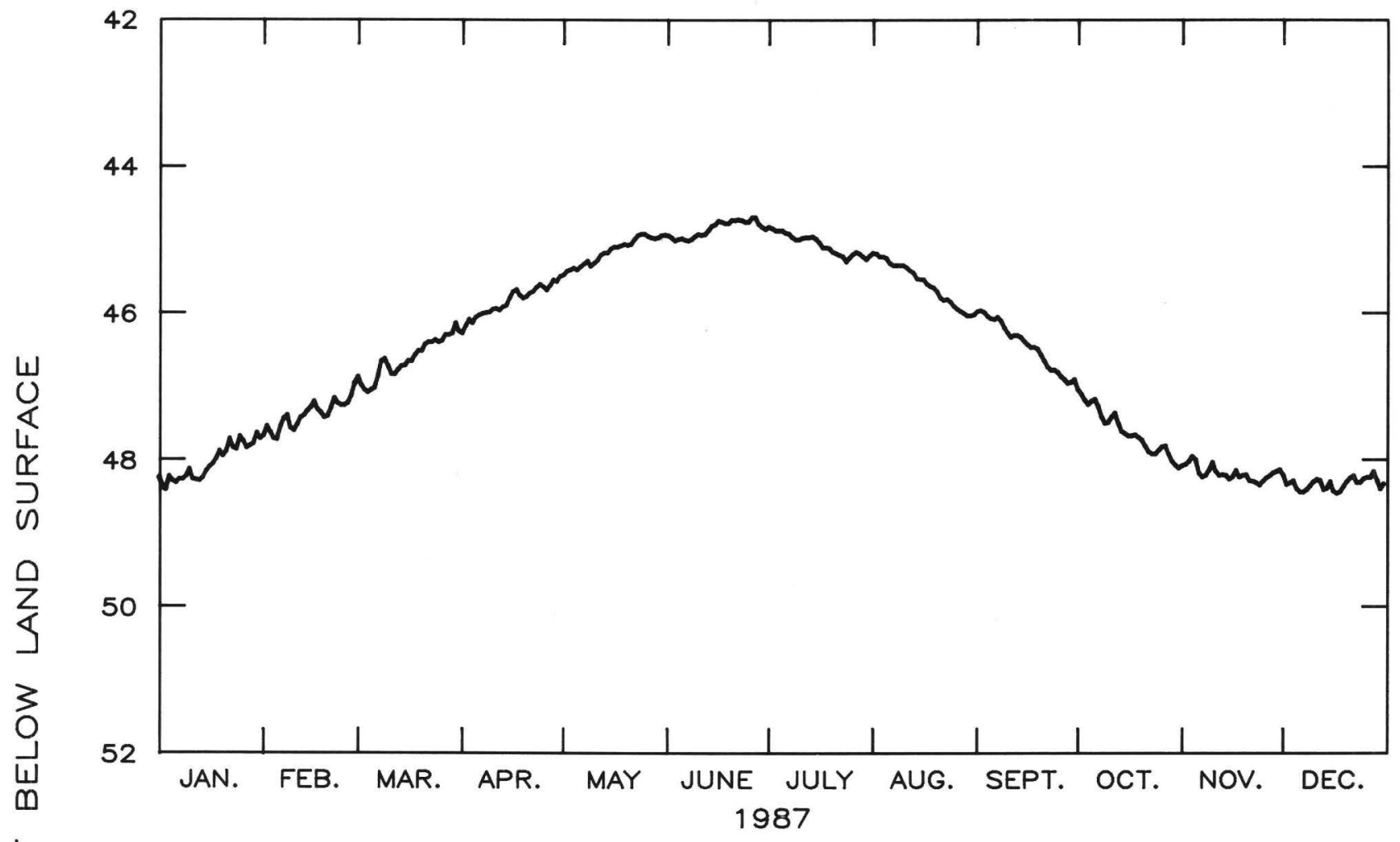

Z

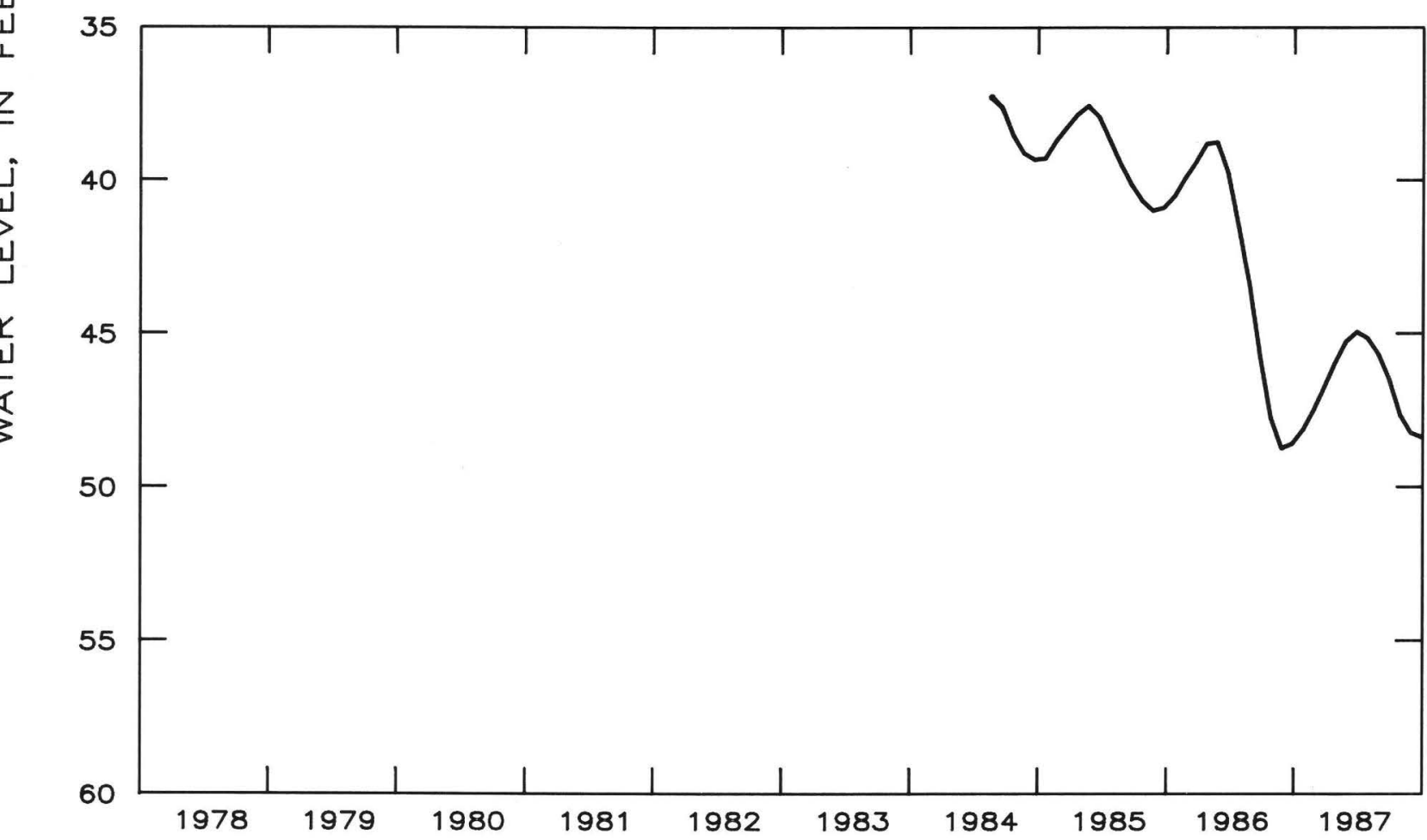

Figure 2.5-2.--Water level in observation well 14P014, Crisp County. 
314602084473701 Local number, 07N001.

LOCATION.--Lat $31^{\circ} 46^{\prime} 09^{\prime \prime}$, long $84^{\circ} 47^{\prime} 43^{\prime \prime}$, Hydrologic Unit 03110204 , south of intersection of College and Andrew Streets, near electric substation.

Owner: City of Cuthbert.

AQUIFER.--Clayton.

WELL CHARACTERISTICS.--Drilled unused municipal well, diameter 8 in., depth $372 \mathrm{ft}$, casing depth unknown. DATUM.--Elevation of land-surface datum is $460 \mathrm{ft}$.

Measuring point: Floor of recorder shelter, $3.30 \mathrm{ft}$ above land-surface datum.

REMARKS.--Well pumped and sounded June 22,1978 , to a depth of $372 \mathrm{ft}$; water-quality sample collected at conclusion of pumping. Well near city wells. Water levels for periods of missing record, August 5, and October 29, were estimated.

PERIOD OF RECORD.--January 1965 to current year.

EXTREMES FOR PERIOD OF RECORD.--Highest water level, $132.00 \mathrm{ft}$ below land-surface datum, December 10, 1967; 1owest, $162.08 \mathrm{ft}$ below land-surface datum, August $4,1986$. DEPTH BELOW LAND SURFACE (WATER LEVEL) ( FEET) CALENDAR YEAR JANUARY 1987 TO DECEMBER 1987
MEAN VALUES

\begin{tabular}{|c|c|c|c|c|c|c|c|c|c|c|c|c|}
\hline DAY & JAN & FEB & MAR & APR & MAY & JUN & JUL & AUG & SEP & OCT & NOV & DEC \\
\hline $\begin{array}{l}1 \\
2 \\
3 \\
4 \\
5\end{array}$ & $\begin{array}{l}151.47 \\
152.96 \\
152.55 \\
152.22 \\
153.70\end{array}$ & $\begin{array}{l}151.77 \\
151.86 \\
151.61 \\
151.94 \\
152.00\end{array}$ & $\begin{array}{l}149.74 \\
150.54 \\
151.07 \\
150.73 \\
151.23\end{array}$ & $\begin{array}{l}148.91 \\
149.02 \\
149.10 \\
149.67 \\
149.21\end{array}$ & $\begin{array}{l}152.59 \\
152.62 \\
151.27 \\
153.23 \\
151.86\end{array}$ & $\begin{array}{l}156.18 \\
154.52 \\
154.18 \\
155.11 \\
155.15\end{array}$ & $\begin{array}{l}154.46 \\
153.16 \\
154.51 \\
153.83 \\
154.77\end{array}$ & $\begin{array}{l}155.68 \\
156.11 \\
155.81 \\
156.64 \\
155.58\end{array}$ & $\begin{array}{l}156.91 \\
158.71 \\
158.05 \\
158.78 \\
157.14\end{array}$ & $\begin{array}{l}155.58 \\
155.64 \\
155.15 \\
155.02 \\
155.63\end{array}$ & $\begin{array}{l}153.18 \\
154.09 \\
153.13 \\
153.15 \\
153.24\end{array}$ & $\begin{array}{l}154.04 \\
152.22 \\
153.53 \\
153.49 \\
152.97\end{array}$ \\
\hline $\begin{array}{r}6 \\
7 \\
8 \\
9 \\
10\end{array}$ & $\begin{array}{l}153.47 \\
152.71 \\
153.09 \\
153.27 \\
151.72\end{array}$ & $\begin{array}{l}151.75 \\
151.75 \\
150.91 \\
152.07 \\
152.51\end{array}$ & $\begin{array}{l}151.60 \\
150.42 \\
149.62 \\
150.25 \\
149.62\end{array}$ & $\begin{array}{l}149.80 \\
150.47 \\
150.03 \\
150.45 \\
151.25\end{array}$ & $\begin{array}{l}152.00 \\
151.56 \\
153.10 \\
153.89 \\
154.04\end{array}$ & $\begin{array}{l}155.92 \\
155.75 \\
157.10 \\
156.74 \\
156.56\end{array}$ & $\begin{array}{l}153.94 \\
153.50 \\
153.47 \\
153.49 \\
153.98\end{array}$ & $\begin{array}{l}154.51 \\
154.76 \\
155.54 \\
156.62 \\
156.82\end{array}$ & $\begin{array}{l}157.16 \\
156.25 \\
155.17 \\
156.76 \\
157.01\end{array}$ & $\begin{array}{l}155.59 \\
155.78 \\
154.78 \\
155.31 \\
155.40\end{array}$ & $\begin{array}{l}152.54 \\
152.86 \\
153.93 \\
153.59 \\
153.84\end{array}$ & $\begin{array}{l}152.56 \\
152.15 \\
153.95 \\
154.89 \\
152.41\end{array}$ \\
\hline $\begin{array}{l}11 \\
12 \\
13 \\
14 \\
15\end{array}$ & $\begin{array}{l}153.31 \\
152.13 \\
153.16 \\
153.14 \\
152.15\end{array}$ & $\begin{array}{l}152.75 \\
151.95 \\
151.18 \\
150.34 \\
150.80\end{array}$ & $\begin{array}{l}150.65 \\
150.72 \\
151.69 \\
150.55 \\
150.50\end{array}$ & $\begin{array}{l}150.75 \\
150.01 \\
150.63 \\
149.31 \\
150.90\end{array}$ & $\begin{array}{l}154.52 \\
153.83 \\
153.49 \\
154.07 \\
153.86\end{array}$ & $\begin{array}{l}156.93 \\
155.43 \\
155.20 \\
154.58 \\
155.88\end{array}$ & $\begin{array}{l}152.57 \\
152.65 \\
155.18 \\
155.27 \\
153.41\end{array}$ & $\begin{array}{l}156.49 \\
157.05 \\
157.55 \\
156.41 \\
157.19\end{array}$ & $\begin{array}{l}157.49 \\
155.60 \\
156.42 \\
154.99 \\
155.46\end{array}$ & $\begin{array}{l}154.36 \\
154.42 \\
155.17 \\
154.71 \\
155.23\end{array}$ & $\begin{array}{l}153.82 \\
152.94 \\
153.77 \\
152.59 \\
153.57\end{array}$ & $\begin{array}{l}151.54 \\
151.53 \\
151.79 \\
152.74 \\
151.33\end{array}$ \\
\hline $\begin{array}{l}16 \\
17 \\
18 \\
19 \\
20\end{array}$ & $\begin{array}{l}151.48 \\
152.34 \\
150.94 \\
152.03 \\
152.25\end{array}$ & $\begin{array}{l}150.52 \\
151.47 \\
151.95 \\
152.67 \\
151.07\end{array}$ & $\begin{array}{l}151.45 \\
150.35 \\
149.21 \\
149.72 \\
149.68\end{array}$ & $\begin{array}{l}149.09 \\
150.09 \\
148.03 \\
150.34 \\
149.92\end{array}$ & $\begin{array}{l}152.87 \\
152.46 \\
153.53 \\
152.72 \\
152.85\end{array}$ & $\begin{array}{l}156.62 \\
156.33 \\
155.43 \\
156.55 \\
156.15\end{array}$ & $\begin{array}{l}155.17 \\
155.67 \\
156.34 \\
155.62 \\
156.17\end{array}$ & $\begin{array}{l}157.17 \\
156.87 \\
156.64 \\
157.18 \\
157.44\end{array}$ & $\begin{array}{l}155.43 \\
156.30 \\
157.91 \\
155.87 \\
155.39\end{array}$ & $\begin{array}{l}155.70 \\
155.74 \\
154.77 \\
155.59 \\
155.27\end{array}$ & $\begin{array}{l}154.12 \\
153.41 \\
153.67 \\
153.18 \\
152.80\end{array}$ & $\begin{array}{l}152.09 \\
152.72 \\
152.22 \\
151.94 \\
151.42\end{array}$ \\
\hline $\begin{array}{l}21 \\
22 \\
23 \\
24 \\
25\end{array}$ & $\begin{array}{l}151.74 \\
151.67 \\
151.60 \\
151.35 \\
151.79\end{array}$ & $\begin{array}{l}151.38 \\
150.50 \\
151.01 \\
150.62 \\
150.30\end{array}$ & $\begin{array}{l}150.23 \\
149.58 \\
150.23 \\
149.38 \\
149.57\end{array}$ & $\begin{array}{l}151.24 \\
151.83 \\
152.17 \\
151.67 \\
150.63\end{array}$ & $\begin{array}{l}152.43 \\
154.16 \\
153.84 \\
153.59 \\
153.90\end{array}$ & $\begin{array}{l}155.54 \\
154.50 \\
155.44 \\
154.29 \\
154.72\end{array}$ & $\begin{array}{l}155.61 \\
156.35 \\
157.51 \\
157.38 \\
156.51\end{array}$ & $\begin{array}{l}157.55 \\
157.84 \\
158.10 \\
159.19 \\
158.32\end{array}$ & $\begin{array}{l}157.08 \\
156.18 \\
156.74 \\
156.40 \\
155.78\end{array}$ & $\begin{array}{l}154.14 \\
154.81 \\
155.61 \\
154.93 \\
154.48\end{array}$ & $\begin{array}{l}153.52 \\
153.42 \\
153.72 \\
153.99 \\
153.22\end{array}$ & $\begin{array}{l}151.57 \\
151.47 \\
151.28 \\
152.09 \\
151.20\end{array}$ \\
\hline $\begin{array}{l}26 \\
27 \\
28 \\
29 \\
30 \\
31\end{array}$ & $\begin{array}{l}151.76 \\
152.21 \\
151.40 \\
152.68 \\
151.71 \\
151.30\end{array}$ & $\begin{array}{r}150.45 \\
151.01 \\
150.86 \\
--- \\
--- \\
---\end{array}$ & $\begin{array}{l}149.42 \\
149.51 \\
149.09 \\
148.88 \\
148.74 \\
148.20\end{array}$ & $\begin{array}{r}151.11 \\
150.61 \\
151.85 \\
153.25 \\
153.08 \\
-.--\end{array}$ & $\begin{array}{l}154.17 \\
153.32 \\
152.60 \\
152.69 \\
153.94 \\
155.32\end{array}$ & $\begin{array}{r}154.63 \\
154.04 \\
152.78 \\
153.12 \\
153.56 \\
.--\end{array}$ & $\begin{array}{l}156.12 \\
155.58 \\
155.90 \\
155.95 \\
156.78 \\
155.37\end{array}$ & $\begin{array}{l}157.40 \\
157.28 \\
157.65 \\
157.54 \\
158.13 \\
156.86\end{array}$ & $\begin{array}{r}155.28 \\
155.43 \\
156.27 \\
156.21 \\
154.89 \\
-.-\end{array}$ & $\begin{array}{l}155.00 \\
154.79 \\
153.25 \\
154.17 \\
154.01 \\
154.47\end{array}$ & $\begin{array}{r}152.25 \\
152.09 \\
153.30 \\
152.51 \\
152.91 \\
. .-\end{array}$ & $\begin{array}{l}152.15 \\
151.87 \\
152.41 \\
151.91 \\
153.09 \\
152.48\end{array}$ \\
\hline MEAN & 152.24 & 151.39 & 150.07 & 150.48 & 153.24 & 155.30 & 155.04 & 156.90 & 156.44 & 154.98 & 153.28 & 152.3 \\
\hline & & & 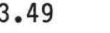 & & & & & & & & & \\
\hline
\end{tabular}



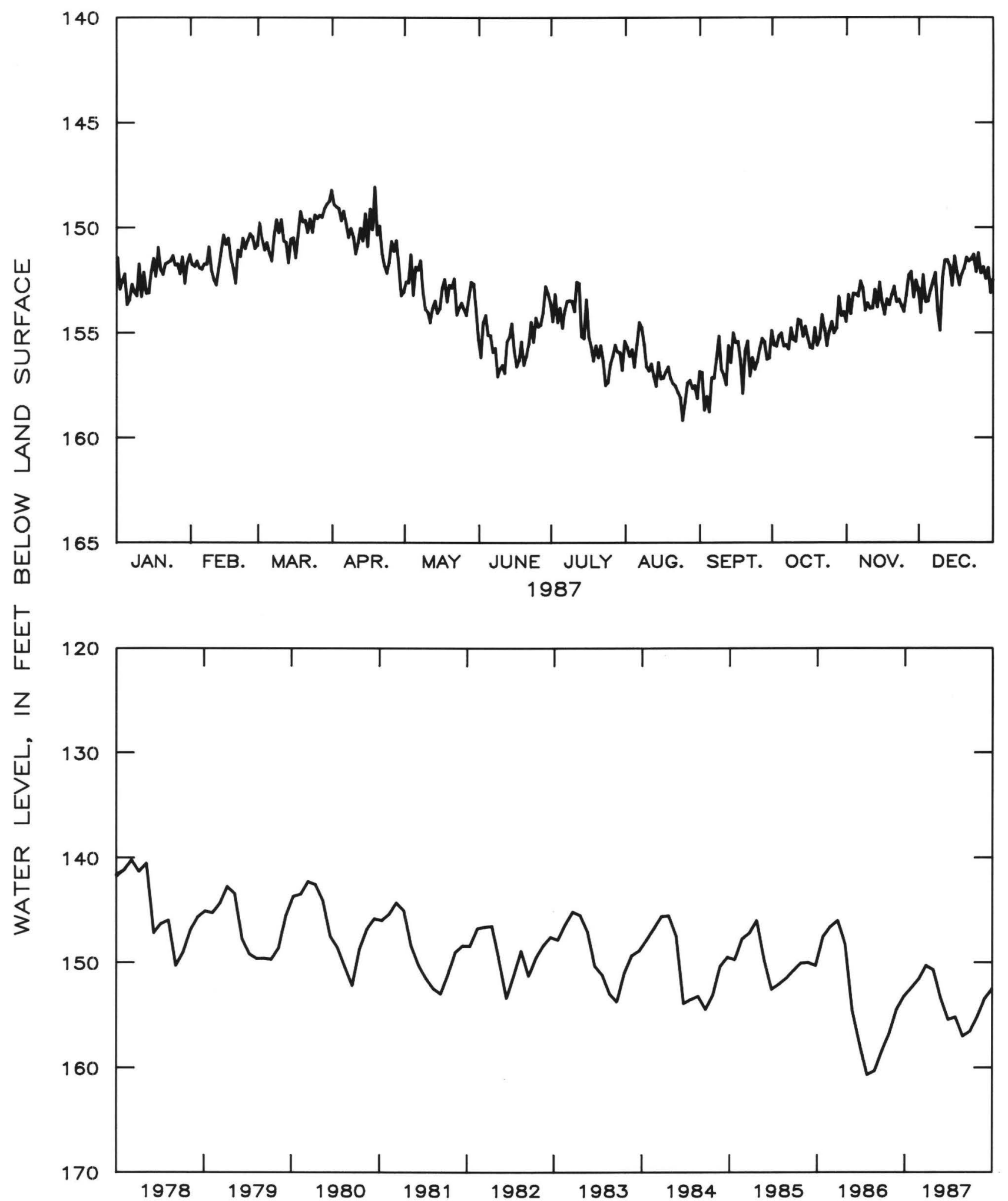

Figure 2.5-3.--Water level in observation well 07N001, Randolph County. 
313530084203201 Local number, 11L002.

LOCATION.--Lat $31^{\circ} 35^{\prime} 32^{\prime \prime}$, long $84^{\circ} 20^{\prime} 35^{\prime \prime}$, Hydrologic Unit 03130008 , Tallahassee Plantation, 10.4 mi west of Albany.

Owner: Georgia Department of Natural Resources, Albany Nursery.

AQUIFER.--Clayton.

WELL CHARACTERISTICS.--Drilled observation well, diameter $3 \mathrm{in}$., depth $656 \mathrm{ft}$, cased to $542 \mathrm{ft}$, open hole. DATUM.--Elevation of land-surface datum is $222 \mathrm{ft}$.

Measuring point: Floor of recorder shelter, $3.02 \mathrm{ft}$ above land-surface datum.

REMARKS.--Well pumped April 1976; water-quality sample collected at conclusion of pumping. Borehole geophysical survey conducted June 3, 1975. Water levels for period of missing record, September 3 to October 8 , were estimated.

PERIOD OF RECORD.--September 1973 to current year.

EXTREMES FOR PERIOD OF RECORD.--Highest water level, $58.90 \mathrm{ft}$ below land-surface datum, April 29, 1975; lowest, $152.61 \mathrm{ft}$ below land-surface datum, August 23, 1986.

DEPTH BELOW LAND SURFACE (WATER LEVEL) (FEET) CALENDAR YEAR JANUARY 1987 TO DECEMBER 1987 MEAN VALUES

\begin{tabular}{|c|c|c|c|c|c|c|c|c|c|c|c|c|}
\hline DAY & JAN & FEB & MAR & APR & MAY & JUN & JUL & AUG & SEP & OCT & NOV & $\mathrm{DEC}$ \\
\hline $\begin{array}{l}1 \\
2 \\
3 \\
4 \\
5\end{array}$ & $\begin{array}{l}115.64 \\
115.56 \\
115.44 \\
115.15 \\
114.99\end{array}$ & $\begin{array}{l}111.37 \\
111.15 \\
111.04 \\
111.07 \\
111.11\end{array}$ & $\begin{array}{l}108.23 \\
108.32 \\
108.27 \\
108.18 \\
108.02\end{array}$ & $\begin{array}{l}104.40 \\
104.35 \\
104.10 \\
103.87 \\
103.90\end{array}$ & $\begin{array}{l}106.43 \\
107.18 \\
107.97 \\
108.84 \\
109.57\end{array}$ & $\begin{array}{l}112.88 \\
113.43 \\
114.03 \\
114.63 \\
115.32\end{array}$ & $\begin{array}{l}115.92 \\
115.68 \\
115.46 \\
115.25\end{array}$ & $\begin{array}{l}130.10 \\
130.55 \\
130.85 \\
131.09 \\
131.27\end{array}$ & $\begin{array}{l}138.59 \\
138.59 \\
137.35 \\
136.31 \\
135.50\end{array}$ & $\begin{array}{l}131.10 \\
130.90 \\
130.77 \\
130.67 \\
130.64\end{array}$ & $\begin{array}{l}125.01 \\
124.64 \\
124.29 \\
124.01 \\
123.86\end{array}$ & $\begin{array}{l}118.63 \\
118.52 \\
118.21 \\
117.91\end{array}$ \\
\hline $\begin{array}{r}6 \\
7 \\
8 \\
9 \\
10\end{array}$ & $\begin{array}{l}4.95 \\
4.84 \\
4.78 \\
4.58 \\
4.33\end{array}$ & $\begin{array}{l}110.91 \\
110.67 \\
110.47 \\
110.57 \\
110.61\end{array}$ & $\begin{array}{l}107.92 \\
107.58 \\
107.20 \\
107.01 \\
106.96\end{array}$ & $\begin{array}{l}103.87 \\
103.75 \\
103.63 \\
103.53 \\
103.42\end{array}$ & $\begin{array}{l}110.2 \\
110.9 \\
111.5 \\
112.1 \\
112.7\end{array}$ & & $\begin{array}{l}14.95 \\
14.73 \\
14.50 \\
14.43 \\
14.40\end{array}$ & $\begin{array}{l}131.75 \\
132.45 \\
133.32 \\
134.36 \\
135.42\end{array}$ & $\begin{array}{l}134.84 \\
134.33 \\
133.88 \\
133.49 \\
133.07\end{array}$ & $\begin{array}{l}130.64 \\
130.64 \\
130.59 \\
130.52 \\
130.31\end{array}$ & $\begin{array}{l}123.85 \\
123.76 \\
123.55 \\
123.25 \\
122.86\end{array}$ & 7.54 \\
\hline $\begin{array}{l}11 \\
12 \\
13 \\
14 \\
15\end{array}$ & $\begin{array}{l}14.27 \\
14.14 \\
13.94 \\
13.77 \\
13.52\end{array}$ & $\begin{array}{l}110.46 \\
110.22 \\
110.07 \\
109.91 \\
109.70\end{array}$ & $\begin{array}{l}106.98 \\
106.93 \\
106.84 \\
106.70 \\
106.66\end{array}$ & $\begin{array}{l}103.36 \\
103.34 \\
103.40 \\
103.33 \\
103.16\end{array}$ & & & $\begin{array}{l}114.40 \\
114.57 \\
114.97 \\
115.51 \\
116.25\end{array}$ & $\begin{array}{l}136.50 \\
137.41 \\
138.17 \\
138.58 \\
138.73\end{array}$ & $\begin{array}{l}132.64 \\
132.23 \\
131.88 \\
131.67 \\
131.44\end{array}$ & & $\begin{array}{l}122.68 \\
122.40 \\
122.16 \\
122.10 \\
122.09\end{array}$ & \\
\hline $\begin{array}{l}16 \\
17 \\
18 \\
19 \\
20\end{array}$ & $\begin{array}{l}13.32 \\
13.26 \\
13.05 \\
12.84 \\
12.90\end{array}$ & $\begin{array}{l}09.56 \\
09.57 \\
09.58 \\
09.60 \\
09.50\end{array}$ & & $\begin{array}{l}102.98 \\
102.90 \\
102.87 \\
102.94 \\
103.02\end{array}$ & & & & & & & $\begin{array}{l}122.09 \\
122.06 \\
122.04 \\
122.04 \\
121.80\end{array}$ & \\
\hline $\begin{array}{l}21 \\
22 \\
23 \\
24 \\
25\end{array}$ & $\begin{array}{l}2.67 \\
12.37 \\
2.49 \\
12.52 \\
2.28\end{array}$ & $\begin{array}{l}109.34 \\
109.08 \\
108.98 \\
108.98 \\
108.92\end{array}$ & $\begin{array}{l}105.77 \\
105.65 \\
105.58 \\
105.49 \\
105.43\end{array}$ & $\begin{array}{l}102.99 \\
103.00 \\
103.11 \\
103.29 \\
103.65\end{array}$ & & & & & & & $\begin{array}{l}121.55 \\
121.19 \\
120.93 \\
120.63 \\
120.30\end{array}$ & \\
\hline $\begin{array}{l}26 \\
27 \\
28 \\
29 \\
30 \\
31\end{array}$ & $\begin{array}{l}112.16 \\
112.17 \\
112.04 \\
111.86 \\
111.62 \\
111.51\end{array}$ & $\begin{array}{r}108.82 \\
108.67 \\
108.37 \\
--- \\
--- \\
---\end{array}$ & $\begin{array}{l}105.28 \\
105.10 \\
104.98 \\
104.83 \\
104.83 \\
104.45\end{array}$ & $\begin{array}{r}104.14 \\
104.55 \\
104.98 \\
105.37 \\
105.77 \\
-.-\end{array}$ & $\begin{array}{l}112.12 \\
112.04 \\
112.00 \\
112.02 \\
112.20 \\
112.47\end{array}$ & $\begin{array}{r}117.09 \\
116.82 \\
116.59 \\
116.33 \\
116.12 \\
---\end{array}$ & $\begin{array}{l}125.59 \\
126.40 \\
127.00 \\
128.00 \\
128.87 \\
129.57\end{array}$ & $\begin{array}{l}136.79 \\
137.18 \\
137.65 \\
138.00 \\
138.30 \\
138.49\end{array}$ & $\begin{array}{r}129.98 \\
130.72 \\
131.14 \\
131.39 \\
131.31 \\
.--\end{array}$ & $\begin{array}{l}126.50 \\
126.23 \\
126.15 \\
125.99 \\
125.73 \\
125.40\end{array}$ & $\begin{array}{r}120.10 \\
119.87 \\
119.50 \\
119.25 \\
118.83 \\
---\end{array}$ & $\begin{array}{l}113.2 \\
113.0\end{array}$ \\
\hline EAN & 13.51 & 09.94 & 106.48 & 103.70 & 112.00 & 116.42 & 119.45 & 135.78 & 132.05 & 128.65 & 122.09 & 115 \\
\hline
\end{tabular}

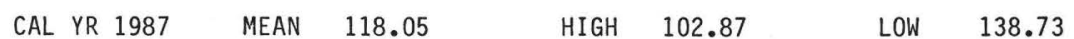



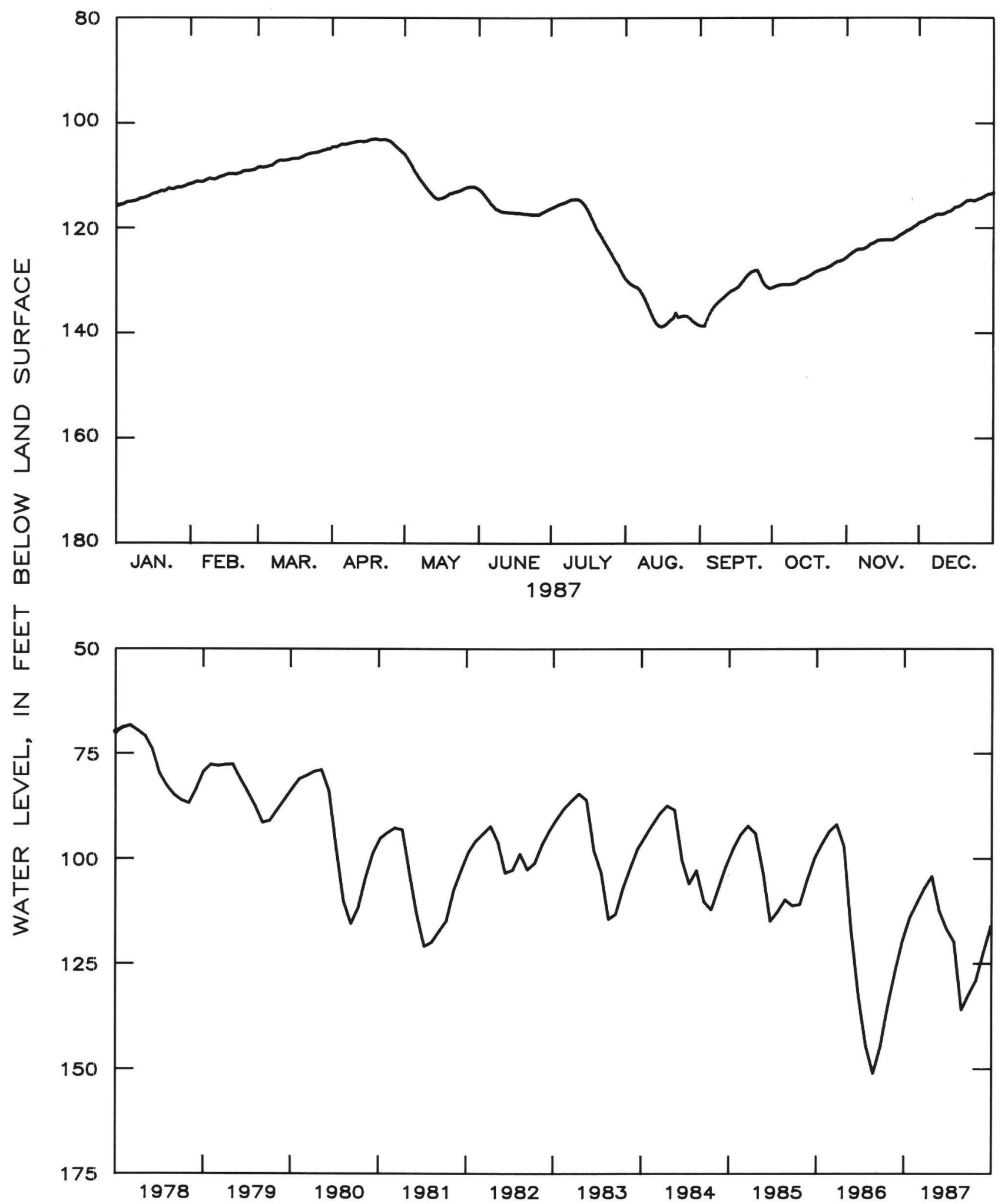

Figure 2.5-4.--Water level in observation well 11LOO2, Dougherty County. 
313554084062601 Local number, 13 L002.

LOCATION.--Lat $31^{\circ} 35^{\prime} 54^{\prime \prime}$, long $84^{\circ} 06^{\prime} 25^{\prime \prime}$, Hydrologic Unit 03130008, Malone and Gardner Avenue near main entrance to Turner Field, Albany.

Owner: City of Albany, Turner City.

AQUIFER.--Clayton.

WELL CHARACTERISTICS.--Drilled unused supply well, diameter $12 \mathrm{in}$. and $8 \mathrm{in}$., depth $760 \mathrm{ft}$, cased to $713 \mathrm{ft}$, open hole.

DATUM.--Elevation of land-surface datum is $212.84 \mathrm{ft}$.

Measuring point: Floor of recorder shelter, $3.2 \mathrm{ft}$ above land-surface datum.

REMARKS.--Well pumped and sounded to a depth of $760 \mathrm{ft}$, June 21, 1978: water-quality sample collected at conclusion of pumping. Borehole geophysical survey conducted March 17, 1977. Water levels for periods of missing record, February 1-19, March 11-26, and October 26, were estimated.

PERIOD OF RECORD.--December 1957 to December 1959. January 1962 to current year.

EXTREMES FOR PERIOD OF RECORD.--Highest water level, $38.19 \mathrm{ft}$ below land-surface datum, April 1, 1959; 10west, $160.88 \mathrm{ft}$ below land-surface datum, July $26,1986$.

DEPTH BELOW LAND SURFACE (WATER LEVEL) (FEET) CALENDAR YEAR JANUARY 1987 TO DECEMBER 1987

\begin{tabular}{|c|c|c|c|c|c|c|c|c|c|c|c|c|}
\hline DAY & JAN & FEB & MAR & APR & MAY & JUN & JUL & AUG & SEP & ОСТ & NOV & DEC \\
\hline $\begin{array}{l}1 \\
2 \\
3 \\
4 \\
5\end{array}$ & $\begin{array}{l}137.73 \\
137.73 \\
137.73 \\
137.73 \\
137.73\end{array}$ & $\begin{array}{l}139.90 \\
139.96 \\
140.04 \\
140.10 \\
140.16\end{array}$ & $\begin{array}{l}140.13 \\
139.88 \\
139.56 \\
139.56 \\
139.60\end{array}$ & $\begin{array}{l}133.63 \\
134.21 \\
134.20 \\
134.23 \\
133.68\end{array}$ & $\begin{array}{l}135.30 \\
136.85 \\
137.75 \\
138.21 \\
138.77\end{array}$ & $\begin{array}{l}135.66 \\
136.21 \\
136.93 \\
137.77 \\
137.46\end{array}$ & $\begin{array}{l}138.58 \\
139.78 \\
140.15 \\
140.02 \\
139.87\end{array}$ & $\begin{array}{l}141.91 \\
142.01 \\
143.19 \\
143.66 \\
144.43\end{array}$ & $\begin{array}{l}148.85 \\
148.81 \\
148.54 \\
148.56 \\
148.77\end{array}$ & $\begin{array}{l}146.91 \\
147.68 \\
147.93 \\
147.81 \\
145.85\end{array}$ & $\begin{array}{l}149.04 \\
148.05 \\
148.63 \\
148.83 \\
147.63\end{array}$ & $\begin{array}{l}142.14 \\
141.81 \\
141.51 \\
140.33 \\
142.00\end{array}$ \\
\hline $\begin{array}{r}6 \\
7 \\
8 \\
9 \\
10\end{array}$ & $\begin{array}{l}137.73 \\
139.10 \\
141.62 \\
142.40 \\
142.82\end{array}$ & $\begin{array}{l}140.22 \\
140.28 \\
140.35 \\
140.41 \\
140.45\end{array}$ & $\begin{array}{l}137.82 \\
135.77 \\
133.50 \\
131.05 \\
130.61\end{array}$ & $\begin{array}{l}132.47 \\
132.42 \\
132.25 \\
130.48 \\
129.52\end{array}$ & $\begin{array}{l}139.31 \\
139.57 \\
139.87 \\
138.60 \\
139.24\end{array}$ & $\begin{array}{l}138.83 \\
139.52 \\
139.98 \\
140.69 \\
141.53\end{array}$ & $\begin{array}{l}139.78 \\
139.59 \\
137.77 \\
138.26 \\
138.55\end{array}$ & $\begin{array}{l}145.29 \\
146.04 \\
146.67 \\
147.10 \\
147.46\end{array}$ & $\begin{array}{l}147.69 \\
148.33 \\
148.13 \\
148.46 \\
148.50\end{array}$ & $\begin{array}{l}145.98 \\
146.89 \\
148.01 \\
148.61 \\
148.81\end{array}$ & $\begin{array}{l}146.28 \\
146.36 \\
147.17 \\
146.71 \\
144.44\end{array}$ & $\begin{array}{l}142.29 \\
142.27 \\
141.51 \\
141.27 \\
141.84\end{array}$ \\
\hline $\begin{array}{l}11 \\
12 \\
13 \\
14 \\
15\end{array}$ & $\begin{array}{l}143.27 \\
143.30 \\
143.48 \\
143.65 \\
143.82\end{array}$ & $\begin{array}{l}140.51 \\
138.46 \\
138.43 \\
138.40 \\
138.36\end{array}$ & $\begin{array}{l}128.84 \\
128.44 \\
133.43 \\
133.57 \\
133.50\end{array}$ & $\begin{array}{l}131.25 \\
131.67 \\
131.13 \\
129.28 \\
130.60\end{array}$ & $\begin{array}{l}139.17 \\
138.68 \\
138.76 \\
137.88 \\
137.19\end{array}$ & $\begin{array}{l}142.47 \\
143.43 \\
144.08 \\
144.12 \\
143.79\end{array}$ & $\begin{array}{l}138.74 \\
138.74 \\
138.56 \\
138.56 \\
138.95\end{array}$ & $\begin{array}{l}147.96 \\
148.51 \\
148.68 \\
148.66 \\
148.88\end{array}$ & $\begin{array}{l}148.45 \\
148.53 \\
147.53 \\
147.68 \\
146.32\end{array}$ & $\begin{array}{l}148.90 \\
148.74 \\
148.98 \\
149.45 \\
148.68\end{array}$ & $\begin{array}{l}145.68 \\
144.07 \\
145.48 \\
145.87 \\
145.95\end{array}$ & $\begin{array}{l}141.07 \\
141.33 \\
138.71 \\
137.07 \\
137.90\end{array}$ \\
\hline $\begin{array}{l}16 \\
17 \\
18 \\
19 \\
20\end{array}$ & $\begin{array}{l}144.22 \\
144.50 \\
144.29 \\
144.01 \\
143.83\end{array}$ & $\begin{array}{l}138.33 \\
138.31 \\
138.29 \\
138.27 \\
136.94\end{array}$ & $\begin{array}{l}129.34 \\
124.87 \\
124.89 \\
126.32 \\
129.83\end{array}$ & $\begin{array}{l}129.58 \\
128.03 \\
128.18 \\
127.94 \\
128.26\end{array}$ & $\begin{array}{l}137.72 \\
137.62 \\
136.75 \\
134.66 \\
135.70\end{array}$ & $\begin{array}{l}143.60 \\
143.62 \\
143.54 \\
143.29 \\
143.12\end{array}$ & $\begin{array}{l}139.60 \\
139.86 \\
139.33 \\
138.33 \\
138.83\end{array}$ & $\begin{array}{l}148.58 \\
148.24 \\
148.18 \\
148.35 \\
148.54\end{array}$ & $\begin{array}{l}146.78 \\
146.77 \\
147.01 \\
147.34 \\
147.27\end{array}$ & $\begin{array}{l}149.10 \\
149.25 \\
149.02 \\
148.82 \\
147.69\end{array}$ & $\begin{array}{l}145.17 \\
142.92 \\
144.32 \\
143.82 \\
144.37\end{array}$ & $\begin{array}{l}139.25 \\
139.16 \\
139.38 \\
139.33 \\
138.17\end{array}$ \\
\hline $\begin{array}{l}21 \\
22 \\
23 \\
24 \\
25\end{array}$ & $\begin{array}{l}143.71 \\
143.61 \\
144.00 \\
144.02 \\
143.43\end{array}$ & $\begin{array}{l}136.76 \\
136.76 \\
138.47 \\
140.20 \\
140.48\end{array}$ & $\begin{array}{l}131.07 \\
131.12 \\
130.95 \\
131.34 \\
131.86\end{array}$ & $\begin{array}{l}129.79 \\
130.77 \\
131.92 \\
132.81 \\
133.23\end{array}$ & $\begin{array}{l}136.10 \\
135.76 \\
136.03 \\
135.88 \\
135.46\end{array}$ & $\begin{array}{l}143.02 \\
142.41 \\
140.89 \\
140.94 \\
140.05\end{array}$ & $\begin{array}{l}139.26 \\
140.73 \\
141.62 \\
142.27 \\
142.96\end{array}$ & $\begin{array}{l}148.74 \\
148.74 \\
148.57 \\
148.25 \\
148.51\end{array}$ & $\begin{array}{l}147.43 \\
147.53 \\
147.42 \\
147.35 \\
146.11\end{array}$ & & $\begin{array}{l}144.59 \\
144.45 \\
144.26 \\
144.14 \\
143.81\end{array}$ & $\begin{array}{l}136.37 \\
136.11 \\
137.35 \\
137.30 \\
136.92\end{array}$ \\
\hline $\begin{array}{l}26 \\
27 \\
28 \\
29 \\
30 \\
31\end{array}$ & $\begin{array}{l}143.23 \\
143.39 \\
143.55 \\
143.55 \\
142.39 \\
139.84\end{array}$ & $\begin{array}{r}139.10 \\
139.50 \\
140.24 \\
--- \\
-.- \\
---\end{array}$ & $\begin{array}{l}132.66 \\
134.50 \\
134.59 \\
134.81 \\
134.57 \\
134.85\end{array}$ & $\begin{array}{r}133.65 \\
133.90 \\
133.10 \\
134.70 \\
134.91 \\
---\end{array}$ & $\begin{array}{l}135.13 \\
134.01 \\
134.86 \\
135.39 \\
135.61 \\
134.91\end{array}$ & $\begin{array}{r}139.12 \\
140.29 \\
138.94 \\
138.11 \\
137.85 \\
----\end{array}$ & $\begin{array}{l}143.20 \\
143.11 \\
143.24 \\
143.62 \\
142.74 \\
143.97\end{array}$ & $\begin{array}{l}148.91 \\
149.12 \\
147.91 \\
148.84 \\
147.46 \\
148.41\end{array}$ & $\begin{array}{r}146.11 \\
145.76 \\
145.38 \\
145.20 \\
145.46 \\
-.-\end{array}$ & $\begin{array}{l}148.91 \\
148.86 \\
149.05 \\
149.16 \\
149.32 \\
149.45\end{array}$ & $\begin{array}{r}142.08 \\
142.30 \\
142.90 \\
142.82 \\
142.50 \\
---\end{array}$ & $\begin{array}{l}136.10 \\
136.53 \\
136.31 \\
135.43 \\
135.42 \\
135.42\end{array}$ \\
\hline MEAN & 142.11 & 139.20 & 132.99 & 131.73 & 136.99 & 140.71 & 140.28 & 147.28 & 147.40 & 148.46 & 145.15 & 138.95 \\
\hline & & EN & 95 & $\mathrm{HIGH}$ & 124.87 & & & & & & & \\
\hline
\end{tabular}



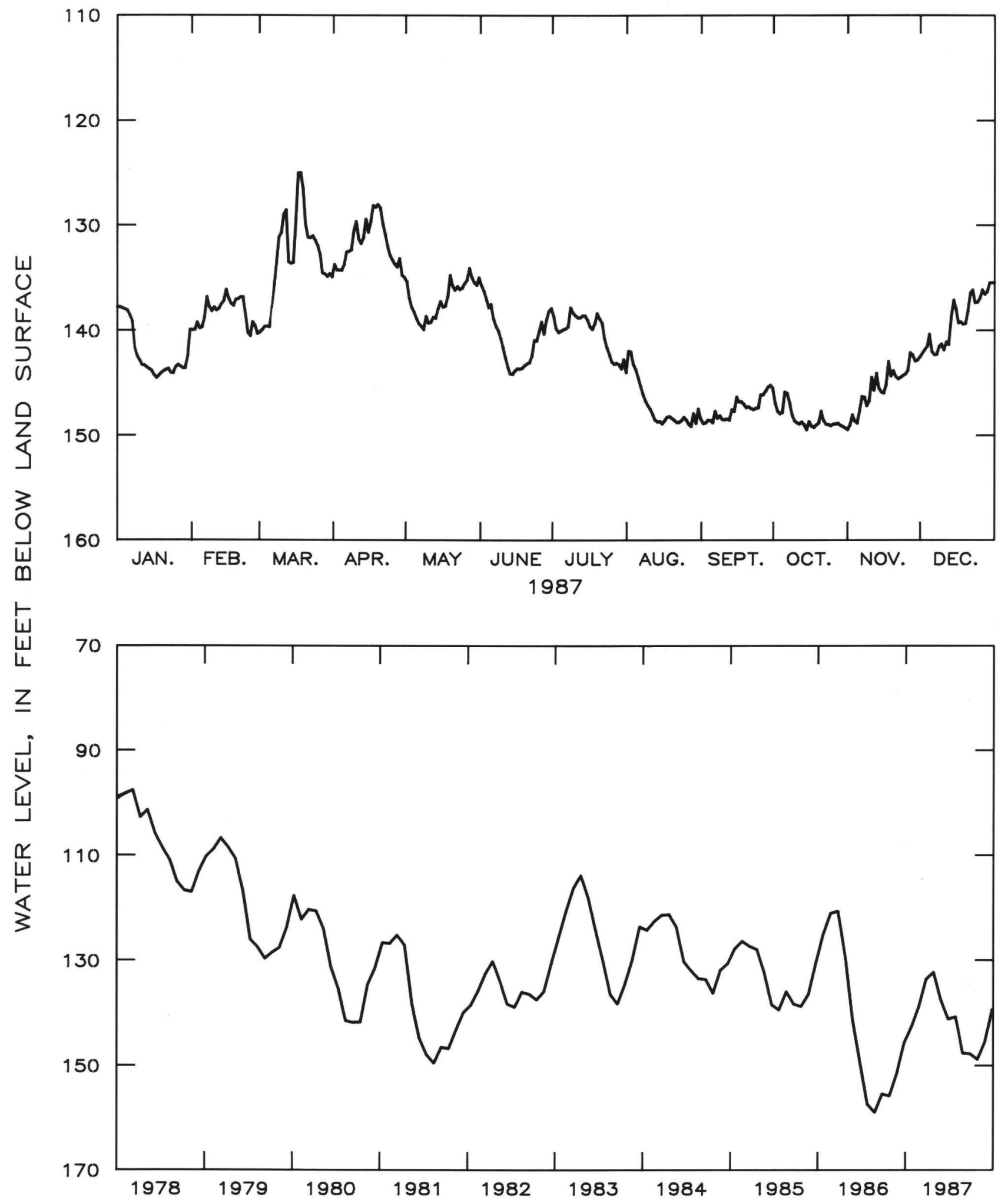

Figure 2.5-5.--Water level in observation well 13LOO2, Dougherty County. 


\subsection{Claiborne Aquifer}

The Claiborne aquifer is a major aquifer in southwestern Georgia where it supplies more than $36 \mathrm{Mgal} / \mathrm{d}$ for municipal, agricultural, and industrial use (McFadden and Perrie110, 1983). The aquifer is comprised of several hydraulically interconnected water-bearing zones of sand, limestone, and coquina. In east-central Georgia, the Claiborne aquifer is part of the Gordon aquifer system (Brooks and others, 1985).

During October, water-level measurements were made in 75 wells tapping the Claiborne aquifer and a potentiometric map was prepared. Pumping from the aquifer has resulted in a cone of depression centered at Albany. The water level in the Claiborne aquifer near Albany, Dougherty County, is affected primarily by local and regional pumping. The water level generally is highest during the winter and spring rainy seasons, and lowest in the fall following the summer irrigation season.

According to McFadden and Perriello (1983), the water level in the Claiborne aquifer at Albany declined about $68 \mathrm{ft}$ during 1951-79. Mean water levels in three wells tapping the Claiborne aquifer near Albany were from 1.8 to $5.1 \mathrm{ft}$ higher in 1987 than in 1986. The rises reversed a downward trend that began in 1984. By the end of April, the water level in three wells tapping the Claiborne aquifer had recovered 4.0 to $12.0 \mathrm{ft}$ from the 1986 lows, and the record low measured during the 1986 drought. At the end of 1987, water levels were from $0.4 \mathrm{ft}$ lower to $5.1 \mathrm{ft}$ higher than at the end of 1986 . 


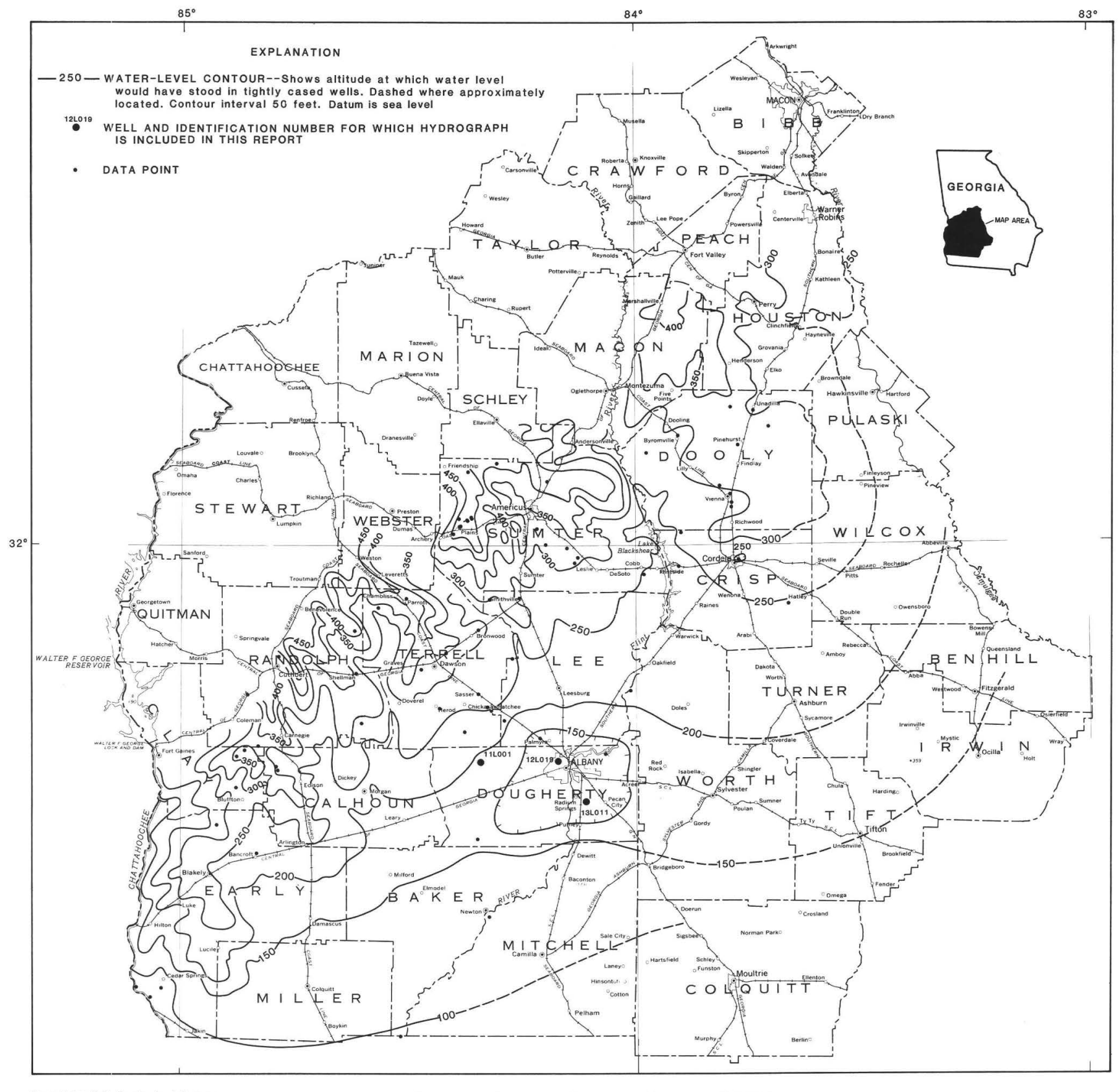

Base from U.S. Geological Survey

10

20

30

40 MILES

Figure 2.6-1.--Observation well locations and the water level in the Claiborne aquifer, October 1987. 
31353084203202 Local number, 11L001.

LOCATION.--Lat $31^{\circ} 35^{\prime} 30^{\prime \prime}$, long $84^{\circ} 20^{\prime} 32^{\prime \prime}$, Hydrologic Unit $03130008,10.4$ mi west of Albany.

Owner: U.S. Geological Survey, test well 4.

AQUIFER.--Claiborne.

WELL CHARACTERISTICS.--Drilled observation well, depth $251 \mathrm{ft}$, cased to $233 \mathrm{ft}$.

DATUM.--Elevation of land-surface datum is $220 \mathrm{ft}$.

Measuring point: Floor of recorder shelter, $3.0 \mathrm{ft}$ above land-surface datum.

REMARKS.--Water level for the period of missing record, October 30 , was estimated.

PERIOD OF RECORD.--March 1978 to current year.

EXTREMES FOR PERIOD OF RECORD.-- Highest water level, $12.11 \mathrm{ft}$ below land-surface datum, June 5-6, 1978; 10west,

$34.75 \mathrm{ft}$ below land-surface datum, October 19-20, 1986.

DEPTH BELOW LAND SURFACE (WATER LEVEL) (FEET) CALENDAR YEAR JANUARY 1987 TO DECEMBER 1987 MEAN VALUES

\begin{tabular}{|c|c|c|c|c|c|c|c|c|c|c|c|c|}
\hline DAY & JAN & FEB & MAR & APR & MAY & JUN & JUL & AUG & SEP & ОСТ & NOV & DEC \\
\hline $\begin{array}{l}1 \\
2 \\
3 \\
4 \\
5\end{array}$ & $\begin{array}{l}28.79 \\
28.65 \\
28.48 \\
28.32 \\
28.15\end{array}$ & $\begin{array}{l}24.47 \\
24.29 \\
24.18 \\
24.11 \\
23.98\end{array}$ & $\begin{array}{l}21.01 \\
20.92 \\
20.84 \\
20.75 \\
20.68\end{array}$ & $\begin{array}{l}18.66 \\
18.66 \\
18.65 \\
18.62 \\
18.60\end{array}$ & $\begin{array}{l}18.47 \\
18.52 \\
18.60 \\
18.69 \\
18.77\end{array}$ & $\begin{array}{l}20.40 \\
20.48 \\
20.60 \\
20.70 \\
20.75\end{array}$ & $\begin{array}{l}21.47 \\
21.40 \\
21.33 \\
21.23 \\
21.15\end{array}$ & $\begin{array}{l}22.59 \\
22.82 \\
22.93 \\
23.02 \\
23.15\end{array}$ & $\begin{array}{l}26.72 \\
26.75 \\
26.77 \\
26.83 \\
26.92\end{array}$ & $\begin{array}{l}28.69 \\
28.75 \\
28.81 \\
28.87 \\
28.88\end{array}$ & $\begin{array}{l}30.02 \\
30.05 \\
30.08 \\
30.06 \\
30.05\end{array}$ & $\begin{array}{l}29.68 \\
29.68 \\
29.60 \\
29.51 \\
29.49\end{array}$ \\
\hline $\begin{array}{r}6 \\
7 \\
8 \\
9 \\
10\end{array}$ & $\begin{array}{l}28.01 \\
27.85 \\
27.72 \\
27.62 \\
27.49\end{array}$ & $\begin{array}{l}23.87 \\
23.80 \\
23.73 \\
23.65 \\
23.54\end{array}$ & $\begin{array}{l}20.62 \\
20.56 \\
20.52 \\
20.43 \\
20.34\end{array}$ & $\begin{array}{l}18.60 \\
18.59 \\
18.58 \\
18.58 \\
18.56\end{array}$ & $\begin{array}{l}18.86 \\
18.92 \\
18.99 \\
19.07 \\
19.16\end{array}$ & $\begin{array}{l}20.85 \\
20.98 \\
21.07 \\
21.18 \\
21.33\end{array}$ & $\begin{array}{l}21.12 \\
21.08 \\
21.05 \\
21.03 \\
21.00\end{array}$ & $\begin{array}{l}23.31 \\
23.53 \\
23.70 \\
23.81 \\
23.93\end{array}$ & $\begin{array}{l}27.02 \\
27.11 \\
27.21 \\
27.36 \\
27.54\end{array}$ & $\begin{array}{l}28.89 \\
28.93 \\
29.02 \\
29.08 \\
29.12\end{array}$ & $\begin{array}{l}30.11 \\
30.13 \\
30.10 \\
30.03 \\
29.94\end{array}$ & $\begin{array}{l}29.47 \\
29.44 \\
29.37 \\
29.28 \\
29.22\end{array}$ \\
\hline $\begin{array}{l}11 \\
12 \\
13 \\
14 \\
15\end{array}$ & $\begin{array}{l}27.42 \\
27.33 \\
27.22 \\
27.10 \\
26.95\end{array}$ & $\begin{array}{l}23.44 \\
23.33 \\
23.22 \\
23.12 \\
23.01\end{array}$ & $\begin{array}{l}20.29 \\
20.21 \\
20.12 \\
20.04 \\
19.92\end{array}$ & $\begin{array}{l}18.55 \\
18.54 \\
18.54 \\
18.58 \\
18.59\end{array}$ & $\begin{array}{l}19.25 \\
19.32 \\
19.42 \\
19.52 \\
19.62\end{array}$ & $\begin{array}{l}21.52 \\
21.67 \\
21.85 \\
21.83 \\
21.80\end{array}$ & $\begin{array}{l}20.97 \\
20.95 \\
20.93 \\
20.90 \\
20.90\end{array}$ & $\begin{array}{l}24.06 \\
24.16 \\
24.25 \\
24.37 \\
24.53\end{array}$ & $\begin{array}{l}27.69 \\
27.80 \\
27.95 \\
28.12 \\
28.25\end{array}$ & $\begin{array}{l}29.14 \\
29.15 \\
29.24 \\
29.32 \\
29.37\end{array}$ & $\begin{array}{l}29.95 \\
29.95 \\
29.94 \\
29.95 \\
29.98\end{array}$ & $\begin{array}{l}29.19 \\
29.20 \\
29.31 \\
29.43 \\
29.49\end{array}$ \\
\hline $\begin{array}{l}16 \\
17 \\
18 \\
19 \\
20\end{array}$ & $\begin{array}{l}26.85 \\
26.77 \\
26.51 \\
26.20 \\
26.07\end{array}$ & $\begin{array}{l}22.89 \\
22.79 \\
22.67 \\
22.57 \\
22.47\end{array}$ & $\begin{array}{l}19.84 \\
19.79 \\
19.65 \\
19.49 \\
19.41\end{array}$ & $\begin{array}{l}18.60 \\
18.64 \\
18.60 \\
18.60 \\
18.58\end{array}$ & $\begin{array}{l}19.71 \\
19.79 \\
19.84 \\
19.88 \\
19.94\end{array}$ & $\begin{array}{l}21.87 \\
21.89 \\
21.92 \\
21.93 \\
21.93\end{array}$ & $\begin{array}{l}20.89 \\
20.89 \\
20.90 \\
20.92 \\
20.97\end{array}$ & $\begin{array}{l}24.70 \\
24.90 \\
25.07 \\
25.12 \\
25.17\end{array}$ & $\begin{array}{l}28.35 \\
28.44 \\
28.49 \\
28.52 \\
28.58\end{array}$ & $\begin{array}{l}29.42 \\
29.48 \\
29.54 \\
29.58 \\
29.65\end{array}$ & $\begin{array}{l}30.01 \\
29.98 \\
30.04 \\
30.09 \\
30.13\end{array}$ & $\begin{array}{l}29.64 \\
29.79 \\
29.89 \\
29.93 \\
29.94\end{array}$ \\
\hline $\begin{array}{l}21 \\
22 \\
23 \\
24 \\
25\end{array}$ & $\begin{array}{l}25.94 \\
25.72 \\
25.57 \\
25.44 \\
25.32\end{array}$ & $\begin{array}{l}22.35 \\
22.23 \\
22.09 \\
21.90 \\
21.67\end{array}$ & $\begin{array}{l}19.35 \\
19.29 \\
19.23 \\
19.16 \\
19.07\end{array}$ & $\begin{array}{l}18.56 \\
18.58 \\
18.60 \\
18.58 \\
18.58\end{array}$ & $\begin{array}{l}19.90 \\
19.87 \\
19.92 \\
19.97 \\
20.01\end{array}$ & $\begin{array}{l}21.92 \\
21.85 \\
21.76 \\
21.69 \\
21.64\end{array}$ & $\begin{array}{l}21.05 \\
21.16 \\
21.32 \\
21.48 \\
21.66\end{array}$ & $\begin{array}{l}25.39 \\
25.62 \\
25.87 \\
26.01 \\
26.18\end{array}$ & $\begin{array}{l}28.69 \\
28.78 \\
28.81 \\
28.79 \\
28.77\end{array}$ & $\begin{array}{l}29.74 \\
29.81 \\
29.85 \\
29.87 \\
29.86\end{array}$ & $\begin{array}{l}30.23 \\
30.29 \\
30.28 \\
30.23 \\
30.13\end{array}$ & $\begin{array}{l}29.94 \\
29.92 \\
29.94 \\
29.88 \\
29.78\end{array}$ \\
\hline $\begin{array}{l}26 \\
27 \\
28 \\
29 \\
30 \\
31\end{array}$ & $\begin{array}{l}25.22 \\
25.13 \\
25.04 \\
24.91 \\
24.73 \\
24.61\end{array}$ & $\begin{array}{r}21.51 \\
21.36 \\
21.19 \\
-\ldots- \\
-.- \\
-.-\end{array}$ & $\begin{array}{l}18.99 \\
18.92 \\
18.82 \\
18.71 \\
18.66 \\
18.68\end{array}$ & $\begin{array}{r}18.56 \\
18.55 \\
18.56 \\
18.46 \\
18.41 \\
. .-\end{array}$ & $\begin{array}{l}20.04 \\
20.17 \\
20.23 \\
20.27 \\
20.30 \\
20.34\end{array}$ & $\begin{array}{r}21.57 \\
21.55 \\
21.54 \\
21.51 \\
21.49 \\
. .-\end{array}$ & $\begin{array}{l}21.80 \\
21.90 \\
22.00 \\
22.10 \\
22.24 \\
22.44\end{array}$ & $\begin{array}{l}26.29 \\
26.39 \\
26.50 \\
26.59 \\
26.64 \\
26.66\end{array}$ & $\begin{array}{r}28.76 \\
28.76 \\
28.76 \\
28.70 \\
28.64 \\
. .-\end{array}$ & $\begin{array}{l}29.84 \\
29.82 \\
29.87 \\
29.91 \\
29.95 \\
29.99\end{array}$ & $\begin{array}{r}30.00 \\
29.90 \\
29.81 \\
29.72 \\
29.68 \\
.\end{array}$ & $\begin{array}{l}29.65 \\
29.55 \\
29.42 \\
29.39 \\
29.34 \\
29.24\end{array}$ \\
\hline MEAN & 26.68 & 22.98 & 19.82 & 18.58 & 19.53 & 21.44 & 21.30 & 24.75 & 28.00 & 29.40 & 30.03 & 29.57 \\
\hline
\end{tabular}

$\begin{array}{lllllll}\text { CAL YR } 1987 & \text { MEAN } & 24.35 & \text { HIGH } & 18.41 & \text { LOW } & 30.29\end{array}$ 

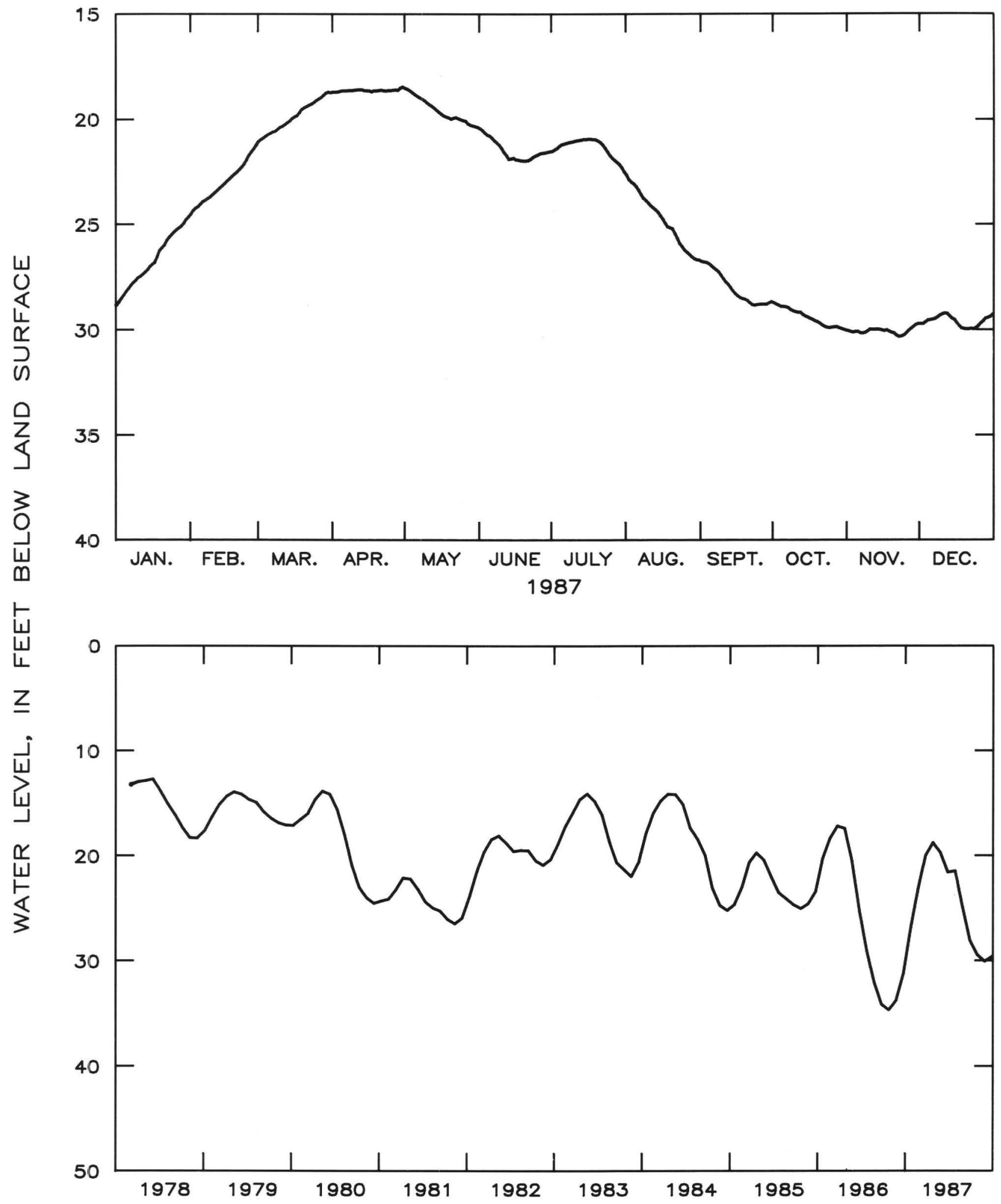

Figure 2.6-2.--Water level in observation well 11LOO1, Dougherty County. 
12 LO19 TEST WELL 5 DOUGHERTY COUNTY

313534084103001 Local number, 12L019.

LOCATION.--Lat $31^{\circ} 35^{\prime} 34^{\prime \prime}$, long $84^{\circ} 10^{\prime} 30^{\prime \prime}$, Hydrologic Unit 03130008 , located in park at intersection of S1appey Drive and Fifth Avenue.

Owner: U.S. Geological Survey, test well 5.

AQUIFER.--Claiborne.

WELL CHARACTERISTICS.--Drilled observation we 11 , depth $257 \mathrm{ft}$, cased and screened to $88 \mathrm{ft}$.

DATUM.--Elevation of land-surface datum is $198 \mathrm{ft}$.

Measuring point: Floor of recorder shelter, $3.0 \mathrm{ft}$ above land-surface datum.

REMARKS.--Water levels for periods of mising record, October 27 and December 1, were estimated.

PERIOD OF RECORD.--March 1978 to current year.

EXTREMES FOR PERIOD OF RECORD.--Highest water level, $72.35 \mathrm{ft}$ below land-surface datum, April 20, 1983; 10west,

$99.53 \mathrm{ft}$ below land-surface datum, August 1-2, 1978.

\section{DEPTH BELOW LAND SURFACE (WATER LEVEL) (FEET) CALENDAR YEAR JANUARY 1987 TO DECEMBER 1987}

\begin{tabular}{|c|c|c|c|c|c|c|c|c|c|c|c|c|}
\hline DAY & JAN & FEB & MAR & APR & MAY & JUN & JUL & AUG & SEP & OCT & NOV & DEC \\
\hline $\begin{array}{l}1 \\
2 \\
3 \\
4 \\
5\end{array}$ & $\begin{array}{l}85.05 \\
85.05 \\
85.05 \\
84.94 \\
84.83\end{array}$ & $\begin{array}{l}83.97 \\
83.91 \\
83.96 \\
84.03 \\
84.00\end{array}$ & $\begin{array}{l}81.94 \\
81.59 \\
80.98 \\
80.87 \\
80.74\end{array}$ & $\begin{array}{l}78.78 \\
78.51 \\
78.25 \\
77.62 \\
77.18\end{array}$ & $\begin{array}{l}78.65 \\
79.25 \\
79.56 \\
79.74 \\
80.18\end{array}$ & $\begin{array}{l}81.99 \\
82.34 \\
82.77 \\
83.12 \\
83.49\end{array}$ & $\begin{array}{l}79.18 \\
79.21 \\
79.11 \\
78.76 \\
78.40\end{array}$ & $\begin{array}{l}80.74 \\
80.83 \\
81.05 \\
81.02 \\
80.65\end{array}$ & $\begin{array}{l}84.48 \\
84.15 \\
83.53 \\
83.07 \\
83.10\end{array}$ & $\begin{array}{l}83.99 \\
84.25 \\
84.53 \\
84.50 \\
84.38\end{array}$ & $\begin{array}{l}85.87 \\
85.91 \\
85.90 \\
85.83 \\
85.98\end{array}$ & $\begin{array}{l}84.52 \\
84.51 \\
84.36 \\
84.23 \\
84.26\end{array}$ \\
\hline $\begin{array}{r}6 \\
7 \\
8 \\
9 \\
10\end{array}$ & $\begin{array}{l}84.74 \\
84.25 \\
83.89 \\
83.30 \\
83.31\end{array}$ & $\begin{array}{l}83.79 \\
82.95 \\
82.84 \\
83.25 \\
83.60\end{array}$ & $\begin{array}{l}80.44 \\
80.12 \\
79.91 \\
80.01 \\
80.16\end{array}$ & $\begin{array}{l}76.87 \\
76.53 \\
76.43 \\
76.43 \\
76.35\end{array}$ & $\begin{array}{l}80.55 \\
80.93 \\
81.32 \\
81.66 \\
81.81\end{array}$ & $\begin{array}{l}83.87 \\
84.17 \\
84.40 \\
84.64 \\
84.74\end{array}$ & $\begin{array}{l}77.88 \\
77.32 \\
77.11 \\
77.38 \\
77.56\end{array}$ & $\begin{array}{l}80.39 \\
80.72 \\
80.81 \\
80.93 \\
80.96\end{array}$ & $\begin{array}{l}83.09 \\
83.13 \\
83.48 \\
83.77 \\
83.84\end{array}$ & $\begin{array}{l}84.34 \\
84.58 \\
84.89 \\
85.22 \\
85.66\end{array}$ & $\begin{array}{l}86.01 \\
86.00 \\
86.00 \\
86.00 \\
85.96\end{array}$ & $\begin{array}{l}84.43 \\
84.42 \\
84.28 \\
84.14 \\
84.08\end{array}$ \\
\hline $\begin{array}{l}11 \\
12 \\
13 \\
14 \\
15\end{array}$ & $\begin{array}{l}83.83 \\
84.17 \\
84.15 \\
83.95 \\
83.62\end{array}$ & $\begin{array}{l}83.79 \\
83.91 \\
84.02 \\
84.12 \\
84.21\end{array}$ & $\begin{array}{l}80.36 \\
80.17 \\
79.62 \\
79.44 \\
79.49\end{array}$ & $\begin{array}{l}76.35 \\
76.39 \\
76.51 \\
76.21 \\
75.97\end{array}$ & $\begin{array}{l}81.94 \\
81.92 \\
81.91 \\
82.04 \\
82.00\end{array}$ & $\begin{array}{l}84.58 \\
84.46 \\
84.40 \\
84.24 \\
83.80\end{array}$ & $\begin{array}{l}77.55 \\
77.50 \\
77.77 \\
78.19 \\
78.28\end{array}$ & $\begin{array}{l}81.27 \\
81.74 \\
82.47 \\
82.91 \\
82.82\end{array}$ & $\begin{array}{l}83.64 \\
83.56 \\
83.46 \\
83.27 \\
83.14\end{array}$ & $\begin{array}{l}85.97 \\
86.15 \\
86.33 \\
86.64 \\
86.68\end{array}$ & $\begin{array}{l}85.93 \\
85.72 \\
85.15 \\
84.83 \\
84.78\end{array}$ & $\begin{array}{l}84.08 \\
84.01 \\
83.55 \\
83.21 \\
82.99\end{array}$ \\
\hline $\begin{array}{l}16 \\
17 \\
18 \\
19 \\
20\end{array}$ & $\begin{array}{l}83.59 \\
83.83 \\
84.07 \\
84.28 \\
84.58\end{array}$ & $\begin{array}{l}84.09 \\
83.65 \\
82.64 \\
82.02 \\
81.70\end{array}$ & $\begin{array}{l}79.18 \\
78.38 \\
77.92 \\
77.62 \\
77.59\end{array}$ & $\begin{array}{l}75.99 \\
75.94 \\
75.89 \\
75.97 \\
75.85\end{array}$ & $\begin{array}{l}82.01 \\
82.00 \\
82.09 \\
82.30 \\
82.50\end{array}$ & $\begin{array}{l}83.51 \\
83.22 \\
82.91 \\
82.43 \\
81.79\end{array}$ & $\begin{array}{l}78.38 \\
78.45 \\
78.48 \\
78.65 \\
78.97\end{array}$ & $\begin{array}{l}82.31 \\
81.96 \\
81.91 \\
82.03 \\
81.97\end{array}$ & $\begin{array}{l}82.90 \\
83.03 \\
82.95 \\
82.77 \\
82.97\end{array}$ & $\begin{array}{l}86.45 \\
86.40 \\
86.65 \\
86.68 \\
86.64\end{array}$ & $\begin{array}{l}84.61 \\
84.17 \\
83.89 \\
83.89 \\
83.84\end{array}$ & $\begin{array}{l}82.95 \\
82.83 \\
82.63 \\
82.69 \\
82.66\end{array}$ \\
\hline $\begin{array}{l}21 \\
22 \\
23 \\
24 \\
25\end{array}$ & $\begin{array}{l}84.71 \\
84.61 \\
84.71 \\
84.67 \\
84.40\end{array}$ & $\begin{array}{l}81.83 \\
82.10 \\
82.47 \\
82.73 \\
82.45\end{array}$ & $\begin{array}{l}77.86 \\
78.18 \\
78.44 \\
78.66 \\
78.86\end{array}$ & $\begin{array}{l}75.90 \\
75.94 \\
75.92 \\
76.45 \\
76.98\end{array}$ & $\begin{array}{l}82.62 \\
82.43 \\
82.24 \\
82.06 \\
81.97\end{array}$ & $\begin{array}{l}81.24 \\
80.87 \\
80.65 \\
80.89 \\
81.02\end{array}$ & $\begin{array}{l}79.29 \\
79.40 \\
79.63 \\
80.11 \\
80.51\end{array}$ & $\begin{array}{l}81.70 \\
81.72 \\
82.05 \\
82.49 \\
82.94\end{array}$ & $\begin{array}{l}83.17 \\
83.17 \\
83.11 \\
83.13 \\
83.41\end{array}$ & $\begin{array}{l}86.73 \\
87.03 \\
87.02 \\
87.00 \\
87.01\end{array}$ & $\begin{array}{l}83.85 \\
84.04 \\
84.12 \\
84.10 \\
84.17\end{array}$ & $\begin{array}{l}82.57 \\
82.45 \\
82.18 \\
82.03 \\
81.90\end{array}$ \\
\hline $\begin{array}{l}26 \\
27 \\
28 \\
29 \\
30 \\
31\end{array}$ & $\begin{array}{l}84.25 \\
84.16 \\
84.08 \\
84.01 \\
83.93 \\
83.95\end{array}$ & $\begin{array}{r}82.13 \\
82.24 \\
82.15 \\
--- \\
-- \\
---\end{array}$ & $\begin{array}{l}78.89 \\
78.74 \\
78.87 \\
78.97 \\
78.97 \\
78.99\end{array}$ & $\begin{array}{r}77.28 \\
77.26 \\
77.38 \\
77.61 \\
78.03 \\
---\end{array}$ & $\begin{array}{l}81.99 \\
82.13 \\
81.89 \\
81.43 \\
81.51 \\
81.77\end{array}$ & $\begin{array}{r}80.68 \\
80.28 \\
79.96 \\
79.69 \\
79.26 \\
---\end{array}$ & $\begin{array}{l}80.47 \\
80.61 \\
80.89 \\
80.94 \\
80.91 \\
80.77\end{array}$ & $\begin{array}{l}83.35 \\
83.83 \\
84.05 \\
83.89 \\
83.90 \\
84.18\end{array}$ & $\begin{array}{r}83.50 \\
83.73 \\
83.93 \\
83.94 \\
83.84 \\
---\end{array}$ & $\begin{array}{l}86.94 \\
86.48 \\
85.99 \\
85.94 \\
85.87 \\
85.82\end{array}$ & $\begin{array}{r}84.52 \\
84.59 \\
84.56 \\
84.52 \\
84.53 \\
---\end{array}$ & $\begin{array}{l}81.42 \\
80.90 \\
80.50 \\
80.38 \\
80.25 \\
79.97\end{array}$ \\
\hline MEAN & 84.26 & 83.16 & 79.42 & 76.76 & 81.50 & 82.51 & 78.96 & 82.05 & 83.41 & 85.90 & 84.98 & 82.8 \\
\hline
\end{tabular}

$\begin{array}{lllllll}\text { CAL YR } 1987 & \text { MEAN } & 82.14 & \text { HIGH } & 75.85 & \text { LOW } & 87.03\end{array}$ 

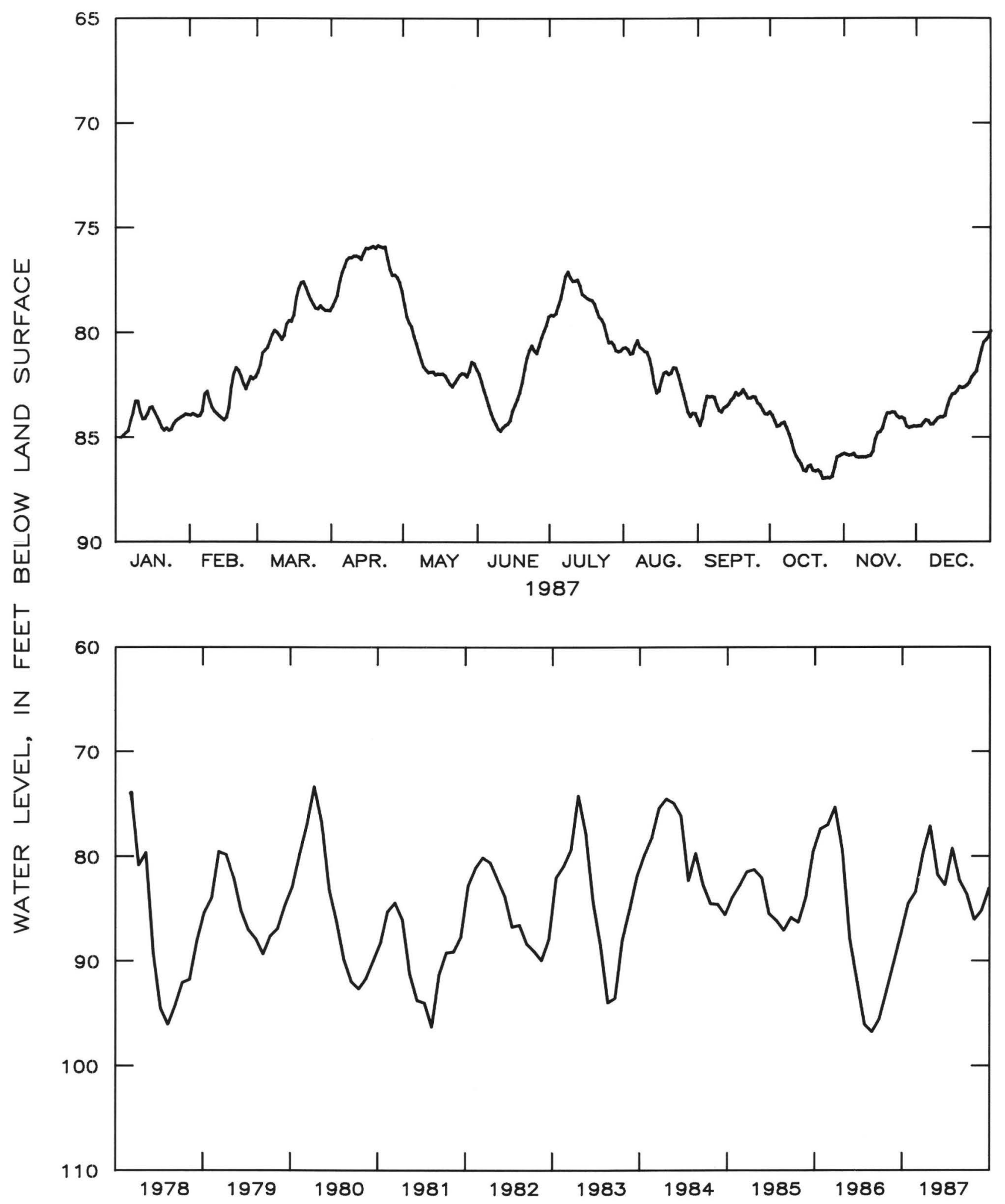

Figure 2.6-3.--Water level in observation well 12LO19, Dougherty County. 
313105084064301 Local number, 13 L011.

LOCATION.--Lat $31^{\circ} 31^{\prime} 05^{\prime \prime}$, long $84^{\circ} 06^{\prime} 43^{\prime \prime}$, Hydrologic Unit 03130008 , about 6.5 mi southeast of Albany off U.S. Highway 19 on School Bus Road.

Owner: U.S. Geological Survey, test well 2.

AQUIFER.--Claiborne.

WELL CHARACTERISTICS.--Drilled observation well, depth $418 \mathrm{ft}$, cased to $398 \mathrm{ft}$.

DATUM.--Elevation of land-surface datum is $195 \mathrm{ft}$.

Measuring point: Floor of recorder shelter, $3.0 \mathrm{ft}$ above land-surface datum.

REMARKS.--None.

PERIOD OF RECORD.--June 1977 to current year.

EXTREMES FOR PERIOD OF RECORD.--Highest water level, $60.01 \mathrm{ft}$ below land-surface datum, April 5, 1978 ; 10west, $95.00 \mathrm{ft}$ below land-surface datum, August 9-11, 1981.

DEPTH BELOW LAND SURFACE (WATER LEVEL) (FEET) CALENDAR YEAR JANUARY 1987 TO DECEMBER 1987 MEAN VALUES

\begin{tabular}{|c|c|c|c|c|c|c|c|c|c|c|c|c|}
\hline DAY & JAN & FEB & MAR & APR & MAY & JUN & JUL & AUG & SEP & OCT & NOV & DEC \\
\hline $\begin{array}{l}1 \\
2 \\
3 \\
4 \\
5\end{array}$ & $\begin{array}{l}79.57 \\
79.46 \\
79.25 \\
78.89 \\
78.70\end{array}$ & $\begin{array}{l}78.45 \\
78.73 \\
79.04 \\
79.17 \\
79.35\end{array}$ & $\begin{array}{l}75.63 \\
75.58 \\
75.65 \\
75.75 \\
75.79\end{array}$ & $\begin{array}{l}73.38 \\
73.38 \\
73.33 \\
73.35 \\
73.38\end{array}$ & $\begin{array}{l}74.75 \\
75.07 \\
75.28 \\
75.55 \\
75.88\end{array}$ & $\begin{array}{l}77.48 \\
77.70 \\
77.98 \\
78.20 \\
78.40\end{array}$ & $\begin{array}{l}78.38 \\
78.36 \\
78.32 \\
78.26 \\
78.12\end{array}$ & $\begin{array}{l}80.88 \\
80.66 \\
80.50 \\
80.43 \\
80.63\end{array}$ & $\begin{array}{l}84.50 \\
84.49 \\
84.52 \\
84.37 \\
84.21\end{array}$ & $\begin{array}{l}86.16 \\
86.20 \\
86.25 \\
86.48 \\
86.61\end{array}$ & $\begin{array}{l}85.42 \\
85.34 \\
85.26 \\
85.12 \\
84.99\end{array}$ & $\begin{array}{l}81.17 \\
81.08 \\
81.05 \\
81.09 \\
81.24\end{array}$ \\
\hline $\begin{array}{r}6 \\
7 \\
8 \\
9 \\
10\end{array}$ & $\begin{array}{l}78.69 \\
78.96 \\
79.31 \\
79.37 \\
79.13\end{array}$ & $\begin{array}{l}79.35 \\
79.12 \\
79.02 \\
79.23 \\
79.39\end{array}$ & $\begin{array}{l}75.74 \\
75.55 \\
75.19 \\
74.94 \\
74.98\end{array}$ & $\begin{array}{l}73.26 \\
73.14 \\
73.22 \\
73.24 \\
73.15\end{array}$ & $\begin{array}{l}76.19 \\
76.37 \\
76.54 \\
76.62 \\
76.71\end{array}$ & $\begin{array}{l}78.46 \\
78.43 \\
78.53 \\
78.73 \\
79.02\end{array}$ & $\begin{array}{l}77.98 \\
77.88 \\
77.90 \\
78.04 \\
78.27\end{array}$ & $\begin{array}{l}81.07 \\
81.56 \\
82.01 \\
82.36 \\
82.64\end{array}$ & $\begin{array}{l}84.09 \\
84.04 \\
84.08 \\
84.16 \\
84.19\end{array}$ & $\begin{array}{l}86.58 \\
86.64 \\
86.89 \\
87.05 \\
87.10\end{array}$ & $\begin{array}{l}84.85 \\
84.61 \\
84.33 \\
84.01 \\
83.73\end{array}$ & $\begin{array}{l}81.42 \\
81.53 \\
81.49 \\
81.50 \\
81.46\end{array}$ \\
\hline $\begin{array}{l}11 \\
12 \\
13 \\
14 \\
15\end{array}$ & $\begin{array}{l}78.98 \\
78.73 \\
78.49 \\
78.42 \\
78.44\end{array}$ & $\begin{array}{l}79.32 \\
79.36 \\
79.45 \\
79.22 \\
78.78\end{array}$ & $\begin{array}{l}75.08 \\
75.08 \\
74.91 \\
74.83 \\
74.77\end{array}$ & $\begin{array}{l}73.01 \\
72.82 \\
72.75 \\
72.85 \\
72.97\end{array}$ & $\begin{array}{l}76.89 \\
77.04 \\
77.02 \\
76.92 \\
76.87\end{array}$ & $\begin{array}{l}79.34 \\
79.62 \\
79.82 \\
79.69 \\
79.35\end{array}$ & $\begin{array}{l}78.27 \\
78.31 \\
78.57 \\
78.95 \\
79.32\end{array}$ & $\begin{array}{l}82.88 \\
83.11 \\
83.36 \\
83.49 \\
83.40\end{array}$ & $\begin{array}{l}84.29 \\
84.51 \\
84.55 \\
84.63 \\
84.76\end{array}$ & $\begin{array}{l}87.10 \\
87.10 \\
87.10 \\
87.10 \\
87.10\end{array}$ & $\begin{array}{l}83.65 \\
83.63 \\
83.59 \\
83.43 \\
83.32\end{array}$ & $\begin{array}{l}81.32 \\
81.23 \\
81.21 \\
81.02 \\
80.77\end{array}$ \\
\hline $\begin{array}{l}16 \\
17 \\
18 \\
19 \\
20\end{array}$ & $\begin{array}{l}78.42 \\
78.30 \\
78.10 \\
77.80 \\
77.70\end{array}$ & $\begin{array}{l}78.27 \\
78.00 \\
77.79 \\
77.55 \\
77.37\end{array}$ & $\begin{array}{l}74.59 \\
74.49 \\
74.39 \\
74.27 \\
74.27\end{array}$ & $\begin{array}{l}72.91 \\
72.76 \\
72.65 \\
72.68 \\
72.72\end{array}$ & $\begin{array}{l}76.86 \\
76.89 \\
77.03 \\
77.04 \\
76.90\end{array}$ & $\begin{array}{l}79.10 \\
78.97 \\
78.84 \\
78.73 \\
78.66\end{array}$ & $\begin{array}{l}79.53 \\
79.82 \\
80.07 \\
80.26 \\
80.46\end{array}$ & $\begin{array}{l}83.24 \\
83.20 \\
83.24 \\
83.23 \\
83.19\end{array}$ & $\begin{array}{l}84.85 \\
84.94 \\
84.84 \\
84.65 \\
84.46\end{array}$ & $\begin{array}{l}87.09 \\
87.09 \\
87.09 \\
87.09 \\
87.08\end{array}$ & $\begin{array}{l}83.33 \\
83.27 \\
83.27 \\
83.27 \\
83.20\end{array}$ & $\begin{array}{l}80.80 \\
80.82 \\
80.74 \\
80.62 \\
80.49\end{array}$ \\
\hline $\begin{array}{l}21 \\
22 \\
23 \\
24 \\
25\end{array}$ & $\begin{array}{l}77.63 \\
77.71 \\
77.87 \\
77.72 \\
77.38\end{array}$ & $\begin{array}{l}77.26 \\
77.06 \\
76.84 \\
76.61 \\
76.46\end{array}$ & $\begin{array}{l}74.24 \\
74.18 \\
74.14 \\
74.13 \\
74.15\end{array}$ & $\begin{array}{l}72.68 \\
72.79 \\
72.99 \\
73.13 \\
73.24\end{array}$ & $\begin{array}{l}76.74 \\
76.67 \\
76.58 \\
76.55 \\
76.55\end{array}$ & $\begin{array}{l}78.69 \\
78.70 \\
78.59 \\
78.51 \\
78.47\end{array}$ & $\begin{array}{l}80.62 \\
80.73 \\
80.78 \\
80.82 \\
80.94\end{array}$ & $\begin{array}{l}83.19 \\
83.23 \\
83.26 \\
83.33 \\
83.54\end{array}$ & $\begin{array}{l}84.38 \\
84.50 \\
84.65 \\
84.89 \\
85.18\end{array}$ & $\begin{array}{l}87.08 \\
87.08 \\
87.08 \\
87.08 \\
87.08\end{array}$ & $\begin{array}{l}83.19 \\
83.08 \\
82.89 \\
82.75 \\
82.56\end{array}$ & $\begin{array}{l}80.25 \\
80.05 \\
80.09 \\
80.11 \\
79.96\end{array}$ \\
\hline $\begin{array}{l}26 \\
27 \\
28 \\
29 \\
30 \\
31\end{array}$ & $\begin{array}{l}77.20 \\
77.22 \\
77.42 \\
77.67 \\
77.84 \\
78.12\end{array}$ & $\begin{array}{r}76.37 \\
76.13 \\
75.86 \\
--- \\
--- \\
---\end{array}$ & $\begin{array}{l}74.17 \\
74.15 \\
74.06 \\
73.85 \\
73.48 \\
73.37\end{array}$ & $\begin{array}{r}73.43 \\
73.63 \\
73.84 \\
74.18 \\
74.42 \\
---\end{array}$ & $\begin{array}{l}76.63 \\
76.72 \\
76.83 \\
76.95 \\
77.12 \\
77.32\end{array}$ & $\begin{array}{r}78.37 \\
78.25 \\
78.16 \\
78.19 \\
78.36 \\
---\end{array}$ & $\begin{array}{l}81.02 \\
81.09 \\
81.29 \\
81.46 \\
81.37 \\
81.15\end{array}$ & $\begin{array}{l}83.73 \\
83.84 \\
83.99 \\
84.14 \\
84.30 \\
84.46\end{array}$ & $\begin{array}{r}85.32 \\
85.45 \\
85.60 \\
85.81 \\
85.96 \\
-.-\end{array}$ & $\begin{array}{l}87.08 \\
87.08 \\
87.08 \\
86.46 \\
85.29 \\
85.41\end{array}$ & $\begin{array}{r}82.36 \\
82.15 \\
81.86 \\
81.60 \\
81.36 \\
.--\end{array}$ & $\begin{array}{l}79.89 \\
79.87 \\
79.85 \\
79.95 \\
80.09 \\
80.14\end{array}$ \\
\hline MEAN & 78.34 & 78.16 & 74.69 & 73.18 & 76.55 & 78.64 & 79.56 & 82.78 & 84.70 & 86.80 & 83.51 & 80.72 \\
\hline CAL & & & & $\mathrm{HIGH}$ & 72.65 & & & & & & & \\
\hline
\end{tabular}



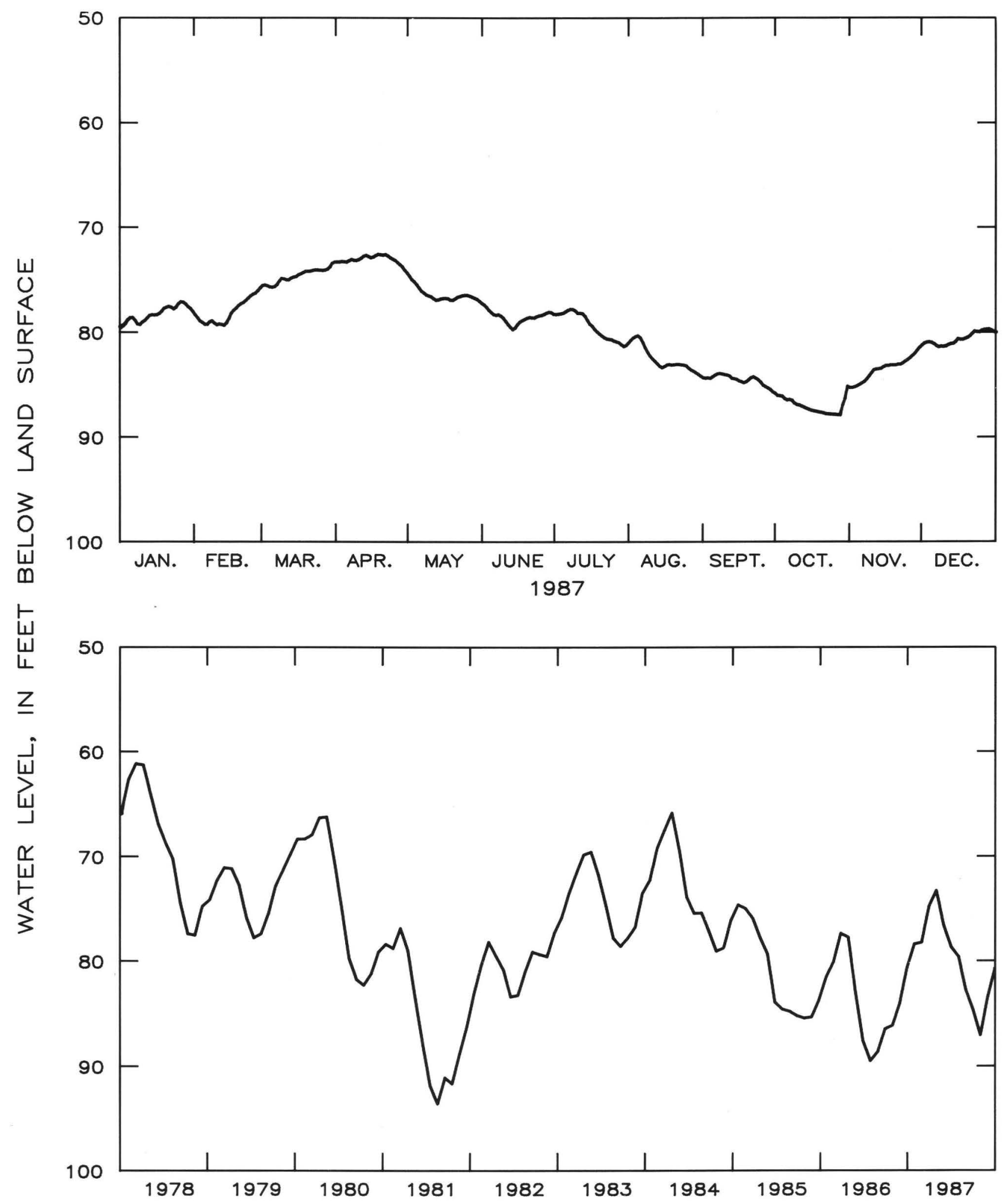

Figure 2.6-4.--Water level in observation well 13L011, Dougherty County. 


\subsection{Upper Floridan Aquifer}

The Upper Floridan aquifer (formerly the principal artesian aquifer) is part of the Floridan aquifer system, which is one of the most productive ground-water reservoirs in the United States. Regionally, the Floridan aquifer system has been divided by Miller (1986) into the Upper and the Lower Floridan aquifers. About $600 \mathrm{Mgal} / \mathrm{d}$ is pumped from the Upper Floridan aquifer in Georgia, mostly for industrial use and for irrigation (Pierce and Barber, 1982).

The Upper Floridan aquifer consists of a sequence of limestone and dolostone that underlies most of the Georgia Coastal Plain. Water in the Upper Floridan is under artesian pressure, except where it crops out at land surface. In some areas, the artesian pressure is sufficient to produce flowing wells.

In outcrop areas, the water level in the Upper Floridan aquifer fluctuates seasonally in response to recharge from precipitation. Near the coast where the aquifer is deeply buried, the water level responds primarily to pumping, and fluctuations related to recharge are less pronounced.

In October 1987, water levels were measured in 124 wells tapping the Upper Floridan aquifer' in Glynn and surrounding counties. From these measurements, a map showing the configuration of the potentiometric surface was drawn. 


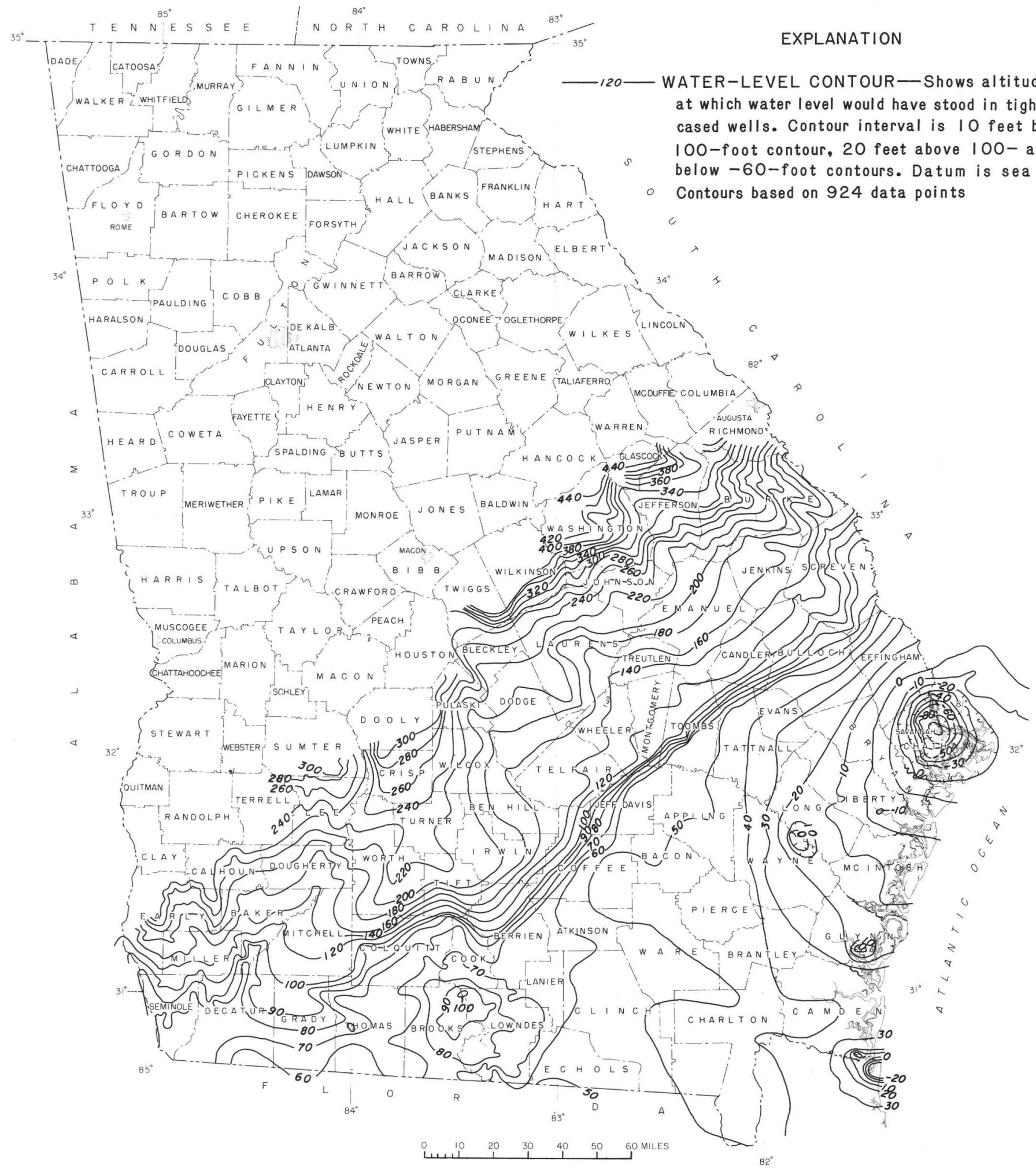

Figure 2.7-1.-Water level in the Upper Floridan aquifer, May 1985. 


\subsubsection{Southwest area}

The water level in the Upper Floridan aquifer in southwestern Georgia (Dougherty Plain area) responds to variations in rainfall, evapotranspiration, stream stage, and pumping. During 1985, an estimated $207 \mathrm{Mgal} / \mathrm{d}$ (Turlington and others, 1987) were withdrawn from aquifers in southwest Georgia. Much of this pumping was from the Upper Floridan aquifer, primarily for irrigation. This large withdrawal, including the more than 2,250 Mgal/d that were pumped during the irrigation season (H.E. Gill, U.S. Geological Survey, written commun., 1981), has not produced a discernable cone of depression because wells in the area are widely separated, and the transmissivity of the aquifer is high. The large withdrawal also has not caused long-term water-level declines because pumping is seasonal and recharge is plentiful during periods of normal rainfall.

The mean water levels in seven wells tapping the Upper Floridan aquifer in the southwest area were from 1.1 to $4.8 \mathrm{ft}$ higher in 1987 than in 1986. Above normal rainfall during the first half of the year resulted in significant recovery from the effects of the 1986 drought. By the end of April, the water levels in the seven wells had recovered 11.9 to $28.7 \mathrm{ft}$ from the lows and the record low recorded during the 1986 drought. Although mean water levels were higher, year-end water levels generally were lower in 1987 than in 1986. At the end of 1987, water levels in five wells were from about the same to $7.3 \mathrm{ft}$ lower than at the end of 1986. 


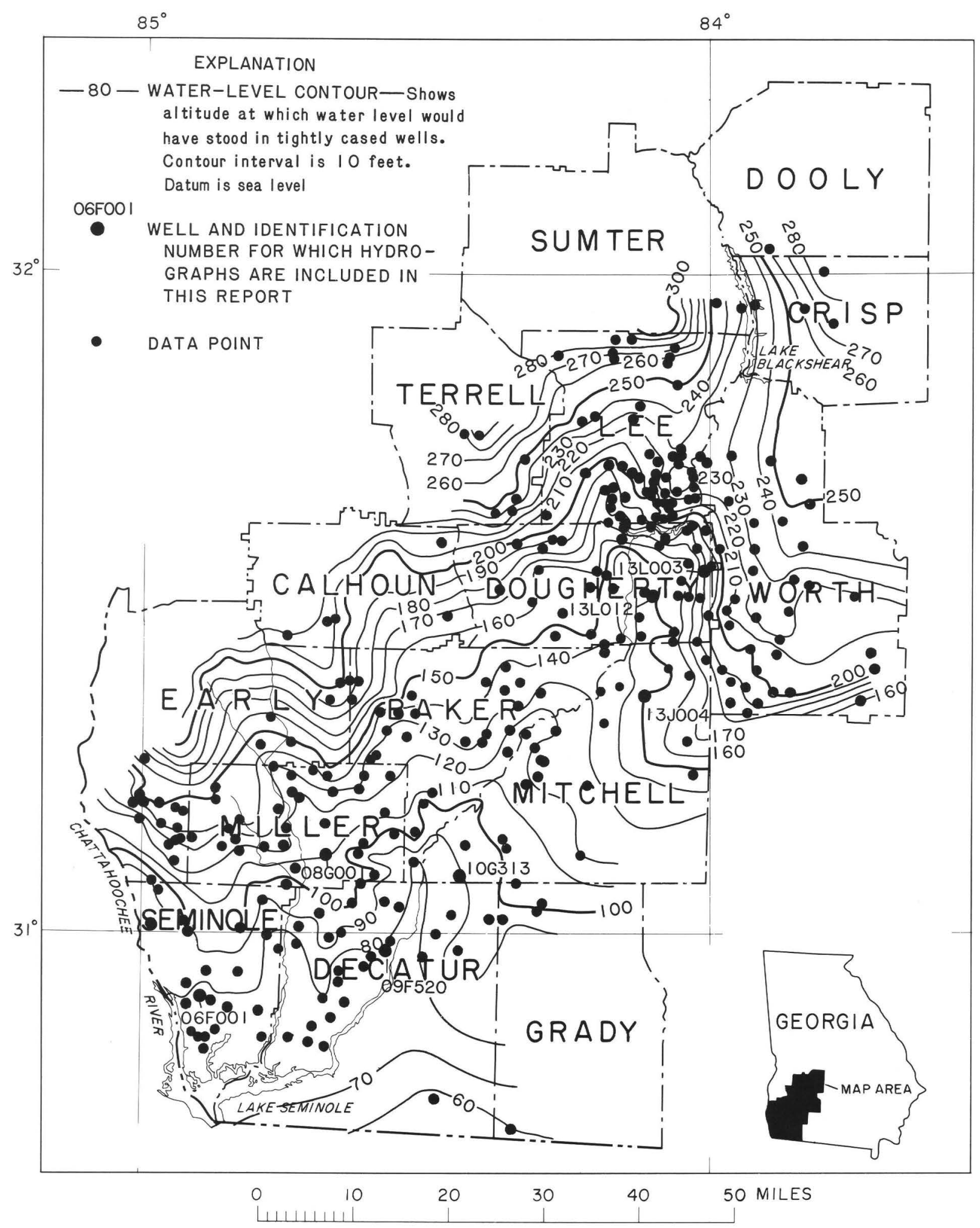

Figure 2.7.1-1.-Observation well locations and the water level in the Upper Floridan aquifer in the southwest area, October 
313748084002901 Local number, 13L003.

LOCATION.--Lat $31^{\circ} 33^{\prime} 13^{\prime \prime}$, long $84^{\circ} 00^{\prime} 21^{\prime \prime}$, Hydrologic Unit 03130008, near northeast corner of Marine Corps Supply Center, Acree, Ga.

Owner: City of Albany and Dougherty County.

AQUIFER.--Upper Floridan aquifer.

WELL CHARACTERISTICS.--Drilled unused supply well, diameter $6 \mathrm{in}$., depth $259 \mathrm{ft}$, cased to $206 \mathrm{ft}$, open hole.

DATUM.--Elevation of 1 and-surface datum is $225 \mathrm{ft}$.

Measuring point: Floor of recorder shelter, $4.10 \mathrm{ft}$ above land-surface datum.

REMARKS.--Well pumped and sounded June 21, 1978; water-quality sample collected at conclusion of pumping. Borehole geophysical survey conducted March 17, 1977. Water levels for period of missing record, January 22-28, and June 25-30, were estimated.

PERIOD OF RECORD.--January 1963 to current year.

EXTREMES FOR PERIOD OF RECORD.--Highest water level, $17.41 \mathrm{ft}$ below land-surface datum, April 2, 1965 ; 10west, $44.89 \mathrm{ft}$ below land-surface datum, December 13, 1981.

DEPTH BELOW LAND SURFACE (WATER LEVEL) (FEET) CALENDAR YEAR JANUARY 1987 TO DECEMBER 1987 MEAN VALUES

\begin{tabular}{|c|c|c|c|c|c|c|c|c|c|c|c|c|}
\hline DAY & JAN & FEB & MAR & APR & MAY & JUN & JUL & AUG & SEP & OCT & NOV & $\mathrm{DE}$ \\
\hline $\begin{array}{l}1 \\
2 \\
3 \\
4 \\
5\end{array}$ & $\begin{array}{l}39.83 \\
39.90 \\
39.80 \\
39.56 \\
39.55\end{array}$ & $\begin{array}{l}32.42 \\
32.25 \\
32.33 \\
32.44 \\
32.48\end{array}$ & $\begin{array}{l}29.85 \\
29.37 \\
29.15 \\
28.90 \\
28.65\end{array}$ & $\begin{array}{l}28.13 \\
27.85 \\
27.62 \\
27.56 \\
27.43\end{array}$ & $\begin{array}{l}30.02 \\
30.08 \\
30.22 \\
30.36 \\
30.51\end{array}$ & $\begin{array}{l}32.15 \\
32.25 \\
32.41 \\
32.55 \\
32.68\end{array}$ & $\begin{array}{l}30.67 \\
30.59 \\
30.86 \\
30.66 \\
30.60\end{array}$ & $\begin{array}{l}32.82 \\
32.90 \\
32.97 \\
33.05 \\
33.23\end{array}$ & $\begin{array}{l}34.59 \\
34.63 \\
34.67 \\
34.71 \\
34.72\end{array}$ & $\begin{array}{l}36.50 \\
36.57 \\
36.65 \\
36.65 \\
36.62\end{array}$ & $\begin{array}{l}38.03 \\
38.03 \\
38.03 \\
38.02 \\
38.12\end{array}$ & $\begin{array}{l}38.9 \\
39.0 \\
38.9 \\
39.0 \\
39.1\end{array}$ \\
\hline $\begin{array}{r}6 \\
7 \\
8 \\
9 \\
10\end{array}$ & $\begin{array}{l}39.35 \\
39.14 \\
39.05 \\
38.88 \\
38.77\end{array}$ & $\begin{array}{l}32.31 \\
32.13 \\
32.03 \\
32.16 \\
32.09\end{array}$ & $\begin{array}{l}28.40 \\
27.97 \\
27.72 \\
27.74 \\
28.00\end{array}$ & $\begin{array}{l}27.36 \\
27.41 \\
27.50 \\
27.57 \\
27.65\end{array}$ & $\begin{array}{l}30.63 \\
30.69 \\
30.77 \\
30.89 \\
30.95\end{array}$ & $\begin{array}{l}32.80 \\
32.90 \\
33.04 \\
33.13 \\
33.25\end{array}$ & $\begin{array}{l}30.54 \\
30.49 \\
30.64 \\
30.75 \\
30.91\end{array}$ & $\begin{array}{l}33.41 \\
33.45 \\
33.57 \\
33.67 \\
33.66\end{array}$ & $\begin{array}{l}34.79 \\
34.83 \\
34.96 \\
35.19 \\
35.22\end{array}$ & $\begin{array}{l}36.66 \\
36.82 \\
36.97 \\
37.04 \\
37.06\end{array}$ & $\begin{array}{l}38.28 \\
38.32 \\
38.32 \\
38.29 \\
38.32\end{array}$ & $\begin{array}{l}39.1 \\
39.1\end{array}$ \\
\hline $\begin{array}{l}11 \\
12 \\
13 \\
14 \\
15\end{array}$ & $\begin{array}{l}38.92 \\
38.87 \\
38.78 \\
38.77 \\
38.70\end{array}$ & $\begin{array}{l}31.89 \\
31.66 \\
31.60 \\
31.57 \\
31.64\end{array}$ & $\begin{array}{l}28.23 \\
28.31 \\
28.28 \\
28.30 \\
28.36\end{array}$ & $\begin{array}{l}27.79 \\
27.95 \\
28.06 \\
28.12 \\
28.05\end{array}$ & $\begin{array}{l}31.05 \\
31.02 \\
31.07 \\
31.10 \\
31.06\end{array}$ & $\begin{array}{l}33.44 \\
33.53 \\
33.53 \\
33.32 \\
32.90\end{array}$ & $\begin{array}{l}31.03 \\
31.07 \\
31.06 \\
30.96 \\
31.38\end{array}$ & $\begin{array}{l}33.75 \\
33.82 \\
33.68 \\
33.52 \\
33.40\end{array}$ & $\begin{array}{l}35.25 \\
35.26 \\
35.33 \\
35.35 \\
35.37\end{array}$ & $\begin{array}{l}37.03 \\
37.05 \\
37.18 \\
37.27 \\
37.29\end{array}$ & $\begin{array}{l}38.45 \\
38.51 \\
38.50 \\
38.53 \\
38.61\end{array}$ & $\begin{array}{l}39.2 \\
39.3\end{array}$ \\
\hline $\begin{array}{l}16 \\
17 \\
18 \\
19 \\
20\end{array}$ & $\begin{array}{l}38.70 \\
38.50 \\
38.13 \\
37.25 \\
36.05\end{array}$ & $\begin{array}{l}31.63 \\
31.79 \\
31.92 \\
32.03 \\
32.01\end{array}$ & $\begin{array}{l}28.43 \\
28.53 \\
28.47 \\
28.32 \\
28.36\end{array}$ & $\begin{array}{l}28.15 \\
28.28 \\
28.49 \\
28.67 \\
28.79\end{array}$ & $\begin{array}{l}31.10 \\
31.16 \\
31.18 \\
31.20 \\
31.30\end{array}$ & $\begin{array}{l}32.52 \\
32.17 \\
31.90 \\
31.65 \\
31.44\end{array}$ & $\begin{array}{l}31.63 \\
31.73 \\
31.98 \\
31.99 \\
31.90\end{array}$ & $\begin{array}{l}33.36 \\
33.32 \\
33.35 \\
33.39 \\
33.48\end{array}$ & $\begin{array}{l}35.41 \\
35.45 \\
35.56 \\
35.65 \\
35.63\end{array}$ & $\begin{array}{l}37.32 \\
37.36 \\
37.39 \\
37.43 \\
37.50\end{array}$ & $\begin{array}{l}38.56 \\
38.54 \\
38.58 \\
38.60 \\
38.62\end{array}$ & 39.6 \\
\hline $\begin{array}{l}21 \\
22 \\
23 \\
24 \\
25\end{array}$ & $\begin{array}{l}35.53 \\
35.13 \\
34.77 \\
34.40 \\
33.99\end{array}$ & $\begin{array}{l}31.85 \\
31.61 \\
31.61 \\
31.53 \\
31.37\end{array}$ & $\begin{array}{l}28.27 \\
28.26 \\
28.28 \\
28.27 \\
28.35\end{array}$ & $\begin{array}{l}28.85 \\
28.98 \\
29.07 \\
29.13 \\
29.32\end{array}$ & $\begin{array}{l}31.40 \\
31.47 \\
31.48 \\
31.50 \\
31.55\end{array}$ & $\begin{array}{l}31.30 \\
31.17 \\
31.00 \\
30.90 \\
30.77\end{array}$ & $\begin{array}{l}32.12 \\
32.53 \\
32.33 \\
32.63 \\
32.78\end{array}$ & $\begin{array}{l}33.60 \\
33.67 \\
33.67 \\
33.82 \\
33.96\end{array}$ & $\begin{array}{l}35.71 \\
35.79 \\
35.91 \\
36.00 \\
36.09\end{array}$ & $\begin{array}{l}37.58 \\
37.64 \\
37.67 \\
37.68 \\
37.67\end{array}$ & $\begin{array}{l}38.64 \\
38.72 \\
38.77 \\
38.76 \\
38.70\end{array}$ & $\begin{array}{l}39.6 \\
39.7 \\
39.7 \\
39.7 \\
39.7\end{array}$ \\
\hline $\begin{array}{l}26 \\
27 \\
28 \\
29 \\
30 \\
31\end{array}$ & $\begin{array}{l}33.59 \\
33.18 \\
32.84 \\
32.41 \\
32.42 \\
32.50\end{array}$ & $\begin{array}{r}31.24 \\
31.02 \\
30.62 \\
--- \\
-.- \\
---\end{array}$ & $\begin{array}{l}28.37 \\
28.37 \\
28.43 \\
28.55 \\
28.25 \\
28.25\end{array}$ & $\begin{array}{r}29.49 \\
29.55 \\
29.66 \\
29.83 \\
29.90 \\
.\end{array}$ & $\begin{array}{l}31.66 \\
31.78 \\
31.88 \\
31.95 \\
32.02 \\
32.10\end{array}$ & $\begin{array}{r}30.59 \\
30.85 \\
30.59 \\
30.55 \\
30.61 \\
.\end{array}$ & $\begin{array}{l}32.83 \\
32.58 \\
32.66 \\
32.84 \\
32.99 \\
32.86\end{array}$ & $\begin{array}{l}34.05 \\
34.16 \\
34.30 \\
34.48 \\
34.57 \\
34.55\end{array}$ & $\begin{array}{r}36.18 \\
36.18 \\
36.20 \\
36.23 \\
36.35 \\
.\end{array}$ & $\begin{array}{l}37.66 \\
37.73 \\
37.85 \\
37.95 \\
37.95 \\
38.03\end{array}$ & $\begin{array}{r}38.68 \\
38.68 \\
38.73 \\
38.78 \\
38.83 \\
.-\end{array}$ & $\begin{array}{l}39.7 \\
39.8\end{array}$ \\
\hline MEAN & 37.01 & 31.83 & 28.42 & 28.41 & 31.13 & 32.06 & 31.63 & 33.63 & 35.44 & 37.28 & 38.49 & $0 y^{\circ}$ \\
\hline
\end{tabular}



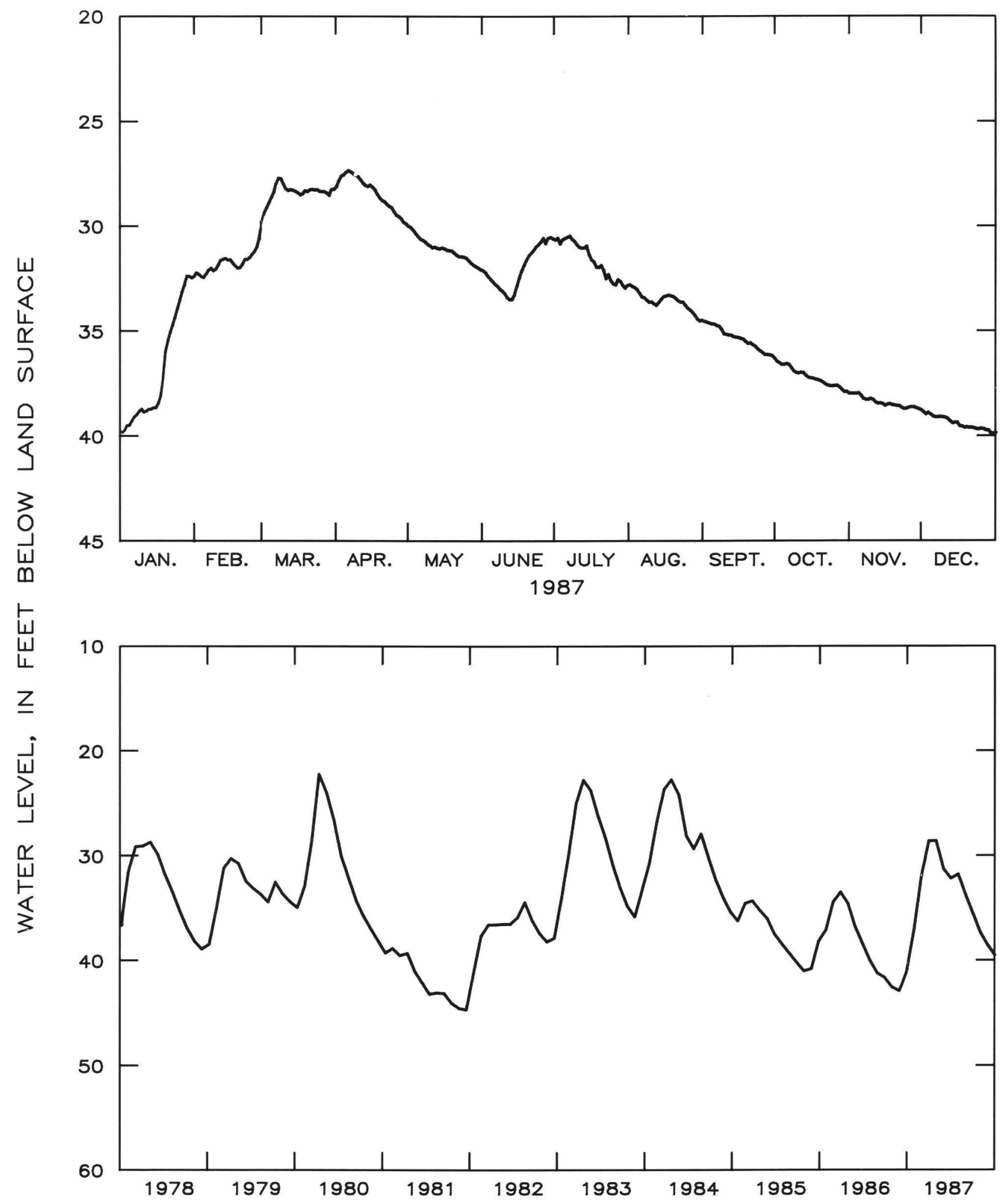

Figure 2.7.1-2.--Water level in observation well 13L0O3, Dougherty County. 
313105084064302 Local number, 13 L 012.

LOCATION.--Lat $31^{\circ} 31^{\prime} 05^{\prime \prime}$, long $84^{\circ} 06^{\prime} 43^{\prime \prime}$, Hydrologic Unit 03130008 , about $6.5 \mathrm{mi}$ southeast of Albany, off U.S. Highway 19 on School Bus Road.

Owner: U.S. Geological Survey, test well 3.

AQUIFER.--Upper Floridan aquifer.

WELL CHARACTERISTICS.--Drilled observation well, depth $218 \mathrm{ft}$, cased to $54 \mathrm{ft}$.

DATUM.--Elevation of land-surface datum is $195 \mathrm{ft}$.

Measuring point: Floor of recorder shelter, $3.0 \mathrm{ft}$ above land-surface datum.

REMARKS.--Water levels for period of missing record, March 25 to April 27, and October 29, were estimated. PERIOD OF RECORD.--June 1977 to current year.

EXTREMES FOR PERIOD OF RECORD.--Highest water level, $21.92 \mathrm{ft}$ below land-surface datum, March 2, 1979 ; 10 west, $48.18 \mathrm{ft}$ below land-surface datum, July 1, 1981 .

DEPTH BELOW LAND SURFACE (WATER LEVEL) (FEET) CALENDAR YEAR JANUARY 1987 TO DECEMBER 1987 MEAN VALUES

\begin{tabular}{|c|c|c|c|c|c|c|c|c|c|c|c|c|}
\hline DAY & JAN & FEB & MAR & APR & MAY & JUN & JUL & AUG & SEP & ОСТ & NOV & $\mathrm{DEC}$ \\
\hline $\begin{array}{l}1 \\
2 \\
3 \\
4 \\
5\end{array}$ & $\begin{array}{l}42.01 \\
41.88 \\
41.93 \\
41.69 \\
41.06\end{array}$ & $\begin{array}{l}33.56 \\
34.19 \\
35.06 \\
35.73 \\
36.53\end{array}$ & $\begin{array}{l}28.80 \\
29.71 \\
29.47 \\
29.01 \\
28.66\end{array}$ & $\begin{array}{l}35.30 \\
35.30 \\
34.93 \\
34.67 \\
34.59\end{array}$ & $\begin{array}{l}41.39 \\
41.24 \\
41.35 \\
41.55 \\
41.60\end{array}$ & $\begin{array}{l}42.18 \\
42.66 \\
42.83 \\
43.12 \\
43.31\end{array}$ & $\begin{array}{l}40.02 \\
40.00 \\
39.92 \\
40.20 \\
39.78\end{array}$ & $\begin{array}{l}42.71 \\
42.86 \\
42.95 \\
43.24 \\
43.41\end{array}$ & $\begin{array}{l}45.15 \\
45.08 \\
45.07 \\
45.00 \\
44.94\end{array}$ & $\begin{array}{l}46.67 \\
46.56 \\
46.42 \\
46.27 \\
46.18\end{array}$ & $\begin{array}{l}45.94 \\
45.78 \\
45.76 \\
45.68 \\
45.58\end{array}$ & $\begin{array}{l}44.78 \\
44.67 \\
44.57 \\
44.70 \\
44.78\end{array}$ \\
\hline $\begin{array}{r}6 \\
7 \\
8 \\
9 \\
10\end{array}$ & $\begin{array}{l}41.04 \\
41.08 \\
41.12 \\
41.25 \\
41.29\end{array}$ & $\begin{array}{l}36.87 \\
36.31 \\
36.00 \\
35.51 \\
35.46\end{array}$ & $\begin{array}{l}28.25 \\
28.05 \\
28.70 \\
29.90 \\
31.59\end{array}$ & $\begin{array}{l}34.65 \\
34.63 \\
34.91 \\
35.18 \\
35.56\end{array}$ & $\begin{array}{l}41.76 \\
41.76 \\
41.67 \\
41.35 \\
41.57\end{array}$ & $\begin{array}{l}43.29 \\
43.29 \\
43.43 \\
43.63 \\
43.77\end{array}$ & $\begin{array}{l}39.99 \\
39.95 \\
39.81 \\
40.18 \\
40.30\end{array}$ & $\begin{array}{l}43.46 \\
43.61 \\
43.77 \\
43.73 \\
43.81\end{array}$ & $\begin{array}{l}44.89 \\
44.93 \\
45.16 \\
45.37 \\
45.47\end{array}$ & $\begin{array}{l}46.26 \\
45.98 \\
46.18 \\
46.33 \\
46.28\end{array}$ & $\begin{array}{l}45.79 \\
45.89 \\
45.86 \\
45.79 \\
45.80\end{array}$ & $\begin{array}{l}44.88 \\
44.78 \\
44.65 \\
44.74 \\
44.81\end{array}$ \\
\hline $\begin{array}{l}11 \\
12 \\
13 \\
14 \\
15\end{array}$ & $\begin{array}{l}41.46 \\
41.53 \\
41.44 \\
41.71 \\
41.80\end{array}$ & $\begin{array}{l}35.45 \\
34.98 \\
35.37 \\
36.29 \\
37.62\end{array}$ & $\begin{array}{l}33.38 \\
33.98 \\
34.87 \\
35.30 \\
35.87\end{array}$ & $\begin{array}{l}36.02 \\
36.26 \\
37.33 \\
38.14 \\
37.49\end{array}$ & $\begin{array}{l}41.50 \\
41.03 \\
41.03 \\
40.81 \\
40.65\end{array}$ & $\begin{array}{l}43.92 \\
43.99 \\
43.86 \\
42.59 \\
41.10\end{array}$ & $\begin{array}{l}40.54 \\
40.75 \\
41.27 \\
41.84 \\
42.35\end{array}$ & $\begin{array}{l}43.97 \\
43.84 \\
43.25 \\
43.06 \\
43.07\end{array}$ & $\begin{array}{l}45.60 \\
45.33 \\
45.21 \\
44.98 \\
44.95\end{array}$ & $\begin{array}{l}46.11 \\
45.99 \\
46.02 \\
45.91 \\
46.11\end{array}$ & $\begin{array}{l}45.88 \\
45.88 \\
45.82 \\
45.80 \\
45.75\end{array}$ & $\begin{array}{l}44.96 \\
45.00 \\
45.03 \\
44.99 \\
44.99\end{array}$ \\
\hline $\begin{array}{l}16 \\
17 \\
18 \\
19 \\
20\end{array}$ & $\begin{array}{l}41.65 \\
40.76 \\
39.85 \\
33.77 \\
30.62\end{array}$ & $\begin{array}{l}37.55 \\
37.50 \\
38.09 \\
38.54 \\
38.99\end{array}$ & $\begin{array}{l}36.81 \\
37.12 \\
36.94 \\
36.00 \\
36.16\end{array}$ & $\begin{array}{l}37.77 \\
38.29 \\
38.48 \\
38.17 \\
39.13\end{array}$ & $\begin{array}{l}40.71 \\
40.75 \\
40.74 \\
40.76 \\
40.87\end{array}$ & $\begin{array}{l}40.63 \\
40.22 \\
40.05 \\
40.13 \\
40.17\end{array}$ & $\begin{array}{l}43.02 \\
43.06 \\
42.84 \\
42.96 \\
42.89\end{array}$ & $\begin{array}{l}43.33 \\
43.22 \\
43.65 \\
43.85 \\
44.01\end{array}$ & $\begin{array}{l}44.90 \\
44.99 \\
45.18 \\
45.07 \\
44.16\end{array}$ & $\begin{array}{l}46.27 \\
46.26 \\
45.97 \\
45.88 \\
45.92\end{array}$ & $\begin{array}{l}45.63 \\
45.36 \\
45.10 \\
45.34 \\
45.20\end{array}$ & $\begin{array}{l}45.03 \\
45.03 \\
45.12 \\
45.21 \\
45.09\end{array}$ \\
\hline $\begin{array}{l}21 \\
22 \\
23 \\
24 \\
25\end{array}$ & $\begin{array}{l}31.83 \\
28.26 \\
27.96 \\
29.22 \\
29.22\end{array}$ & $\begin{array}{l}38.34 \\
38.16 \\
37.39 \\
37.12 \\
36.72\end{array}$ & $\begin{array}{l}36.23 \\
36.01 \\
36.32 \\
36.46 \\
36.53\end{array}$ & $\begin{array}{l}40.27 \\
39.81 \\
40.15 \\
40.42 \\
40.52\end{array}$ & $\begin{array}{l}41.10 \\
40.96 \\
40.84 \\
40.78 \\
40.93\end{array}$ & $\begin{array}{l}40.45 \\
40.20 \\
39.71 \\
39.41 \\
39.60\end{array}$ & $\begin{array}{l}43.05 \\
42.69 \\
42.10 \\
42.19 \\
42.51\end{array}$ & $\begin{array}{l}44.09 \\
44.04 \\
43.98 \\
44.45 \\
44.89\end{array}$ & $\begin{array}{l}44.17 \\
44.46 \\
45.20 \\
45.80 \\
45.96\end{array}$ & $\begin{array}{l}45.89 \\
45.86 \\
45.90 \\
45.91 \\
45.90\end{array}$ & $\begin{array}{l}44.91 \\
45.29 \\
45.28 \\
44.99 \\
44.67\end{array}$ & $\begin{array}{l}45.02 \\
44.93 \\
44.83 \\
44.84 \\
44.76\end{array}$ \\
\hline $\begin{array}{l}26 \\
27 \\
28 \\
29 \\
30 \\
31\end{array}$ & $\begin{array}{l}30.11 \\
30.48 \\
30.63 \\
31.08 \\
32.10 \\
32.64\end{array}$ & $\begin{array}{r}36.68 \\
36.55 \\
35.64 \\
--- \\
--- \\
---\end{array}$ & $\begin{array}{l}36.58 \\
36.58 \\
36.68 \\
36.71 \\
36.10 \\
35.47\end{array}$ & $\begin{array}{r}40.63 \\
40.76 \\
41.10 \\
40.95 \\
41.22 \\
. .-\end{array}$ & $\begin{array}{l}41.38 \\
41.62 \\
41.78 \\
41.91 \\
41.86 \\
41.95\end{array}$ & $\begin{array}{r}39.78 \\
39.19 \\
39.01 \\
39.30 \\
39.46 \\
-.-\end{array}$ & $\begin{array}{l}42.52 \\
42.86 \\
43.26 \\
43.11 \\
42.81 \\
42.33\end{array}$ & $\begin{array}{l}44.93 \\
45.17 \\
45.16 \\
45.09 \\
45.15 \\
45.39\end{array}$ & $\begin{array}{r}45.96 \\
45.96 \\
45.96 \\
46.28 \\
46.57 \\
---\end{array}$ & $\begin{array}{l}45.78 \\
45.69 \\
45.83 \\
45.87 \\
45.91 \\
45.92\end{array}$ & $\begin{array}{r}44.69 \\
44.66 \\
44.71 \\
44.82 \\
44.77 \\
---\end{array}$ & $\begin{array}{l}44.71 \\
44.74 \\
44.78 \\
44.84 \\
44.79 \\
44.80\end{array}$ \\
\hline MEAN & 36.85 & 36.51 & 33.81 & 37.75 & 41.26 & 41.48 & 41.65 & 43.91 & 45.26 & 46.07 & 45.41 & 44.8 \\
\hline
\end{tabular}

$\begin{array}{lllllll}\text { CAL YR } 1987 & \text { MEAN } & 41.26 & \text { HIGH } & 27.96 & \text { LOW } & 46.67\end{array}$ 


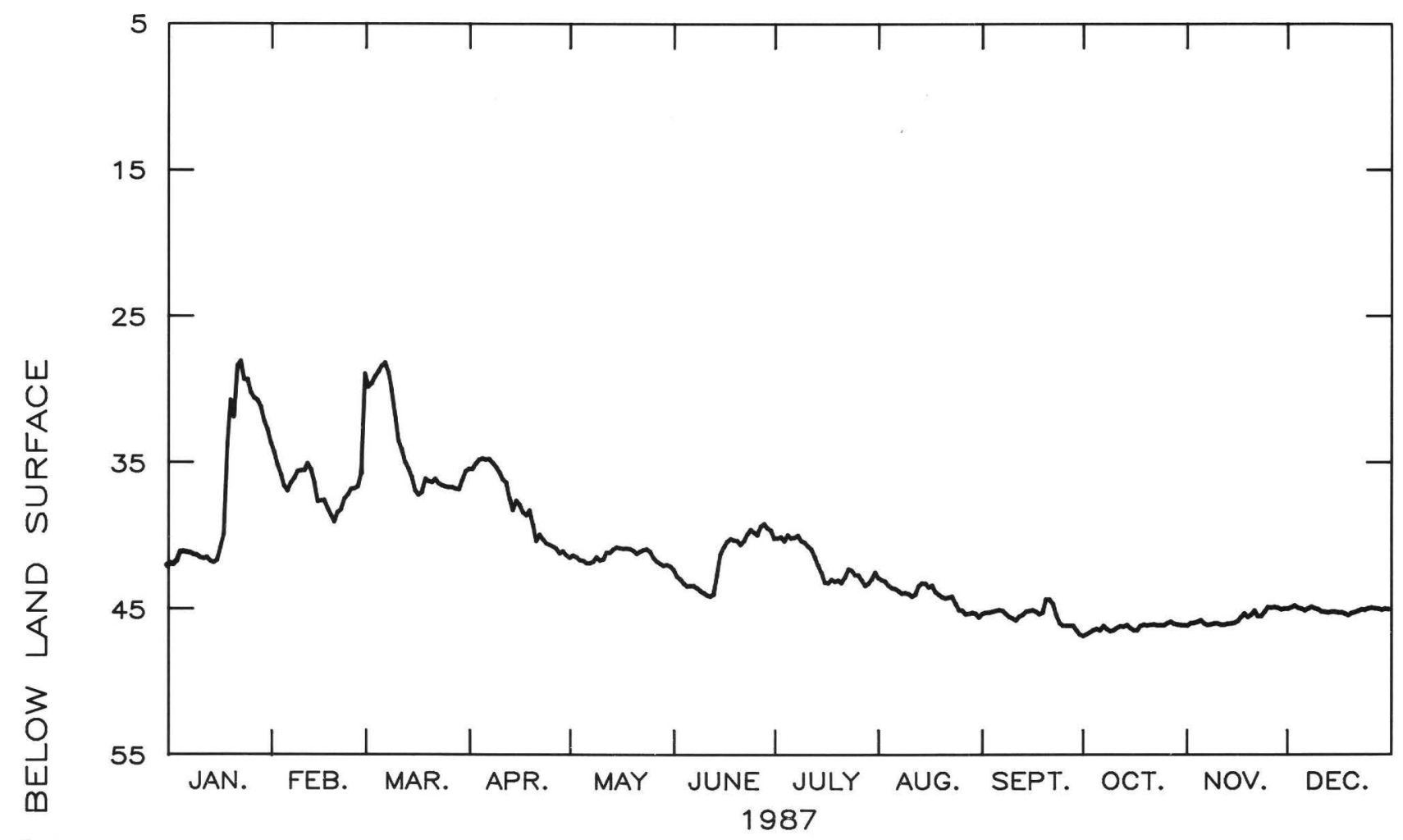

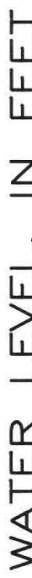

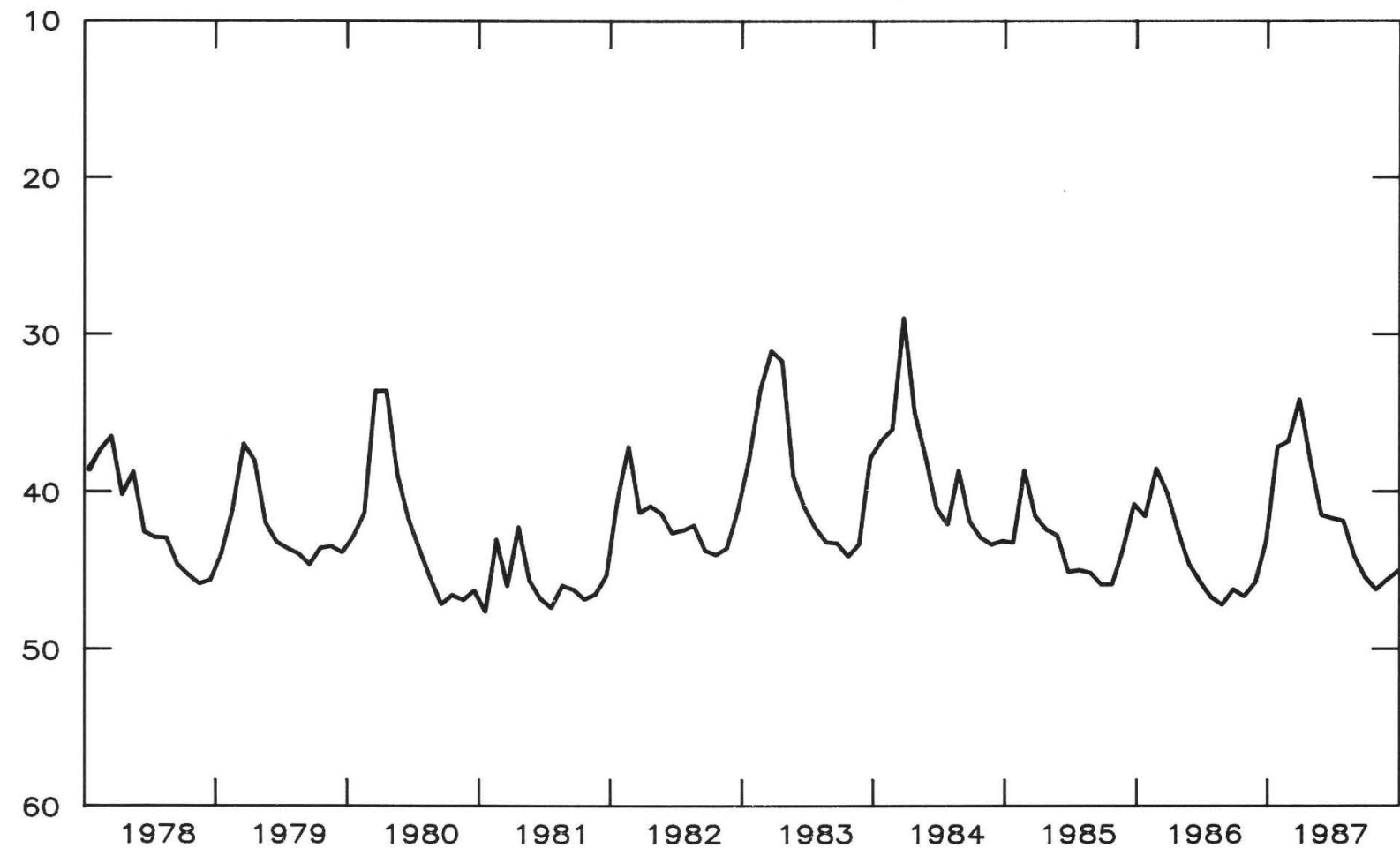

Figure 2.7.1-3.--Water level in observation well 13L012, Dougherty County. 
312127084065801 Local number, 13J004.

LOCATION.--Lat $31^{\circ} 21^{\prime} 27^{\prime \prime}$, long $84^{\circ} 06^{\prime} 58^{\prime \prime}$, Hydrologic Unit $03130008,2.7$ mi north of intersection of U.S. Highway 19 and Georgia Highway $112,0.7$ mi west of Stagecoach Road.

Owner: Henry Wright.

AQUIFER.--Upper Floridan aquifer.

WELL CHARACTERISTICS.--Drilled observation well, diameter $12 \mathrm{in.,}$ depth $208 \mathrm{ft}$, cased to $77 \mathrm{ft}$, open hole. DATUM.--Elevation of land-surface datum is $200 \mathrm{ft}$.

Measuring point: Top front edge of recorder shelter, $3.60 \mathrm{ft}$ above land-surface datum.

REMARKS.--Water level for period of missing record, October 26, was estimated.

PERIOD OF RECORD.--June 1978 to current year.

EXTREMES FOR PERIOD OF RECORD.--Highest water level, $36.90 \mathrm{ft}$ below land-surface datum, April 13, 1980; 1owest, $54.00 \mathrm{ft}$ below land-surface datum, September $25,1981$.

DEPTH BELOW LAND SURFACE (WATER LEVEL) (FEET) CALENDAR YEAR JANUARY 1987 TO DECEMBER 1987 MEAN VALUES

\begin{tabular}{|c|c|c|c|c|c|c|c|c|c|c|c|c|}
\hline DAY & JAN & FEB & MAR & APR & MAY & JUN & JUL & AUG & SEP & ОСТ & NOV & DEC \\
\hline $\begin{array}{l}1 \\
2 \\
3 \\
4 \\
5\end{array}$ & $\begin{array}{l}48.80 \\
48.89 \\
49.03 \\
48.88 \\
48.70\end{array}$ & $\begin{array}{l}42.52 \\
42.32 \\
42.30 \\
42.13 \\
41.99\end{array}$ & $\begin{array}{l}39.80 \\
39.60 \\
39.51 \\
39.47 \\
39.41\end{array}$ & $\begin{array}{l}38.59 \\
38.38 \\
38.22 \\
38.35 \\
38.22\end{array}$ & $\begin{array}{l}41.55 \\
41.63 \\
41.60 \\
41.70 \\
42.25\end{array}$ & $\begin{array}{l}43.87 \\
43.68 \\
43.93 \\
44.20 \\
43.82\end{array}$ & $\begin{array}{l}43.32 \\
43.30 \\
43.35 \\
43.39 \\
43.42\end{array}$ & $\begin{array}{l}44.32 \\
44.20 \\
44.30 \\
44.36 \\
44.40\end{array}$ & $\begin{array}{l}45.70 \\
45.78 \\
45.60 \\
45.51 \\
45.38\end{array}$ & $\begin{array}{l}46.70 \\
47.41 \\
46.88 \\
46.73 \\
46.56\end{array}$ & $\begin{array}{l}47.54 \\
47.48 \\
47.41 \\
47.29 \\
47.35\end{array}$ & $\begin{array}{l}47.91 \\
48.12 \\
48.09 \\
47.97 \\
48.15\end{array}$ \\
\hline $\begin{array}{r}6 \\
7 \\
8 \\
9 \\
10\end{array}$ & $\begin{array}{l}48.85 \\
48.80 \\
48.64 \\
48.55 \\
48.35\end{array}$ & $\begin{array}{l}41.80 \\
41.71 \\
41.62 \\
41.81 \\
41.97\end{array}$ & $\begin{array}{l}39.30 \\
39.00 \\
38.71 \\
38.69 \\
38.90\end{array}$ & $\begin{array}{l}38.20 \\
38.25 \\
38.35 \\
38.40 \\
38.53\end{array}$ & $\begin{array}{l}42.28 \\
42.40 \\
42.35 \\
42.65 \\
42.80\end{array}$ & $\begin{array}{l}44.20 \\
44.53 \\
44.13 \\
44.14 \\
44.63\end{array}$ & $\begin{array}{l}43.47 \\
43.45 \\
43.55 \\
43.77 \\
43.95\end{array}$ & $\begin{array}{l}44.60 \\
44.74 \\
44.82 \\
44.82 \\
45.10\end{array}$ & $\begin{array}{l}45.39 \\
45.51 \\
46.38 \\
46.51 \\
45.92\end{array}$ & $\begin{array}{l}46.53 \\
46.65 \\
46.87 \\
46.95 \\
46.93\end{array}$ & $\begin{array}{l}47.65 \\
47.68 \\
47.61 \\
47.51 \\
47.41\end{array}$ & $\begin{array}{l}48.19 \\
48.15 \\
48.30 \\
48.56 \\
48.10\end{array}$ \\
\hline $\begin{array}{l}11 \\
12 \\
13 \\
14 \\
15\end{array}$ & $\begin{array}{l}48.25 \\
48.43 \\
48.42 \\
48.30 \\
48.23\end{array}$ & $\begin{array}{l}41.96 \\
41.89 \\
41.95 \\
41.95 \\
41.97\end{array}$ & $\begin{array}{l}39.12 \\
39.17 \\
39.40 \\
39.46 \\
39.30\end{array}$ & $\begin{array}{l}38.70 \\
38.90 \\
39.05 \\
39.15 \\
39.05\end{array}$ & $\begin{array}{l}42.70 \\
42.60 \\
42.60 \\
42.49 \\
42.55\end{array}$ & $\begin{array}{l}45.30 \\
45.23 \\
45.13 \\
44.53 \\
44.34\end{array}$ & $\begin{array}{l}44.15 \\
44.30 \\
44.85 \\
45.22 \\
45.65\end{array}$ & $\begin{array}{l}45.22 \\
44.97 \\
44.89 \\
44.39 \\
43.90\end{array}$ & $\begin{array}{l}45.88 \\
45.78 \\
45.71 \\
45.72 \\
45.70\end{array}$ & $\begin{array}{l}46.79 \\
46.69 \\
46.91 \\
47.06 \\
47.08\end{array}$ & $\begin{array}{l}47.67 \\
47.76 \\
47.72 \\
47.72 \\
47.81\end{array}$ & $\begin{array}{l}48.50 \\
48.37 \\
48.39 \\
48.33 \\
48.20\end{array}$ \\
\hline $\begin{array}{l}16 \\
17 \\
18 \\
19 \\
20\end{array}$ & $\begin{array}{l}48.10 \\
48.10 \\
48.10 \\
47.75 \\
47.20\end{array}$ & $\begin{array}{l}41.88 \\
41.84 \\
41.63 \\
41.65 \\
41.67\end{array}$ & $\begin{array}{l}39.38 \\
39.48 \\
39.50 \\
39.30 \\
39.05\end{array}$ & $\begin{array}{l}39.11 \\
39.25 \\
39.52 \\
39.75 \\
40.20\end{array}$ & $\begin{array}{l}42.60 \\
42.37 \\
42.33 \\
42.65 \\
42.42\end{array}$ & $\begin{array}{l}44.26 \\
44.28 \\
44.30 \\
44.21 \\
44.12\end{array}$ & $\begin{array}{l}45.50 \\
44.98 \\
44.90 \\
44.84 \\
45.50\end{array}$ & $\begin{array}{l}43.65 \\
43.80 \\
43.93 \\
44.14 \\
44.25\end{array}$ & $\begin{array}{l}45.71 \\
45.78 \\
46.04 \\
46.43 \\
46.00\end{array}$ & $\begin{array}{l}47.12 \\
47.03 \\
47.00 \\
47.02 \\
47.11\end{array}$ & $\begin{array}{l}47.75 \\
47.70 \\
47.85 \\
47.79 \\
47.77\end{array}$ & $\begin{array}{l}48.45 \\
48.50 \\
48.48 \\
48.39 \\
48.35\end{array}$ \\
\hline $\begin{array}{l}21 \\
22 \\
23 \\
24 \\
25\end{array}$ & $\begin{array}{l}46.50 \\
45.60 \\
44.85 \\
44.30 \\
43.95\end{array}$ & $\begin{array}{l}41.60 \\
41.31 \\
41.13 \\
40.84 \\
40.72\end{array}$ & $\begin{array}{l}38.90 \\
38.89 \\
38.90 \\
38.99 \\
39.12\end{array}$ & $\begin{array}{l}40.46 \\
40.60 \\
40.70 \\
40.77 \\
40.95\end{array}$ & $\begin{array}{l}42.58 \\
42.75 \\
42.60 \\
42.56 \\
42.60\end{array}$ & $\begin{array}{l}44.10 \\
44.06 \\
43.97 \\
43.72 \\
43.43\end{array}$ & $\begin{array}{l}46.10 \\
46.41 \\
46.46 \\
46.33 \\
46.15\end{array}$ & $\begin{array}{l}44.47 \\
44.51 \\
44.99 \\
45.65 \\
45.70\end{array}$ & $\begin{array}{l}46.02 \\
45.98 \\
45.98 \\
45.98 \\
46.35\end{array}$ & $\begin{array}{l}47.29 \\
47.40 \\
47.40 \\
47.36 \\
47.26\end{array}$ & $\begin{array}{l}47.88 \\
47.88 \\
47.90 \\
47.90 \\
47.79\end{array}$ & $\begin{array}{l}48.36 \\
48.37 \\
48.81 \\
48.58 \\
48.48\end{array}$ \\
\hline $\begin{array}{l}26 \\
27 \\
28 \\
29 \\
30 \\
31\end{array}$ & $\begin{array}{l}43.00 \\
42.25 \\
42.28 \\
42.29 \\
42.35 \\
42.51\end{array}$ & $\begin{array}{r}40.62 \\
40.48 \\
40.13 \\
--- \\
--- \\
---\end{array}$ & $\begin{array}{l}38.94 \\
38.80 \\
38.81 \\
38.80 \\
38.57 \\
38.60\end{array}$ & $\begin{array}{r}40.93 \\
40.36 \\
41.60 \\
42.15 \\
41.66 \\
-.-\end{array}$ & $\begin{array}{l}42.80 \\
43.00 \\
43.04 \\
43.18 \\
43.19 \\
43.56\end{array}$ & $\begin{array}{r}43.33 \\
43.33 \\
43.38 \\
43.40 \\
43.35 \\
---\end{array}$ & $\begin{array}{l}46.50 \\
46.75 \\
46.62 \\
45.60 \\
45.40 \\
44.90\end{array}$ & $\begin{array}{l}45.48 \\
45.55 \\
45.52 \\
45.92 \\
45.62 \\
45.66\end{array}$ & $\begin{array}{r}46.97 \\
46.48 \\
46.79 \\
46.38 \\
46.18 \\
---\end{array}$ & $\begin{array}{l}47.21 \\
47.16 \\
47.37 \\
47.49 \\
47.55 \\
47.60\end{array}$ & $\begin{array}{r}47.75 \\
47.75 \\
47.72 \\
47.76 \\
47.75 \\
. .-\end{array}$ & $\begin{array}{l}48.47 \\
48.49 \\
48.49 \\
48.67 \\
48.79 \\
48.67\end{array}$ \\
\hline MEAN & 46.72 & 41.62 & 39.12 & 39.55 & 42.53 & 44.10 & 44.91 & 44.77 & 45.98 & 47.07 & 47.69 & 48.38 \\
\hline
\end{tabular}




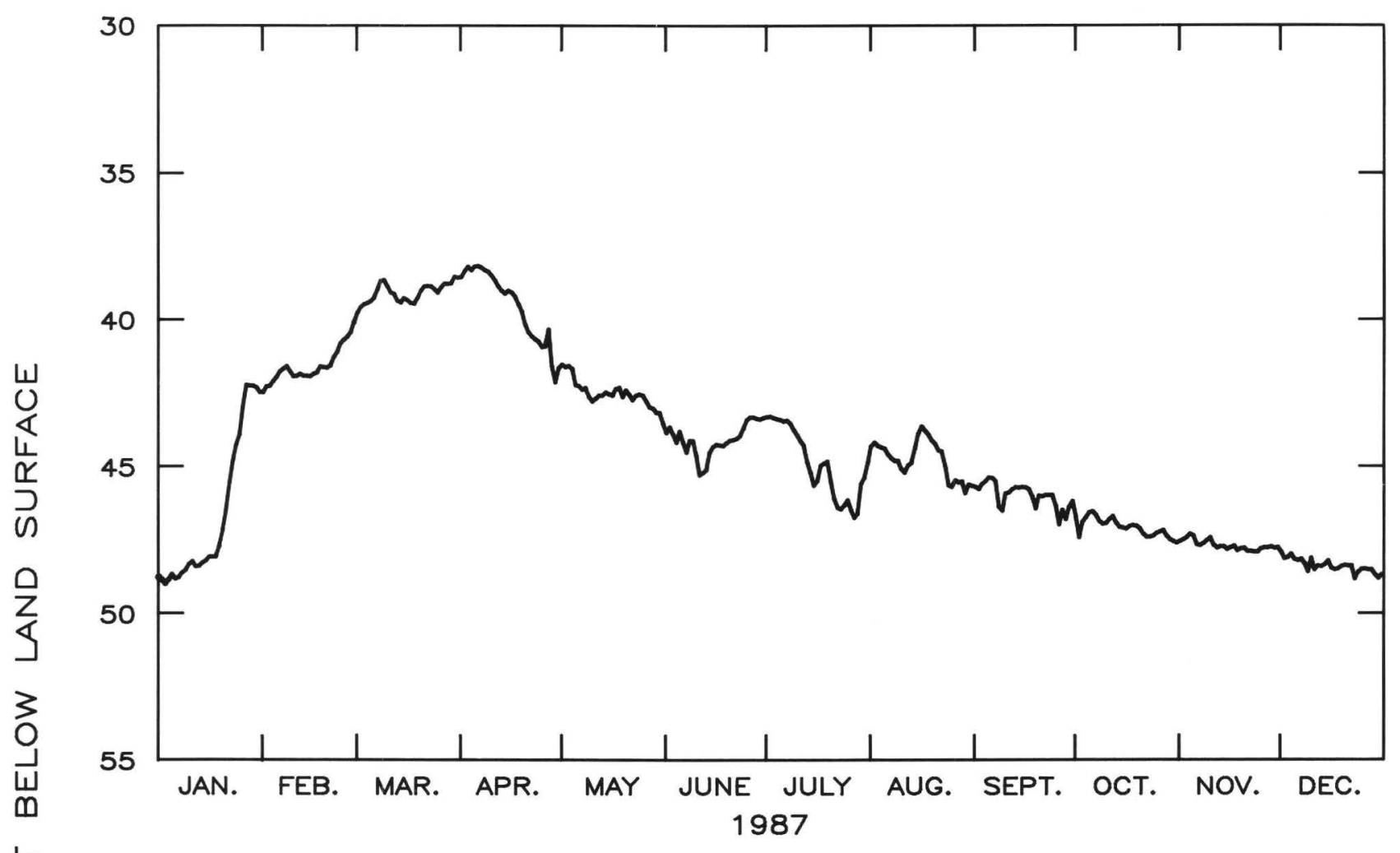

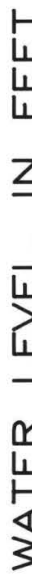

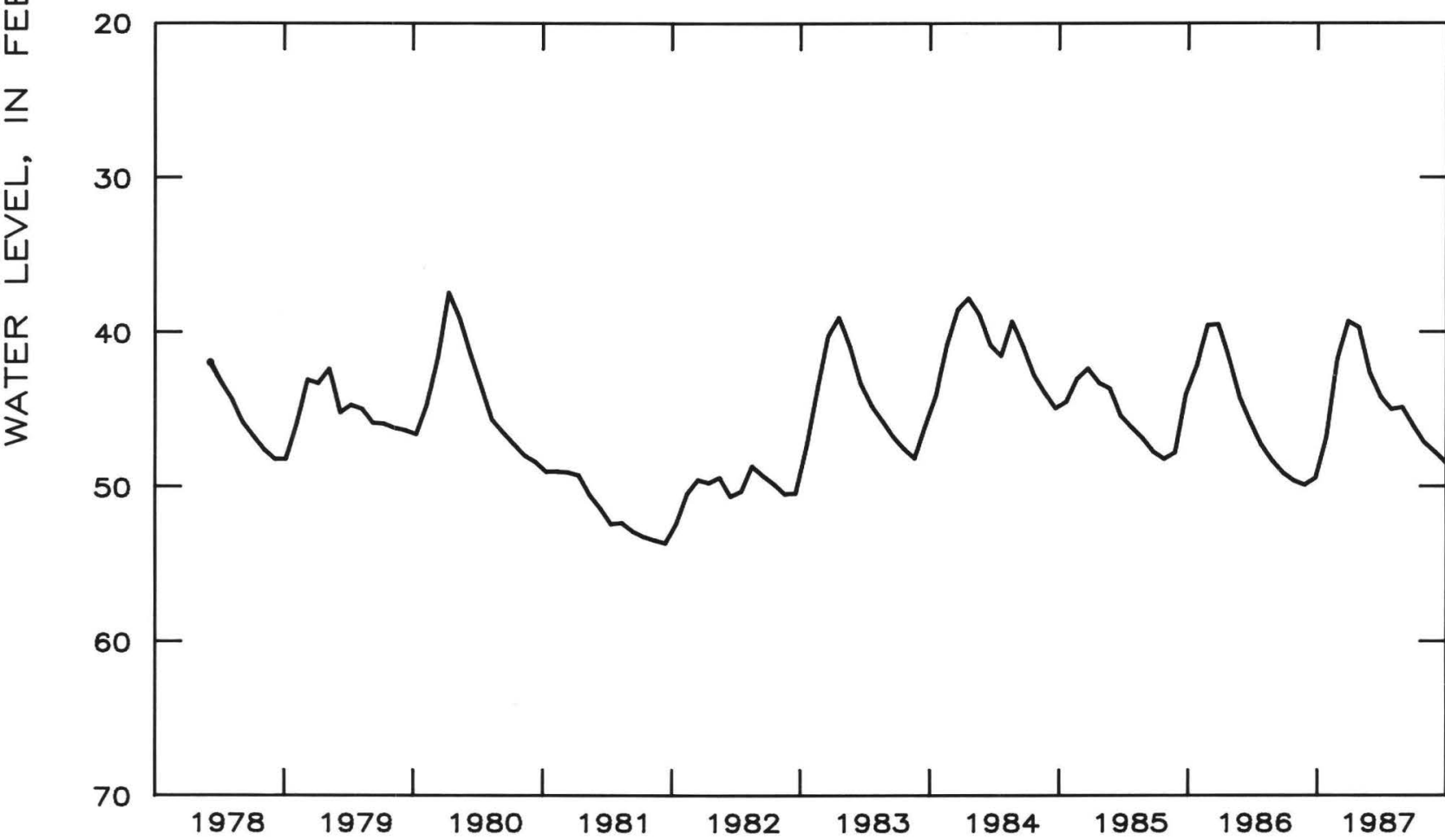

Figure 2.7.1-4.--Water level in observation well 13J004, Mitchell County. 
310507084262201 Local number, 10G313.

LOCATION.--Lat $31^{\circ} 05^{\prime} 07^{\prime \prime}$, long $84^{\circ} 26^{\prime} 22^{\prime \prime}$, Hydrologic Unit $03130008,1.95 \mathrm{mi}$ west of Vada off of Decatur-Mitchell County line road, on right.

Owner: Harvey Meinders.

AQUIFER.---Upper Floridan aquifer.

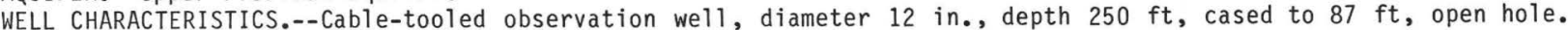
DATUM.--Elevation of land-surface datum is $145 \mathrm{ft}$.

Measuring point: Floor of recorder shelter, $1.35 \mathrm{ft}$ above land-surface datum. REMARKS.--None.

PERIOD OF RECORD.--November 1961 to September 1968; April 1976 to current year.

EXTREMES FOR PERIOD OF RECORD.---Highest water level, $32.98 \mathrm{ft}$ below land-surface datum, April 9, 1984; 10west, $60.26 \mathrm{ft}$ below land-surface datum, January 1, 1982.

DEPTH BELOW LAND SURFACE (WATER LEVEL) (FEET) CALENDAR YEAR JANUARY 1987 TO DECEMBER 1987 MEAN VALUES

\begin{tabular}{|c|c|c|c|c|c|c|c|c|c|c|c|c|}
\hline DAY & JAN & FEB & MAR & APR & MAY & JUN & JUL & AUG & SEP & OCT & NOV & $\mathrm{DEC}$ \\
\hline $\begin{array}{l}1 \\
2 \\
3 \\
4 \\
5\end{array}$ & $\begin{array}{l}55.22 \\
55.18 \\
55.12 \\
55.11 \\
55.11\end{array}$ & $\begin{array}{l}50.52 \\
50.39 \\
50.38 \\
50.24 \\
50.04\end{array}$ & $\begin{array}{l}47.12 \\
46.93 \\
46.63 \\
46.33 \\
46.03\end{array}$ & $\begin{array}{l}40.66 \\
40.18 \\
39.85 \\
39.78 \\
39.55\end{array}$ & $\begin{array}{l}40.55 \\
40.65 \\
40.78 \\
40.90 \\
41.02\end{array}$ & $\begin{array}{l}43.73 \\
43.86 \\
43.96 \\
44.06 \\
44.13\end{array}$ & $\begin{array}{l}45.08 \\
45.06 \\
45.08 \\
45.08 \\
45.11\end{array}$ & $\begin{array}{l}46.37 \\
46.41 \\
46.46 \\
46.51 \\
46.56\end{array}$ & $\begin{array}{l}48.35 \\
48.40 \\
48.46 \\
48.52 \\
48.56\end{array}$ & $\begin{array}{l}50.12 \\
50.19 \\
50.27 \\
50.32 \\
50.36\end{array}$ & $\begin{array}{l}51.96 \\
52.00 \\
52.03 \\
52.07 \\
52.14\end{array}$ & $\begin{array}{l}53.39 \\
53.45 \\
53.46 \\
53.53 \\
53.58\end{array}$ \\
\hline $\begin{array}{r}6 \\
7 \\
8 \\
9 \\
10\end{array}$ & $\begin{array}{l}55.16 \\
55.07 \\
54.94 \\
54.94 \\
54.94\end{array}$ & $\begin{array}{l}49.55 \\
49.22 \\
49.30 \\
49.18 \\
49.12\end{array}$ & $\begin{array}{l}45.72 \\
45.29 \\
45.01 \\
44.81 \\
44.71\end{array}$ & $\begin{array}{l}39.41 \\
39.31 \\
39.27 \\
39.19 \\
39.14\end{array}$ & $\begin{array}{l}41.11 \\
41.20 \\
41.28 \\
41.45 \\
41.55\end{array}$ & $\begin{array}{l}44.23 \\
44.32 \\
44.41 \\
44.48 \\
44.61\end{array}$ & $\begin{array}{l}45.13 \\
45.12 \\
45.14 \\
45.17 \\
45.19\end{array}$ & $\begin{array}{l}46.64 \\
46.72 \\
46.80 \\
46.87 \\
46.94\end{array}$ & $\begin{array}{l}48.62 \\
48.67 \\
48.74 \\
48.81 \\
48.87\end{array}$ & $\begin{array}{l}50.41 \\
50.50 \\
50.58 \\
50.64 \\
50.68\end{array}$ & $\begin{array}{l}52.23 \\
52.27 \\
52.31 \\
52.33 \\
52.38\end{array}$ & $\begin{array}{l}53.63 \\
53.66 \\
53.69 \\
53.73 \\
53.76\end{array}$ \\
\hline $\begin{array}{l}11 \\
12 \\
13 \\
14 \\
15\end{array}$ & $\begin{array}{l}54.94 \\
54.96 \\
54.92 \\
54.87 \\
54.87\end{array}$ & $\begin{array}{l}48.98 \\
48.92 \\
48.92 \\
48.91 \\
48.86\end{array}$ & $\begin{array}{l}44.58 \\
44.36 \\
44.12 \\
43.91 \\
43.74\end{array}$ & $\begin{array}{l}39.14 \\
39.15 \\
39.15 \\
39.12 \\
39.03\end{array}$ & $\begin{array}{l}41.64 \\
41.71 \\
41.83 \\
41.93 \\
42.00\end{array}$ & $\begin{array}{l}44.72 \\
44.82 \\
44.89 \\
44.95 \\
45.03\end{array}$ & $\begin{array}{l}45.22 \\
45.26 \\
45.30 \\
45.36 \\
45.42\end{array}$ & $\begin{array}{l}47.02 \\
47.09 \\
47.15 \\
47.21 \\
47.26\end{array}$ & $\begin{array}{l}48.94 \\
48.99 \\
49.05 \\
49.12 \\
49.17\end{array}$ & $\begin{array}{l}50.72 \\
50.76 \\
50.86 \\
50.92 \\
50.98\end{array}$ & $\begin{array}{l}52.47 \\
52.52 \\
52.56 \\
52.62 \\
52.67\end{array}$ & $\begin{array}{l}53.79 \\
53.85 \\
53.91 \\
53.93 \\
53.97\end{array}$ \\
\hline $\begin{array}{l}16 \\
17 \\
18 \\
19 \\
20\end{array}$ & $\begin{array}{l}54.88 \\
54.87 \\
54.74 \\
54.61 \\
54.61\end{array}$ & $\begin{array}{l}48.85 \\
48.80 \\
48.75 \\
48.75 \\
48.74\end{array}$ & $\begin{array}{l}43.57 \\
43.42 \\
43.23 \\
43.04 \\
42.86\end{array}$ & $\begin{array}{l}39.01 \\
39.09 \\
39.24 \\
39.40 \\
39.45\end{array}$ & $\begin{array}{l}42.10 \\
42.21 \\
42.28 \\
42.36 \\
42.46\end{array}$ & $\begin{array}{l}45.09 \\
45.16 \\
45.23 \\
45.23 \\
45.19\end{array}$ & $\begin{array}{l}45.53 \\
45.59 \\
45.62 \\
45.67 \\
45.74\end{array}$ & $\begin{array}{l}47.32 \\
47.38 \\
47.43 \\
47.48 \\
47.55\end{array}$ & $\begin{array}{l}49.22 \\
49.28 \\
49.33 \\
49.38 \\
49.46\end{array}$ & $\begin{array}{l}51.03 \\
51.08 \\
51.13 \\
51.19 \\
51.25\end{array}$ & $\begin{array}{l}52.70 \\
52.75 \\
52.82 \\
52.85 \\
52.90\end{array}$ & $\begin{array}{l}54.05 \\
54.08 \\
54.12 \\
54.14 \\
54.19\end{array}$ \\
\hline $\begin{array}{l}21 \\
22 \\
23 \\
24 \\
25\end{array}$ & $\begin{array}{l}54.52 \\
53.67 \\
52.35 \\
51.80 \\
51.81\end{array}$ & $\begin{array}{l}48.74 \\
48.64 \\
48.40 \\
48.28 \\
47.89\end{array}$ & $\begin{array}{l}42.58 \\
42.37 \\
42.20 \\
42.02 \\
41.93\end{array}$ & $\begin{array}{l}39.51 \\
39.56 \\
39.66 \\
39.75 \\
39.89\end{array}$ & $\begin{array}{l}42.58 \\
42.69 \\
42.76 \\
42.83 \\
42.94\end{array}$ & $\begin{array}{l}45.16 \\
45.15 \\
45.16 \\
45.16 \\
45.17\end{array}$ & $\begin{array}{l}45.82 \\
45.85 \\
45.92 \\
46.04 \\
46.10\end{array}$ & $\begin{array}{l}47.64 \\
47.70 \\
47.77 \\
47.85 \\
47.95\end{array}$ & $\begin{array}{l}49.53 \\
49.59 \\
49.65 \\
49.69 \\
49.76\end{array}$ & $\begin{array}{l}51.33 \\
51.39 \\
51.45 \\
51.49 \\
51.53\end{array}$ & $\begin{array}{l}52.96 \\
53.01 \\
53.06 \\
53.11 \\
53.14\end{array}$ & $\begin{array}{l}54.22 \\
54.26 \\
54.32 \\
54.35 \\
54.38\end{array}$ \\
\hline $\begin{array}{l}26 \\
27 \\
28 \\
29 \\
30 \\
31\end{array}$ & $\begin{array}{l}51.85 \\
51.90 \\
51.34 \\
51.10 \\
51.09 \\
50.88\end{array}$ & $\begin{array}{r}47.73 \\
47.52 \\
47.27 \\
--- \\
--- \\
---\end{array}$ & $\begin{array}{l}41.76 \\
41.58 \\
41.51 \\
41.38 \\
41.07 \\
41.01\end{array}$ & $\begin{array}{r}40.06 \\
40.11 \\
40.16 \\
40.34 \\
40.42 \\
-.-\end{array}$ & $\begin{array}{l}43.08 \\
43.20 \\
43.31 \\
43.41 \\
43.50 \\
43.60\end{array}$ & $\begin{array}{r}45.19 \\
45.19 \\
45.18 \\
45.14 \\
45.12 \\
. .-\end{array}$ & $\begin{array}{l}46.13 \\
46.16 \\
46.20 \\
46.25 \\
46.32 \\
46.35\end{array}$ & $\begin{array}{l}48.02 \\
48.08 \\
48.14 \\
48.20 \\
48.25 \\
48.30\end{array}$ & $\begin{array}{r}49.84 \\
49.90 \\
49.95 \\
49.99 \\
50.03 \\
.--\end{array}$ & $\begin{array}{l}51.57 \\
51.64 \\
51.72 \\
51.79 \\
51.84 \\
51.91\end{array}$ & $\begin{array}{r}53.18 \\
53.21 \\
53.25 \\
53.29 \\
53.32 \\
. .-\end{array}$ & $\begin{array}{l}54.42 \\
54.46 \\
54.49 \\
54.57 \\
54.60 \\
54.62\end{array}$ \\
\hline MEAN & 53.92 & 48.96 & 43.70 & 39.59 & 42.09 & 44.79 & 45.58 & 47.32 & 49.20 & 51.02 & 52.67 & 54.02 \\
\hline
\end{tabular}



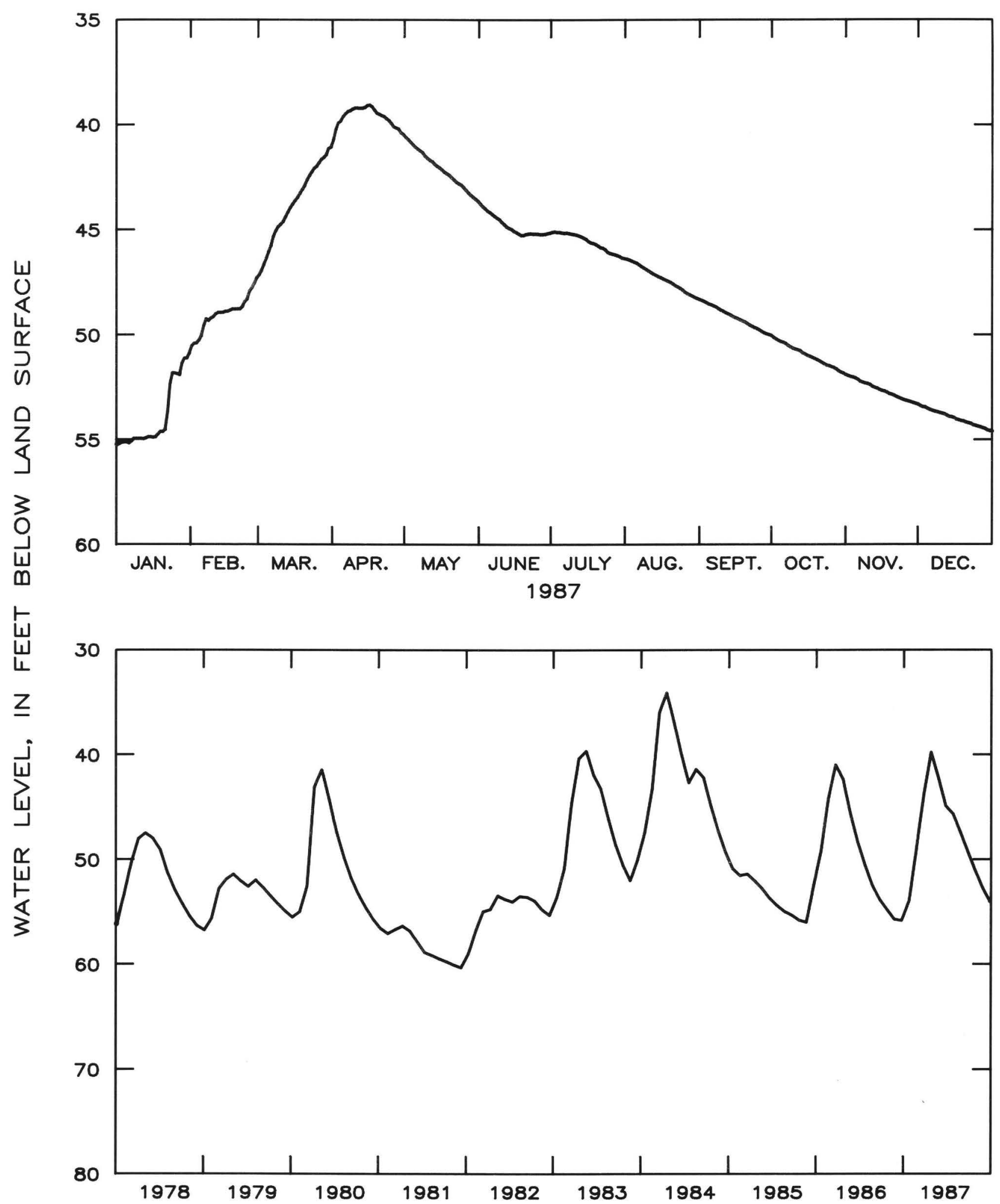

Figure 2.7.1-5.--Water level in observation well 10G313, Mitchell County. 
09F520 BOLTON DECATUR COUNTY

305736084355801 Local number, 09F520.

LOCATION.--Lat $30^{\circ} 57^{\prime} 42^{\prime \prime}$, long $84^{\circ} 35^{\prime} 46^{\prime \prime}$, Hydrologic Unit 03130008 , U.S. Highway 27 north of Bainbridge, right on dirt road near John Deere tractor dealership.

Owner: Graham Bolton.

AQUIFER.--Upper Floridan aquifer.

WELL CHARACTERISTICS.---Unused private irrigation well, diameter $12 \mathrm{in}$., depth $251 \mathrm{ft}$, cased to $130 \mathrm{ft}$, open hole. DATUM.--Elevation of land-surface datum is $128 \mathrm{ft}$.

Measuring point: Floor of recorder shelter, $3.50 \mathrm{ft}$ above land-surface datum.

REMARKS.--This well is about $15 \mathrm{ft}$ from pumped well.

PERIOD OF RECORD.--June 1969 to current year.

EXTREMES FOR PERIOD OF RECORD.---Highest water level, $34.86 \mathrm{ft}$ below land-surface datum, April 15, 1984; 10west, $54.78 \mathrm{ft}$ below land-surface datum, August 20, 1981 .

DEPTH BELOW LAND SURFACE (WATER LEVEL) (FEET) CALENDAR YEAR JANUARY 1987 TO DECEMBER 1987 MEAN VALUES

\begin{tabular}{|c|c|c|c|c|c|c|c|c|c|c|c|c|}
\hline DAY & JAN & FEB & MAR & APR & MAY & JUN & JUL & AUG & SEP & ОСТ & NOV & DEC \\
\hline $\begin{array}{l}1 \\
2 \\
3 \\
4 \\
5\end{array}$ & $\begin{array}{l}47.73 \\
47.72 \\
47.66 \\
47.60 \\
47.59\end{array}$ & $\begin{array}{l}42.34 \\
42.19 \\
42.15 \\
42.09 \\
42.03\end{array}$ & $\begin{array}{l}41.28 \\
41.20 \\
41.08 \\
40.98 \\
40.87\end{array}$ & $\begin{array}{l}39.26 \\
39.00 \\
38.87 \\
38.83 \\
38.70\end{array}$ & $\begin{array}{l}40.05 \\
44.15 \\
43.86 \\
43.07 \\
42.89\end{array}$ & $\begin{array}{l}44.19 \\
43.43 \\
44.90 \\
41.49 \\
43.93\end{array}$ & $\begin{array}{l}42.06 \\
42.01 \\
41.96 \\
41.92 \\
41.89\end{array}$ & $\begin{array}{l}42.95 \\
42.98 \\
43.02 \\
43.07 \\
43.16\end{array}$ & $\begin{array}{l}44.54 \\
44.57 \\
44.61 \\
44.64 \\
44.67\end{array}$ & $\begin{array}{l}45.80 \\
45.85 \\
45.91 \\
45.94 \\
45.98\end{array}$ & $\begin{array}{l}47.14 \\
47.16 \\
47.18 \\
47.20 \\
47.25\end{array}$ & $\begin{array}{l}47.88 \\
47.92 \\
47.92 \\
47.96 \\
47.99\end{array}$ \\
\hline $\begin{array}{r}6 \\
7 \\
8 \\
9 \\
10\end{array}$ & $\begin{array}{l}47.49 \\
47.41 \\
47.33 \\
47.23 \\
47.16\end{array}$ & $\begin{array}{l}41.94 \\
41.89 \\
41.86 \\
41.92 \\
41.88\end{array}$ & $\begin{array}{l}40.74 \\
40.54 \\
40.43 \\
40.35 \\
40.33\end{array}$ & $\begin{array}{l}38.61 \\
38.55 \\
38.53 \\
38.48 \\
38.46\end{array}$ & $\begin{array}{l}40.34 \\
40.38 \\
43.56 \\
42.47 \\
40.66\end{array}$ & $\begin{array}{l}45.16 \\
41.81 \\
43.93 \\
45.38 \\
44.39\end{array}$ & $\begin{array}{l}41.87 \\
41.85 \\
41.85 \\
41.85 \\
41.86\end{array}$ & $\begin{array}{l}43.24 \\
43.32 \\
43.38 \\
45.38 \\
47.09\end{array}$ & $\begin{array}{l}44.71 \\
44.74 \\
44.78 \\
44.82 \\
44.87\end{array}$ & $\begin{array}{l}46.03 \\
46.08 \\
46.14 \\
46.19 \\
46.24\end{array}$ & $\begin{array}{l}47.30 \\
47.32 \\
47.34 \\
47.35 \\
47.38\end{array}$ & $\begin{array}{l}48.02 \\
48.05 \\
48.08 \\
48.10 \\
48.12\end{array}$ \\
\hline $\begin{array}{l}11 \\
12 \\
13 \\
14 \\
15\end{array}$ & $\begin{array}{l}47.15 \\
47.07 \\
47.04 \\
47.00 \\
46.95\end{array}$ & $\begin{array}{l}41.81 \\
41.74 \\
41.71 \\
41.66 \\
41.63\end{array}$ & $\begin{array}{l}40.31 \\
40.27 \\
40.21 \\
40.17 \\
40.16\end{array}$ & $\begin{array}{l}38.50 \\
38.53 \\
38.53 \\
38.54 \\
38.51\end{array}$ & $\begin{array}{l}40.69 \\
40.72 \\
40.76 \\
40.81 \\
40.83\end{array}$ & $\begin{array}{l}45.97 \\
45.78 \\
46.67 \\
43.44 \\
43.44\end{array}$ & $\begin{array}{l}41.88 \\
41.91 \\
41.99 \\
42.14 \\
42.19\end{array}$ & $\begin{array}{l}43.60 \\
43.64 \\
43.69 \\
43.70 \\
43.73\end{array}$ & $\begin{array}{l}44.92 \\
44.95 \\
44.99 \\
45.83 \\
45.08\end{array}$ & $\begin{array}{l}46.25 \\
46.28 \\
46.36 \\
46.41 \\
46.44\end{array}$ & $\begin{array}{l}47.44 \\
47.47 \\
47.49 \\
47.52 \\
47.55\end{array}$ & $\begin{array}{l}48.15 \\
48.19 \\
48.23 \\
48.24 \\
48.28\end{array}$ \\
\hline $\begin{array}{l}16 \\
17 \\
18 \\
19 \\
20\end{array}$ & $\begin{array}{l}46.93 \\
46.89 \\
46.78 \\
46.55 \\
45.95\end{array}$ & $\begin{array}{l}41.62 \\
41.70 \\
41.75 \\
41.78 \\
41.77\end{array}$ & $\begin{array}{l}40.14 \\
40.14 \\
40.11 \\
40.11 \\
40.08\end{array}$ & $\begin{array}{l}38.72 \\
38.76 \\
38.77 \\
38.84 \\
38.88\end{array}$ & $\begin{array}{l}40.84 \\
40.84 \\
40.82 \\
40.72 \\
40.63\end{array}$ & $\begin{array}{l}43.47 \\
43.51 \\
43.54 \\
43.53 \\
43.54\end{array}$ & $\begin{array}{l}42.26 \\
42.30 \\
42.34 \\
42.40 \\
45.12\end{array}$ & $\begin{array}{l}43.75 \\
43.79 \\
43.82 \\
43.86 \\
43.90\end{array}$ & $\begin{array}{l}45.12 \\
45.16 \\
45.19 \\
45.24 \\
45.29\end{array}$ & $\begin{array}{l}46.47 \\
46.52 \\
46.55 \\
46.59 \\
46.64\end{array}$ & $\begin{array}{l}47.58 \\
47.62 \\
47.65 \\
47.66 \\
47.67\end{array}$ & $\begin{array}{l}48.33 \\
48.35 \\
48.37 \\
48.39 \\
48.42\end{array}$ \\
\hline $\begin{array}{l}21 \\
22 \\
23 \\
24 \\
25\end{array}$ & $\begin{array}{l}45.42 \\
45.02 \\
44.76 \\
44.29 \\
43.79\end{array}$ & $\begin{array}{l}41.74 \\
41.69 \\
41.72 \\
41.63 \\
41.57\end{array}$ & $\begin{array}{l}40.01 \\
39.97 \\
39.95 \\
39.92 \\
39.93\end{array}$ & $\begin{array}{l}38.90 \\
38.96 \\
39.04 \\
41.01 \\
42.53\end{array}$ & $\begin{array}{l}40.59 \\
40.59 \\
40.58 \\
40.59 \\
42.50\end{array}$ & $\begin{array}{l}43.54 \\
43.54 \\
42.79 \\
42.28 \\
42.22\end{array}$ & $\begin{array}{l}45.26 \\
42.62 \\
42.70 \\
42.77 \\
42.78\end{array}$ & $\begin{array}{l}43.97 \\
44.05 \\
46.07 \\
47.80 \\
44.28\end{array}$ & $\begin{array}{l}45.36 \\
45.42 \\
45.48 \\
45.51 \\
45.55\end{array}$ & $\begin{array}{l}46.97 \\
46.76 \\
46.80 \\
46.83 \\
46.85\end{array}$ & $\begin{array}{l}47.69 \\
47.70 \\
47.72 \\
47.74 \\
47.76\end{array}$ & $\begin{array}{l}48.44 \\
48.47 \\
48.51 \\
48.53 \\
48.55\end{array}$ \\
\hline $\begin{array}{l}26 \\
27 \\
28 \\
29 \\
30 \\
31\end{array}$ & $\begin{array}{l}43.65 \\
43.37 \\
43.10 \\
42.86 \\
42.63 \\
42.50\end{array}$ & $\begin{array}{r}41.50 \\
41.42 \\
41.32 \\
--- \\
--- \\
---\end{array}$ & $\begin{array}{l}39.89 \\
39.84 \\
39.86 \\
39.82 \\
39.63 \\
39.51\end{array}$ & $\begin{array}{r}39.44 \\
39.47 \\
41.37 \\
43.45 \\
39.72 \\
-.-\end{array}$ & $\begin{array}{l}43.49 \\
42.18 \\
43.90 \\
41.01 \\
41.05 \\
42.98\end{array}$ & $\begin{array}{r}42.19 \\
42.15 \\
42.12 \\
42.10 \\
42.08 \\
. .-\end{array}$ & $\begin{array}{l}42.79 \\
42.81 \\
42.83 \\
42.86 \\
42.91 \\
42.94\end{array}$ & $\begin{array}{l}44.30 \\
44.33 \\
44.36 \\
44.40 \\
44.45 \\
44.51\end{array}$ & $\begin{array}{r}45.59 \\
45.64 \\
45.68 \\
45.71 \\
45.74 \\
---\end{array}$ & $\begin{array}{l}46.88 \\
46.92 \\
46.97 \\
47.03 \\
47.08 \\
47.11\end{array}$ & $\begin{array}{r}47.78 \\
47.79 \\
47.80 \\
47.83 \\
47.84 \\
---\end{array}$ & $\begin{array}{l}48.57 \\
48.58 \\
48.59 \\
48.64 \\
48.65 \\
48.65\end{array}$ \\
\hline MEAN & 45.96 & 41.79 & 40.25 & 39.26 & 41.57 & 43.55 & 42.48 & 44.12 & 45.15 & 46.48 & 47.53 & 48.30 \\
\hline
\end{tabular}

$\begin{array}{lllllll}\text { CAL YR } 1987 & \text { MEAN } & 43.89 & \text { HIGH } & 38.46 & \text { LOW } & 48.65\end{array}$ 

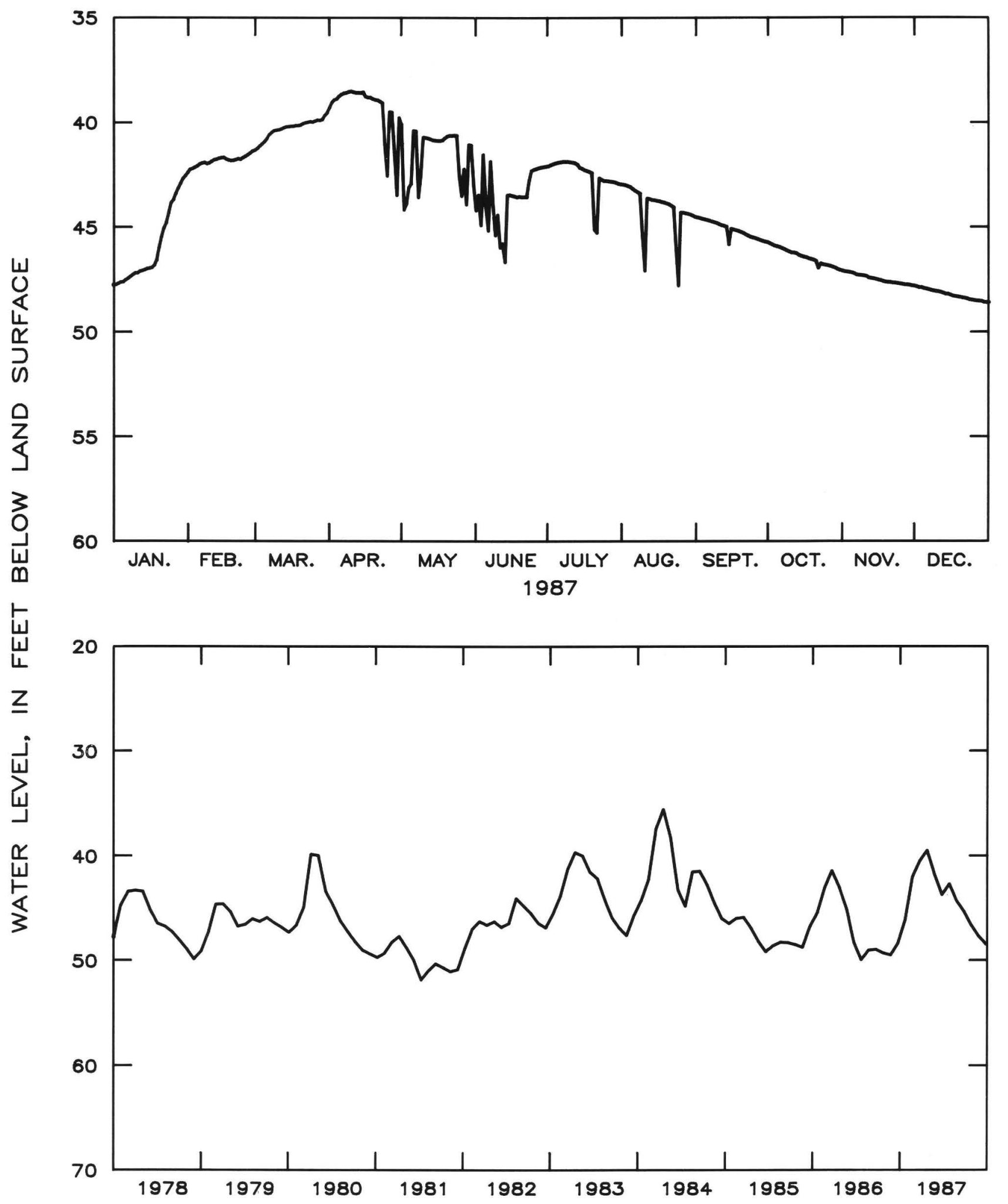

Figure 2.7.1-6.--Water level in observation well 09F520, Decatur County. 


\section{G001 FLEET MILLER COUNTY}

310651084404501 Local number, 08G001.

LOCATION.--Lat $31^{\circ} 06^{\prime} 51^{\prime \prime}$, long $84^{\circ} 40^{\prime} 45^{\prime \prime}$, Hydrologic Unit 03130010, 1 mi northeast of Boykin, Ga.

Owner: Jack Fleet.

AQUIFER.--Upper Floridan aquifer.

WELL CHARACTERISTICS.--Drilled unused irrigation well, diameter $12 \mathrm{in.,}$ depth $255 \mathrm{ft}$, cased to $130 \mathrm{ft}$, open hole. DATUM.--Elevation of land-surface datum is $150 \mathrm{ft}$.

Measuring point: Top front edge of recorder shelter, $3.0 \mathrm{ft}$ above land-surface datum.

REMARKS.--None.

PERIOD OF RECORD.--February 1977 to current year.

EXTREMES FOR PERIOD OF RECORD.--Highest water level, $11.18 \mathrm{ft}$ below land-surface datum, April 11, 1984 ; 10west, $43.88 \mathrm{ft}$ below land-surface datum, July $17,1981$. DEPTH BELOW LAND SURFACE (WATER LEVEL) ( FEET) C CALENDAR YEAR JANUARY 1987 TO DECEMBER 1987

\begin{tabular}{|c|c|c|c|c|c|c|c|c|c|c|c|c|}
\hline DAY & JAN & FEB & MAR & APR & MAY & JUN & JUL & AUG & SEP & ОСТ & NOV & DEC \\
\hline $\begin{array}{l}1 \\
2 \\
3 \\
4 \\
5\end{array}$ & $\begin{array}{l}32.60 \\
32.88 \\
32.96 \\
32.70 \\
32.53\end{array}$ & $\begin{array}{l}14.65 \\
14.89 \\
15.13 \\
15.29 \\
15.40\end{array}$ & $\begin{array}{l}16.05 \\
15.49 \\
15.17 \\
15.06 \\
15.06\end{array}$ & $\begin{array}{l}13.98 \\
13.74 \\
13.73 \\
13.86 \\
13.98\end{array}$ & $\begin{array}{l}18.45 \\
19.05 \\
18.97 \\
19.05 \\
20.05\end{array}$ & $\begin{array}{l}25.20 \\
26.44 \\
26.00 \\
26.39 \\
24.82\end{array}$ & $\begin{array}{l}23.30 \\
23.42 \\
23.60 \\
23.75 \\
23.87\end{array}$ & $\begin{array}{l}29.30 \\
29.33 \\
29.37 \\
29.48 \\
30.52\end{array}$ & $\begin{array}{l}34.66 \\
34.59 \\
34.43 \\
34.37 \\
34.31\end{array}$ & $\begin{array}{l}37.56 \\
38.05 \\
37.75 \\
37.05 \\
36.87\end{array}$ & $\begin{array}{l}38.59 \\
38.52 \\
38.48 \\
38.45 \\
38.48\end{array}$ & $\begin{array}{l}39.19 \\
39.24 \\
39.26 \\
39.27 \\
39.32\end{array}$ \\
\hline $\begin{array}{r}6 \\
7 \\
8 \\
9 \\
10\end{array}$ & $\begin{array}{l}31.65 \\
30.15 \\
28.95 \\
27.75 \\
27.04\end{array}$ & $\begin{array}{l}15.57 \\
15.70 \\
15.88 \\
16.08 \\
16.20\end{array}$ & $\begin{array}{l}15.05 \\
15.05 \\
15.15 \\
15.30 \\
15.45\end{array}$ & $\begin{array}{l}14.13 \\
14.28 \\
14.42 \\
14.55 \\
14.72\end{array}$ & $\begin{array}{l}21.00 \\
21.65 \\
20.60 \\
20.79 \\
21.15\end{array}$ & $\begin{array}{l}24.30 \\
24.20 \\
24.50 \\
25.57 \\
26.50\end{array}$ & $\begin{array}{l}24.07 \\
24.40 \\
24.60 \\
24.60 \\
25.80\end{array}$ & $\begin{array}{l}30.23 \\
30.03 \\
30.41 \\
30.99 \\
32.56\end{array}$ & $\begin{array}{l}34.30 \\
34.29 \\
34.34 \\
34.40 \\
34.44\end{array}$ & $\begin{array}{l}37.39 \\
37.79 \\
37.45 \\
37.34 \\
37.26\end{array}$ & $\begin{array}{l}38.56 \\
38.58 \\
38.63 \\
38.63 \\
38.67\end{array}$ & $\begin{array}{l}39.3 \\
39.3\end{array}$ \\
\hline $\begin{array}{l}11 \\
12 \\
13 \\
14 \\
15\end{array}$ & $\begin{array}{l}27.15 \\
27.23 \\
27.25 \\
27.45 \\
27.60\end{array}$ & $\begin{array}{l}16.31 \\
16.45 \\
16.57 \\
16.71 \\
16.84\end{array}$ & $\begin{array}{l}15.55 \\
15.65 \\
15.75 \\
15.83 \\
15.93\end{array}$ & $\begin{array}{l}14.88 \\
15.00 \\
15.12 \\
15.20 \\
15.31\end{array}$ & $\begin{array}{l}21.30 \\
21.16 \\
21.27 \\
21.40 \\
21.48\end{array}$ & $\begin{array}{l}26.20 \\
27.21 \\
27.40 \\
25.90 \\
25.31\end{array}$ & $\begin{array}{l}27.31 \\
27.32 \\
27.36 \\
26.86 \\
27.71\end{array}$ & $\begin{array}{l}34.50 \\
33.89 \\
32.71 \\
31.96 \\
31.77\end{array}$ & $\begin{array}{l}34.48 \\
34.50 \\
34.64 \\
34.64 \\
34.64\end{array}$ & $\begin{array}{l}37.10 \\
37.02 \\
37.10 \\
37.37 \\
37.76\end{array}$ & $\begin{array}{l}38.77 \\
38.80 \\
38.80 \\
38.84 \\
38.89\end{array}$ & $\begin{array}{l}39.4 \\
39.5 \\
39.5 \\
39.5 \\
39.5\end{array}$ \\
\hline $\begin{array}{l}16 \\
17 \\
18 \\
19 \\
20\end{array}$ & $\begin{array}{l}27.82 \\
27.35 \\
25.95 \\
20.52 \\
15.13\end{array}$ & $\begin{array}{l}16.96 \\
17.15 \\
17.33 \\
17.51 \\
17.67\end{array}$ & $\begin{array}{l}16.05 \\
16.16 \\
16.10 \\
15.88 \\
15.62\end{array}$ & $\begin{array}{l}15.43 \\
15.60 \\
15.78 \\
15.83 \\
16.08\end{array}$ & $\begin{array}{l}21.65 \\
21.75 \\
21.97 \\
22.05 \\
21.75\end{array}$ & $\begin{array}{l}24.50 \\
23.52 \\
22.80 \\
22.27 \\
21.97\end{array}$ & $\begin{array}{l}28.35 \\
28.86 \\
29.97 \\
30.22 \\
29.82\end{array}$ & $\begin{array}{l}31.82 \\
31.82 \\
31.92 \\
32.98 \\
34.44\end{array}$ & $\begin{array}{l}34.66 \\
34.73 \\
34.76 \\
34.81 \\
34.91\end{array}$ & $\begin{array}{l}37.81 \\
37.94 \\
37.67 \\
37.62 \\
37.71\end{array}$ & $\begin{array}{l}38.90 \\
38.91 \\
38.94 \\
38.92 \\
38.93\end{array}$ & $\begin{array}{l}39.6 \\
39.6 \\
39.6 \\
39.6 \\
39.7\end{array}$ \\
\hline $\begin{array}{l}21 \\
22 \\
23 \\
24 \\
25\end{array}$ & $\begin{array}{l}14.31 \\
14.03 \\
13.47 \\
13.17 \\
13.15\end{array}$ & $\begin{array}{l}17.80 \\
17.87 \\
17.95 \\
17.72 \\
17.50\end{array}$ & $\begin{array}{l}15.39 \\
15.25 \\
15.16 \\
15.12 \\
15.17\end{array}$ & $\begin{array}{l}16.13 \\
16.25 \\
16.41 \\
17.00 \\
17.87\end{array}$ & $\begin{array}{l}21.70 \\
21.70 \\
22.13 \\
22.30 \\
21.98\end{array}$ & $\begin{array}{l}21.87 \\
21.87 \\
21.96 \\
22.10 \\
22.25\end{array}$ & $\begin{array}{l}29.21 \\
29.44 \\
30.36 \\
30.27 \\
31.71\end{array}$ & $\begin{array}{l}34.33 \\
33.65 \\
33.07 \\
33.06 \\
33.59\end{array}$ & $\begin{array}{l}35.01 \\
35.45 \\
36.68 \\
36.58 \\
36.85\end{array}$ & $\begin{array}{l}38.24 \\
38.42 \\
38.57 \\
38.29 \\
38.30\end{array}$ & $\begin{array}{l}38.97 \\
38.99 \\
39.01 \\
39.03 \\
39.05\end{array}$ & $\begin{array}{l}39.7 \\
39.7 \\
39.7 \\
39.8 \\
39.8\end{array}$ \\
\hline $\begin{array}{l}26 \\
27 \\
28 \\
29 \\
30 \\
31\end{array}$ & $\begin{array}{l}13.39 \\
13.69 \\
13.95 \\
14.10 \\
14.30 \\
14.49\end{array}$ & $\begin{array}{r}17.25 \\
17.03 \\
16.70 \\
--- \\
---\end{array}$ & $\begin{array}{l}15.17 \\
15.20 \\
15.28 \\
15.27 \\
15.00 \\
14.48\end{array}$ & $\begin{array}{c}17.90 \\
17.62 \\
18.00 \\
18.20 \\
18.30 \\
-.-\end{array}$ & $\begin{array}{l}22.45 \\
23.08 \\
22.75 \\
23.05 \\
23.75 \\
24.13\end{array}$ & $\begin{array}{r}22.40 \\
22.60 \\
22.80 \\
23.02 \\
23.17 \\
. . .\end{array}$ & $\begin{array}{l}29.91 \\
29.51 \\
29.43 \\
29.39 \\
29.41 \\
29.37\end{array}$ & $\begin{array}{l}34.31 \\
36.29 \\
36.40 \\
35.80 \\
34.89 \\
34.78\end{array}$ & $\begin{array}{r}36.94 \\
36.68 \\
36.60 \\
37.09 \\
36.58 \\
. .-\end{array}$ & $\begin{array}{l}38.19 \\
38.15 \\
38.22 \\
38.33 \\
38.85 \\
38.78\end{array}$ & $\begin{array}{r}39.08 \\
39.11 \\
39.11 \\
39.14 \\
39.15 \\
.--\end{array}$ & $\begin{array}{l}39.9 \\
39.9\end{array}$ \\
\hline MEAN & 23.06 & 16.58 & 15.41 & 15.64 & 21.47 & 24.17 & 27.52 & 32.59 & 35.18 & 37.80 & 38.83 & 39.6 \\
\hline CAL & & & & $\mathrm{HIGH}$ & 13.15 & & & & & & & \\
\hline
\end{tabular}



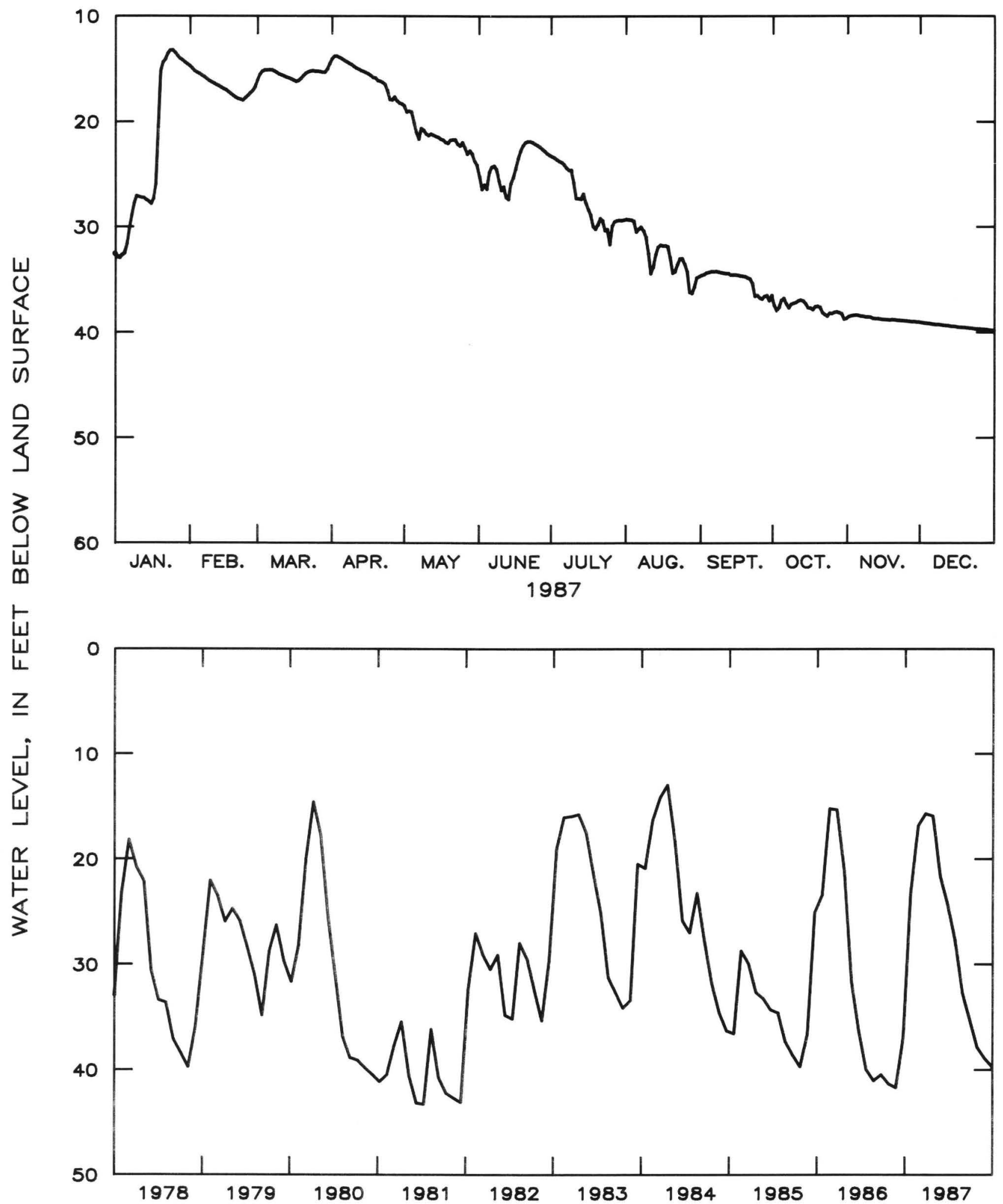

Figure 2.7.1-7.--Water level in observation well 08G001, Miller County. 
305356084534601 Local number, 06F001.

LOCATION.--Lat $30^{\circ} 54^{\prime} 01^{\prime \prime}$, long $84^{\circ} 53^{\prime} 40^{\prime \prime}$, Hydrologic Unit $03130004,9.8 \mathrm{mi}$ south of Donalsonville, $0.85 \mathrm{mi}$ west of Georgia Highway 39.

Owner: Roddenberry Company.

AQUIFER.--Upper Floridan aquifer.

WELL CHARACTERISTICS.--Drilled observation well, diameter $4 \mathrm{in.,}$ depth $150 \mathrm{ft}$, cased to $98.5 \mathrm{ft}$, open hole. DATUM.--Elevation of land-surface datum is $110 \mathrm{ft}$.

Measuring point: Top front edge of recorder shelter, $3.14 \mathrm{ft}$ above land-surface datum.

REMARKS.--Borehole geophysical survey conducted August 10, 1983. Water levels for period of missing record, January 20-27, were estimated.

PERIOD OF RECORD.--March 1979 to July 1982, August 1983 to current year.

EXTREMES FOR PERIOD OF RECORD.--Highest water level, $4.13 \mathrm{ft}$ below land-surface datum, March 8, 1984; 1owest, $35.65 \mathrm{ft}$ below land-surface datum, October 5,1986 .

DEPTH BELOW LAND SURFACE (WATER LEVEL) (FEET) CALENDAR YEAR JANUARY 1987 TO DECEMBER 1987 MEAN VALUES

\begin{tabular}{|c|c|c|c|c|c|c|c|c|c|c|c|c|}
\hline DAY & JAN & FEB & MAR & APR & MAY & JUN & JUL & AUG & SEP & OCT & NOV & DEC \\
\hline $\begin{array}{l}1 \\
2 \\
3 \\
4 \\
5\end{array}$ & $\begin{array}{l}29.50 \\
29.76 \\
29.83 \\
29.45 \\
29.12\end{array}$ & $\begin{array}{r}9.82 \\
10.00 \\
10.21 \\
10.52 \\
10.81\end{array}$ & $\begin{array}{l}9.91 \\
9.26 \\
9.15 \\
9.30 \\
9.55\end{array}$ & $\begin{array}{l}7.31 \\
7.42 \\
7.62 \\
7.93 \\
8.20\end{array}$ & $\begin{array}{l}18.74 \\
19.26 \\
19.67 \\
19.47 \\
19.40\end{array}$ & $\begin{array}{l}26.34 \\
26.82 \\
26.63 \\
27.34 \\
27.32\end{array}$ & $\begin{array}{l}21.98 \\
22.19 \\
21.90 \\
21.26 \\
21.10\end{array}$ & $\begin{array}{l}27.54 \\
27.67 \\
28.01 \\
28.37 \\
28.26\end{array}$ & $\begin{array}{l}27.25 \\
27.43 \\
27.59 \\
27.69 \\
27.86\end{array}$ & $\begin{array}{l}30.29 \\
30.37 \\
30.45 \\
30.52 \\
30.52\end{array}$ & $\begin{array}{l}32.12 \\
32.14 \\
32.15 \\
32.15 \\
32.22\end{array}$ & $\begin{array}{l}32.68 \\
32.76 \\
32.76 \\
32.78 \\
32.86\end{array}$ \\
\hline $\begin{array}{r}6 \\
7 \\
8 \\
9 \\
10\end{array}$ & $\begin{array}{l}28.78 \\
28.40 \\
28.17 \\
27.96 \\
27.59\end{array}$ & $\begin{array}{l}10.94 \\
11.11 \\
11.33 \\
11.78 \\
12.08\end{array}$ & $\begin{array}{r}9.84 \\
10.00 \\
10.10 \\
10.36 \\
10.76\end{array}$ & $\begin{array}{l}8.48 \\
8.79 \\
9.14 \\
9.45 \\
9.76\end{array}$ & $\begin{array}{l}19.46 \\
19.57 \\
19.64 \\
19.87 \\
20.08\end{array}$ & $\begin{array}{l}27.51 \\
27.35 \\
27.52 \\
28.26 \\
28.27\end{array}$ & $\begin{array}{l}21.09 \\
21.13 \\
21.29 \\
21.51 \\
21.76\end{array}$ & $\begin{array}{l}28.49 \\
28.82 \\
29.28 \\
29.06 \\
29.25\end{array}$ & $\begin{array}{l}27.98 \\
28.10 \\
28.22 \\
28.06 \\
28.09\end{array}$ & $\begin{array}{l}30.57 \\
30.68 \\
30.85 \\
30.96 \\
31.03\end{array}$ & $\begin{array}{l}32.34 \\
32.37 \\
32.37 \\
32.36 \\
32.36\end{array}$ & $\begin{array}{l}32.88 \\
32.89 \\
32.92 \\
32.94 \\
32.95\end{array}$ \\
\hline $\begin{array}{l}11 \\
12 \\
13 \\
14 \\
15\end{array}$ & $\begin{array}{l}27.54 \\
27.32 \\
27.13 \\
27.04 \\
26.94\end{array}$ & $\begin{array}{l}12.26 \\
12.45 \\
12.73 \\
13.01 \\
13.29\end{array}$ & $\begin{array}{l}11.18 \\
11.47 \\
11.71 \\
11.96 \\
12.26\end{array}$ & $\begin{array}{l}10.12 \\
10.47 \\
10.93 \\
11.30 \\
11.46\end{array}$ & $\begin{array}{l}20.24 \\
20.41 \\
20.66 \\
20.96 \\
21.21\end{array}$ & $\begin{array}{l}28.38 \\
28.65 \\
28.81 \\
28.73 \\
28.60\end{array}$ & $\begin{array}{l}22.02 \\
22.32 \\
22.65 \\
23.48 \\
24.24\end{array}$ & $\begin{array}{l}29.70 \\
29.25 \\
28.78 \\
24.64 \\
20.78\end{array}$ & $\begin{array}{l}28.17 \\
28.05 \\
28.07 \\
28.18 \\
28.26\end{array}$ & $\begin{array}{l}31.04 \\
31.06 \\
31.20 \\
31.33 \\
31.39\end{array}$ & $\begin{array}{l}32.46 \\
32.52 \\
32.54 \\
32.57 \\
32.61\end{array}$ & $\begin{array}{l}32.98 \\
33.01 \\
33.10 \\
33.11 \\
33.12\end{array}$ \\
\hline $\begin{array}{l}16 \\
17 \\
18 \\
19 \\
20\end{array}$ & $\begin{array}{r}26.77 \\
24.04 \\
21.43 \\
14.13 \\
8.84\end{array}$ & $\begin{array}{l}13.50 \\
14.00 \\
14.42 \\
14.80 \\
15.06\end{array}$ & $\begin{array}{l}12.55 \\
12.84 \\
12.81 \\
11.14 \\
10.35\end{array}$ & $\begin{array}{l}11.75 \\
12.37 \\
12.66 \\
13.15 \\
13.63\end{array}$ & $\begin{array}{l}21.45 \\
21.65 \\
21.79 \\
21.91 \\
22.09\end{array}$ & $\begin{array}{l}28.05 \\
26.02 \\
25.01 \\
23.26 \\
21.44\end{array}$ & $\begin{array}{l}24.89 \\
25.72 \\
25.76 \\
26.00 \\
26.35\end{array}$ & $\begin{array}{l}19.71 \\
19.78 \\
20.24 \\
20.84 \\
21.50\end{array}$ & $\begin{array}{l}28.34 \\
28.45 \\
28.53 \\
28.62 \\
28.77\end{array}$ & $\begin{array}{l}31.43 \\
31.47 \\
31.52 \\
31.56 \\
31.67\end{array}$ & $\begin{array}{l}32.62 \\
32.56 \\
32.53 \\
32.52 \\
32.53\end{array}$ & $\begin{array}{l}33.22 \\
33.24 \\
33.26 \\
33.26 \\
33.27\end{array}$ \\
\hline $\begin{array}{l}21 \\
22 \\
23 \\
24 \\
25\end{array}$ & $\begin{array}{l}8.12 \\
7.95 \\
7.49 \\
7.29 \\
7.37\end{array}$ & $\begin{array}{l}15.07 \\
14.51 \\
12.64 \\
11.74 \\
11.45\end{array}$ & $\begin{array}{l}10.11 \\
10.18 \\
10.40 \\
10.68 \\
10.91\end{array}$ & $\begin{array}{l}13.88 \\
14.19 \\
14.55 \\
14.87 \\
15.42\end{array}$ & $\begin{array}{l}22.30 \\
22.63 \\
22.85 \\
23.48 \\
23.87\end{array}$ & $\begin{array}{l}20.74 \\
20.62 \\
20.45 \\
20.36 \\
20.38\end{array}$ & $\begin{array}{l}26.65 \\
27.02 \\
26.85 \\
27.46 \\
27.61\end{array}$ & $\begin{array}{l}22.14 \\
22.65 \\
23.09 \\
23.70 \\
24.50\end{array}$ & $\begin{array}{l}28.91 \\
29.03 \\
29.17 \\
29.49 \\
29.69\end{array}$ & $\begin{array}{l}31.81 \\
31.89 \\
31.79 \\
31.75 \\
31.77\end{array}$ & $\begin{array}{l}32.59 \\
32.59 \\
32.62 \\
32.63 \\
32.61\end{array}$ & $\begin{array}{l}33.30 \\
33.33 \\
33.38 \\
33.39 \\
33.40\end{array}$ \\
\hline $\begin{array}{l}26 \\
27 \\
28 \\
29 \\
30 \\
31\end{array}$ & $\begin{array}{l}7.72 \\
8.12 \\
8.48 \\
8.81 \\
9.08 \\
9.48\end{array}$ & $\begin{array}{r}11.38 \\
11.33 \\
11.25 \\
--- \\
--- \\
---\end{array}$ & $\begin{array}{r}11.00 \\
11.10 \\
11.09 \\
10.76 \\
8.72 \\
7.44\end{array}$ & $\begin{array}{r}16.14 \\
16.20 \\
17.09 \\
17.95 \\
18.31 \\
-.-\end{array}$ & $\begin{array}{l}23.69 \\
24.24 \\
25.38 \\
25.40 \\
25.85 \\
25.69\end{array}$ & $\begin{array}{r}20.55 \\
20.79 \\
21.16 \\
21.47 \\
21.75 \\
. .-\end{array}$ & $\begin{array}{l}27.57 \\
27.67 \\
27.74 \\
28.19 \\
27.85 \\
27.54\end{array}$ & $\begin{array}{l}24.84 \\
25.32 \\
25.87 \\
26.36 \\
26.98 \\
27.01\end{array}$ & $\begin{array}{r}29.70 \\
29.83 \\
29.91 \\
29.95 \\
30.07 \\
. .-\end{array}$ & $\begin{array}{l}31.76 \\
31.81 \\
31.91 \\
31.98 \\
32.04 \\
32.09\end{array}$ & $\begin{array}{r}32.61 \\
32.62 \\
32.61 \\
32.62 \\
32.62 \\
. .-\end{array}$ & $\begin{array}{l}33.41 \\
33.43 \\
33.43 \\
33.54 \\
33.59 \\
33.58\end{array}$ \\
\hline MEAN & 19.67 & 12.27 & 10.61 & 12.02 & 21.71 & 24.95 & 24.54 & 25.56 & 28.58 & 31.31 & 32.47 & 33.15 \\
\hline
\end{tabular}



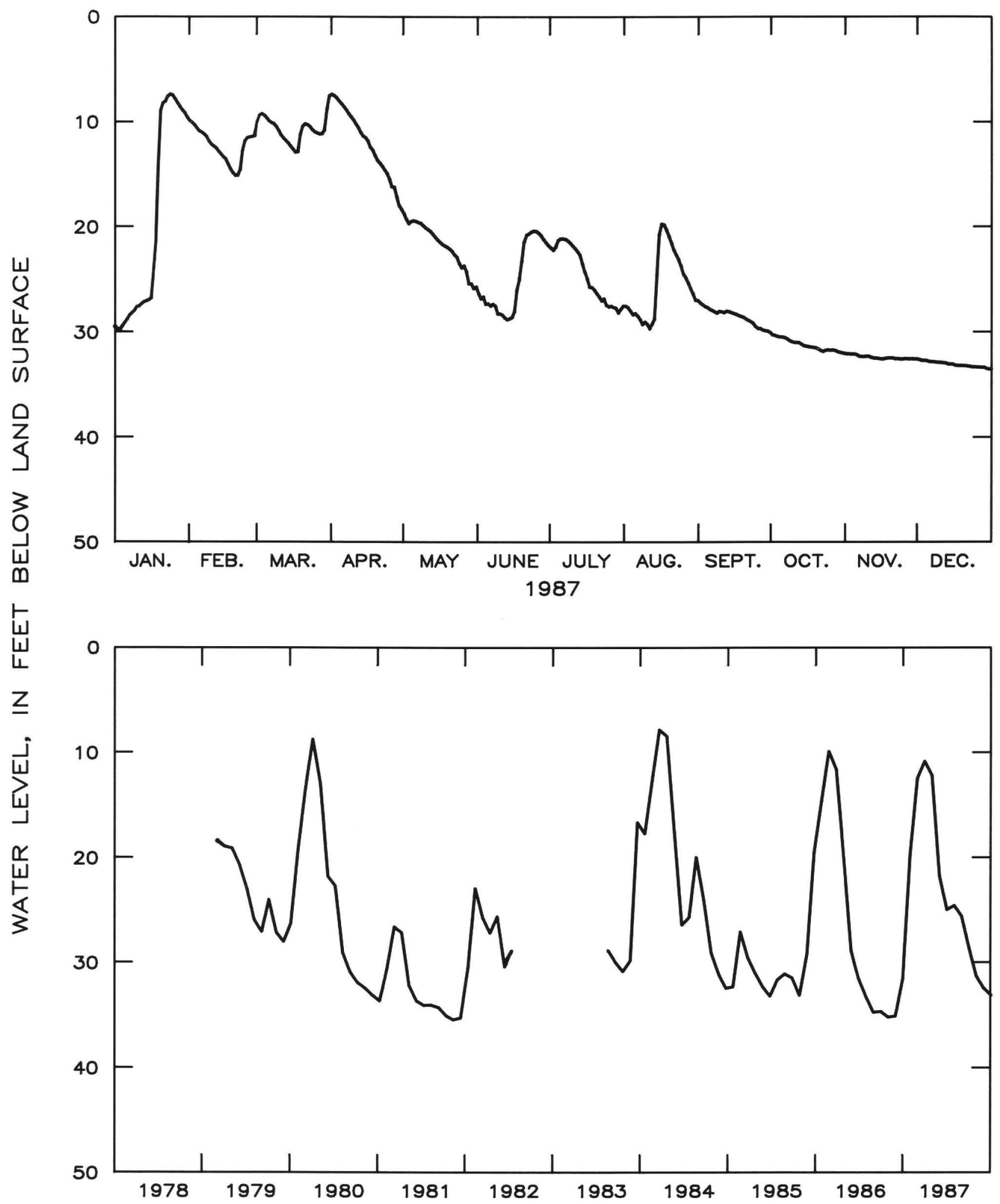

Figure 2.7.1-8--Water level in observation well 06F001, Seminole County. 


\subsubsection{South-central area}

The water level in the Upper Floridan aquifer in south-central Georgia is affected by rainfall, evapotranspiration, stream stage, and pumping. In this area, the water level generally is highest in the winter and spring rainy seasons, and lowest in the fall following the summer irrigation season.

Water levels in three wells tapping the Upper Floridan aquifer in Tift, Worth, and Cook Counties, at the end of April, had recovered 3.8 to $6.6 \mathrm{ft}$ from the lows and record lows recorded during the 1986 drought. Although there was some recovery from the 1986 drought, the mean water levels in the three wells were from about the same to $1.0 \mathrm{ft}$ lower in 1987 than in 1986. These declines continued a general downward trend since 1977. At the end of 1987, water levels were from 0.4 to $1.6 \mathrm{ft}$ lower than at the end of 1986.

The ground-water level in the Valdosta area is controlled mainly by local recharge (Krause, 1979). The water level is highest north of the city, where the Upper Floridan aquifer receives recharge from the Withlacoochee River. The river flows into sinkholes and cave openings in the aquifer, and the ground-water level responds to this recharge. Increased rainfall and streamflow in winter and early spring cause a high water level. Decreased rainfall and increased evapotranspiration in summer and fall result in low streamflow and a correspondingly low water level. In the Valdosta area, the mean water levels in two wells were from 1.1 to $1.6 \mathrm{ft}$ higher in 1987 than in 1986. By the end of March, the water level in the two wells had recovered 26.0 to 27.0 ft from the lows measured during the 1986 drought. Although there was some recovery from the drought, water levels were from 10.5 to $14.2 \mathrm{ft}$ lower at the end of 1987 than at the end of 1986 . 


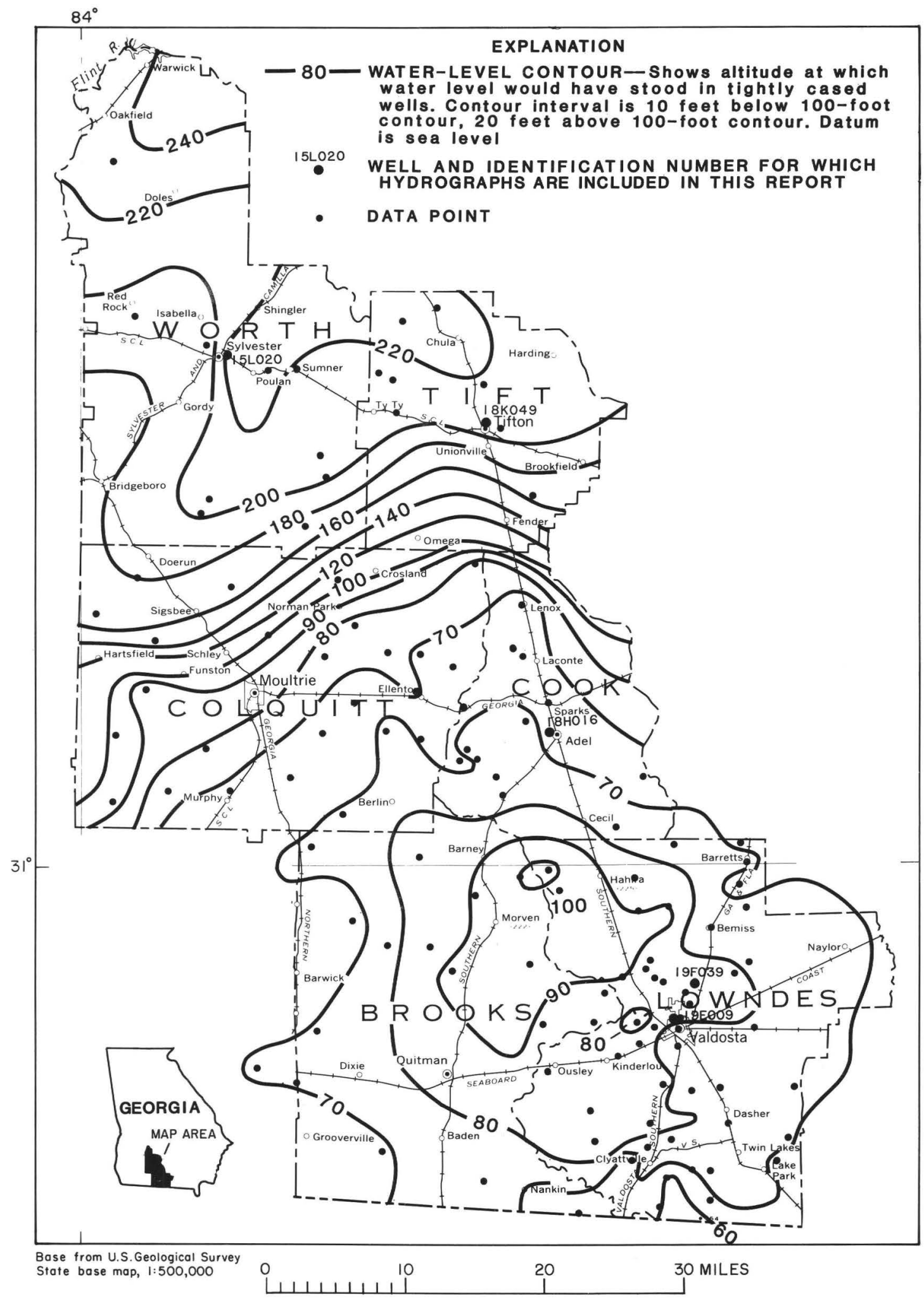

Figure 2.7.2-1.--Observation well locations and the water level in the Upper Floridan aquifer in the south-central area, May 1985. 
313146083491601 Local number, 15L020.

LOCATION.--Lat $31^{\circ} 31^{\prime} 46^{\prime \prime}$, long $83^{\circ} 49^{\prime} 16^{\prime \prime}$, Hydrologic Unit 03110204 , near water tank, behind the VFW on U.S. Highway 82 east, Sylvester, Ga.

Owner: City of Sylvester.

AQUIFER.--Upper Floridan aquifer.

WELL CHARACTERISTICS.--Drilled unused municipal well, diameter $18 \mathrm{in}$., depth $450 \mathrm{ft}$, cased to $212 \mathrm{ft}$, open hole. DATUM.--Elevation of land-surface datum is $420 \mathrm{ft}$.

Measuring point: Floor of recorder shelter, $2.90 \mathrm{ft}$ above land-surface datum.

REMARKS.--Well pumped and sounded July 19, 1978. Borehole geophysical survey conducted June 5, 1975.

PERIOD OF RECORD.--May 1972 to current year.

EXTREMES FOR PERIOD OF RECORD.--Highest water level, $191.50 \mathrm{ft}$ below land-surface datum, May 17, 1973; lowest, $204.67 \mathrm{ft}$ below land-surface datum, August 10, 1986.

DEPTH BELOW LAND SURFACE (WATER LEVEL) (FEET) CALENDAR YEAR JANUARY 1987 TO DECEMBER 1987 MEAN VALUES

\begin{tabular}{|c|c|c|c|c|c|c|c|c|c|c|c|c|}
\hline DAY & JAN & FEB & MAR & APR & MAY & JUN & JUL & AUG & SEP & OCT & NOV & DEC \\
\hline $\begin{array}{l}1 \\
2 \\
3 \\
4 \\
5\end{array}$ & $\begin{array}{l}201.73 \\
201.96 \\
202.03 \\
201.76 \\
201.90\end{array}$ & $\begin{array}{l}201.58 \\
201.33 \\
201.44 \\
201.58 \\
201.65\end{array}$ & $\begin{array}{l}201.00 \\
201.19 \\
201.30 \\
201.39 \\
201.45\end{array}$ & $\begin{array}{l}200.89 \\
200.79 \\
200.64 \\
200.75 \\
200.68\end{array}$ & $\begin{array}{l}201.53 \\
201.54 \\
201.56 \\
201.61 \\
201.68\end{array}$ & $\begin{array}{l}201.58 \\
201.71 \\
201.85 \\
201.84 \\
201.81\end{array}$ & $\begin{array}{l}201.77 \\
201.74 \\
201.76 \\
201.74 \\
201.75\end{array}$ & $\begin{array}{l}201.77 \\
201.72 \\
201.74 \\
201.73 \\
201.72\end{array}$ & $\begin{array}{l}202.08 \\
202.08 \\
202.10 \\
202.11 \\
202.07\end{array}$ & $\begin{array}{l}202.10 \\
202.20 \\
202.27 \\
202.37 \\
202.25\end{array}$ & $\begin{array}{l}202.63 \\
202.54 \\
202.41 \\
202.21 \\
202.21\end{array}$ & $\begin{array}{l}201.86 \\
202.09 \\
202.07 \\
201.94 \\
202.11\end{array}$ \\
\hline $\begin{array}{r}6 \\
7 \\
8 \\
9 \\
10\end{array}$ & $\begin{array}{l}202.00 \\
201.93 \\
201.93 \\
201.88 \\
201.67\end{array}$ & $\begin{array}{l}201.48 \\
201.30 \\
201.26 \\
201.55 \\
201.70\end{array}$ & $\begin{array}{l}201.45 \\
201.16 \\
200.86\end{array}$ & $\begin{array}{l}200 . \\
200 . \\
200 .\end{array}$ & $\begin{array}{l}201.68 \\
201.62 \\
201.57 \\
201.70 \\
201.74\end{array}$ & $\begin{array}{l}201.85 \\
201.90 \\
201.93 \\
202.01 \\
202.09\end{array}$ & $\begin{array}{l}201.76 \\
201.74 \\
201.75 \\
201.78 \\
201.75\end{array}$ & $\begin{array}{l}201.77 \\
201.84 \\
201.86 \\
201.82 \\
201.77\end{array}$ & $\begin{array}{l}202.08 \\
202.07 \\
202.09 \\
202.13 \\
202.17\end{array}$ & & $\begin{array}{l}202.51 \\
202.59 \\
202.55 \\
202.44 \\
202.24\end{array}$ & $\begin{array}{l}202.19 \\
202.18 \\
202.12 \\
202.04 \\
201.91\end{array}$ \\
\hline $\begin{array}{l}11 \\
12 \\
13 \\
14 \\
15\end{array}$ & $\begin{array}{l}201.90 \\
201.94 \\
201.91 \\
201.89 \\
201.77\end{array}$ & $\begin{array}{l}201.61 \\
201.42 \\
201.35 \\
201.22 \\
201.13\end{array}$ & $\begin{array}{l}201.24 \\
201.27 \\
201.22 \\
201.16 \\
201.13\end{array}$ & $\begin{array}{l}200.55 \\
200.60 \\
200.65 \\
200.65 \\
200.51\end{array}$ & $\begin{array}{l}01.69 \\
01.62\end{array}$ & $\begin{array}{l}202.20 \\
202.30 \\
202.28 \\
202.13 \\
202.06\end{array}$ & $\begin{array}{l}201.71 \\
201.65 \\
201.63 \\
201.61 \\
201.66\end{array}$ & $\begin{array}{l}201.73 \\
201.77 \\
201.83 \\
201.89 \\
201.91\end{array}$ & & & $\begin{array}{l}202.52 \\
202.46 \\
202.43 \\
202.51\end{array}$ & $\begin{array}{l}201.82 \\
201.79 \\
202.01 \\
202.04 \\
201.88\end{array}$ \\
\hline $\begin{array}{l}16 \\
17 \\
18 \\
19 \\
20\end{array}$ & $\begin{array}{l}201.75 \\
201.60 \\
201.51 \\
201.77\end{array}$ & $\begin{array}{l}200.96 \\
201.14 \\
201.29 \\
201.48 \\
201.49\end{array}$ & $\begin{array}{l}200.93 \\
200.79 \\
200.84\end{array}$ & $\begin{array}{l}200.43 \\
200.54 \\
200.72 \\
200.79\end{array}$ & $\begin{array}{l}201.48 \\
201.44 \\
201.44\end{array}$ & $\begin{array}{l}202.00 \\
202.00 \\
202.03 \\
201.98 \\
201.89\end{array}$ & $\begin{array}{l}201.77 \\
201.89 \\
201.92 \\
201.91 \\
201.94\end{array}$ & $\begin{array}{l}201.93 \\
201.93 \\
201.92 \\
201.94\end{array}$ & $\begin{array}{l}202.10 \\
202.10\end{array}$ & $\begin{array}{l}202.38 \\
202.33 \\
202.31 \\
202.33 \\
202.38\end{array}$ & $\begin{array}{l}202.34 \\
202.44 \\
202.40 \\
202.31\end{array}$ & $\begin{array}{l}202.11 \\
202.26 \\
202.32 \\
202.26 \\
202.17\end{array}$ \\
\hline $\begin{array}{l}21 \\
22 \\
23 \\
24 \\
25\end{array}$ & $\begin{array}{l}201.68 \\
201.35 \\
201.73 \\
201.82 \\
201.47\end{array}$ & $\begin{array}{l}201.29 \\
201.06 \\
201.24 \\
201.40 \\
201.43\end{array}$ & $\begin{array}{l}200.74 \\
200.76 \\
200.71 \\
200.75\end{array}$ & $\begin{array}{l}200.76 \\
200.76 \\
200.77 \\
200.89\end{array}$ & $\begin{array}{l}201.54 \\
201.52 \\
201.47 \\
201.46\end{array}$ & $\begin{array}{l}201.85 \\
201.80 \\
201.74 \\
201.71 \\
201.64\end{array}$ & $\begin{array}{l}201.95 \\
201.90 \\
201.75 \\
201.75 \\
201.78\end{array}$ & $\begin{array}{l}202.04 \\
202.05 \\
201.99 \\
201.98 \\
202.04\end{array}$ & $\begin{array}{l}202.18 \\
202.21 \\
202.19 \\
202.14 \\
202.15\end{array}$ & $\begin{array}{l}202.50 \\
202.65 \\
202.67 \\
202.66 \\
202.56\end{array}$ & $\begin{array}{l}202.3 \\
202.4 \\
202.4 \\
202.3\end{array}$ & $\begin{array}{l}202.12 \\
202.07 \\
202.21 \\
202.23 \\
202.17\end{array}$ \\
\hline $\begin{array}{l}26 \\
27 \\
28 \\
29 \\
30 \\
31\end{array}$ & $\begin{array}{l}201.55 \\
201.78 \\
201.80 \\
201.77 \\
201.53 \\
201.59\end{array}$ & $\begin{array}{r}201.43 \\
201.32 \\
201.10 \\
--- \\
--- \\
---\end{array}$ & $\begin{array}{l}200.75 \\
200.65 \\
200.69 \\
200.72 \\
200.49 \\
200.69\end{array}$ & $\begin{array}{r}201.07 \\
201.09 \\
201.10 \\
201.35 \\
201.43 \\
---\end{array}$ & $\begin{array}{l}201.52 \\
201.57 \\
201.58 \\
201.57 \\
201.54 \\
201.54\end{array}$ & $\begin{array}{r}201.62 \\
201.63 \\
201.73 \\
201.78 \\
201.80 \\
.--\end{array}$ & $\begin{array}{l}201.74 \\
201.72 \\
201.72 \\
201.75 \\
201.82 \\
201.84\end{array}$ & $\begin{array}{l}202.08 \\
202.07 \\
202.05 \\
202.04 \\
202.05 \\
202.07\end{array}$ & $\begin{array}{r}202.24 \\
202.32 \\
202.33 \\
202.21 \\
201.99 \\
---\end{array}$ & $\begin{array}{l}202.41 \\
202.29 \\
202.43 \\
202.55 \\
202.62 \\
202.67\end{array}$ & $\begin{array}{r}202.11 \\
201.98 \\
201.88 \\
201.83 \\
201.76 \\
---\end{array}$ & $\begin{array}{l}202.14 \\
202.13 \\
201.98 \\
202.16 \\
202.39 \\
202.34\end{array}$ \\
\hline & 1 & 01.37 & 00. & 00.75 & 01.56 & 9 & 201. & 0 & 202 & 02 & 4 & \\
\hline
\end{tabular}

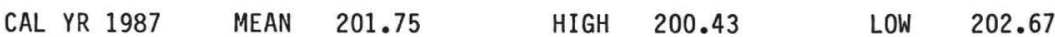




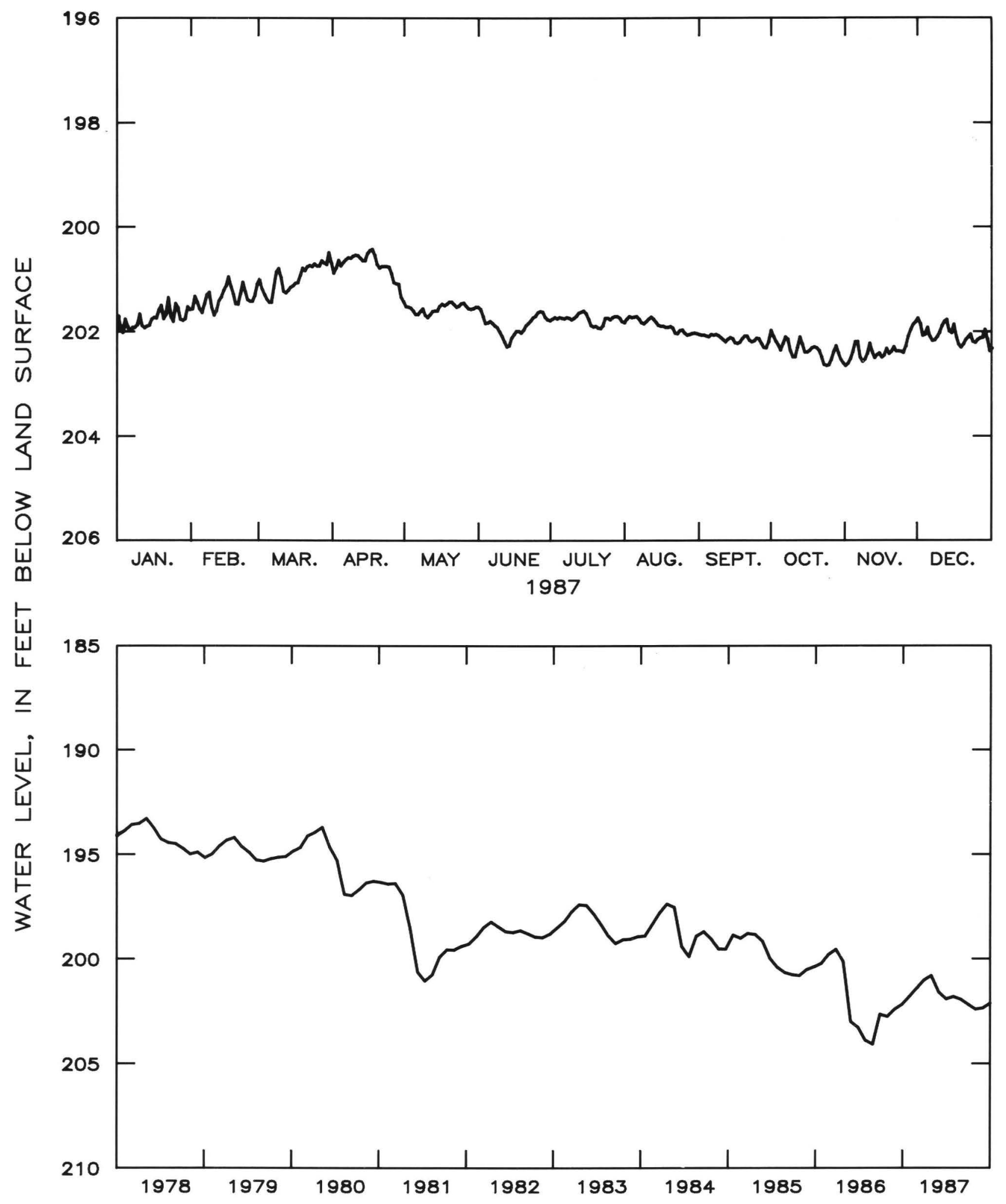

Figure 2.7.2-2.--Water level in observation well 15LO20, Worth County. 
312712082593301 , Local number, 18K049.

LOCATION.--Lat $31^{\circ} 27^{\prime} 12^{\prime \prime}$, long $82^{\circ} 59^{\prime} 33^{\prime \prime}$, Hydrologic Unit 03110203 , near the intersection of Goff Street and Ferry Lake Road, at city of Tifton Maintence and Water Works, on east. side of Tifton.

Owner: U.S. Geological Survey

AQUIFER.--Upper Floridan aquifer.

WELL CHARACTERISTICS.--Drilled observation well, diameter 6 in., depth $620 \mathrm{ft}$, cased to $270 \mathrm{ft}$.

DATUM.--Elevation of land-surface datum is $330 \mathrm{ft}$.

Measuring point: Floor of recorder shelter, $3.0 \mathrm{ft}$ above land-surface datum.

REMARKS.--Well sounded March 10, 1978. Borehole geophysical survey conducted.

PERIOD OF RECORD.--March 28, 1978 to current year.

EXTREMES FOR PERIOD OF RECORD.--Highest water level, $102.70 \mathrm{ft}$ below land-surface datum, May 14, 1978; 1owest, $120.77 \mathrm{ft}$ below land-surface datum, July 22, 1986.

DEPTH BELOW LAND SURFACE (WATER LEVEL) (FEET), CALENDAR YEAR JANUARY 1987 TO DECEMBER 1987 MEAN VALUES

\begin{tabular}{|c|c|c|c|c|c|c|c|c|c|c|c|c|}
\hline AY & JAN & FEB & MAR & APR & MAY & JUN & JUL & AUG & SEP & OCT & NOV & \\
\hline $\begin{array}{l}1 \\
2 \\
3 \\
4 \\
5\end{array}$ & $\begin{array}{l}115.97 \\
116.21 \\
116.31 \\
116.04 \\
116.15\end{array}$ & $\begin{array}{l}115.53 \\
115.33 \\
115.44 \\
115.56 \\
115.62\end{array}$ & $\begin{array}{l}114.63 \\
114.81 \\
114.95 \\
115.04 \\
115.11\end{array}$ & $\begin{array}{l}114.56 \\
114.52 \\
114.43 \\
114.37 \\
114.23\end{array}$ & $\begin{array}{l}117.10 \\
117.20 \\
117.20 \\
117.48 \\
117.67\end{array}$ & $\begin{array}{l}116.44 \\
116.93 \\
117.23 \\
117.27 \\
117.44\end{array}$ & $\begin{array}{l}116.65 \\
116.73 \\
116.60 \\
116.41 \\
116.25\end{array}$ & $\begin{array}{l}118.85 \\
118.74 \\
118.81 \\
118.87 \\
118.97\end{array}$ & $\begin{array}{l}119.20 \\
119.14 \\
119.05 \\
118.90 \\
118.54\end{array}$ & $\begin{array}{l}118.38 \\
118.44 \\
118.37 \\
118.34 \\
118.36\end{array}$ & $\begin{array}{l}119.19 \\
119.23 \\
119.16 \\
118.86 \\
118.76\end{array}$ & 117 \\
\hline $\begin{array}{r}6 \\
7 \\
8 \\
9 \\
10\end{array}$ & $\begin{array}{l}116.29 \\
116.27 \\
116.27 \\
116.18 \\
115.94\end{array}$ & $\begin{array}{l}5.50 \\
5.30 \\
5.19 \\
5.42 \\
5.62\end{array}$ & $\begin{array}{l}82 \\
47 \\
43 \\
63\end{array}$ & $\begin{array}{l}114.30 \\
114.41 \\
114.59 \\
114.68 \\
114.84\end{array}$ & $\begin{array}{l}117.60 \\
117.65 \\
117.20 \\
116.98 \\
116.93\end{array}$ & & $\begin{array}{l}16.32 \\
16.52 \\
16.57 \\
16.48 \\
16.68\end{array}$ & $\begin{array}{l}19.05 \\
19.43 \\
19.67 \\
19.80 \\
20.06\end{array}$ & $\begin{array}{l}18.24 \\
18.04 \\
18.16 \\
18.32 \\
18.54\end{array}$ & $\begin{array}{l}8.44 \\
8.60 \\
8.91 \\
9.11 \\
8.98\end{array}$ & $\begin{array}{l}118.89 \\
118.91 \\
118.90 \\
118.93 \\
118.67\end{array}$ & 1 \\
\hline $\begin{array}{l}11 \\
12 \\
13 \\
14 \\
15\end{array}$ & $\begin{array}{l}115.99 \\
116.05 \\
116.09 \\
116.09 \\
115.98\end{array}$ & $\begin{array}{l}115.55 \\
115.40 \\
115.35 \\
115.19 \\
115.00\end{array}$ & $\begin{array}{l}114.87 \\
114.91 \\
114.90 \\
114.81 \\
114.68\end{array}$ & $\begin{array}{l}114.81 \\
114.78 \\
114.94 \\
114.96 \\
114.74\end{array}$ & & & & & & $\begin{array}{l}118.69 \\
118.63 \\
118.90 \\
119.12 \\
119.24\end{array}$ & $\begin{array}{l}118.69 \\
118.74 \\
118.68 \\
118.53 \\
118.51\end{array}$ & 117 \\
\hline $\begin{array}{l}16 \\
17 \\
18 \\
19 \\
20\end{array}$ & $\begin{array}{l}115.91 \\
115.85 \\
115.64 \\
115.61 \\
115.82\end{array}$ & $\begin{array}{l}114.84 \\
115.02 \\
115.16 \\
115.31 \\
115.32\end{array}$ & $\begin{array}{l}114.68 \\
114.76 \\
114.61 \\
114.47 \\
114.53\end{array}$ & $\begin{array}{l}114.75 \\
114.68 \\
114.76 \\
114.88 \\
115.15\end{array}$ & & & & & & & $\begin{array}{l}118.58 \\
118.51 \\
118.54 \\
118.49 \\
118.32\end{array}$ & \\
\hline $\begin{array}{l}21 \\
22 \\
23 \\
24 \\
25\end{array}$ & $\begin{array}{l}115.76 \\
115.50 \\
115.81 \\
115.83 \\
115.47\end{array}$ & $\begin{array}{l}115.11 \\
114.85 \\
114.98 \\
115.12 \\
115.16\end{array}$ & $\begin{array}{l}114.44 \\
114.32 \\
114.47 \\
114.52 \\
114.61\end{array}$ & $\begin{array}{l}115.47 \\
115.70 \\
115.71 \\
115.79 \\
115.94\end{array}$ & $\begin{array}{l}116 \\
116 \\
115 \\
115 \\
115\end{array}$ & $\begin{array}{l}117.15 \\
117.04 \\
116.89 \\
116.79 \\
116.70\end{array}$ & $\begin{array}{l}119.13 \\
119.08 \\
119.28 \\
119.61 \\
119.43\end{array}$ & & & & $\begin{array}{l}118.29 \\
118.22 \\
118.41 \\
118.38 \\
118.24\end{array}$ & $\begin{array}{l}117 \\
117\end{array}$ \\
\hline $\begin{array}{l}26 \\
27 \\
28 \\
29 \\
30 \\
31\end{array}$ & $\begin{array}{l}115.54 \\
115.77 \\
115.81 \\
115.81 \\
115.64 \\
115.63\end{array}$ & $\begin{array}{r}115.17 \\
115.09 \\
114.85 \\
--- \\
--- \\
---\end{array}$ & $\begin{array}{l}114.57 \\
114.42 \\
114.37 \\
114.31 \\
114.18 \\
114.38\end{array}$ & $\begin{array}{r}116.08 \\
116.13 \\
116.41 \\
116.69 \\
116.94 \\
---\end{array}$ & $\begin{array}{l}115.97 \\
116.11 \\
116.09 \\
116.18 \\
116.29 \\
116.24\end{array}$ & $\begin{array}{r}116.57 \\
116.41 \\
116.34 \\
116.43 \\
116.55 \\
---\end{array}$ & $\begin{array}{l}119.19 \\
119.02 \\
118.80 \\
118.84 \\
119.11 \\
119.17\end{array}$ & $\begin{array}{l}118.82 \\
119.12 \\
119.30 \\
119.36 \\
119.31 \\
119.40\end{array}$ & $\begin{array}{r}118.26 \\
118.24 \\
118.38 \\
118.44 \\
118.31 \\
---\end{array}$ & $\begin{array}{l}119.35 \\
119.31 \\
119.27 \\
119.33 \\
119.47 \\
119.41\end{array}$ & $\begin{array}{r}117.99 \\
117.79 \\
117.65 \\
117.55 \\
117.69 \\
---\end{array}$ & $\begin{array}{l}117 \\
117\end{array}$ \\
\hline EAN & 115.91 & 115.25 & 114.64 & 115.14 & 116.51 & 117.54 & 117.88 & 118.96 & 118.41 & 119.03 & 118.51 & 111 \\
\hline
\end{tabular}

$\begin{array}{lllllll}\text { CAL YR } 1987 & \text { MEAN } & 117.14 & \text { HIGH } & 114.18 & \text { LOW } & 120.35\end{array}$ 


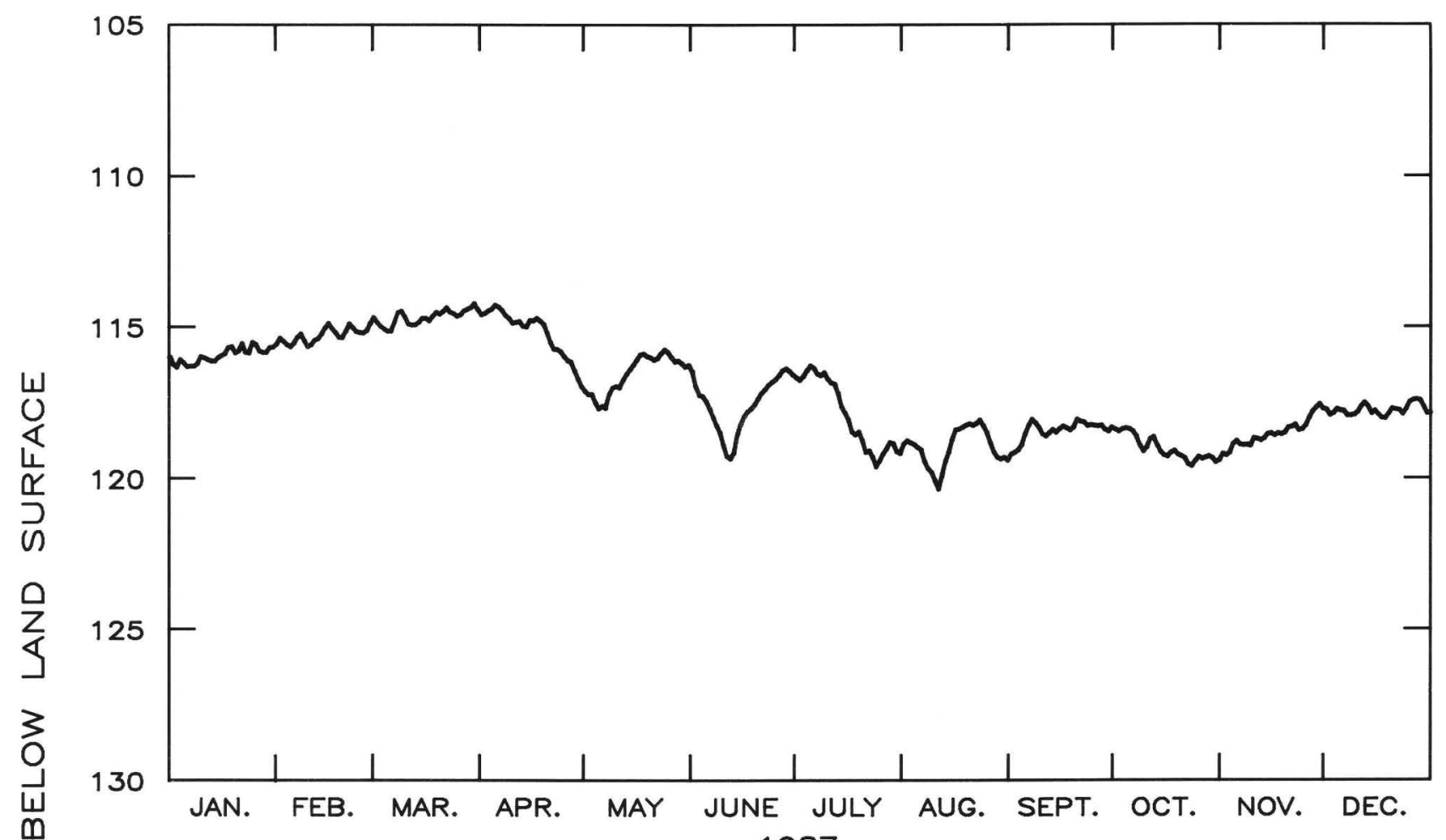
1987

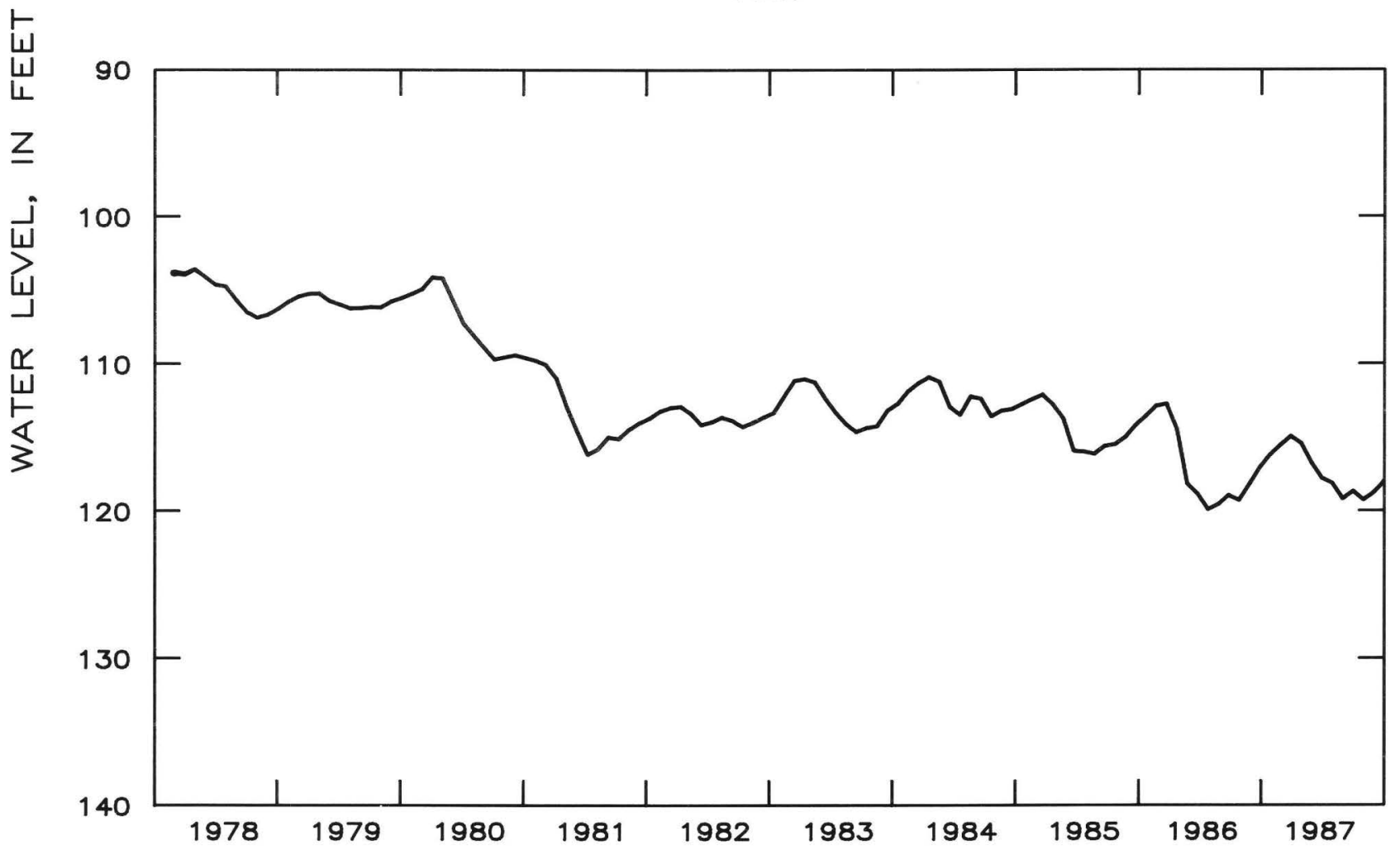

Figure 2.7.2-3.--Water level in observation well 18K049, Tift County. 
18 H016 ADEL COOK COUNTY

310813083260301 Local number, $18 \mathrm{H} 016$.

LOCATION.--Lat $31^{\circ} 08^{\prime} 13^{\prime \prime}$, long $83^{\circ} 26^{\prime} 03^{\prime \prime}$, Hydrologic Unit 03110203, on West Second Street near intersection of Georgia Highways 76 and 37.

Owner: U.S. Geological Survey, Adel test well.

AQUIFER.--Upper Floridan aquifer.

WELL CHARACTERISTICS.--Drilled observation well, diameter $8 \mathrm{in}$., depth $865 \mathrm{ft}$, cased to $207 \mathrm{ft}$, open hole. DATUM.--Elevation of land-surface datum is $241 \mathrm{ft}$.

Measuring point: Floor of recorder shelter, $2.66 \mathrm{ft}$ above land-surface datum.

REMARKS.--Well pumped July 19, 1978; water-quality sample collected at conclusion of pumping. Borehole geophysical survey conducted October 24, 1974.

PERIOD OF RECORD.--December 1964 to current year.

EXTREMES FOR PERIOD OF RECORD.--Highest water level, $163.34 \mathrm{ft}$ below land-surface datum, July 5, 1966; 1owest, $175.14 \mathrm{ft}$ below land-surface datum, July 23, 1985.

DEPTH BELOW LAND SURFACE (WATER LEVEL) (FEET) CALENDAR YEAR JANUARY 1987 TO DECEMBER 1987 MEAN VALUES

\begin{tabular}{|c|c|c|c|c|c|c|c|c|c|c|c|c|}
\hline DAY & JAN & FEB & MAR & APR & MAY & JUN & JUL & AUG & SEP & OCT & NOV & DEC \\
\hline $\begin{array}{l}1 \\
2 \\
3 \\
4 \\
5\end{array}$ & $\begin{array}{l}173.03 \\
173.32 \\
173.42 \\
173.12 \\
173.37\end{array}$ & $\begin{array}{l}172.91 \\
172.73 \\
172.79 \\
172.86 \\
172.91\end{array}$ & $\begin{array}{l}172.04 \\
172.23 \\
172.39 \\
172.45 \\
172.53\end{array}$ & $\begin{array}{l}172.01 \\
171.89 \\
171.70 \\
171.79 \\
171.64\end{array}$ & $\begin{array}{l}172.34 \\
172.29 \\
172.29 \\
172.44 \\
172.64\end{array}$ & $\begin{array}{l}172.93 \\
173.11 \\
173.13 \\
172.83 \\
172.75\end{array}$ & $\begin{array}{l}172.83 \\
172.85 \\
172.86 \\
172.80 \\
172.82\end{array}$ & $\begin{array}{l}173.47 \\
173.37 \\
173.46 \\
173.50 \\
173.51\end{array}$ & $\begin{array}{l}173.52 \\
173.51 \\
173.45 \\
173.45 \\
173.38\end{array}$ & $\begin{array}{l}173.40 \\
173.50 \\
173.49 \\
173.56 \\
173.54\end{array}$ & $\begin{array}{l}174.32 \\
174.22 \\
174.13 \\
173.75 \\
173.74\end{array}$ & $\begin{array}{l}173.48 \\
173.67 \\
173.69 \\
173.55 \\
173.69\end{array}$ \\
\hline $\begin{array}{r}6 \\
7 \\
8 \\
9 \\
10\end{array}$ & $\begin{array}{l}173.44 \\
173.39 \\
173.36 \\
173.31 \\
172.96\end{array}$ & $\begin{array}{l}172.77 \\
172.64 \\
172.58 \\
172.85 \\
173.03\end{array}$ & $\begin{array}{l}172.54 \\
172.21 \\
171.91 \\
171.92 \\
171.99\end{array}$ & $\begin{array}{l}171.67 \\
171.67 \\
171.68 \\
171.66 \\
171.62\end{array}$ & $\begin{array}{l}172.59 \\
172.58 \\
172.30 \\
172.38 \\
172.56\end{array}$ & $\begin{array}{l}172.72 \\
172.76 \\
172.94 \\
173.01 \\
173.07\end{array}$ & $\begin{array}{l}172.78 \\
172.76 \\
172.79 \\
172.85 \\
172.93\end{array}$ & $\begin{array}{l}173.52 \\
173.64 \\
173.71 \\
173.70 \\
173.76\end{array}$ & $\begin{array}{l}173.28 \\
173.19 \\
173.25 \\
173.31 \\
173.37\end{array}$ & $\begin{array}{l}173.44 \\
173.49 \\
173.67 \\
173.95 \\
174.07\end{array}$ & $\begin{array}{l}174.04 \\
174.20 \\
174.14 \\
174.05 \\
173.75\end{array}$ & 173.83 \\
\hline $\begin{array}{l}11 \\
12 \\
13 \\
14 \\
15\end{array}$ & $\begin{array}{l}173.19 \\
173.38 \\
173.33 \\
173.36 \\
173.17\end{array}$ & $\begin{array}{l}172.96 \\
172.76 \\
172.68 \\
172.51 \\
172.32\end{array}$ & $\begin{array}{l}172.25 \\
172.31 \\
172.27 \\
172.16 \\
172.04\end{array}$ & $\begin{array}{l}171.61 \\
171.60 \\
171.69 \\
171.70 \\
171.57\end{array}$ & $\begin{array}{l}172.56 \\
172.56 \\
172.54 \\
172.19 \\
172.10\end{array}$ & $\begin{array}{l}173.22 \\
173.35 \\
173.15 \\
172.81 \\
172.82\end{array}$ & $\begin{array}{l}172.93 \\
172.92 \\
173.07 \\
173.14 \\
173.02\end{array}$ & $\begin{array}{l}173.53 \\
173.50 \\
173.45 \\
173.47 \\
173.39\end{array}$ & $\begin{array}{l}173.39 \\
173.34 \\
173.31 \\
173.30 \\
173.38\end{array}$ & $\begin{array}{l}174.01 \\
173.58 \\
173.69 \\
173.85 \\
173.92\end{array}$ & $\begin{array}{l}173.90 \\
174.14 \\
174.14 \\
174.12 \\
174.11\end{array}$ & $\begin{array}{l}173.47 \\
173.49\end{array}$ \\
\hline $\begin{array}{l}16 \\
17 \\
18 \\
19 \\
20\end{array}$ & $\begin{array}{l}173.12 \\
173.12 \\
172.80 \\
172.83 \\
173.10\end{array}$ & $\begin{array}{l}172.12 \\
172.36 \\
172.50 \\
172.68 \\
172.70\end{array}$ & $\begin{array}{l}172.06 \\
172.28 \\
172.14 \\
171.93 \\
171.95\end{array}$ & $\begin{array}{l}171.55 \\
171.44 \\
171.39 \\
171.58 \\
171.79\end{array}$ & $\begin{array}{l}172.07 \\
172.08 \\
172.08 \\
172.05 \\
172.07\end{array}$ & $\begin{array}{l}172.80 \\
172.77 \\
172.83 \\
172.81 \\
172.71\end{array}$ & $\begin{array}{l}173.15 \\
173.40 \\
173.43 \\
173.39 \\
173.57\end{array}$ & $\begin{array}{l}173.35 \\
173.42 \\
173.40 \\
173.37 \\
173.37\end{array}$ & $\begin{array}{l}173.38 \\
173.41 \\
173.35 \\
173.29 \\
173.27\end{array}$ & $\begin{array}{l}173.93 \\
173.94 \\
174.06 \\
174.09 \\
174.11\end{array}$ & $\begin{array}{l}174.14 \\
174.11 \\
174.11 \\
173.99 \\
173.81\end{array}$ & $\begin{array}{l}173.7 \\
173.7\end{array}$ \\
\hline $\begin{array}{l}21 \\
22 \\
23 \\
24 \\
25\end{array}$ & $\begin{array}{l}173.00 \\
172.72 \\
173.20 \\
173.24 \\
172.77\end{array}$ & $\begin{array}{l}172.53 \\
172.25 \\
172.37 \\
172.54 \\
172.57\end{array}$ & $\begin{array}{l}171.89 \\
171.74 \\
171.83 \\
171.87 \\
171.88\end{array}$ & $\begin{array}{l}171.88 \\
171.90 \\
171.88 \\
171.88 \\
171.93\end{array}$ & $\begin{array}{l}172.13 \\
172.20 \\
172.22 \\
172.13 \\
172.25\end{array}$ & $\begin{array}{l}172.60 \\
172.57 \\
172.57 \\
172.59 \\
172.56\end{array}$ & $\begin{array}{l}173.71 \\
173.64 \\
173.70 \\
173.89 \\
173.67\end{array}$ & $\begin{array}{l}173.43 \\
173.47 \\
173.34 \\
173.41 \\
173.48\end{array}$ & $\begin{array}{l}173.30 \\
173.34 \\
173.36 \\
173.35 \\
173.41\end{array}$ & $\begin{array}{l}174.15 \\
174.25 \\
174.25 \\
174.29 \\
174.13\end{array}$ & $\begin{array}{l}174.01 \\
174.03 \\
174.03 \\
174.12 \\
174.04\end{array}$ & $\begin{array}{l}173.6 \\
173.6 \\
173.7 \\
173.7 \\
173.6\end{array}$ \\
\hline $\begin{array}{l}26 \\
27 \\
28 \\
29 \\
30 \\
31\end{array}$ & $\begin{array}{l}172.86 \\
173.30 \\
173.25 \\
173.23 \\
172.98 \\
172.94\end{array}$ & $\begin{array}{r}172.58 \\
172.47 \\
172.22 \\
--- \\
--- \\
---\end{array}$ & $\begin{array}{l}171.91 \\
171.73 \\
171.75 \\
171.85 \\
171.59 \\
171.78\end{array}$ & $\begin{array}{r}171.98 \\
172.06 \\
172.06 \\
172.20 \\
172.29 \\
----\end{array}$ & $\begin{array}{l}172.49 \\
172.65 \\
172.71 \\
172.81 \\
172.86 \\
172.79\end{array}$ & $\begin{array}{r}172.52 \\
172.49 \\
172.61 \\
172.67 \\
172.77 \\
---\end{array}$ & $\begin{array}{l}173.53 \\
173.56 \\
173.60 \\
173.59 \\
173.62 \\
173.59\end{array}$ & $\begin{array}{l}173.54 \\
173.59 \\
173.61 \\
173.57 \\
173.54 \\
173.56\end{array}$ & $\begin{array}{r}173.49 \\
173.56 \\
173.66 \\
173.54 \\
173.32 \\
---\end{array}$ & $\begin{array}{l}174.06 \\
173.80 \\
174.03 \\
174.18 \\
174.36 \\
174.36\end{array}$ & $\begin{array}{r}173.77 \\
173.64 \\
173.61 \\
173.51 \\
173.43 \\
-\cdots\end{array}$ & $\begin{array}{l}173.5 \\
173.5 \\
173.5 \\
173.6 \\
174.0 \\
174.0\end{array}$ \\
\hline MEAI & 173.15 & 72.61 & 172.05 & 171.78 & 172.39 & 172.82 & 173.24 & 173.50 & 173.38 & 173.91 & 173.97 & 173.6 \\
\hline CAL & 87 & TLAIV & .04 & $\mathrm{HIGH}$ & 171.39 & & & & & & & \\
\hline
\end{tabular}



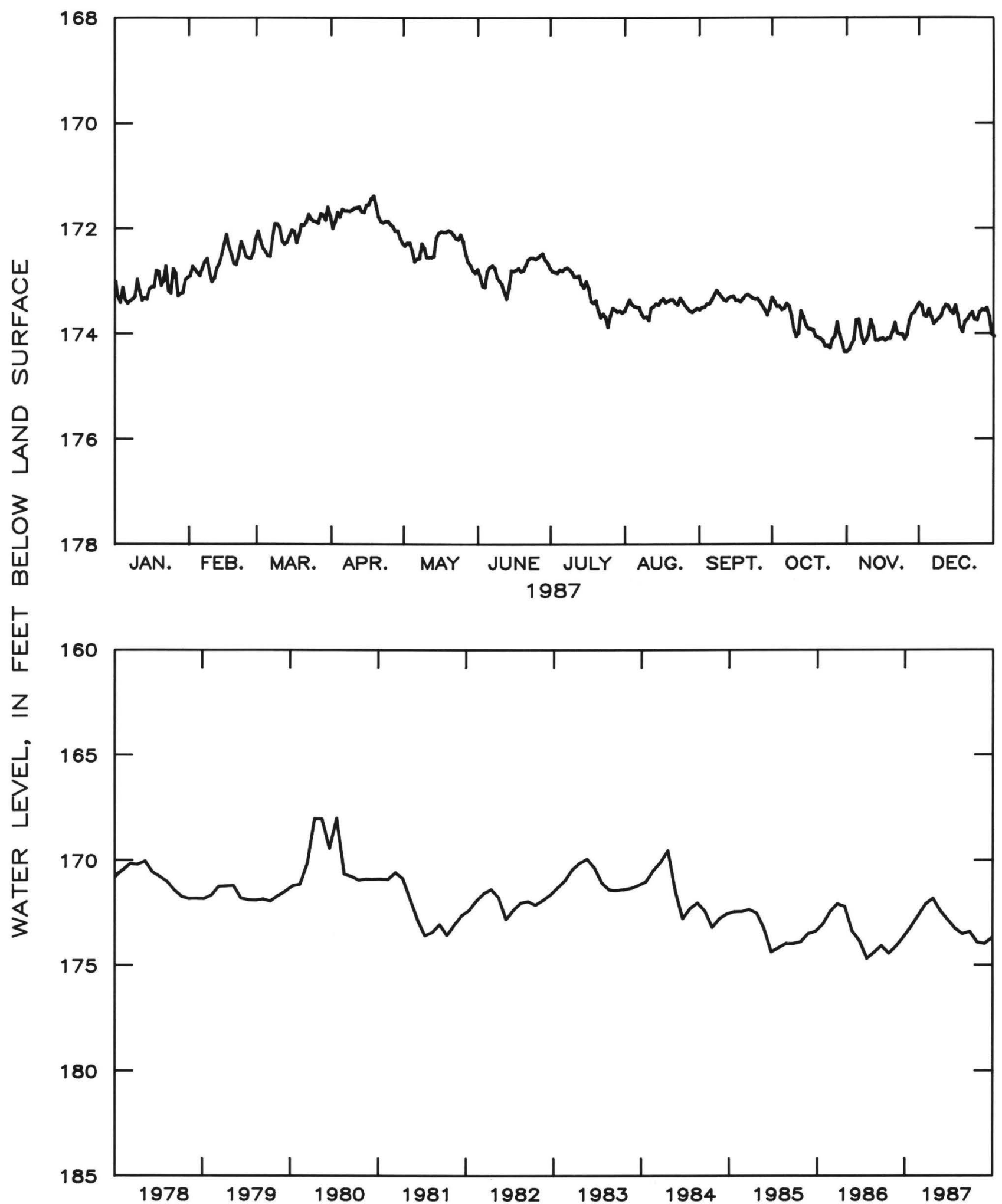

Figure 2.7.2-4.--Water level in observation well 18H016, Cook County. 
305241083154401 Local number, $19 \mathrm{~F} 039$.

LOCATION.--Lat $30^{\circ} 52^{\prime} 41^{\prime \prime}$, long $83^{\circ} 15^{\prime} 44^{\prime \prime}$, Hydrologic Unit 03110203, at water tank by Valdosta High School. Owner: City of Valdosta, well 8.

AQUIFER.--Upper Floridan aquifer.

WELL CHARACTERISTICS.--Drilled observation well, depth $450 \mathrm{ft}$, cased to $350 \mathrm{ft}$, open hole.

DATUM.--Elevation of land-surface datum is $222 \mathrm{ft}$.

Measuring point: Pump base, $1.40 \mathrm{ft}$ above land-surface datum.

REMARKS.--Water levels for period of missing record, July 2-9, were estimated.

PERIOD OF RECORD.--February 1979 to current year.

EXTREMES FOR PERIOD OF RECORD.--Highest water level, $114.28 \mathrm{ft}$ below land-surface datum, April 9, 1984 ; 10west, $145.67 \mathrm{ft}$ below land-surface datum, October 24, 1981.

DEPTH BELOW LAND SURFACE (WATER LEVEL) (FEET) CALENDAR YEAR JANUARY 1987 TO DECEMBER 1987 MEAN VALUES

\begin{tabular}{|c|c|c|c|c|c|c|c|c|c|c|c|c|}
\hline$A Y$ & JAN & FEB & MAR & APR & MAY & JUN & JUL & AUG & SEP & ОСТ & NOV & DEC \\
\hline $\begin{array}{l}1 \\
2 \\
3 \\
4 \\
5\end{array}$ & $\begin{array}{l}122.06 \\
121.96 \\
121.71 \\
121.23 \\
120.95\end{array}$ & $\begin{array}{l}118.26 \\
118.22 \\
118.28 \\
118.41 \\
118.52\end{array}$ & $\begin{array}{l}116.52 \\
116.58 \\
116.58 \\
116.57 \\
116.53\end{array}$ & $\begin{array}{l}117.14 \\
116.84 \\
116.57 \\
116.46 \\
116.34\end{array}$ & $\begin{array}{l}122.48 \\
122.65 \\
122.93 \\
123.53 \\
124.69\end{array}$ & $\begin{array}{l}124.67 \\
126.23 \\
127.35 \\
128.04 \\
126.34\end{array}$ & $\begin{array}{l}126.61 \\
126.50 \\
126.33 \\
126.10 \\
125.90\end{array}$ & $\begin{array}{l}130.34 \\
131.33 \\
131.88 \\
132.74 \\
133.40\end{array}$ & $\begin{array}{l}131.74 \\
132.13 \\
132.47 \\
132.52 \\
132.73\end{array}$ & $\begin{array}{l}136.78 \\
137.13 \\
137.46 \\
137.90 \\
138.03\end{array}$ & $\begin{array}{l}144.48 \\
144.58 \\
144.59 \\
144.29 \\
144.20\end{array}$ & $\begin{array}{l}140.00 \\
140.09 \\
140.20 \\
140.10 \\
140.17\end{array}$ \\
\hline $\begin{array}{r}6 \\
7 \\
8 \\
9 \\
10\end{array}$ & $\begin{array}{l}120.48 \\
120.61 \\
120.50 \\
120.43 \\
120.22\end{array}$ & $\begin{array}{l}118.63 \\
118.45 \\
118.35 \\
118.25 \\
118.42\end{array}$ & $\begin{array}{l}116.39 \\
116.22 \\
116.17 \\
116.29 \\
116.56\end{array}$ & $\begin{array}{l}116.35 \\
116.50 \\
116.77 \\
117.06 \\
117.39\end{array}$ & $\begin{array}{l}125.48 \\
126.09 \\
126.13 \\
126.33 \\
126.65\end{array}$ & $\begin{array}{l}125.65 \\
124.91 \\
124.52 \\
124.56 \\
124.72\end{array}$ & $\begin{array}{l}125.70 \\
125.58 \\
125.51 \\
125.50 \\
125.56\end{array}$ & $\begin{array}{l}134.29 \\
135.05 \\
135.72 \\
136.26 \\
136.76\end{array}$ & $\begin{array}{l}132.08 \\
129.99 \\
129.13 \\
128.88 \\
128.72\end{array}$ & $\begin{array}{l}138.23 \\
138.60 \\
139.00 \\
139.54 \\
139.83\end{array}$ & $\begin{array}{l}144.46 \\
144.69 \\
144.65 \\
144.76 \\
144.60\end{array}$ & $\begin{array}{l}140.18 \\
140.20\end{array}$ \\
\hline $\begin{array}{l}11 \\
12 \\
13 \\
14 \\
15\end{array}$ & $\begin{array}{l}120.28 \\
120.32 \\
120.35 \\
120.43 \\
120.44\end{array}$ & $\begin{array}{l}118.42 \\
118.38 \\
118.40 \\
118.42 \\
118.45\end{array}$ & $\begin{array}{l}116.85 \\
116.96 \\
116.94 \\
116.91 \\
116.99\end{array}$ & $\begin{array}{l}117.87 \\
118.20 \\
118.40 \\
118.42 \\
118.57\end{array}$ & $\begin{array}{l}126.93 \\
127.10 \\
127.59 \\
127.23 \\
124.68\end{array}$ & $\begin{array}{l}124.96 \\
125.25 \\
126.40 \\
127.23 \\
127.83\end{array}$ & $\begin{array}{l}125.91 \\
127.63 \\
129.08 \\
130.36 \\
131.23\end{array}$ & $\begin{array}{l}136.91 \\
136.67 \\
131.97 \\
129.92 \\
129.22\end{array}$ & $\begin{array}{l}128.67 \\
129.47 \\
129.71 \\
129.78 \\
130.55\end{array}$ & $\begin{array}{l}140.02 \\
140.23 \\
140.43 \\
140.86 \\
141.18\end{array}$ & $\begin{array}{l}144.80 \\
144.89 \\
145.06 \\
145.08 \\
145.10\end{array}$ & $\begin{array}{l}140.81 \\
141.06\end{array}$ \\
\hline $\begin{array}{l}16 \\
17 \\
18 \\
19 \\
20\end{array}$ & $\begin{array}{l}120.36 \\
120.14 \\
119.80 \\
119.54 \\
119.43\end{array}$ & $\begin{array}{l}118.39 \\
118.42 \\
118.41 \\
118.41 \\
118.35\end{array}$ & $\begin{array}{l}117.14 \\
117.37 \\
117.52 \\
117.53 \\
117.62\end{array}$ & $\begin{array}{l}119.07 \\
119.23 \\
119.40 \\
119.66 \\
119.94\end{array}$ & $\begin{array}{l}123.95 \\
123.58 \\
123.26 \\
123.16 \\
123.02\end{array}$ & $\begin{array}{l}128.37 \\
128.75 \\
128.77 \\
128.54 \\
127.70\end{array}$ & $\begin{array}{l}132.10 \\
132.93 \\
133.62 \\
134.14 \\
134.54\end{array}$ & $\begin{array}{l}128.81 \\
128.37 \\
128.21 \\
128.14 \\
128.17\end{array}$ & $\begin{array}{l}131.45 \\
132.37 \\
132.88 \\
133.25 \\
133.45\end{array}$ & $\begin{array}{l}141.48 \\
141.54 \\
141.88 \\
141.99 \\
142.26\end{array}$ & $\begin{array}{l}145.29 \\
143.14 \\
138.48 \\
138.90 \\
139.84\end{array}$ & $\begin{array}{l}138 . \\
139 . \\
140 . \\
139 . \\
139 .\end{array}$ \\
\hline $\begin{array}{l}21 \\
22 \\
23 \\
24 \\
25\end{array}$ & $\begin{array}{l}119.13 \\
118.66 \\
118.66 \\
118.65 \\
118.33\end{array}$ & $\begin{array}{l}118.12 \\
117.65 \\
117.32 \\
117.12 \\
117.02\end{array}$ & $\begin{array}{l}117.72 \\
117.94 \\
118.16 \\
118.26 \\
118.37\end{array}$ & $\begin{array}{l}120.19 \\
120.40 \\
120.63 \\
120.80 \\
121.04\end{array}$ & $\begin{array}{l}122.90 \\
122.71 \\
122.70 \\
122.83 \\
123.04\end{array}$ & $\begin{array}{l}126.88 \\
126.44 \\
126.09 \\
125.62 \\
125.57\end{array}$ & $\begin{array}{l}134.81 \\
135.20 \\
135.64 \\
136.15 \\
136.34\end{array}$ & $\begin{array}{l}128.17 \\
127.76 \\
127.67 \\
127.88 \\
128.09\end{array}$ & $\begin{array}{l}133.93 \\
134.21 \\
134.26 \\
134.26 \\
134.68\end{array}$ & $\begin{array}{l}142.36 \\
142.82 \\
143.11 \\
143.23 \\
143.52\end{array}$ & $\begin{array}{l}140.04 \\
139.96 \\
140.13 \\
140.35 \\
140.57\end{array}$ & $\begin{array}{l}139 . \\
139 . \\
138 . \\
138 . \\
137 .\end{array}$ \\
\hline $\begin{array}{l}26 \\
27 \\
28 \\
29 \\
30 \\
31\end{array}$ & $\begin{array}{l}118.25 \\
118.35 \\
118.42 \\
118.30 \\
118.25 \\
118.46\end{array}$ & $\begin{array}{r}116.91 \\
116.77 \\
116.62 \\
-.- \\
-.-\end{array}$ & $\begin{array}{l}118.42 \\
118.42 \\
118.42 \\
118.36 \\
117.76 \\
117.37\end{array}$ & $\begin{array}{r}121.32 \\
121.58 \\
121.75 \\
121.98 \\
122.23 \\
-\end{array}$ & $\begin{array}{l}123.32 \\
123.54 \\
123.62 \\
123.71 \\
123.87 \\
124.08\end{array}$ & $\begin{array}{r}125.51 \\
125.44 \\
125.97 \\
127.36 \\
127.77 \\
-\ldots\end{array}$ & $\begin{array}{l}135.91 \\
134.93 \\
135.56 \\
135.51 \\
132.12 \\
129.96\end{array}$ & $\begin{array}{l}128.14 \\
128.34 \\
128.99 \\
130.25 \\
130.95 \\
131.59\end{array}$ & $\begin{array}{r}135.35 \\
135.81 \\
136.19 \\
136.59 \\
136.64 \\
---\end{array}$ & $\begin{array}{l}143.39 \\
143.51 \\
143.78 \\
143.94 \\
144.14 \\
144.28\end{array}$ & $\begin{array}{r}140.46 \\
139.96 \\
139.92 \\
139.92 \\
139.87 \\
.--\end{array}$ & $\begin{array}{l}137 . \\
137 . \\
137 . \\
137 . \\
137 . \\
136 .\end{array}$ \\
\hline MEAN & 119.83 & 118.05 & 117.27 & 118.94 & 124.32 & 126.45 & 130.74 & 131.03 & 132.46 & 141.05 & 142.57 & 139. \\
\hline
\end{tabular}




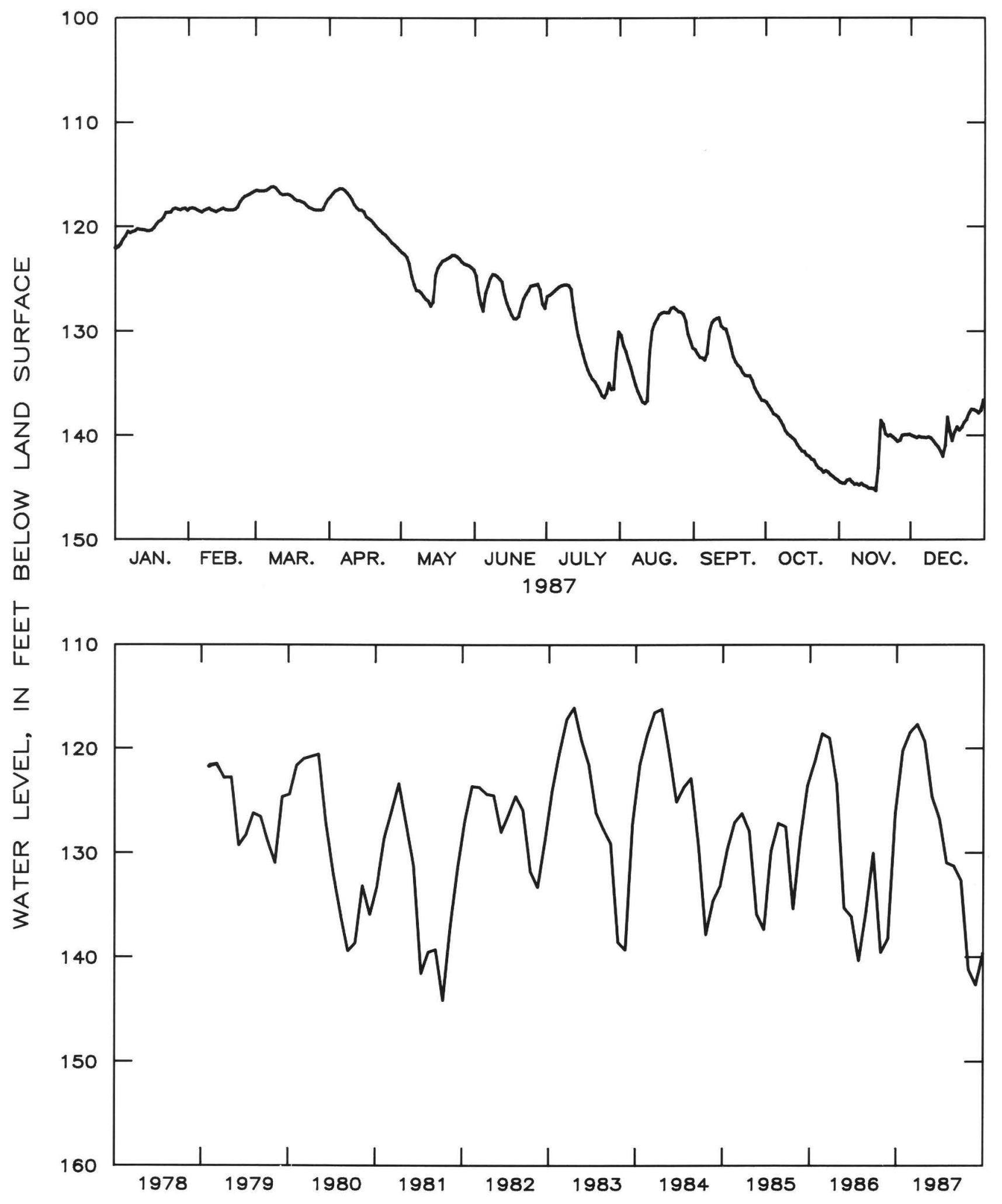

Figure 2.7.2-5.--Water level in observation well 19F039, Lowndes County. 
304949083165301 Local number, $19 \mathrm{E} 009$.

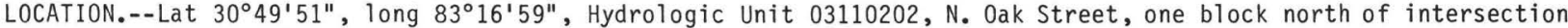
with U.S. Highway 84 , Valdosta, Ga. Owner: City of Valdosta.

AQUIFER.--Upper Floridan aquifer.

WELL CHARACTERISTICS.--Drilled unused municipal supply well, diameter $20 \mathrm{in}$., depth $342 \mathrm{ft}$, cased to $200 \mathrm{ft}$, open hole.

DATUM.--Elevation of land-surface datum is $217 \mathrm{ft}$.

Measuring point: Top of casing, $1.7 \mathrm{ft}$ above land-surface datum.

REMARKS.--Well pumped July 18, 1978; water-quality sample collected at conclusion of pumping. Borehole geophysical survey conducted April 11, 1963. Water level affected by city pumping.

PERIOD OF RECORD.--February 1957 to current year.

EXTREMES FOR PERIOD OF RECORD.--Highest water level, $112.69 \mathrm{ft}$ below land-surface datum, March 9, 1964; 1owest, $146.60 \mathrm{ft}$ below land-surface datum, July $18,1981$.

DEPTH BELOW LAND SURFACE (WATER LEVEL) (FEET) CALENDAR YEAR JANUARY 1987 TO DECEMBER 1987 MEAN VALUES

\begin{tabular}{|c|c|c|c|c|c|c|c|c|c|c|c|c|}
\hline DAY & JAN & FEB & MAR & APR & MAY & JUN & JUL & AUG & SEP & OCT & NOV & DEC \\
\hline $\begin{array}{l}1 \\
2 \\
3 \\
4 \\
5\end{array}$ & $\begin{array}{l}28.48 \\
28.44 \\
27.80 \\
27.93 \\
27.59\end{array}$ & $\begin{array}{l}124.59 \\
123.48 \\
123.48 \\
124.50 \\
124.79\end{array}$ & $\begin{array}{l}121.87 \\
121.39 \\
121.22 \\
121.20 \\
121.02\end{array}$ & $\begin{array}{l}121.55 \\
121.08 \\
121.08 \\
121.16 \\
120.48\end{array}$ & $\begin{array}{l}127.59 \\
128.26 \\
127.96 \\
129.14 \\
127.98\end{array}$ & $\begin{array}{l}129.82 \\
131.02 \\
131.51 \\
131.76 \\
128.17\end{array}$ & $\begin{array}{l}130.47 \\
130.30 \\
130.59 \\
130.46 \\
130.51\end{array}$ & $\begin{array}{l}134.90 \\
134.57 \\
134.60 \\
135.35 \\
135.77\end{array}$ & $\begin{array}{l}134.78 \\
134.82 \\
135.20 \\
135.61 \\
135.62\end{array}$ & $\begin{array}{l}138.50 \\
139.04 \\
139.75 \\
139.49 \\
139.44\end{array}$ & $\begin{array}{l}143.88 \\
144.53 \\
144.33 \\
143.61 \\
143.41\end{array}$ & $\begin{array}{l}141.14 \\
140.98 \\
141.42 \\
140.89 \\
141.94\end{array}$ \\
\hline $\begin{array}{r}6 \\
7 \\
8 \\
9 \\
10\end{array}$ & $\begin{array}{l}127.64 \\
127.50 \\
128.20 \\
127.39 \\
127.92\end{array}$ & $\begin{array}{l}125.17 \\
124.77 \\
123.99 \\
123.68 \\
123.99\end{array}$ & $\begin{array}{l}120.95 \\
120.63 \\
120.41 \\
120.31 \\
120.81\end{array}$ & $\begin{array}{l}120.95 \\
120.95 \\
121.07 \\
121.20 \\
122.24\end{array}$ & $\begin{array}{l}128.74 \\
129.06 \\
129.50 \\
128.37 \\
128.34\end{array}$ & $\begin{array}{l}130.16 \\
130.22 \\
129.66 \\
130.79 \\
131.17\end{array}$ & $\begin{array}{l}130.81 \\
130.64 \\
130.43 \\
130.24 \\
130.29\end{array}$ & $\begin{array}{l}135.89 \\
137.88 \\
137.96 \\
138.07 \\
138.87\end{array}$ & 135.41 & $\begin{array}{l}139.39 \\
140.01 \\
140.31 \\
141.15 \\
140.98\end{array}$ & 144.54 & $\begin{array}{l}141.94 \\
141.23\end{array}$ \\
\hline $\begin{array}{l}11 \\
12 \\
13 \\
14 \\
15\end{array}$ & $\begin{array}{l}126.32 \\
126.58 \\
126.80 \\
126.56 \\
126.50\end{array}$ & $\begin{array}{l}124.40 \\
123.86 \\
123.68 \\
123.93 \\
123.73\end{array}$ & $\begin{array}{l}121.42 \\
121.12 \\
120.79 \\
121.54 \\
121.62\end{array}$ & $\begin{array}{l}122.49 \\
122.78 \\
122.34 \\
122.26 \\
122.61\end{array}$ & $\begin{array}{l}129.59 \\
129.29 \\
128.38\end{array}$ & $\begin{array}{l}131.54 \\
131.40 \\
129.86 \\
130.11 \\
130.49\end{array}$ & & & & $\begin{array}{l}140.82 \\
140.76 \\
141.20 \\
141.15 \\
141.71\end{array}$ & $\begin{array}{l}143.97 \\
143.93 \\
144.86 \\
144.52 \\
144.32\end{array}$ & $\begin{array}{l}142.48 \\
141.68 \\
141.70 \\
141.80 \\
141.63\end{array}$ \\
\hline $\begin{array}{l}16 \\
17 \\
18 \\
19 \\
20\end{array}$ & $\begin{array}{l}126.33 \\
125.96 \\
125.62 \\
125.41 \\
125.48\end{array}$ & $\begin{array}{l}123.14 \\
123.37 \\
123.03 \\
123.29 \\
123.12\end{array}$ & $\begin{array}{l}21.11 \\
21.16 \\
21.71 \\
21.26 \\
21.64\end{array}$ & $\begin{array}{l}122.43 \\
122.38 \\
122.80 \\
122.93 \\
123.58\end{array}$ & $\begin{array}{l}128.17 \\
128.25 \\
127.96 \\
128.14 \\
127.81\end{array}$ & $\begin{array}{l}130.79 \\
131.19 \\
131.99 \\
132.08 \\
131.74\end{array}$ & & & & & & $\begin{array}{l}140.52 \\
140.71 \\
141.04 \\
140.42\end{array}$ \\
\hline $\begin{array}{l}21 \\
22 \\
23 \\
24 \\
25\end{array}$ & $\begin{array}{l}125.57 \\
125.54 \\
125.99 \\
126.70 \\
125.40\end{array}$ & $\begin{array}{l}123.24 \\
122.47 \\
122.57 \\
122.85 \\
122.47\end{array}$ & $\begin{array}{l}122.39 \\
122.42 \\
122.23 \\
122.03 \\
121.91\end{array}$ & $\begin{array}{l}124.14 \\
125.02 \\
124.88 \\
125.44 \\
126.05\end{array}$ & $\begin{array}{l}128.21 \\
127.14 \\
127.64 \\
127.53 \\
127.32\end{array}$ & $\begin{array}{l}131.23 \\
130.96 \\
130.86 \\
130.76 \\
130.09\end{array}$ & $\begin{array}{l}135.86 \\
136.30 \\
138.07 \\
138.92 \\
137.92\end{array}$ & & & & $\begin{array}{l}142.31 \\
142.08 \\
141.52 \\
141.31 \\
141.19\end{array}$ & $\begin{array}{l}140.69 \\
140.58 \\
140.24 \\
140.08 \\
139.91\end{array}$ \\
\hline $\begin{array}{l}26 \\
27 \\
28 \\
29 \\
30 \\
31\end{array}$ & $\begin{array}{l}125.01 \\
126.70 \\
125.69 \\
124.74 \\
124.15 \\
125.09\end{array}$ & $\begin{array}{r}122.79 \\
122.46 \\
122.18 \\
--- \\
--- \\
-.-\end{array}$ & $\begin{array}{l}121.80 \\
122.77 \\
122.75 \\
122.12 \\
122.02 \\
122.13\end{array}$ & $\begin{array}{r}126.13 \\
125.94 \\
126.47 \\
126.92 \\
127.97 \\
.--\end{array}$ & $\begin{array}{l}127.81 \\
128.15 \\
128.77 \\
129.16 \\
129.17 \\
129.48\end{array}$ & $\begin{array}{r}129.45 \\
129.43 \\
129.67 \\
130.28 \\
130.75 \\
\ldots-.-\end{array}$ & $\begin{array}{l}137.14 \\
136.82 \\
137.20 \\
136.99 \\
135.76 \\
134.83\end{array}$ & $\begin{array}{l}133 \\
134 \\
135 \\
135 \\
135 \\
134\end{array}$ & $\begin{array}{l}138.45 \\
138.44 \\
138.79 \\
138.88 \\
138.09\end{array}$ & $\begin{array}{l}144.11 \\
143.38 \\
143.57 \\
144.00 \\
144.59 \\
144.70\end{array}$ & $\begin{array}{r}140.98 \\
141.45 \\
141.82 \\
141.60 \\
140.77 \\
.--\end{array}$ & $\begin{array}{l}139.97 \\
139.41 \\
139.59 \\
139.27 \\
139.30\end{array}$ \\
\hline A & 126.48 & 123.54 & 121.54 & 123.24 & 128.43 & 130.63 & 133.87 & 135.26 & 135.89 & 141.93 & 143.03 & 140.84 \\
\hline
\end{tabular}

$\begin{array}{lllllll}\text { CAL YR } 1987 & \text { MEAN } & 132.12 & \text { HIGH } & 120.31 & \text { LOW } & 144.86\end{array}$ 


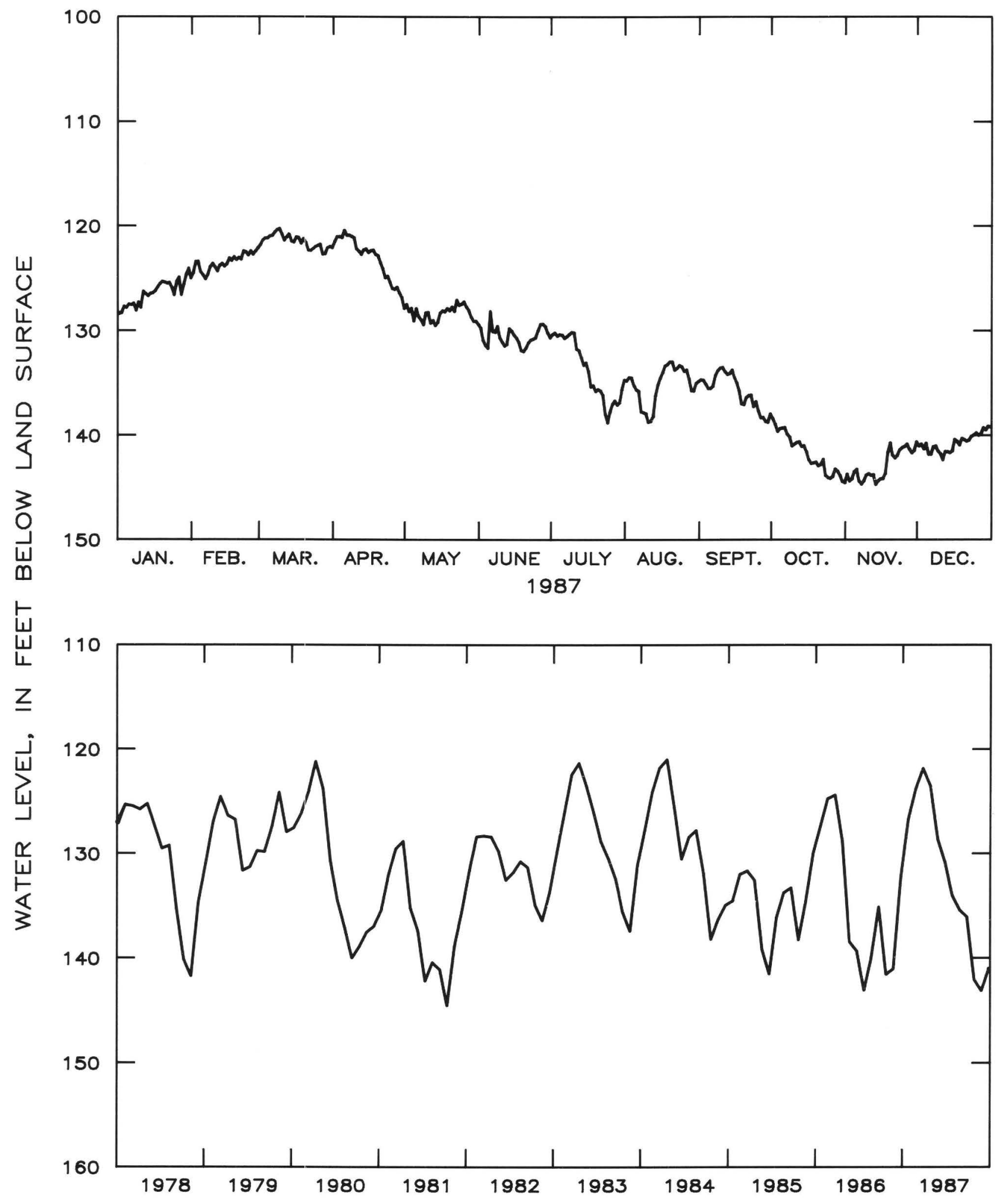

Figure 2.7.2-6.--Water level in observation well 19E009, Lowndes County. 


\subsubsection{East-central area}

The water level in the Upper Floridan aquifer in east-central Georgia is affected by rainfall, evapotranspiration, stream stage, and pumping. The water level generally is highest in the winter and spring rainy seasons and lowest in the fall following the summer irrigation season.

We11 21 T001 in Laurens County is near the recharge area for the Upper Floridan aquifer, and the water level responds primarily to seasonal fluctuations in rainfall and evapotranspiration. The mean water level in well 21 T001 was $1.8 \mathrm{ft}$ higher in 1987 than in 1986, and a record high was measured in January 1987. By the end of March, the water level had recovered $14.0 \mathrm{ft}$ from the low measured during the 1986 drought. Although there was some recovery from the drought, at the end of 1987, the water level in well 21 T001 was 10.4 ft lower than at the end of 1986 .

In Montgomery and Toombs Counties, the mean water levels in wells 25Q001 and 26R001 were from $0.4 \mathrm{ft}$ lower to $1.1 \mathrm{ft}$ higher in 1987 than in 1986 . The water levels in these two wells had recovered 4.4 to $9.8 \mathrm{ft}$ by the end of March 1987 from the record lows measured during the 1986 drought. At the end of 1987, water levels were from $0.3 \mathrm{ft}$ lower to $0.9 \mathrm{ft}$ higher than at the end of 1986 .

In well 26R001 at Vidalia, the mean water level was $1.1 \mathrm{ft}$ higher in 1987 than in 1986, which reversed a downward water-level trend since 1978. At the end of 1987, the water level in the well was $0.9 \mathrm{ft}$ higher than at the end of 1986. The mean water level in well 250001 at Uvalda, however, was $0.4 \mathrm{ft}$ lower than in 1986, which continued the downward trend in that area. At the end of 1987, the water level in the well was $0.3 \mathrm{ft} l$ lower than at the end of 1986. 


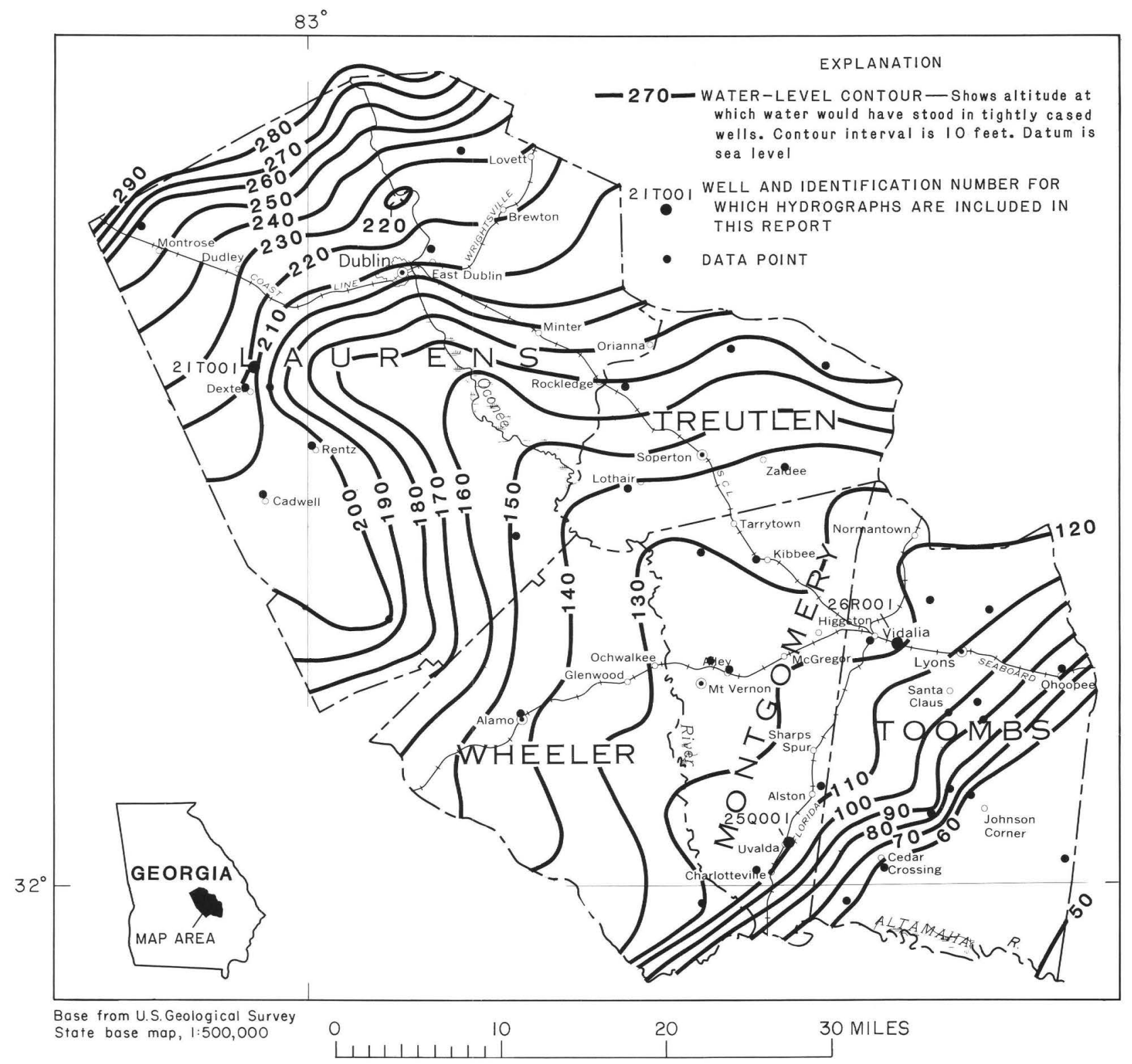

Figure 2.7.3-1.-Observation well locations and the water level in the Upper Floridan aquifer in the east-central area, May 1985. 
322652083033001 Local number, 21T001.

LOCATION.--Lat $32^{\circ} 27^{\prime} 06^{\prime \prime}$, long $83^{\circ} 03^{\prime} 28^{\prime \prime}$, Hydrologic Unit 03070102 , approximately 1.8 mi northeast of Dexter, Ga. Owner: Danny Hogan.

AQUIFER.--Upper Floridan aquifer.

WELL CHARACTERISTICS.--Drilled unused domestic well, diameter $4 \mathrm{in.,}$ depth $123 \mathrm{ft}$, cased to $89 \mathrm{ft}$, open hole.

DATUM.--Elevation of land-surface datum is $259 \mathrm{ft}$.

Measuring point: Floor of recorder shelter, $2.57 \mathrm{ft}$ above land-surface datum.

REMARKS.--Borehole geophysical survey conducted November 1973. Water level for period of missing record, August 31 , was estimated.

PERIOD OF RECORD.--March 1964 to current year.

EXTREMES FOR PERIOD OF RECORD.--Highest water 1evel, $23.62 \mathrm{ft}$ below land-surface datum, January 26, 1987; 1owest, $39.58 \mathrm{ft}$ below land-surface datum, November 12, 1968.

DEPTH BELOW LAND SURFACE (WATER LEVEL) (FEET) CALENDAR YEAR JANUARY 1987 TO DECEMBER 1987 MEAN VALUES

\begin{tabular}{|c|c|c|c|c|c|c|c|c|c|c|c|c|}
\hline DAY & JAN & FEB & MAR & APR & MAY & JUN & JUL & AUG & SEP & OCT & NOV & DEC \\
\hline $\begin{array}{l}1 \\
2 \\
3 \\
4 \\
5\end{array}$ & $\begin{array}{l}26.54 \\
26.15 \\
25.88 \\
25.59 \\
25.31\end{array}$ & $\begin{array}{l}24.01 \\
24.00 \\
24.03 \\
24.12 \\
24.22\end{array}$ & $\begin{array}{l}24.20 \\
24.09 \\
24.08 \\
24.08 \\
24.13\end{array}$ & $\begin{array}{l}25.25 \\
25.23 \\
25.16 \\
25.15 \\
25.17\end{array}$ & $\begin{array}{l}28.10 \\
28.26 \\
28.47 \\
28.66 \\
28.84\end{array}$ & $\begin{array}{l}32.03 \\
32.16 \\
32.29 \\
32.41 \\
32.55\end{array}$ & $\begin{array}{l}27.04 \\
27.03 \\
26.84 \\
26.60 \\
26.44\end{array}$ & $\begin{array}{l}29.94 \\
30.07 \\
30.20 \\
30.27 \\
30.37\end{array}$ & $\begin{array}{l}33.01 \\
32.93 \\
32.91 \\
32.92 \\
32.93\end{array}$ & $\begin{array}{l}34.34 \\
34.45 \\
34.52 \\
34.62 \\
34.64\end{array}$ & $\begin{array}{l}36.11 \\
36.13 \\
36.14 \\
36.15 \\
36.16\end{array}$ & $\begin{array}{l}36.50 \\
36.58 \\
36.61 \\
36.58 \\
36.65\end{array}$ \\
\hline $\begin{array}{r}6 \\
7 \\
8 \\
9 \\
10\end{array}$ & $\begin{array}{l}25.16 \\
25.03 \\
24.97 \\
24.94 \\
24.87\end{array}$ & $\begin{array}{l}24.23 \\
24.12 \\
24.04 \\
24.09 \\
24.16\end{array}$ & $\begin{array}{l}24.17 \\
24.17 \\
24.06 \\
24.02 \\
24.09\end{array}$ & $\begin{array}{l}25.23 \\
25.29 \\
25.37 \\
25.44 \\
25.53\end{array}$ & $\begin{array}{l}28.95 \\
29.05 \\
29.16 \\
29.35 \\
29.50\end{array}$ & $\begin{array}{l}32.69 \\
32.84 \\
32.97 \\
33.06 \\
33.17\end{array}$ & $\begin{array}{l}26.36 \\
26.20 \\
26.06 \\
25.98 \\
25.96\end{array}$ & $\begin{array}{l}30.51 \\
30.67 \\
30.78 \\
30.87 \\
30.95\end{array}$ & $\begin{array}{l}32.93 \\
32.95 \\
32.98 \\
33.06 \\
33.13\end{array}$ & $\begin{array}{l}34.64 \\
34.70 \\
34.83 \\
34.94 \\
34.99\end{array}$ & $\begin{array}{l}36.26 \\
36.34 \\
36.36 \\
36.37 \\
36.34\end{array}$ & $\begin{array}{l}36.69 \\
36.73 \\
36.72 \\
36.75 \\
36.70\end{array}$ \\
\hline $\begin{array}{l}11 \\
12 \\
13 \\
14 \\
15\end{array}$ & $\begin{array}{l}24.91 \\
24.97 \\
25.01 \\
25.06 \\
25.07\end{array}$ & $\begin{array}{l}24.18 \\
24.18 \\
24.22 \\
24.27 \\
24.33\end{array}$ & $\begin{array}{l}24.24 \\
24.32 \\
24.37 \\
24.42 \\
24.48\end{array}$ & $\begin{array}{l}25.63 \\
25.75 \\
25.86 \\
25.94 \\
25.96\end{array}$ & $\begin{array}{l}29.63 \\
29.72 \\
29.82 \\
29.92 \\
29.97\end{array}$ & $\begin{array}{l}33.29 \\
33.38 \\
33.40 \\
33.16 \\
32.78\end{array}$ & $\begin{array}{l}25.97 \\
26.04 \\
26.15 \\
26.28 \\
26.46\end{array}$ & $\begin{array}{l}31.06 \\
31.19 \\
31.31 \\
31.46 \\
31.51\end{array}$ & $\begin{array}{l}33.20 \\
33.23 \\
33.22 \\
33.21 \\
33.23\end{array}$ & $\begin{array}{l}34.99 \\
34.99 \\
35.08 \\
35.18 \\
35.24\end{array}$ & $\begin{array}{l}36.38 \\
36.46 \\
36.47 \\
36.49 \\
36.55\end{array}$ & $\begin{array}{l}36.67 \\
36.68 \\
36.77 \\
36.81 \\
36.76\end{array}$ \\
\hline $\begin{array}{l}16 \\
17 \\
18 \\
19 \\
20\end{array}$ & $\begin{array}{l}25.08 \\
25.06 \\
24.94 \\
24.54 \\
24.29\end{array}$ & $\begin{array}{l}24.33 \\
24.38 \\
24.44 \\
24.54 \\
24.61\end{array}$ & $\begin{array}{l}24.54 \\
24.64 \\
24.70 \\
24.70 \\
24.77\end{array}$ & $\begin{array}{l}25.98 \\
26.08 \\
26.22 \\
26.40 \\
26.54\end{array}$ & $\begin{array}{l}30.04 \\
30.16 \\
30.25 \\
30.34 \\
30.45\end{array}$ & $\begin{array}{l}32.09 \\
31.20 \\
30.46 \\
29.83 \\
29.34\end{array}$ & $\begin{array}{l}26.66 \\
26.89 \\
27.11 \\
27.30 \\
27.51\end{array}$ & $\begin{array}{l}31.73 \\
31.83 \\
31.94 \\
32.07 \\
32.17\end{array}$ & $\begin{array}{l}33.25 \\
33.29 \\
33.33 \\
33.39 \\
33.49\end{array}$ & $\begin{array}{l}35.28 \\
35.31 \\
35.36 \\
35.42 \\
35.47\end{array}$ & $\begin{array}{l}36.58 \\
36.54 \\
36.50 \\
36.52 \\
36.50\end{array}$ & $\begin{array}{l}36.78 \\
36.83 \\
36.86 \\
36.87 \\
36.85\end{array}$ \\
\hline $\begin{array}{l}21 \\
22 \\
23 \\
24 \\
25\end{array}$ & $\begin{array}{l}24.14 \\
23.79 \\
23.71 \\
23.72 \\
23.66\end{array}$ & $\begin{array}{l}24.62 \\
24.59 \\
24.59 \\
24.68 \\
24.74\end{array}$ & $\begin{array}{l}24.83 \\
24.92 \\
25.01 \\
25.10 \\
25.15\end{array}$ & $\begin{array}{l}26.65 \\
26.77 \\
26.91 \\
27.04 \\
27.20\end{array}$ & $\begin{array}{l}30.58 \\
30.71 \\
30.83 \\
30.95 \\
31.09\end{array}$ & $\begin{array}{l}28.97 \\
28.65 \\
28.25 \\
27.89 \\
27.62\end{array}$ & $\begin{array}{l}27.75 \\
27.94 \\
28.12 \\
28.38 \\
28.64\end{array}$ & $\begin{array}{l}32.28 \\
32.39 \\
32.46 \\
32.58 \\
32.69\end{array}$ & $\begin{array}{l}33.62 \\
33.72 \\
33.80 \\
33.86 \\
33.93\end{array}$ & $\begin{array}{l}35.55 \\
35.65 \\
35.71 \\
35.75 \\
35.77\end{array}$ & $\begin{array}{l}36.51 \\
36.55 \\
36.58 \\
36.62 \\
36.63\end{array}$ & $\begin{array}{l}36.85 \\
36.82 \\
36.82 \\
36.82 \\
36.82\end{array}$ \\
\hline $\begin{array}{l}26 \\
27 \\
28 \\
29 \\
30 \\
31\end{array}$ & $\begin{array}{l}23.62 \\
23.72 \\
23.79 \\
23.87 \\
23.87 \\
23.92\end{array}$ & $\begin{array}{r}24.78 \\
24.71 \\
24.51 \\
--- \\
--- \\
---\end{array}$ & $\begin{array}{l}25.21 \\
25.25 \\
25.31 \\
25.40 \\
25.32 \\
25.25\end{array}$ & $\begin{array}{r}27.40 \\
27.58 \\
27.71 \\
27.87 \\
27.98 \\
---\end{array}$ & $\begin{array}{l}31.23 \\
31.36 \\
31.50 \\
31.62 \\
31.73 \\
31.88\end{array}$ & $\begin{array}{r}27.39 \\
27.19 \\
27.10 \\
27.06 \\
27.05 \\
.--\end{array}$ & $\begin{array}{l}28.85 \\
29.04 \\
29.24 \\
29.45 \\
29.69 \\
29.83\end{array}$ & $\begin{array}{l}32.79 \\
32.84 \\
32.92 \\
32.99 \\
33.07 \\
33.04\end{array}$ & $\begin{array}{r}34.05 \\
34.14 \\
34.24 \\
34.28 \\
34.27 \\
---\end{array}$ & $\begin{array}{l}35.78 \\
35.79 \\
35.87 \\
35.95 \\
36.02 \\
36.08\end{array}$ & $\begin{array}{r}36.57 \\
36.54 \\
36.51 \\
36.49 \\
36.47 \\
-.-\end{array}$ & $\begin{array}{l}36.82 \\
36.82 \\
36.82 \\
36.84 \\
36.90 \\
36.91\end{array}$ \\
\hline MEAN & 24.68 & 24.35 & 24.61 & 26.21 & 30.07 & 30.74 & 27.35 & 31.65 & 33.42 & 35.26 & 36.43 & 36.76 \\
\hline
\end{tabular}




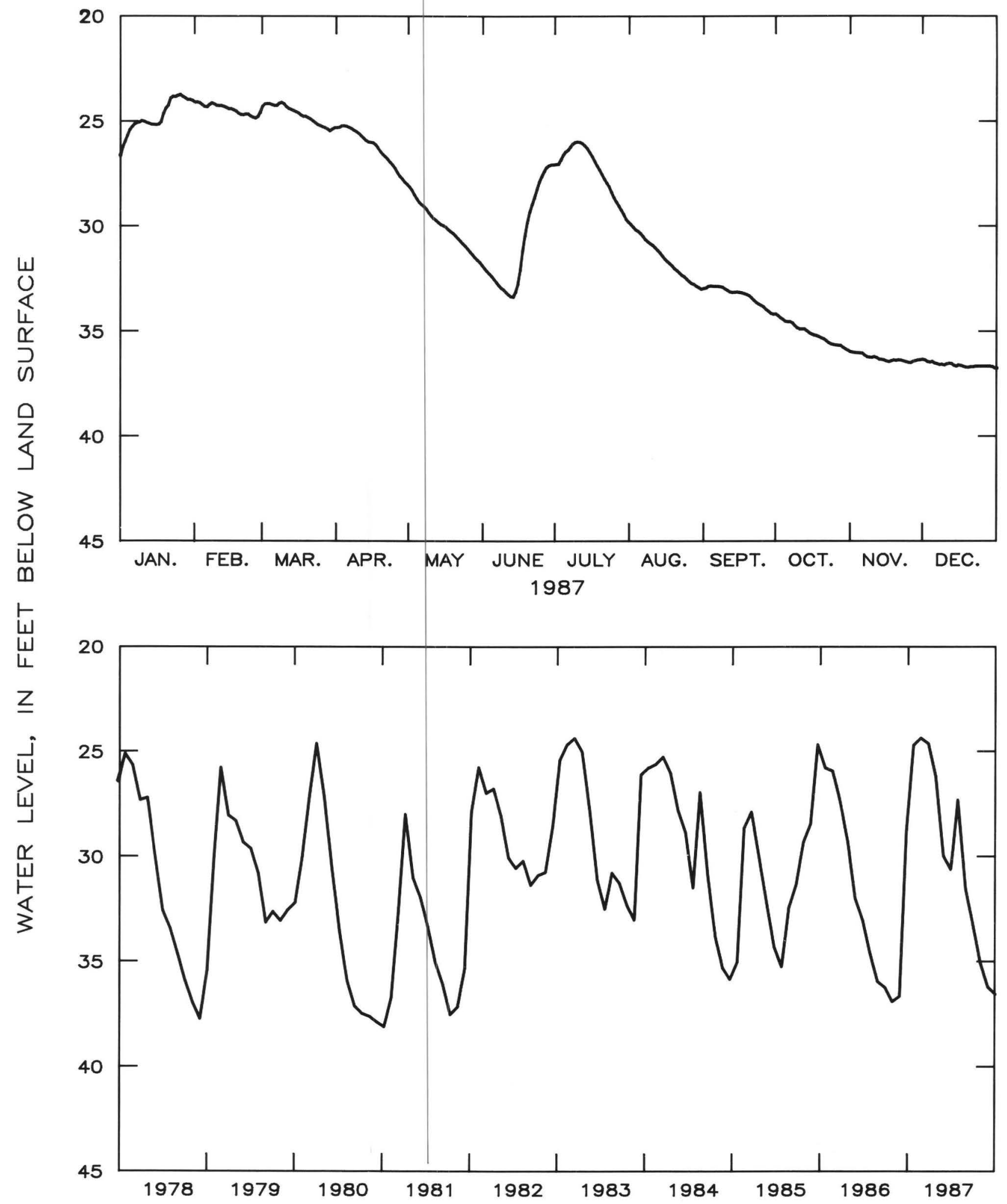

Figure 2.7.3-2.--Water level in observation well 21T001, Laurens County. 


\section{UVALDA SCHOOL MONTGOMERY COUNTY}

320226082301101 Local number, 250001.

LOCATION.--Lat $32^{\circ} 02^{\prime} 25^{\prime \prime}$, long $82^{\circ} 30^{\circ} 05^{\prime \prime}$. Hydrologic Unit 03070106, well is located behind the Uvalda School in the city of Uvalda.

Owner: Montgomery County Board of Education.

AQUIFER.---Upper Floridan aquifer.

WELL CHARACTERISTICS.--Drilled unused supply well, diameter $6 \mathrm{in}$., depth $536 \mathrm{ft}$, cased to $421 \mathrm{ft}$, open hole. DATUM.--Elevation of land-surface datum is $190 \mathrm{ft}$.

Measuring point: Top of $6-i n$. casing at land surface.

REMARKS.--Borehole geophysical survey conducted April 22, 1966. Water levels for periods of missing record, June 7-16, and June 21-25, were estimated.

PERIOD OF RECORD.--June 1966 to current year.

EXTREMES FOR PERIOD OF RECORD.--Highest water level, $64.13 \mathrm{ft}$ below land-surface datum, June 10, 1966; 10west, $82.27 \mathrm{ft}$ below land-surface datum, July $17,1986$.

DEPTH BELOW LAND SURFACE (WATER LEVEL) (FEET) CALENDAR YEAR JANUARY 1987 TO DECEMBER 1987 MEAN VALUES

\begin{tabular}{|c|c|c|c|c|c|c|c|c|c|c|c|c|}
\hline DAY & JAN & FEB & MAR & APR & MAY & JUN & JUL & AUG & SEP & OCT & NOV & DEC \\
\hline $\begin{array}{l}1 \\
2 \\
3 \\
4 \\
5\end{array}$ & $\begin{array}{l}79.21 \\
79.33 \\
79.40 \\
79.22 \\
79.20\end{array}$ & $\begin{array}{l}78.83 \\
78.68 \\
78.71 \\
78.79 \\
78.83\end{array}$ & $\begin{array}{l}78.08 \\
78.20 \\
78.29 \\
78.34 \\
78.39\end{array}$ & $\begin{array}{l}78.05 \\
77.99 \\
77.89 \\
77.92 \\
77.89\end{array}$ & $\begin{array}{l}79.67 \\
79.69 \\
79.73 \\
79.79 \\
79.92\end{array}$ & $\begin{array}{l}79.99 \\
80.10 \\
80.20 \\
80.23 \\
80.25\end{array}$ & $\begin{array}{l}80.14 \\
80.12 \\
80.10 \\
80.06 \\
80.03\end{array}$ & $\begin{array}{l}81.08 \\
81.03 \\
80.97 \\
80.88 \\
80.81\end{array}$ & $\begin{array}{l}79.97 \\
79.91 \\
79.90 \\
79.87 \\
79.83\end{array}$ & $\begin{array}{l}79.52 \\
79.58 \\
79.61 \\
79.69 \\
79.62\end{array}$ & $\begin{array}{l}79.94 \\
79.92 \\
79.87 \\
79.76 \\
79.73\end{array}$ & $\begin{array}{l}79.95 \\
80.04 \\
79.98 \\
79.88 \\
79.96\end{array}$ \\
\hline $\begin{array}{r}6 \\
7 \\
8 \\
9 \\
10\end{array}$ & $\begin{array}{l}79.27 \\
79.21 \\
79.21 \\
79.19 \\
79.04\end{array}$ & $\begin{array}{l}78.69 \\
78.54 \\
78.49 \\
78.66 \\
78.74\end{array}$ & $\begin{array}{l}78.38 \\
78.22 \\
78.02 \\
77.97 \\
78.08\end{array}$ & $\begin{array}{l}77.89 \\
77.86 \\
77.85 \\
77.85 \\
77.88\end{array}$ & $\begin{array}{l}79.99 \\
79.99 \\
79.99 \\
80.16 \\
80.26\end{array}$ & $\begin{array}{l}80.27 \\
80.28 \\
80.27 \\
80.26 \\
80.25\end{array}$ & $\begin{array}{l}79.99 \\
79.90 \\
79.84 \\
79.80 \\
79.74\end{array}$ & $\begin{array}{l}80.78 \\
80.76 \\
80.71 \\
80.69 \\
80.68\end{array}$ & $\begin{array}{l}79.81 \\
79.75 \\
79.75 \\
79.78 \\
79.80\end{array}$ & $\begin{array}{l}79.55 \\
79.56 \\
79.68 \\
79.73 \\
79.73\end{array}$ & $\begin{array}{l}79.90 \\
79.96 \\
79.93 \\
79.86 \\
79.73\end{array}$ & $\begin{array}{l}80.05 \\
80.09 \\
80.06 \\
79.99 \\
79.89\end{array}$ \\
\hline $\begin{array}{l}11 \\
12 \\
13 \\
14 \\
15\end{array}$ & $\begin{array}{l}79.13 \\
79.14 \\
79.13 \\
79.13 \\
79.07\end{array}$ & $\begin{array}{l}78.70 \\
78.59 \\
78.56 \\
78.49 \\
78.48\end{array}$ & $\begin{array}{l}78.22 \\
78.21 \\
78.15 \\
78.11 \\
78.10\end{array}$ & $\begin{array}{l}77.91 \\
77.98 \\
78.04 \\
78.07 \\
78.03\end{array}$ & $\begin{array}{l}80.33 \\
80.42 \\
80.55 \\
80.67 \\
80.70\end{array}$ & $\begin{array}{l}80.24 \\
80.25 \\
80.26 \\
80.25 \\
80.25\end{array}$ & $\begin{array}{l}79.69 \\
79.66 \\
79.69 \\
79.78 \\
79.93\end{array}$ & $\begin{array}{l}80.68 \\
80.67 \\
80.63 \\
80.61 \\
80.56\end{array}$ & $\begin{array}{l}79.82 \\
79.77 \\
79.70 \\
79.69 \\
79.68\end{array}$ & $\begin{array}{l}79.64 \\
79.56 \\
79.65 \\
79.73 \\
79.73\end{array}$ & $\begin{array}{l}79.82 \\
79.92 \\
79.88 \\
79.93 \\
80.11\end{array}$ & $\begin{array}{l}79.88 \\
79.89 \\
80.02 \\
80.02 \\
79.88\end{array}$ \\
\hline $\begin{array}{l}16 \\
17 \\
18 \\
19 \\
20\end{array}$ & $\begin{array}{l}79.01 \\
78.98 \\
78.89 \\
78.83 \\
78.94\end{array}$ & $\begin{array}{l}78.36 \\
78.44 \\
78.49 \\
78.57 \\
78.58\end{array}$ & $\begin{array}{l}78.07 \\
78.09 \\
78.02 \\
77.90 \\
77.92\end{array}$ & $\begin{array}{l}78.08 \\
78.15 \\
78.26 \\
78.45 \\
78.56\end{array}$ & $\begin{array}{l}80.70 \\
80.67 \\
80.55 \\
80.40 \\
80.29\end{array}$ & $\begin{array}{l}80.27 \\
80.27 \\
80.28 \\
80.31 \\
80.30\end{array}$ & $\begin{array}{l}80.11 \\
80.30 \\
80.44 \\
80.55 \\
80.66\end{array}$ & $\begin{array}{l}80.48 \\
80.41 \\
80.33 \\
80.27 \\
80.25\end{array}$ & $\begin{array}{l}79.65 \\
79.61 \\
79.56 \\
79.54 \\
79.55\end{array}$ & $\begin{array}{l}79.71 \\
79.68 \\
79.69 \\
79.70 \\
79.71\end{array}$ & $\begin{array}{l}80.23 \\
80.27 \\
80.42 \\
80.45 \\
80.40\end{array}$ & $\begin{array}{l}79.95 \\
79.99 \\
80.00 \\
79.94 \\
79.86\end{array}$ \\
\hline $\begin{array}{l}21 \\
22 \\
23 \\
24 \\
25\end{array}$ & $\begin{array}{l}78.89 \\
78.64 \\
78.84 \\
78.93 \\
78.76\end{array}$ & $\begin{array}{l}78.46 \\
78.33 \\
78.37 \\
78.45 \\
78.46\end{array}$ & $\begin{array}{l}77.87 \\
77.88 \\
77.92 \\
77.90 \\
77.90\end{array}$ & $\begin{array}{l}78.56 \\
78.58 \\
78.65 \\
78.75 \\
78.96\end{array}$ & $\begin{array}{l}80.22 \\
80.16 \\
80.07 \\
79.98 \\
79.90\end{array}$ & $\begin{array}{l}80.29 \\
80.27 \\
80.25 \\
80.23 \\
80.21\end{array}$ & $\begin{array}{l}80.77 \\
80.83 \\
80.85 \\
80.91 \\
80.91\end{array}$ & $\begin{array}{l}80.25 \\
80.20 \\
80.12 \\
80.09 \\
80.10\end{array}$ & $\begin{array}{l}79.60 \\
79.61 \\
79.59 \\
79.56 \\
79.57\end{array}$ & $\begin{array}{l}79.77 \\
79.90 \\
79.92 \\
79.91 \\
79.87\end{array}$ & $\begin{array}{l}80.46 \\
80.47 \\
80.44 \\
80.40 \\
80.29\end{array}$ & $\begin{array}{l}79.82 \\
79.75 \\
79.80 \\
79.78 \\
79.72\end{array}$ \\
\hline $\begin{array}{l}26 \\
27 \\
28 \\
29 \\
30 \\
31\end{array}$ & $\begin{array}{l}78.78 \\
78.91 \\
78.93 \\
78.93 \\
78.78 \\
78.81\end{array}$ & $\begin{array}{r}78.45 \\
78.37 \\
78.23 \\
--- \\
--- \\
---\end{array}$ & $\begin{array}{l}77.94 \\
77.91 \\
77.95 \\
78.00 \\
77.85 \\
77.94\end{array}$ & $\begin{array}{r}79.18 \\
79.32 \\
79.38 \\
79.53 \\
79.60 \\
.--\end{array}$ & $\begin{array}{l}79.86 \\
79.83 \\
79.80 \\
79.78 \\
79.78 \\
79.87\end{array}$ & $\begin{array}{r}80.19 \\
80.17 \\
80.19 \\
80.17 \\
80.15 \\
---\end{array}$ & $\begin{array}{l}80.93 \\
80.89 \\
80.88 \\
80.95 \\
81.06 \\
81.12\end{array}$ & $\begin{array}{l}80.09 \\
80.05 \\
80.01 \\
79.99 \\
79.99 \\
80.00\end{array}$ & $\begin{array}{r}79.63 \\
79.66 \\
79.67 \\
79.59 \\
79.45 \\
.--\end{array}$ & $\begin{array}{l}79.81 \\
79.75 \\
79.82 \\
79.88 \\
79.92 \\
79.95\end{array}$ & $\begin{array}{r}80.18 \\
80.11 \\
80.05 \\
79.98 \\
79.93 \\
-.-\end{array}$ & $\begin{array}{l}79.68 \\
79.67 \\
79.57 \\
79.63 \\
79.78 \\
79.74\end{array}$ \\
\hline MEAN & 79.02 & 78.55 & 78.06 & 78.37 & 80.12 & 80.23 & 80.34 & 80.46 & 79.70 & 79.73 & 80.08 & 79.8 \\
\hline
\end{tabular}




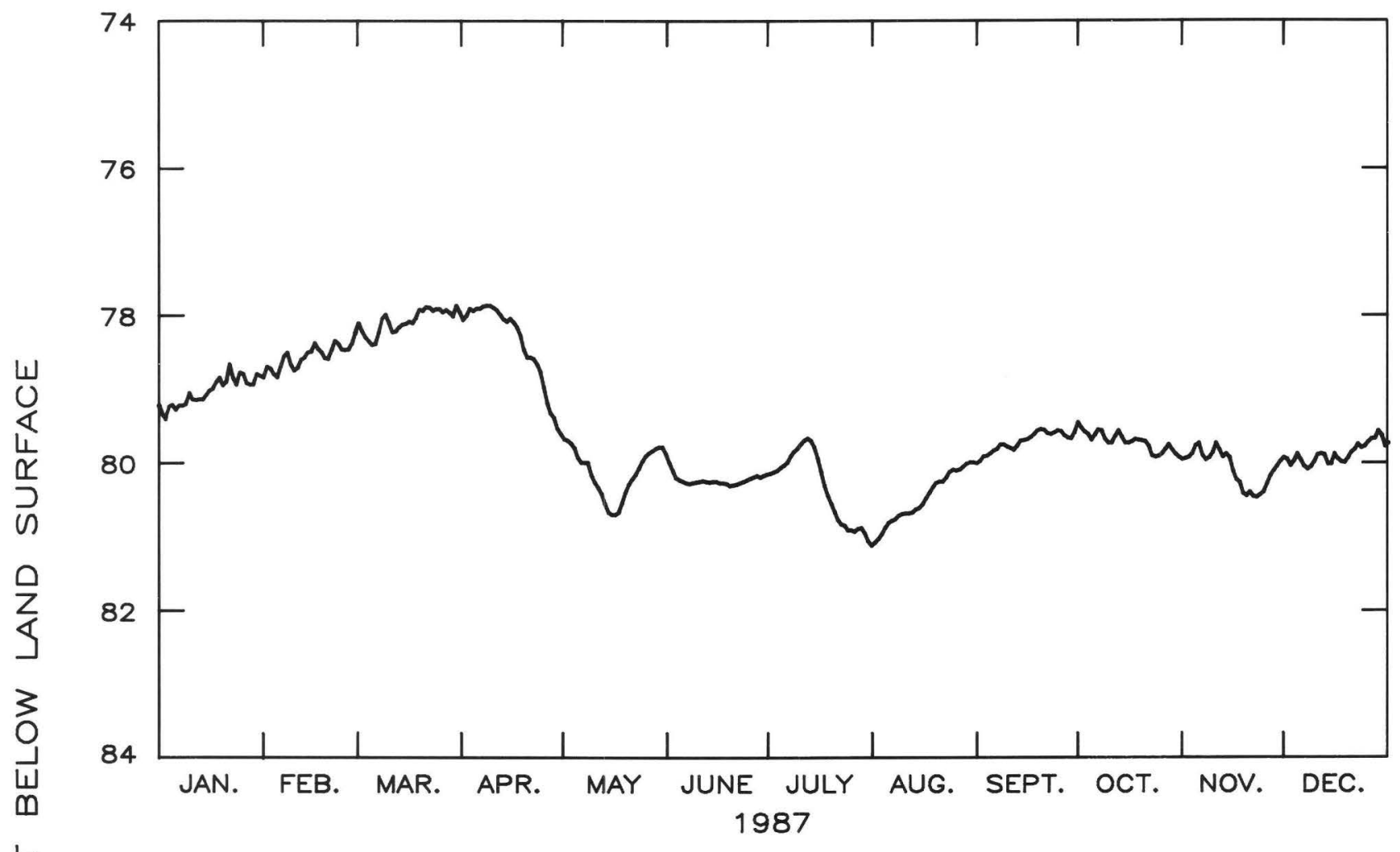

岁

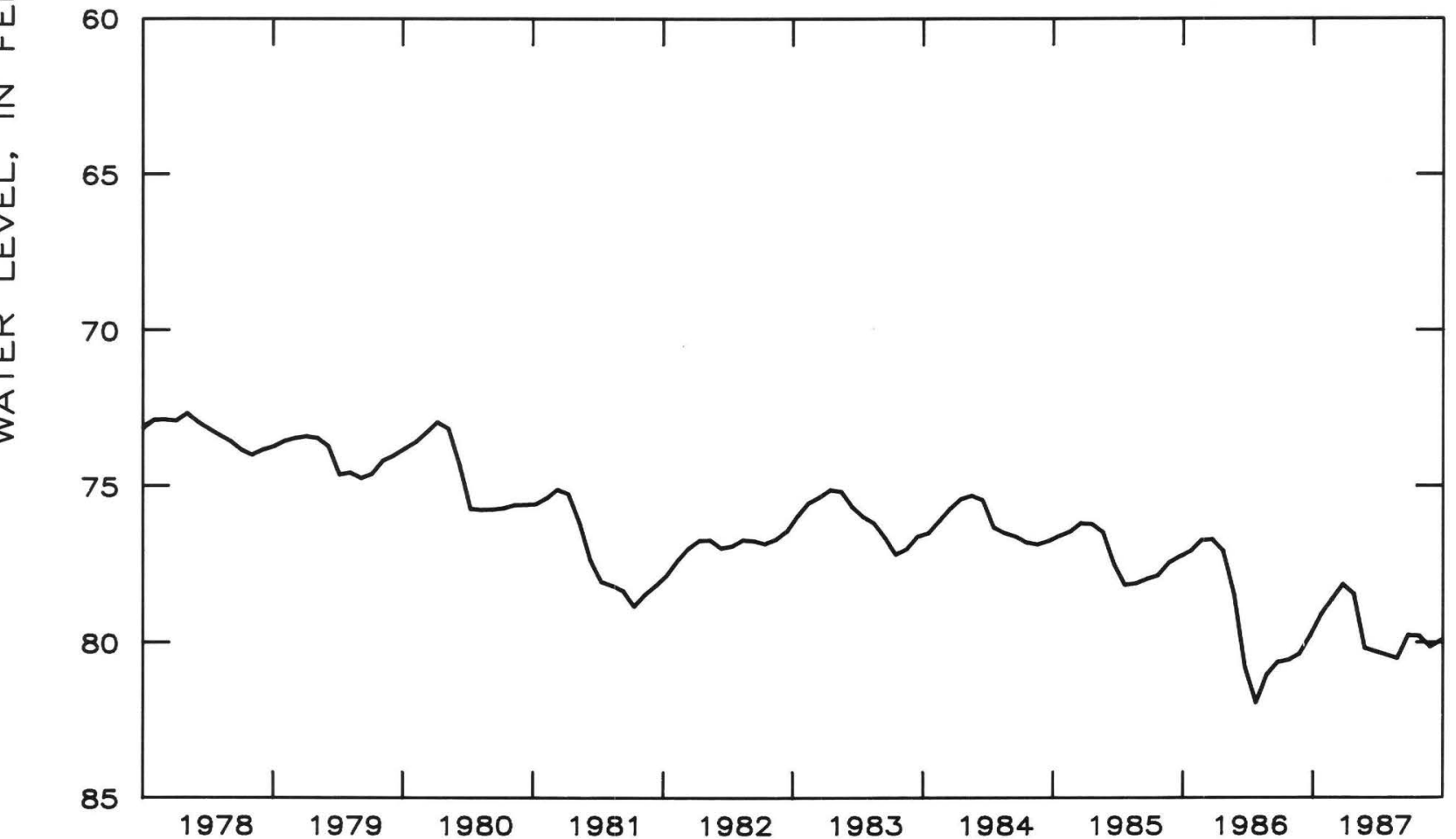

Figure 2.7.3-3.--Water level in observation well 25Q001, Montgomery County. 
321302082243601 Local number, 26R001.

LOCATION.--Lat $32^{\circ} 13^{\prime} 02^{\prime \prime}$, long $82^{\circ} 24^{\prime} 36^{\prime \prime}$, Hydrologic Unit $03070107,15 \mathrm{ft}$ south of the Vidalia Water and Street Department and Fire Station.

Owner: City of Vidalia, well 2.

AQUIFER.--Upper Floridan aquifer.

WELL CHARACTERISTICS.--Drilled municipal well, diameter $12 \mathrm{in.,} \mathrm{depth} \mathrm{1,000} \mathrm{ft,} \mathrm{cased} \mathrm{to} 720 \mathrm{ft}$, open hole. DATUM.--Elevation of land-surface datum is $285 \mathrm{ft}$.

Measuring point: Top of 12-in. casing.

REMARKS.--Water level affected by city pumping. Water levels for periods of missing record, May 25, June 28, September 1-8, and December 9, were estimated.

PERIOD OF RECORD.--April 1974 to current year.

EXTREMES FOR PERIOD OF RECORD.--Highest water level, $151.64 \mathrm{ft}$ below land-surface datum, April 15, 1974 ; 10west, $171.94 \mathrm{ft}$ below land-surface datum, July 10, 1986 .

DEPTH BELOW LAND SURFACE (WATER LEVEL) (FEET) CALENDAR YEAR JANUARY 1987 TO DECEMBER 1987 MEAN VALUES

\begin{tabular}{|c|c|c|c|c|c|c|c|c|c|c|c|c|}
\hline DAY & JAN & FEB & MAR & APR & MAY & JUN & JUL & AUG & SEP & OCT & NOV & DEC \\
\hline $\begin{array}{l}1 \\
2 \\
3 \\
4 \\
5\end{array}$ & $\begin{array}{l}165.13 \\
165.28 \\
165.32 \\
165.01 \\
165.23\end{array}$ & $\begin{array}{l}164.66 \\
164.62 \\
164.69 \\
164.87 \\
164.85\end{array}$ & $\begin{array}{l}163.83 \\
163.91 \\
164.00 \\
164.08 \\
164.09\end{array}$ & $\begin{array}{l}163.05 \\
162.68 \\
162.33 \\
162.47 \\
162.23\end{array}$ & $\begin{array}{l}165.46 \\
165.78 \\
166.02 \\
166.16 \\
165.66\end{array}$ & $\begin{array}{l}167.19 \\
166.87 \\
166.55 \\
166.51 \\
165.83\end{array}$ & $\begin{array}{l}165.55 \\
165.65 \\
165.40 \\
165.15 \\
165.10\end{array}$ & $\begin{array}{l}167.42 \\
167.14 \\
167.00 \\
166.99 \\
167.12\end{array}$ & $\begin{array}{l}167.55 \\
167.39 \\
167.21 \\
166.96 \\
166.50\end{array}$ & $\begin{array}{l}165.90 \\
166.61 \\
166.01 \\
165.68 \\
165.63\end{array}$ & $\begin{array}{l}166.33 \\
166.39 \\
166.31 \\
166.25 \\
166.08\end{array}$ & $\begin{array}{l}165.19 \\
165.30 \\
165.36 \\
165.33 \\
165.23\end{array}$ \\
\hline $\begin{array}{r}6 \\
7 \\
8 \\
9 \\
10\end{array}$ & $\begin{array}{l}165.42 \\
165.35 \\
165.47 \\
165.33 \\
164.95\end{array}$ & $\begin{array}{l}164.67 \\
164.42 \\
164.26 \\
164.42 \\
164.80\end{array}$ & $\begin{array}{l}164.02 \\
163.94 \\
163.46 \\
163.31 \\
162.83\end{array}$ & $\begin{array}{l}162.62 \\
162.93 \\
162.96 \\
162.98 \\
163.01\end{array}$ & $\begin{array}{l}165 . \\
165 . \\
165 . \\
165 . \\
165 .\end{array}$ & $\begin{array}{l}165.68 \\
165.72 \\
165.75 \\
166.35 \\
166.71\end{array}$ & $\begin{array}{l}165.20 \\
165.35 \\
165.66 \\
165.97 \\
166.10\end{array}$ & $\begin{array}{l}167.29 \\
167.43 \\
167.33 \\
167.28 \\
167.37\end{array}$ & $\begin{array}{l}166.11 \\
165.81 \\
165.84 \\
165.90 \\
165.67\end{array}$ & $\begin{array}{l}165.73 \\
165.94 \\
166.14 \\
165.28 \\
166.25\end{array}$ & $\begin{array}{l}166.10 \\
166.29 \\
166.13 \\
166.25 \\
166.07\end{array}$ & $\begin{array}{l}165.12 \\
165.30 \\
165.45 \\
165.54 \\
165.63\end{array}$ \\
\hline $\begin{array}{l}11 \\
12 \\
13 \\
14 \\
15\end{array}$ & $\begin{array}{l}165.00 \\
165.08 \\
165.10 \\
165.19 \\
165.51\end{array}$ & $\begin{array}{l}164.83 \\
164.69 \\
164.64 \\
164.44 \\
164.21\end{array}$ & $\begin{array}{l}162.95 \\
163.00 \\
162.92 \\
162.76 \\
162.75\end{array}$ & $\begin{array}{l}163.07 \\
163.10 \\
163.18 \\
162.94 \\
163.21\end{array}$ & $\begin{array}{l}164.96 \\
164.80 \\
164.48 \\
164.39 \\
164.07\end{array}$ & $\begin{array}{l}166.85 \\
167.07 \\
166.86 \\
165.77 \\
165.50\end{array}$ & $\begin{array}{l}166.18 \\
166.22 \\
166.52 \\
166.78 \\
167.19\end{array}$ & $\begin{array}{l}167.21 \\
167.12 \\
166.95 \\
166.96 \\
166.56\end{array}$ & $\begin{array}{l}165.67 \\
165.58 \\
165.31 \\
165.38 \\
165.45\end{array}$ & $\begin{array}{l}165.90 \\
165.77 \\
165.98 \\
166.24 \\
166.15\end{array}$ & $\begin{array}{l}166.18 \\
166.19 \\
166.06 \\
166.14 \\
166.06\end{array}$ & $\begin{array}{l}165.54 \\
165.38 \\
165.26 \\
165.30 \\
165.18\end{array}$ \\
\hline $\begin{array}{l}16 \\
17 \\
18 \\
19 \\
20\end{array}$ & $\begin{array}{l}165.64 \\
165.75 \\
165.66 \\
165.50 \\
165.37\end{array}$ & $\begin{array}{l}164.05 \\
164.34 \\
164.57 \\
164.53 \\
164.67\end{array}$ & $\begin{array}{l}163.04 \\
162.64 \\
162.57 \\
162.61\end{array}$ & $\begin{array}{l}162.76 \\
162.94 \\
163.07 \\
162.70\end{array}$ & $\begin{array}{l}164.18 \\
164.42 \\
164.39 \\
164.21\end{array}$ & $\begin{array}{l}165.45 \\
165.46 \\
165.33 \\
165.36 \\
165.22\end{array}$ & $\begin{array}{l}167.45 \\
167.80 \\
168.06 \\
168.16 \\
168.53\end{array}$ & $\begin{array}{l}166.29 \\
166.29 \\
166.41 \\
166.46 \\
166.50\end{array}$ & $\begin{array}{l}165.63 \\
165.78 \\
165.90 \\
165.79 \\
165.52\end{array}$ & & $\begin{array}{l}165.95 \\
165.77 \\
165.62 \\
165.50 \\
165.50\end{array}$ & $\begin{array}{l}165.20 \\
165.24 \\
165.22 \\
165.17 \\
165.17\end{array}$ \\
\hline $\begin{array}{l}21 \\
22 \\
23 \\
24 \\
25\end{array}$ & $\begin{array}{l}165.10 \\
164.82 \\
165.15 \\
165.52 \\
164.80\end{array}$ & $\begin{array}{l}164.30 \\
164.07 \\
164.18 \\
164.42 \\
164.41\end{array}$ & $\begin{array}{l}162.63 \\
162.48 \\
162.60 \\
162.63 \\
162.78\end{array}$ & $\begin{array}{l}163.45 \\
164.06 \\
164.16 \\
163.78 \\
163.81\end{array}$ & & $\begin{array}{l}165.05 \\
165.02 \\
165.28 \\
165.47 \\
165.97\end{array}$ & & $\begin{array}{l}166.59 \\
166.80 \\
167.24 \\
167.47 \\
167.85\end{array}$ & & & $\begin{array}{l}165.71 \\
165.54 \\
165.56 \\
165.80 \\
165.93\end{array}$ & $\begin{array}{l}165.05 \\
164.96 \\
165.02 \\
164.99 \\
164.68\end{array}$ \\
\hline $\begin{array}{l}26 \\
27 \\
28 \\
29 \\
30 \\
31\end{array}$ & $\begin{array}{l}164.92 \\
165.11 \\
165.26 \\
165.17 \\
164.84 \\
164.87\end{array}$ & $\begin{array}{r}164.54 \\
164.29 \\
164.12 \\
--- \\
--- \\
-.-\end{array}$ & $\begin{array}{l}162.60 \\
162.48 \\
162.45 \\
162.13 \\
162.10 \\
162.34\end{array}$ & $\begin{array}{r}163.98 \\
164.30 \\
164.66 \\
164.87 \\
165.18 \\
---\end{array}$ & $\begin{array}{l}165.36 \\
165.55 \\
165.72 \\
165.98 \\
166.23 \\
166.70\end{array}$ & $\begin{array}{r}165.56 \\
165.14 \\
165.29 \\
165.44 \\
165.48 \\
---\end{array}$ & $\begin{array}{l}167.50 \\
167.50 \\
167.51 \\
167.46 \\
167.07 \\
166.89\end{array}$ & $\begin{array}{l}167.76 \\
167.87 \\
168.17 \\
168.20 \\
167.85 \\
167.84\end{array}$ & $\begin{array}{r}166.13 \\
166.07 \\
166.49 \\
166.46 \\
165.95 \\
---\end{array}$ & $\begin{array}{l}166 \\
166 \\
166 \\
166 \\
166 \\
166\end{array}$ & $\begin{array}{r}165.68 \\
165.24 \\
165.20 \\
164.98 \\
165.03 \\
---\end{array}$ & $\begin{array}{l}164.67 \\
164.67 \\
164.53 \\
164.61 \\
164.90 \\
164.70\end{array}$ \\
\hline 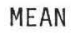 & 65.22 & 164.48 & 163.00 & 163.31 & 165.08 & 165.86 & 167.02 & 167.19 & 166.07 & 166.17 & 165.86 & 165.1 \\
\hline
\end{tabular}




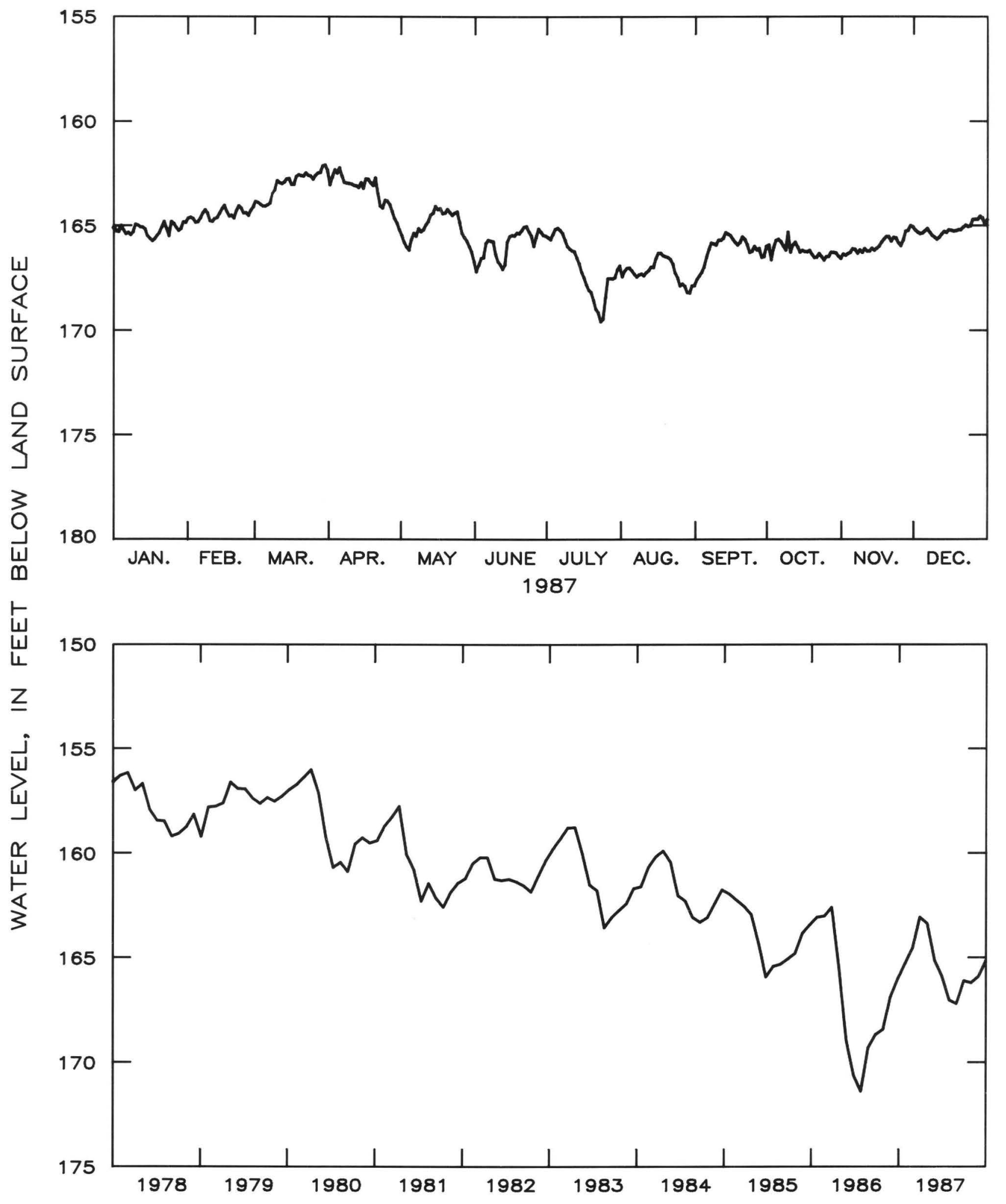

Figure 2.7.3-4.--Water level in observation well 26R001, Toombs County. 
2.7.4 Coastal area

In the coastal area of Georgia and adjacent parts of Florida and South Carolina, the potentiometric surface of the Upper Floridan aquifer is characterized by cones of depression that are caused by large ground-water withdrawals. The combined pumpage in the coastal area of Georgia in 1985 was about $306 \mathrm{Mgal} / \mathrm{d}$ (Turlington and others, 1987), about 69 percent of which was used for industrial purposes. In the coastal areas of Georgia, nearly all the ground water is pumped from the Upper Floridan aquifer. Ground-water pumping from the Upper Floridan, primarily in the Savannah, Jesup, Brunswick, and St Marys-Fernandina Beach areas, has resulted in water-level declines and in the development of cones of depression. Because the Upper Floridan aquifer in the coastal area is deeply buried and far from the outcrop area, the ground-water level is not influenced by concurrent rainfall, but rather by increased withdrawals during dry periods. 


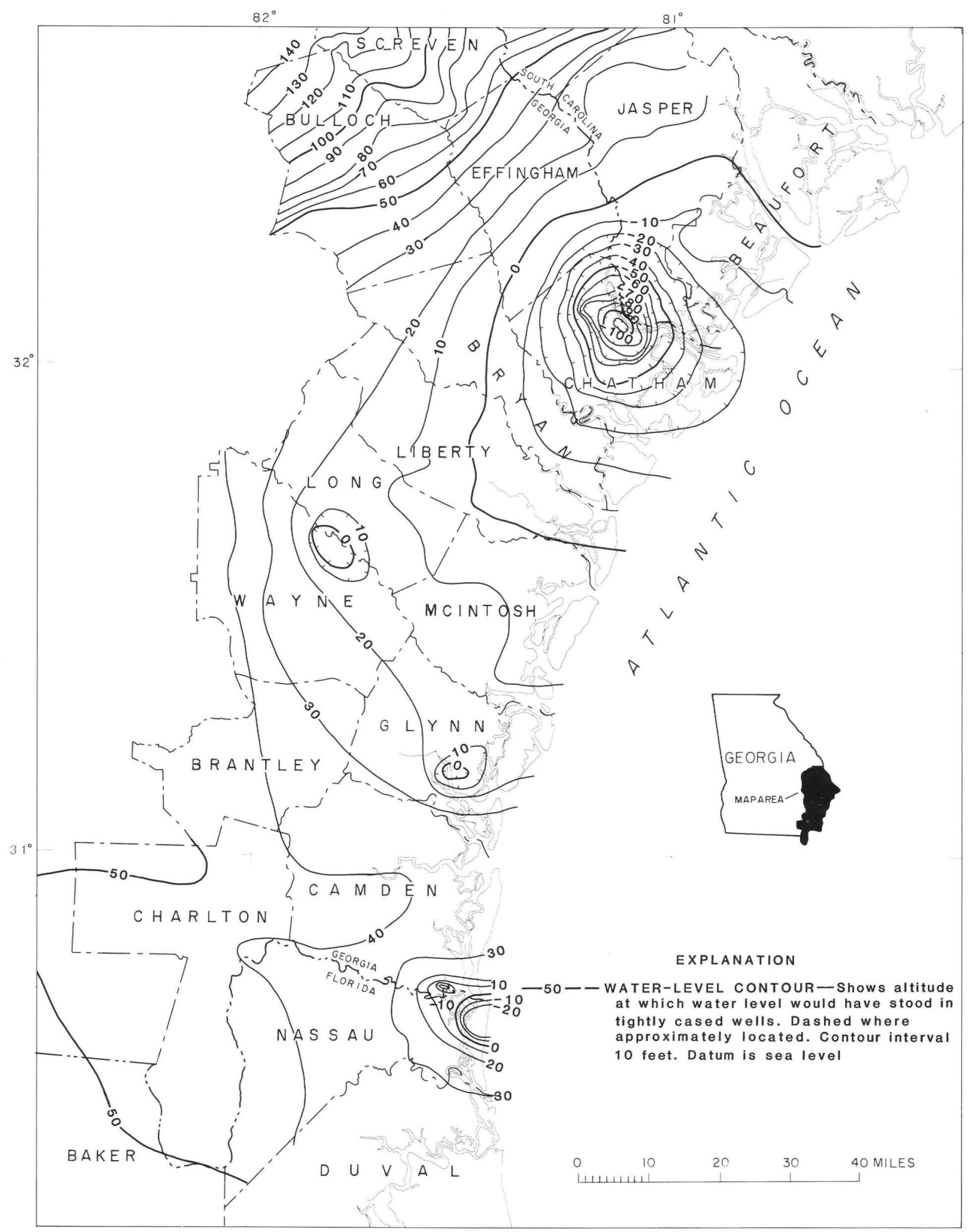

Figure 2.7.4-1.-Water level in the Upper Floridan aquifer in the coastal area, May 1985. 


\subsubsection{Savannah area}

The water level in the Upper Floridan aquifer in the Savannah area is affected by pumping for municipal and industrial uses, and in 1985, withdrawals exceeded $82 \mathrm{Mgal} / \mathrm{d}$ (Turlington and others, 1987). As a result of this pumping, a cone of depression has developed in the potentiometric surface around Savannah. Hydrographs for observation wells near the center of pumping, and in outlying areas, illustrate the effects of pumping on the ground-water level.

During 1987, water levels near the center of pumping showed a recovery from the effects of the 1986 drought. The 1987 mean water levels in wells 380002,360020 , and 36Q008 were from 0.2 to $4.4 \mathrm{ft}$ higher than in 1986, which reversed the downward trend that began in 1983. By the end of March, the water levels in wells 360020, 380002, and 39Q003, had recovered 6.4 to $8.4 \mathrm{ft}$ from the low and record lows measured during the 1986 drought. By the middle of April, the water level in well $36 Q 008$ had recovered $20.8 \mathrm{ft}$ from the low measured in August 1986. The mean water level in well 390003 at Savannah Beach was $0.4 \mathrm{ft}$ lower in 1987 than in 1986, which continued the downward trend in that area. Although there was some recovery from the effects of the 1986 drought, water levels in three of the four wells were lower at the end of 1987 than at the end of 1986 .

Observation well 32R002, located west of the pumping center at Savannah, also responds to changes in pumping at Savannah, but to a lesser degree than wells in the area of the cone of depression. During 1987, the mean water level in the well was about the same as in 1986, and water levels showed a recovery from the effects of the 1986 drought. By the end of April, the water level had recovered $3.3 \mathrm{ft}$ from the $10 \mathrm{w}$ measured in August during the 1986 drought. At the end of 1987, the water level was about the same as at the end of 1986 . 


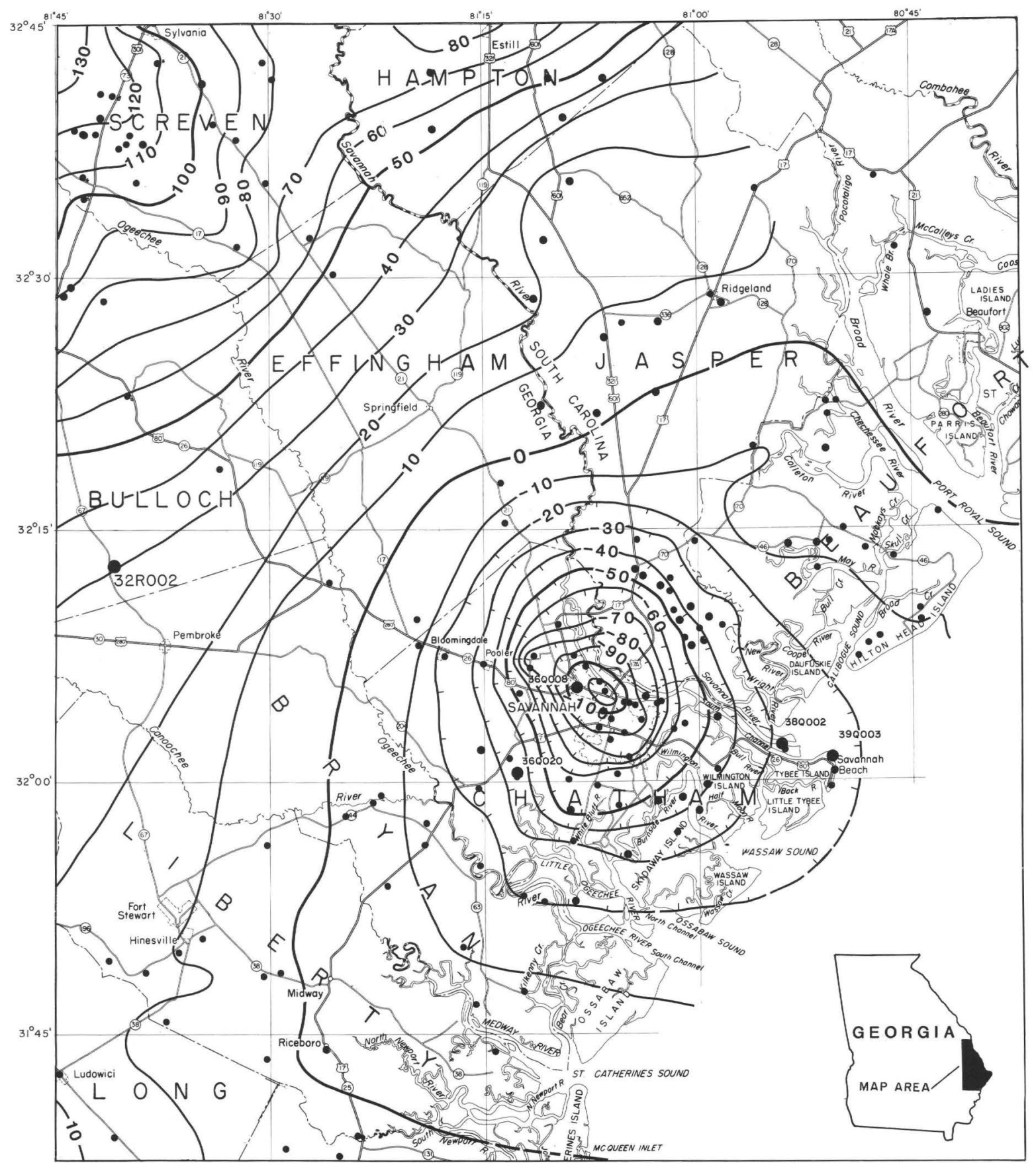

Base from U.S.Geological Survey 1:250, 000 quadrangles

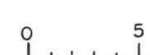

10

$15 \quad 20$

25 MILES

EXPLANATION

-10-- WATER-LEVEL CONTOUR - Shows altitude at which water level would have stood in tightly cased wells. Dashed where approximately located. Contour interval is 10 feet. Datum is sea level
380002

- well and identification number for WHICH HYDROGRAPHS ARE INCLUDED IN THIS REPORT

- data POINT

Figure 2.7.4.1-1.-Observation well locations and the water level in the Upper Floridan aquifer in the Savannah area, May 1985. 
320530081085001 Local number, 360008 .

LOCATION.--Lat $32^{\circ} 05^{\prime} 30^{\prime \prime}$, long $81^{\circ} 08^{\prime} 50^{\prime \prime}$, Hydrologic Unit $03060204,0.19 \mathrm{mi}$ southeast of intersection of Alfred Street and U.S. Highway 80.

Owner: Layne-Atlantic Co.

AQUIFER.--Upper Floridan aquifer.

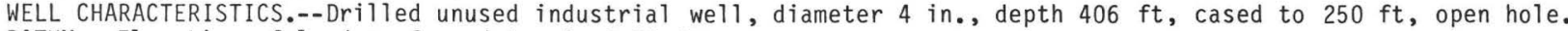
DATUM.--Elevation of land-surface datum is $9.91 \mathrm{ft}$.

Measuring point: Top of $3-i n$. casing, $1.0 \mathrm{ft}$ above land-surface datum.

REMARKS.--Water levels for periods of missing record, January 30, February 3-18, and March 22, were estimated. PERIOD OF RECORD.--February 1954 to current year.

EXTREMES FOR PERIOD OF RECORD.--Highest water level, $49.17 \mathrm{ft}$ below land-surface datum, July 11, 1954; 1owest, $124.40 \mathrm{ft}$ below land-surface datum, August 30, 1980.

DEPTH BELOW LAND SURFACE (WATER LEVEL) (FEET) CALENDAR YEAR JANUARY 1987 TO DECEMBER 1987 MEAN VALUES

\begin{tabular}{|c|c|c|c|c|c|c|c|c|c|c|c|c|}
\hline DAY & JAN & FEB & MAR & APR & MAY & JUN & JUL & AUG & SEP & OCT & NOV & $\mathrm{DE}$ \\
\hline $\begin{array}{l}1 \\
2 \\
3 \\
4 \\
5\end{array}$ & $\begin{array}{l}108.03 \\
108.26 \\
107.70 \\
107.30 \\
108.06\end{array}$ & $\begin{array}{l}105.17 \\
104.42 \\
104.30 \\
104.54 \\
104.59\end{array}$ & $\begin{array}{l}101.22 \\
101.50 \\
102.48 \\
102.78 \\
102.70\end{array}$ & $\begin{array}{l}104.54 \\
103.82 \\
103.70 \\
102.78 \\
101.30\end{array}$ & $\begin{array}{l}107.56 \\
108.51 \\
108.42 \\
108.66 \\
109.86\end{array}$ & $\begin{array}{l}107.10 \\
108.70 \\
109.70 \\
110.22 \\
109.76\end{array}$ & $\begin{array}{l}110.81 \\
111.03 \\
110.75 \\
109.92 \\
109.61\end{array}$ & $\begin{array}{l}112.36 \\
111.81 \\
112.04 \\
113.17 \\
113.25\end{array}$ & $\begin{array}{l}115.20 \\
115.14 \\
115.29 \\
115.70 \\
114.81\end{array}$ & $\begin{array}{l}115.16 \\
115.19 \\
115.37 \\
115.45 \\
115.60\end{array}$ & $\begin{array}{l}112.48 \\
112.45 \\
112.91 \\
112.38 \\
111.71\end{array}$ & 107.8 \\
\hline $\begin{array}{r}6 \\
7 \\
8 \\
9 \\
10\end{array}$ & $\begin{array}{l}109.28 \\
108.61 \\
107.44 \\
108.02 \\
106.99\end{array}$ & $\begin{array}{l}104.04 \\
103.68 \\
103.64 \\
103.83 \\
104.59\end{array}$ & $\begin{array}{l}102.69 \\
102.38 \\
101.56 \\
101.74 \\
102.98\end{array}$ & $\begin{array}{l}101.55 \\
102.37 \\
102.36 \\
102.90 \\
103.25\end{array}$ & $\begin{array}{l}109.76 \\
109.20 \\
108.24 \\
107.78 \\
106.72\end{array}$ & $\begin{array}{l}108.83 \\
107.78 \\
108.39 \\
108.94 \\
109.00\end{array}$ & $\begin{array}{l}109.65 \\
110.81 \\
112.27 \\
113.56 \\
114.36\end{array}$ & $\begin{array}{l}113.85 \\
114.12 \\
113.37 \\
113.11 \\
113.21\end{array}$ & $\begin{array}{l}113.48 \\
113.00 \\
113.27 \\
114.38 \\
114.54\end{array}$ & $\begin{array}{l}115.75 \\
115.60 \\
115.70 \\
115.52 \\
115.36\end{array}$ & $\begin{array}{l}110.94 \\
110.45 \\
110.44 \\
109.34 \\
108.83\end{array}$ & 110. \\
\hline $\begin{array}{l}11 \\
12 \\
13 \\
14 \\
15\end{array}$ & $\begin{array}{l}105.29 \\
106.37 \\
108.14 \\
108.56 \\
108.16\end{array}$ & $\begin{array}{l}104.39 \\
104.20 \\
104.20 \\
103.77 \\
103.47\end{array}$ & $\begin{array}{l}103.38 \\
103.47 \\
103.61 \\
102.80 \\
101.52\end{array}$ & $\begin{array}{l}102.90 \\
102.20 \\
101.76 \\
102.99 \\
102.49\end{array}$ & $\begin{array}{l}106.61 \\
107.60 \\
108.10 \\
107.60 \\
108.05\end{array}$ & $\begin{array}{l}109.47 \\
109.51 \\
109.60 \\
108.54 \\
108.06\end{array}$ & $\begin{array}{l}114.98 \\
115.46 \\
116.25 \\
116.97 \\
117.25\end{array}$ & $\begin{array}{l}113.69 \\
113.94 \\
113.92 \\
114.11 \\
114.44\end{array}$ & $\begin{array}{l}115.18 \\
115.08 \\
114.78 \\
114.59 \\
114.85\end{array}$ & $\begin{array}{l}114.74 \\
114.68 \\
115.01 \\
115.38 \\
115.38\end{array}$ & $\begin{array}{l}108.76 \\
108.83 \\
108.22 \\
106.92 \\
105.80\end{array}$ & 109. \\
\hline $\begin{array}{l}16 \\
17 \\
18 \\
19 \\
20\end{array}$ & $\begin{array}{l}106.75 \\
105.57 \\
105.76 \\
106.14 \\
105.54\end{array}$ & $\begin{array}{l}102.92 \\
103.06 \\
103.20 \\
103.57 \\
103.32\end{array}$ & $\begin{array}{l}101.48 \\
102.18 \\
102.37 \\
102.35 \\
102.84\end{array}$ & $\begin{array}{l}102.56 \\
102.06 \\
101.41 \\
101.20 \\
101.84\end{array}$ & $\begin{array}{l}107.46 \\
106.76 \\
107.18 \\
107.80 \\
107.80\end{array}$ & $\begin{array}{l}108.07 \\
109.02 \\
109.52 \\
109.66 \\
109.78\end{array}$ & & & $\begin{array}{l}115.59 \\
116.10 \\
116.75 \\
116.40 \\
116.21\end{array}$ & $\begin{array}{l}115.52 \\
114.70 \\
114.05 \\
114.08 \\
114.79\end{array}$ & $\begin{array}{l}106.18 \\
107.22 \\
106.89 \\
106.84 \\
107.18\end{array}$ & 107 \\
\hline $\begin{array}{l}21 \\
22 \\
23 \\
24 \\
25\end{array}$ & $\begin{array}{l}105.36 \\
105.62 \\
105.50 \\
106.19 \\
105.92\end{array}$ & $\begin{array}{l}102.56 \\
101.54 \\
101.94 \\
102.39 \\
102.81\end{array}$ & $\begin{array}{l}103.15 \\
102.92 \\
102.74 \\
103.62 \\
104.23\end{array}$ & $\begin{array}{l}101.76 \\
102.86 \\
103.28 \\
103.63 \\
103.77\end{array}$ & $\begin{array}{l}106.80 \\
107.16 \\
106.38 \\
105.83 \\
105.35\end{array}$ & $\begin{array}{l}108.68 \\
107.85 \\
107.76 \\
108.80 \\
110.45\end{array}$ & & & $\begin{array}{l}116.27 \\
116.38 \\
115.86 \\
115.37 \\
115.28\end{array}$ & $\begin{array}{l}115.00 \\
114.44 \\
113.81 \\
113.52 \\
112.82\end{array}$ & $\begin{array}{l}108.19 \\
106.66 \\
106.40 \\
108.14 \\
108.78\end{array}$ & 106. \\
\hline $\begin{array}{l}26 \\
27 \\
28 \\
29 \\
30 \\
31\end{array}$ & $\begin{array}{l}105.93 \\
106.69 \\
108.08 \\
108.42 \\
107.50 \\
106.66\end{array}$ & $\begin{array}{r}103.15 \\
103.10 \\
102.26 \\
-.- \\
-.- \\
---\end{array}$ & $\begin{array}{l}104.00 \\
103.92 \\
103.44 \\
102.68 \\
101.82 \\
102.97\end{array}$ & $\begin{array}{r}103.81 \\
103.83 \\
104.49 \\
105.02 \\
105.77 \\
-.-\end{array}$ & $\begin{array}{l}105.58 \\
106.20 \\
106.92 \\
107.38 \\
106.78 \\
106.48\end{array}$ & $\begin{array}{r}110.53 \\
109.76 \\
109.40 \\
109.61 \\
110.55 \\
-.-\end{array}$ & $\begin{array}{l}112.17 \\
112.32 \\
112.85 \\
112.71 \\
112.35 \\
111.90\end{array}$ & $\begin{array}{l}113.91 \\
114.53 \\
115.05 \\
115.15 \\
115.15 \\
115.02\end{array}$ & $\begin{array}{r}114.98 \\
114.46 \\
114.74 \\
115.60 \\
115.47 \\
---\end{array}$ & $\begin{array}{l}112.71 \\
113.11 \\
113.19 \\
113.47 \\
113.32 \\
113.30\end{array}$ & $\begin{array}{r}107.41 \\
105.38 \\
105.23 \\
106.39 \\
106.77 \\
---\end{array}$ & 107. \\
\hline 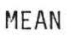 & 107.03 & 103.52 & 102.69 & 102.94 & 107.44 & 109.10 & 113.13 & 113.20 & 115.16 & 114.57 & 108.47 & \\
\hline
\end{tabular}



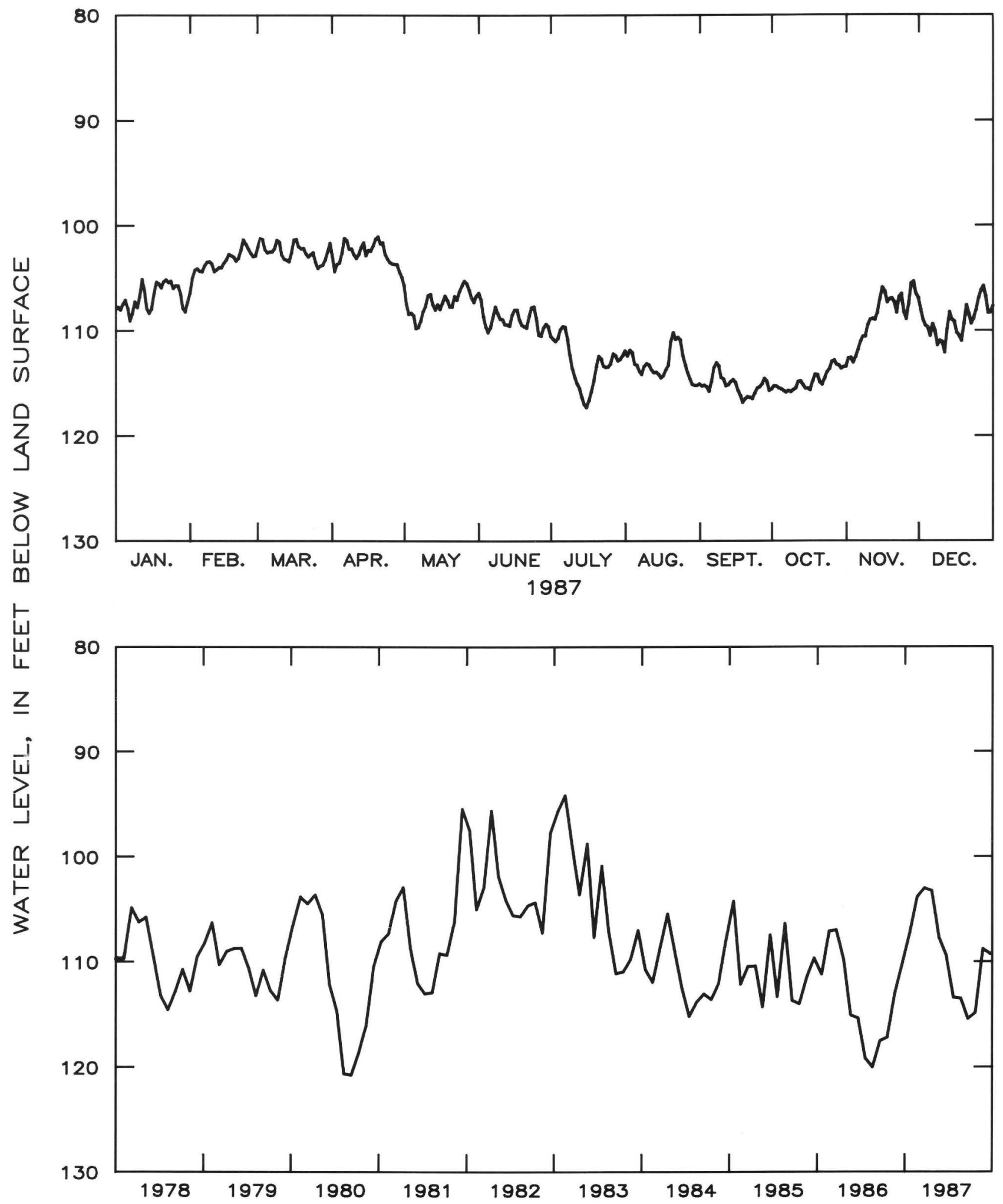

Figure 2.7.4.1-2.--Water level in observation well 36Q008, Chatham County. 
320021081124801 Local number, 360020 .

LOCATION.--Lat $32^{\circ} 00^{\prime} 18^{\prime \prime}$, long $81^{\circ} 12^{\prime} 48^{\prime \prime}$, Hydrologic Unit $03060204,2.7$ mi south of intersection of U.S. Highway 17 with Dean Forest Road.

Owner: H. J. Morrison.

AQUIFER.--Upper Floridan aquifer.

WELL CHARACTERISTICS.--Drilled unused domestic we1l, diameter $3 \mathrm{in.,}$ depth $365 \mathrm{ft}$, cased to $330 \mathrm{ft}$, open hole. DATUM.--Elevation of land-surface datum is $13 \mathrm{ft}$.

Measuring point: Floor of recorder shelter, $3.88 \mathrm{ft}$ above land-surface datum.

REMARKS.--Borehole geophysical survey, May 7, 1985.

PERIOD OF RECORD.--March 1958 to current year.

EXTREMES FOR PERIOD OF RECORD.--Highest water level, $17.66 \mathrm{ft}$ below land-surface datum, June 28, 1958; 1owest, $54.45 \mathrm{ft}$ below land-surface datum, July 23,1986 .

DEPTH BELOW LAND SURFACE (WATER LEVEL) (FEET) CALENDAR YEAR JANUARY 1987 TO DECEMBER 1987 MEAN VALUES

\begin{tabular}{|c|c|c|c|c|c|c|c|c|c|c|c|c|}
\hline DAY & JAN & FEB & MAR & APR & MAY & JUN & JUL & AUG & SEP & OCT & NOV & $\mathrm{DEC}$ \\
\hline $\begin{array}{l}1 \\
2 \\
3 \\
4 \\
5\end{array}$ & $\begin{array}{l}47.79 \\
48.06 \\
48.14 \\
47.87 \\
47.85\end{array}$ & $\begin{array}{l}47.68 \\
47.46 \\
47.46 \\
47.56 \\
47.60\end{array}$ & $\begin{array}{l}46.40 \\
46.57 \\
46.66 \\
46.69 \\
46.75\end{array}$ & $\begin{array}{l}46.52 \\
46.47 \\
46.34 \\
46.41 \\
46.40\end{array}$ & $\begin{array}{l}47.49 \\
47.76 \\
48.11 \\
48.38 \\
48.61\end{array}$ & $\begin{array}{l}50.53 \\
50.62 \\
50.72 \\
50.80 \\
50.76\end{array}$ & $\begin{array}{l}50.31 \\
50.29 \\
50.33 \\
50.31 \\
50.31\end{array}$ & $\begin{array}{l}52.28 \\
52.28 \\
52.29 \\
52.31 \\
52.29\end{array}$ & $\begin{array}{l}52.70 \\
52.59 \\
52.50 \\
52.37 \\
52.20\end{array}$ & $\begin{array}{l}51.48 \\
51.52 \\
51.52 \\
51.58 \\
51.47\end{array}$ & $\begin{array}{l}51.38 \\
51.28 \\
51.13 \\
50.90 \\
50.81\end{array}$ & $\begin{array}{l}49.41 \\
49.53 \\
49.47 \\
49.35 \\
49.51\end{array}$ \\
\hline $\begin{array}{r}6 \\
7 \\
8 \\
9 \\
10\end{array}$ & $\begin{array}{l}47.96 \\
47.90 \\
47.91 \\
47.86 \\
47.65\end{array}$ & $\begin{array}{l}47.38 \\
47.16 \\
47.08 \\
47.30 \\
47.37\end{array}$ & $\begin{array}{l}46.75 \\
46.54 \\
46.27 \\
46.23 \\
46.30\end{array}$ & $\begin{array}{l}46.39 \\
46.34 \\
46.31 \\
46.28 \\
46.27\end{array}$ & $\begin{array}{l}48.59 \\
48.59 \\
48.65 \\
48.90 \\
49.08\end{array}$ & $\begin{array}{l}50.72 \\
50.77 \\
50.78 \\
50.79 \\
50.84\end{array}$ & $\begin{array}{l}50.32 \\
50.23 \\
50.19 \\
50.25 \\
50.28\end{array}$ & $\begin{array}{l}52.35 \\
52.38 \\
52.44 \\
52.50 \\
52.51\end{array}$ & $\begin{array}{l}52.09 \\
51.93 \\
51.95 \\
51.95 \\
51.94\end{array}$ & $\begin{array}{l}51.34 \\
51.36 \\
51.54 \\
51.64 \\
51.60\end{array}$ & $\begin{array}{l}51.02 \\
51.04 \\
50.98 \\
50.85 \\
50.61\end{array}$ & $\begin{array}{l}49.61 \\
49.63 \\
49.56 \\
49.48 \\
49.35\end{array}$ \\
\hline $\begin{array}{l}11 \\
12 \\
13 \\
14 \\
15\end{array}$ & $\begin{array}{l}47.80 \\
47.82 \\
47.80 \\
47.77 \\
47.66\end{array}$ & $\begin{array}{l}47.28 \\
47.11 \\
47.08 \\
46.98 \\
46.96\end{array}$ & $\begin{array}{l}46.48 \\
46.46 \\
46.41 \\
46.39 \\
46.36\end{array}$ & $\begin{array}{l}46.32 \\
46.46 \\
46.62 \\
46.63 \\
46.63\end{array}$ & $\begin{array}{l}49.16 \\
49.14 \\
49.10 \\
49.12 \\
49.12\end{array}$ & $\begin{array}{l}50.93 \\
51.02 \\
51.04 \\
50.95 \\
50.87\end{array}$ & $\begin{array}{l}50.38 \\
50.53 \\
50.65 \\
50.78 \\
50.98\end{array}$ & $\begin{array}{l}52.39 \\
52.39 \\
52.42 \\
52.46 \\
52.50\end{array}$ & $\begin{array}{l}51.95 \\
51.92 \\
51.86 \\
51.83 \\
51.80\end{array}$ & $\begin{array}{l}51.44 \\
51.30 \\
51.34 \\
51.43 \\
51.40\end{array}$ & $\begin{array}{l}50.65 \\
50.71 \\
50.60 \\
50.53 \\
50.56\end{array}$ & $\begin{array}{l}49.29 \\
49.29 \\
49.48 \\
49.50 \\
49.30\end{array}$ \\
\hline $\begin{array}{l}16 \\
17 \\
18 \\
19 \\
20\end{array}$ & $\begin{array}{l}47.60 \\
47.57 \\
47.44 \\
47.39 \\
47.49\end{array}$ & $\begin{array}{l}46.70 \\
46.84 \\
46.94 \\
47.08 \\
47.07\end{array}$ & $\begin{array}{l}46.33 \\
46.35 \\
46.21 \\
46.07 \\
46.15\end{array}$ & $\begin{array}{l}46.63 \\
46.63 \\
46.63 \\
46.63 \\
46.63\end{array}$ & $\begin{array}{l}49.18 \\
49.31 \\
49.40 \\
49.44 \\
49.44\end{array}$ & $\begin{array}{l}50.71 \\
50.64 \\
50.65 \\
50.58 \\
50.51\end{array}$ & $\begin{array}{l}51.19 \\
51.41 \\
51.55 \\
51.67 \\
51.81\end{array}$ & $\begin{array}{l}52.54 \\
52.54 \\
52.52 \\
52.48 \\
52.51\end{array}$ & $\begin{array}{l}51.75 \\
51.72 \\
51.69 \\
51.68 \\
51.67\end{array}$ & $\begin{array}{l}51.36 \\
51.32 \\
51.35 \\
51.36 \\
51.35\end{array}$ & $\begin{array}{l}50.48 \\
50.28 \\
50.26 \\
50.13 \\
49.95\end{array}$ & $\begin{array}{l}49.40 \\
49.53 \\
49.56 \\
49.51 \\
49.42\end{array}$ \\
\hline $\begin{array}{l}21 \\
22 \\
23 \\
24 \\
25\end{array}$ & $\begin{array}{l}47.40 \\
47.07 \\
47.39 \\
47.58 \\
47.37\end{array}$ & $\begin{array}{l}46.72 \\
46.58 \\
46.75 \\
46.73 \\
46.74\end{array}$ & $\begin{array}{l}46.10 \\
46.15 \\
46.23 \\
46.22 \\
46.19\end{array}$ & $\begin{array}{l}46.67 \\
46.70 \\
46.67 \\
46.61 \\
46.81\end{array}$ & $\begin{array}{l}49.43 \\
49.46 \\
49.52 \\
49.61 \\
49.75\end{array}$ & $\begin{array}{l}50.48 \\
50.44 \\
50.35 \\
50.26 \\
50.19\end{array}$ & $\begin{array}{l}51.91 \\
51.97 \\
52.04 \\
52.22 \\
52.32\end{array}$ & $\begin{array}{l}52.50 \\
52.45 \\
52.42 \\
52.44 \\
52.46\end{array}$ & $\begin{array}{l}51.71 \\
51.70 \\
51.65 \\
51.60 \\
51.64\end{array}$ & $\begin{array}{l}51.40 \\
51.53 \\
51.57 \\
51.55 \\
51.48\end{array}$ & $\begin{array}{l}50.06 \\
50.11 \\
50.11 \\
50.07 \\
49.93\end{array}$ & $\begin{array}{l}49.37 \\
49.29 \\
49.40 \\
49.38 \\
49.30\end{array}$ \\
\hline $\begin{array}{l}26 \\
27 \\
28 \\
29 \\
30 \\
31\end{array}$ & $\begin{array}{l}47.41 \\
47.62 \\
47.72 \\
47.77 \\
47.62 \\
47.69\end{array}$ & $\begin{array}{r}46.73 \\
46.68 \\
46.54 \\
--- \\
--- \\
---\end{array}$ & $\begin{array}{l}46.17 \\
46.13 \\
46.17 \\
46.27 \\
46.12 \\
46.29\end{array}$ & $\begin{array}{r}47.05 \\
47.13 \\
47.13 \\
47.32 \\
47.38 \\
-\end{array}$ & $\begin{array}{l}49.90 \\
50.02 \\
50.08 \\
50.16 \\
50.27 \\
50.42\end{array}$ & $\begin{array}{r}50.13 \\
50.12 \\
50.23 \\
50.32 \\
50.36 \\
. .\end{array}$ & $\begin{array}{l}52.33 \\
52.37 \\
52.40 \\
52.39 \\
52.32 \\
52.33\end{array}$ & $\begin{array}{l}52.52 \\
52.59 \\
52.68 \\
52.78 \\
52.84 \\
52.82\end{array}$ & $\begin{array}{r}51.72 \\
51.77 \\
51.77 \\
51.66 \\
51.44 \\
.-\end{array}$ & $\begin{array}{l}51.33 \\
51.23 \\
51.28 \\
51.35 \\
51.39 \\
51.37\end{array}$ & $\begin{array}{r}49.83 \\
49.72 \\
49.59 \\
49.47 \\
49.36 \\
-\ldots\end{array}$ & $\begin{array}{l}49.24 \\
49.22 \\
49.05 \\
49.13 \\
49.34 \\
49.28\end{array}$ \\
\hline MEAN & 47.68 & 47.06 & 46.34 & 46.63 & 49.20 & 50.60 & 51.24 & 52.48 & 51.89 & 51.43 & 50.41 & 49. \\
\hline
\end{tabular}

$\begin{array}{llllll}\text { CAL YR } 1987 \text { MEAN } & 49.55 & \text { HIGH } & 46.07 & \text { LOW } & 52.84\end{array}$ 


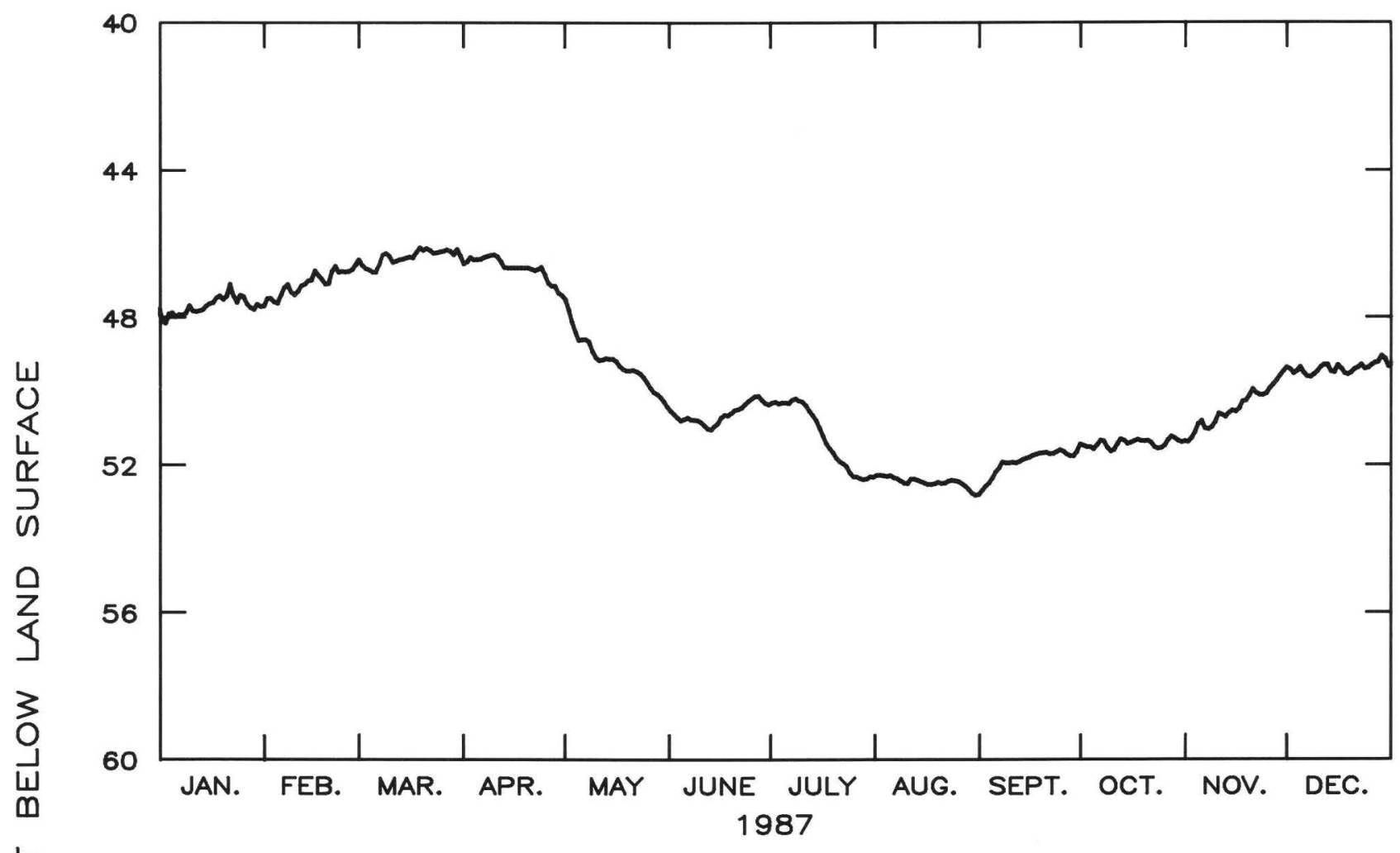

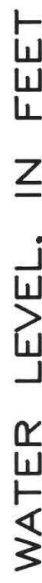

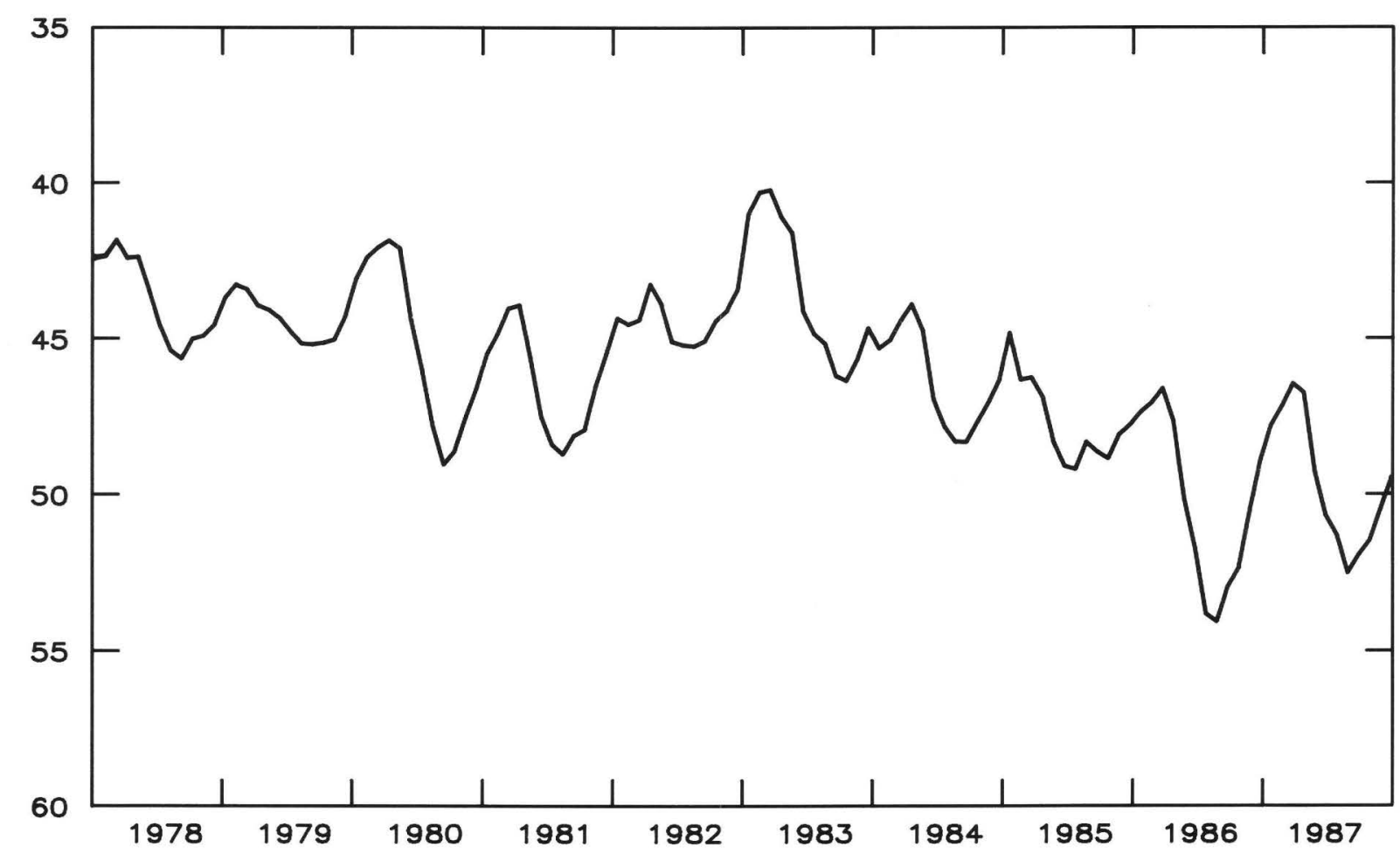

Figure 2.7.4.1-3.--Water level in observation well 36Q020, Chatham County. 
320202080541201 Local number, 380002.

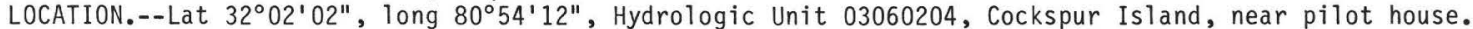

Owner: U.S. Department of the Interior, National Park Service.

AQUIFER.--Upper Floridan aquifer.

WELL CHARACTERISTICS.--Drilled observation well, diameter $8 \mathrm{in.,}$ depth $348 \mathrm{ft}$, cased to $110 \mathrm{ft}$, open hole. DATUM.--Elevation of land-surface datum is $8.0 \mathrm{ft}$.

Measuring point: Floor of recorder shelter, $3.62 \mathrm{ft}$ above land-surface datum.

REMARKS.--Borehole geophysical survey conducted June 16, 1961. Water levels for period of missing record, May 1-3, were estimated.

PERIOD OF RECORD.--February 1956 to current year.

EXTREMES FOR PERIOD OF RECORD.--Highest water leve1, $16.00 \mathrm{ft}$ below land-surface datum, March 5, 1956; 10west, $38.48 \mathrm{ft}$ below land-surface datum, August $4,1986$.

DEPTH BELOW LAND SURFACE (WATER LEVEL) (FEET) CALENDAR YEAR JANUARY 1987 TO DECEMBER 1987 MEAN VALUES

\begin{tabular}{|c|c|c|c|c|c|c|c|c|c|c|c|c|}
\hline DAY & JAN & FEB & MAR & APR & MAY & JUN & JUL & AUG & SEP & ОСТ & NOV & DEC \\
\hline $\begin{array}{l}1 \\
2 \\
3 \\
4 \\
5\end{array}$ & $\begin{array}{l}31.38 \\
31.53 \\
31.73 \\
31.39 \\
31.38\end{array}$ & $\begin{array}{l}33.05 \\
32.98 \\
33.00 \\
32.97 \\
32.89\end{array}$ & $\begin{array}{l}32.24 \\
32.46 \\
32.50 \\
32.54 \\
32.52\end{array}$ & $\begin{array}{l}32.28 \\
32.18 \\
32.16 \\
32.43 \\
32.29\end{array}$ & $\begin{array}{l}33.01 \\
33.17 \\
33.33 \\
33.49 \\
33.25\end{array}$ & $\begin{array}{l}35.54 \\
35.70 \\
35.78 \\
35.79 \\
35.71\end{array}$ & $\begin{array}{l}35.85 \\
35.85 \\
35.92 \\
35.88 \\
35.89\end{array}$ & $\begin{array}{l}37.36 \\
37.37 \\
37.38 \\
37.41 \\
37.36\end{array}$ & $\begin{array}{l}37.58 \\
37.54 \\
37.39 \\
37.11 \\
36.97\end{array}$ & $\begin{array}{l}36.45 \\
36.47 \\
36.50 \\
36.43 \\
36.30\end{array}$ & $\begin{array}{l}36.09 \\
35.88 \\
35.83 \\
35.68 \\
35.69\end{array}$ & $\begin{array}{l}35.28 \\
35.35 \\
35.26 \\
35.40 \\
35.18\end{array}$ \\
\hline $\begin{array}{r}6 \\
7 \\
8 \\
9 \\
10\end{array}$ & $\begin{array}{l}31.93 \\
32.09 \\
32.01 \\
32.75 \\
33.34\end{array}$ & $\begin{array}{l}32.69 \\
32.56 \\
32.69 \\
33.20 \\
33.06\end{array}$ & $\begin{array}{l}32.50 \\
32.16 \\
32.17 \\
32.26 \\
32.18\end{array}$ & $\begin{array}{l}32.10 \\
32.11 \\
31.79 \\
32.15 \\
32.07\end{array}$ & $\begin{array}{l}33.30 \\
33.35 \\
33.40 \\
33.38 \\
33.65\end{array}$ & $\begin{array}{l}35.64 \\
35.76 \\
35.85 \\
35.91 \\
35.96\end{array}$ & $\begin{array}{l}35.85 \\
35.72 \\
35.68 \\
35.71 \\
35.68\end{array}$ & $\begin{array}{l}37.36 \\
37.28 \\
37.16 \\
37.13 \\
37.24\end{array}$ & $\begin{array}{l}36.97 \\
36.88 \\
36.97 \\
37.02 \\
37.00\end{array}$ & $\begin{array}{l}36.18 \\
36.38 \\
36.46 \\
36.48 \\
36.43\end{array}$ & $\begin{array}{l}35.85 \\
36.01 \\
36.03 \\
35.99 \\
35.87\end{array}$ & $\begin{array}{l}35.26 \\
35.19 \\
35.09 \\
35.10 \\
35.01\end{array}$ \\
\hline $\begin{array}{l}11 \\
12 \\
13 \\
14 \\
15\end{array}$ & $\begin{array}{l}33.73 \\
33.64 \\
33.41 \\
33.36 \\
33.32\end{array}$ & $\begin{array}{l}33.08 \\
32.99 \\
32.81 \\
32.70 \\
32.61\end{array}$ & $\begin{array}{l}32.17 \\
32.17 \\
32.09 \\
31.98 \\
32.03\end{array}$ & $\begin{array}{l}32.13 \\
32.38 \\
32.52 \\
32.41 \\
32.18\end{array}$ & $\begin{array}{l}33.71 \\
33.71 \\
33.83 \\
33.91 \\
33.92\end{array}$ & $\begin{array}{l}35.80 \\
35.92 \\
36.12 \\
36.17 \\
36.16\end{array}$ & $\begin{array}{l}35.69 \\
35.73 \\
35.74 \\
35.85 \\
35.97\end{array}$ & $\begin{array}{l}37.14 \\
36.94 \\
36.85 \\
36.97 \\
37.12\end{array}$ & $\begin{array}{l}37.01 \\
36.96 \\
36.95 \\
36.89 \\
36.78\end{array}$ & $\begin{array}{l}36.27 \\
36.06 \\
35.97 \\
35.99 \\
35.92\end{array}$ & $\begin{array}{l}36.04 \\
35.91 \\
35.76 \\
35.70 \\
35.74\end{array}$ & $\begin{array}{l}34.97 \\
35.01 \\
35.02 \\
34.96 \\
34.91\end{array}$ \\
\hline $\begin{array}{l}16 \\
17 \\
18 \\
19 \\
20\end{array}$ & $\begin{array}{l}33.22 \\
33.04 \\
32.90 \\
33.18 \\
33.32\end{array}$ & $\begin{array}{l}32.18 \\
32.56 \\
32.68 \\
32.79 \\
32.75\end{array}$ & $\begin{array}{l}32.08 \\
31.94 \\
31.92 \\
31.81 \\
31.79\end{array}$ & $\begin{array}{l}32.22 \\
32.21 \\
32.23 \\
32.31 \\
32.38\end{array}$ & $\begin{array}{l}34.04 \\
34.12 \\
34.27 \\
34.36 \\
34.38\end{array}$ & $\begin{array}{l}36.12 \\
36.10 \\
36.09 \\
35.99 \\
35.99\end{array}$ & $\begin{array}{l}36.00 \\
36.01 \\
36.12 \\
36.30 \\
36.47\end{array}$ & $\begin{array}{l}37.20 \\
37.25 \\
37.24 \\
37.19 \\
37.16\end{array}$ & $\begin{array}{l}36.70 \\
36.71 \\
36.72 \\
36.65 \\
36.51\end{array}$ & $\begin{array}{l}35.88 \\
35.88 \\
35.95 \\
35.94 \\
35.97\end{array}$ & $\begin{array}{l}35.69 \\
35.58 \\
35.68 \\
35.51 \\
35.51\end{array}$ & $\begin{array}{l}35.40 \\
35.11 \\
34.92 \\
34.86 \\
34.83\end{array}$ \\
\hline $\begin{array}{l}21 \\
22 \\
23 \\
24 \\
25\end{array}$ & $\begin{array}{l}33.10 \\
32.85 \\
33.45 \\
33.34 \\
32.98\end{array}$ & $\begin{array}{l}32.53 \\
32.33 \\
32.75 \\
32.63 \\
32.48\end{array}$ & $\begin{array}{l}31.86 \\
31.80 \\
31.80 \\
31.76 \\
31.70\end{array}$ & $\begin{array}{l}32.38 \\
32.34 \\
32.18 \\
32.13 \\
32.26\end{array}$ & $\begin{array}{l}34.21 \\
34.25 \\
34.38 \\
34.53 \\
34.66\end{array}$ & $\begin{array}{l}36.08 \\
36.10 \\
36.02 \\
36.00 \\
35.78\end{array}$ & $\begin{array}{l}36.59 \\
36.64 \\
36.61 \\
36.72 \\
36.86\end{array}$ & $\begin{array}{l}37.16 \\
37.18 \\
37.29 \\
37.24 \\
37.26\end{array}$ & $\begin{array}{l}36.43 \\
36.47 \\
36.43 \\
36.37 \\
36.45\end{array}$ & $\begin{array}{l}36.12 \\
36.08 \\
36.15 \\
36.10 \\
36.17\end{array}$ & $\begin{array}{l}35.59 \\
35.56 \\
35.56 \\
35.62 \\
35.57\end{array}$ & $\begin{array}{l}34.84 \\
34.98 \\
34.81 \\
34.83 \\
34.87\end{array}$ \\
\hline $\begin{array}{l}26 \\
27 \\
28 \\
29 \\
30 \\
31\end{array}$ & $\begin{array}{l}32.99 \\
32.94 \\
33.06 \\
32.97 \\
33.04 \\
33.13\end{array}$ & $\begin{array}{r}32.25 \\
32.20 \\
32.11 \\
--- \\
--- \\
---\end{array}$ & $\begin{array}{l}31.75 \\
31.73 \\
31.82 \\
31.84 \\
31.76 \\
32.28\end{array}$ & $\begin{array}{r}32.34 \\
32.32 \\
32.36 \\
32.51 \\
32.86 \\
\ldots\end{array}$ & $\begin{array}{l}34.78 \\
34.74 \\
34.88 \\
35.06 \\
35.21 \\
35.40\end{array}$ & $\begin{array}{r}35.74 \\
35.81 \\
35.72 \\
35.75 \\
35.85 \\
\ldots\end{array}$ & $\begin{array}{l}37.03 \\
37.22 \\
37.31 \\
37.30 \\
37.24 \\
37.34\end{array}$ & $\begin{array}{l}37.39 \\
37.51 \\
37.63 \\
37.71 \\
37.66 \\
37.65\end{array}$ & $\begin{array}{r}36.53 \\
36.57 \\
36.55 \\
36.56 \\
36.46 \\
. .-\end{array}$ & $\begin{array}{l}35.88 \\
35.91 \\
36.10 \\
36.16 \\
36.24 \\
36.25\end{array}$ & $\begin{array}{r}35.48 \\
35.34 \\
35.14 \\
35.10 \\
35.08 \\
\ldots .\end{array}$ & $\begin{array}{l}34.95 \\
34.91 \\
34.91 \\
35.14 \\
35.09 \\
34.96\end{array}$ \\
\hline MEAN & 32.79 & 32.70 & 32.05 & 32.27 & 34.05 & 35.90 & 36.28 & 37.28 & 36.80 & 36.16 & 35.67 & 35.05 \\
\hline
\end{tabular}

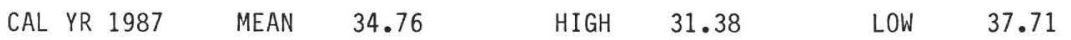




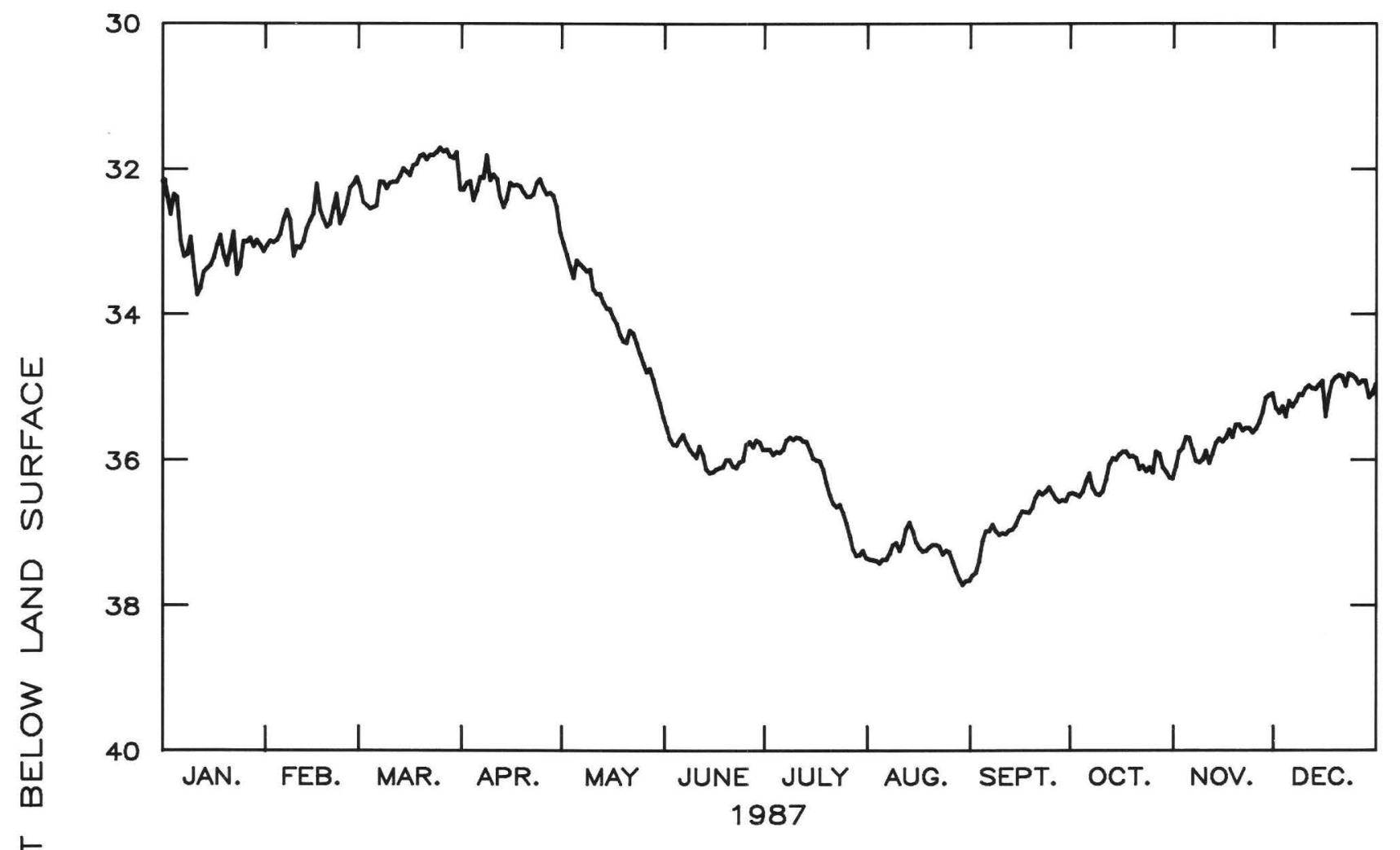

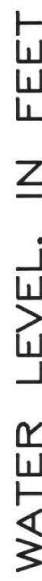

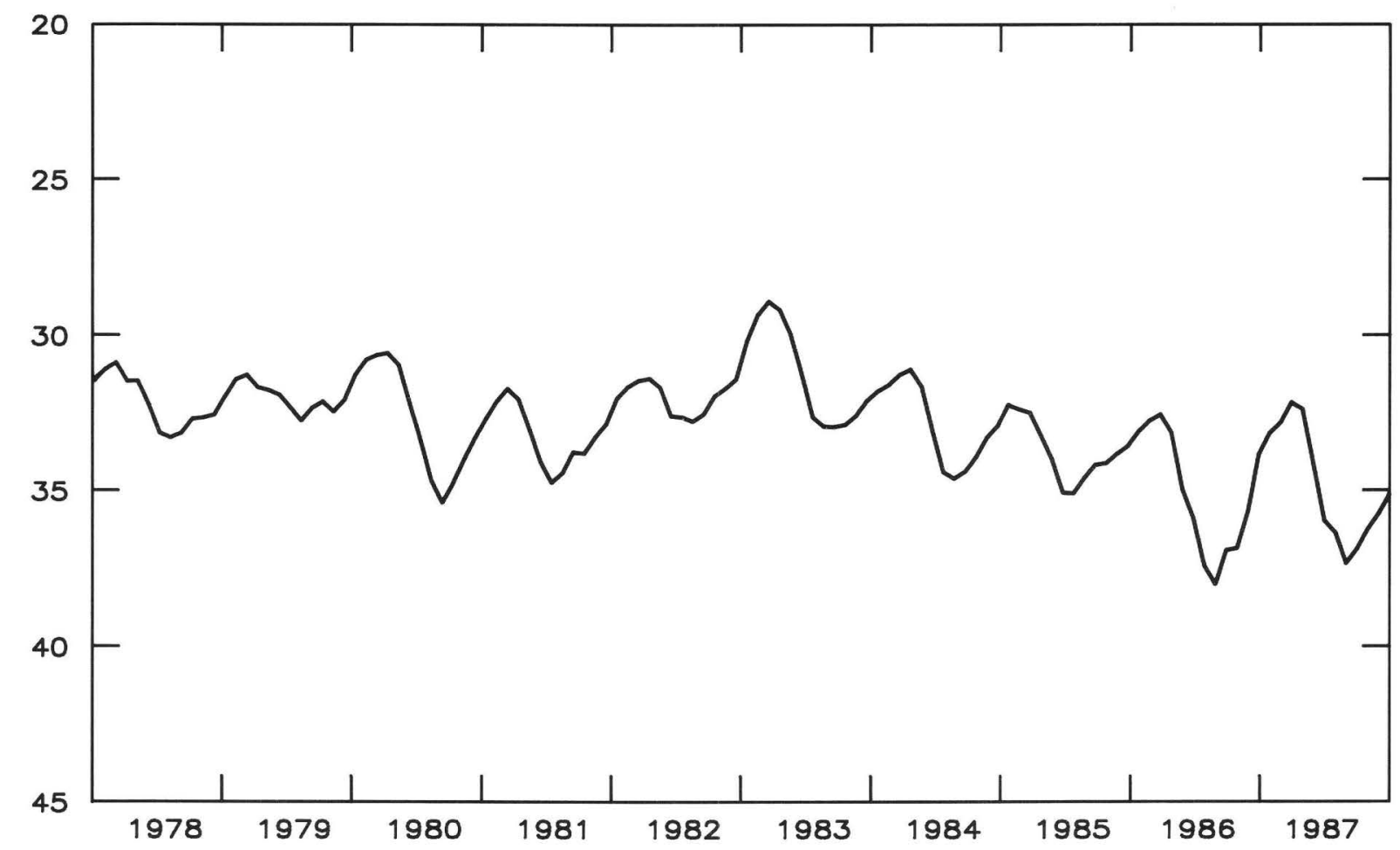

Figure 2.7.4.1-4.--Water level in observation well 38Q002, Chatham County. 
320122080510202 Local number, 390003.

LOCATION.--Lat $32^{\circ} 01^{\prime} 22^{\prime \prime}$, long $80^{\circ} 51^{\prime} 02^{\prime \prime}$, Hydrologic Unit 03060204 , Tybee Island near Fort Screven.

Owner: U.S. Geological Survey, test well 7.

AQUIFER.--Upper Floridan aquifer.

WELL CHARACTERISTICS.--Drilled observation well, diameter $10 \mathrm{in.,}$ depth $600 \mathrm{ft}$, cased to $129 \mathrm{ft}$, open hole. DATUM.--Elevation of land-surface datum is $7.0 \mathrm{ft}$.

Measuring point: Top of 10-in. casing, $2.0 \mathrm{ft}$ above land-surface datum.

REMARKS.--Borehole geophysical survey conducted January 24, 1962.

PERIOD OF RECORD.--May 1952 to current year.

EXTREMES FOR PERIOD OF RECORD.--Highest water level, $17.80 \mathrm{ft}$ below land-surface datum, April 11, 1963 ; 1owest, $34.33 \mathrm{ft}$ below 1and-surface datum, August 3, 1986 .

DEPTH BELOW LAND SURFACE (WATER LEVEL) (FEET) CALENDAR YEAR JANUARY 1987 TO DECEMBER 1987 MEAN VALUES

\begin{tabular}{|c|c|c|c|c|c|c|c|c|c|c|c|c|}
\hline DAY & JAN & FEB & MAR & APR & MAY & JUN & JUL & AUG & SEP & OCT & NOV & DEC \\
\hline $\begin{array}{l}1 \\
2 \\
3 \\
4 \\
5\end{array}$ & $\begin{array}{l}29.01 \\
29.83 \\
29.82 \\
29.15 \\
29.02\end{array}$ & $\begin{array}{l}29.12 \\
28.99 \\
29.01 \\
28.90 \\
28.78\end{array}$ & $\begin{array}{l}28.38 \\
28.63 \\
28.63 \\
28.67 \\
28.61\end{array}$ & $\begin{array}{l}28.60 \\
28.46 \\
28.47 \\
28.87 \\
28.70\end{array}$ & $\begin{array}{l}29.10 \\
29.12 \\
29.32 \\
29.39 \\
29.15\end{array}$ & $\begin{array}{l}31.82 \\
32.07 \\
32.09 \\
31.91 \\
31.68\end{array}$ & $\begin{array}{l}32.12 \\
32.17 \\
32.31 \\
32.30 \\
32.36\end{array}$ & $\begin{array}{l}33.40 \\
33.22 \\
33.12 \\
33.29 \\
33.27\end{array}$ & $\begin{array}{l}33.18 \\
33.07 \\
33.01 \\
32.62 \\
32.56\end{array}$ & $\begin{array}{l}32.17 \\
32.23 \\
32.32 \\
32.44 \\
32.16\end{array}$ & $\begin{array}{l}32.03 \\
31.76 \\
31.76 \\
31.57 \\
31.55\end{array}$ & $\begin{array}{l}31.15 \\
31.30 \\
31.23 \\
31.52 \\
31.27\end{array}$ \\
\hline $\begin{array}{r}6 \\
7 \\
8 \\
9 \\
10\end{array}$ & $\begin{array}{l}29.27 \\
29.52 \\
29.49 \\
29.35 \\
29.32\end{array}$ & $\begin{array}{l}28.56 \\
28.44 \\
28.65 \\
29.35 \\
29.18\end{array}$ & $\begin{array}{l}28.60 \\
28.20 \\
28.30 \\
28.47 \\
28.30\end{array}$ & $\begin{array}{l}28.41 \\
28.40 \\
28.39 \\
28.45 \\
28.26\end{array}$ & $\begin{array}{l}29.25 \\
29.34 \\
29.47 \\
29.42 \\
29.80\end{array}$ & $\begin{array}{l}31.72 \\
31.99 \\
32.12 \\
32.32 \\
32.37\end{array}$ & $\begin{array}{l}32.20 \\
32.03 \\
32.01 \\
32.18 \\
32.18\end{array}$ & $\begin{array}{l}33.27 \\
33.21 \\
33.08 \\
33.02 \\
33.18\end{array}$ & $\begin{array}{l}33.11 \\
32.76 \\
32.61 \\
32.69 \\
32.62\end{array}$ & $\begin{array}{l}32.01 \\
32.27 \\
32.36 \\
32.34 \\
32.26\end{array}$ & $\begin{array}{l}31.72 \\
31.92 \\
31.96 \\
31.93 \\
31.78\end{array}$ & $\begin{array}{l}31.28 \\
31.15 \\
31.04 \\
31.10 \\
31.04\end{array}$ \\
\hline $\begin{array}{l}11 \\
12 \\
13 \\
14 \\
15\end{array}$ & $\begin{array}{l}29.88 \\
29.76 \\
29.40 \\
29.34 \\
29.32\end{array}$ & $\begin{array}{l}29.20 \\
29.10 \\
28.91 \\
28.74 \\
28.66\end{array}$ & $\begin{array}{l}28.21 \\
28.25 \\
28.18 \\
28.10 \\
28.19\end{array}$ & $\begin{array}{l}28.35 \\
28.71 \\
28.85 \\
28.64 \\
28.38\end{array}$ & $\begin{array}{l}29.76 \\
29.72 \\
29.79 \\
29.91 \\
29.96\end{array}$ & $\begin{array}{l}32.07 \\
32.18 \\
32.35 \\
32.35 \\
32.34\end{array}$ & $\begin{array}{l}32.17 \\
32.08 \\
32.05 \\
32.24 \\
32.33\end{array}$ & $\begin{array}{l}32.92 \\
32.67 \\
32.56 \\
32.73 \\
32.98\end{array}$ & $\begin{array}{l}32.64 \\
32.57 \\
32.60 \\
32.51 \\
32.38\end{array}$ & $\begin{array}{l}32.03 \\
31.81 \\
31.67 \\
31.67 \\
31.66\end{array}$ & $\begin{array}{l}31.98 \\
31.81 \\
31.60 \\
31.55 \\
31.64\end{array}$ & $\begin{array}{l}31.02 \\
31.07 \\
31.10 \\
30.97 \\
30.90\end{array}$ \\
\hline $\begin{array}{l}16 \\
17 \\
18 \\
19 \\
20\end{array}$ & $\begin{array}{l}29.21 \\
28.96 \\
28.83 \\
29.28 \\
29.41\end{array}$ & $\begin{array}{l}28.10 \\
28.54 \\
28.69 \\
28.81 \\
28.76\end{array}$ & $\begin{array}{l}28.29 \\
28.07 \\
28.04 \\
27.97 \\
27.94\end{array}$ & $\begin{array}{l}28.43 \\
28.46 \\
28.44 \\
28.52 \\
28.59\end{array}$ & $\begin{array}{l}30.12 \\
30.17 \\
30.26 \\
30.29 \\
30.28\end{array}$ & $\begin{array}{l}32.35 \\
32.35 \\
32.35 \\
32.23 \\
32.27\end{array}$ & $\begin{array}{l}32.33 \\
32.27 \\
32.30 \\
32.48 \\
32.58\end{array}$ & $\begin{array}{l}33.11 \\
33.23 \\
33.16 \\
33.07 \\
33.02\end{array}$ & $\begin{array}{l}32.29 \\
32.37 \\
32.43 \\
32.42 \\
32.16\end{array}$ & $\begin{array}{l}31.65 \\
31.70 \\
31.85 \\
31.79 \\
31.80\end{array}$ & $\begin{array}{l}31.56 \\
31.45 \\
31.61 \\
31.43 \\
31.43\end{array}$ & $\begin{array}{l}31.40 \\
31.23 \\
30.97 \\
30.93 \\
30.91\end{array}$ \\
\hline $\begin{array}{l}21 \\
22 \\
23 \\
24 \\
25\end{array}$ & $\begin{array}{l}29.10 \\
28.79 \\
29.65 \\
29.51 \\
29.05\end{array}$ & $\begin{array}{l}28.50 \\
28.30 \\
28.82 \\
28.73 \\
28.53\end{array}$ & $\begin{array}{l}28.08 \\
27.99 \\
27.98 \\
27.90 \\
27.89\end{array}$ & $\begin{array}{l}28.60 \\
28.56 \\
28.39 \\
28.40 \\
28.57\end{array}$ & $\begin{array}{l}30.06 \\
30.14 \\
30.40 \\
30.68 \\
30.82\end{array}$ & $\begin{array}{l}32.40 \\
32.37 \\
32.15 \\
32.23 \\
31.92\end{array}$ & $\begin{array}{l}32.59 \\
32.64 \\
32.52 \\
32.58 \\
32.71\end{array}$ & $\begin{array}{l}33.01 \\
33.07 \\
33.27 \\
33.19 \\
33.11\end{array}$ & $\begin{array}{l}31.97 \\
32.10 \\
32.12 \\
32.05 \\
32.20\end{array}$ & $\begin{array}{l}31.97 \\
31.88 \\
31.98 \\
31.95 \\
32.13\end{array}$ & $\begin{array}{l}31.59 \\
31.48 \\
31.52 \\
31.61 \\
31.56\end{array}$ & $\begin{array}{l}30.93 \\
30.72 \\
30.77 \\
30.81 \\
30.87\end{array}$ \\
\hline $\begin{array}{l}26 \\
27 \\
28 \\
29 \\
30 \\
31\end{array}$ & $\begin{array}{l}29.06 \\
29.01 \\
29.18 \\
29.05 \\
29.16 \\
29.27\end{array}$ & $\begin{array}{r}28.38 \\
28.17 \\
28.03 \\
--- \\
-.- \\
--\end{array}$ & $\begin{array}{l}27.98 \\
27.98 \\
28.11 \\
28.13 \\
28.01 \\
28.70\end{array}$ & $\begin{array}{r}28.62 \\
28.52 \\
28.54 \\
28.67 \\
29.03 \\
\ldots . .\end{array}$ & $\begin{array}{l}30.72 \\
30.59 \\
30.68 \\
31.12 \\
31.39 \\
31.71\end{array}$ & $\begin{array}{r}31.69 \\
31.87 \\
31.83 \\
31.94 \\
32.09 \\
\ldots\end{array}$ & $\begin{array}{l}32.95 \\
33.17 \\
33.29 \\
33.07 \\
33.07 \\
33.24\end{array}$ & $\begin{array}{l}33.31 \\
33.43 \\
33.59 \\
33.64 \\
33.51 \\
33.38\end{array}$ & $\begin{array}{r}32.38 \\
32.39 \\
32.24 \\
32.28 \\
32.17 \\
. .-\end{array}$ & $\begin{array}{l}31.61 \\
31.63 \\
31.93 \\
32.01 \\
32.21 \\
32.23\end{array}$ & $\begin{array}{r}31.46 \\
31.35 \\
31.24 \\
31.14 \\
31.01 \\
-.-\end{array}$ & $\begin{array}{l}31.01 \\
30.92 \\
30.51 \\
31.00 \\
30.68 \\
30.67\end{array}$ \\
\hline MEAN & 29.29 & 28.71 & 28.22 & 28.54 & 30.06 & 32.11 & 32.47 & 33.16 & 32.48 & 31.99 & 31.60 & 31.02 \\
\hline
\end{tabular}

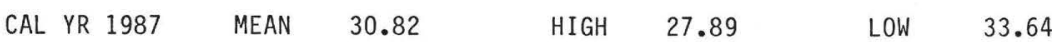




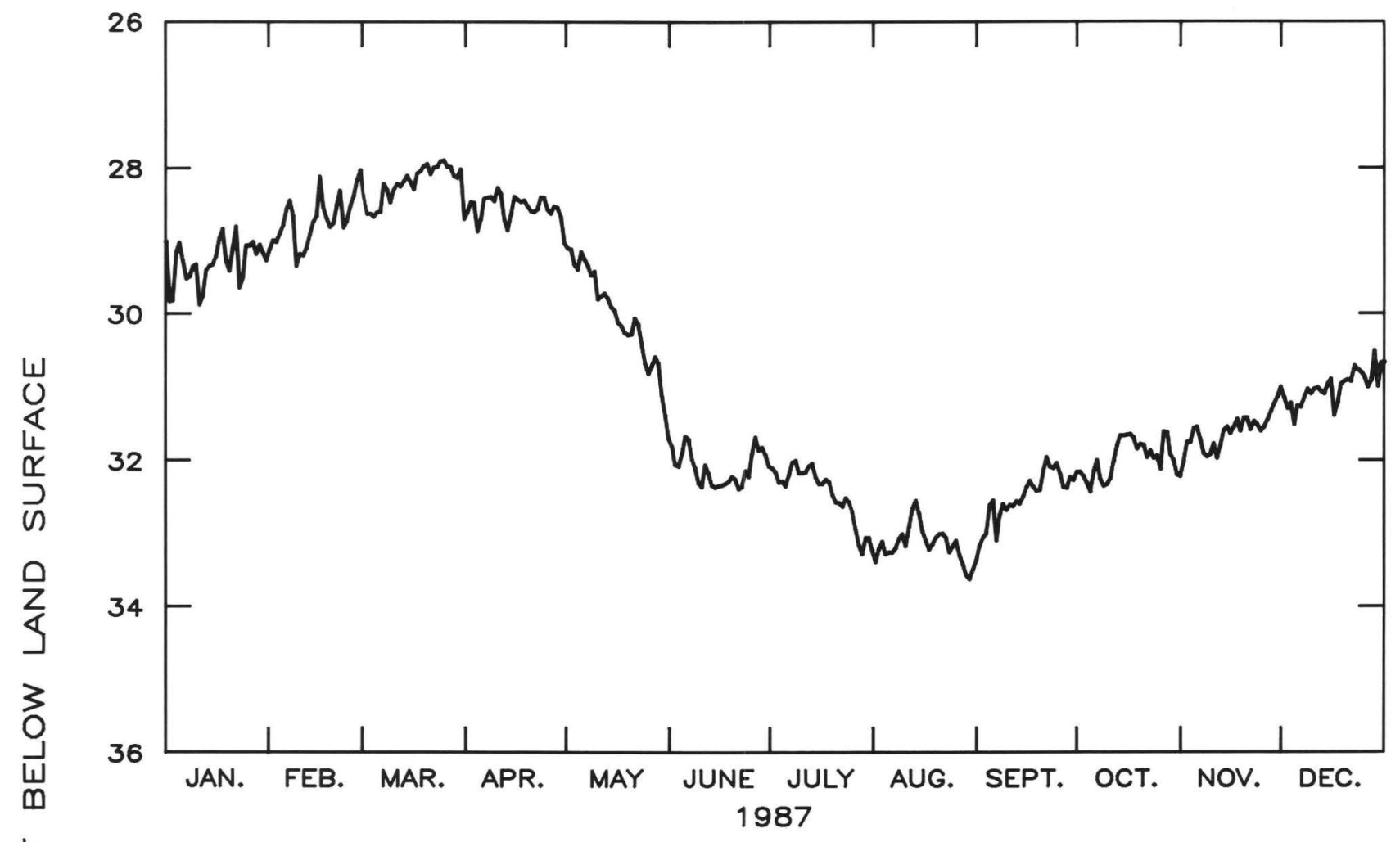

岁

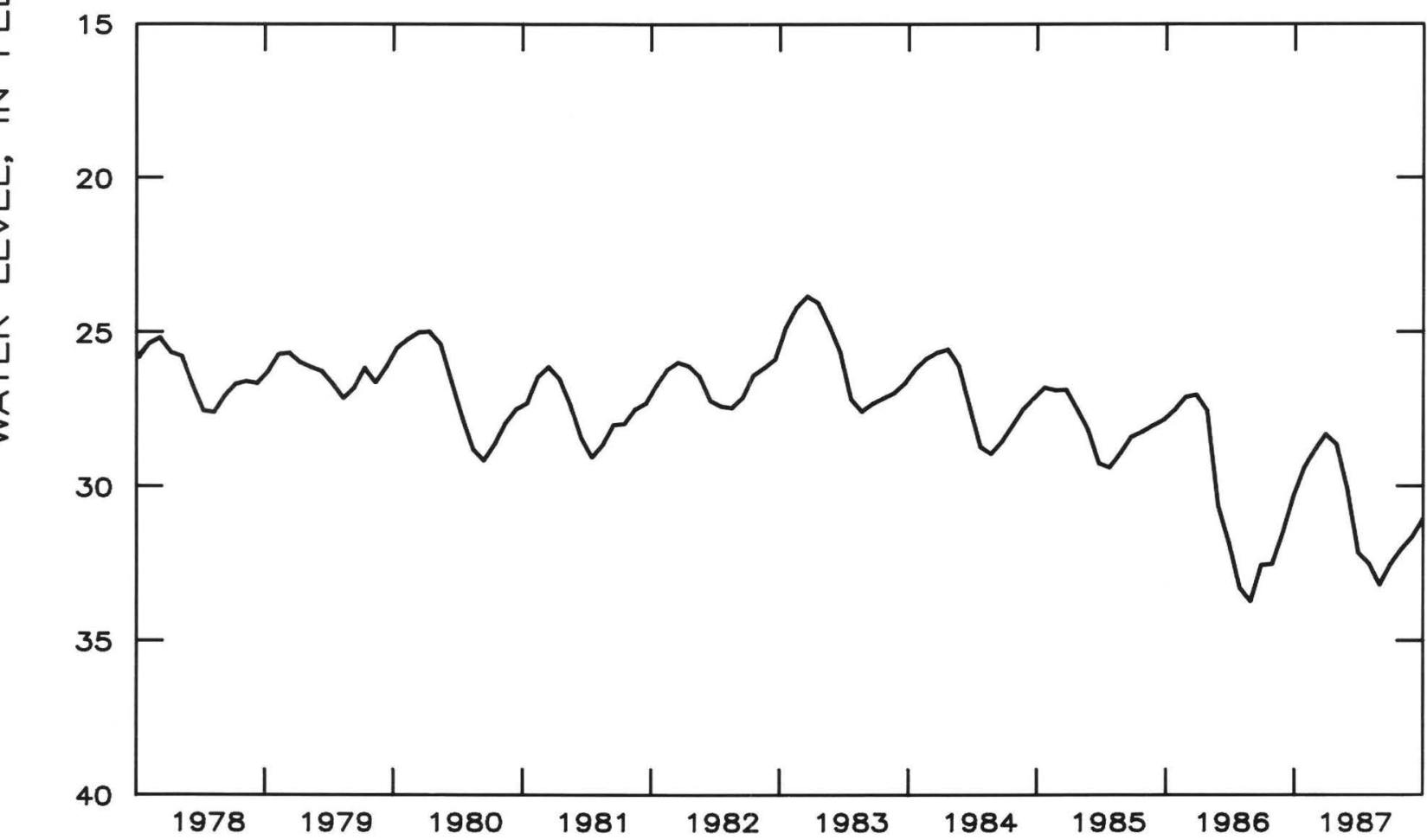

Figure 2.7.4.1-5.--Water level in observation well 39Q003, Chatham County. 
321240081411501 , Local number, 32R002.

LOCATION.--Lat $32^{\circ} 12^{\prime} 40^{\prime \prime}$, long $81^{\circ} 41^{\prime} 15^{\prime \prime}$, Hydrologic Unit 03060202, 2.6 mi north along Georgia Road 67 from the Bulloch-Bryan County line, on east side of, and approximately $100 \mathrm{ft}$ from center line of road.

Owner: Georgia Geologic Survey.

AQUIFER.--Upper Floridan aquifer.

WELL CHARACTERISTICS.--Drilled observation well, diameter $6 \mathrm{in}$., depth $804 \mathrm{ft}$, cased to $420 \mathrm{ft}$.

DATUM.--Elevation of land-surface datum is $120 \mathrm{ft}$.

Measuring point: Floor of recorder shelter, $3.0 \mathrm{ft}$ above land-surface datum.

REMARKS.--Borehole geophysical survey and well sounded August 1982. Water levels for periods of missing record, May 6 to June 4, and September 3, were estimated.

PERIOD OF RECORD.--February 1983 to current year.

EXTREMES FOR PERIOD OF RECORD.--Highest water level, $85.08 \mathrm{ft}$ below land-surface datum, April 24, 1983; 10west, $92.32 \mathrm{ft}$ below land-surface datum, August $5-6,1986$.

DEPTH BELOW LAND SURFACE (WATER LEVEL) (FEET) CALENDAR YEAR JANUARY 1987 TO DECEMBER 1987 MEAN VALUES

\begin{tabular}{|c|c|c|c|c|c|c|c|c|c|c|c|c|}
\hline DAY & JAN & FEB & MAR & APR & MAY & JUN & JUL & AUG & SEP & OCT & NOV & DEC \\
\hline $\begin{array}{l}1 \\
2 \\
3 \\
4 \\
5\end{array}$ & $\begin{array}{l}90.68 \\
90.82 \\
90.91 \\
90.76 \\
90.70\end{array}$ & $\begin{array}{l}90.31 \\
90.16 \\
90.20 \\
90.30 \\
90.40\end{array}$ & $\begin{array}{l}89.66 \\
89.76 \\
89.85 \\
89.88 \\
89.93\end{array}$ & $\begin{array}{l}89.41 \\
89.36 \\
89.26 \\
89.22 \\
89.22\end{array}$ & $\begin{array}{l}89.24 \\
89.24 \\
89.31 \\
89.39 \\
89.44\end{array}$ & $\begin{array}{l}89.70 \\
89.73 \\
89.83 \\
89.85 \\
89.94\end{array}$ & $\begin{array}{l}90.09 \\
90.02 \\
89.96 \\
89.96 \\
90.00\end{array}$ & $\begin{array}{l}90.37 \\
90.37 \\
90.40 \\
90.40 \\
90.41\end{array}$ & $\begin{array}{l}90.80 \\
90.82 \\
90.82 \\
90.82 \\
90.78\end{array}$ & $\begin{array}{l}90.64 \\
90.75 \\
90.79 \\
90.87 \\
90.78\end{array}$ & $\begin{array}{l}91.09 \\
91.03 \\
90.96 \\
90.82 \\
90.81\end{array}$ & $\begin{array}{l}90.67 \\
90.80 \\
90.77 \\
90.71 \\
90.83\end{array}$ \\
\hline $\begin{array}{r}6 \\
7 \\
8 \\
9 \\
10\end{array}$ & $\begin{array}{l}90.80 \\
90.72 \\
90.73 \\
90.73 \\
90.57\end{array}$ & $\begin{array}{l}90.26 \\
90.09 \\
90.02 \\
90.23 \\
90.35\end{array}$ & $\begin{array}{l}89.95 \\
89.78 \\
89.56 \\
89.44 \\
89.59\end{array}$ & $\begin{array}{l}89.22 \\
89.20 \\
89.19 \\
89.16 \\
89.18\end{array}$ & $\begin{array}{l}89.47 \\
89.40 \\
89.41 \\
89.51 \\
89.53\end{array}$ & $\begin{array}{l}90.01 \\
90.10 \\
90.12 \\
90.09 \\
90.06\end{array}$ & $\begin{array}{l}90.05 \\
90.06 \\
90.04 \\
90.03 \\
90.02\end{array}$ & $\begin{array}{l}90.45 \\
90.52 \\
90.54 \\
90.53 \\
90.49\end{array}$ & $\begin{array}{l}90.76 \\
90.69 \\
90.70 \\
90.73 \\
90.77\end{array}$ & $\begin{array}{l}90.68 \\
90.72 \\
90.87 \\
90.97 \\
90.95\end{array}$ & $\begin{array}{l}90.99 \\
91.09 \\
91.07 \\
90.97 \\
90.81\end{array}$ & $\begin{array}{l}90.91 \\
90.93 \\
90.89 \\
90.80 \\
90.68\end{array}$ \\
\hline $\begin{array}{l}11 \\
12 \\
13 \\
14 \\
15\end{array}$ & $\begin{array}{l}90.64 \\
90.68 \\
90.68 \\
90.68 \\
90.71\end{array}$ & $\begin{array}{l}90.28 \\
90.14 \\
90.06 \\
90.00 \\
89.96\end{array}$ & $\begin{array}{l}89.78 \\
89.78 \\
89.72 \\
89.70 \\
89.67\end{array}$ & $\begin{array}{l}89.19 \\
89.25 \\
89.28 \\
89.28 \\
89.28\end{array}$ & $\begin{array}{l}89.50 \\
89.46 \\
89.48 \\
89.49 \\
89.44\end{array}$ & $\begin{array}{l}90.08 \\
90.14 \\
90.13 \\
90.11 \\
90.09\end{array}$ & $\begin{array}{l}90.00 \\
89.98 \\
89.96 \\
89.94 \\
89.98\end{array}$ & $\begin{array}{l}90.48 \\
90.51 \\
90.55 \\
90.58 \\
90.58\end{array}$ & $\begin{array}{l}90.80 \\
90.77 \\
90.72 \\
90.72 \\
90.73\end{array}$ & $\begin{array}{l}90.84 \\
90.71 \\
90.80 \\
90.91 \\
90.91\end{array}$ & $\begin{array}{l}90.86 \\
90.98 \\
90.94 \\
90.94 \\
91.01\end{array}$ & $\begin{array}{l}90.65 \\
90.66 \\
90.78 \\
90.84 \\
90.70\end{array}$ \\
\hline $\begin{array}{l}16 \\
17 \\
18 \\
19 \\
20\end{array}$ & $\begin{array}{l}90.54 \\
90.54 \\
90.46 \\
90.35 \\
90.45\end{array}$ & $\begin{array}{l}89.88 \\
89.90 \\
90.00 \\
90.10 \\
90.13\end{array}$ & $\begin{array}{l}89.62 \\
89.67 \\
89.56 \\
89.44 \\
89.45\end{array}$ & $\begin{array}{l}89.28 \\
89.12 \\
89.02 \\
89.14 \\
89.20\end{array}$ & $\begin{array}{l}89.44 \\
89.47 \\
89.45 \\
89.42 \\
89.43\end{array}$ & $\begin{array}{l}90.10 \\
90.12 \\
90.18 \\
90.17 \\
90.12\end{array}$ & $\begin{array}{l}90.06 \\
90.16 \\
90.22 \\
90.24 \\
90.27\end{array}$ & $\begin{array}{l}90.61 \\
90.62 \\
90.63 \\
90.63 \\
90.68\end{array}$ & $\begin{array}{l}90.72 \\
90.69 \\
90.63 \\
90.62 \\
90.62\end{array}$ & $\begin{array}{l}90.89 \\
90.85 \\
90.86 \\
90.85 \\
90.85\end{array}$ & $\begin{array}{l}91.01 \\
90.91 \\
90.93 \\
90.91 \\
90.81\end{array}$ & $\begin{array}{l}90.72 \\
90.89 \\
90.95 \\
90.94 \\
90.86\end{array}$ \\
\hline $\begin{array}{l}21 \\
22 \\
23 \\
24 \\
25\end{array}$ & $\begin{array}{l}90.38 \\
90.13 \\
90.31 \\
90.45 \\
90.28\end{array}$ & $\begin{array}{l}90.07 \\
89.85 \\
89.86 \\
90.00 \\
90.01\end{array}$ & $\begin{array}{l}89.42 \\
89.39 \\
89.44 \\
89.44 \\
89.41\end{array}$ & $\begin{array}{l}89.19 \\
89.16 \\
89.12 \\
89.07 \\
89.16\end{array}$ & $\begin{array}{l}89.49 \\
89.54 \\
89.56 \\
89.54 \\
89.55\end{array}$ & $\begin{array}{l}90.10 \\
90.08 \\
90.04 \\
90.02 \\
89.98\end{array}$ & $\begin{array}{l}90.31 \\
90.28 \\
90.27 \\
90.33 \\
90.38\end{array}$ & $\begin{array}{l}90.77 \\
90.80 \\
90.76 \\
90.78 \\
90.87\end{array}$ & $\begin{array}{l}90.69 \\
90.72 \\
90.71 \\
90.69 \\
90.72\end{array}$ & $\begin{array}{l}90.92 \\
91.04 \\
91.03 \\
91.02 \\
90.98\end{array}$ & $\begin{array}{l}90.92 \\
90.99 \\
91.01 \\
91.02 \\
90.96\end{array}$ & $\begin{array}{l}90.83 \\
90.79 \\
90.86 \\
90.90 \\
90.86\end{array}$ \\
\hline $\begin{array}{l}26 \\
27 \\
28 \\
29 \\
30 \\
31\end{array}$ & $\begin{array}{l}90.22 \\
90.38 \\
90.43 \\
90.43 \\
90.28 \\
90.26\end{array}$ & $\begin{array}{r}90.02 \\
89.96 \\
89.80 \\
--- \\
-- \\
--\end{array}$ & $\begin{array}{l}89.38 \\
89.32 \\
89.32 \\
89.36 \\
89.20 \\
89.25\end{array}$ & $\begin{array}{r}89.29 \\
89.32 \\
89.35 \\
89.30 \\
89.25 \\
.--\end{array}$ & $\begin{array}{l}89.58 \\
89.61 \\
89.62 \\
89.61 \\
89.61 \\
89.65\end{array}$ & $\begin{array}{r}89.92 \\
89.92 \\
90.01 \\
90.10 \\
90.12 \\
. .-\end{array}$ & $\begin{array}{l}90.36 \\
90.31 \\
90.28 \\
90.28 \\
90.34 \\
90.39\end{array}$ & $\begin{array}{l}90.89 \\
90.90 \\
90.88 \\
90.87 \\
90.88 \\
90.88\end{array}$ & $\begin{array}{r}90.78 \\
90.82 \\
90.84 \\
90.74 \\
90.58 \\
. .-\end{array}$ & $\begin{array}{l}90.86 \\
90.78 \\
90.86 \\
90.96 \\
91.05 \\
91.11\end{array}$ & $\begin{array}{r}90.85 \\
90.79 \\
90.73 \\
90.67 \\
90.59 \\
.--\end{array}$ & $\begin{array}{l}90.8 \\
90.8 \\
90.7 \\
90.8 \\
90.98 \\
90.98\end{array}$ \\
\hline MEAN & 90.54 & 90.08 & 89.57 & 89.22 & 89.48 & 90.03 & 90.15 & 90.63 & 90.73 & 90.87 & 90.92 & 90.8 \\
\hline
\end{tabular}




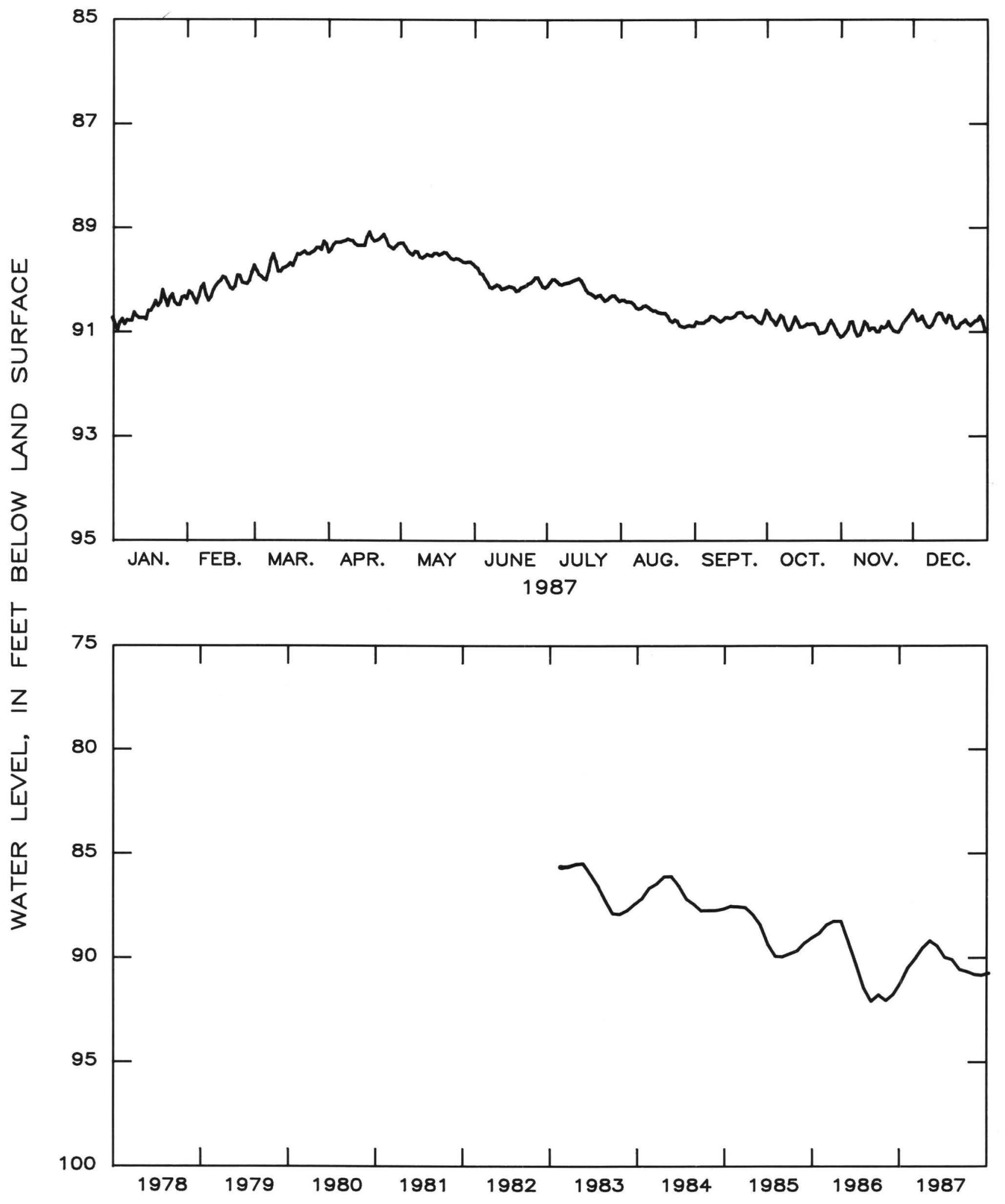

Figure 2.7.4.1-6.--Water level in observation well 32R002, Bulloch County. 


\subsubsection{Jesup-Riceboro area}

The water level in the Upper Floridan aquifer in the Jesup-Riceboro area is affected by industrial pumping at Doctortown near Jesup and at Riceboro. In 1985, pumpage was about $70 \mathrm{Mgal} / \mathrm{d}$ (Turlington and others, 1987) at Doctortown and about $16 \mathrm{Mgal} / \mathrm{d}$ (Turlington and others, 1987) at Riceboro. The 1987 hydrographs for wells $30 \mathrm{~L} 003$ and 32L015 illustrate the effects that a partial industrial shutdown in July near Jesup had on the water level in these areas. Similarly, the 1987 hydrograph for well 34M054 illustrates the effects that partial industrial shutdowns in April and October at Riceboro had on the water level there.

In the Jesup-Riceboro area, water levels showed some recovery from the effects of the 1986 drought. By the end of April, the water levels in five observation wells tapping the Upper Floridan aquifer had recovered 2.2 to $4.7 \mathrm{ft}$ from the record lows measured during the 1986 drought. Although there was a slight recovery in the early part of the year, mean water levels during 1987 were from 0.1 to $0.6 \mathrm{ft}$ lower than in 1986. Record low water levels were measured in we11s 35M013 and 32L015 during December of 1987. At the end of 1987, water levels were from 0.6 to $2.7 \mathrm{ft}$ lower than at the end of 1986. These declines continued a downward trend that began in 1983, which can be attributed to increased regional pumping as a result of from below-normal precipitation. 


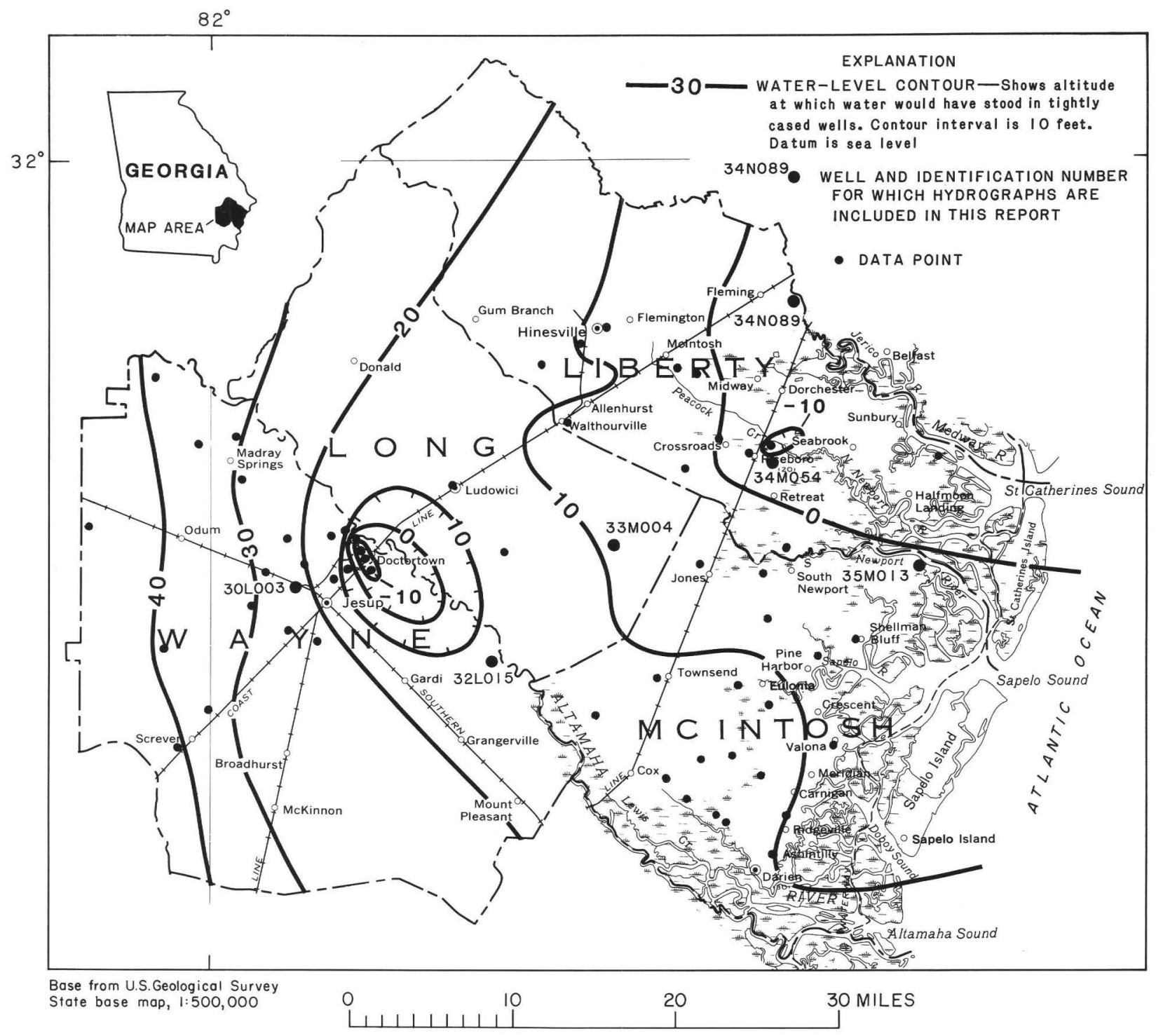

Figure 2.7.4.2-1.--Observation well locations and the water level in the Upper Floridan aquifer in the Jesup-Riceboro area, May 1985. 
313701081543501 Local number, 30L003.

LOCATION.--Lat $31^{\circ} 37^{\prime} 01^{\prime \prime}$, long $81^{\circ} 54^{\prime} 35^{\prime \prime}$, Hydrologic Unit 03070106 , about $0.5 \mathrm{mi}$ west of Jesup city 1 imits near intersection of Highway 341 and Sunset Drive.

Owner: City of Jesup Housing Authority.

AQUIFER.--Upper Floridan aquifer.

WELL CHARACTERISTICS.--Drilled unused domestic well, diameter $4 \mathrm{in}$, depth $584 \mathrm{ft}$, cased to $472 \mathrm{ft}$, open hole. DATUM.--Elevation of land-surface datum is $107 \mathrm{ft}$.

Measuring point: Floor of recorder shelter, $2.88 \mathrm{ft}$ above land-surface datum.

REMARKS.--Borehole geophysical survey conducted August 19, 1963.

PERIOD OF RECORD.--January 1964 to March 1967. February 1976 to current year

EXTREMES FOR PERIOD OF RECORD.--Highest water level, $59.98 \mathrm{ft}$ below land-surface datum, Apri1 19, 1964 ; 10 west $85.27 \mathrm{ft}$ below land-surface datum, June 29,1981 .

DEPTH BELOW LAND SURFACE (WATER LEVEL) (FEET) CALENDAR YEAR JANUARY 1987 TO DECEMBER 1987 MEAN VALUES

\begin{tabular}{|c|c|c|c|c|c|c|c|c|c|c|c|c|}
\hline DAY & JAN & FEB & MAR & APR & MAY & JUN & JUL & AUG & SEP & ОСТ & NOV & DEC \\
\hline $\begin{array}{l}1 \\
2 \\
3 \\
4 \\
5\end{array}$ & $\begin{array}{l}80.82 \\
81.24 \\
81.50 \\
81.48 \\
81.52\end{array}$ & $\begin{array}{l}80.47 \\
80.48 \\
80.80 \\
81.08 \\
81.17\end{array}$ & $\begin{array}{l}80.86 \\
80.90 \\
80.98 \\
81.03 \\
81.05\end{array}$ & $\begin{array}{l}80.52 \\
80.55 \\
80.49 \\
80.44 \\
80.38\end{array}$ & $\begin{array}{l}80.11 \\
80.24 \\
80.44 \\
80.52 \\
80.30\end{array}$ & $\begin{array}{l}81.00 \\
81.13 \\
81.17 \\
81.27 \\
81.26\end{array}$ & $\begin{array}{l}81.94 \\
81.94 \\
81.95 \\
82.02 \\
82.07\end{array}$ & $\begin{array}{l}80.58 \\
80.76 \\
80.88 \\
81.00 \\
81.15\end{array}$ & $\begin{array}{l}82.42 \\
82.42 \\
82.45 \\
82.44 \\
82.43\end{array}$ & $\begin{array}{l}82.74 \\
82.70 \\
82.50 \\
82.10 \\
81.90\end{array}$ & $\begin{array}{l}83.43 \\
83.35 \\
83.27 \\
83.16 \\
83.14\end{array}$ & $\begin{array}{l}83.53 \\
53.68 \\
83.78 \\
83.75 \\
83.80\end{array}$ \\
\hline $\begin{array}{r}6 \\
7 \\
8 \\
9 \\
10\end{array}$ & $\begin{array}{l}81.62 \\
81.55 \\
81.64 \\
81.73 \\
81.68\end{array}$ & $\begin{array}{l}81.14 \\
81.03 \\
80.90 \\
80.95 \\
81.17\end{array}$ & $\begin{array}{l}81.08 \\
80.98 \\
80.76 \\
80.64 \\
80.81\end{array}$ & $\begin{array}{l}80.31 \\
80.26 \\
80.27 \\
80.33 \\
80.32\end{array}$ & $\begin{array}{l}79.94 \\
79.60 \\
79.41 \\
79.60 \\
79.92\end{array}$ & $\begin{array}{l}81.37 \\
81.54 \\
81.58 \\
81.55 \\
81.57\end{array}$ & $\begin{array}{l}82.06 \\
82.06 \\
82.06 \\
82.05 \\
81.98\end{array}$ & $\begin{array}{l}81.34 \\
81.47 \\
81.58 \\
81.68 \\
81.73\end{array}$ & $\begin{array}{l}82.45 \\
82.40 \\
82.37 \\
82.45 \\
82.48\end{array}$ & $\begin{array}{l}82.14 \\
82.45 \\
82.78 \\
83.00 \\
83.10\end{array}$ & $\begin{array}{l}83.34 \\
83.48 \\
83.51 \\
83.47 \\
83.26\end{array}$ & $\begin{array}{l}83.74 \\
83.69 \\
83.67 \\
83.65 \\
83.65\end{array}$ \\
\hline $\begin{array}{l}11 \\
12 \\
13 \\
14 \\
15\end{array}$ & $\begin{array}{l}81.92 \\
82.10 \\
82.10 \\
82.11 \\
82.02\end{array}$ & $\begin{array}{l}81.24 \\
81.14 \\
81.13 \\
81.25 \\
81.25\end{array}$ & $\begin{array}{l}81.02 \\
81.04 \\
80.94 \\
80.83 \\
80.77\end{array}$ & $\begin{array}{l}80.28 \\
80.30 \\
80.32 \\
80.22 \\
79.98\end{array}$ & $\begin{array}{l}80.08 \\
80.18 \\
80.20 \\
80.16 \\
80.22\end{array}$ & $\begin{array}{l}81.67 \\
81.78 \\
81.88 \\
81.88 \\
82.00\end{array}$ & $\begin{array}{l}81.28 \\
80.00 \\
79.08 \\
78.72 \\
78.64\end{array}$ & $\begin{array}{l}81.84 \\
81.91 \\
81.93 \\
81.95 \\
81.95\end{array}$ & $\begin{array}{l}82.45 \\
82.40 \\
82.36 \\
82.38 \\
82.45\end{array}$ & $\begin{array}{l}83.05 \\
82.88 \\
82.70 \\
82.46 \\
82.14\end{array}$ & $\begin{array}{l}83.22 \\
83.37 \\
83.42 \\
83.43 \\
83.48\end{array}$ & $\begin{array}{l}83.61 \\
83.63 \\
83.81 \\
83.95 \\
83.88\end{array}$ \\
\hline $\begin{array}{l}16 \\
17 \\
18 \\
19 \\
20\end{array}$ & $\begin{array}{l}82.00 \\
82.06 \\
82.02 \\
81.84 \\
81.90\end{array}$ & $\begin{array}{l}81.14 \\
81.12 \\
81.24 \\
81.41 \\
81.44\end{array}$ & $\begin{array}{l}80.73 \\
80.70 \\
80.60 \\
80.47 \\
80.43\end{array}$ & $\begin{array}{l}79.81 \\
79.75 \\
79.85 \\
79.92 \\
79.87\end{array}$ & $\begin{array}{l}80.34 \\
80.38 \\
80.37 \\
80.26 \\
80.14\end{array}$ & $\begin{array}{l}82.05 \\
82.04 \\
82.04 \\
81.97 \\
81.86\end{array}$ & $\begin{array}{l}78.76 \\
78.96 \\
79.07 \\
79.32 \\
79.65\end{array}$ & $\begin{array}{l}81.98 \\
82.03 \\
82.02 \\
82.01 \\
82.02\end{array}$ & $\begin{array}{l}82.47 \\
82.49 \\
82.50 \\
82.50 \\
82.49\end{array}$ & $\begin{array}{l}81.84 \\
81.76 \\
82.00 \\
82.24 \\
82.44\end{array}$ & $\begin{array}{l}83.53 \\
83.51 \\
83.55 \\
83.56 \\
83.51\end{array}$ & $\begin{array}{l}83.94 \\
84.11 \\
84.12 \\
84.02 \\
83.92\end{array}$ \\
\hline $\begin{array}{l}21 \\
22 \\
23 \\
24 \\
25\end{array}$ & $\begin{array}{l}81.94 \\
81.73 \\
81.86 \\
81.93 \\
81.72\end{array}$ & $\begin{array}{l}81.29 \\
81.11 \\
81.08 \\
81.15 \\
81.14\end{array}$ & $\begin{array}{l}80.34 \\
80.28 \\
80.40 \\
80.48 \\
80.39\end{array}$ & $\begin{array}{l}79.66 \\
79.44 \\
79.21 \\
79.30 \\
79.56\end{array}$ & $\begin{array}{l}80.33 \\
80.53 \\
80.61 \\
80.63 \\
80.64\end{array}$ & $\begin{array}{l}81.76 \\
81.72 \\
81.69 \\
81.61 \\
81.57\end{array}$ & $\begin{array}{l}79.86 \\
79.93 \\
79.88 \\
79.96 \\
80.10\end{array}$ & $\begin{array}{l}82.06 \\
82.06 \\
82.05 \\
82.12 \\
82.16\end{array}$ & $\begin{array}{l}82.60 \\
82.70 \\
82.80 \\
82.85 \\
82.85\end{array}$ & $\begin{array}{l}82.70 \\
82.88 \\
82.91 \\
82.99 \\
82.98\end{array}$ & $\begin{array}{l}83.58 \\
83.62 \\
83.64 \\
83.67 \\
83.60\end{array}$ & $\begin{array}{l}83.85 \\
83.77 \\
83.82 \\
83.83 \\
83.74\end{array}$ \\
\hline $\begin{array}{l}26 \\
27 \\
28 \\
29 \\
30 \\
31\end{array}$ & $\begin{array}{l}81.51 \\
81.54 \\
81.45 \\
81.15 \\
80.75 \\
80.58\end{array}$ & $\begin{array}{r}81.24 \\
81.28 \\
81.10 \\
--- \\
--- \\
---\end{array}$ & $\begin{array}{l}80.30 \\
80.33 \\
80.46 \\
80.43 \\
80.20 \\
80.31\end{array}$ & $\begin{array}{r}79.69 \\
79.76 \\
79.80 \\
79.93 \\
80.04 \\
-\end{array}$ & $\begin{array}{l}80.61 \\
80.59 \\
80.65 \\
80.66 \\
80.68 \\
80.81\end{array}$ & $\begin{array}{r}81.55 \\
81.50 \\
81.60 \\
81.78 \\
81.90 \\
. .\end{array}$ & $\begin{array}{l}80.16 \\
80.17 \\
80.17 \\
80.14 \\
80.26 \\
80.42\end{array}$ & $\begin{array}{l}82.18 \\
82.22 \\
82.24 \\
82.32 \\
82.43 \\
82.45\end{array}$ & $\begin{array}{r}82.78 \\
82.75 \\
82.82 \\
82.85 \\
82.79 \\
. .-\end{array}$ & $\begin{array}{l}82.94 \\
82.86 \\
82.97 \\
83.14 \\
83.26 \\
83.36\end{array}$ & $\begin{array}{r}83.52 \\
83.49 \\
83.49 \\
83.46 \\
83.43 \\
. \ldots\end{array}$ & $\begin{array}{l}83.59 \\
83.50 \\
83.45 \\
83.45 \\
83.58 \\
83.49\end{array}$ \\
\hline MEAN & 81.65 & 81.11 & 80.66 & 80.02 & 80.30 & 81.64 & 80.47 & 81.81 & 82.55 & 82.64 & 83.45 & 82.7 \\
\hline
\end{tabular}

$\begin{array}{lllllll}\text { CAL YR } 1987 & \text { MEAN } & 81.59 & \text { HIGH } & 53.68 & \text { LOW } & 84.12\end{array}$ 

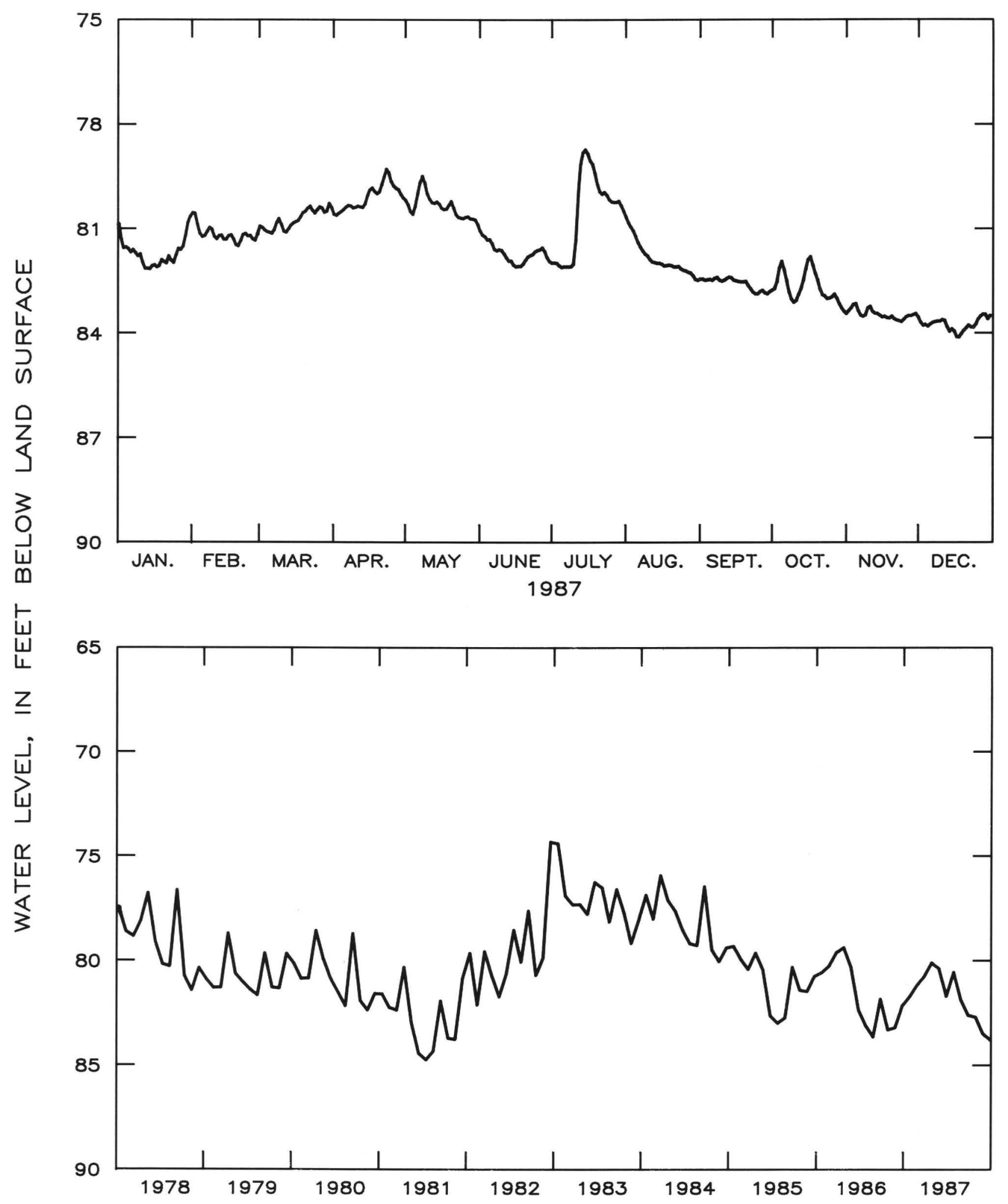

Figure 2.7.4.2-2.--Water level in observation well 30LOO3, Wayne County. 
313253081433502 , Local number, 32L015.

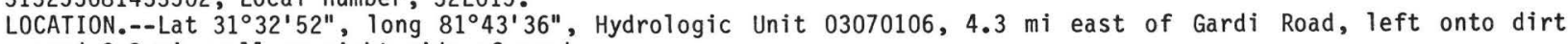
road $0.8 \mathrm{mi}$, well on right side of road.

Owner: Georgia Geologic Survey.

AQUIFER.--Upper Floridan aquifer.

WELL CHARACTERISTICS.--Drilled observation well, diameter $4 \mathrm{in}$., depth $750 \mathrm{ft}$, cased to $545 \mathrm{ft}$.

DATUM.--Elevation of land-surface datum is $74 \mathrm{ft}$.

Measuring point: Floor of recorder shelter, $4.0 \mathrm{ft}$ above land-surface datum.

REMARKS.--Borehole geophysical survey and well sounded April 20, 1983. Water level for period of missing record, January 19 , was estimated.

PERIOD OF RECORD.--April 20, 1983 to current year.

EXTREMES FOR PERIOD OF RECORD.--Highest water level, $49.12 \mathrm{ft}$ below land-surface datum, March 19, 1984; 1owest, $59.43 \mathrm{ft}$ below land-surface datum, December $18,1987$.

DEPTH BELOW LAND SURFACE (WATER LEVEL) (FEET) CALENDAR YEAR JANUARY 1987 TO DECEMBER 1987 MEAN VALUES

\begin{tabular}{|c|c|c|c|c|c|c|c|c|c|c|c|c|}
\hline DAY & JAN & FEB & MAR & APR & MAY & JUN & JUL & AUG & SEP & ОСТ & NOV & DEC \\
\hline $\begin{array}{l}1 \\
2 \\
3 \\
4 \\
5\end{array}$ & $\begin{array}{l}56.56 \\
56.98 \\
57.17 \\
57.01 \\
57.14\end{array}$ & $\begin{array}{l}56.92 \\
56.77 \\
56.93 \\
57.14 \\
57.28\end{array}$ & $\begin{array}{l}56.98 \\
57.10 \\
57.20 \\
57.21 \\
57.21\end{array}$ & $\begin{array}{l}56.66 \\
56.60 \\
56.50 \\
56.50 \\
56.44\end{array}$ & $\begin{array}{l}56.07 \\
56.12 \\
56.22 \\
56.31 \\
56.28\end{array}$ & $\begin{array}{l}56.53 \\
56.59 \\
56.59 \\
56.64 \\
56.67\end{array}$ & $\begin{array}{l}57.45 \\
57.46 \\
57.48 \\
57.54 \\
57.59\end{array}$ & $\begin{array}{l}56.40 \\
56.53 \\
56.66 \\
56.76 \\
56.86\end{array}$ & $\begin{array}{l}58.02 \\
58.04 \\
58.08 \\
58.10 \\
58.11\end{array}$ & $\begin{array}{l}58.45 \\
58.51 \\
58.43 \\
58.34 \\
58.15\end{array}$ & $\begin{array}{l}58.81 \\
58.76 \\
58.69 \\
58.52 \\
58.52\end{array}$ & $\begin{array}{l}58.84 \\
59.04 \\
59.07 \\
58.98 \\
59.16\end{array}$ \\
\hline $\begin{array}{r}6 \\
7 \\
8 \\
9 \\
10\end{array}$ & $\begin{array}{l}57.31 \\
57.30 \\
57.31 \\
57.28 \\
57.18\end{array}$ & $\begin{array}{l}57.16 \\
57.05 \\
56.98 \\
57.18 \\
57.37\end{array}$ & $\begin{array}{l}57.19 \\
56.98 \\
56.72 \\
56.64 \\
56.81\end{array}$ & $\begin{array}{l}56.40 \\
56.35 \\
56.34 \\
56.36 \\
56.35\end{array}$ & $\begin{array}{l}56.12 \\
55.77 \\
55.63 \\
55.76 \\
55.88\end{array}$ & $\begin{array}{l}56.80 \\
56.94 \\
56.99 \\
56.97 \\
56.98\end{array}$ & $\begin{array}{l}57.62 \\
57.62 \\
57.62 \\
57.64 \\
57.59\end{array}$ & $\begin{array}{l}57.03 \\
57.13 \\
57.21 \\
57.25 \\
57.28\end{array}$ & $\begin{array}{l}58.13 \\
58.09 \\
58.11 \\
58.17 \\
58.19\end{array}$ & $\begin{array}{l}58.08 \\
58.17 \\
58.42 \\
58.61 \\
58.66\end{array}$ & $\begin{array}{l}58.80 \\
58.91 \\
58.90 \\
58.79 \\
58.62\end{array}$ & $\begin{array}{l}59.18 \\
59.19 \\
59.13 \\
59.05 \\
58.96\end{array}$ \\
\hline $\begin{array}{l}11 \\
12 \\
13 \\
14 \\
15\end{array}$ & $\begin{array}{l}57.41 \\
57.52 \\
57.63 \\
57.69 \\
57.65\end{array}$ & $\begin{array}{l}57.38 \\
57.21 \\
57.16 \\
57.21 \\
57.21\end{array}$ & $\begin{array}{l}57.01 \\
57.00 \\
56.91 \\
56.88 \\
56.84\end{array}$ & $\begin{array}{l}56.37 \\
56.40 \\
56.44 \\
56.40 \\
56.20\end{array}$ & $\begin{array}{l}55.92 \\
55.95 \\
56.01 \\
56.03 \\
56.03\end{array}$ & $\begin{array}{l}57.03 \\
57.08 \\
57.08 \\
57.10 \\
57.19\end{array}$ & $\begin{array}{l}57.37 \\
56.76 \\
56.16 \\
55.83 \\
55.67\end{array}$ & $\begin{array}{l}57.32 \\
57.43 \\
57.50 \\
57.61 \\
57.65\end{array}$ & $\begin{array}{l}58.17 \\
58.15 \\
58.13 \\
58.15 \\
58.20\end{array}$ & $\begin{array}{l}58.56 \\
58.43 \\
58.45 \\
58.45 \\
58.31\end{array}$ & $\begin{array}{l}58.69 \\
58.85 \\
58.83 \\
58.84 \\
58.90\end{array}$ & $\begin{array}{l}58.90 \\
58.91 \\
59.14 \\
59.20 \\
59.07\end{array}$ \\
\hline $\begin{array}{l}16 \\
17 \\
18 \\
19 \\
20\end{array}$ & $\begin{array}{l}57.68 \\
57.73 \\
57.67 \\
57.73 \\
57.80\end{array}$ & $\begin{array}{l}57.12 \\
57.19 \\
57.27 \\
57.41 \\
57.46\end{array}$ & $\begin{array}{l}56.82 \\
56.86 \\
56.72 \\
56.62 \\
56.64\end{array}$ & $\begin{array}{l}56.05 \\
56.03 \\
56.12 \\
56.22 \\
56.24\end{array}$ & $\begin{array}{l}56.08 \\
56.15 \\
56.13 \\
56.06 \\
56.02\end{array}$ & $\begin{array}{l}57.22 \\
57.29 \\
57.40 \\
57.36 \\
57.29\end{array}$ & $\begin{array}{l}55.63 \\
55.66 \\
55.66 \\
55.70 \\
55.79\end{array}$ & $\begin{array}{l}57.72 \\
57.77 \\
57.78 \\
57.79 \\
57.84\end{array}$ & $\begin{array}{l}58.22 \\
58.23 \\
58.20 \\
58.20 \\
58.23\end{array}$ & $\begin{array}{l}58.15 \\
58.00 \\
58.03 \\
58.08 \\
58.14\end{array}$ & $\begin{array}{l}58.91 \\
58.84 \\
58.91 \\
58.89 \\
58.82\end{array}$ & $\begin{array}{l}59.20 \\
59.37 \\
59.43 \\
59.35 \\
59.26\end{array}$ \\
\hline $\begin{array}{l}21 \\
22 \\
23 \\
24 \\
25\end{array}$ & $\begin{array}{l}57.67 \\
57.42 \\
57.66 \\
57.78 \\
57.54\end{array}$ & $\begin{array}{l}57.34 \\
57.16 \\
57.16 \\
57.28 \\
57.29\end{array}$ & $\begin{array}{l}56.55 \\
56.55 \\
56.62 \\
56.63 \\
56.57\end{array}$ & $\begin{array}{l}56.11 \\
55.98 \\
55.83 \\
55.75 \\
55.89\end{array}$ & $\begin{array}{l}56.09 \\
56.22 \\
56.28 \\
56.31 \\
56.33\end{array}$ & $\begin{array}{l}57.27 \\
57.24 \\
57.18 \\
57.15 \\
57.13\end{array}$ & $\begin{array}{l}55.88 \\
55.88 \\
55.84 \\
55.89 \\
55.93\end{array}$ & $\begin{array}{l}57.92 \\
57.93 \\
57.89 \\
57.91 \\
57.97\end{array}$ & $\begin{array}{l}58.33 \\
58.41 \\
58.45 \\
58.46 \\
58.51\end{array}$ & $\begin{array}{l}58.26 \\
58.49 \\
58.52 \\
58.55 \\
58.50\end{array}$ & $\begin{array}{l}58.97 \\
59.03 \\
59.05 \\
59.09 \\
58.99\end{array}$ & $\begin{array}{l}59.22 \\
59.17 \\
59.29 \\
59.27 \\
59.19\end{array}$ \\
\hline $\begin{array}{l}26 \\
27 \\
28 \\
29 \\
30 \\
31\end{array}$ & $\begin{array}{l}57.38 \\
57.48 \\
57.47 \\
57.34 \\
57.04 \\
56.98\end{array}$ & $\begin{array}{r}57.33 \\
57.30 \\
57.14 \\
-.- \\
-.- \\
-.-\end{array}$ & $\begin{array}{l}56.54 \\
56.46 \\
56.56 \\
56.54 \\
56.34 \\
56.48\end{array}$ & $\begin{array}{r}56.01 \\
56.04 \\
55.96 \\
56.07 \\
56.05 \\
.--\end{array}$ & $\begin{array}{l}56.34 \\
56.35 \\
56.36 \\
56.37 \\
56.37 \\
56.42\end{array}$ & $\begin{array}{r}57.13 \\
57.12 \\
57.21 \\
57.36 \\
57.44 \\
. .-\end{array}$ & $\begin{array}{l}55.93 \\
55.98 \\
56.01 \\
56.03 \\
56.15 \\
56.32\end{array}$ & $\begin{array}{l}57.99 \\
58.00 \\
58.00 \\
57.99 \\
58.06 \\
58.07\end{array}$ & $\begin{array}{r}58.55 \\
58.58 \\
58.61 \\
58.53 \\
58.36 \\
.--\end{array}$ & $\begin{array}{l}58.42 \\
58.35 \\
58.47 \\
58.63 \\
58.75 \\
58.81\end{array}$ & $\begin{array}{r}58.88 \\
58.83 \\
58.81 \\
58.78 \\
58.75 \\
.--\end{array}$ & $\begin{array}{l}59.12 \\
59.07 \\
58.94 \\
59.01 \\
59.20 \\
59.09\end{array}$ \\
\hline IEAN & 57.40 & 57.19 & 56.78 & 56.22 & 56.13 & 57.07 & 56.51 & 57.52 & 58.26 & 58.39 & 58.83 & 59.13 \\
\hline
\end{tabular}

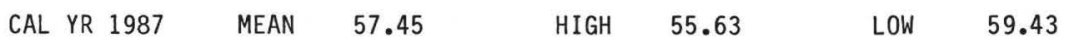




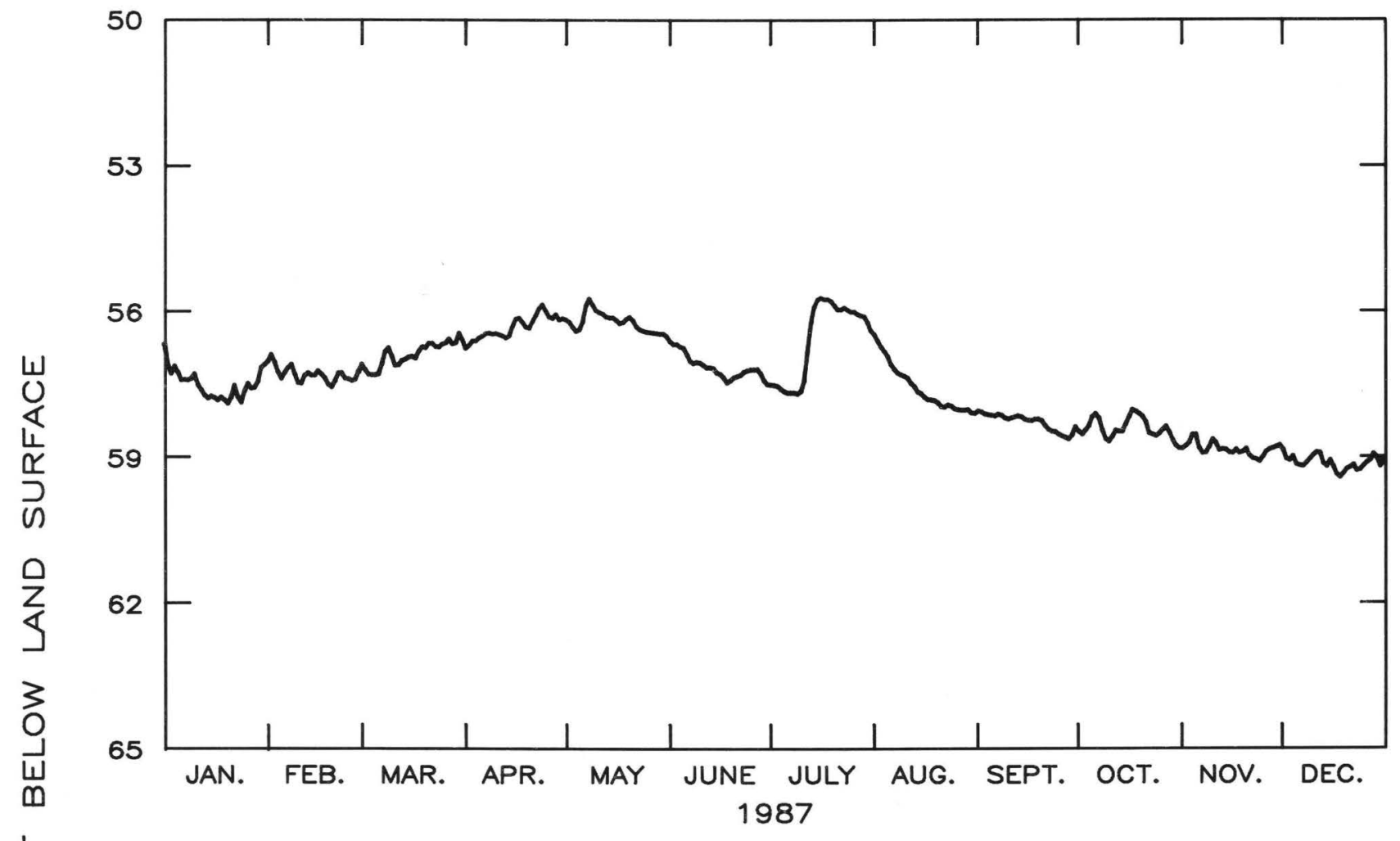

虫

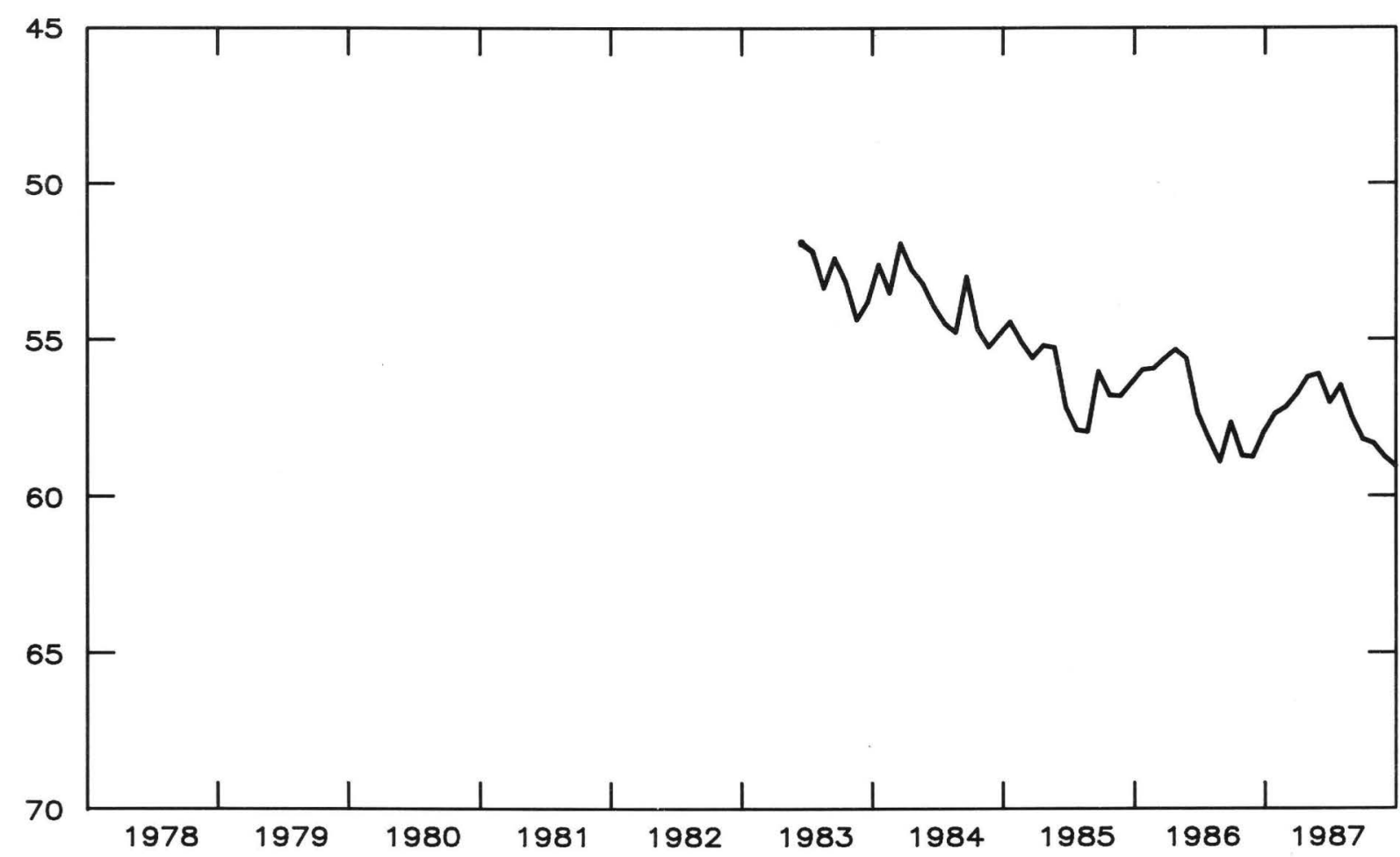

Figure 2.7.4.2-3.--Water level in observation well 32LO15, Wayne County. 
313845081361701 Local number, 33M004.

LOCATION.--Lat $31^{\circ} 38^{\prime} 54^{\prime \prime}$, long $81^{\circ} 36^{\prime} 04^{\prime \prime}$, Hydrologic Unit 03070106, 9 mi southeast of Ludowici, at Hope Cemetery. Owner: U.S. Geological Survey, test well 3.

AQUIFER.--Upper Floridan aquifer.

WELL CHARACTERISTICS.--Drilled observation well, diameter 4-3 in., depth $872 \mathrm{ft}$, cased to $538 \mathrm{ft}$, open hole. DATUM.--Elevation of land-surface datum is $61.2 \mathrm{ft}$.

Measuring point: Floor of recorder shelter, $3.5 \mathrm{ft}$ above land-surface datum.

REMARKS.--Well pumped and sounded June 17, 1976, to depth of $861 \mathrm{ft}$; water-quality sample collected. Borehole geophysical survey conducted July 28, 1976. Water levels for period of missing record, Auglist 23-29, were estimated.

PERIOD OF RECORD.--January 1968 to current year.

EXTREMES FOR PERIOD OF RECORD.--Highest water level, $34.04 \mathrm{ft}$ below land-surface datum, January 14, 1968; 10west, $54.18 \mathrm{ft}$ below land-surface datum, August 30 and November $14,1986$.

DEPTH BELOW LAND SURFACE (WATER LEVEL) (FEET) CALENDAR YEAR JANUARY 1987 TO DECEMBER 1987 MEAN VALUES

\begin{tabular}{|c|c|c|c|c|c|c|c|c|c|c|c|c|}
\hline DAY & JAN & FEB & MAR & APR & MAY & JUN & JUL & AUG & SEP & OCT & NOV & $\mathrm{DEC}$ \\
\hline $\begin{array}{l}1 \\
2 \\
3 \\
4 \\
5\end{array}$ & $\begin{array}{l}52.30 \\
52.56 \\
52.68 \\
52.49 \\
52.44\end{array}$ & $\begin{array}{l}52.63 \\
52.47 \\
52.46 \\
52.60 \\
52.70\end{array}$ & $\begin{array}{l}52.28 \\
52.39 \\
52.50 \\
52.54 \\
52.62\end{array}$ & $\begin{array}{l}52.05 \\
51.94 \\
51.84 \\
51.85 \\
51.80\end{array}$ & $\begin{array}{l}51.56 \\
51.58 \\
51.64 \\
51.71 \\
51.79\end{array}$ & $\begin{array}{l}51.91 \\
51.92 \\
51.94 \\
51.94 \\
51.97\end{array}$ & $\begin{array}{l}52.64 \\
52.63 \\
52.66 \\
52.67 \\
52.68\end{array}$ & $\begin{array}{l}52.08 \\
52.07 \\
52.11 \\
52.16 \\
52.19\end{array}$ & $\begin{array}{l}53.12 \\
53.13 \\
53.14 \\
53.14 \\
53.10\end{array}$ & $\begin{array}{l}53.40 \\
53.55 \\
53.61 \\
53.72 \\
53.60\end{array}$ & $\begin{array}{l}53.84 \\
53.80 \\
53.70 \\
53.54 \\
53.50\end{array}$ & $\begin{array}{l}53.64 \\
53.79 \\
53.84 \\
53.77 \\
53.90\end{array}$ \\
\hline $\begin{array}{r}6 \\
7 \\
8 \\
9 \\
10\end{array}$ & $\begin{array}{l}52.62 \\
52.62 \\
52.64 \\
52.64 \\
52.50\end{array}$ & $\begin{array}{l}52.54 \\
52.40 \\
52.32 \\
52.57 \\
52.76\end{array}$ & $\begin{array}{l}52.60 \\
52.36 \\
52.11 \\
52.04 \\
52.20\end{array}$ & $\begin{array}{l}51.76 \\
51.72 \\
51.69 \\
51.64 \\
51.62\end{array}$ & $\begin{array}{l}51.81 \\
51.73 \\
51.66 \\
51.78 \\
51.80\end{array}$ & $\begin{array}{l}52.05 \\
52.16 \\
52.22 \\
52.20 \\
52.19\end{array}$ & $\begin{array}{l}52.75 \\
52.72 \\
52.74 \\
52.77 \\
52.74\end{array}$ & $\begin{array}{l}52.28 \\
52.38 \\
52.44 \\
52.45 \\
52.44\end{array}$ & $\begin{array}{l}53.10 \\
53.07 \\
53.11 \\
53.15 \\
53.22\end{array}$ & $\begin{array}{l}53.44 \\
53.45 \\
53.62 \\
53.73 \\
53.75\end{array}$ & $\begin{array}{l}53.72 \\
53.86 \\
53.85 \\
53.80 \\
53.64\end{array}$ & $\begin{array}{l}53.98 \\
54.00 \\
53.98 \\
53.93 \\
53.84\end{array}$ \\
\hline $\begin{array}{l}11 \\
12 \\
13 \\
14 \\
15\end{array}$ & $\begin{array}{l}52.62 \\
52.70 \\
52.76 \\
52.79 \\
52.76\end{array}$ & $\begin{array}{l}52.71 \\
52.55 \\
52.51 \\
52.45 \\
52.44\end{array}$ & $\begin{array}{l}52.40 \\
52.40 \\
52.33 \\
52.32 \\
52.29\end{array}$ & $\begin{array}{l}51.68 \\
51.72 \\
51.77 \\
51.78 \\
51.61\end{array}$ & $\begin{array}{l}51.76 \\
51.72 \\
51.74 \\
51.78 \\
51.74\end{array}$ & $\begin{array}{l}52.23 \\
52.28 \\
52.26 \\
52.23 \\
52.28\end{array}$ & $\begin{array}{l}52.71 \\
52.64 \\
52.52 \\
52.41 \\
52.33\end{array}$ & $\begin{array}{l}52.47 \\
52.55 \\
52.63 \\
52.68 \\
52.74\end{array}$ & $\begin{array}{l}53.26 \\
53.26 \\
53.23 \\
53.26 \\
53.28\end{array}$ & $\begin{array}{l}53.64 \\
53.48 \\
53.59 \\
53.67 \\
53.70\end{array}$ & $\begin{array}{l}53.65 \\
53.80 \\
53.82 \\
53.82 \\
53.86\end{array}$ & $\begin{array}{l}53.76 \\
53.75 \\
53.86 \\
53.93 \\
53.84\end{array}$ \\
\hline $\begin{array}{l}16 \\
17 \\
18 \\
19 \\
20\end{array}$ & $\begin{array}{l}52.76 \\
52.78 \\
52.76 \\
52.68 \\
52.82\end{array}$ & $\begin{array}{l}52.35 \\
52.40 \\
52.50 \\
52.60 \\
52.67\end{array}$ & $\begin{array}{l}52.23 \\
52.26 \\
52.12 \\
52.03 \\
52.03\end{array}$ & $\begin{array}{l}51.48 \\
51.47 \\
51.53 \\
51.65 \\
51.72\end{array}$ & $\begin{array}{l}51.74 \\
51.78 \\
51.78 \\
51.76 \\
51.75\end{array}$ & $\begin{array}{l}52.30 \\
52.36 \\
52.44 \\
52.46 \\
52.44\end{array}$ & $\begin{array}{l}52.30 \\
52.31 \\
52.26 \\
52.20 \\
52.18\end{array}$ & $\begin{array}{l}52.78 \\
52.82 \\
52.85 \\
52.88 \\
52.95\end{array}$ & $\begin{array}{l}53.28 \\
53.27 \\
53.24 \\
53.23 \\
53.26\end{array}$ & $\begin{array}{l}53.67 \\
53.56 \\
53.47 \\
53.42 \\
53.41\end{array}$ & $\begin{array}{l}53.88 \\
53.81 \\
53.82 \\
53.82 \\
53.74\end{array}$ & $\begin{array}{l}53.91 \\
54.06 \\
54.14 \\
54.14 \\
54.08\end{array}$ \\
\hline $\begin{array}{l}21 \\
22 \\
23 \\
24 \\
25\end{array}$ & $\begin{array}{l}52.71 \\
52.48 \\
52.75 \\
52.92 \\
52.75\end{array}$ & $\begin{array}{l}52.58 \\
52.40 \\
52.42 \\
52.58 \\
52.61\end{array}$ & $\begin{array}{l}51.98 \\
52.00 \\
52.03 \\
52.02 \\
51.98\end{array}$ & $\begin{array}{l}51.69 \\
51.66 \\
51.60 \\
51.51 \\
51.58\end{array}$ & $\begin{array}{l}51.78 \\
51.82 \\
51.83 \\
51.82 \\
51.84\end{array}$ & $\begin{array}{l}52.43 \\
52.46 \\
52.43 \\
52.42 \\
52.41\end{array}$ & $\begin{array}{l}52.18 \\
52.10 \\
52.06 \\
52.10 \\
52.11\end{array}$ & $\begin{array}{l}53.02 \\
53.06 \\
53.02 \\
53.04 \\
53.10\end{array}$ & $\begin{array}{l}53.35 \\
53.40 \\
53.40 \\
53.38 \\
53.44\end{array}$ & $\begin{array}{l}53.48 \\
53.62 \\
53.71 \\
53.74 \\
53.67\end{array}$ & $\begin{array}{l}53.85 \\
53.92 \\
53.95 \\
53.96 \\
53.92\end{array}$ & $\begin{array}{l}54.02 \\
53.99 \\
54.06 \\
54.10 \\
54.07\end{array}$ \\
\hline $\begin{array}{l}26 \\
27 \\
28 \\
29 \\
30 \\
31\end{array}$ & $\begin{array}{l}52.69 \\
52.82 \\
52.85 \\
52.84 \\
52.64 \\
52.61\end{array}$ & $\begin{array}{r}52.62 \\
52.56 \\
52.41 \\
-.- \\
-.- \\
-.-\end{array}$ & $\begin{array}{l}51.96 \\
51.88 \\
51.97 \\
51.90 \\
51.77 \\
51.98\end{array}$ & $\begin{array}{r}51.68 \\
51.68 \\
51.58 \\
51.64 \\
51.59 \\
.\end{array}$ & $\begin{array}{l}51.88 \\
51.90 \\
51.92 \\
51.91 \\
51.89 \\
51.90\end{array}$ & $\begin{array}{r}52.40 \\
52.42 \\
52.52 \\
52.62 \\
52.67 \\
. .-\end{array}$ & $\begin{array}{l}52.07 \\
52.02 \\
51.99 \\
52.00 \\
52.04 \\
52.10\end{array}$ & $\begin{array}{l}53.11 \\
53.12 \\
53.12 \\
53.11 \\
53.18 \\
53.16\end{array}$ & $\begin{array}{r}53.52 \\
53.58 \\
53.61 \\
53.50 \\
53.34 \\
. \ldots\end{array}$ & $\begin{array}{l}53.52 \\
53.44 \\
53.54 \\
53.66 \\
53.76 \\
53.83\end{array}$ & $\begin{array}{r}53.87 \\
53.81 \\
53.75 \\
53.64 \\
53.52 \\
.\end{array}$ & $\begin{array}{l}54.05 \\
54.00 \\
53.91 \\
53.98 \\
54.14 \\
54.14\end{array}$ \\
\hline EAI & 52.68 & 52.53 & 52.18 & 51.68 & 51.78 & 52.29 & 52.38 & 52.71 & 53.28 & 53.60 & 53.78 & 53 \\
\hline
\end{tabular}

$\begin{array}{lllllll}\text { CAL YR } 1987 & \text { MEAN } & 52.74 & \text { HIGH } & 51.47 & \text { LOW } & 54.14\end{array}$ 


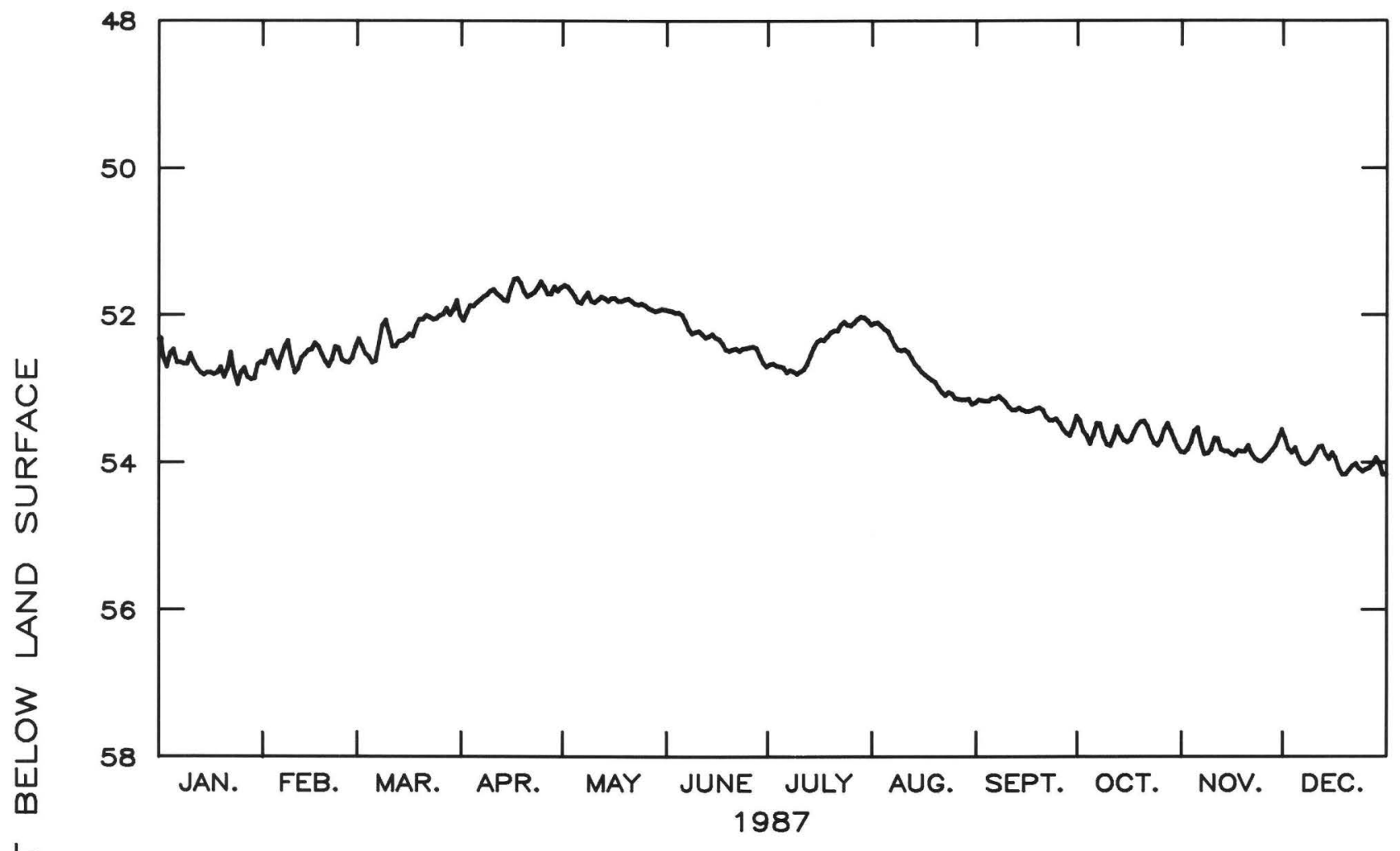

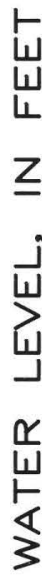

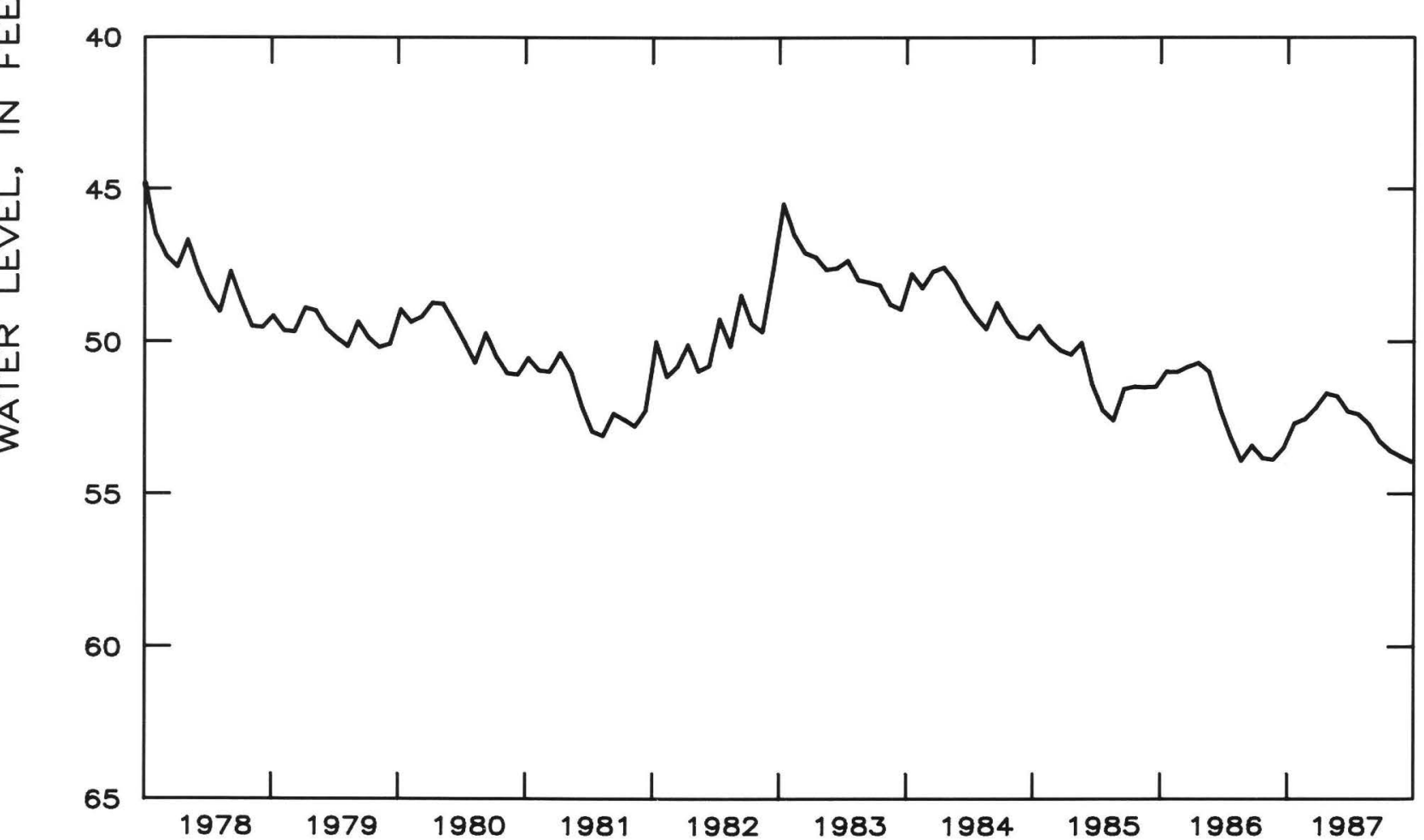

Figure 2.7.4.2-4.--Water level in observation well 33M004, Long County. 
314343081251901 Local number, 34 M054

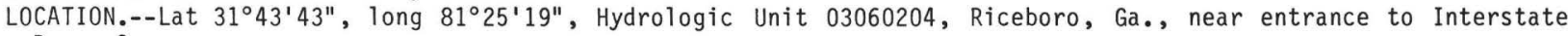
Paper Company.

Owner: U.S. Geological Survey, test well 2.

AQUIFER.--Upper Floridan aquifer.

WELL CHARACTERISTICS.--Drilled observation well, diameter 4 in., depth $802 \mathrm{ft}$, cased to $467 \mathrm{ft}$, open hole. DATUM.--Elevation of land-surface datum is $19 \mathrm{ft}$.

Measuring point: Floor of recorder shelter, $3.4 \mathrm{ft}$ above land-surface datum.

REMARKS.--Well pumped July 11, 1979; water-quality sample collected at conclusion of pumping. Borehole geophysical survey conducted June 15, 1976. Water levels for periods of missing record, August 27 to September 28 , and October 26, were estimated.

PERIOD OF RECORD.--February 1967 to current year.

EXTREMES FOR PERIOD OF RECORD.--Highest water level, $0.85 \mathrm{ft}$ below land-surface datum, February 5 , 1967; 1owest, $27.13 \mathrm{ft}$ below 1 and-surface datum, November 1, 1986.

\section{DEPTH BELOW LAND SURFACE (WATER LEVEL) (FEET) CALENDAR YEAR JANUARY 1987 TO DECEMBER 1987}

\begin{tabular}{|c|c|c|c|c|c|c|c|c|c|c|c|c|}
\hline DAY & JAN & FEB & MAR & APR & MAY & JUN & JUL & AUG & SEP & OCT & NOV & DEC \\
\hline $\begin{array}{l}1 \\
2 \\
3 \\
4 \\
5\end{array}$ & $\begin{array}{l}25.72 \\
25.96 \\
26.00 \\
25.67 \\
25.70\end{array}$ & $\begin{array}{l}25.81 \\
25.66 \\
25.73 \\
25.89 \\
25.97\end{array}$ & $\begin{array}{l}25.24 \\
25.30 \\
25.21 \\
25.22 \\
25.30\end{array}$ & $\begin{array}{l}22.49 \\
22.41 \\
22.41 \\
22.41 \\
22.66\end{array}$ & $\begin{array}{l}24.57 \\
24.52 \\
24.54 \\
24.67 \\
24.87\end{array}$ & $\begin{array}{l}24.91 \\
25.01 \\
25.06 \\
25.06 \\
25.08\end{array}$ & $\begin{array}{l}25.67 \\
25.65 \\
25.63 \\
25.64 \\
25.71\end{array}$ & $\begin{array}{l}25.52 \\
25.49 \\
25.49 \\
25.42 \\
25.45\end{array}$ & $\begin{array}{l}26.23 \\
26.19 \\
26.16 \\
26.11 \\
25.99\end{array}$ & $\begin{array}{l}25.93 \\
26.06 \\
25.73 \\
25.62 \\
25.43\end{array}$ & $\begin{array}{l}26.28 \\
26.21 \\
26.31 \\
26.35 \\
26.05\end{array}$ & $\begin{array}{l}26.32 \\
26.47 \\
26.37 \\
26.23 \\
26.49\end{array}$ \\
\hline $\begin{array}{r}6 \\
7 \\
8 \\
9 \\
10\end{array}$ & $\begin{array}{l}25.86 \\
25.82 \\
25.82 \\
25.77 \\
25.61\end{array}$ & $\begin{array}{l}25.80 \\
25.66 \\
25.60 \\
25.34 \\
25.25\end{array}$ & $\begin{array}{l}25.28 \\
25.02 \\
24.71 \\
24.66 \\
24.73\end{array}$ & $\begin{array}{l}23.17 \\
23.44 \\
23.59 \\
23.82 \\
23.93\end{array}$ & $\begin{array}{l}24.83 \\
24.80 \\
24.77 \\
24.85 \\
24.80\end{array}$ & $\begin{array}{l}25.16 \\
25.23 \\
25.17 \\
25.21 \\
25.22\end{array}$ & $\begin{array}{l}25.78 \\
25.80 \\
25.71 \\
25.60 \\
25.78\end{array}$ & $\begin{array}{l}25.58 \\
25.84 \\
25.94 \\
26.00 \\
26.03\end{array}$ & $\begin{array}{l}25.87 \\
25.75 \\
25.75 \\
25.81 \\
25.86\end{array}$ & $\begin{array}{l}25.08 \\
25.20 \\
25.48 \\
25.62 \\
25.52\end{array}$ & $\begin{array}{l}26.29 \\
26.44 \\
26.38 \\
26.30 \\
26.15\end{array}$ & $\begin{array}{l}26.57 \\
26.56 \\
26.49 \\
26.43 \\
26.23\end{array}$ \\
\hline $\begin{array}{l}11 \\
12 \\
13 \\
14 \\
15\end{array}$ & $\begin{array}{l}25.76 \\
25.88 \\
25.77 \\
25.82 \\
25.62\end{array}$ & $\begin{array}{l}25.81 \\
25.80 \\
25.77 \\
25.81 \\
25.74\end{array}$ & $\begin{array}{l}25.02 \\
25.01 \\
25.03 \\
25.08 \\
25.02\end{array}$ & $\begin{array}{l}23.90 \\
24.08 \\
24.12 \\
24.20 \\
24.10\end{array}$ & $\begin{array}{l}24.51 \\
24.61 \\
24.83 \\
24.69 \\
24.68\end{array}$ & $\begin{array}{l}25.35 \\
25.38 \\
25.34 \\
25.15 \\
25.17\end{array}$ & $\begin{array}{l}25.65 \\
25.56 \\
25.48 \\
25.62 \\
25.73\end{array}$ & $\begin{array}{l}26.08 \\
26.08 \\
26.11 \\
26.18 \\
26.20\end{array}$ & $\begin{array}{l}25.89 \\
25.84 \\
25.77 \\
25.77 \\
25.76\end{array}$ & $\begin{array}{l}25.41 \\
25.24 \\
25.07 \\
25.19 \\
25.16\end{array}$ & $\begin{array}{l}26.42 \\
26.64 \\
26.56 \\
26.63 \\
26.71\end{array}$ & $\begin{array}{l}26.23 \\
26.20 \\
26.35 \\
26.42 \\
26.26\end{array}$ \\
\hline $\begin{array}{l}16 \\
17 \\
18 \\
19 \\
20\end{array}$ & $\begin{array}{l}25.62 \\
25.67 \\
25.53 \\
25.47 \\
25.75\end{array}$ & $\begin{array}{l}25.41 \\
25.49 \\
25.62 \\
25.76 \\
25.71\end{array}$ & $\begin{array}{l}25.00 \\
24.87 \\
24.67 \\
24.43 \\
24.43\end{array}$ & $\begin{array}{l}24.18 \\
24.18 \\
24.37 \\
24.44 \\
24.42\end{array}$ & $\begin{array}{l}24.73 \\
24.79 \\
24.67 \\
24.62 \\
24.51\end{array}$ & $\begin{array}{l}25.19 \\
25.20 \\
25.03 \\
25.26 \\
25.30\end{array}$ & $\begin{array}{l}25.91 \\
26.26 \\
26.28 \\
26.30 \\
25.88\end{array}$ & $\begin{array}{l}26.16 \\
26.10 \\
26.01 \\
26.16 \\
26.16\end{array}$ & $\begin{array}{l}25.73 \\
25.69 \\
25.63 \\
25.62 \\
25.61\end{array}$ & $\begin{array}{l}25.10 \\
25.07 \\
25.04 \\
25.06 \\
25.04\end{array}$ & $\begin{array}{l}26.51 \\
26.34 \\
26.46 \\
26.38 \\
26.30\end{array}$ & $\begin{array}{l}26.48 \\
26.48 \\
26.44 \\
26.40 \\
26.32\end{array}$ \\
\hline $\begin{array}{l}21 \\
22 \\
23 \\
24 \\
25\end{array}$ & $\begin{array}{l}25.74 \\
25.44 \\
25.79 \\
25.96 \\
25.67\end{array}$ & $\begin{array}{l}25.55 \\
25.37 \\
25.40 \\
25.71 \\
25.62\end{array}$ & $\begin{array}{l}24.38 \\
24.42 \\
24.43 \\
24.38 \\
24.32\end{array}$ & $\begin{array}{l}24.34 \\
24.40 \\
24.30 \\
24.25 \\
24.27\end{array}$ & $\begin{array}{l}24.56 \\
24.64 \\
24.74 \\
24.71 \\
24.71\end{array}$ & $\begin{array}{l}25.47 \\
25.48 \\
25.45 \\
25.46 \\
25.47\end{array}$ & $\begin{array}{l}25.82 \\
25.67 \\
25.73 \\
25.80 \\
25.81\end{array}$ & $\begin{array}{l}26.24 \\
26.22 \\
26.14 \\
26.11 \\
26.20\end{array}$ & $\begin{array}{l}25.67 \\
25.70 \\
25.67 \\
25.64 \\
25.65\end{array}$ & $\begin{array}{l}25.10 \\
25.20 \\
25.23 \\
25.20 \\
25.05\end{array}$ & $\begin{array}{l}26.46 \\
26.62 \\
26.62 \\
26.57 \\
26.54\end{array}$ & $\begin{array}{l}26.23 \\
26.22 \\
26.35 \\
26.37 \\
26.34\end{array}$ \\
\hline $\begin{array}{l}26 \\
27 \\
28 \\
29 \\
30 \\
31\end{array}$ & $\begin{array}{l}25.69 \\
25.77 \\
25.92 \\
25.99 \\
25.80 \\
25.79\end{array}$ & $\begin{array}{r}25.65 \\
25.53 \\
25.42 \\
--- \\
--- \\
---\end{array}$ & $\begin{array}{l}24.32 \\
24.22 \\
24.18 \\
24.22 \\
23.39 \\
22.71\end{array}$ & $\begin{array}{r}24.34 \\
24.39 \\
24.41 \\
24.54 \\
24.54 \\
-.--\end{array}$ & $\begin{array}{l}24.83 \\
24.83 \\
24.90 \\
25.00 \\
25.01 \\
24.94\end{array}$ & $\begin{array}{r}25.38 \\
25.32 \\
25.37 \\
25.58 \\
25.72 \\
. .-\end{array}$ & $\begin{array}{l}25.61 \\
25.54 \\
25.51 \\
25.52 \\
25.63 \\
25.63\end{array}$ & $\begin{array}{l}26.24 \\
26.22 \\
26.26 \\
26.37 \\
26.44 \\
26.35\end{array}$ & $\begin{array}{r}25.74 \\
25.78 \\
25.87 \\
25.76 \\
25.72 \\
. .-\end{array}$ & $\begin{array}{l}25.58 \\
26.02 \\
26.22 \\
26.24 \\
26.30 \\
26.32\end{array}$ & $\begin{array}{r}26.52 \\
26.52 \\
26.40 \\
26.33 \\
26.27 \\
.--\end{array}$ & $\begin{array}{l}26.30 \\
26.29 \\
26.15 \\
26.31 \\
26.53 \\
26.42\end{array}$ \\
\hline MEAN & 25.75 & 25.64 & 24.65 & 23.87 & 24.73 & 25.27 & 25.74 & 26.03 & 25.81 & 25.47 & 26.42 & 26.36 \\
\hline CAL & & & & HIGH & 22.41 & & & & & & & \\
\hline
\end{tabular}




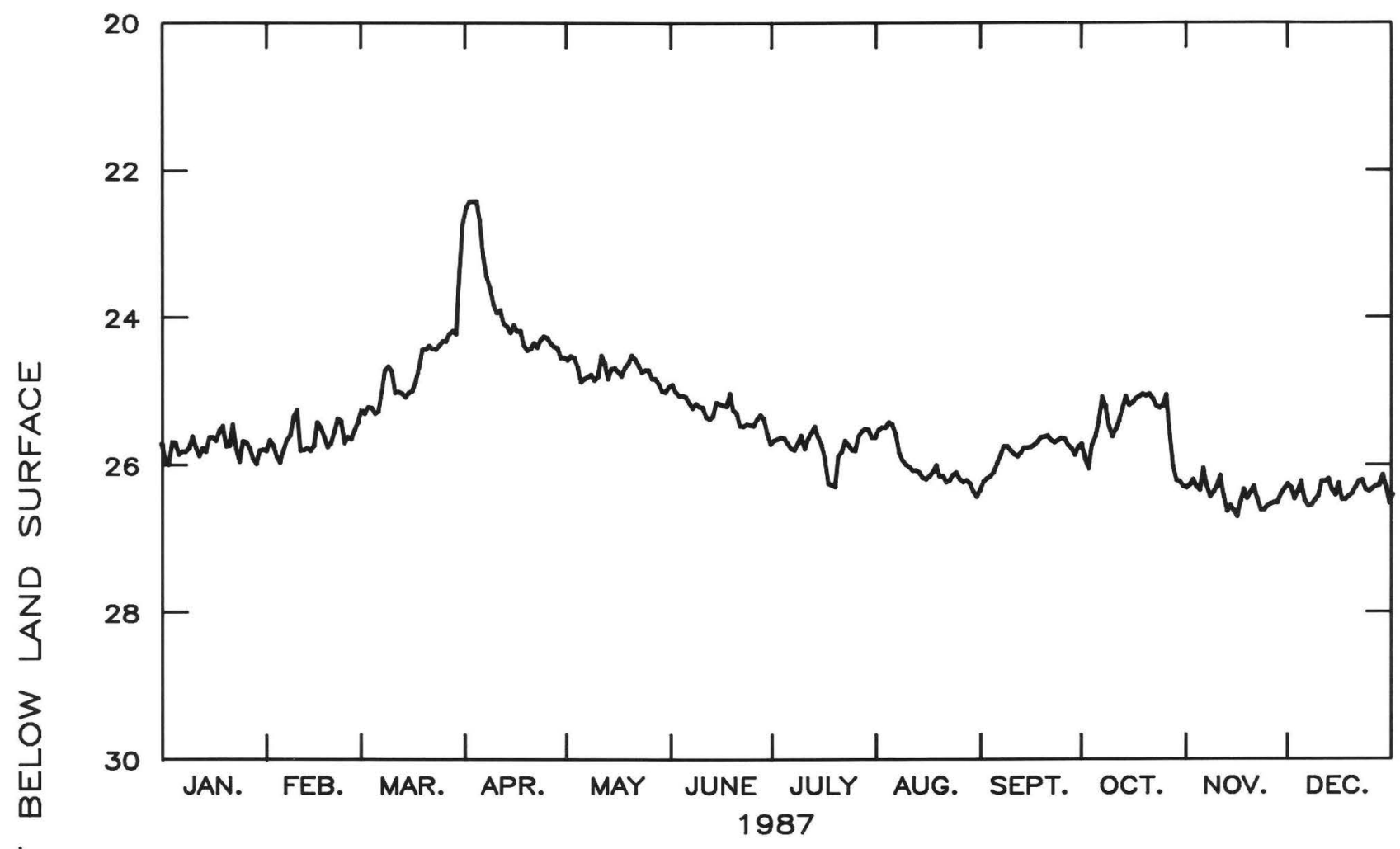

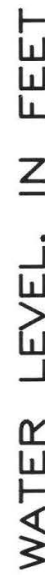

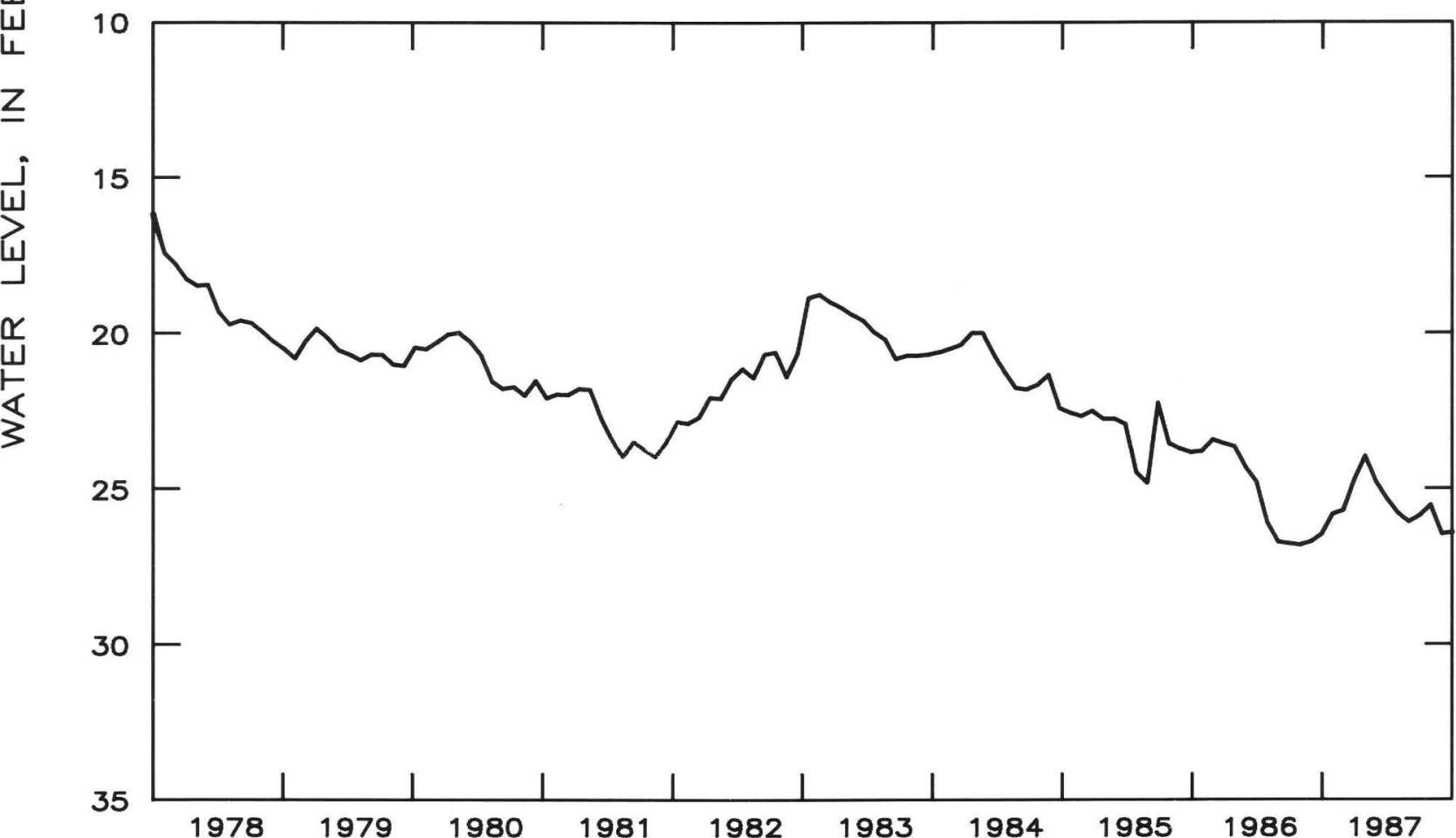

Figure 2.7.4.2-5.--Water level in observation well 34M054, Liberty County. 
315214081235301 Local number, 34N089.

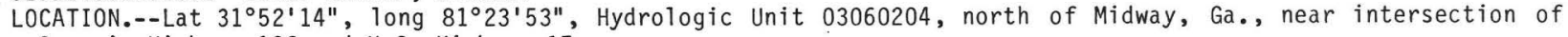
Georgia Highway 196 and U.S. Highway 17.

Owner: U.S. Geological Survey, test well 1.

AQUIFER.--Upper Floridan aquifer.

WELL CHARACTERISTICS.--Drilled observation well, diameter $4 \mathrm{in.,}$ depth $789 \mathrm{ft}$, cased to $410 \mathrm{ft}$, open hole. DATUM.--Elevation of land-surface datum is $17 \mathrm{ft}$.

Measuring point: Top of $4-i n$. casing, $1.33 \mathrm{ft}$ above land-surface datum.

REMARKS.--Well pumped July 11, 1979; water-quality sample collected at conclusion of pumping. Borehole geophysical survey conducted June $15,1976$.

PERIOD OF RECORD.--February 1967 to current year.

EXTREMES FOR PERIOD OF RECORD.--Highest water level, $2.34 \mathrm{ft}$ below land-surface datum, March 6, 1967; 1owest, $25.16 \mathrm{ft}$ below land-surface datum, August 29, 1986 .

DEPTH BELOW LAND SURFACE (WATER LEVEL) (FEET) CALENDAR YEAR JANUARY 1987 TO DECEMBER 1987 MEAN VALUES

\begin{tabular}{|c|c|c|c|c|c|c|c|c|c|c|c|c|}
\hline DAY & JAN & FEB & MAR & APR & MAY & JUN & JUL & AUG & SEP & OCT & NOV & DEC \\
\hline $\begin{array}{l}1 \\
2 \\
3 \\
4 \\
5\end{array}$ & $\begin{array}{l}23.21 \\
23.45 \\
23.60 \\
23.30 \\
23.23\end{array}$ & $\begin{array}{l}23.02 \\
22.85 \\
22.90 \\
23.04 \\
23.11\end{array}$ & $\begin{array}{l}22.43 \\
22.56 \\
22.73 \\
22.77 \\
22.81\end{array}$ & $\begin{array}{l}22.23 \\
22.16 \\
21.97 \\
21.95 \\
21.88\end{array}$ & $\begin{array}{l}22.18 \\
22.23 \\
22.35 \\
22.44 \\
22.54\end{array}$ & $\begin{array}{l}22.99 \\
23.03 \\
23.03 \\
23.03 \\
23.03\end{array}$ & $\begin{array}{l}23.51 \\
23.49 \\
23.52 \\
23.51 \\
23.52\end{array}$ & $\begin{array}{l}23.80 \\
23.85 \\
23.87 \\
23.86 \\
23.88\end{array}$ & $\begin{array}{l}24.54 \\
24.51 \\
24.49 \\
24.45 \\
24.34\end{array}$ & $\begin{array}{l}24.22 \\
24.32 \\
24.35 \\
24.48 \\
24.41\end{array}$ & $\begin{array}{l}24.43 \\
24.38 \\
24.29 \\
24.10 \\
24.06\end{array}$ & $\begin{array}{l}23.84 \\
24.06 \\
24.07 \\
23.95 \\
24.14\end{array}$ \\
\hline $\begin{array}{r}6 \\
7 \\
8 \\
9 \\
10\end{array}$ & $\begin{array}{l}23.36 \\
23.28 \\
23.28 \\
23.24 \\
23.06\end{array}$ & $\begin{array}{l}22.95 \\
22.80 \\
22.77 \\
22.96 \\
23.11\end{array}$ & $\begin{array}{l}22.81 \\
22.55 \\
22.22 \\
22.17 \\
22.28\end{array}$ & $\begin{array}{l}21.83 \\
21.78 \\
21.78 \\
21.77 \\
21.77\end{array}$ & $\begin{array}{l}22.50 \\
22.45 \\
22.44 \\
22.60 \\
22.73\end{array}$ & $\begin{array}{l}23.08 \\
23.13 \\
23.15 \\
23.13 \\
23.12\end{array}$ & $\begin{array}{l}23.53 \\
23.50 \\
23.49 \\
23.48 \\
23.44\end{array}$ & $\begin{array}{l}23.96 \\
24.01 \\
24.12 \\
24.18 \\
24.16\end{array}$ & $\begin{array}{l}24.23 \\
24.12 \\
24.13 \\
24.20 \\
24.26\end{array}$ & $\begin{array}{l}24.29 \\
24.28 \\
24.43 \\
24.52 \\
24.50\end{array}$ & $\begin{array}{l}24.30 \\
24.36 \\
24.32 \\
24.24 \\
24.05\end{array}$ & $\begin{array}{l}24.26 \\
24.28 \\
24.22 \\
24.08 \\
23.93\end{array}$ \\
\hline $\begin{array}{l}11 \\
12 \\
13 \\
14 \\
15\end{array}$ & $\begin{array}{l}23.18 \\
23.20 \\
23.22 \\
23.23 \\
23.15\end{array}$ & $\begin{array}{l}23.03 \\
22.86 \\
22.84 \\
22.80 \\
22.79\end{array}$ & $\begin{array}{l}22.51 \\
22.51 \\
22.46 \\
22.45 \\
22.43\end{array}$ & $\begin{array}{l}21.84 \\
21.91 \\
21.95 \\
21.95 \\
21.78\end{array}$ & $\begin{array}{l}22.76 \\
22.76 \\
22.77 \\
22.79 \\
22.70\end{array}$ & $\begin{array}{l}23.20 \\
23.26 \\
23.25 \\
23.18 \\
23.21\end{array}$ & $\begin{array}{l}23.41 \\
23.39 \\
23.34 \\
23.33 \\
23.38\end{array}$ & $\begin{array}{l}24.11 \\
24.12 \\
24.15 \\
24.19 \\
24.24\end{array}$ & $\begin{array}{l}24.30 \\
24.27 \\
24.21 \\
24.22 \\
24.22\end{array}$ & $\begin{array}{l}24.39 \\
24.24 \\
24.32 \\
24.42 \\
24.40\end{array}$ & $\begin{array}{l}24.14 \\
24.27 \\
24.23 \\
24.24 \\
24.30\end{array}$ & $\begin{array}{l}23.84 \\
23.83 \\
24.02 \\
24.03 \\
23.82\end{array}$ \\
\hline $\begin{array}{l}16 \\
17 \\
18 \\
19 \\
20\end{array}$ & $\begin{array}{l}23.12 \\
23.11 \\
23.00 \\
22.94 \\
23.09\end{array}$ & $\begin{array}{l}22.59 \\
22.74 \\
22.81 \\
22.92 \\
22.96\end{array}$ & $\begin{array}{l}22.40 \\
22.46 \\
22.36 \\
22.18 \\
22.22\end{array}$ & $\begin{array}{l}21.70 \\
21.69 \\
21.79 \\
21.91 \\
21.96\end{array}$ & $\begin{array}{l}22.61 \\
22.69 \\
22.72 \\
22.57 \\
22.55\end{array}$ & $\begin{array}{l}23.24 \\
23.26 \\
23.34 \\
23.34 \\
23.29\end{array}$ & $\begin{array}{l}23.44 \\
23.55 \\
23.57 \\
23.58 \\
23.65\end{array}$ & $\begin{array}{l}24.28 \\
24.29 \\
24.28 \\
24.28 \\
24.34\end{array}$ & $\begin{array}{l}24.20 \\
24.17 \\
24.12 \\
24.12 \\
24.12\end{array}$ & $\begin{array}{l}24.37 \\
24.32 \\
24.32 \\
24.31 \\
24.30\end{array}$ & $\begin{array}{l}24.29 \\
24.18 \\
24.22 \\
24.17 \\
24.07\end{array}$ & $\begin{array}{l}23.94 \\
24.08 \\
24.12 \\
24.08 \\
23.98\end{array}$ \\
\hline $\begin{array}{l}21 \\
22 \\
23 \\
24 \\
25\end{array}$ & $\begin{array}{l}23.03 \\
22.73 \\
23.02 \\
23.14 \\
22.91\end{array}$ & $\begin{array}{l}22.82 \\
22.67 \\
22.70 \\
22.83 \\
22.83\end{array}$ & $\begin{array}{l}22.16 \\
22.16 \\
22.21 \\
22.18 \\
22.15\end{array}$ & $\begin{array}{l}21.95 \\
21.95 \\
21.94 \\
21.88 \\
21.99\end{array}$ & $\begin{array}{l}22.60 \\
22.70 \\
22.76 \\
22.78 \\
22.80\end{array}$ & $\begin{array}{l}23.26 \\
23.29 \\
23.31 \\
23.35 \\
23.40\end{array}$ & $\begin{array}{l}23.69 \\
23.66 \\
23.64 \\
23.73 \\
23.75\end{array}$ & $\begin{array}{l}24.40 \\
24.40 \\
24.38 \\
24.41 \\
24.48\end{array}$ & $\begin{array}{l}24.19 \\
24.23 \\
24.21 \\
24.19 \\
24.21\end{array}$ & $\begin{array}{l}24.32 \\
24.48 \\
24.50 \\
24.49 \\
24.43\end{array}$ & $\begin{array}{l}24.21 \\
24.27 \\
24.28 \\
24.28 \\
24.18\end{array}$ & $\begin{array}{l}23.93 \\
23.89 \\
24.02 \\
24.03 \\
23.94\end{array}$ \\
\hline $\begin{array}{l}26 \\
27 \\
28 \\
29 \\
30 \\
31\end{array}$ & $\begin{array}{l}22.88 \\
23.06 \\
23.09 \\
23.11 \\
22.93 \\
22.97\end{array}$ & $\begin{array}{r}22.83 \\
22.78 \\
22.59 \\
-\ldots \\
-.- \\
-.-\end{array}$ & $\begin{array}{l}22.13 \\
22.07 \\
22.08 \\
22.15 \\
21.95 \\
22.05\end{array}$ & $\begin{array}{r}22.12 \\
22.15 \\
22.05 \\
22.13 \\
22.13 \\
\quad . .\end{array}$ & $\begin{array}{l}22.82 \\
22.85 \\
22.85 \\
22.84 \\
22.84 \\
22.88\end{array}$ & $\begin{array}{r}23.44 \\
23.43 \\
23.50 \\
23.54 \\
23.54 \\
. .-\end{array}$ & $\begin{array}{l}23.73 \\
23.76 \\
23.79 \\
23.79 \\
23.81 \\
23.82\end{array}$ & $\begin{array}{l}24.49 \\
24.48 \\
24.53 \\
24.65 \\
24.73 \\
24.65\end{array}$ & $\begin{array}{r}24.31 \\
24.36 \\
24.46 \\
24.36 \\
24.14 \\
. \ldots\end{array}$ & $\begin{array}{l}24.30 \\
24.16 \\
24.26 \\
24.36 \\
24.43 \\
24.45\end{array}$ & $\begin{array}{r}24.08 \\
24.02 \\
23.94 \\
23.85 \\
23.78 \\
. .--\end{array}$ & $\begin{array}{l}23.91 \\
23.91 \\
23.78 \\
23.86 \\
24.12 \\
24.08\end{array}$ \\
\hline EAN & 23.13 & 22.85 & 22.34 & 21.93 & 22.65 & 23.25 & 23.57 & 24.24 & 24.26 & 24.37 & 24.18 & 24.00 \\
\hline
\end{tabular}

$\begin{array}{llllll}\text { CAL YR } 1987 & \text { MEAN } 23.40 & \text { HIGH } & 21.69 & \text { LOW } & 24.73\end{array}$ 


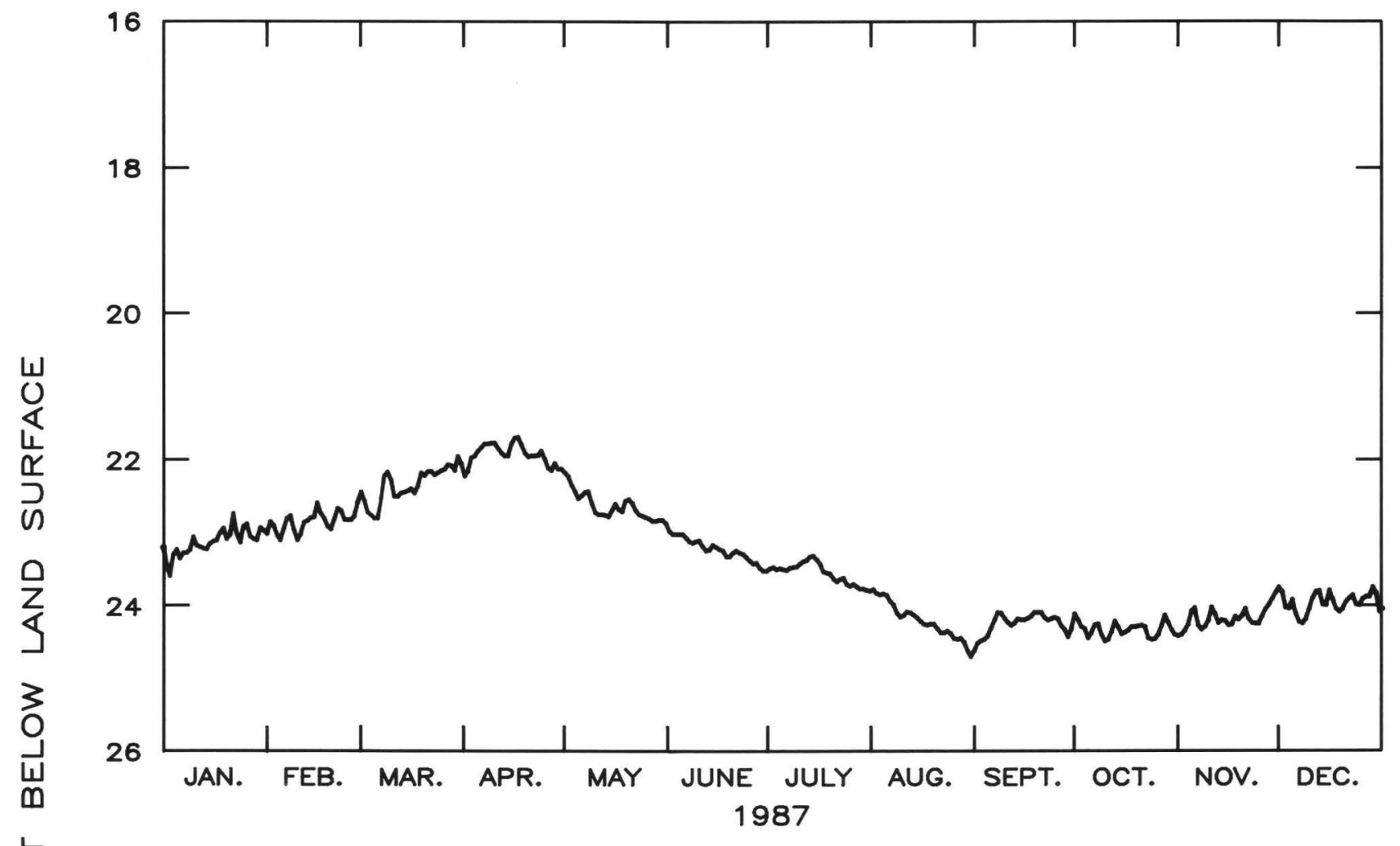

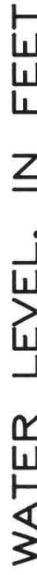

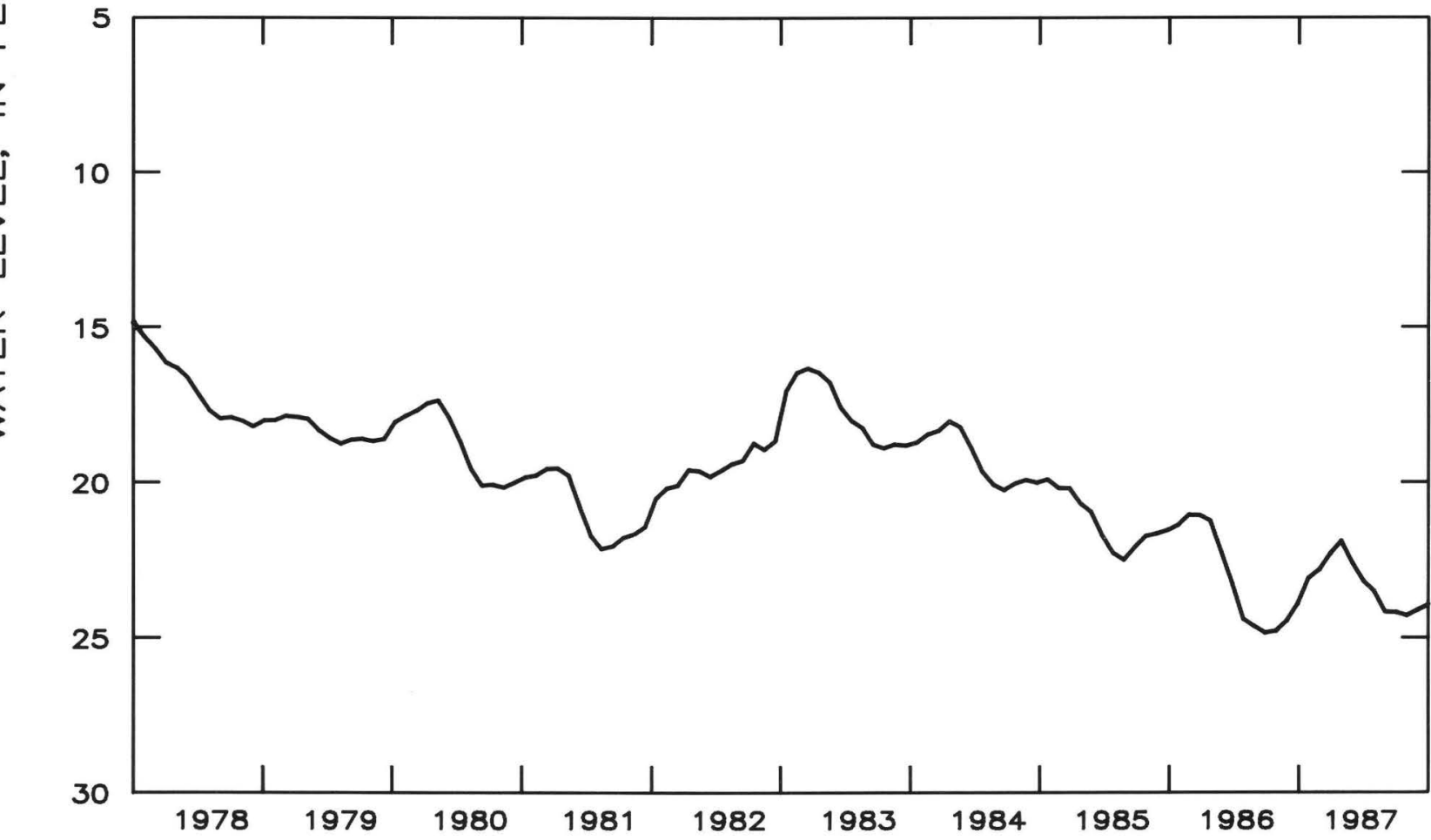

Figure 2.7.4.2-6.--Water level in observation well 34N089, Liberty County. 
313826081152601 Local number, 35M013.

LOCATION.--Lat $31^{\circ} 38^{\prime} 23^{\prime \prime}$, long $81^{\circ} 15^{\prime} 42^{\prime \prime}$, Hydrologic Unit $03060204,8.5$ mi east of U.S. Highway 17 at Harris Neck Wildl ife Refuge.

Owner: U.S. Department of the Interior, Fish and Wildlife Service.

AQUIFER.--Upper Floridan aquifer.

WELL CHARACTERISTICS.--Drilled unused supply well, diameter $10 \mathrm{in.,}$ depth $553 \mathrm{ft}$, cased to $376 \mathrm{ft}$, open hole. DATUM.--Elevation of land-surface datum is $16.3 \mathrm{ft}$.

Measuring point: Floor of recorder shelter, $3.2 \mathrm{ft}$ above land-surface datum.

REMARKS.--Well pumped August 3, 1976; water-quality sample collected at conclusion of pumping. Borehole geophysical survey conducted June 16, 1976. Water level for period of missing record, January 28, was estimated. PERIOD OF RECORD.--September 1966 to current year.

EXTREMES FOR PERIOD OF RECORD.--Highest water level, $4.35 \mathrm{ft}$ below land-surface datum, 0ctober 4, 1966; 10west, $22.41 \mathrm{ft}$ below land-surface datum, December $16,1987$.

DEPTH BELOW LAND SURFACE (WATER LEVEL) (FEET) CALENDAR YEAR JANUARY 1987 TO DECEMBER 1987 MEAN VALUES

\begin{tabular}{|c|c|c|c|c|c|c|c|c|c|c|c|c|}
\hline DAY & JAN & FEB & MAR & APR & MAY & JUN & JUL & AUG & SEP & OCT & NOV & $\mathrm{DE}$ \\
\hline $\begin{array}{l}1 \\
2 \\
3 \\
4 \\
5\end{array}$ & $\begin{array}{l}21.66 \\
21.74 \\
21.30 \\
21.26 \\
21.46\end{array}$ & $\begin{array}{l}21.34 \\
21.21 \\
21.28 \\
21.34 \\
21.31\end{array}$ & $\begin{array}{l}20.84 \\
21.06 \\
21.13 \\
21.20 \\
21.24\end{array}$ & $\begin{array}{l}20.73 \\
20.64 \\
20.54 \\
20.67 \\
20.55\end{array}$ & $\begin{array}{l}20.40 \\
20.40 \\
20.47 \\
20.51 \\
20.45\end{array}$ & $\begin{array}{l}20.81 \\
20.84 \\
20.86 \\
20.83 \\
20.77\end{array}$ & $\begin{array}{l}21.18 \\
21.19 \\
21.25 \\
21.25 \\
21.27\end{array}$ & $\begin{array}{l}21.45 \\
21.44 \\
21.46 \\
21.48 \\
21.46\end{array}$ & $\begin{array}{l}21.77 \\
21.67 \\
21.28 \\
21.75 \\
21.89\end{array}$ & $\begin{array}{l}21.75 \\
21.84 \\
21.91 \\
21.90 \\
21.80\end{array}$ & $\begin{array}{l}22.02 \\
21.86 \\
21.79 \\
21.64 \\
21.67\end{array}$ & $\begin{array}{l}21.87 \\
22.00 \\
21.95 \\
21.99 \\
21.99\end{array}$ \\
\hline $\begin{array}{r}6 \\
7 \\
8 \\
9 \\
10\end{array}$ & $\begin{array}{l}21.56 \\
21.60 \\
21.52 \\
21.39 \\
21.68\end{array}$ & $\begin{array}{l}21.10 \\
21.00 \\
21.05 \\
21.44 \\
21.46\end{array}$ & $\begin{array}{l}21.20 \\
20.91 \\
20.80 \\
20.84 \\
20.87\end{array}$ & $\begin{array}{l}20.41 \\
20.38 \\
20.34 \\
20.33 \\
20.24\end{array}$ & $\begin{array}{l}20.46 \\
20.42 \\
20.42 \\
20.42 \\
20.53\end{array}$ & $\begin{array}{l}20.76 \\
20.85 \\
20.90 \\
20.92 \\
20.93\end{array}$ & $\begin{array}{l}21.26 \\
21.18 \\
21.16 \\
21.18 \\
21.13\end{array}$ & $\begin{array}{l}21.52 \\
21.52 \\
21.47 \\
21.42 \\
21.46\end{array}$ & $\begin{array}{l}21.99 \\
21.75 \\
21.93 \\
21.24 \\
21.33\end{array}$ & $\begin{array}{l}21.71 \\
21.87 \\
22.02 \\
22.09 \\
22.04\end{array}$ & $\begin{array}{l}21.87 \\
22.04 \\
22.07 \\
22.01 \\
21.89\end{array}$ & $\begin{array}{l}22.0 \\
22.0 \\
21.9 \\
22.0 \\
21.9\end{array}$ \\
\hline $\begin{array}{l}11 \\
12 \\
13 \\
14 \\
15\end{array}$ & $\begin{array}{l}21.67 \\
21.56 \\
21.52 \\
21.47 \\
21.41\end{array}$ & $\begin{array}{l}21.42 \\
21.27 \\
21.17 \\
21.07 \\
21.04\end{array}$ & $\begin{array}{l}20.94 \\
20.92 \\
20.85 \\
20.78 \\
20.79\end{array}$ & $\begin{array}{l}20.24 \\
20.47 \\
20.44 \\
20.41 \\
20.11\end{array}$ & $\begin{array}{l}20.65 \\
20.03 \\
20.41 \\
20.43 \\
20.35\end{array}$ & $\begin{array}{l}20.85 \\
20.88 \\
20.95 \\
20.96 \\
20.98\end{array}$ & $\begin{array}{l}21.10 \\
21.08 \\
21.04 \\
21.08 \\
21.16\end{array}$ & $\begin{array}{l}21.43 \\
21.38 \\
21.35 \\
21.44 \\
21.57\end{array}$ & $\begin{array}{l}21.24 \\
21.51 \\
21.82 \\
21.70 \\
21.61\end{array}$ & $\begin{array}{l}21.90 \\
21.76 \\
21.78 \\
21.86 \\
21.85\end{array}$ & $\begin{array}{l}22.04 \\
22.06 \\
21.98 \\
21.96 \\
22.01\end{array}$ & $\begin{array}{l}22.1 \\
21.9 \\
22.0 \\
22.0 \\
22.0\end{array}$ \\
\hline $\begin{array}{l}16 \\
17 \\
18 \\
19 \\
20\end{array}$ & $\begin{array}{l}21.32 \\
21.20 \\
21.33 \\
21.51 \\
21.37\end{array}$ & $\begin{array}{l}20.70 \\
20.99 \\
21.13 \\
21.27 \\
21.25\end{array}$ & $\begin{array}{l}20.80 \\
20.72 \\
20.63 \\
20.54 \\
20.58\end{array}$ & $\begin{array}{l}20.07 \\
20.07 \\
20.12 \\
20.23 \\
20.27\end{array}$ & $\begin{array}{l}20.43 \\
20.46 \\
20.50 \\
20.51 \\
20.51\end{array}$ & $\begin{array}{l}20.97 \\
20.99 \\
21.03 \\
20.98 \\
20.97\end{array}$ & $\begin{array}{l}21.19 \\
21.22 \\
21.23 \\
21.31 \\
21.39\end{array}$ & $\begin{array}{l}21.66 \\
21.70 \\
21.70 \\
21.69 \\
21.79\end{array}$ & $\begin{array}{l}21.48 \\
21.31 \\
21.02 \\
20.81 \\
20.79\end{array}$ & $\begin{array}{l}21.81 \\
21.77 \\
21.82 \\
21.79 \\
21.79\end{array}$ & $\begin{array}{l}21.98 \\
21.89 \\
22.03 \\
21.98 \\
21.94\end{array}$ & $\begin{array}{l}22.4 \\
22.2 \\
22.2 \\
22.1 \\
22.0\end{array}$ \\
\hline $\begin{array}{l}21 \\
22 \\
23 \\
24 \\
25\end{array}$ & $\begin{array}{l}21.06 \\
21.55 \\
21.56 \\
21.03 \\
21.23\end{array}$ & $\begin{array}{l}21.07 \\
20.88 \\
21.18 \\
21.18 \\
21.08\end{array}$ & $\begin{array}{l}20.56 \\
20.52 \\
20.52 \\
20.47 \\
20.43\end{array}$ & $\begin{array}{l}20.26 \\
20.22 \\
20.17 \\
20.13 \\
20.19\end{array}$ & $\begin{array}{l}20.43 \\
20.42 \\
20.48 \\
20.51 \\
20.55\end{array}$ & $\begin{array}{l}21.02 \\
21.02 \\
20.99 \\
20.99 \\
20.90\end{array}$ & $\begin{array}{l}21.42 \\
21.37 \\
21.27 \\
21.31 \\
21.35\end{array}$ & $\begin{array}{l}21.76 \\
21.75 \\
21.77 \\
21.85 \\
21.88\end{array}$ & $\begin{array}{l}20.86 \\
20.94 \\
21.05 \\
21.31 \\
21.50\end{array}$ & $\begin{array}{l}21.90 \\
21.92 \\
21.97 \\
21.94 \\
21.92\end{array}$ & $\begin{array}{l}22.02 \\
22.00 \\
22.11 \\
22.11 \\
22.03\end{array}$ & $\begin{array}{l}22.1 \\
22.1 \\
22.1 \\
22.1 \\
22.1\end{array}$ \\
\hline $\begin{array}{l}26 \\
27 \\
28 \\
29 \\
30 \\
31\end{array}$ & $\begin{array}{l}21.30 \\
21.34 \\
21.25 \\
21.17 \\
21.28 \\
21.37\end{array}$ & $\begin{array}{r}20.96 \\
20.89 \\
20.79 \\
--- \\
-- \\
--\end{array}$ & $\begin{array}{l}20.44 \\
20.37 \\
20.42 \\
20.43 \\
20.30 \\
20.66\end{array}$ & $\begin{array}{r}20.29 \\
20.29 \\
20.22 \\
20.28 \\
20.39 \\
. .-\end{array}$ & $\begin{array}{l}20.59 \\
20.53 \\
20.59 \\
20.68 \\
20.71 \\
20.77\end{array}$ & $\begin{array}{r}20.90 \\
20.98 \\
21.02 \\
21.08 \\
21.17 \\
\ldots . .\end{array}$ & $\begin{array}{l}21.39 \\
21.45 \\
21.47 \\
21.44 \\
21.44 \\
21.48\end{array}$ & $\begin{array}{l}21.87 \\
21.57 \\
21.69 \\
21.53 \\
21.39 \\
21.93\end{array}$ & $\begin{array}{r}21.21 \\
21.67 \\
21.71 \\
21.78 \\
21.68 \\
.--\end{array}$ & $\begin{array}{l}21.81 \\
21.71 \\
21.89 \\
22.01 \\
22.10 \\
22.13\end{array}$ & $\begin{array}{r}21.94 \\
21.86 \\
21.72 \\
21.69 \\
21.68 \\
\ldots\end{array}$ & $\begin{array}{l}22.14 \\
22.12 \\
21.92 \\
22.18 \\
22.27 \\
22.25\end{array}$ \\
\hline MEAN & 21.42 & 21.14 & 20.73 & 20.32 & 20.48 & 20.94 & 21.27 & 21.59 & 21.45 & 21.88 & 21.93 & 2.0 \\
\hline
\end{tabular}

$\begin{array}{llllll}\text { CAL YR } 1987 & \text { MEAN } 21.27 & \text { HIGH } & 20.03 & \text { LOW } & 22.41\end{array}$ 


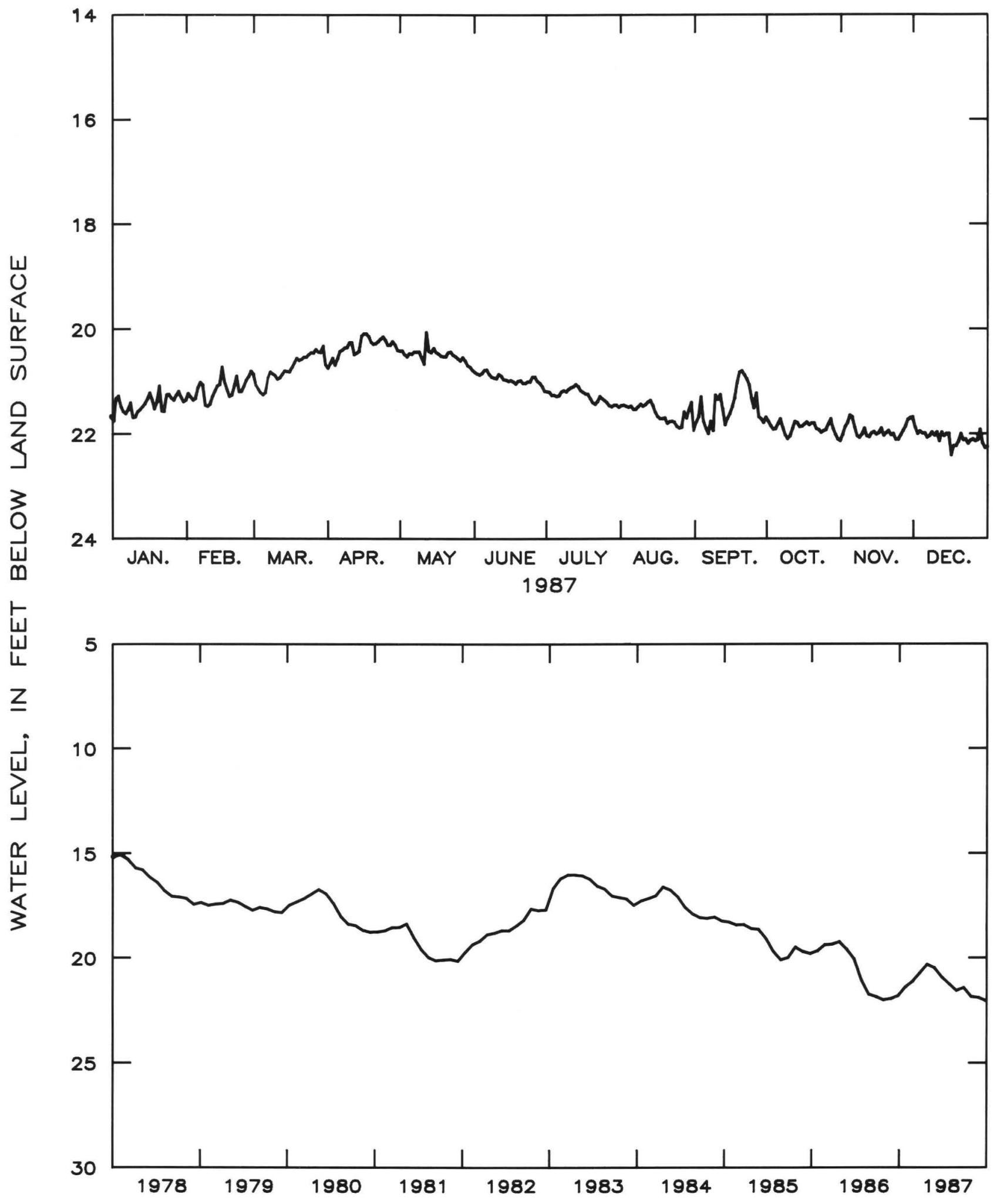

Figure 2.7.4.2-7.--Water level in observation well 35M013, Mclntosh County. 


\subsubsection{Brunswick area}

The water level in the Upper Floridan aquifer in the Brunswick area is affected primarily by industrial pumpage that was about $80 \mathrm{Mgal} / \mathrm{d}$ in 1985 (Turlington and others, 1987). This pumping has resulted in the development of a cone of depression centered at Brunswick. In 1987 two partial industrial shutdowns are reflected by sharp water-level rises during mid May and early November.

In October 1987, water levels were measured in 103 wells tapping the Upper Floridan aquifer in the Brunswick area and a map showing the potentiometric surface was prepared.

Mean water levels in water-bearing zones of the Upper Floridan aquifer in the Brunswick area were about $1.0 \mathrm{ft}$ lower in 1987 than in 1986. Mean water levels in the brackish-water zone of the Lower Floridan aquifer were from 0.8 to $1.0 \mathrm{ft}$ lower in 1987 than in 1986. By the end of March the water levels in all zones had recovered 3.9 to $6.1 \mathrm{ft}$ from the lows measured during the 1986 drought. Although mean water levels were lower during 1987, at the end of the year, water levels in the Upper and Lower Floridan were from about the same to $2.5 \mathrm{ft}$ higher than at the end of 1986 . 


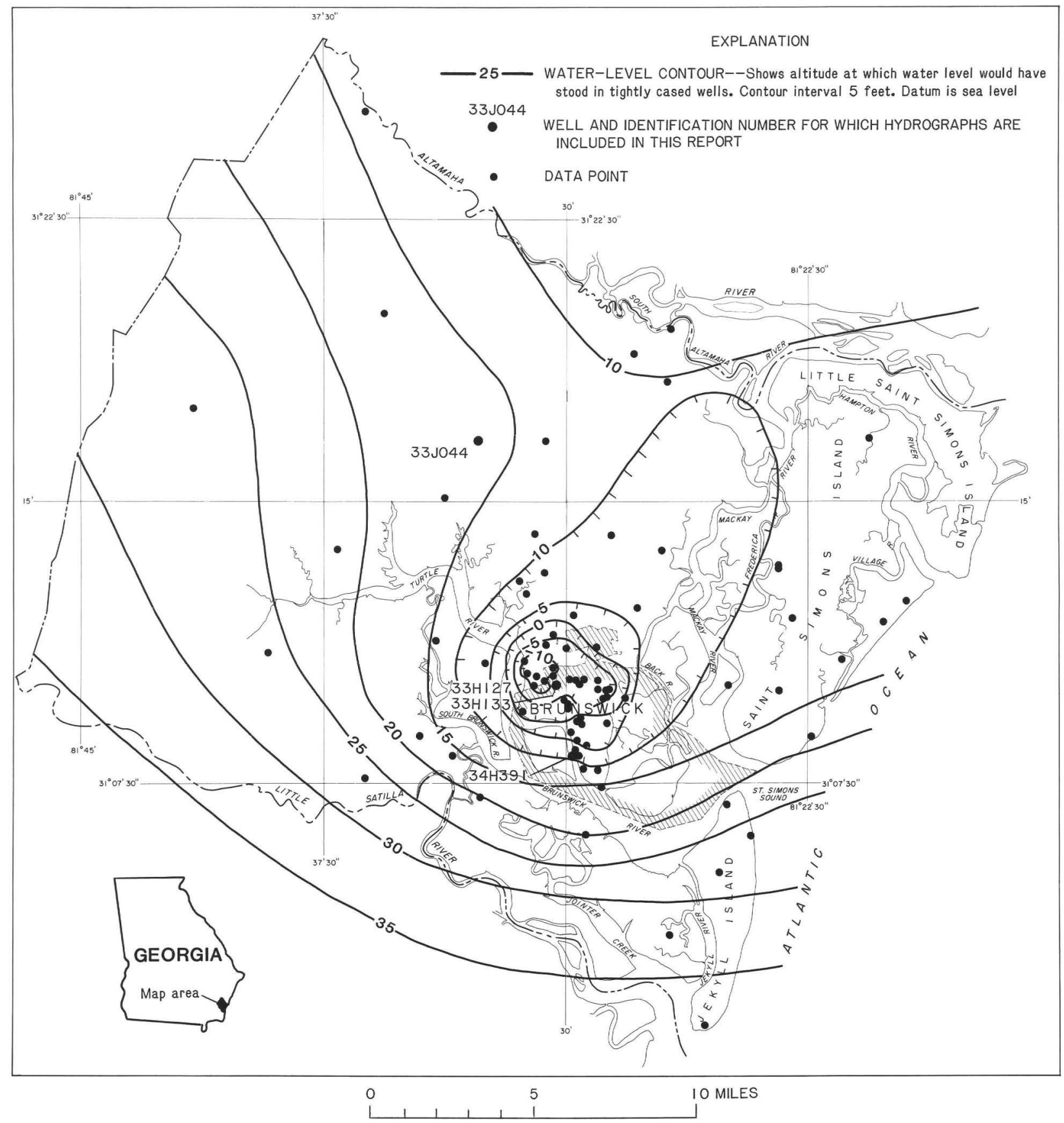

Figure 2.7.4.3-1.--Observation well locations and the water level in the Upper Floridan aquifer in the Brunswick area, October 1987. 
311007081301701 Local number, 33H127.

LOCATION.--Lat $31^{\circ} 10^{\prime} 07^{\prime \prime}$, long $81^{\circ} 30^{\prime} 17^{\prime \prime}$, Hydrologic Unit 03070203, in south corner of Greenwood Cemetery in Brunswick.

Owner: U.S. Geological Survey, test well 3.

AQUIFER.--Upper Floridan aquifer.

WELL CHARACTERISTICS.--Drilled observation well, diameter $4 \mathrm{in}$., depth 1,002 ft, cased to $823 \mathrm{ft}$, open hole. DATUM.--Elevation of 1 and-surface datum is $6.2 \mathrm{ft}$.

Measuring point: Floor of recorder shelter, $8.00 \mathrm{ft}$ above land-surface datum.

REMARKS.--Water levels for periods of missing record, February 5-9, March 28 to April 1, September 28-30, and November 21-23, were estimated. Well pumped and sampled semi-annually. Well flowed May 14-15. PERIOD OF RECORD.--August 1962 to current year.

EXTREMES FOR PERIOD OF RECORD.--Highest water leve1, $14.00 \mathrm{ft}$ above land-surface datum, 0ctober 9, 1962; 10west, $11.19 \mathrm{ft}$ below land-surface datum, July 14, 1977.

WATER LEVEL, IN FEET ABOVE OR BELOW (-) LAND SURFACE DATUM

CALENDAR YEAR JANUARY 1987 TO DECEMBER 1987 MEAN VALUES

\begin{tabular}{|c|c|c|c|c|c|c|c|c|c|c|c|c|}
\hline DAY & JAN & FEB & MAR & APR & MAY & JUN & JUL & AUG & SEP & ОСТ & NOV & DEC \\
\hline $\begin{array}{l}1 \\
2 \\
3 \\
4 \\
5\end{array}$ & $\begin{array}{l}-4.36 \\
-4.14 \\
-4.43 \\
-4.34 \\
-3.68\end{array}$ & $\begin{array}{l}-3.93 \\
-3.68 \\
-3.40 \\
-3.46 \\
-3.37\end{array}$ & $\begin{array}{l}-2.34 \\
-2.34 \\
-2.05 \\
-1.90 \\
-2.27\end{array}$ & $\begin{array}{l}-2.02 \\
-2.04 \\
-2.10 \\
-2.56 \\
-2.50\end{array}$ & $\begin{array}{l}-3.20 \\
-3.27 \\
-3.63 \\
-3.84 \\
-4.02\end{array}$ & $\begin{array}{l}-5.03 \\
-4.89 \\
-5.29 \\
-4.52 \\
-5.11\end{array}$ & $\begin{array}{l}-4.06 \\
-3.51 \\
-4.04 \\
-3.78 \\
-3.90\end{array}$ & $\begin{array}{l}-6.35 \\
-5.46 \\
-6.63 \\
-6.94 \\
-6.49\end{array}$ & $\begin{array}{l}-7.04 \\
-7.15 \\
-7.34 \\
-7.74 \\
-7.57\end{array}$ & $\begin{array}{l}-4.46 \\
-4.55 \\
-4.33 \\
-4.34 \\
-4.12\end{array}$ & $\begin{array}{r}2.24 \\
-0.22 \\
-1.70 \\
-0.95 \\
-1.76\end{array}$ & $\begin{array}{l}-3.34 \\
-3.76 \\
-3.53 \\
-3.29 \\
-3.34\end{array}$ \\
\hline $\begin{array}{r}6 \\
7 \\
8 \\
9 \\
10\end{array}$ & $\begin{array}{l}-3.81 \\
-3.81 \\
-3.79 \\
-4.16 \\
-3.79\end{array}$ & $\begin{array}{l}-3.29 \\
-3.21 \\
-3.12 \\
-3.04 \\
-2.96\end{array}$ & $\begin{array}{l}-2.52 \\
-1.86 \\
-2.12 \\
-2.10 \\
-2.21\end{array}$ & $\begin{array}{l}-2.22 \\
-2.33 \\
-2.82 \\
-2.19 \\
-2.76\end{array}$ & $\begin{array}{l}-4.18 \\
-3.93 \\
-3.94 \\
-3.88 \\
-2.99\end{array}$ & $\begin{array}{l}-5.58 \\
-5.85 \\
-6.00 \\
-6.10 \\
-6.04\end{array}$ & $\begin{array}{l}-4.38 \\
-4.82 \\
-6.04 \\
-5.79 \\
-6.12\end{array}$ & $\begin{array}{l}-6.71 \\
-7.28 \\
-7.59 \\
-7.09 \\
-6.59\end{array}$ & $\begin{array}{l}-7.49 \\
-7.39 \\
-7.30 \\
-7.12 \\
-7.34\end{array}$ & $\begin{array}{l}-4.38 \\
-4.74 \\
-5.21 \\
-5.84 \\
-5.97\end{array}$ & $\begin{array}{l}-1.39 \\
-1.34 \\
-1.07 \\
-0.89 \\
-1.56\end{array}$ & $\begin{array}{l}-3.56 \\
-3.08 \\
-3.05 \\
-3.02 \\
-2.62\end{array}$ \\
\hline $\begin{array}{l}11 \\
12 \\
13 \\
14 \\
15\end{array}$ & $\begin{array}{l}-3.81 \\
-3.79 \\
-3.55 \\
-3.96 \\
-3.31\end{array}$ & $\begin{array}{l}-3.56 \\
-3.00 \\
-3.18 \\
-3.60 \\
-3.61\end{array}$ & $\begin{array}{l}-2.70 \\
-2.20 \\
-2.71 \\
-2.39 \\
-2.64\end{array}$ & $\begin{array}{l}-2.66 \\
-2.68 \\
-2.82 \\
-2.36 \\
-2.64\end{array}$ & $\begin{array}{r}-1.16 \\
-0.98 \\
3.35 \\
--- \\
---\end{array}$ & $\begin{array}{l}-5.36 \\
-5.73 \\
-5.70 \\
-5.84 \\
-5.56\end{array}$ & $\begin{array}{l}-6.41 \\
-6.60 \\
-6.74 \\
-6.17 \\
-6.39\end{array}$ & $\begin{array}{l}-6.27 \\
-5.82 \\
-6.31 \\
-6.82 \\
-6.51\end{array}$ & $\begin{array}{l}-7.67 \\
-7.57 \\
-7.94 \\
-7.39 \\
-7.32\end{array}$ & $\begin{array}{l}-6.02 \\
-4.52 \\
-2.70 \\
-2.46 \\
-3.50\end{array}$ & $\begin{array}{l}-1.95 \\
-2.16 \\
-2.98 \\
-4.02 \\
-3.32\end{array}$ & $\begin{array}{l}-3.43 \\
-3.61 \\
-3.50 \\
-3.68 \\
-3.20\end{array}$ \\
\hline $\begin{array}{l}16 \\
17 \\
18 \\
19 \\
20\end{array}$ & $\begin{array}{l}-3.51 \\
-3.58 \\
-3.28 \\
-3.34 \\
-3.73\end{array}$ & $\begin{array}{l}-2.75 \\
-3.24 \\
-2.87 \\
-2.33 \\
-2.33\end{array}$ & $\begin{array}{l}-2.32 \\
-2.60 \\
-2.03 \\
-1.77 \\
-1.81\end{array}$ & $\begin{array}{l}-2.32 \\
-2.40 \\
-2.39 \\
-2.32 \\
-2.92\end{array}$ & $\begin{array}{r}3.35 \\
-1.32 \\
-1.78 \\
-2.92 \\
-3.43\end{array}$ & $\begin{array}{l}-5.62 \\
-5.28 \\
-5.20 \\
-5.70 \\
-5.26\end{array}$ & $\begin{array}{l}-7.36 \\
-6.12 \\
-6.59 \\
-5.83 \\
-6.59\end{array}$ & $\begin{array}{l}-7.26 \\
-6.84 \\
-6.83 \\
-6.90 \\
-6.62\end{array}$ & $\begin{array}{l}-6.44 \\
-6.88 \\
-6.75 \\
-7.56 \\
-7.73\end{array}$ & $\begin{array}{l}-4.78 \\
-4.78 \\
-5.12 \\
-4.97 \\
-4.55\end{array}$ & $\begin{array}{l}-2.70 \\
-2.94 \\
-2.53 \\
-2.50 \\
-2.73\end{array}$ & $\begin{array}{l}-4.20 \\
-3.85 \\
-3.87 \\
-3.72 \\
-3.04\end{array}$ \\
\hline $\begin{array}{l}21 \\
22 \\
23 \\
24 \\
25\end{array}$ & $\begin{array}{l}-3.69 \\
-2.97 \\
-3.64 \\
-3.92 \\
-3.53\end{array}$ & $\begin{array}{l}-2.62 \\
-2.99 \\
-2.42 \\
-2.59 \\
-2.11\end{array}$ & $\begin{array}{l}-2.25 \\
-2.30 \\
-1.89 \\
-2.14 \\
-2.40\end{array}$ & $\begin{array}{l}-3.08 \\
-3.16 \\
-3.00 \\
-3.05 \\
-3.32\end{array}$ & $\begin{array}{l}-1.57 \\
-0.78 \\
-3.90 \\
-4.17 \\
-4.60\end{array}$ & $\begin{array}{l}-4.96 \\
-5.13 \\
-5.14 \\
-4.37 \\
-3.85\end{array}$ & $\begin{array}{l}-7.43 \\
-7.36 \\
-7.29 \\
-7.97 \\
-8.28\end{array}$ & $\begin{array}{l}-6.84 \\
-7.20 \\
-6.69 \\
-7.02 \\
-7.14\end{array}$ & $\begin{array}{l}-7.26 \\
-6.82 \\
-6.46 \\
-4.62 \\
-3.78\end{array}$ & $\begin{array}{l}-4.44 \\
-4.24 \\
-4.90 \\
-5.44 \\
-5.94\end{array}$ & $\begin{array}{l}-2.59 \\
-3.27 \\
-3.37 \\
-3.39 \\
-3.26\end{array}$ & $\begin{array}{l}-2.49 \\
-2.61 \\
-2.93 \\
-3.01 \\
-3.47\end{array}$ \\
\hline $\begin{array}{l}26 \\
27 \\
28 \\
29 \\
30 \\
31\end{array}$ & $\begin{array}{l}-3.34 \\
-3.84 \\
-3.83 \\
-3.46 \\
-3.73 \\
-3.89\end{array}$ & $\begin{array}{r}-1.58 \\
-2.20 \\
-1.74 \\
-.- \\
--- \\
---\end{array}$ & $\begin{array}{l}-1.69 \\
-1.91 \\
-1.93 \\
-1.95 \\
-1.97 \\
-1.99\end{array}$ & $\begin{array}{r}-2.89 \\
-3.42 \\
-3.06 \\
-3.58 \\
-2.60 \\
-\ldots\end{array}$ & $\begin{array}{l}-4.15 \\
-4.07 \\
-3.97 \\
-4.81 \\
-4.78 \\
-5.03\end{array}$ & $\begin{array}{r}-3.51 \\
-3.56 \\
-3.70 \\
-3.92 \\
-3.99 \\
---\end{array}$ & $\begin{array}{l}-8.39 \\
-7.93 \\
-7.57 \\
-7.27 \\
-7.03 \\
-7.16\end{array}$ & $\begin{array}{l}-7.05 \\
-6.59 \\
-7.35 \\
-8.20 \\
-7.68 \\
-7.67\end{array}$ & $\begin{array}{r}-4.84 \\
-5.18 \\
-5.52 \\
-5.38 \\
-5.10 \\
. .-\end{array}$ & $\begin{array}{r}-4.98 \\
-3.42 \\
-3.10 \\
-4.12 \\
-2.84 \\
.65\end{array}$ & $\begin{array}{r}-2.93 \\
-2.93 \\
-2.82 \\
-2.80 \\
-3.14 \\
-.-\end{array}$ & $\begin{array}{l}-3.32 \\
-2.96 \\
-3.23 \\
-3.84 \\
-3.52 \\
-3.42\end{array}$ \\
\hline MEAN & -3.74 & -2.94 & -2.17 & -2.67 & -2.88 & -5.06 & -6.29 & -6.86 & -6.72 & -4.33 & -2.23 & -3.34 \\
\hline
\end{tabular}



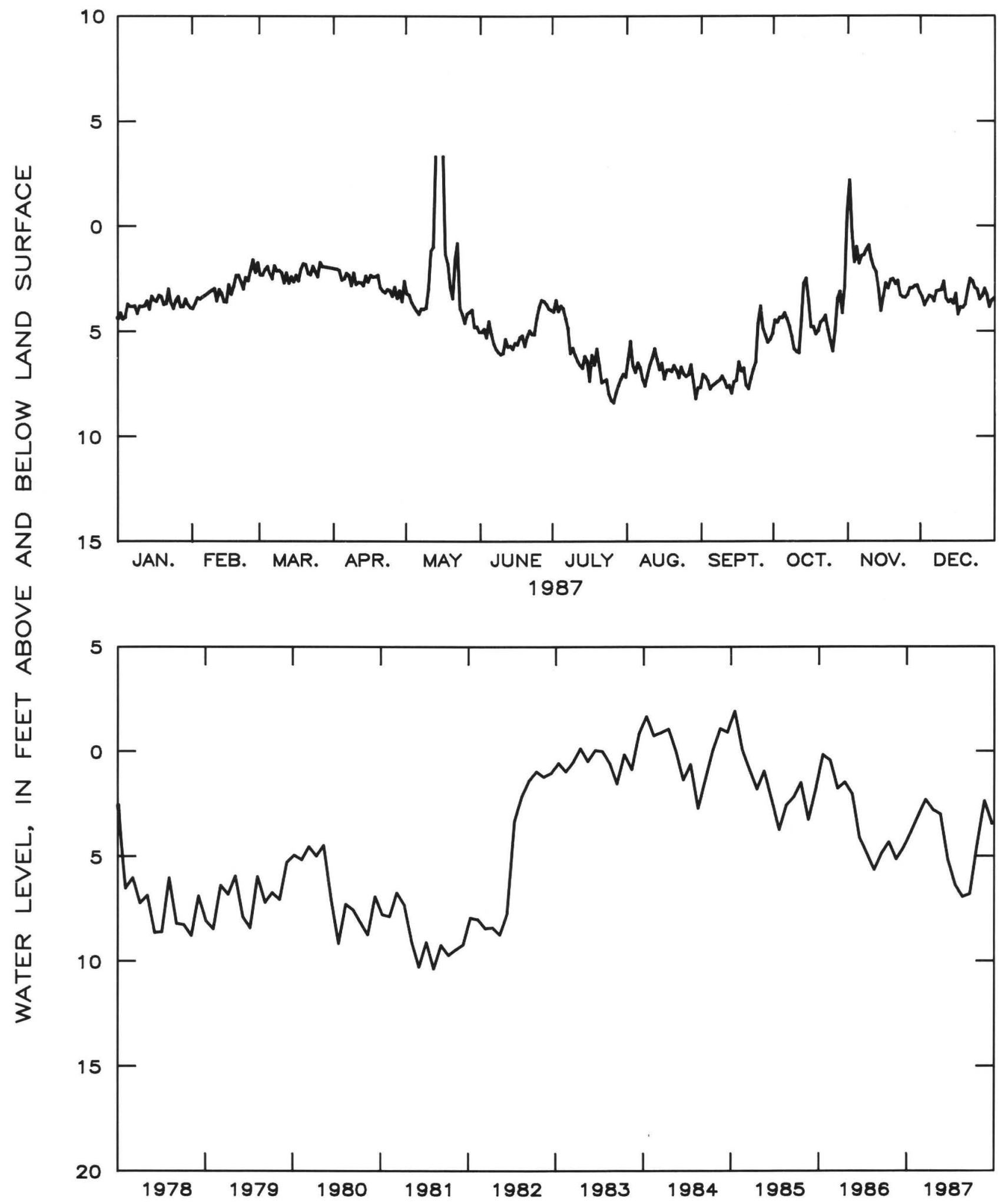

Figure 2.7.4.3-2.--Water level in observation well 33H127, lower water-bearing zone, Glynn County. 
311007081301702 Local number, 33 H133.

LOCATION.--Lat $31^{\circ} 10^{\prime} 07^{\prime \prime}$, long $81^{\circ} 30^{\prime} 17^{\prime \prime}$, Hydrologic Unit 03070203, in south corner of Greenwood Cemetery in Brunswick.

Owner: U.S. Geological Survey, test well 6.

AQUIFER.--Upper Floridan aquifer.

WELL CHARACTERISTICS.--Drilled observation well, diameter $4 \mathrm{in.,}$ depth $790 \mathrm{ft}$, cased to $520 \mathrm{ft}$, open hole. DATUM.--Elevation of land-surface datum is $6.7 \mathrm{ft}$.

Measuring point: Floor of recorder shelter, $5.1 \mathrm{ft}$ above land-surface datum.

REMARKS.--Wel1 pumped and sampled semi-annually. Borehole geophysical survey conducted September 26, 1977. Water levels for period of missing record, November 1-4, were estimated. Well flowed May 13-14.

PERIOD OF RECORD.--January 1963 to current year.

EXTREMES FOR PERIOD OF RECORD.---Highest water 1evel, $9.07 \mathrm{ft}$ above 1and-surface datum, December 26, 1965; 10west, $21.87 \mathrm{ft}$ below land-surface datum, July 22, 1977.

WATER LEVEL, IN FEET ABOVE OR BELOW (-) LAND SURFACE DATUM CALENDAR YEAR JANUARY 1987 TO DECEMBER 1987 MEAN VALUES

\begin{tabular}{|c|c|c|c|c|c|c|c|c|c|c|c|c|}
\hline DAY & JAN & FEB & MAR & APR & MAY & JUN & JUL & AUG & SEP & OCT & NOV & DEC \\
\hline $\begin{array}{l}1 \\
2 \\
3 \\
4 \\
5\end{array}$ & $\begin{array}{l}-14.35 \\
-13.75 \\
-14.28 \\
-14.64 \\
-13.51\end{array}$ & $\begin{array}{l}-13.86 \\
-13.69 \\
-12.82 \\
-12.54 \\
-11.36\end{array}$ & $\begin{array}{l}-11.83 \\
-11.64 \\
-11.49 \\
-10.94 \\
-11.30\end{array}$ & $\begin{array}{l}-11.58 \\
-11.63 \\
-11.58 \\
-12.18 \\
-12.11\end{array}$ & $\begin{array}{l}-12.53 \\
-12.77 \\
-13.14 \\
-13.36 \\
-13.78\end{array}$ & $\begin{array}{l}-14.94 \\
-14.86 \\
-15.12 \\
-14.25\end{array}$ & $\begin{array}{l}-13.06 \\
-11.96 \\
-13.06 \\
-12.74 \\
-12.93\end{array}$ & $\begin{array}{l}-16.41 \\
-15.59 \\
-17.04 \\
-17.36 \\
-16.69\end{array}$ & $\begin{array}{l}22 \\
54 \\
51 \\
20 \\
37\end{array}$ & $\begin{array}{l}-13.08 \\
-13.44 \\
-13.20 \\
-13.18 \\
-13.06\end{array}$ & $\begin{array}{l}-5.88 \\
-8.25 \\
-9.78 \\
-8.82 \\
-9.55\end{array}$ & $\begin{array}{l}-12.48 \\
-13.19 \\
-12.67 \\
-12.72 \\
-12.14\end{array}$ \\
\hline $\begin{array}{r}6 \\
7 \\
8 \\
9 \\
10\end{array}$ & $\begin{array}{l}-13.25 \\
-13.36 \\
-13.32 \\
-14.20 \\
-13.27\end{array}$ & $\begin{array}{l}-12.71 \\
-12.79 \\
-12.58 \\
-13.32 \\
-12.70\end{array}$ & $\begin{array}{l}-11.72 \\
-10.92 \\
-11.22 \\
-11.30 \\
-11.66\end{array}$ & $\begin{array}{l}-12.10 \\
-11.93 \\
-12.64 \\
-11.66 \\
-12.38\end{array}$ & $\begin{array}{l}-13.74 \\
-13.58 \\
-13.26 \\
-13.38 \\
-12.08\end{array}$ & $\begin{array}{l}-15.51 \\
-15.94 \\
-16.06 \\
-16.21\end{array}$ & & & & & $\begin{array}{r}-9.26 \\
-9.66 \\
-9.37 \\
-9.60 \\
-10.75\end{array}$ & $\begin{array}{l}-12.28 \\
-11.82 \\
-11.76 \\
-12.00 \\
-11.35\end{array}$ \\
\hline $\begin{array}{l}11 \\
12 \\
13 \\
14 \\
15\end{array}$ & $\begin{array}{l}-13.24 \\
-13.38 \\
-13.03 \\
-13.72 \\
-12.70\end{array}$ & $\begin{array}{l}-13.25 \\
-12.18 \\
-13.09 \\
-13.36 \\
-12.29\end{array}$ & $\begin{array}{l}-12.43 \\
-11.44 \\
-12.32 \\
-11.85 \\
-12.17\end{array}$ & $\begin{array}{l}-11.73 \\
-11.76 \\
-12.06 \\
-12.12 \\
-12.00\end{array}$ & $\begin{array}{r}-4.20 \\
1.70 \\
--- \\
-\cdots \\
1.70\end{array}$ & $\begin{array}{l}-15.27 \\
-15.16 \\
-15.62 \\
-15.68 \\
-15.42\end{array}$ & $\begin{array}{l}-16.46 \\
-17.02 \\
-16.50 \\
-17.83 \\
-17.10\end{array}$ & $\begin{array}{l}-16.05 \\
-16.30 \\
-16.10 \\
-16.72 \\
-16.24\end{array}$ & $\begin{array}{l}-18.17 \\
-18.04 \\
-18.33 \\
-17.36 \\
-17.53\end{array}$ & $\begin{array}{l}-15.40 \\
-13.10 \\
-10.75 \\
-10.40 \\
-11.72\end{array}$ & $\begin{array}{l}-11.21 \\
-11.56 \\
-12.99 \\
-14.30 \\
-12.98\end{array}$ & $\begin{array}{l}-12.18 \\
-12.36 \\
-12.20 \\
-12.78 \\
-12.35\end{array}$ \\
\hline $\begin{array}{l}16 \\
17 \\
18 \\
19 \\
20\end{array}$ & $\begin{array}{l}-13.16 \\
-13.23 \\
-12.79 \\
-12.81 \\
-13.45\end{array}$ & $\begin{array}{l}-12.18 \\
-12.53 \\
-12.10 \\
-11.34 \\
-12.10\end{array}$ & $\begin{array}{l}-11.19 \\
-12.50 \\
-11.34 \\
-10.91 \\
-11.08\end{array}$ & $\begin{array}{l}-11.34 \\
-11.52 \\
-11.33 \\
-11.90 \\
-12.54\end{array}$ & $\begin{array}{r}-2.00 \\
-7.30 \\
-11.25 \\
-12.85 \\
-12.95\end{array}$ & $\begin{array}{l}-15.55 \\
-14.80 \\
-14.58 \\
-15.38 \\
-14.69\end{array}$ & $\begin{array}{l}-15.57 \\
-16.95 \\
-16.86 \\
-17.84 \\
-17.60\end{array}$ & $\begin{array}{l}-17.32 \\
-16.86 \\
-16.79 \\
-16.98 \\
-16.80\end{array}$ & $\begin{array}{l}-16.26 \\
-16.77 \\
-17.31 \\
-17.80 \\
-18.06\end{array}$ & $\begin{array}{l}-12.92 \\
-12.27 \\
-11.68 \\
-11.90 \\
-12.68\end{array}$ & $\begin{array}{l}-12.14 \\
-12.33 \\
-11.99 \\
-11.83 \\
-12.05\end{array}$ & $\begin{array}{l}-13.36 \\
-12.89 \\
-12.69 \\
-12.62 \\
-11.47\end{array}$ \\
\hline $\begin{array}{l}21 \\
22 \\
23 \\
24 \\
25\end{array}$ & $\begin{array}{l}-13.27 \\
-12.09 \\
-13.22 \\
-13.71 \\
-13.20\end{array}$ & $\begin{array}{l}-12.52 \\
-12.63 \\
-11.89 \\
-12.08 \\
-11.58\end{array}$ & $\begin{array}{l}-11.65 \\
-11.68 \\
-11.31 \\
-11.37 \\
-11.83\end{array}$ & $\begin{array}{l}-12.63 \\
-12.54 \\
-12.18 \\
-12.40 \\
-13.11\end{array}$ & $\begin{array}{l}-12.00 \\
-11.35 \\
-13.40 \\
-13.40 \\
-14.20\end{array}$ & $\begin{array}{l}-14.50 \\
-14.00 \\
-13.39 \\
-13.04 \\
-12.66\end{array}$ & $\begin{array}{l}-17.62 \\
-17.38 \\
-17.79 \\
-18.64 \\
-18.83\end{array}$ & $\begin{array}{l}-17.22 \\
-17.35 \\
-16.55 \\
-17.02 \\
-17.30\end{array}$ & $\begin{array}{l}-17.37 \\
-16.40 \\
-15.86 \\
-14.96 \\
-14.85\end{array}$ & $\begin{array}{l}-12.78 \\
-13.09 \\
-11.99 \\
-11.35 \\
-10.80\end{array}$ & $\begin{array}{l}-11.96 \\
-12.68 \\
-12.82 \\
-12.89 \\
-12.38\end{array}$ & $\begin{array}{l}-10.80 \\
-10.97 \\
-11.48 \\
-11.39 \\
-11.86\end{array}$ \\
\hline $\begin{array}{l}26 \\
27 \\
28 \\
29 \\
30 \\
31\end{array}$ & $\begin{array}{l}-12.85 \\
-13.52 \\
-13.33 \\
-12.99 \\
-13.58 \\
-13.76\end{array}$ & $\begin{array}{r}-10.64 \\
-11.52 \\
-11.42 \\
--- \\
---\end{array}$ & $\begin{array}{l}-10.40 \\
-10.59 \\
-10.70 \\
-11.29 \\
-10.58 \\
-11.38\end{array}$ & $\begin{array}{r}-12.68 \\
-13.10 \\
-12.80 \\
-13.50 \\
-11.70 \\
---\end{array}$ & $\begin{array}{l}-14.05 \\
-13.96 \\
-13.52 \\
-14.76 \\
-14.77 \\
-15.03\end{array}$ & $\begin{array}{r}-12.44 \\
-12.54 \\
-12.77 \\
-12.98 \\
-13.02 \\
-.--\end{array}$ & $\begin{array}{l}-18.71 \\
-18.89 \\
-18.39 \\
-17.55 \\
-17.20 \\
-17.11\end{array}$ & $\begin{array}{l}-17.45 \\
-16.96 \\
-18.05 \\
-18.95 \\
-18.44 \\
-18.28\end{array}$ & $\begin{array}{r}-13.51 \\
-13.66 \\
-13.84 \\
-14.59 \\
-14.02 \\
---\end{array}$ & $\begin{array}{l}-11.96 \\
-11.09 \\
-11.33 \\
-12.39 \\
-11.12 \\
-7.75\end{array}$ & $\begin{array}{r}-11.98 \\
-11.86 \\
-11.70 \\
-12.07 \\
-12.50 \\
---\end{array}$ & $\begin{array}{l}-11.96 \\
-11.76 \\
-11.95 \\
-12.77 \\
-12.50 \\
-12.41\end{array}$ \\
\hline EAN & -13.39 & -12.40 & -11.42 & -12.16 & -11.09 & -14.58 & -16.43 & -17.06 & -16.72 & -12.47 & -11.24 & -12.1 \\
\hline
\end{tabular}



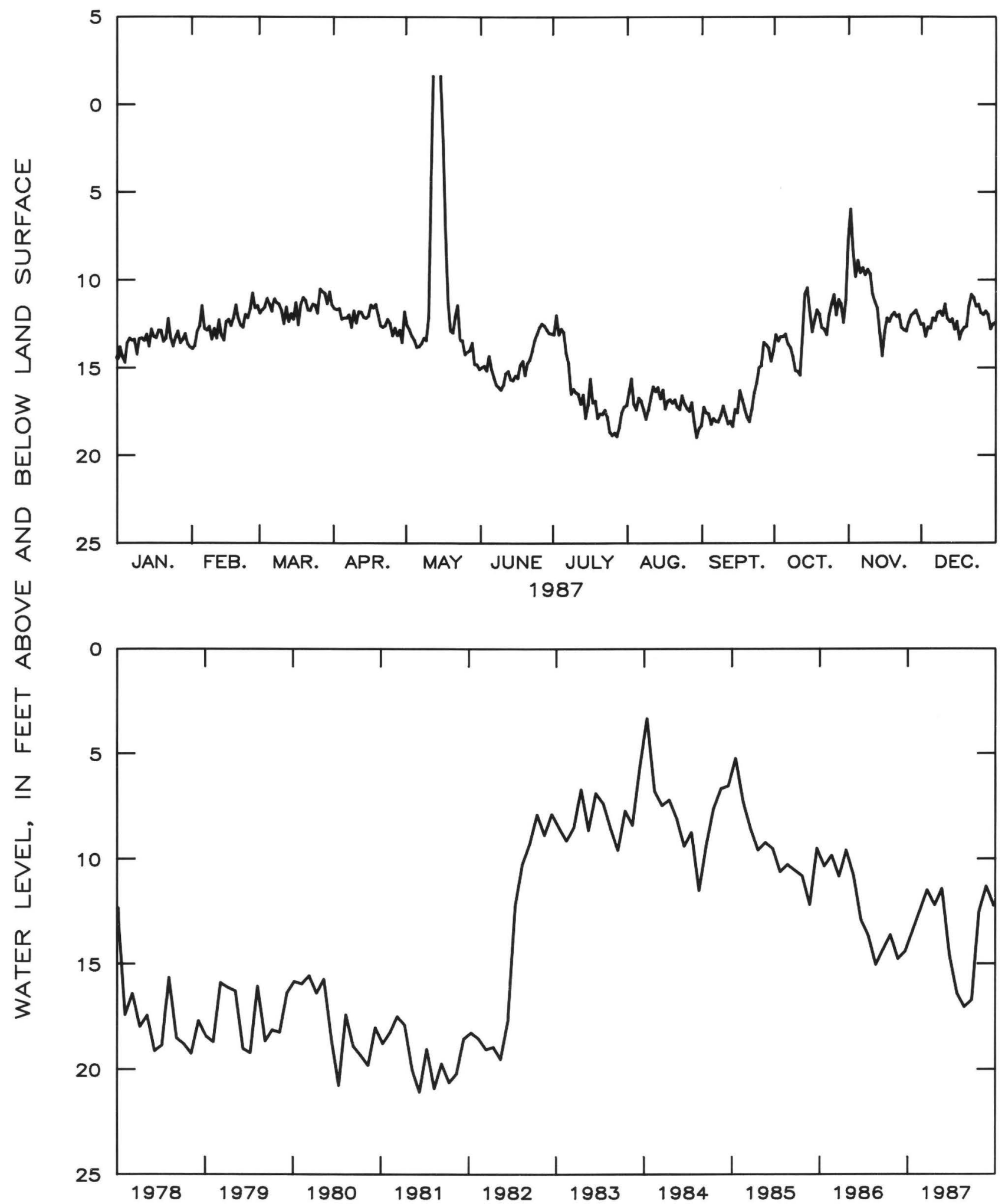

Figure 2.7.4.3-3.--Water level in observation well $33 \mathrm{H} 133$, upper water-bearing zone, Glynn County. 
310818081294201 Local number, $34 \mathrm{H} 391$.

LOCATION.--Lat $31^{\circ} 08^{\prime} 18^{\prime \prime}$, long $81^{\circ} 29^{\prime} 42^{\prime \prime}$, Hydrologic Unit 03070203, located near intersection of Albermarle Street and Bay Street in Brunswick.

Owner: U.S. Geological Survey, test well 16.

AQUIFER.--Lower Floridan aquifer.

WELL CHARACTERISTICS.--Drilled observation well, diameter $6 \mathrm{in}$., depth $1,150 \mathrm{ft}$, cased to 1,070 ft, open hole. DATUM.--Elevation of land-surface datum is $7.13 \mathrm{ft}$.

Measuring point: Floor of recorder shelter $12.5 \mathrm{ft}$ above land-surface datum.

REMARKS.--Well pumped and sampled semi-annually. Water levels for periods of missing record, April 10-27, May 14-15, and September 24 to October 13, were estimated.

PERIOD OF RECORD.--August 1975 to current year.

EXTREMES FOR PERIOD OF RECORD.---Highest water level, $11.65 \mathrm{ft}$ above land-surface datum, October 13-14, 1985; lowest, $2.96 \mathrm{ft}$ below land-surface datum, July 27, 1977.

WATER LEVEL, IN FEET ABOVE LAND SURFACE DATUM CALENDAR YEAR JANUARY 1987 TO DECEMBER 1987 MEAN VALUES

\begin{tabular}{|c|c|c|c|c|c|c|c|c|c|c|c|c|}
\hline DAY & JAN & FEB & MAR & APR & MAY & JUN & JUL & AUG & SEP & ОСТ & NOV & DE \\
\hline $\begin{array}{l}1 \\
2 \\
3 \\
4 \\
5\end{array}$ & $\begin{array}{l}4.08 \\
3.91 \\
3.88 \\
4.13 \\
4.28\end{array}$ & $\begin{array}{l}4.53 \\
4.68 \\
4.68 \\
4.79 \\
4.98\end{array}$ & $\begin{array}{l}5.86 \\
5.71 \\
5.65 \\
5.75 \\
5.58\end{array}$ & $\begin{array}{l}5.91 \\
5.96 \\
6.02 \\
5.85 \\
5.85\end{array}$ & $\begin{array}{l}5.08 \\
5.02 \\
4.85 \\
4.71 \\
4.47\end{array}$ & $\begin{array}{l}3.15 \\
3.10 \\
3.00 \\
3.08 \\
3.10\end{array}$ & $\begin{array}{l}3.66 \\
3.81 \\
3.75 \\
3.77 \\
3.90\end{array}$ & $\begin{array}{l}1.50 \\
1.89 \\
1.84 \\
1.40 \\
1.50\end{array}$ & $\begin{array}{l}1.12 \\
1.26 \\
1.32 \\
1.41 \\
1.52\end{array}$ & $\begin{array}{l}3.51 \\
3.63 \\
3.81 \\
3.95 \\
4.02\end{array}$ & $\begin{array}{l}6.88 \\
6.65 \\
5.83 \\
5.89 \\
5.89\end{array}$ & $\begin{array}{l}4.4 \\
4.1 \\
4.1 \\
4.2 \\
4.2\end{array}$ \\
\hline $\begin{array}{r}6 \\
7 \\
8 \\
9 \\
10\end{array}$ & $\begin{array}{l}4.18 \\
4.19 \\
4.22 \\
4.18 \\
4.43\end{array}$ & $\begin{array}{l}5.15 \\
5.19 \\
5.24 \\
4.88 \\
4.85\end{array}$ & $\begin{array}{l}5.52 \\
5.94 \\
6.05 \\
5.95 \\
5.81\end{array}$ & $\begin{array}{l}5.93 \\
5.90 \\
5.75 \\
5.78 \\
5.36\end{array}$ & $\begin{array}{l}4.43 \\
4.45 \\
4.48 \\
4.52 \\
4.51\end{array}$ & $\begin{array}{l}2.99 \\
2.79 \\
2.62 \\
2.48 \\
2.45\end{array}$ & $\begin{array}{l}3.58 \\
3.25 \\
2.53 \\
2.16 \\
2.01\end{array}$ & $\begin{array}{l}1.35 \\
1.20 \\
1.08 \\
1.14 \\
1.27\end{array}$ & $\begin{array}{l}1.48 \\
1.57 \\
1.45 \\
1.48 \\
1.40\end{array}$ & $\begin{array}{l}3.96 \\
3.74 \\
3.41 \\
3.15 \\
3.32\end{array}$ & $\begin{array}{l}5.67 \\
5.57 \\
5.65 \\
5.73 \\
5.39\end{array}$ & 4.5 \\
\hline $\begin{array}{l}11 \\
12 \\
13 \\
14 \\
15\end{array}$ & $\begin{array}{l}4.37 \\
4.34 \\
4.42 \\
4.38 \\
4.52\end{array}$ & $\begin{array}{l}4.79 \\
4.98 \\
5.04 \\
5.00 \\
5.04\end{array}$ & $\begin{array}{l}5.43 \\
5.49 \\
5.51 \\
5.54 \\
5.58\end{array}$ & $\begin{array}{l}5.78 \\
5.73 \\
5.68 \\
5.37 \\
5.78\end{array}$ & $\begin{array}{l}5.48 \\
6.30 \\
5.62 \\
8.44 \\
8.80\end{array}$ & $\begin{array}{l}2.58 \\
2.57 \\
2.52 \\
2.60 \\
2.66\end{array}$ & $\begin{array}{l}1.86 \\
1.87 \\
1.85 \\
1.48 \\
1.44\end{array}$ & $\begin{array}{l}1.63 \\
1.71 \\
1.79 \\
1.57 \\
1.64\end{array}$ & $\begin{array}{l}1.23 \\
1.23 \\
1.22 \\
1.36 \\
1.53\end{array}$ & $\begin{array}{l}2.87 \\
2.66 \\
3.46 \\
3.96 \\
4.04\end{array}$ & $\begin{array}{l}5.02 \\
4.83 \\
4.47 \\
3.98 \\
4.06\end{array}$ & 4.3 \\
\hline $\begin{array}{l}16 \\
17 \\
18 \\
19 \\
20\end{array}$ & $\begin{array}{l}4.64 \\
4.68 \\
4.83 \\
4.81 \\
4.55\end{array}$ & $\begin{array}{l}5.46 \\
5.11 \\
5.21 \\
5.29 \\
5.35\end{array}$ & $\begin{array}{l}5.61 \\
5.56 \\
5.77 \\
6.03 \\
6.08\end{array}$ & $\begin{array}{l}6.17 \\
6.14 \\
6.31 \\
5.94 \\
5.49\end{array}$ & $\begin{array}{l}8.84 \\
7.45 \\
5.96 \\
5.10 \\
4.67\end{array}$ & $\begin{array}{l}2.67 \\
2.72 \\
2.91 \\
2.78 \\
2.84\end{array}$ & $\begin{array}{l}1.69 \\
1.58 \\
1.55 \\
1.40 \\
1.21\end{array}$ & $\begin{array}{l}1.61 \\
1.65 \\
1.68 \\
1.64 \\
1.59\end{array}$ & $\begin{array}{l}1.64 \\
1.82 \\
1.64 \\
1.52 \\
1.52\end{array}$ & $\begin{array}{l}3.60 \\
3.33 \\
3.16 \\
3.06 \\
3.17\end{array}$ & $\begin{array}{l}4.22 \\
4.44 \\
4.42 \\
4.57 \\
4.67\end{array}$ & 4.5 \\
\hline $\begin{array}{l}21 \\
22 \\
23 \\
24 \\
25\end{array}$ & $\begin{array}{l}4.62 \\
4.98 \\
4.57 \\
4.39 \\
4.76\end{array}$ & $\begin{array}{l}5.43 \\
5.49 \\
5.40 \\
5.39 \\
5.53\end{array}$ & $\begin{array}{l}6.07 \\
6.03 \\
6.06 \\
6.10 \\
5.98\end{array}$ & $\begin{array}{l}5.37 \\
5.37 \\
5.81 \\
5.61 \\
5.15\end{array}$ & $\begin{array}{l}4.37 \\
4.86 \\
4.41 \\
4.18 \\
3.89\end{array}$ & $\begin{array}{l}3.09 \\
3.29 \\
3.39 \\
3.73 \\
3.86\end{array}$ & $\begin{array}{r}1.24 \\
1.17 \\
1.24 \\
.91 \\
.68\end{array}$ & $\begin{array}{l}1.58 \\
1.48 \\
1.73 \\
1.65 \\
1.43\end{array}$ & $\begin{array}{l}1.46 \\
1.70 \\
1.89 \\
2.25 \\
2.40\end{array}$ & $\begin{array}{l}3.22 \\
3.30 \\
3.03 \\
2.92 \\
2.85\end{array}$ & $\begin{array}{l}4.51 \\
4.43 \\
4.14 \\
4.15 \\
4.06\end{array}$ & $\begin{array}{l}4.8 \\
4.9 \\
4.8\end{array}$ \\
\hline $\begin{array}{l}26 \\
27 \\
28 \\
29 \\
30 \\
31\end{array}$ & $\begin{array}{l}4.76 \\
4.53 \\
4.47 \\
4.47 \\
4.59 \\
4.51\end{array}$ & $\begin{array}{r}5.85 \\
5.92 \\
5.97 \\
--- \\
-- \\
---\end{array}$ & $\begin{array}{l}6.16 \\
6.35 \\
6.37 \\
6.29 \\
6.42 \\
6.06\end{array}$ & $\begin{array}{c}5.42 \\
5.19 \\
5.26 \\
4.99 \\
5.13 \\
---\end{array}$ & $\begin{array}{l}3.64 \\
3.57 \\
3.70 \\
3.46 \\
3.38 \\
3.27\end{array}$ & $\begin{array}{r}4.04 \\
4.07 \\
4.05 \\
3.90 \\
3.73 \\
-.-\end{array}$ & $\begin{array}{r}.70 \\
.58 \\
.57 \\
.83 \\
1.02 \\
1.16\end{array}$ & $\begin{array}{r}1.23 \\
1.40 \\
1.13 \\
.85 \\
.83 \\
.91\end{array}$ & $\begin{array}{c}2.80 \\
3.25 \\
3.28 \\
3.13 \\
3.26 \\
-.-\end{array}$ & $\begin{array}{l}3.21 \\
3.80 \\
4.13 \\
3.84 \\
3.82 \\
5.38\end{array}$ & $\begin{array}{c}4.47 \\
4.54 \\
4.75 \\
4.74 \\
4.64 \\
---\end{array}$ & $\begin{array}{l}4.8 \\
4.8 \\
4.5 \\
4.5 \\
4.5 \\
4.5\end{array}$ \\
\hline MEAN & 4.44 & 5.19 & 5.88 & 5.67 & 5.03 & 3.09 & 1.89 & 1.45 & 1.80 & 3.53 & 4.94 & \\
\hline CAL Y & & & & MAX & 8.84 & & & & & & & \\
\hline
\end{tabular}



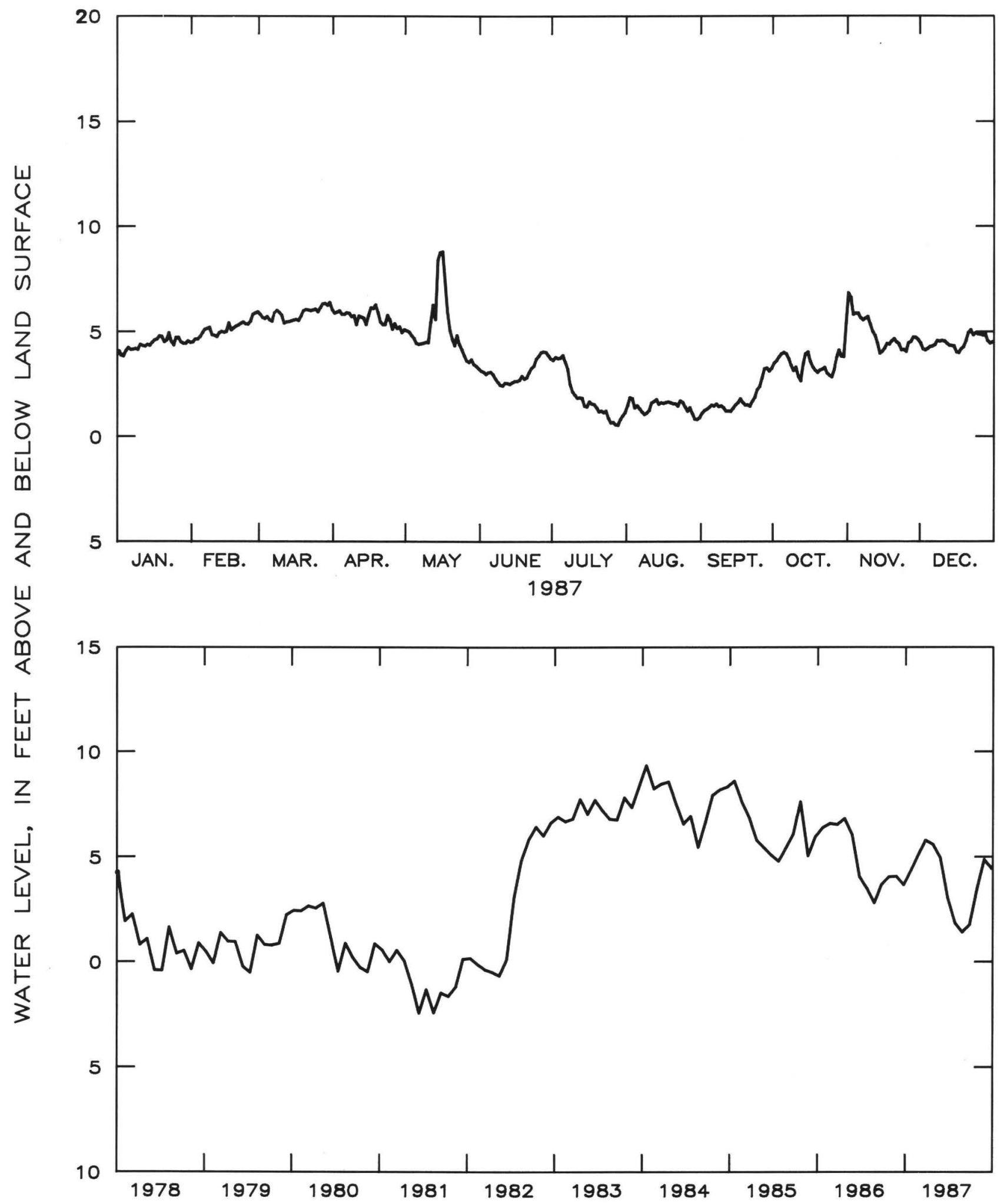

Figure 2.7.4.3-4.--Water level in observation well 34H391, brackish water zone, Glynn County. 
311633081324001 Local number, 33 J044.

LOCATION.--Lat $31^{\circ} 16^{\prime} 33^{\prime \prime}$, long $81^{\circ} 32^{\prime} 40^{\prime \prime}$, Hydrologic Unit $03070203,1.2$ mi east of Sterling, off State Highway 99 at the Brunswick Pulp and Paper Company, Sterling Wood Products Division.

Owner: Brunswick Pulp and Paper Co., USGS test well 27.

AQUIFER.--Lower Floridan aquifer.

WELL CHARACTERISTICS.--Drilled unused oil-test well converted to observation well, diameter 9 in., depth 2,260 $\mathrm{ft}$, cased to $1,079 \mathrm{ft}$, open hole.

DATUM.--Elevation of 1 and-surface datum is $20 \mathrm{ft}$.

Measuring point: Floor of recorder shelter, $9.5 \mathrm{ft}$ above land-surface datum.

REMARKS.--This is the Sterling oil-test well. Water levels for period of missing record, July 1-2, were estimated.

PERIOD OF RECORD.--May 1979 to current year.

EXTREMES FOR PERIOD OF RECORD.--Highest water level, $3.09 \mathrm{ft}$ above land-surface datum, October 13, 1985; 10west, $6.30 \mathrm{ft}$ below land-surface datum, August 11, 1981.

WATER LEVEL, IN FEET ABOVE OR BELOW (-) LAND SURFACE DATUM

CALENDAR YEAR JANUARY 1987 TO DECEMBER

1987

MEAN VALUES

\begin{tabular}{|c|c|c|c|c|c|c|c|c|c|c|c|c|}
\hline DAY & JAN & FEB & MAR & APR & MAY & JUN & JUL & AUG & SEP & ОСТ & NOV & DEC \\
\hline $\begin{array}{l}1 \\
2 \\
3 \\
4 \\
5\end{array}$ & $\begin{array}{l}-2.22 \\
-2.42 \\
-2.50 \\
-2.18 \\
-2.19\end{array}$ & $\begin{array}{l}-1.77 \\
-1.60 \\
-1.66 \\
-1.71 \\
-1.65\end{array}$ & $\begin{array}{l}-0.54 \\
-0.74 \\
-0.82 \\
-0.84 \\
-0.94\end{array}$ & $\begin{array}{l}-0.39 \\
-0.36 \\
-0.24 \\
-0.34 \\
-0.33\end{array}$ & $\begin{array}{l}-0.86 \\
-0.90 \\
-1.00 \\
-1.12 \\
-1.28\end{array}$ & $\begin{array}{l}-1.95 \\
-2.04 \\
-2.16 \\
-2.22 \\
-2.24\end{array}$ & $\begin{array}{l}-2.19 \\
-2.20 \\
-2.22 \\
-2.17 \\
-2.22\end{array}$ & $\begin{array}{l}-4.05 \\
-3.86 \\
-3.74 \\
-3.79 \\
-3.86\end{array}$ & $\begin{array}{l}-4.35 \\
-4.23 \\
-4.20 \\
-4.14 \\
-4.08\end{array}$ & $\begin{array}{l}-3.26 \\
-3.20 \\
-3.16 \\
-3.15 \\
-3.00\end{array}$ & $\begin{array}{l}-1.54 \\
-1.00 \\
-0.95 \\
-0.94 \\
-1.00\end{array}$ & $\begin{array}{l}-1.91 \\
-2.09 \\
-2.12 \\
-2.08 \\
-2.24\end{array}$ \\
\hline $\begin{array}{r}6 \\
7 \\
8 \\
9 \\
10\end{array}$ & $\begin{array}{l}-2.39 \\
-2.22 \\
-2.19 \\
-2.16 \\
-2.03\end{array}$ & $\begin{array}{l}-1.40 \\
-1.26 \\
-1.25 \\
-1.56 \\
-1.64\end{array}$ & $\begin{array}{l}-0.95 \\
-0.67 \\
-0.42 \\
-0.40 \\
-0.58\end{array}$ & $\begin{array}{l}-0.32 \\
-0.30 \\
-0.36 \\
-0.36 \\
-0.34\end{array}$ & $\begin{array}{l}-1.35 \\
-1.36 \\
-1.38 \\
-1.54 \\
-1.50\end{array}$ & $\begin{array}{l}-2.33 \\
-2.47 \\
-2.59 \\
-2.66 \\
-2.73\end{array}$ & $\begin{array}{l}-2.30 \\
-2.42 \\
-2.74 \\
-2.90 \\
-3.00\end{array}$ & $\begin{array}{l}-3.92 \\
-4.00 \\
-4.06 \\
-4.14 \\
-4.13\end{array}$ & $\begin{array}{l}-4.09 \\
-4.02 \\
-4.08 \\
-4.12 \\
-4.14\end{array}$ & $\begin{array}{l}-2.90 \\
-2.95 \\
-3.15 \\
-3.38 \\
-3.44\end{array}$ & $\begin{array}{l}-1.30 \\
-1.41 \\
-1.38 \\
-1.24 \\
-1.15\end{array}$ & $\begin{array}{l}-2.25 \\
-2.22 \\
-2.08 \\
-1.96 \\
-1.92\end{array}$ \\
\hline $\begin{array}{l}11 \\
12 \\
13 \\
14 \\
15\end{array}$ & $\begin{array}{l}-2.08 \\
-2.09 \\
-2.04 \\
-2.03 \\
-1.96\end{array}$ & $\begin{array}{l}-1.58 \\
-1.44 \\
-1.36 \\
-1.34 \\
-1.30\end{array}$ & $\begin{array}{l}-0.81 \\
-0.84 \\
-0.76 \\
-0.75 \\
-0.70\end{array}$ & $\begin{array}{l}-0.42 \\
-0.48 \\
-0.53 \\
-0.51 \\
-0.32\end{array}$ & $\begin{array}{r}-1.20 \\
-0.67 \\
.01 \\
.90 \\
1.60\end{array}$ & $\begin{array}{l}-2.77 \\
-2.75 \\
-2.77 \\
-2.74 \\
-2.74\end{array}$ & $\begin{array}{l}-3.11 \\
-3.17 \\
-3.27 \\
-3.47 \\
-3.60\end{array}$ & $\begin{array}{l}-4.07 \\
-3.93 \\
-3.84 \\
-3.78 \\
-3.84\end{array}$ & $\begin{array}{l}-4.08 \\
-4.22 \\
-4.24 \\
-4.26 \\
-4.20\end{array}$ & $\begin{array}{l}-3.36 \\
-3.23 \\
-3.05 \\
-2.84 \\
-2.60\end{array}$ & $\begin{array}{l}-1.43 \\
-1.62 \\
-1.76 \\
-2.02 \\
-2.21\end{array}$ & $\begin{array}{l}-1.85 \\
-1.93 \\
-2.12 \\
-2.15 \\
-2.06\end{array}$ \\
\hline $\begin{array}{l}16 \\
17 \\
18 \\
19 \\
20\end{array}$ & $\begin{array}{l}-1.82 \\
-1.82 \\
-1.70 \\
-1.65 \\
-1.79\end{array}$ & $\begin{array}{l}-1.06 \\
-1.20 \\
-1.28 \\
-1.34 \\
-1.24\end{array}$ & $\begin{array}{l}-0.68 \\
-0.70 \\
-0.50 \\
-0.43 \\
-0.44\end{array}$ & $\begin{array}{l}-0.24 \\
-0.20 \\
-0.28 \\
-0.39 \\
-0.49\end{array}$ & $\begin{array}{r}1.76 \\
1.44 \\
.79 \\
.20 \\
-0.22\end{array}$ & $\begin{array}{l}-2.73 \\
-2.78 \\
-2.76 \\
-2.73 \\
-2.72\end{array}$ & $\begin{array}{l}-3.64 \\
-3.70 \\
-3.72 \\
-3.76 \\
-3.89\end{array}$ & $\begin{array}{l}-3.86 \\
-3.86 \\
-3.88 \\
-3.86 \\
-3.87\end{array}$ & $\begin{array}{l}-4.17 \\
-4.02 \\
-4.00 \\
-4.06 \\
-4.12\end{array}$ & $\begin{array}{l}-2.64 \\
-2.74 \\
-2.84 \\
-2.96 \\
-2.93\end{array}$ & $\begin{array}{l}-2.18 \\
-2.03 \\
-2.03 \\
-1.98 \\
-1.86\end{array}$ & $\begin{array}{l}-2.28 \\
-2.40 \\
-2.36 \\
-2.32 \\
-2.20\end{array}$ \\
\hline $\begin{array}{l}21 \\
22 \\
23 \\
24 \\
25\end{array}$ & $\begin{array}{l}-1.71 \\
-1.47 \\
-1.78 \\
-1.92 \\
-1.73\end{array}$ & $\begin{array}{l}-1.08 \\
-0.94 \\
-1.06 \\
-1.12 \\
-1.06\end{array}$ & $\begin{array}{l}-0.34 \\
-0.37 \\
-0.44 \\
-0.40 \\
-0.39\end{array}$ & $\begin{array}{l}-0.55 \\
-0.62 \\
-0.68 \\
-0.56 \\
-0.74\end{array}$ & $\begin{array}{l}-0.60 \\
-0.68 \\
-0.82 \\
-1.01 \\
-1.20\end{array}$ & $\begin{array}{l}-2.66 \\
-2.58 \\
-2.47 \\
-2.31 \\
-2.20\end{array}$ & $\begin{array}{l}-3.92 \\
-3.94 \\
-3.92 \\
-4.08 \\
-4.22\end{array}$ & $\begin{array}{l}-3.92 \\
-4.00 \\
-4.03 \\
-4.02 \\
-4.02\end{array}$ & $\begin{array}{l}-4.24 \\
-4.20 \\
-4.07 \\
-3.90 \\
-3.81\end{array}$ & $\begin{array}{l}-2.94 \\
-3.02 \\
-3.02 \\
-3.05 \\
-3.05\end{array}$ & $\begin{array}{l}-1.97 \\
-2.01 \\
-2.18 \\
-2.24 \\
-2.20\end{array}$ & $\begin{array}{l}-2.01 \\
-1.84 \\
-1.95 \\
-1.92 \\
-1.89\end{array}$ \\
\hline $\begin{array}{l}26 \\
27 \\
28 \\
29 \\
30 \\
31\end{array}$ & $\begin{array}{l}-1.68 \\
-1.79 \\
-1.90 \\
-1.92 \\
-1.73 \\
-1.74\end{array}$ & $\begin{array}{r}-1.00 \\
-0.77 \\
-0.61 \\
--- \\
--- \\
---\end{array}$ & $\begin{array}{l}-0.36 \\
-0.22 \\
-0.19 \\
-0.22 \\
-0.01 \\
-0.10\end{array}$ & $\begin{array}{r}-0.86 \\
-0.84 \\
-0.81 \\
-0.88 \\
-0.90 \\
---\end{array}$ & $\begin{array}{l}-1.40 \\
-1.54 \\
-1.58 \\
-1.64 \\
-1.74 \\
-1.84\end{array}$ & $\begin{array}{r}-2.08 \\
-2.03 \\
-2.05 \\
-2.14 \\
-2.17 \\
-.-\end{array}$ & $\begin{array}{l}-4.23 \\
-4.30 \\
-4.32 \\
-4.30 \\
-4.28 \\
-4.21\end{array}$ & $\begin{array}{l}-4.10 \\
-4.14 \\
-4.14 \\
-4.27 \\
-4.41 \\
-4.39\end{array}$ & $\begin{array}{r}-3.71 \\
-3.54 \\
-3.44 \\
-3.34 \\
-3.21 \\
---\end{array}$ & $\begin{array}{l}-2.97 \\
-2.74 \\
-2.51 \\
-2.66 \\
-2.72 \\
-2.35\end{array}$ & $\begin{array}{r}-2.04 \\
-1.95 \\
-1.86 \\
-1.78 \\
-1.75 \\
---\end{array}$ & $\begin{array}{l}-1.92 \\
-1.98 \\
-1.92 \\
-2.12 \\
-2.34 \\
-2.34\end{array}$ \\
\hline MEAN & -1.96 & -1.30 & -0.53 & -0.49 & -0.70 & -2.45 & -3.40 & -3.99 & -4.01 & -2.96 & -1.70 & -2 \\
\hline CAL & & & & MAX & 1.76 & & & & & & & \\
\hline
\end{tabular}



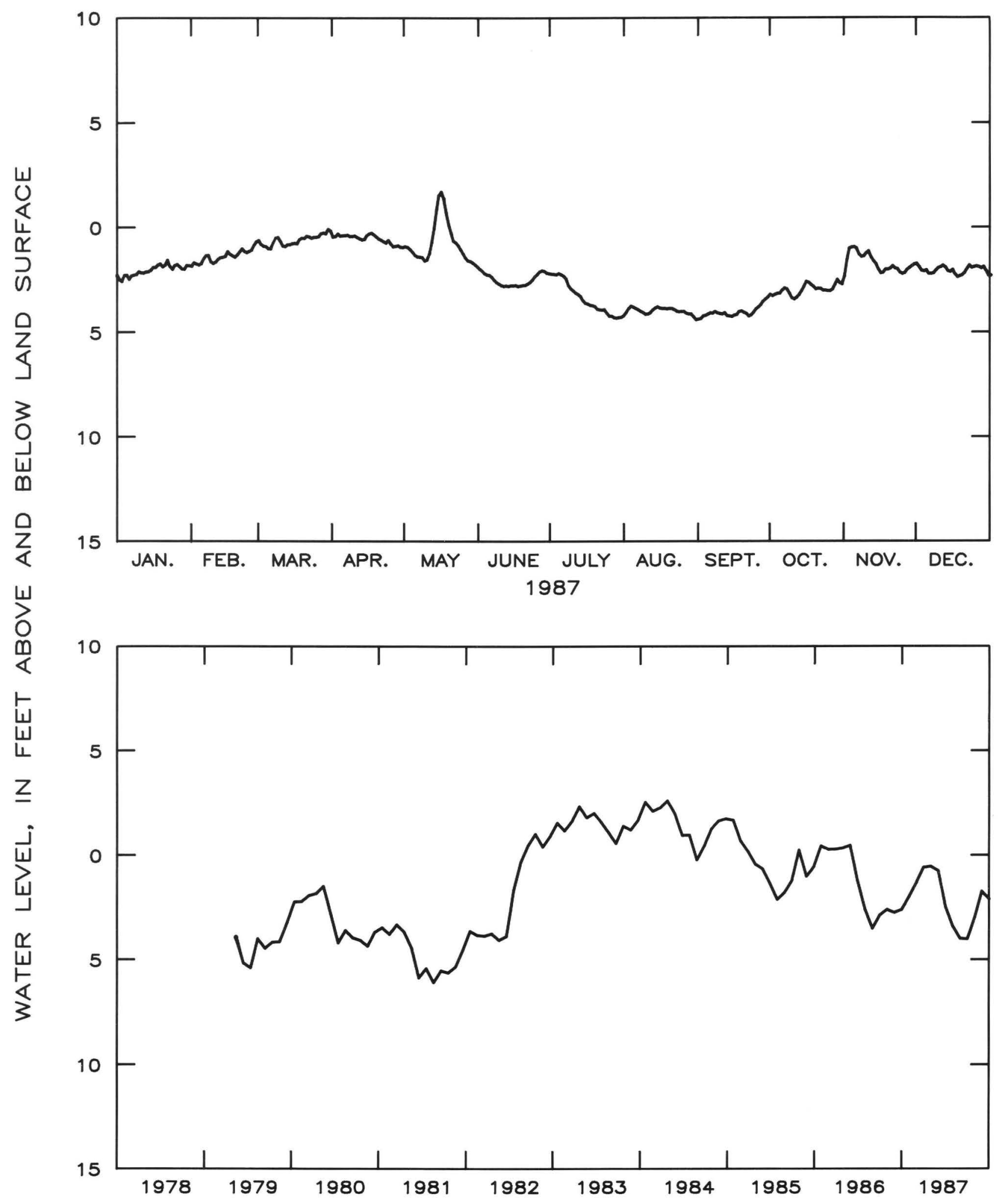

Figure 2.7.4.3-5.--Water level in observation well 33J044, brackish water zone, Glynn County. 


\subsubsection{Kings Bay-Okefenokee Swamp area}

The water level in the Upper Floridan aquifer in the Kings Bay area is affected by industrial pumpage of about $32 \mathrm{Mgal} / \mathrm{d}$ (Turlington and others, 1987) at St Marys and about 37 Mgal/d (Marella, 1986) at Fernandina Beach, Fla. Pumping in these areas has resulted in the formation of a cone of depression centered at Fernandina Beach, Fla., and to a lesser extent at St Marys, Ga.

The mean water level in well 33E027 at Kings Bay was $0.4 \mathrm{ft}$ lower in 1987 than in 1986. Although the mean water level was lower in 1987 than in 1986, the water level in the well had recovered $5.2 \mathrm{ft}$ by the end of April from the low measured during the 1986 drought. At the end of 1987, the water level was $1.7 \mathrm{ft}$ lower than at the end of 1986. These declines continue the downward trend in the area since 1985.

The water table in the Okefenokee Swamp fluctuates seasonally in response to rainfall and evapotranspiration. This fluctuation probably affects the water level in the underlying confined Upper Floridan aquifer (Callahan, 1964). The mean water level at well 27E004 in western Charlton County was about the same in 1987 as in 1986, but by the end of April, the daily mean was level had recovered $6.0 \mathrm{ft}$ from the low measured during the 1986 drought. At the end of 1987, the water level was $1.3 \mathrm{ft}$ lower than at the end of 1986. 


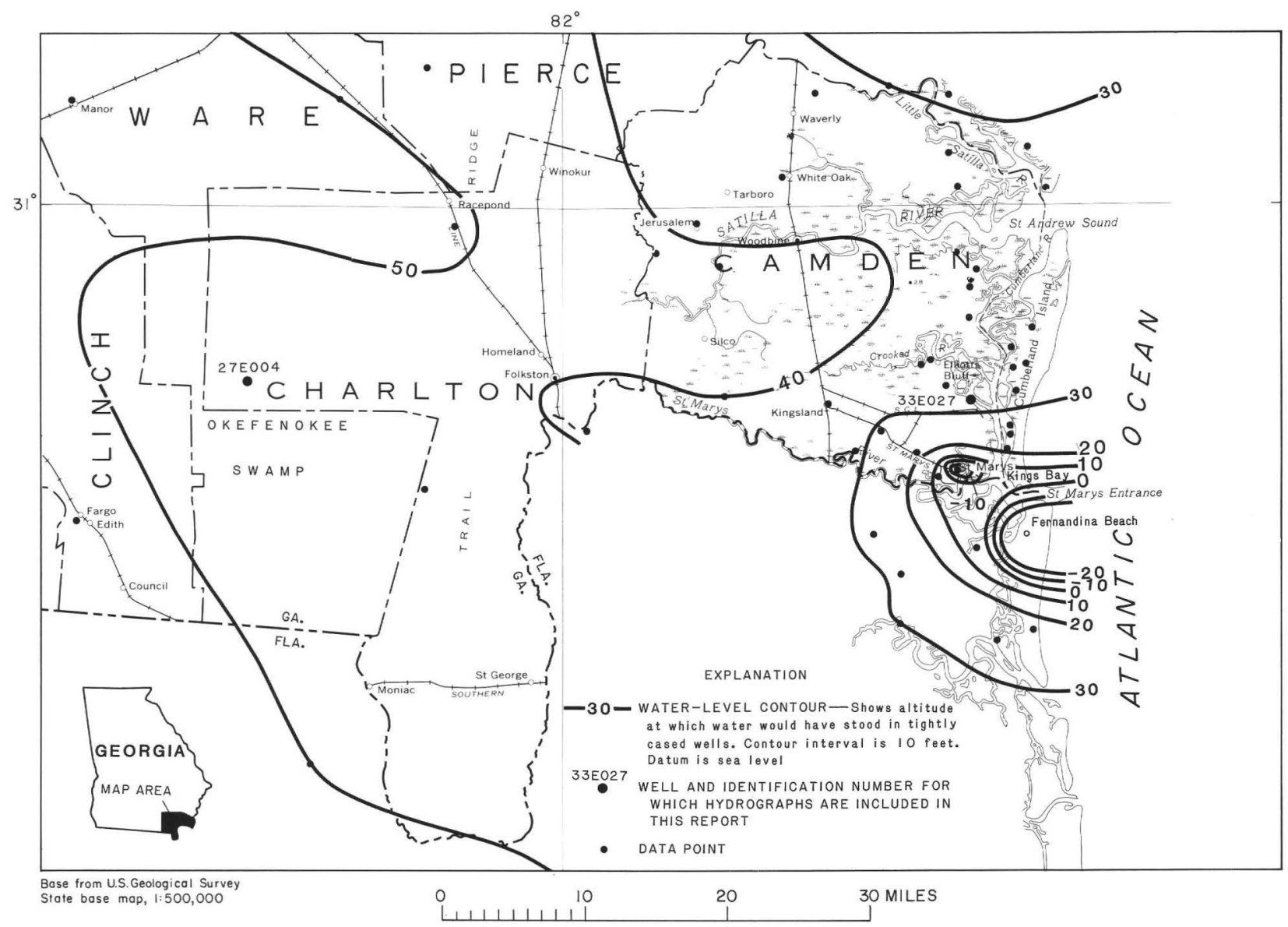

Figure 2.7.4.4-1.-Observation well locations and the water level in the Upper Floridan aquifer in the Kings Bay-Okefenokee Swamp area, May 1985. 
304756081311101 Local number, 33E027.

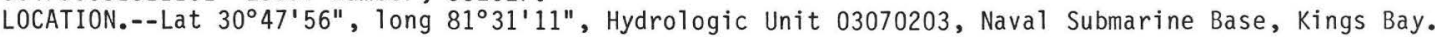

Owner: U.S. Department of the Navy.

AQUIFER.--Upper Floridan aquifer.

WELL CHARACTERISTICS.--Drilled test well, diameter $8 \mathrm{in}$., depth 1,306 ft, cased to $555 \mathrm{ft}$, backfilled to $990 \mathrm{ft}$. DATUM.--Elevation of land-surface datum is $10.0 \mathrm{ft}$.

Measuring point: Top of flange at land-surface datum.

REMARKS.-- Water levels for periods of missing record, January 12-29, March 14-25, and 0ctober 4-14, were estimated. PERIOD OF RECORD.--August 1979 to current year.

EXTREMES FOR PERIOD OF RECORD.--Highest water leve1, $24.71 \mathrm{ft}$ above land-surface datum, March 28 , 1984 , and March 17, 1983; lowest, $13.90 \mathrm{ft}$ above land-surface datum, June 10-11, 1985.

WATER LEVEL, IN FEET ABOVE LAND SURFACE DATUM CALENDAR YEAR JANUARY 1987 TO DECEMBER 1987

MEAN VALUES

\begin{tabular}{|c|c|c|c|c|c|c|c|c|c|c|c|c|}
\hline DAY & JAN & FEB & MAR & APR & MAY & JUN & JUL & AUG & SEP & OCT & NOV & $\mathrm{DEC}$ \\
\hline $\begin{array}{l}1 \\
2 \\
3 \\
4 \\
5\end{array}$ & $\begin{array}{l}20.30 \\
20.10 \\
20.10 \\
20.30 \\
20.90\end{array}$ & $\begin{array}{l}19.40 \\
19.60 \\
19.50 \\
19.10 \\
19.00\end{array}$ & $\begin{array}{l}20.50 \\
20.30 \\
20.20 \\
20.00 \\
19.90\end{array}$ & $\begin{array}{l}20.60 \\
20.90 \\
21.00 \\
20.20 \\
20.50\end{array}$ & $\begin{array}{l}20.80 \\
20.70 \\
20.60 \\
20.80 \\
20.70\end{array}$ & $\begin{array}{l}18.40 \\
18.30 \\
18.00 \\
18.10 \\
17.80\end{array}$ & $\begin{array}{l}18.20 \\
18.20 \\
17.90 \\
17.90 \\
18.00\end{array}$ & $\begin{array}{l}17.30 \\
17.30 \\
17.40 \\
17.30 \\
17.30\end{array}$ & $\begin{array}{l}17.20 \\
17.00 \\
17.10 \\
17.10 \\
17.20\end{array}$ & $\begin{array}{l}16.30 \\
16.20 \\
16.50 \\
16.60 \\
16.70\end{array}$ & $\begin{array}{l}17.50 \\
17.70 \\
17.70 \\
17.90 \\
18.10\end{array}$ & $\begin{array}{l}18.70 \\
18.40 \\
18.40 \\
18.50 \\
18.40\end{array}$ \\
\hline $\begin{array}{r}6 \\
7 \\
8 \\
9 \\
10\end{array}$ & $\begin{array}{l}20.50 \\
20.50 \\
20.30 \\
20.00 \\
20.30\end{array}$ & $\begin{array}{l}19.40 \\
19.70 \\
19.80 \\
19.40 \\
19.20\end{array}$ & $\begin{array}{l}19.90 \\
20.10 \\
20.30 \\
20.40 \\
20.20\end{array}$ & $\begin{array}{l}20.50 \\
21.10 \\
21.10 \\
21.20 \\
21.20\end{array}$ & $\begin{array}{l}20.70 \\
20.80 \\
20.90 \\
20.80 \\
20.90\end{array}$ & $\begin{array}{l}18.00 \\
18.10 \\
17.70 \\
17.90 \\
18.10\end{array}$ & $\begin{array}{l}18.10 \\
18.10 \\
17.90 \\
17.80 \\
17.80\end{array}$ & $\begin{array}{l}17.30 \\
17.30 \\
17.40 \\
17.50 \\
17.60\end{array}$ & $\begin{array}{l}17.10 \\
17.40 \\
17.20 \\
16.90 \\
16.90\end{array}$ & $\begin{array}{l}16.60 \\
16.50 \\
16.40 \\
16.30 \\
16.40\end{array}$ & $\begin{array}{l}17.70 \\
17.60 \\
17.70 \\
18.00 \\
18.10\end{array}$ & $\begin{array}{l}18.4 \\
18.5 \\
18.8 \\
18.8 \\
18.8\end{array}$ \\
\hline $\begin{array}{l}11 \\
12 \\
13 \\
14 \\
15\end{array}$ & $\begin{array}{l}20.30 \\
20.19 \\
20.12 \\
20.05 \\
19.94\end{array}$ & $\begin{array}{l}19.40 \\
19.50 \\
19.60 \\
19.60 \\
19.80\end{array}$ & $\begin{array}{l}20.10 \\
20.20 \\
20.30 \\
20.36 \\
20.44\end{array}$ & $\begin{array}{l}21.10 \\
21.00 \\
21.00 \\
21.00 \\
21.20\end{array}$ & $\begin{array}{l}20.80 \\
20.80 \\
20.60 \\
20.60 \\
20.60\end{array}$ & $\begin{array}{l}18.40 \\
18.80 \\
18.90 \\
19.00 \\
18.80\end{array}$ & $\begin{array}{l}17.80 \\
17.80 \\
17.80 \\
17.80 \\
17.80\end{array}$ & $\begin{array}{l}17.60 \\
17.60 \\
17.50 \\
17.50 \\
17.50\end{array}$ & $\begin{array}{l}16.80 \\
16.80 \\
16.70 \\
16.70 \\
16.30\end{array}$ & $\begin{array}{l}16.50 \\
16.60 \\
16.60 \\
16.70 \\
16.80\end{array}$ & $\begin{array}{l}17.80 \\
17.70 \\
18.00 \\
17.90 \\
17.90\end{array}$ & $\begin{array}{l}18.9 \\
18.8 \\
18.6 \\
18.8 \\
18.8\end{array}$ \\
\hline $\begin{array}{l}16 \\
17 \\
18 \\
19 \\
20\end{array}$ & $\begin{array}{l}20.04 \\
19.97 \\
19.98 \\
20.02 \\
19.85\end{array}$ & $\begin{array}{l}20.20 \\
20.10 \\
19.90 \\
19.70 \\
19.70\end{array}$ & $\begin{array}{l}20.53 \\
20.52 \\
20.68 \\
20.84 \\
20.87\end{array}$ & $\begin{array}{l}21.30 \\
21.00 \\
21.20 \\
21.20 \\
21.20\end{array}$ & $\begin{array}{l}20.50 \\
20.40 \\
20.40 \\
20.30 \\
20.40\end{array}$ & $\begin{array}{l}18.70 \\
18.80 \\
18.70 \\
18.80 \\
18.60\end{array}$ & $\begin{array}{l}17.80 \\
17.50 \\
17.70 \\
17.80 \\
17.80\end{array}$ & $\begin{array}{l}17.40 \\
17.50 \\
17.30 \\
17.30 \\
17.30\end{array}$ & $\begin{array}{l}16.20 \\
16.30 \\
16.30 \\
16.20 \\
16.30\end{array}$ & $\begin{array}{l}17.40 \\
17.50 \\
17.60 \\
17.50 \\
17.50\end{array}$ & $\begin{array}{l}17.90 \\
18.00 \\
18.00 \\
17.90 \\
18.00\end{array}$ & $\begin{array}{l}18.2 \\
18.2 \\
18.2 \\
18.3 \\
18.4\end{array}$ \\
\hline $\begin{array}{l}21 \\
22 \\
23 \\
24 \\
25\end{array}$ & $\begin{array}{l}19.84 \\
20.02 \\
19.77 \\
19.56 \\
19.66\end{array}$ & $\begin{array}{l}20.00 \\
20.20 \\
20.30 \\
20.20 \\
20.30\end{array}$ & $\begin{array}{l}20.94 \\
21.02 \\
21.01 \\
21.05 \\
21.13\end{array}$ & $\begin{array}{l}21.30 \\
21.20 \\
20.80 \\
20.60 \\
20.80\end{array}$ & $\begin{array}{l}20.60 \\
20.80 \\
20.60 \\
20.30 \\
20.20\end{array}$ & $\begin{array}{l}18.80 \\
18.70 \\
18.70 \\
18.70 \\
18.60\end{array}$ & $\begin{array}{l}17.60 \\
17.60 \\
17.60 \\
17.40 \\
17.30\end{array}$ & $\begin{array}{l}17.20 \\
17.20 \\
17.20 \\
17.20 \\
17.10\end{array}$ & $\begin{array}{l}16.30 \\
16.30 \\
16.30 \\
16.20 \\
16.00\end{array}$ & $\begin{array}{l}17.50 \\
17.50 \\
17.50 \\
17.60 \\
17.80\end{array}$ & $\begin{array}{l}17.70 \\
17.90 \\
18.00 \\
18.00 \\
18.30\end{array}$ & $\begin{array}{l}18.5 \\
18.6 \\
18.5 \\
18.5 \\
18.6\end{array}$ \\
\hline $\begin{array}{l}26 \\
27 \\
28 \\
29 \\
30 \\
31\end{array}$ & $\begin{array}{l}19.65 \\
19.41 \\
19.29 \\
19.22 \\
19.30 \\
19.20\end{array}$ & $\begin{array}{r}20.30 \\
20.30 \\
20.40 \\
-\ldots- \\
-- \\
---\end{array}$ & $\begin{array}{l}21.20 \\
21.20 \\
21.10 \\
20.80 \\
21.20 \\
21.00\end{array}$ & $\begin{array}{r}20.80 \\
20.70 \\
20.80 \\
20.70 \\
20.80 \\
. .-\end{array}$ & $\begin{array}{l}20.00 \\
19.50 \\
19.10 \\
18.90 \\
18.90 \\
18.60\end{array}$ & $\begin{array}{r}18.80 \\
18.60 \\
18.70 \\
18.40 \\
18.30 \\
. .-\end{array}$ & $\begin{array}{l}17.30 \\
17.30 \\
17.30 \\
17.20 \\
17.20 \\
17.10\end{array}$ & $\begin{array}{l}17.10 \\
17.00 \\
17.00 \\
17.20 \\
17.10 \\
17.10\end{array}$ & $\begin{array}{r}16.00 \\
16.10 \\
16.00 \\
16.40 \\
16.40 \\
-.-\end{array}$ & $\begin{array}{l}17.80 \\
17.90 \\
17.50 \\
17.10 \\
17.10 \\
17.20\end{array}$ & $\begin{array}{r}18.60 \\
18.70 \\
18.80 \\
18.80 \\
18.80 \\
-.-\end{array}$ & $\begin{array}{l}18.7 \\
18.6 \\
18.8 \\
18.5 \\
18.4 \\
18.3\end{array}$ \\
\hline MEAN & 19.96 & 19.77 & 20.59 & 20.93 & 20.34 & 18.47 & 7.69 & 17.31 & 16.59 & 17.02 & 18.02 & . \\
\hline
\end{tabular}




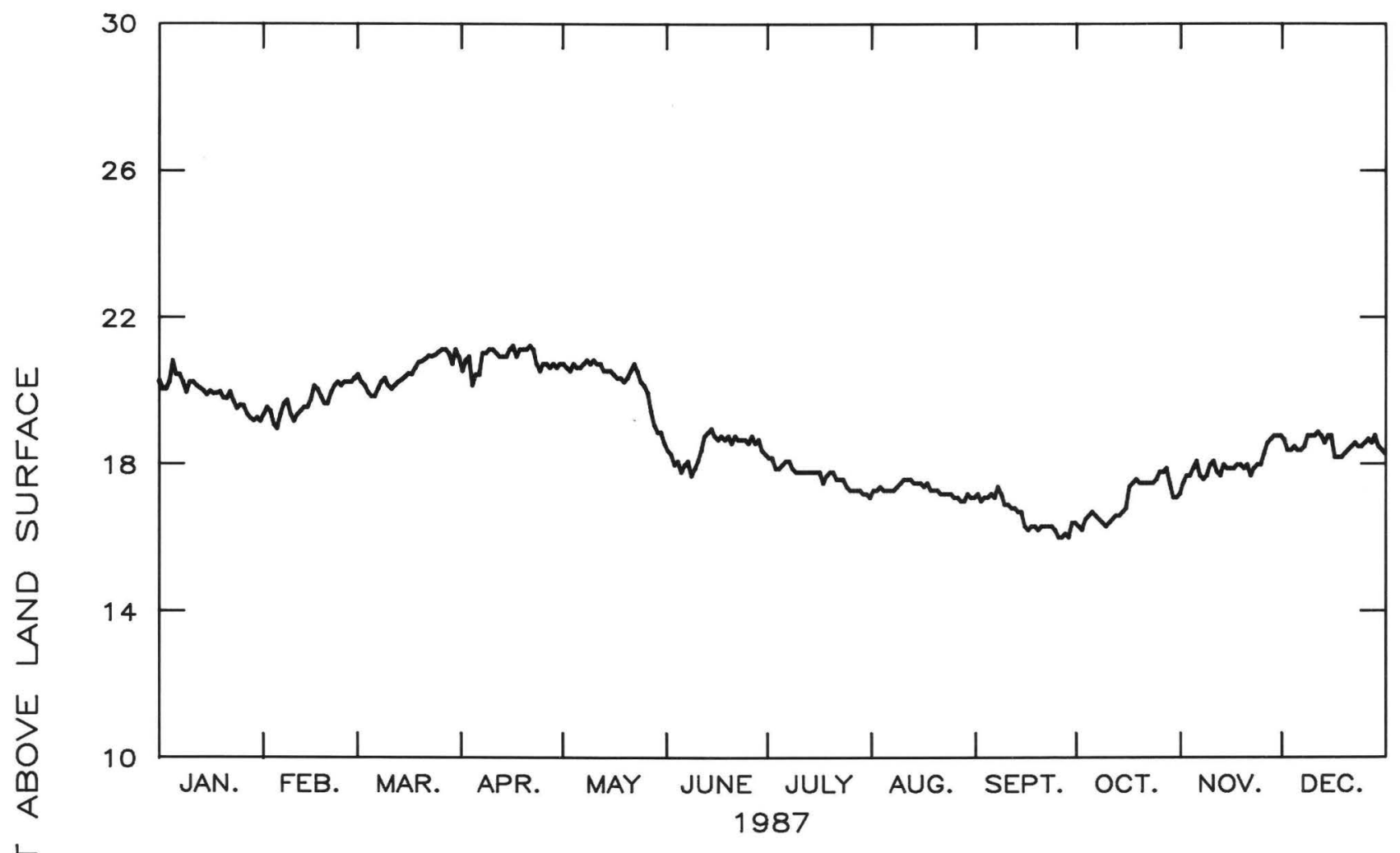

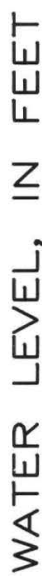

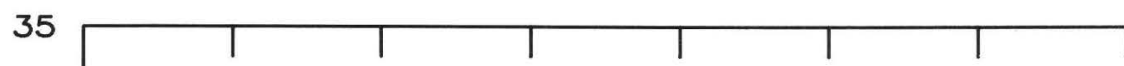

30

$\frac{1}{4}$ 
304942082213801 Local number, 27 E004.

LOCATION.--Lat $30^{\circ} 49^{\prime} 43^{\prime \prime}$, long $82^{\circ} 21^{\prime} 38^{\prime \prime}$, Hydrologic Unit 03110201 , end of Georgia Highway 177 east of Stephen C. Foster State Park.

Owner: U.S. Geological Survey, test well oK 9.

AQUIFER.--Upper Floridan aquifer.

WELL CHARACTERISTICS.--Drilled observation well, diameter 4 in., depth $700 \mathrm{ft}$, cased to $498 \mathrm{ft}$, open hole. DATUM.--Elevation of land-surface datum is $116 \mathrm{ft}$.

Measuring point: Floor of recorder shelter, $4.3 \mathrm{ft}$ above land-surface datum.

REMARKS.--Well drilled in May, 1978 to replace U.S. Geological Survey test well 0K 8 (27E002).

PERIOD OF RECORD.--June 14, 1978 to January 26, 1979; January 1, 1980 to current year.

EXTREMES FOR PERIOD OF RECORD.--Highest water leve1, $62.30 \mathrm{ft}$ below land-surface datum, May 9, 1984 ; 1 lowest, $71.60 \mathrm{ft}$ below land-surface datum, July $27,1981$.

DEPTH BELOW LAND SURFACE (WATER LEVEL) (FEET) CALENDAR YEAR JANUARY 1987 TO DECEMBER 1987 MEAN VALUES

\begin{tabular}{|c|c|c|c|c|c|c|c|c|c|c|c|c|}
\hline DAY & JAN & FEB & MAR & APR & MAY & JUN & JUL & AUG & SEP & OCT & NOV & DEC \\
\hline $\begin{array}{l}1 \\
2 \\
3 \\
4 \\
5\end{array}$ & $\begin{array}{l}68.00 \\
68.19 \\
68.24 \\
67.94 \\
67.96\end{array}$ & $\begin{array}{l}66.55 \\
66.33 \\
66.33 \\
66.40 \\
66.38\end{array}$ & $\begin{array}{l}64.98 \\
65.09 \\
65.13 \\
65.16 \\
65.20\end{array}$ & $\begin{array}{l}64.21 \\
64.13 \\
64.01 \\
64.06 \\
64.00\end{array}$ & $\begin{array}{l}64.45 \\
64.51 \\
64.62 \\
64.71 \\
64.83\end{array}$ & $\begin{array}{l}66.26 \\
66.31 \\
66.38 \\
66.43 \\
66.50\end{array}$ & $\begin{array}{l}67.60 \\
67.58 \\
67.63 \\
67.66 \\
67.71\end{array}$ & $\begin{array}{l}68.29 \\
68.29 \\
68.32 \\
68.33 \\
68.34\end{array}$ & $\begin{array}{l}68.65 \\
68.65 \\
68.66 \\
68.64 \\
68.60\end{array}$ & $\begin{array}{l}68.75 \\
68.86 \\
68.91 \\
69.01 \\
68.93\end{array}$ & $\begin{array}{l}69.53 \\
69.47 \\
69.39 \\
69.26 \\
69.27\end{array}$ & $\begin{array}{l}69.23 \\
69.40 \\
69.38 \\
69.26 \\
69.42\end{array}$ \\
\hline $\begin{array}{r}6 \\
7 \\
8 \\
9 \\
10\end{array}$ & $\begin{array}{l}68.04 \\
67.96 \\
67.90 \\
67.82 \\
67.61\end{array}$ & $\begin{array}{l}66.17 \\
66.01 \\
65.95 \\
66.12 \\
66.21\end{array}$ & $\begin{array}{l}65.13 \\
64.83 \\
64.60 \\
64.51 \\
64.62\end{array}$ & $\begin{array}{l}63.95 \\
63.91 \\
63.90 \\
63.86 \\
63.83\end{array}$ & $\begin{array}{l}64.89 \\
64.90 \\
64.92 \\
65.11 \\
65.20\end{array}$ & $\begin{array}{l}66.63 \\
66.74 \\
66.80 \\
66.81 \\
66.82\end{array}$ & $\begin{array}{l}67.74 \\
67.75 \\
67.74 \\
67.76 \\
67.73\end{array}$ & $\begin{array}{l}68.41 \\
68.48 \\
68.50 \\
68.49 \\
68.46\end{array}$ & $\begin{array}{l}68.60 \\
68.58 \\
68.61 \\
68.65 \\
68.68\end{array}$ & $\begin{array}{l}68.85 \\
68.90 \\
69.07 \\
69.18 \\
69.17\end{array}$ & $\begin{array}{l}69.51 \\
69.58 \\
69.55 \\
69.47 \\
69.32\end{array}$ & $\begin{array}{l}69.48 \\
69.47 \\
69.42 \\
69.34 \\
69.23\end{array}$ \\
\hline $\begin{array}{l}11 \\
12 \\
13 \\
14 \\
15\end{array}$ & $\begin{array}{l}67.71 \\
67.70 \\
67.63 \\
67.59 \\
67.45\end{array}$ & $\begin{array}{l}66.09 \\
65.89 \\
65.79 \\
65.68 \\
65.60\end{array}$ & $\begin{array}{l}64.79 \\
64.75 \\
64.67 \\
64.61 \\
64.55\end{array}$ & $\begin{array}{l}63.86 \\
63.89 \\
63.90 \\
63.88 \\
63.72\end{array}$ & $\begin{array}{l}65.22 \\
65.24 \\
65.34 \\
65.44 \\
65.43\end{array}$ & $\begin{array}{l}66.90 \\
66.97 \\
67.00 \\
67.02 \\
67.08\end{array}$ & $\begin{array}{l}67.71 \\
67.69 \\
67.72 \\
67.74 \\
67.79\end{array}$ & $\begin{array}{l}68.45 \\
68.48 \\
68.49 \\
68.53 \\
68.56\end{array}$ & $\begin{array}{l}68.72 \\
68.68 \\
68.65 \\
68.66 \\
68.65\end{array}$ & $\begin{array}{l}69.04 \\
68.91 \\
69.05 \\
69.18 \\
69.22\end{array}$ & $\begin{array}{l}69.43 \\
69.54 \\
69.51 \\
69.50 \\
69.56\end{array}$ & $\begin{array}{l}69.16 \\
69.18 \\
69.35 \\
69.37 \\
69.23\end{array}$ \\
\hline $\begin{array}{l}16 \\
17 \\
18 \\
19 \\
20\end{array}$ & $\begin{array}{l}67.37 \\
67.32 \\
67.21 \\
67.13 \\
67.23\end{array}$ & $\begin{array}{l}65.38 \\
65.51 \\
65.59 \\
65.69 \\
65.67\end{array}$ & $\begin{array}{l}64.48 \\
64.46 \\
64.33 \\
64.15 \\
64.19\end{array}$ & $\begin{array}{l}63.63 \\
63.63 \\
63.73 \\
63.89 \\
63.94\end{array}$ & $\begin{array}{l}65.48 \\
65.57 \\
65.59 \\
65.60 \\
65.64\end{array}$ & $\begin{array}{l}67.12 \\
67.21 \\
67.29 \\
67.29 \\
67.29\end{array}$ & $\begin{array}{l}67.88 \\
67.99 \\
68.02 \\
68.04 \\
68.10\end{array}$ & $\begin{array}{l}68.58 \\
68.60 \\
68.60 \\
68.58 \\
68.61\end{array}$ & $\begin{array}{l}68.63 \\
68.61 \\
68.56 \\
68.55 \\
68.57\end{array}$ & $\begin{array}{l}69.19 \\
69.15 \\
69.16 \\
69.17 \\
69.19\end{array}$ & $\begin{array}{l}69.53 \\
69.45 \\
69.49 \\
69.45 \\
69.40\end{array}$ & $\begin{array}{l}69.37 \\
69.47 \\
69.51 \\
69.47 \\
69.42\end{array}$ \\
\hline $\begin{array}{l}21 \\
22 \\
23 \\
24 \\
25\end{array}$ & $\begin{array}{l}67.12 \\
66.80 \\
67.06 \\
67.11 \\
66.78\end{array}$ & $\begin{array}{l}65.47 \\
65.26 \\
65.31 \\
65.39 \\
65.39\end{array}$ & $\begin{array}{l}64.11 \\
64.10 \\
64.14 \\
64.11 \\
64.13\end{array}$ & $\begin{array}{l}63.93 \\
63.95 \\
63.97 \\
63.97 \\
64.09\end{array}$ & $\begin{array}{l}65.71 \\
65.81 \\
65.84 \\
65.84 \\
65.88\end{array}$ & $\begin{array}{l}67 \cdot 30 \\
67 \cdot 32 \\
67 \cdot 35 \\
67 \cdot 36 \\
67.36\end{array}$ & $\begin{array}{l}68.13 \\
68.11 \\
68.07 \\
68.15 \\
68.20\end{array}$ & $\begin{array}{l}68.66 \\
68.66 \\
68.62 \\
68.61 \\
68.65\end{array}$ & $\begin{array}{l}68.65 \\
68.69 \\
68.68 \\
68.66 \\
68.69\end{array}$ & $\begin{array}{l}69.27 \\
69.42 \\
69.45 \\
69.45 \\
69.37\end{array}$ & $\begin{array}{l}69.50 \\
69.54 \\
69.55 \\
69.55 \\
69.47\end{array}$ & $\begin{array}{l}69.37 \\
69.34 \\
69.46 \\
69.47 \\
69.43\end{array}$ \\
\hline $\begin{array}{l}26 \\
27 \\
28 \\
29 \\
30 \\
31\end{array}$ & $\begin{array}{l}66.76 \\
66.90 \\
66.87 \\
66.85 \\
66.61 \\
66.58\end{array}$ & $\begin{array}{r}65.38 \\
65.27 \\
65.11 \\
\ldots-- \\
\ldots- \\
\ldots-\end{array}$ & $\begin{array}{l}64.08 \\
64.01 \\
64.05 \\
64.09 \\
63.93 \\
64.07\end{array}$ & $\begin{array}{r}64.26 \\
64.31 \\
64.27 \\
64.39 \\
64.41 \\
. .-\end{array}$ & $\begin{array}{l}65.96 \\
66.03 \\
66.07 \\
66.11 \\
66.13 \\
66.19\end{array}$ & $\begin{array}{r}67.38 \\
67.40 \\
67.53 \\
67.62 \\
67.65 \\
\ldots .-\end{array}$ & $\begin{array}{l}68.19 \\
68.19 \\
68.18 \\
68.21 \\
68.29 \\
68.33\end{array}$ & $\begin{array}{l}68.67 \\
68.66 \\
68.65 \\
68.65 \\
68.66 \\
68.65\end{array}$ & $\begin{array}{r}68.79 \\
68.84 \\
68.86 \\
68.78 \\
68.63 \\
. .-\end{array}$ & $\begin{array}{l}69.27 \\
69.24 \\
69.32 \\
69.46 \\
69.52 \\
69.56\end{array}$ & $\begin{array}{r}69.39 \\
69.33 \\
69.27 \\
69.21 \\
69.16 \\
\ldots . .-\end{array}$ & $\begin{array}{l}69.41 \\
69.41 \\
69.30 \\
69.42 \\
69.62 \\
69.57\end{array}$ \\
\hline MEAN & 67.40 & 65.78 & 64.49 & 63.98 & 65.43 & 67.04 & 67.92 & 68.53 & 68.66 & 69.17 & 69.44 & 69.39 \\
\hline
\end{tabular}

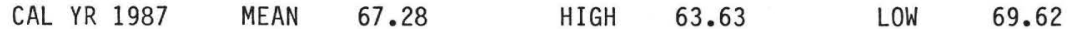



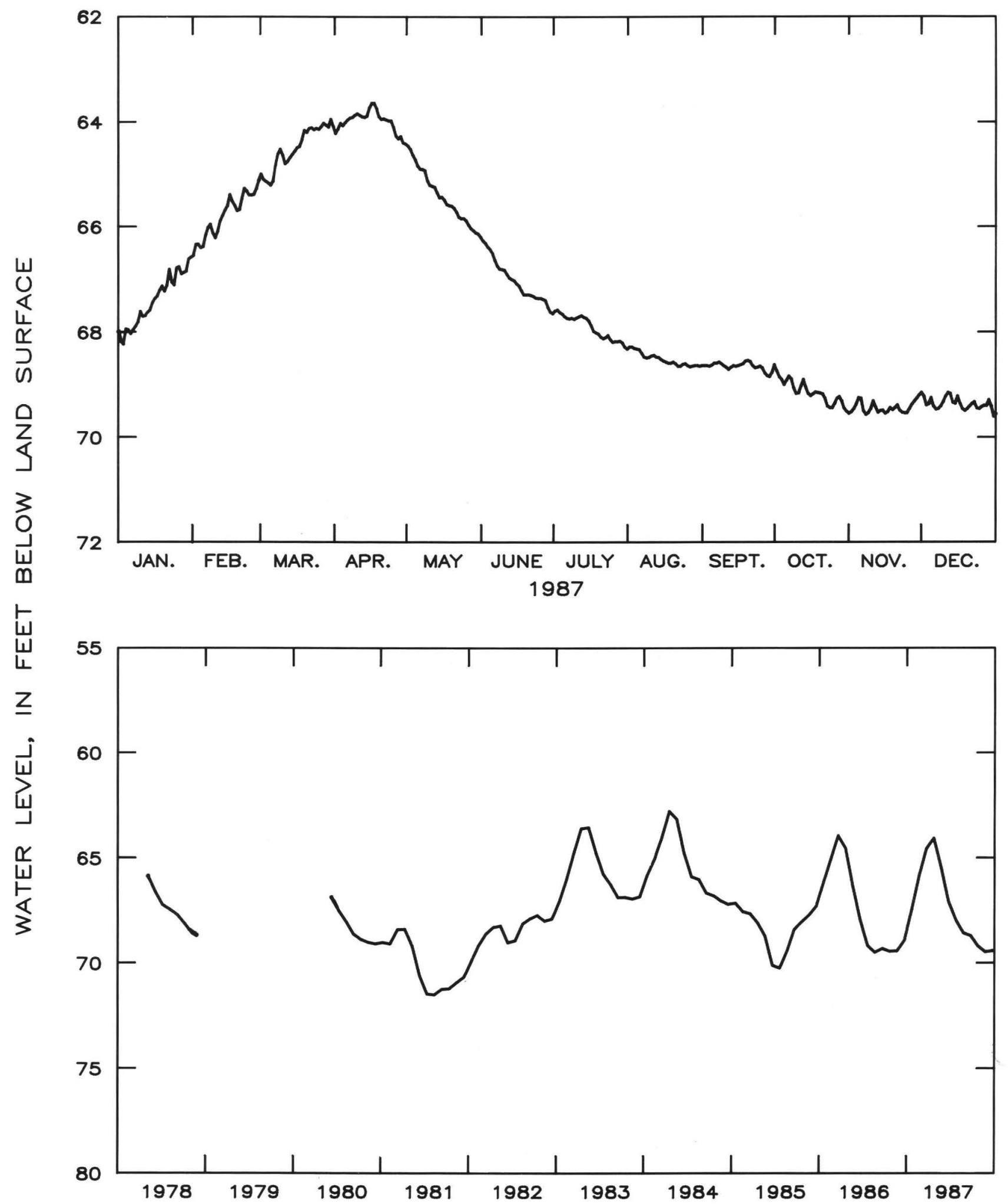

Figure 2.7.4.4-3.--Water level in observation well 27E004, Charlton County. 


\subsection{Miocene aquifers}

There are two principal aquifers of Miocene age in the coastal area of Georgia. The aquifers consist of poorly sorted, fine to coarse, slightly phosphatic and dolomitic quartz sand that ranges in thickness from about 20 to $100 \mathrm{ft}$ (J.S. Clarke, U.S. Geological Survey, written commun., 1988).

Water-level fluctuations and trends in the the two Miocene aquifers are similar. Water levels respond to pumping in areas where the Miocene aquifers are deeply buried and where pumping centers for the Upper Floridan aquifer are nearby (J.S. Clarke, U.S. Geological Survey, written commun., 1988). Although regional pumping has some influence, water levels primarily respond to seasonal climatic changes in areas where the aquifers are not as deeply buried and where they are distant from Upper Floridan pumping centers.

In Bulloch County, the uppermost Miocene aquifer is found near the area where it crops out. Water levels in this area are influenced by both evapotranspiration and by pumping from the Upper Floridan aquifer. During 1987, the water level in well 314009 near Hopeulikit showed a recovery from the effects of the 1986 drought. By the end of April, the water 1evel in the wel1 had recovered $6.6 \mathrm{ft}$ from the record 10w measured in August 1986. The mean water level in well 31 U009 was $1.3 \mathrm{ft}$ higher in 1987 than in 1986, and the water level at the end of the year was about $1.0 \mathrm{ft}$ higher than at the end of 1986. These rises, which reversed a downward trend since 1983, can be attributed to above-normal rainfall during 1 ate 1986 and early 1987.

In the Wayne County area, the water-level trend in the uppermost Miocene aquifer is similar to that in the underlying Upper Floridan aquifer. During 1987, the water level in well 32 L016 near Gardi showed a recovery from the effects of the 1986 drought. By early February, the water level in the well had recovered $2.2 \mathrm{ft}$ from the record 10w measured in November 1986 . However, by December 1987, a new record low was measured in the well that was $0.5 \mathrm{ft}$ lower than the previous record. The mean water level in well 32 L016 was $0.5 \mathrm{ft}$ lower in 1987 than in 1986, and the water level at the end of the year was $1.6 \mathrm{ft}$ lower than at the end of 1986. These declines correspond to the downward trend in the Upper Floridan aquifer since 1983 (see hydrograph for well 32L015, fig. 2.7.4.2-3). 


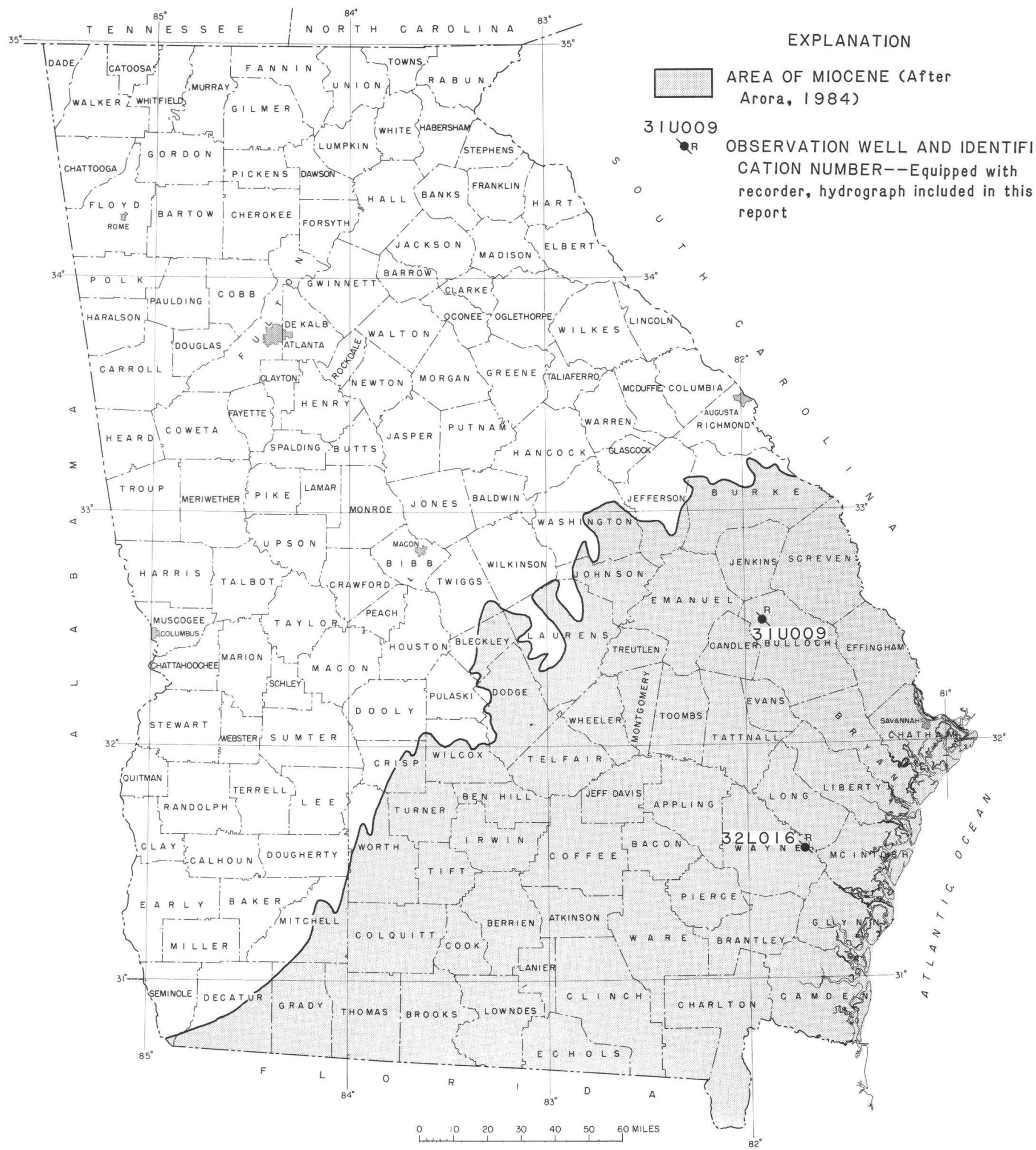

Figure 2.8-1.--Location of observation wells in the Miocene aquifers. 
310009 HOPEULIKIT TW2 BULLOCH COUNTY

323123081511602 , Local number, 310009.

LOCATION.--Lat $32^{\circ} 31^{\prime} 23^{\prime \prime}$, long $81^{\circ} 51^{\prime} 16^{\prime \prime}$, Hydrologic Unit 03060202 , in roadside park on west side of Hopeulikit community, U.S. Highways 25 and 80.

Owner: Georgia Geologic Survey.

AQUIFER.--Miocene.

WELL CHARACTERISTICS.--Drilled observation well, diameter $6 \mathrm{in}$, depth $210 \mathrm{ft}$, cased to $160 \mathrm{ft}$, stainless steel screen 160 to $210 \mathrm{ft}$.

DATUM.--Elevation of land-surface datum is $205 \mathrm{ft}$.

Measuring point: Floor of recorder shelter, $3.0 \mathrm{ft}$ above land-surface datum.

REMARKS.--We11 sounded August 1982. Water levels for period of missing record, January 27-28, were estimated. PERIOD OF RECORD.---October 1982 to current year.

EXTREMES FOR PERIOD OF RECORD.--Highest water level, $70.77 \mathrm{ft}$ below land-surface datum, April 24, 1983; 10west, $78.87 \mathrm{ft}$ below land-surface datum, August 4, 1986.

DEPTH BELOW LAND SURFACE (WATER LEVEL) (FEET) CALENDAR YEAR JANUARY 1987 TO DECEMBER 1987 MEAN VALUES

\begin{tabular}{|c|c|c|c|c|c|c|c|c|c|c|c|c|}
\hline DAY & JAN & FEB & MAR & APR & MAY & JUN & JUL & AUG & SEP & OCT & NOV & $\mathrm{DEC}$ \\
\hline $\begin{array}{l}1 \\
2 \\
3 \\
4 \\
5\end{array}$ & $\begin{array}{l}75.28 \\
75.22 \\
75.24 \\
75.19 \\
75.13\end{array}$ & $\begin{array}{l}74.42 \\
74.34 \\
74.31 \\
74.27 \\
74.27\end{array}$ & $\begin{array}{l}73.19 \\
73.22 \\
73.32 \\
73.34 \\
73.35\end{array}$ & $\begin{array}{l}72.60 \\
72.59 \\
72.58 \\
72.60 \\
72.59\end{array}$ & $\begin{array}{l}72.25 \\
72.26 \\
72.27 \\
72.29 \\
72.28\end{array}$ & $\begin{array}{l}72.47 \\
72.49 \\
72.53 \\
72.56 \\
72.60\end{array}$ & $\begin{array}{l}73.76 \\
73.74 \\
73.69 \\
73.71 \\
73.74\end{array}$ & $\begin{array}{l}74.38 \\
74.39 \\
74.42 \\
74.44 \\
74.46\end{array}$ & $\begin{array}{l}75.07 \\
75.02 \\
74.96 \\
74.93 \\
74.90\end{array}$ & $\begin{array}{l}74.72 \\
74.74 \\
74.76 \\
74.78 \\
74.74\end{array}$ & $\begin{array}{l}74.76 \\
74.73 \\
74.71 \\
74.66 \\
74.65\end{array}$ & $\begin{array}{l}74.48 \\
74.52 \\
74.49 \\
74.47 \\
74.50\end{array}$ \\
\hline $\begin{array}{r}6 \\
7 \\
8 \\
9 \\
10\end{array}$ & $\begin{array}{l}75.14 \\
75.12 \\
75.11 \\
75.09 \\
75.01\end{array}$ & $\begin{array}{l}74.18 \\
74.08 \\
74.02 \\
74.07 \\
74.10\end{array}$ & $\begin{array}{l}73.34 \\
73.27 \\
73.15 \\
73.06 \\
73.12\end{array}$ & $\begin{array}{l}72.58 \\
72.56 \\
72.54 \\
72.52 \\
72.50\end{array}$ & $\begin{array}{l}72.28 \\
72.29 \\
72.30 \\
72.33 \\
72.35\end{array}$ & $\begin{array}{l}72.66 \\
72.72 \\
72.77 \\
72.80 \\
72.84\end{array}$ & $\begin{array}{l}73.78 \\
73.80 \\
73.82 \\
73.85 \\
73.87\end{array}$ & $\begin{array}{l}74.51 \\
74.55 \\
74.58 \\
74.59 \\
74.61\end{array}$ & $\begin{array}{l}74.88 \\
74.86 \\
74.86 \\
74.88 \\
74.89\end{array}$ & $\begin{array}{l}74.71 \\
74.72 \\
74.78 \\
74.80 \\
74.79\end{array}$ & $\begin{array}{l}74.71 \\
74.73 \\
74.72 \\
74.68 \\
74.64\end{array}$ & $\begin{array}{l}74.5 \\
74.5 \\
74.5 \\
74.4 \\
74.4\end{array}$ \\
\hline $\begin{array}{l}11 \\
12 \\
13 \\
14 \\
15\end{array}$ & $\begin{array}{l}74.98 \\
74.98 \\
74.96 \\
74.96 \\
74.92\end{array}$ & $\begin{array}{l}74.07 \\
74.00 \\
73.94 \\
73.87 \\
73.84\end{array}$ & $\begin{array}{l}73.18 \\
73.17 \\
73.14 \\
73.12 \\
73.09\end{array}$ & $\begin{array}{l}72.50 \\
72.49 \\
72.49 \\
72.48 \\
72.42\end{array}$ & $\begin{array}{l}72.35 \\
72.32 \\
72.31 \\
72.29 \\
72.29\end{array}$ & $\begin{array}{l}72.90 \\
72.96 \\
73.00 \\
73.00 \\
73.04\end{array}$ & $\begin{array}{l}73.88 \\
73.89 \\
73.90 \\
73.92 \\
73.94\end{array}$ & $\begin{array}{l}74.60 \\
74.59 \\
74.64 \\
74.68 \\
74.73\end{array}$ & $\begin{array}{l}74.88 \\
74.82 \\
74.78 \\
74.79 \\
74.80\end{array}$ & $\begin{array}{l}74.75 \\
74.72 \\
74.74 \\
74.77 \\
74.78\end{array}$ & $\begin{array}{l}74.65 \\
74.68 \\
74.66 \\
74.66 \\
74.68\end{array}$ & $\begin{array}{l}74 . \\
74 . \\
74 . \\
74 . \\
74 .\end{array}$ \\
\hline $\begin{array}{l}16 \\
17 \\
18 \\
19 \\
20\end{array}$ & $\begin{array}{l}74.85 \\
74.78 \\
74.72 \\
74.65 \\
74.66\end{array}$ & $\begin{array}{l}73.76 \\
73.69 \\
73.71 \\
73.74 \\
73.74\end{array}$ & $\begin{array}{l}73.06 \\
73.07 \\
73.01 \\
72.90 \\
72.84\end{array}$ & $\begin{array}{l}72.37 \\
72.34 \\
72.35 \\
72.36 \\
72.37\end{array}$ & $\begin{array}{l}72.30 \\
72.31 \\
72.32 \\
72.31 \\
72.32\end{array}$ & $\begin{array}{l}73.10 \\
73.17 \\
73.26 \\
73.32 \\
73.37\end{array}$ & $\begin{array}{l}73.98 \\
74.03 \\
74.06 \\
74.08 \\
74.12\end{array}$ & $\begin{array}{l}74.75 \\
74.76 \\
74.79 \\
74.81 \\
74.85\end{array}$ & $\begin{array}{l}74.79 \\
74.78 \\
74.76 \\
74.75 \\
74.71\end{array}$ & $\begin{array}{l}74.76 \\
74.75 \\
74.74 \\
74.74 \\
74.73\end{array}$ & $\begin{array}{l}74.68 \\
74.62 \\
74.62 \\
74.62 \\
74.58\end{array}$ & 74. \\
\hline $\begin{array}{l}21 \\
22 \\
23 \\
24 \\
25\end{array}$ & $\begin{array}{l}74.63 \\
74.48 \\
74.48 \\
74.56 \\
74.50\end{array}$ & $\begin{array}{l}73.70 \\
73.58 \\
73.52 \\
73.56 \\
73.57\end{array}$ & $\begin{array}{l}72.84 \\
72.84 \\
72.85 \\
72.84 \\
72.75\end{array}$ & $\begin{array}{l}72.35 \\
72.34 \\
72.31 \\
72.28 \\
72.30\end{array}$ & $\begin{array}{l}72.33 \\
72.35 \\
72.36 \\
72.37 \\
72.39\end{array}$ & $\begin{array}{l}73.42 \\
73.47 \\
73.52 \\
73.56 \\
73.56\end{array}$ & $\begin{array}{l}74.14 \\
74.16 \\
74.18 \\
74.24 \\
74.28\end{array}$ & $\begin{array}{l}74.90 \\
74.93 \\
74.94 \\
74.98 \\
75.02\end{array}$ & $\begin{array}{l}74.73 \\
74.74 \\
74.74 \\
74.74 \\
74.74\end{array}$ & $\begin{array}{l}74.75 \\
74.79 \\
74.78 \\
74.78 \\
74.75\end{array}$ & $\begin{array}{l}74.61 \\
74.63 \\
74.62 \\
74.63 \\
74.60\end{array}$ & 74. \\
\hline $\begin{array}{l}26 \\
27 \\
28 \\
29 \\
30 \\
31\end{array}$ & $\begin{array}{l}74.44 \\
74.49 \\
74.53 \\
74.57 \\
74.47 \\
74.43\end{array}$ & $\begin{array}{r}73.55 \\
73.47 \\
73.36 \\
-.- \\
--- \\
---\end{array}$ & $\begin{array}{l}72.67 \\
72.66 \\
72.66 \\
72.66 \\
72.58 \\
72.56\end{array}$ & $\begin{array}{r}72.32 \\
72.31 \\
72.27 \\
72.28 \\
72.26 \\
---\end{array}$ & $\begin{array}{l}72.44 \\
72.49 \\
72.45 \\
72.44 \\
72.45 \\
72.46\end{array}$ & $\begin{array}{r}73.51 \\
73.52 \\
73.59 \\
73.66 \\
73.72 \\
---\end{array}$ & $\begin{array}{l}74.31 \\
74.32 \\
74.34 \\
74.31 \\
74.33 \\
74.36\end{array}$ & $\begin{array}{l}75.04 \\
75.06 \\
75.08 \\
75.11 \\
75.12 \\
75.09\end{array}$ & $\begin{array}{r}74.76 \\
74.78 \\
74.78 \\
74.75 \\
74.70 \\
---\end{array}$ & $\begin{array}{l}74.73 \\
74.69 \\
74.72 \\
74.74 \\
74.76 \\
74.77\end{array}$ & $\begin{array}{r}74.58 \\
74.55 \\
74.53 \\
74.49 \\
74.46 \\
---\end{array}$ & 74. \\
\hline MEAN & 74.82 & 73.88 & 73.00 & 72.43 & 72.34 & 73.10 & 74.02 & 74.75 & 74.82 & 74.75 & 74.64 & 8 \\
\hline
\end{tabular}




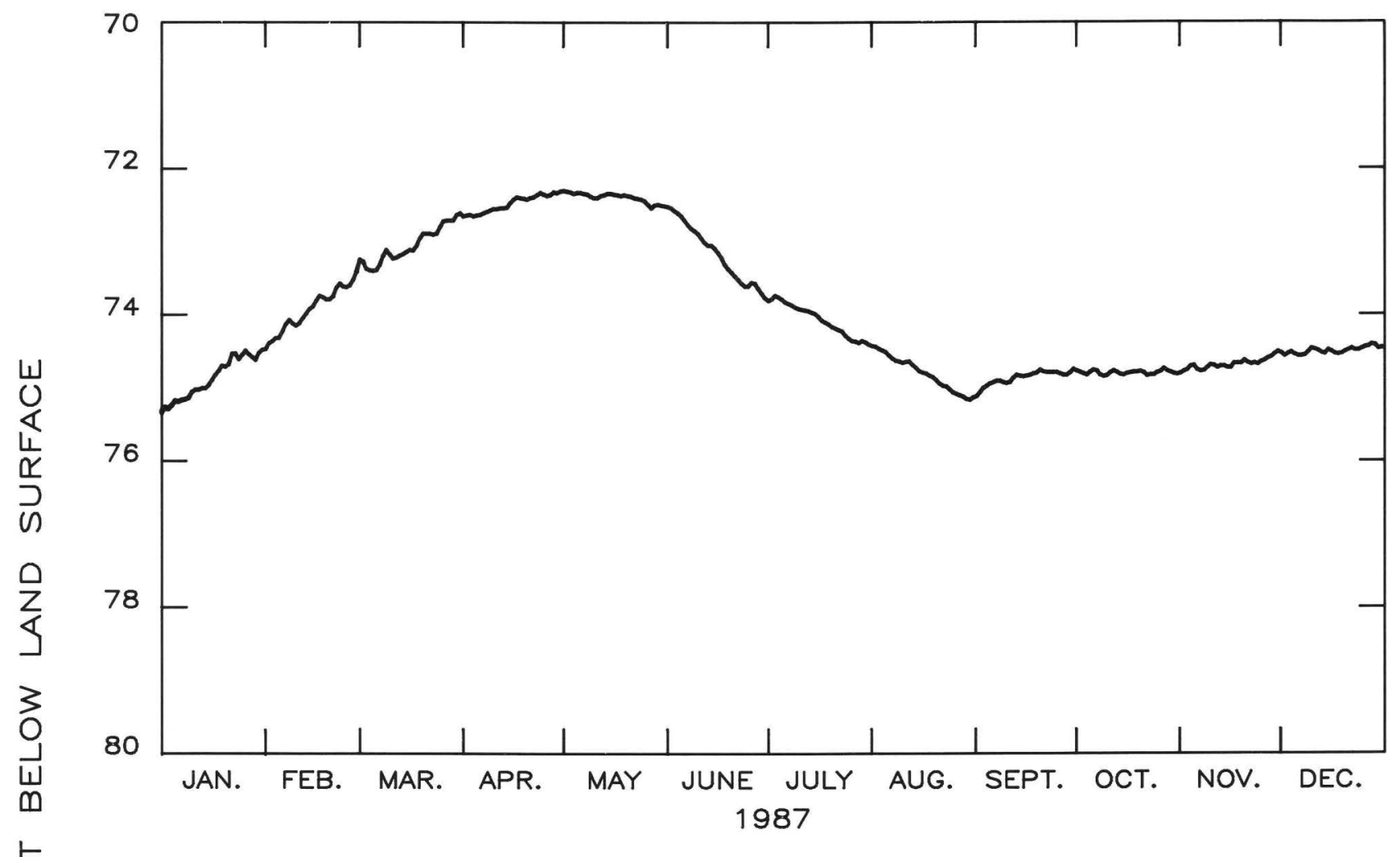

虫

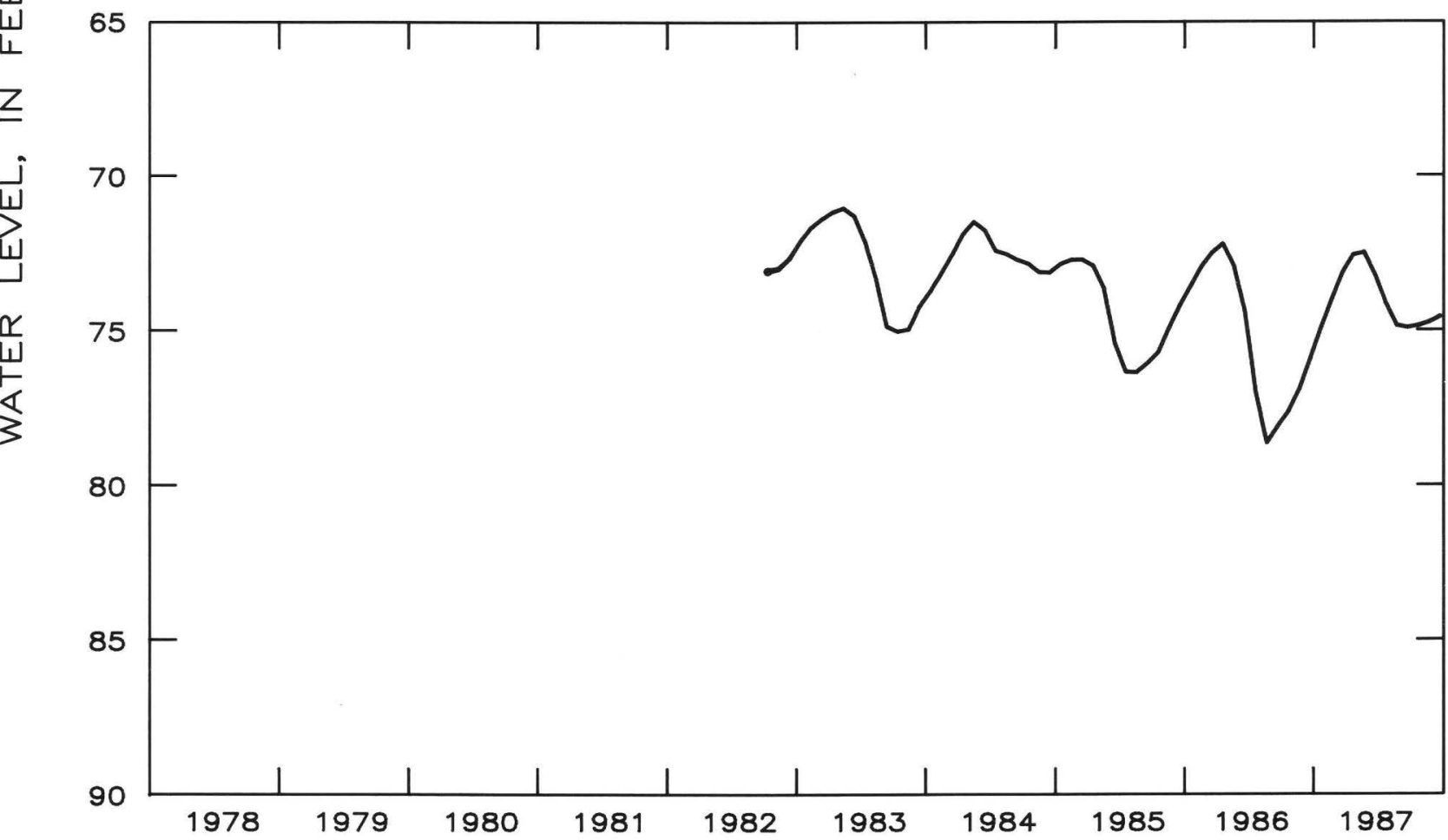

Figure 2.8-2.--Water level in observation well 314009, Bulloch County. 
313253081433503 , Local number, 32L016.

LOCATION.--Lat $31^{\circ} 32^{\prime} 53^{\prime \prime}$, long $81^{\circ} 43^{\prime} 35^{\prime \prime}$, Hydrologic Unit 03070106, $4.3 \mathrm{mi}$ east of Gardi Road, left onto dirt road $0.8 \mathrm{mi}$, well on right side of road.

Owner: Georgia Geologic Survey.

AQUIFER.--Miocene.

WELL CHARACTERISTICS.--Drilled observation well, diameter $4 \mathrm{in}$., depth $340 \mathrm{ft}$, cased to $320 \mathrm{ft}$, PVC screen 320 to $340 \mathrm{ft}$.

DATUM.--Elevation of land-surface datum is $74 \mathrm{ft}$.

Measuring point: Floor of recorder shelter, $4.0 \mathrm{ft}$ above land-surface datum.

REMARKS.--Well sounded April 26, 1983. Water level for period of missing record, January 19, was estimated. PERIOD OF RECORD.--June 1983 to current year.

EXTREMES FOR PERIOD OF RECORD.--Highest water level, $49.26 \mathrm{ft}$ below 1and-surface datum, March 20, 1984; 1owest, $53.43 \mathrm{ft}$ below land-surface datum, November $14,1986$.

DEPTH BELOW LAND SURFACE (WATER LEVEL) (FEET) CALENDAR YEAR JANUARY 1987 TO DECEMBER 1987 MEAN VALUES

\begin{tabular}{|c|c|c|c|c|c|c|c|c|c|c|c|}
\hline DAY & JAN & FEB & MAR & APR & MAY & JUN & JUL & AUG & SEP & OCT & NOV \\
\hline $\begin{array}{l}1 \\
2 \\
3 \\
4 \\
5\end{array}$ & $\begin{array}{l}52.14 \\
52.16 \\
52.19 \\
52.02 \\
51.89\end{array}$ & $\begin{array}{l}51.41 \\
51.33 \\
51.30 \\
51.35 \\
51.37\end{array}$ & $\begin{array}{l}51.64 \\
51.71 \\
51.77 \\
51.81 \\
51.86\end{array}$ & $\begin{array}{l}51.95 \\
51.96 \\
51.95 \\
51.97 \\
51.98\end{array}$ & $\begin{array}{l}52.76 \\
52.80 \\
52.87 \\
52.92 \\
52.98\end{array}$ & $\begin{array}{l}53.45 \\
53.46 \\
53.49 \\
53.49 \\
53.45\end{array}$ & $\begin{array}{l}53.49 \\
53.48 \\
53.46 \\
53.44 \\
53.44\end{array}$ & $\begin{array}{l}53.71 \\
53.71 \\
53.72 \\
53.74 \\
53.75\end{array}$ & $\begin{array}{l}53.81 \\
53.64 \\
53.59 \\
53.60 \\
53.57\end{array}$ & $\begin{array}{l}53.62 \\
53.66 \\
53.67 \\
53.71 \\
53.69\end{array}$ & $\begin{array}{l}53.94 \\
53.93 \\
53.91 \\
53.82 \\
53.78\end{array}$ \\
\hline $\begin{array}{r}6 \\
7 \\
8 \\
9 \\
10\end{array}$ & $\begin{array}{l}51.93 \\
51.91 \\
51.88 \\
51.85 \\
51.76\end{array}$ & $\begin{array}{l}51.29 \\
51.24 \\
51.25 \\
51.34 \\
51.42\end{array}$ & $\begin{array}{l}51.87 \\
51.76 \\
51.66 \\
51.62 \\
51.66\end{array}$ & $\begin{array}{l}51.99 \\
51.99 \\
51.99 \\
52.02 \\
52.03\end{array}$ & $\begin{array}{l}53.01 \\
53.01 \\
53.03 \\
53.12 \\
53.15\end{array}$ & $\begin{array}{l}53.49 \\
53.53 \\
53.55 \\
53.55 \\
53.56\end{array}$ & $\begin{array}{l}53.44 \\
53.44 \\
53.45 \\
53.45 \\
53.43\end{array}$ & $\begin{array}{l}53.78 \\
53.80 \\
53.82 \\
53.82 \\
53.82\end{array}$ & $\begin{array}{l}53.53 \\
53.49 \\
53.44 \\
53.45 \\
53.49\end{array}$ & $\begin{array}{l}53.66 \\
53.68 \\
53.74 \\
53.79 \\
53.79\end{array}$ & $\begin{array}{l}53.87 \\
53.91 \\
53.90 \\
53.87 \\
53.81\end{array}$ \\
\hline $\begin{array}{l}11 \\
12 \\
13 \\
14 \\
15\end{array}$ & $\begin{array}{l}51.76 \\
51.76 \\
51.74 \\
51.74 \\
51.70\end{array}$ & $\begin{array}{l}51.44 \\
51.43 \\
51.45 \\
51.47 \\
51.50\end{array}$ & $\begin{array}{l}51.70 \\
51.67 \\
51.63 \\
51.60 \\
51.58\end{array}$ & $\begin{array}{l}52.05 \\
52.07 \\
52.08 \\
52.09 \\
52.04\end{array}$ & $\begin{array}{l}53.17 \\
53.19 \\
53.20 \\
53.23 \\
53.23\end{array}$ & $\begin{array}{l}53.59 \\
53.60 \\
53.59 \\
53.56 \\
53.57\end{array}$ & $\begin{array}{l}53.43 \\
53.44 \\
53.43 \\
53.44 \\
53.46\end{array}$ & $\begin{array}{l}53.82 \\
53.83 \\
53.84 \\
53.79 \\
53.79\end{array}$ & $\begin{array}{l}53.50 \\
53.50 \\
53.49 \\
53.50 \\
53.52\end{array}$ & $\begin{array}{l}53.75 \\
53.71 \\
53.76 \\
53.80 \\
53.80\end{array}$ & $\begin{array}{l}53.85 \\
53.90 \\
53.88 \\
53.89 \\
53.92\end{array}$ \\
\hline $\begin{array}{l}16 \\
17 \\
18 \\
19 \\
20\end{array}$ & $\begin{array}{l}51.69 \\
51.67 \\
51.66 \\
51.70 \\
51.74\end{array}$ & $\begin{array}{l}51.44 \\
51.50 \\
51.56 \\
51.64 \\
51.68\end{array}$ & $\begin{array}{l}51.56 \\
51.58 \\
51.53 \\
51.51 \\
51.55\end{array}$ & $\begin{array}{l}52.03 \\
52.04 \\
52.10 \\
52.16 \\
52.24\end{array}$ & $\begin{array}{l}53.25 \\
53.29 \\
53.31 \\
53.31 \\
53.30\end{array}$ & $\begin{array}{l}53.58 \\
53.60 \\
53.62 \\
53.62 \\
53.61\end{array}$ & $\begin{array}{l}53.50 \\
53.54 \\
53.56 \\
53.59 \\
53.61\end{array}$ & $\begin{array}{l}53.79 \\
53.79 \\
53.80 \\
53.80 \\
53.81\end{array}$ & $\begin{array}{l}53.52 \\
53.52 \\
53.52 \\
53.52 \\
53.53\end{array}$ & $\begin{array}{l}53.80 \\
53.79 \\
53.80 \\
53.81 \\
53.81\end{array}$ & $\begin{array}{l}53.92 \\
53.87 \\
53.84 \\
53.84 \\
53.81\end{array}$ \\
\hline $\begin{array}{l}21 \\
22 \\
23 \\
24 \\
25\end{array}$ & $\begin{array}{l}51.69 \\
51.58 \\
51.68 \\
51.74 \\
51.66\end{array}$ & $\begin{array}{l}51.64 \\
51.58 \\
51.60 \\
51.68 \\
51.71\end{array}$ & $\begin{array}{l}51.57 \\
51.62 \\
51.70 \\
51.75 \\
51.74\end{array}$ & $\begin{array}{l}52.28 \\
52.32 \\
52.36 \\
52.40 \\
52.48\end{array}$ & $\begin{array}{l}53.28 \\
53.31 \\
53.32 \\
53.33 \\
53.34\end{array}$ & $\begin{array}{l}53.62 \\
53.58 \\
53.47 \\
53.46 \\
53.46\end{array}$ & $\begin{array}{l}53.63 \\
53.64 \\
53.65 \\
53.67 \\
53.68\end{array}$ & $\begin{array}{l}53.84 \\
53.87 \\
53.87 \\
53.86 \\
53.89\end{array}$ & $\begin{array}{l}53.56 \\
53.58 \\
53.59 \\
53.59 \\
53.60\end{array}$ & $\begin{array}{l}53.83 \\
53.89 \\
53.90 \\
53.90 \\
53.88\end{array}$ & $\begin{array}{l}53.86 \\
53.89 \\
53.90 \\
53.91 \\
53.88\end{array}$ \\
\hline $\begin{array}{l}26 \\
27 \\
28 \\
29 \\
30 \\
31\end{array}$ & $\begin{array}{l}51.63 \\
51.65 \\
51.63 \\
51.58 \\
51.48 \\
51.43\end{array}$ & $\begin{array}{r}51.72 \\
51.71 \\
51.68 \\
-.- \\
-.- \\
-.-\end{array}$ & $\begin{array}{l}51.78 \\
51.78 \\
51.84 \\
51.87 \\
51.82 \\
51.88\end{array}$ & $\begin{array}{r}52.56 \\
52.62 \\
52.63 \\
52.68 \\
52.72 \\
. .-\end{array}$ & $\begin{array}{l}53.37 \\
53.39 \\
53.41 \\
53.41 \\
53.41 \\
53.42\end{array}$ & $\begin{array}{r}53.45 \\
53.46 \\
53.49 \\
53.51 \\
53.51 \\
. .-\end{array}$ & $\begin{array}{l}53.68 \\
53.69 \\
53.69 \\
53.66 \\
53.69 \\
53.71\end{array}$ & $\begin{array}{l}53.91 \\
53.92 \\
53.92 \\
53.92 \\
53.92 \\
53.92\end{array}$ & $\begin{array}{r}53.63 \\
53.65 \\
53.67 \\
53.64 \\
53.59 \\
.-\end{array}$ & $\begin{array}{l}53.86 \\
53.84 \\
53.87 \\
53.91 \\
53.93 \\
53.94\end{array}$ & $\begin{array}{r}53.85 \\
53.84 \\
53.83 \\
53.80 \\
53.79 \\
.\end{array}$ \\
\hline MEAN & 51.76 & 51.49 & 51.70 & 52.19 & 53.20 & 53.53 & 53.55 & 53.82 & 53.56 & 53.79 & 53.87 \\
\hline
\end{tabular}

$\begin{array}{llllll}\text { CAL YR } 1987 \text { MEAN } 53.04 & \text { HIGH } & 51.24 & \text { LOW } & 53.97\end{array}$ 

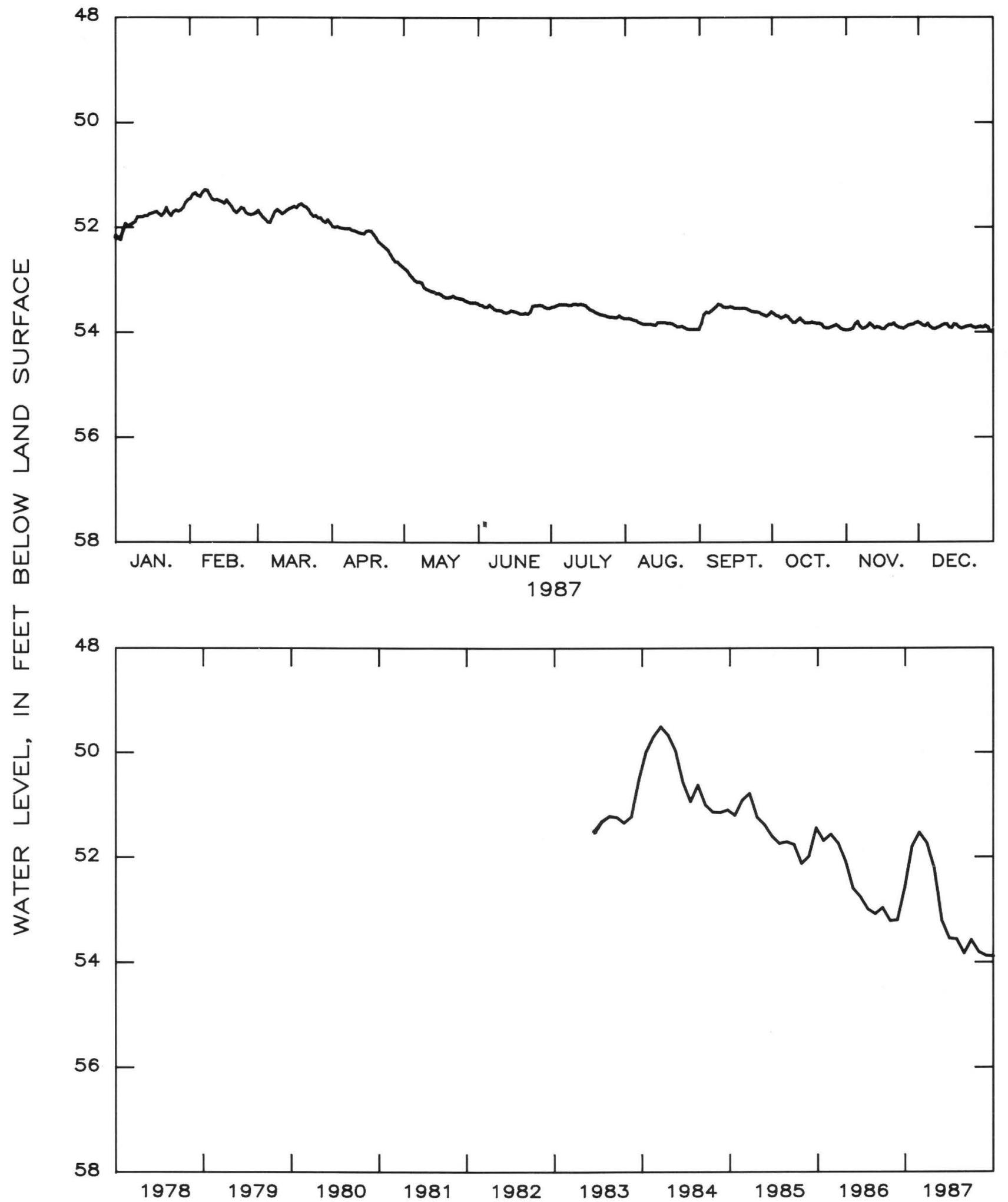

Figure 2.8-3.--Water level in observation well 32LO16, Wayne County. 


\subsection{GROUND-WATER QUALITY}

Water samples are collected periodically throughout Georgia and are analyzed as part of areal and regional ground-water studies. Wells along the coast have been monitored for chloride concentration since the late fifties. Chloride is indicative of saltwater contamination and is readily analyzed in the field. Selected wells in the water-level monitoring networks also are pumped and sampled periodically to note any changes in water quality that may occur in the various aquifers of the State.

Where water-quality problems are noted or are considered likely to occur, samples are collected more frequently and analyzed for water-quality constituents indicative of the problem. Streams also are sampled for water quality in those areas where the stream water recharges an aquifer. Ground-water pumping can induce water-quality problems that otherwise might not have occurred.

\subsection{Savannah area}

Saltwater has the potential to enter the Upper Floridan aquifer in the Savannah area by encroachment from the sea or by upconing (upward leakage) from deeper zones. Twelve wells in the Savannah area are pumped and sampled periodically to monitor changes in chloride concentration in the area. There has been no significant increase in chloride concentration in the wells monitored during the past 20 years. 


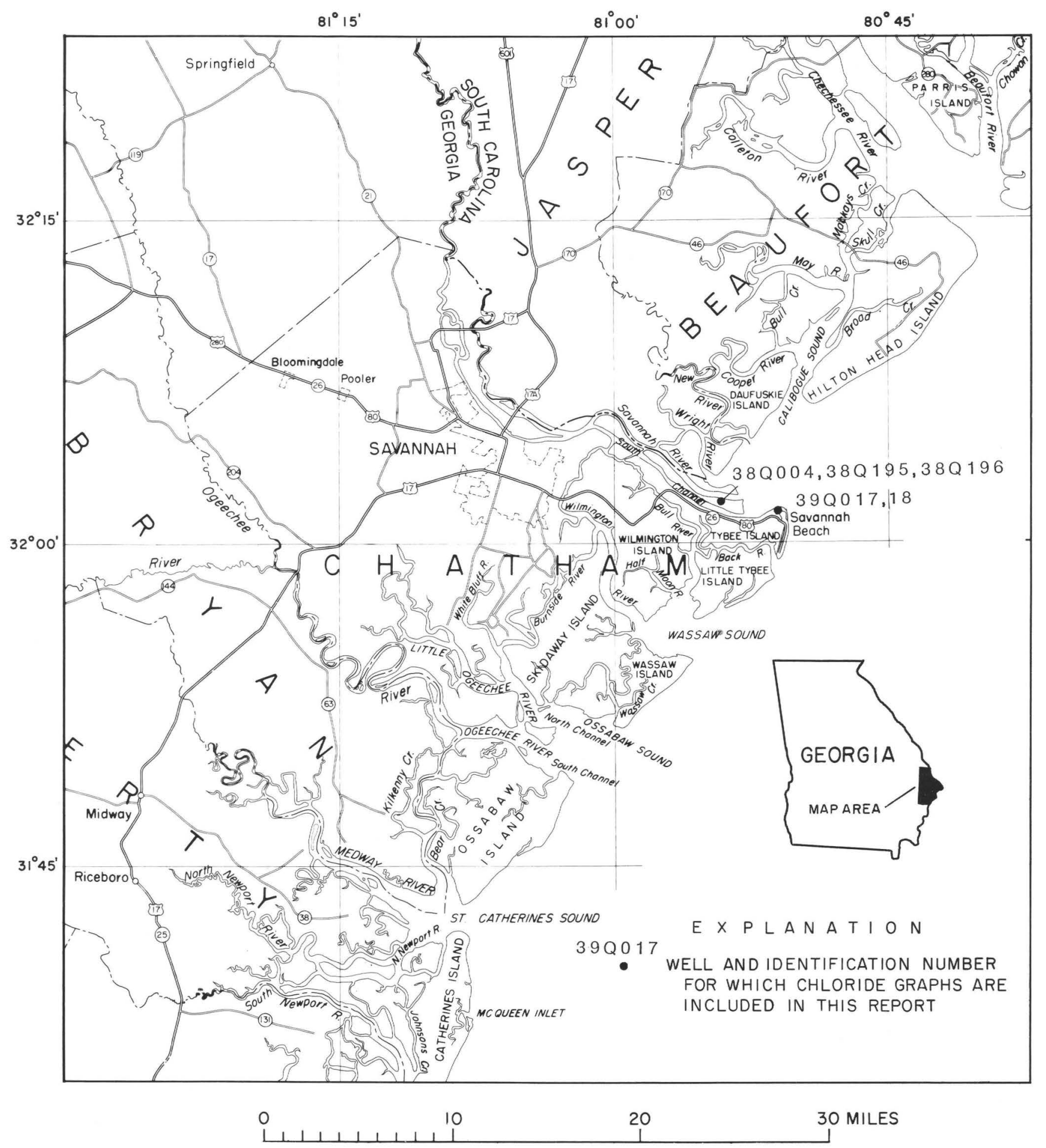

Figure 3.1-1.-Locations of chloride-monitoring wells in the Savannah area. 
Chloride concentration generally increases with depth in the Savannah area. During 1987, well 38Q004 (interval tapped, 606-657 ft) had a concentration of less than $200 \mathrm{mg} / \mathrm{L}$, and wells 390018 (interval tapped, 630-670 ft) and 39Q017 (interval tapped, 710-745 ft) had chloride concentrations of less than $900 \mathrm{mg} / \mathrm{L}$. Well 380196 (interval tapped, 870-925 ft) had a chloride concentration of about $5,300 \mathrm{mg} / \mathrm{L}$ in 1987 . A monitoring well was constructed in 1986 to replace an old monitoring well (38Q195) in which the casing had failed. The new monitoring well (38Q201, interval tapped, 1,358-1,546 ft) was constructed to tap a deep zone underlying the Floridan aquifer system. Analyses of samples collected during early 1987 indicated that the chloride concentration in the well was about $18,000 \mathrm{mg} /$ L (W.L. Stayton, U.S. Geological Survey, written commun., 1987). 


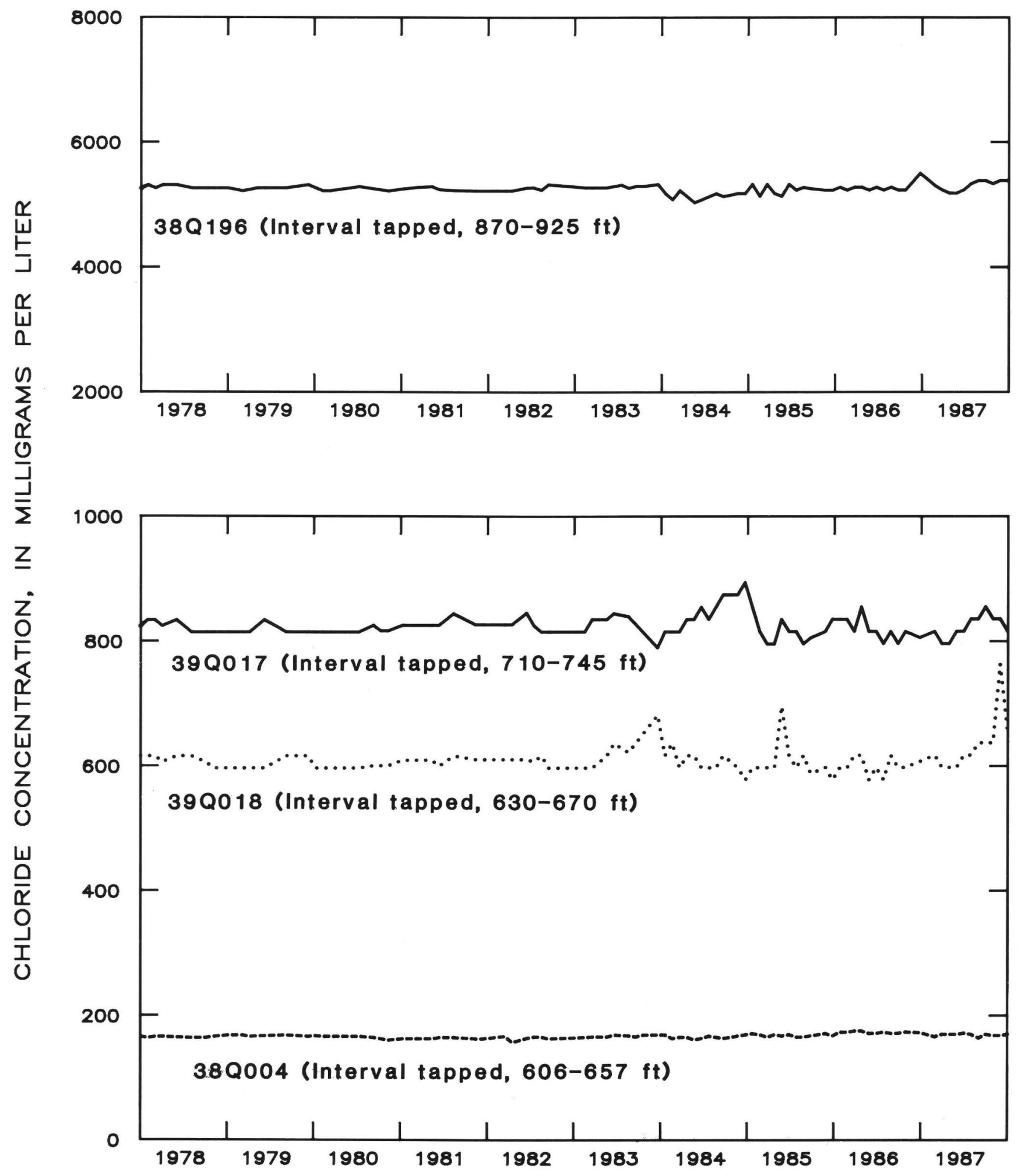

Figure 3.1-2.--Chloride concentrations in the Savannah area. 


\subsection{Brunswick Area}

In the Brunswick area, the Floridan aquifer system is divided into the Upper Floridan aquifer and the Lower Floridan aquifer. The Upper Floridan aquifer includes two freshwater-bearing zones: the upper water-bearing zone and the lower water-bearing zone described by Wait and Gregg (1973, p. 16) and by Gregg and Zimmerman (1974, p. D17 and p1. 1). The upper part of the Lower Floridan aquifer includes a zone of water that has a chloride concentration of about $6,000 \mathrm{mg} / \mathrm{L}$, which Gregg and Zimmerman (1974, p1. 1) referred to as the brackish-water zone. The lower part of the Lower Floridan aquifer is referred to as the Fernandina permeable zone (Krause and Randolph, 1985). In 1978, water in this zone had a chloride concentration of more than 20,000 mg/L (Gill and Mitchel1, 1979).

Since pumping began in the late 1800 's, ground-water withdrawal in the Brunswick area has lowered the water level in the Upper Floridan aquifer by as much as 25 to $65 \mathrm{ft}$. This water-level decline has allowed saltwater to migrate upward into the aquifer at three known locations in Brunswick, and also to move downgradient toward centers of pumping. Changes in chloride concentration may be attributed to shifting water-level gradients that alter the direction of chloride migration. About 80 wells in Glynn County, mostly in the Brunswick area, are pumped and sampled periodically for chloride analysis. At two locations in Brunswick, the chloride concentration in the upper water-bearing zone has risen to more than $2,000 \mathrm{mg} / \mathrm{L}$.

In the Bay Street area, the chloride-concentration distribution has been better defined owing to the addition of a new well to the chloride-monitoring network in 1987. As a result, the $1,500 \mathrm{mg} / \mathrm{L}$ line of equal chloride concentration extends further northward than previously interpreted. 


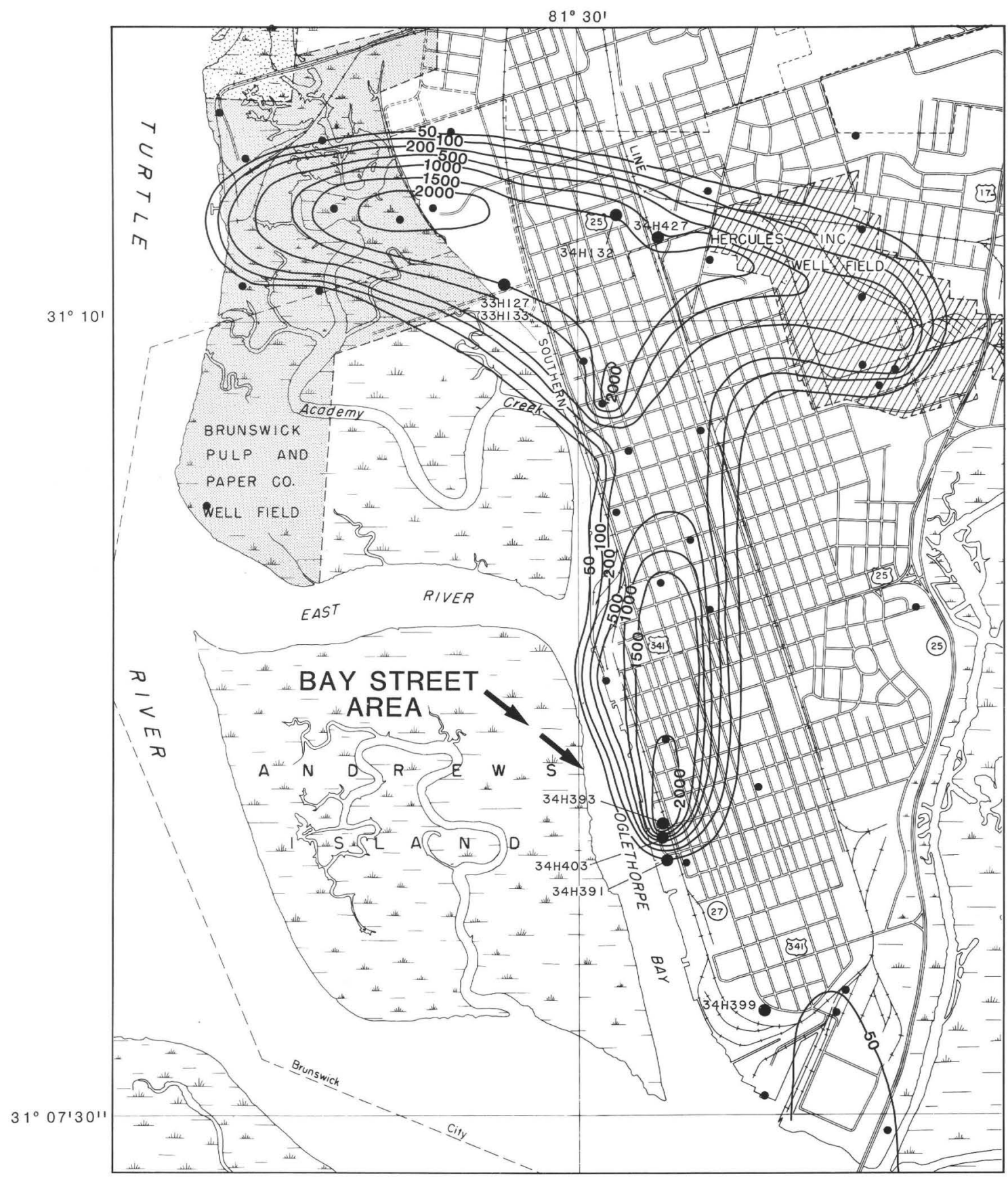

Base from U.S. Geological Survey

$1: 24,000$ quadrangles

0 2 MILES

EXPLANATION

-50-- LINE OF EQIJAL CHLORIDE CONCENTRATIONDashed where approximately located. Interval varies, in milligrams per liter
${ }^{34}{ }^{399}$ WELL FOR WHICH CHLORIDE GRAPHS ARE INCLUDED IN THIS REPORT

- DATA POINT

Figure 3.2-1.--Location of the chloride-monitoring wells and chloride concentrations in the upper water-bearing zone in the Brunswick area, October 1987. 
The chloride concentration in well 34H393, which taps the upper water-bearing zone of the Upper Floridan aquifer in the Bay Street area, has remained about the same since 1976. At the end of 1987, the chloride concentration in this well was $2,400 \mathrm{mg} / \mathrm{L}$. Well $34 \mathrm{H} 403$, which taps the lower waterbearing zone of the Upper Floridan aquifer, yields water that had a chloride concentration of about $1,400 \mathrm{mg} / \mathrm{L}$ at the end of 1987 , indicating a slight decline over recent years. The chloride concentration in well $34 \mathrm{H} 391$, which taps the brackish-water zone, has remained about the same since 1982 . At the end of 1987, water in this well had a chloride concentration of $2,400 \mathrm{mg} / \mathrm{L}$. Well $34 \mathrm{H} 399$ at the southern tip of the Brunswick Peninsula also taps the brackish-water zone. Chloride concentrations in this well continue to increase, and record high chloride concentration of $7,100 \mathrm{mg} / \mathrm{L}$ was found in 1987. 


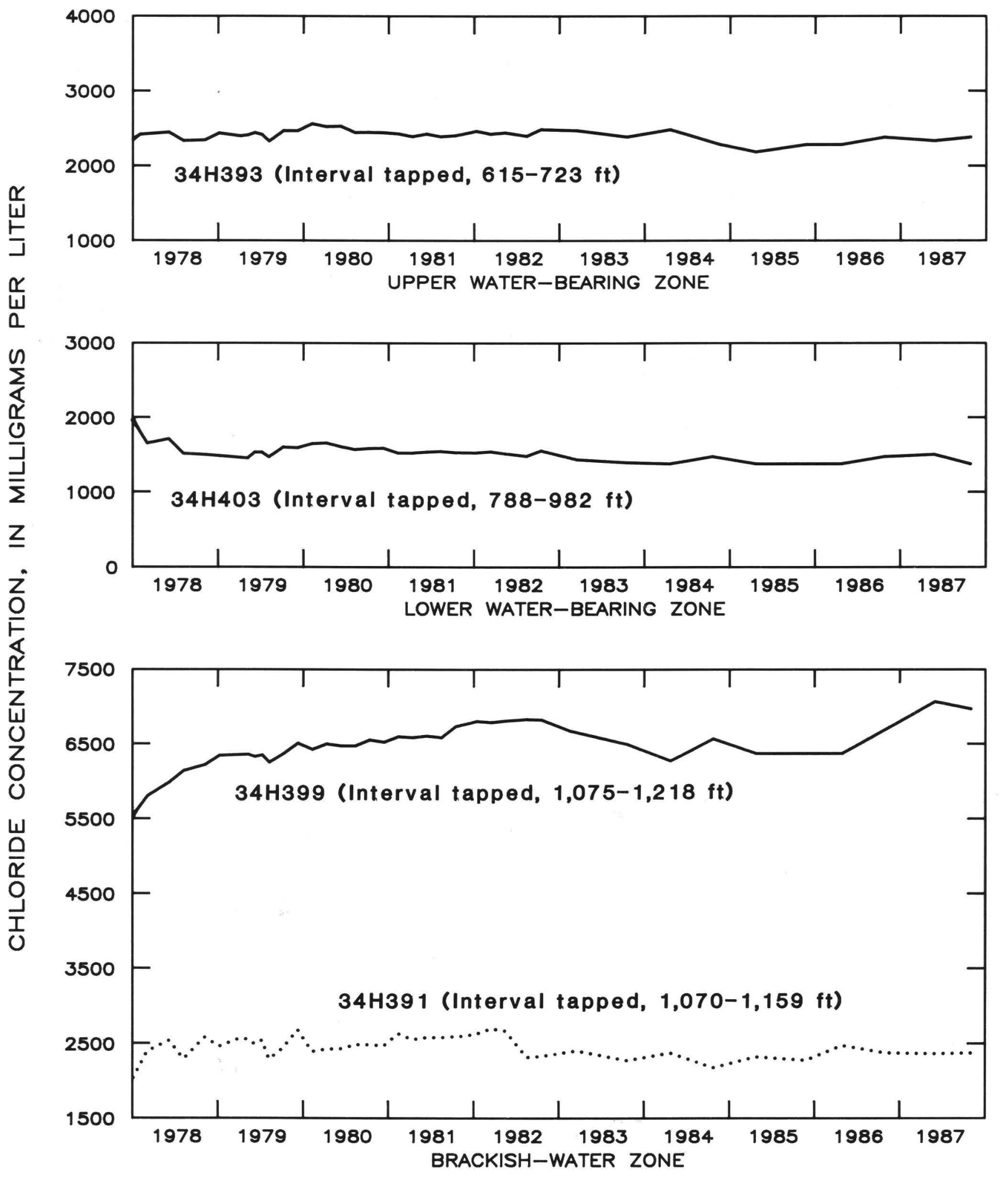

Figure 3.2-2.--Chloride concentrations in the Bay Street area of Brunswick. 
Chloride concentrations in the upper water-bearing zone in the northwestern part of Brunswick continue to increase. Well $33 \mathrm{H} 133$ had a record high chloride concentration of $1,520 \mathrm{mg} / \mathrm{L}$ in 1987 . Chloride concentrations found in the lower water-bearing zone remained about the same in 1987 as in 1986, as indicated by well $33 \mathrm{H} 127$, which had a concentration of $550 \mathrm{mg} / \mathrm{L}$ at the end of 1987.

In the northeastern part of Brunswick, the chloride concentration in well $34 \mathrm{H} 132$ continued to decrease, and in the fall of 1987, the concentration was $1,480 \mathrm{mg} / \mathrm{L}$. In well $34 \mathrm{H} 427$, the chloride concentration during 1987 remained at about $1,000 \mathrm{mg} / \mathrm{L}$, which indicated that the decreasing concentrations found since 1980 had level off. 


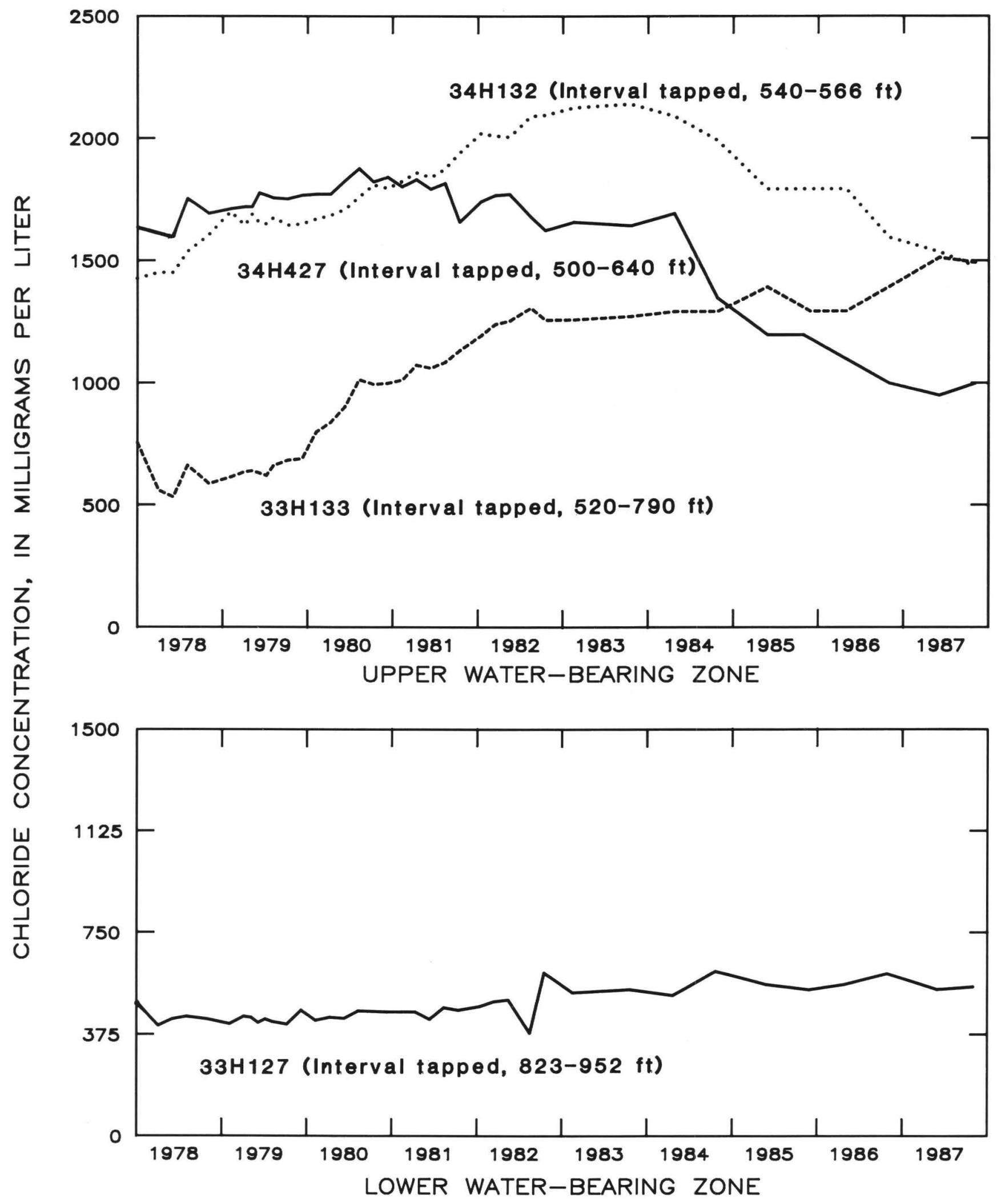

Figure 3.2-3.--Chloride concentrations in the north Brunswick area. 
Arora, Ram, editor, 1984, Hydrogeologic evaluation for underground injection control in the Coastal Plain of Georgia: Georgia Geologic Survey, Hydrologic Atlas 10, $45 \mathrm{p}$.

Brooks, Rebekah, Clarke, J.S., and Faye, R.E., 1985, Hydrogeology of the Gordon aquifer system of east-central Georgia: Georgia Geologic Survey Information Circular 75, $41 \mathrm{p}$.

Callahan, J.T., 1964, The yield of sedimentary aquifers of the Coastal Plain, Southeast River Basins: U.S. Geological Survey Water-Supply Paper 1669-W, $56 \mathrm{p}$.

Carter, R.F., 1983, Effects of the drought of 1980-81 on streamflow and on groundwater levels in Georgia: U.S. Geological Survey Water-Resources Investigations Report 83-4158, $46 \mathrm{p}$.

Clarke, J.S., 1987, Potentiometric surface of the Upper Floridan aquifer in Georgia, May 1985, and water-level trends, 1980-85: Georgia Geologic Survey Hydrologic Atlas 16, 1 sheet, scale 1:1,000,000.

Clarke, J.S., Brooks, Rebekah, and Faye, R.E., 1985a, Hydrogeology of the Dublin and Midville aquifer systems of east-central Georgia: Georgia Geologic Survey Information Circular 74, 62 p.

Clarke, J.S., Faye, R.E., and Brooks, Rebekah, 1983, Hydrogeology of the Providence aquifer of southwest Georgia: Georgia Geologic Survey Hydrologic Atlas 11,5 sheets.

----- 1984a, Hydrogeology of the Clayton aquifer of southwest Georgia: Georgia Geologic Survey Hydrologic Atlas 13, 6 sheets.

Clarke, J.S., Joiner, C.N., Longsworth, S.A., McFadden, K.W., and Peck, M.F., 1986, Ground-water data for Georgia, 1985: U.S. Geological Survey OpenFile Report 86-304, 159 p.

Clarke, J.S., Hester, W.G., and 0'Byrne, M.P., 1979, Ground-water levels and quality data for Georgia, 1978: U.S. Geological Survey Open-File Report $79-1290,94 \mathrm{p}$.

Clarke, J.S., Longsworth, S.A., Joiner, C.N., Peck, M.F., McFadden, K.W., and Milby, B.J., 1987, Ground-water data for Georgia, 1986: U.S. Geological Survey Open-File Report 87-376, 177 p.

Clarke, J.S., Longsworth, S.A., McFadden, K.W., and Peck, M.F., 1985b, GroundWater Data for Georgia, 1984: U.S. Geological Survey Open-File Report $85-331,150$ p.

Clarke, J.S., and McConnell, J.B., 1987, Georgia ground-water quality: U.S. Geological Survey Open-File Report 87-0720, 9 p. 


\section{SELECTED REFERENCES--Continued}

Clarke, J.S., Peck, M.F., Longsworth, S.A., and McFadden, K.W., 1984b, Groundwater data for Georgia, 1983: U.S. Geological Survey Open-File Report 84-605, $145 \mathrm{p}$.

Clarke, J.S., and Pierce, R.R., 1984, Ground-water resources of Georgia: Georgia Operator, v. 21 , no. 4, p. 10-39.

Counts, H.B., and Donsky, Ellis, 1963, Salt-water encroachment, geology, and ground-water resources of Savannah area, Georgia and South Carolina: U.S. Geological Survey Water-Supply Paper 1611, 100 p.

Cressler, C.W., Thurmond, C.J., and Hester, W.G., 1983, Ground water in the Greater Atlanta Region, Georgia: Georgia Geologic Survey Information Circular 63, 144 p.

Cressler, C.W., Franklin, M.A., and Hester, W.G., 1976, Availability of water supplies in northwest Georgia: Georgia Geological Survey Bulletin 91, $140 \mathrm{p}$.

Gill, H.E., Mitchel1, G.D., and Bisdorf, Robert, 1978, Saltwater encroachment in a carbonate aquifer system at Brunswick, Georgia [abs., Southeastern Section of the Geological Society of America, 28th annual meeting]: Abstracts with programs, 1979, v. 11, no. 4, p. 180.

Gill, H.E., and Mitchell, G.D., 1979, Results of Colonels Island deep hydrologic test well, in Investigations of alternative sources of ground water in the coastal area of Georgia: Georgia Geologic Survey Open-File Report $80-3$, p. C1-C13.

Gregg, D.0., and Zimmerman, E.A., 1974, Geologic and hydrologic control of chloride contamination in aquifers in Brunswick, Glynn County, Georgia: U.S. Geological Survey Water-Supply Paper 2029-D, 44 p.

Hayes, R.H., Maslia, M.L., and Meeks, W.C., 1983, Hydrology and model evaluation of the principal artesian aquifer, Dougherty Plain, southwest Georgia: Georgia Geologic Survey Bulletin 97, 93 p.

Hicks, D.W., Krause, R.E., and Clarke, J.S., 1981, Geohydrology of the Albany area, Georgia: Georgia Geologic Survey Information Circular 57, 31 .

Hicks, D.W., Gill, H.E., and Longsworth, S.A., 1987, Hydrogeology, chemical quality, and availability of ground water in the Upper Floridan aquifer, Albany area: U.S. Geological Survey Water-Resources Investigations Report $87-4145,52 \mathrm{p}$.

Johnston, R.H., Healy, H.G., and Hayes, L.R., 1981, Potentiometric surface of the Tertiary limestone aquifer system, Southeastern United States, May 1980: U.S. Geological Survey Open-File Report 81-486, 1 sheet. 


\section{SELECTED REFERENCES--Continued}

Krause, R.E., 1972, Effects of ground-water pumping in parts of Liberty and McIntosh Counties, Georgia, 1966-70: Georgia Geological Survey Information Circular 45, 15 p.

---- 1976, Occurrence and distribution of color and hydrogen sulfide in water from the principal artesian aquifer in the Valdosta area, Georgia: U.S. Geological Survey Open-File Report 76-378, 11 p.

-.--- 1979, Geohydrology of Brooks, Lowndes, and western Echols Counties, Georgia: U.S. Geological Survey Water-Resources Investigations 78-117, $48 \mathrm{p}$.

1982, Digital model evaluation of the predevelopment flow system of the Tertiary limestone aquifer, southeast Georgia, northeast Florida, and southern South Carolina: U.S. Geological Survey Water-Resources Investigations $82-173,27 \mathrm{p}$.

Krause, R.E., and Counts, H.B., 1975, Digital model analysis of the principal artesian aquifer, Glynn County, Georgia: U.S. Geological Survey WaterResources Investigations $1-75,4$ sheets.

Krause, R.E., and Gregg, D.0., 1972, Water from the principal artesian aquifer in coastal Georgia: Georgia Geological Survey Hydrologic Atlas 1, 1 sheet.

Krause, R.E., and Hayes, L.R., 1981, Potentiometric surface of the principal artesian aquifer in Georgia, May 1980: Georgia Geologic Survey Hydrologic Atlas 6, 1 sheet.

Krause, R.E., Matthews, S.E., and Gill, H.E., 1984, Evaluation of the groundwater resources of coastal Georgia--Preliminary report on the data available as of July 1983: Georgia Geologic Survey Information Circular 62, $55 \mathrm{p}$.

Krause, R.E., and Randolph, R.B., 1985, Hydrology of the Floridan aquifer system in southeast Georgia and adjacent parts of Florida and South Carolina: U.S. Geological Survey Professional Paper 1403-D (in press).

Marella, R., 1986, Annual water use survey: 1985: St. Johns River Water Management District, Technical Publication SJ 86-5, August 1986, 117 p.

McCollum, M.J., 1966, Ground-water resources and geology of Rockdale County, Georgia: Georgia Geological Survey Information Circular 33, 17 p.

McFadden, S.S., and Perriello, P.D., 1983, Hydrogeology of the Clayton and Claiborne aquifers in southwestern Georgia: Georgia Geologic Survey Information Circular 55, $59 \mathrm{p}$. 
Matthews, S.E., Hester, W.G., and McFadden, K.W., 1982, Ground-water data for Georgia, 1981: U.S. Geological Survey Open-File Report 82-904, 110 p.

Matthews, S.E., Hester, W.G., and O'Byrne, M.P., 1980, Ground-water data for Georgia, 1979: U.S. Geological Survey Open-File Report 80-501, 93 p.

-.-- 1981, Ground-water data for Georgia, 1980: U.S. Geological Survey OpenFile Report 81-1068, 94 p.

Miller, J.A., 1986, Hydrogeologic framework of the Floridan aquifer system in Florida and in parts of Georgia, South Carolina, and Alabama: U.S. Geological Survey Professional Paper 1403-B, 91 p.

Mitchel1, G.D., 1980, Potentiometric surface of the principal artesian aquifer in Georgia, November 1979: Georgia Geologic Survey Hydrologic Atlas 4, 1 sheet.

Pierce, R.R., and Barber, N.L., 1982, Water use in Georgia, 1980--Summary: Georgia Geologic Survey Circular 4-A, 17 p.

Pierce, R.R., Barber, N.L., and Stiles, H.R., 1982, Water use in Georgia by county for 1980: Georgia Geologic Survey Information Circular 59, 180 p.

Pollard, L.D., Grantham, R.G., and Blanchard, H.E., Jr., 1978, A preliminary appraisal of the impact of agriculture on ground-water availability in southwest Georgia: U.S. Geological Survey Water-Resources Investigations $79-7,22$ p.

Pollard, L.D., and Vorhis, R.C., 1979, Geohydrology of the Cretaceous aquifer system in Georgia: Georgia Geologic Survey Hydrologic Atlas 3, 5 sheets.

Sever, C.W., 1964, Geology and ground-water resources of crystalline rocks, Dawson County, Georgia: Georgia Geological Survey Information Circular $30,32 \mathrm{p}$.

Stiles, H.R., and Matthews, S.E., 1983, Ground-water data for Georgia, 1982: U.S. Geological Survey Open-File Report 83-678, 147 p.

Stringfield, V.T., 1966, Artesian water in Tertiary limestone in the Southeastern States: U.S. Geological Survey Professional Paper 517, 226 p.

Thomson, M.T., Herrick, S.M., Brown, Eugene, and others, 1956, Availability and use of water in Georgia: Georgia Geological Survey Bulletin 65, 329 p.

Turlington, M.C., Fanning, J.L., and Doonan, G.A., 1987, Water use in Georgia by county for 1985: Georgia Geologic Survey Information Circular 81, 110 p. 


\section{SELECTED REFERENCES--Continued}

U.S. Geological Survey, 1978, Ground-water levels and quality data for Georgia, 1977: U.S. Geological Survey Open-File Report 79-123, 88 p.

Watson, T.W., 1982, Aquifer potential of the shallow sediments of the coastal area of Georgia, in Proceedings of the Second Symposium on the Geology of the Southeastern Coastal Plain: Georgia Geologic Survey Information Circular 53, p. 183-194.

Wait, R.L., 1963, Geology and ground-water resources of Dougherty County Georgia: U.S. Geological Survey Water-Supply Paper 1539-P, 102 p.

1965, Geology and occurrence of fresh and brackish ground water in Glynn County, Georgia: U.S. Geological Survey Water-Supply Paper 1613-E, 89 p.

Wait, R.L., and Gregg, D.0., 1973, Hydrology and chloride contamination of the principal artesian aquifer in Glynn County, Georgia: Georgia Geological Survey Hydrologic Report 1, 93 p. 